\title{
Climate Impact of Solar Variability
}

Proceedings of a conference held at NASA Goddard Space Flight Center

Greenbelt, Maryland April 24-27, 1990

\section{NתSก}





\section{Climate Impact of Solar Variability}

Edited by

Kenneth H. Schatten and Albert Arking

Goddard Space Flight Center

Greenbelt, Maryland

Proceedings of a conference held at NASA Goddard Space Flight Center

Greenbelt, Maryland April 24-27, 1990

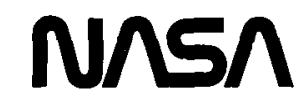

National Aeronautics and Space Administration

Office of Management

Scientific and Technical Information Division 



\section{Preface}

The conference on "The Climate Impact of Solar Variability" was held at Goddard Space Flight Center from April 24-27, 1990. It was intended as a sequel to a conference held a decade ago on "Variations of the Solar Constant" (see NASA Conference Publication 2191). At that time, there were only preliminary indications that the "solar constant" might not really be constant and thus could play a role in climate change. The "solar constant" is the common name for the total solar irradiance which, through the advent of spacecraft observations, was shown in the last decade to vary at a level on the order of $0.1 \%$.

In recent years there developed a renewed interest in the potential effects of increasing greenhouse gases on climate. Carbon dioxide, methane, nitrous oxide, and the chlorofluorocarbons have been increasing at rates that could significantly change climate. There is considerable uncertainty over the magnitude of this anthropogenic change for a number of reasons. One is that the climate system is very complex, with feedback processes that are not fully understood. A second reason is that there are two sources of natural climate variability (volcanic aerosols and solar variability) added to the anthropogenic changes which may confuse our interpretation of the observed temperature record. Thus, if we could understand the climatic impact of the natural variability, it would aid our interpretation and understanding of the manmade climate changes.

Approximately 90 scientists from around the world participated in this international conference which was run jointly by the Climate and Radiation Branch and the Solar Radiation Office of the Laboratory for Atmospheres. The present proceedings contain all of the papers or abstracts presented at the meeting; roughly 50 papers focused on solar activity, variations in the solar constant, climate--past, present and future, solar variability in the context of stellar variability, and correlations between terrestrial climate and solar activity.

\section{Executive Summary}

The conference was opened with the following comment and question. "There is worldwide concern about the global warming...can we answer or shed light on the question, 'What is the role of solar variability on global warming?" " Many of the talks addressed themselves to this question. The principal findings, which can be read about in more detail in this report, can be summarized as follows:

1) The solar irradiance can be modelled on daily to decadal time scales by examining the surface of the Sun for photospheric blemishes; e.g., sunspots and faculae, which affect the Sun's continuum radiative output. Many of the researchers who developed the models, observations, etc. that led to these understandings are represented at the conference. To mention but a few, the work of Willson, Hickey, Hudson, Frohlich, Chapman, Lean, Foukal, Hoyt, Eddy, Oster, Sofia, and others was instrumental in the development of our present understandings. The models provide two important contributions. First, they enable us to recognize the degree to which we understand present-day solar constant variations and second, the models may allow us to calculate past or future solar variability.

2) Other exotic solar variability factors (e.g., flares, coronal effects, etc.) have little influence upon the solar irrandiance variations. The solar ultraviolet variability (discussed in a number of conference papers), however, is an important contributor to solar irradiance variations, and unlike short-lived flares, cannot be ignored for two reasons. First, the variations are large and cause significant fluctuations in the solar constant, and second, the energy is deposited in the ozone layer where it may have a magnified 
impact on terrestrial climate. Our knowledge of solar UV variability has been furthered by the contributions of Brueckner, Rottman, Heath, Pap, Hood, Hinteregger, Lean, and Donnelly.

3) The secular variation in the solar constant has a solar cycle component, which is more or less in phase with solar activity, and although scientists may argue heatedly about the details--slight asymmetries or differences in this decadal variation from the solar cycle, it seems clear that the trend is primarily due to global faculae (from the network and other areas outside centers of activity). It should be kept in mind, however, that much of the network is the result of features which are the remnants of activity centers.

4) A number of models can be used to calculate the long-term trend in the solar constant (in the past or future) from these known variations induced by photospheric blemishes. These, however, do not include the less certain solar constant functions (induced by more global changes of the solar structure originating deep within the Sun, and/or influences from other non-local blemishes). This work was pioneered by Sofia, and furthered by Eddy, Endal, Gilliland, and others. These less well understood variations are likely important when we come to understand solar variations longer than 11 years.

5) The solar constant variations typically observed by spacecraft amount to less than $0.1 \%$ variability, however, it is possible that larger variations occur on time scales longer than the solar cycle. This would make it plausible that past changes in climate--e.g., the warm period in the 12th century, the cold 14th century, and the "little ice age" of the 17th century (which coincide with minima (cool periods) and maxima (warm periods) of the solar cycle amplitude) were induced by changes in solar irradiance.

6) Examining the problem (the impact of solar variability on climate) from the climate model viewpoint provides a different perspective. North reviewed aspects concerning the climate sensitivity. Typically, one finds that solar constant variations on the order of $0.5 \%$ over century time scales would be sufficient to explain the observed changes during the past millennium. If one could confirm that such solar constant changes caused the cold and warm spells of the past thousand years, then this would support the climate models which show that a $\mathrm{CO}_{2}$ doubling would cause a warming, larger in magnitude than the past changes (since a doubling of $\mathrm{CO}_{2}$ in the models is roughly equivalent to a $2 \%$ increase in the solar constant).

7) Viewed in the light of paleoclimate studies, Crowley reports there is only a "flickering relationship" between solar variability (indicated by ${ }^{14} \mathrm{C}$ and ${ }^{10} \mathrm{Be}$ ) and the climate, and the climate variations (indicated by alpine glaciers, tree rings, ice cores, corals, etc.). Further, a 1-1.5 C climate change requires a $0.5-1 \%$ variation in the solar constant. This is an order of magnitude more variation than has been observed from the recent satellite observations. This factor of 10 may be best viewed as a puzzle. What are the sources for these climate variations, and is there solar variability significantly larger than has been observed in the past decade? Interestingly, stellar variability may shed light on this question, which studies of the Sun alone cannot answer now, due to our limited experience with solar constant variations.

8) Studies by Baliunas, Lockwood, Skiff, Radick, Jastrow, and others of the magnetic activity in stars similar to the Sun, indicate that the recently observed solar activity behavior (active cycles and periods of dormance--e.g., the Maunder Minimum, etc.) with attendant variations in the solar constant, is typical of other stars, supporting the view that there may be a real association of reduced activity with "little ice ages." More exciting, however, are the findings of Lockwood and Skiff that the "recent solar variability may be unusual only in its present restraint." Coupled both with the generally increasing levels of solar activity that we have seen in the past 400 years, and the paleoclimate findings just alluded to, one wonders: What will solar activity do in the future? 
9) There is growing support for the idea that the Sun has been influencing the climate, and active researchers in these fields are narrowing down the extent of this influence. From this, we expect to provide better understandings (less uncertainty) concerning the anthropogenic influences upon the climate. Certainly, if we could establish the amount by which the solar constant varied in the past, we could obtain a high-quality estimate concerning the sensitivity of climate to solar radiative variations. Equally, this would enhance our ability to understand and predict the expected effects of varying greenhouse gases. A number of speakers at the conference added to the field of "Sun-climate connections"--Reid, Barnston, Hood, and others. It is important to distinguish the field of Sun-climate relationships from Sun-weather relationships, reviewed by Goldberg. A number of possible Sunweather effects have been reported, but at present, these are "statistical" in nature, and often they disappear with new data. A statistical relationship is not always associated with a physical relationship.

10) In addition to a review of the three main present-day climate forcings (greenhouse gases, volcanic aerosols, and solar activity variations), Jim Hansen pointed out that during the present epoch, all three are high. Greenhouse gases have been increasing; lately there has not been a large amount of volcanically induced stratospheric aerosols; and solar activity is near its peak in the solar cycle (which, additionally, has shown a general increase since the 17th century). Thus, Hansen predicts, "at least 1 year in the period 1990-1992 will be warmer than any year in the previous century." He states that this will "measurably exceed the already-high levels of the 1980s."

To conclude, it is an exciting time to study the blending of these two diverse fields (solar physics and climatology) which is needed to understand "the climate impact of solar variability." As the reader of this proceedings will discover, there is much now to report, and even more yet to be discovered!

We thank Luann Giese for technical help with the publication of this proceedings. We also appreciate the support of Marcy August during the conference. Special thanks go to Jack Eddy for his stimulating after-dinner talk on Langley, and to Vincent Salomonson for his closing remarks concerning the meeting.

K.H. Schatten
A. Arking

The chairpersons for the meeting were Drs. Albert Arking, Kenneth Schatten, and Robert Schiffer. Additionally, members of the organizing committee were Richard Donnelly, Jack Eddy, Claus Frohlich, James Hansen, Robert Kandel, Gerald North, V. Ramanathan, Sabatino Sofia, and T.M. Wigley. 
NASA-Goddard Space Flight Center

April 24-27, 1990

Page

SESSION 1: SUN-CLIMATE OVERVIEW I

Chair: Sabatino Sofia $\ldots \ldots \ldots \ldots \ldots \ldots \ldots \ldots \ldots \ldots \ldots \ldots \ldots \ldots \ldots$

The History of Sun-Climate Relationships

John A. Eddy . . . . . . . . . . . . . . . . . . . . . . . . . . .

SESSION 2: THE SUN AS A STAR

Chair: Sabatino Sofia

Studies of Solar Type Stars

Sallie Baliunas and Robert Jastrow

Some Insights on Solar Variability from Precision Stellar Astronomical Photometry

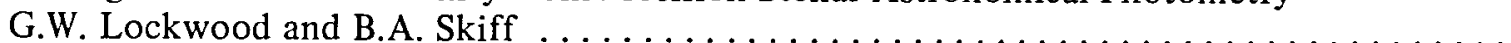

A Program of Photometric Measurements of Solar Irradiance Fluctuations from

Ground-Based Observations

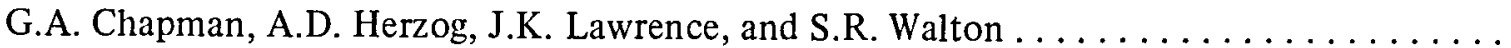

SESSION 3: "THE MONSTER" IN THE SUN-EFFECTS OF INTERNAL FIELDS AND SOLAR ACTIVITY

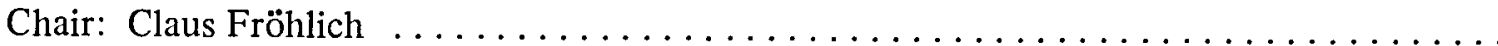

Basic Mechanisms of Solar Variability

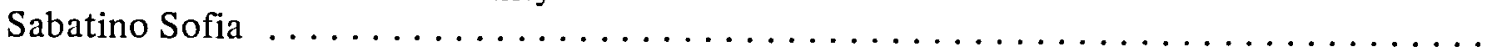

Global Models of Intermediate Timescale Variability on the Sun

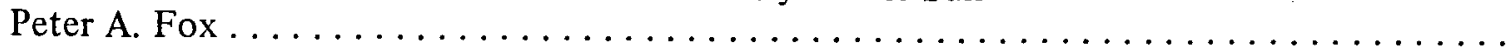

An Estimate of Changes in the Sun's Total Irradiance Caused by UV Irradiance

Variations from 1874 to 1988

J. Lean . . . . . . . . . . . . . . . . . . . . . . . . . . . .

Using the Boundary Conditions of Sunspots as a Technique for Monitoring Solar

Luminosity Variations

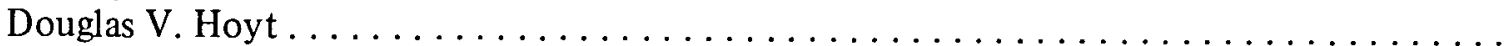

Energy Transport below Sunspots and Faculae

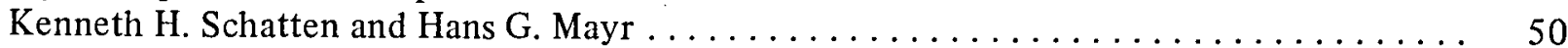

Solar Irradiance Observed from PVO and Inferred Solar Rotation

Charles L. Wolff and Walter R. Hoegy

A Correlation between Changes in Solar Luminosity and Differential Radius

Measurements

R.J. Kroll, H.A. Hill, and B.J. Beardsley .

Solar Response to Luminosity Variations

S. Arendt

SESSION 4: LONG-TERM SOLAR VARIABILITY AND CLIMATE

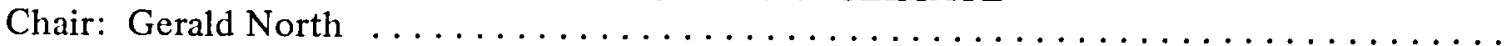

Testing the Sun-Climate Connection with Paleoclimate Data

Thomas J. Crowley and Matthew K. Howard

Sensitivity of the Oceanic Turbulent Boundary Layer to Cyclic Insolation Change with

Response Periods of 23 to $2.5 \mathrm{Ky}$ : An Equatorial Atlantic Record for the Last $200 \mathrm{Ka}$

Andrew McIntyre . 
A Probable $\sim 2400$ Solar Quasi-Cycle in Atmospheric $\Delta^{14} \mathrm{C}$

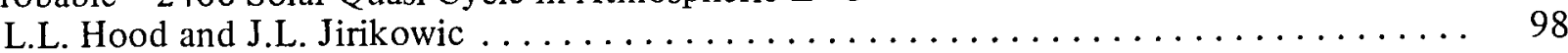

Is the Sun A Long-Period Variable?

Charles P. Sonett ............................... 106

Trends in Solar Variability

Robert Jastow and Sallie Baliunas $\ldots \ldots \ldots \ldots \ldots \ldots \ldots \ldots \ldots \ldots \ldots \ldots \ldots$

The Program Radiative Inputs of the Sun to the Earth (RISE)

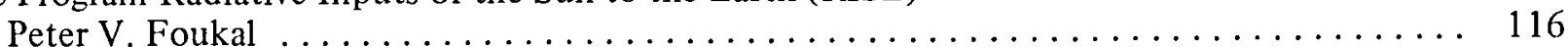

Solar Astronomy Data Base: Packaged Information on Diskette

John A. McKinnon . . . . . . . . . . . . . . . . . . . . . . . .

NOAA/TIROS and GOES Observations of Galactic and Solar Cosmic Rays Over a

Solar Cycle

Herbert H. Sauer . . . . . . . . . . . . . . . . . . . . . . . . . .

Dynamic Power Spectral Analysis of Solar Measurements from Photospheric,

Chromospheric, and Coronal Sources

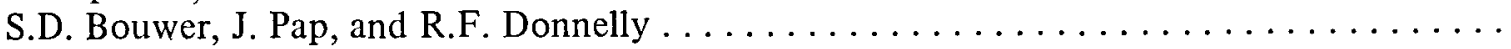

SESSION 5: CLIMATE CONNECTIONS I

Chair: Albert Arking . . . . . . . . . . . . . . . . . . . . . . . . .

Comparison of Solar and Other Influences on Long-Term Climate

James E. Hansen, Andrew A. Lacis, and Reto A. Ruedy . . . . . . . . . . . . . .

Solar Activity and the Sea-Surface Temperature Record-Evidence of a Long-Period

Variation in Solar Total Irradiance?

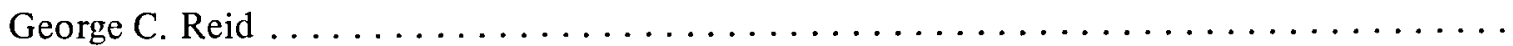

January-February Tropospheric Climate for the Northern Hemisphere and the 11-Year

Solar Cycle, the QBO and the Southern Oscillation

Anthony G. Barnston and Robert E. Livezey . . . . . . . . . . . . . . . . .

A Mechanism Involving Solar Ultraviolet Variations for Modulating the Interannual

Climatology of the Middle Atmosphere

L.L. Hood and J.L. Jirikowic ...........

135

146

149

164

SESSION 6: MODELLING SOLAR VARIABILITY

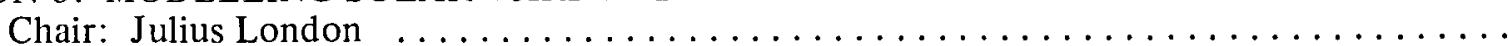

A Solar Constant Model for Sun-Climate Studies: 1600-2000 AD

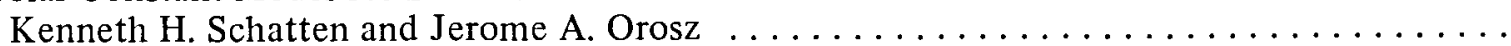

A Solar-Luminosity Model and Climate

Charles A. Perry . . . . . . . . . . . . . . . . . . . . . . . . . . .

Modeling Solar Lyman Alpha Irradiance

J.Pap, H.S. Hudson, G.J. Rottman, R.C. Willson, R.F. Donnelly, and J. London . . . . . . .

Solar Cycle Modulation of Total Irradiance: An Empirical Model from 1874 to 1988

J. Lean and P. Foukal . . . . . . . . . . . . . . . . . . . . . . . . .

SESSION 7: CLIMATE CONNECTIONS II

Chair: Richard Goldberg . . . . . . . . . . . . . . . . . . . . . . .

Climate Fluctuations and Climate Sensitivity

Gerald R. North and Kuor-Jier Joseph Yip . . . . . . . . . . . . . . . . .

Feedback Processes and Climate Response

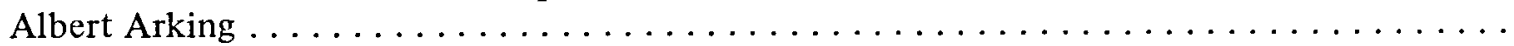

On the Decadal Modes of Oscillation of an Idealized Ocean-Atmosphere System

Vikram M. Mehta . . . . . . . . . . . . . . . . . . . . . . . .

What Could Be Causing Global Ozone Depletion?

S. Fred Singer . 
On the Variability of the Solar Integral Radiation Constituents

G.A. Nikolsky

Forcing of Climate Variations by MeV-GeV Particles?

Brian A. Tinsley

Relationship between Rainfall in the Northern Hemisphere and Impulses

of the Torque in the Sun's Motion

T. Landscheidt

\section{SESSION 8: SOLAR IRRADIANCE VARIATIONS I}

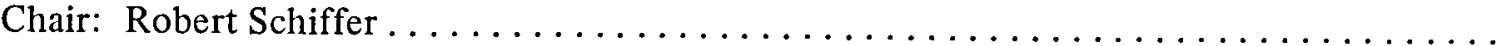

Irradiance Variability of the Sun

Claus Fröhlich

Solar Irradiance Variability During Solar Cycles 21 and 22

Richard C. Willson

The ACRIM Data in the Context of Stellar Variability

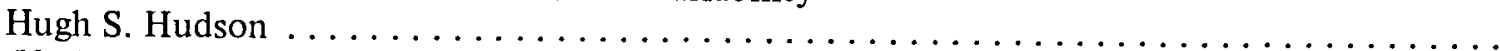

The Variation of Solar Fe XIV and Fe X Flux Over 1.5 Solar Activity Cycles

Richard C. Altrock

An Alternative Derivation of the NIMBUS 7 Total Solar Irradiance Variations

Douglas V. Hoyt and H. Lee Kyle

Long-Term Solar Irradiance Variability: 1984-1989 Observations

Robert B. Lee III

Solar Constant Data from Earth Radiation Budget Measurements

A.T. Mecherikunnel, H.L. Kyle, and R.B. Lee III

\section{SESSION 9: SUN-CLIMATE OVERVIEW II}

Chair: Richard Donnelly

A Review and Reflections on the Sun-Climate Connection

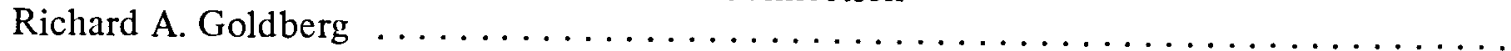

SESSION 10: SOLAR IRRADIANCE VARIATIONS II

Chair: Richard Donnelly

Wavelength Dependence of Solar Rotation and Solar Cycle UV Irradiance Variations

Julius London and Gary J. Rottman

Solar UV Temporal Variations during Solar Cycle 22 and the Twentieth Century

Richard F. Donnelly

Secular Change in Equivalent Width of C 5380, 1978-90

William C. Livingston

Ten Years of Solar Change as Monitored by SBUV and SBUV/2

B.M. Schlesinger, R.P. Cebula, D.F. Heath, M.T. DeLand, and R.D. Hudson

PAPERS ACCEPTED BUT NOT PRESENTED

Some Results from Studies on Relationships between the Optical-Meteorological Param-

eters and Solar Activity. 2. Development of the Problem of Solar Forcing

K.Y. Kondratyev and G.A. Nikolsky

POSTER PAPERS

Speculation on a Solar Chronometer for Climate

Charles A. Perry

Tracking Atmospheric Trace Gases by Spectroscopy from Kitt Peak

Lloyd Wallace and William Livingston

APPENDIX A

Conference Attendees 


\section{SESSION 1: SUN-CLIMATE OVERVIEW I}





\title{
THE HISTORY OF SUN-CLIMATE RELATIONSHIPS
}

\author{
John A. Eddy \\ Office for Interdisciplinary Earth Studies \\ University Corporation for Atmospheric Research \\ Boulder, CO 90307
}

Attempts to connect solar variations with the variability of terrestrial climate have enjoyed a long history, and predate the discovery of the 11-year solar cycle: William Herschel felt that a convincing case was already in hand in 1801, 40 years before there was any evidence of regular solar variations. Nearly every discovery regarding the sun since Herschel's day has been in the direction of greater solar inconstancy, yet the connection with weather or climate has remained elusive and controversial. A part of this apparent paradox can be explained by the parallel evolution of atmospheric science, in which nearly every discovery has emphasized a greater complexity of the atmosphere and its responses, discounting simpler cause and effect relations. Through the years, climatologists have been reluctant to accept proffered theses of significant solar perturbations, and particularly without the buttress of quantitative forcing factors involving energies that are appreciable in terms of the dynamics of the lower atmosphere, or demonstrable mechanisms that might amplify lesser perturbations to compete with intrinsic and more energetic forcing factors. Several recent developments promise to change the light in which solar variations are seen. The first is the availability of hard data on the varying solar inputs to the earth, specifically the more energetic photon fluxes now established in total irradiance and in the intermediate ultraviolet. Another is the quickening pace of understanding of the workings of the upper and middle atmosphere, where most of the known solar variations expend their force. A third is the ascendance of an earth system science that erodes the walls that have separated conventional meteorology from adjoining disciplines and that will demand more information on solar inputs, whether or not they are climatically dominant. Last is the growing level of public and policy attention, worldwide, to issues of climate change, which, though focused on impending greenhouse warming, will necessarily exact a deeper understanding of any factor which could diminish or enhance the anticipated effects of increased levels of radiatively important trace gases. 
SESSION 2: THE SUN AS A STAR 



\title{
STUDIES OF SOLAR TYPE STARS
}

\author{
Sallie Baliunas, Harvard-Smithsonian Center for Astrophysics \\ and Dartmouth College \\ and \\ Robert Jastrow, Dartmouth College
}

Satellite observations show a change of $0.1 \%$ in solar irradiance over the past decade. The question arises as to whether larger changes in irradiance may occur over century time scales. We have approached this question by looking at changes in surface magnetism, since the satellite observations also show that irradiance and surface magnetism are correlated.

To obtain information on possible variations in surface magnetism over century-long intervals, we have looked at records of surface magnetism spanning up to 20 years for a sample of 74 stars of solar type, i.e., of similar age and mass to the sun. The histories of these stars, observed in random phases of their long-term variability, give a snapshot at any one time of the behavior of a solar-type star over long periods of time. Evidence on Maunder Minimum phases and levels of magnetic activity in these solar-type stars will be compared with information on solar magnetism. Implications for solar irradiance changes will be discussed. 


\title{
SOME INSIGHTS ON SOLAR VARIABILITY FROM PRECISION STELLAR ASTRONOMICAL PHOTOMETRY
}

\author{
G. W. Lockwood and B. A. Skiff \\ Lowell Observatory \\ 1400 W. Mars Hill Road, Flagstaff, Arizona 86001
}

\begin{abstract}
At Lowell Observatory, precision photoelectric photometry was directed toward the study of solar variability and the variability of sun-like stars beginning in 1949 and continuing until now. The ubiquity and range of low-level variability, including some that appears to be cyclic, among solar-type stars shows that recent solar variability may be unusual only in its present restraint.
\end{abstract}

\section{SOLAR VARIABILITY STUDIES AT LOWELL OBSERVATORY}

Astronomers at Lowell Observatory have been studying solar variability for more than forty years. A 1955 report, The Study of the Variability of Solar Energy Output from 1949 to 1954 by Differential Methods, describes the application of photoelectric photometry to the study of solar variability. The aim of the Lowell program was to measure solar variability indirectly by comparing the brightness of reflected sunlight from the planets Uranus and Neptune with that of nearby "constant" sun-like comparison stars. By working differentially, they hoped to avoid the well-known limitations of absolute solar radiometry that had plagued Charles Abbott in his pioneering long-term studies of the solar constant. Despite the limitations of their primitive equipment, the Lowell observers produced a significant early result: the Sun was constant to $1 \%$ or better over a five-year interval (1).

A 1966 report, The Sun as a Variable Star, was inconclusive: changes in the brightness of Neptune, less than $3 \%$ overall from 1950 to 1966, could have been caused by changes in Neptune's atmosphere, by solar variability, or by both (2). However, there was a significant by-product resulting from repeated measurements of a small group of sun-like stars, called the "10-year standards," from 1955 to 1966:

...this long series of photoelectric measurements has taught us more about the variations of solar-type stars than about the Sun itself. The observations of 15 stars of spectral types $F$ and $G$ in the years 1955-1966 indicate that for none of these stars does the standard deviation of the yearly mean magnitudes exceed 0.008 [0.8\%] and for [three stars] this deviation is less than 0.004 [0.4\%]. No evidence of variability in the stars which are similar to the Sun has been detected during this program. If we assume the Sun acts in similar fashion to each of these stars, its variability over a fifteen-year period probably does not exceed one-half of one percent.

\section{RECENT SOLAR VARIABILITY}

With the 1980 spacecraft measurements of small dips in the solar "constant" corresponding to the passage of large sunspot groups across the face of the Sun, the existence of solar variability was finally established (3) and led, incorrectly, to an irradiance variability model dominated by sunspot blockage (4). As solar minimum approached, however, the total irradiance decreased by $0.1 \%$ rather than increasing as expected, showing that the excess flux from bright faculae overcomes the flux deficits in sunspots to produce a long-term irradiance variation in phase with solar activity (5).

A new model of solar irradiance variations based on the observed variability in cycle 21 estimates the variation of the Sun's output back to 1874 (6). Surprisingly, because of the unusual ratio of sunspot area to sunspot number in cycle 21, that cycle produced the largest variation in the past 100 years. However, because of the uncertainties in the model, arising especially from the short timebase of the regressions upon which it was based, secular variations as large as $1 \%$ 
over the last century are not ruled out.

We will show below that if the long-term variability of solar irradiance is always as small as the $0.1 \%$ amplitude recorded over the last decade, then the Sun is unusually stable compared with its stellar age, mass, and temperature cohort.

\section{LONG-TERM STELLAR STUDIES}

\section{a) Variations of Young Solar-Type Stars in the Hyades Open Cluster}

Nearly a decade ago, we began measuring the variability of ordinary F-, G-, and K-type dwarf stars similar to the Sun. Since the precision of stellar photoelectric photometry as commonly practiced is normally no better than $1 \%$ or so, extreme care was required to do much better. Using differential methods, we routinely attained a precision of $0.2-0.3 \%$ $r m s$ from night to night and, depending mainly on the intrinsic stability of comparison stars, sometimes as good as $0.1 \%$ from year to year.

The first detection of variations came from measurements of young solar-type stars in the Hyades open cluster (7). With ten seasons of observation of the Hyades stars now completed, two at the Cloudcroft Observatory followed by eight at Lowell, we determined that nearly all the stars cooler than spectral type F8 are slightly variable from day to day and from year to year (8). Brightness variations, typically with amplitudes of $1 \%$ or more, result from the stars' rotation and have allowed us to determine rotation periods for 23 stars.

A comparison of the photometric time series with the near-simultaneous measurements of chromospheric emission in the $\mathrm{Ca} \mathrm{II} \mathrm{H}$ and $\mathrm{K}$ lines made by the Mount Wilson Observatory "HK project" showed that enhanced HK fluxes coincide with the temporary dimming of starlight caused by the disk transit of starspots. Thus, spots and active regions are associated, just like on the Sun. The amplitudes of the rotational lightcurves change from year to year, indicating evolution of the spot coverage. Larger rotational amplitudes correspond to fainter average magnitudes and vice versa. In young stars, the irradiance budget is evidently dominated by spots rather than by bright faculae.

Figure 1 shows the 10-year light curve of one of the Hyades stars, van Bueren 64, a G2 dwarf with a photometric rotation period of 8 days. This star, which is often cited as one of the best spectrophotometric solar analog stars known (9), may have a long-term cycle with an amplitude of about $3 \%$ as indicated by the smoothing spline fit to the data.

\section{b) Luminosity Variations of Ordinary Solar-Type Stars}

Olin Wilson began the renowned Mount Wilson "HK project" in 1966, using the 100-inch telescope to monitor chromospheric emission in 91 solar-type dwarf and subgiant stars. By the time he stopped observing in 1977, he had discovered stellar activity cycles reminiscent of the 11-year sunspot cycle (10). This program continued, using the 60-inch telescope, and showed nearly a decade ago that the younger stars display strong rotational modulation of the HK index indicating the presence of nonuniformly distributed active regions (11). Older, more slowly rotating stars, monitored for nearly 20 years now, tend to be fairly quiescent on rotational timescales but undergo sunlike activity cycles (12).

Since 1984 we have been monitoring the brightness variations of 33 of these "Wilson stars" using the $b(472 \mathrm{~nm})$ and $y$ $(551 \mathrm{~nm})$ filters of the Strömgren photometric system. The goal of the photometry, as in the Lowell program a quarter century earlier, was to determine if ordinary field F-, G-, and K-type main-sequence stars, especially those of solar age, vary detectably, and if so, how that variability compares with solar irradiance variations. Parallel HK observations continue to be made at Mount Wilson.

To attain the highest possible photometric precision, the stars are observed differentially, grouped into trios and quartets containing nearby solar-type field comparison stars. Details of the methodology and complete results through the first four seasons are given in (13). The nightly $m s$ dispersion of the differential magnitudes is typically $0.2-0.3 \%$, depending mainly upon the stability of the comparison stars (whose constancy must, of course, be determined at the same time during the course of the program--a major complication). Over a single season the standard error of the mean approaches $0.1 \%$. Thus, the observations are precise enough to show stellar variability not much larger than that observed for the Sun in cycle 21. 
In the regime of photometric variation below $1 \%$, now accessible through careful differential photometry, many stars previously thought to be stable turn out to vary slightly. As an unexpected by-product of the unusually high precision attained in this program, we have detected low-level brightness variations in several well-known photometric standard stars. Of the more than $100 \mathrm{~F}, \mathrm{G}$, and $\mathrm{K}$ type stars observed since 1984, including the $33 \mathrm{Wilson}$ stars and their nearby comparison stars, one-third varies slightly from night to night with a median $r m s$ fluctuation of $0.5 \%$. Two-thirds of the $\mathrm{K}$ stars are variable. Few exceed $1.0 \%$. For individual stars, the amount of variability tends strongly to repeat consistently from season to season. There are also some--but not many--stars that are stable to $0.2 \%$ or so year after year (13).

For the program stars, the "chromospheric emission ratio," $\log \mathrm{R}^{\prime} \mathrm{HK}$, an estimate of the fraction of the star's total luminosity originating in the chromosphere (14), turns out to be a good predictor of photometric variability as well as of chromospheric activity (13). Using $\log \mathrm{R}^{\prime} \mathrm{HK}$, we can order the stars by increasing activity and compare the corresponding photometric and HK variations (15).

Figure 2 shows the six-year range of variation of the program stars as a function of $\log R^{\prime} H K$. The Sun is plotted on this figure, using a nominal cycle amplitude of $0.1 \%(0.001 \mathrm{mag})$ determined by its variability in cycle 21 . In the lower panel of Figure 3 we show the corresponding range of variation of the most stable pair of stars (observed differentially) in the groups whose program stars are plotted above. If the star pair containing the program pair was the most quiescent pair of the group, there is no corresponding data point on the lower figure.

Photometric activity rises rapidly with increasing $\log \mathrm{R}^{\prime} \mathrm{HK}$ and begins to exceed $1 \%$ when the chromospheric emission ratio is about twice the solar value. The variation in $b$ is consistently greater than in $y$, which is to be expected because of the relative positions of $b$ and $y$ on the stellar energy distribution. The dotted line at $0.5 \%(0.005 \mathrm{mag})$ is a plausible upper limit for the range of variation of the Sun's close neighbors on this figure; and since most of the corresponding comparison star pairs lie below this line with smaller ranges of variation, we conclude that many of the program stars are demonstrably variable by much larger amounts than the Sun.

\section{c) A Sampler of Light Curves}

Figures 3-7 are a sampler of differential light curves showing the individual nightly data points on the left-hand panels ( $b$ and $y$ averaged) and the annual mean magnitudes on the right-hand panels. The six panels for a quartet of stars and three panels for a trio of stars display all the possible differential pairings of the stars (that is: star 1 minus star 2,1-3, $1-4,2-3,2-4$, and $3-4$ for a quartet and $1-2,1-3$, and $2-3$ for a trio). The error bars are $95 \%$ confidence intervals.

Pairs of light curves showing similar variation and sharing a star in common usually reveal unambiguously which star is varying. The sense of a brightness change (positive or negative) on the different panels depends on which star is varying (the first or the second named on the ordinate); so certain light curve pairs, for example (1-2) and (2-3), will show mirror-image variations if the star in common is variable.

Figure 3 shows a star with a small linear trend. Figure 4 shows the photometry of an unusually stable group with little or no variation. Figures 5 and 6 show cyclic variations, and Figure 7 shows a star that became very active during one season only.

\section{CONCLUSIONS}

Nearly 200 years of daily sunspot records teach us that the most visible manifestation of solar activity vary unpredictably. Every 11-year cycle is unique. The variation of the total solar output, measured only for slightly less than one 11-year solar cycle, leads us to think that long-term variations are quite small--only $0.1 \%$ or so. But to contain this miniscule variation requires the delicate and continual balancing between larger competing effects, the flux deficits associated with sunspots and the flux excesses associated with faculae. In addition, subtle temperature effects may add to whatever other variations occur. Secular trends may accompany the tendency of cycle amplitudes to rise and fall over many cycles. With less than 20 cycles elapsed since records began, there is little to guide us. It would be presumptious for us to assume that what we observed in cycle 21 is an accurate Rosetta stone to even the recent past. 
Stellar photometry offers little assurance that the solar variability actually measured thus far provides an accurate longterm prognosis. Indeed, many stars quite similar to the Sun demonstrably vary by amounts much larger than the Sun has over the last decade. To be sure, these stars rotate faster than the Sun, but otherwise, in terms of age, mass, temperature, and chromospheric activity, they appear to be very much like the Sun. The observed stellar variations mostly appear to be "regular" in some sense: either they exhibit a linear trend, a curve with a single maximum or minimum, or what looks like a cycle. Rarely is the variation random, as would be the case if photometric errors dominated the long-term records. Thus we conclude, considering the Sun among the stars, that the present short record of solar variability is remarkable only in its present restraint.

\section{ACKNOWLEGEMENT}

This work was supported by the Air Force Geophysics Laboratory and the Lowell Research Fund.

\section{REFERENCES}

1. Hardie, R. H., and Giclas, H. L. "A Search for Solar Variations." Astrophys. J. 122, 460-465, 1955.

2. Jerzykiewicz, M., and Serkowski, K. "The Sun as a Variable Star III." Lowell Observ. Bull. No. 137 (Vol VI, No. 18), 295-323, 1966.

3. Willson, R. C., Gulkis, S., Janssen, M., Hudson, H. S., and Chapman, G. A. "Observations of Solar Irradiance Variability." Science 211, 700-702, 1981.

4. Hoyt, D. V., and Eddy, J. A. "Solar Irradiance Modulation by Active Regions from 1969 through 1981." Geophys. Res. Lett. 10, 509-512, 1983.

5. Willson, R. C., and Hudson, H. S. "Solar Luminosity Variations in Solar Cycle 21." Nature 332, 810-812, 1988.

6. Foukal, P., and Lean, J. "An Empirical Model of Total Solar Irradiance Variation Between 1874 and 1988." Science 247, 556-558, 1990.

7. Radick, R. R., Hartmann, L., Mihalas, D., Worden, S. P., Africano, J. L., Klimke, A., and Tyson, E. T. "The Photometric Variability of Solar-Type Stars. I. Preliminary Results for the Pleiades, Hyades, and the Malmquist Field." Publ. Astron. Soc. Pacific 94, 934-944, 1982.

8. Radick, R. R., Thompson, D. T., Lockwood, G. W., Duncan, D. K., and Baggett, W. E. "The Activity, Variability, and Rotation of Lower Main-Sequence Hyades Stars." Astrophys. J. 321, 459-472, 1987.

9. Hardorp, J. "The Sun among the Stars." Astron. Astrophys. 91, 221-232, 1980.

10. Wilson, O. C. "Chromospheric Variations in Main-Sequence Stars." Astrophys. J. 226, 379-396, 1978.

11. Vaughan, A. H., Baliunas, S. L., Middelkoop, F., Hartmann, L., Mihalas, D., Noyes, R. W., and Preston, G. W. "Stellar Rotation in Lower Main-Sequence Stars Measured from Time Variations in $\mathrm{H}$ and $\mathrm{K}$ Emission-Line Fluxes. I. Initial Results." Astrophys. J. 250, 276-283,1981.

12. Baliunas, S. L., and Vaughan, A. H. "Stellar Activity Cycles." Ann. Rev. Astron. Astrophys. 23, 379-412, 1985.

13. Lockwood, G. W., and Skiff, B. A. Luminosity Variations of Stars Similar to the Sun. Air Force Geophysics Laboratory final report AFGL-TR-88-0221, 101 pp.,1988.

14. Noyes, R. W., Hartmann, L. W., Baliunas, S. L., Duncan, D. K., and Vaughan, A. H. "Rotation, Convection, and Magnetic Activity in Lower Main-Sequence Stars." Astrophys. J. 279, 763-777, 1984. 
15. Radick, R. R., Lockwood, G. W., and Baliunas, S. L. "Stellar Activity and Brightness Variations: A Glimpse at the Sun's History." Science 247, 39-44, 1990.

16. Skiff, B. A, and Lockwood, G. W. "The Photometric Variability of Solar-Type Stars. V. The Standard Stars 10 and 11 Leonis Minoris." Publ. Astron. Soc. Pacific 96, 714-722, 1984.

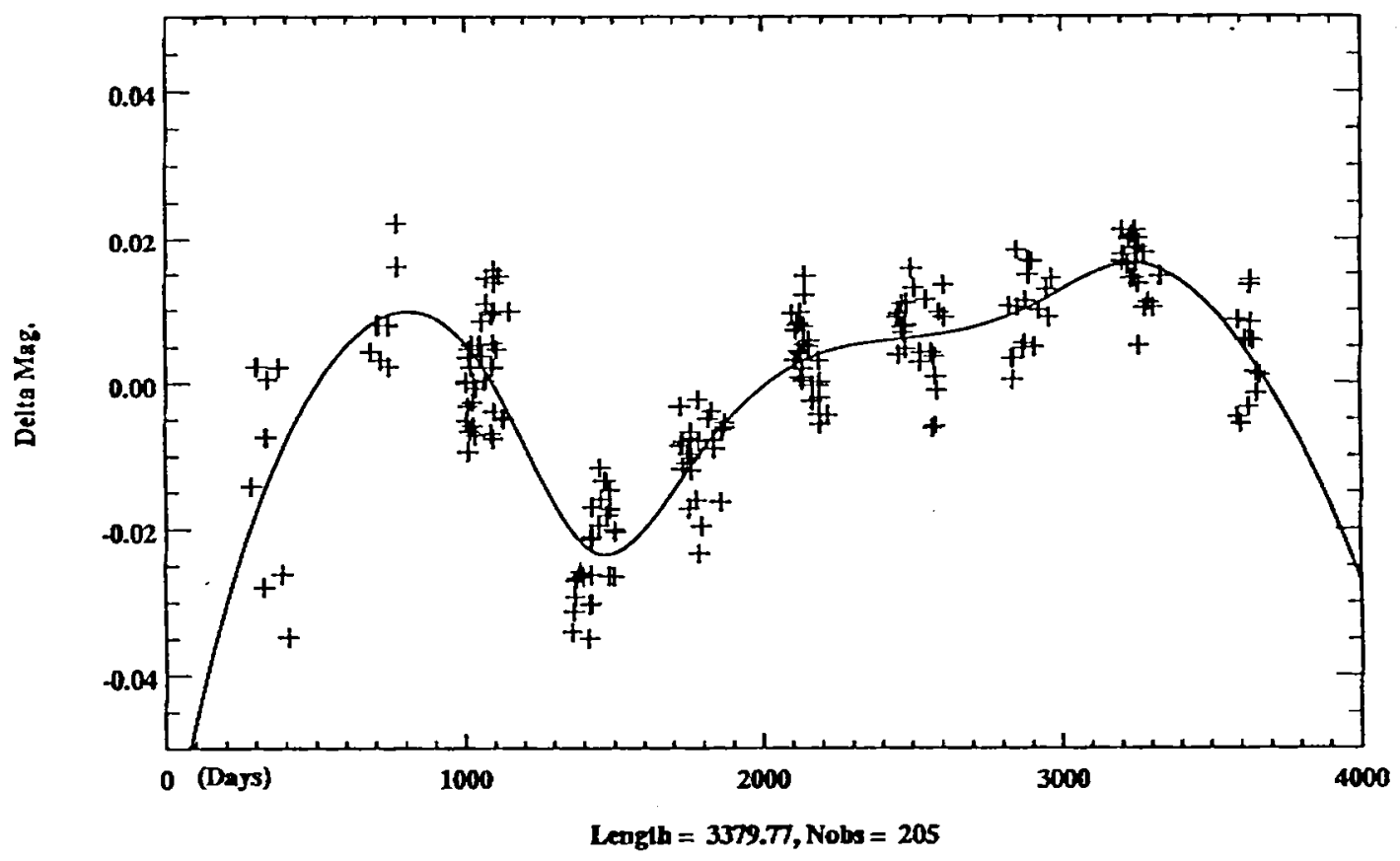

Figure 1. Long-term brightness variations of the young, solar analog, G2 V star, Van Bueren 64, in the Hyades open cluster. The first two seasons' data are from the Cloudcroft Observatory and the remainder are from the Lowell Observatory. vB64 has a rotation period of about 8.7 days based on the photometry. The smoothing spline fit through the data indicates an amplitude of about $0.03 \mathrm{mag}$ and the possibile existence of a stellar luminosity cycle. 

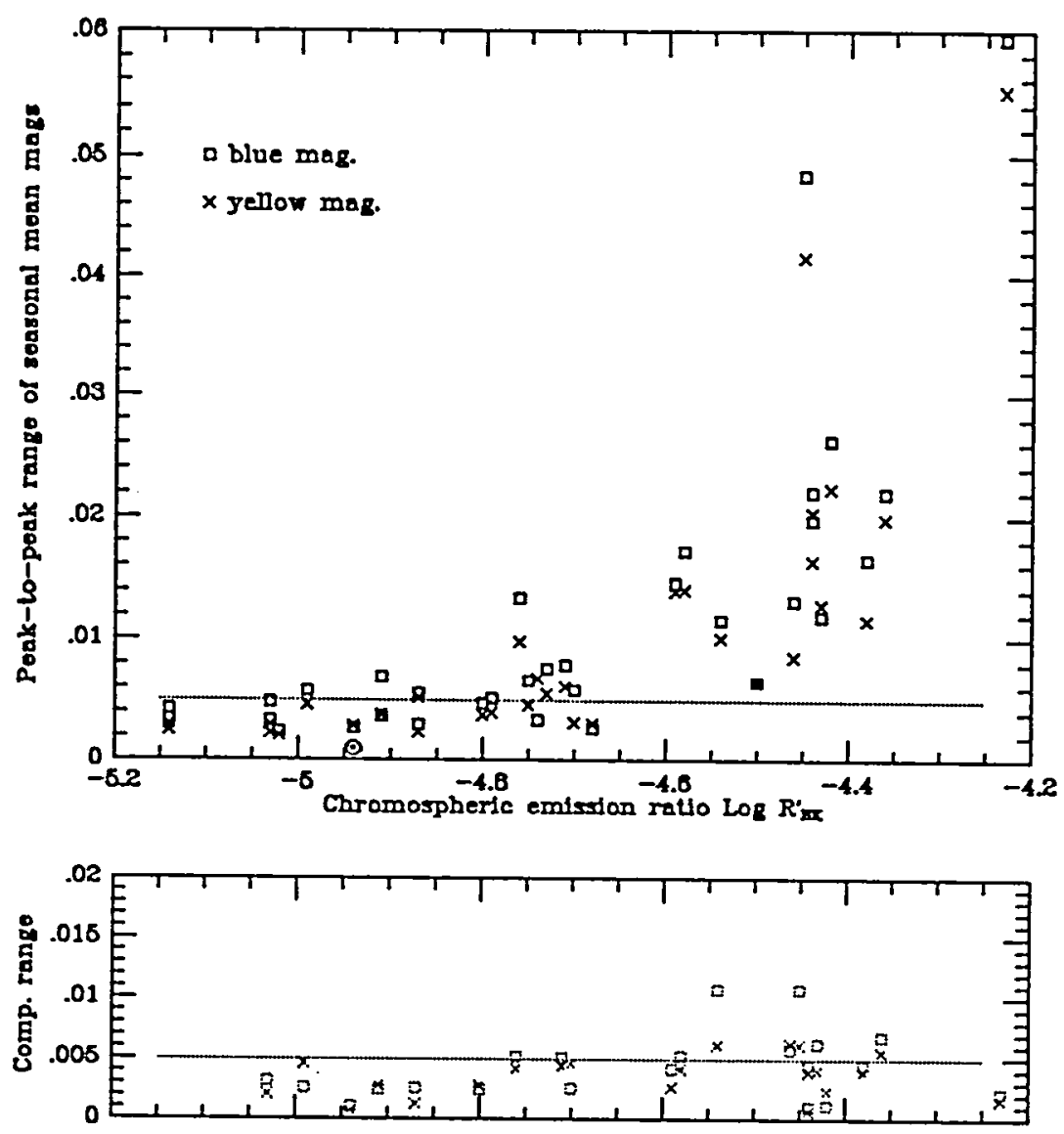

Figure 2. (top). The 6-year range of the annual mean $b$ and $y$ magnitudes of the program stars as a function of the chromospheric emission ratio, $\log \mathrm{R}^{\prime} \mathrm{HK}$. Note the position of the Sun at $(-4.94,0.001)$. None of the Sun's neighbors on this figure vary by more than $0.5 \%$, indicated by the dotted line at $0.005 \mathrm{mag}$. (bottom). The range of the least-variable pair of comparison stars in the groups corresponding to the program stars plotted above. Since the average variability of the comparison stars tends generally to be on the order of $0.0025 \mathrm{mag}$, the indicated variability of the corresponding program stars in excess of that amount is likely to be intrinsic rather than observational.
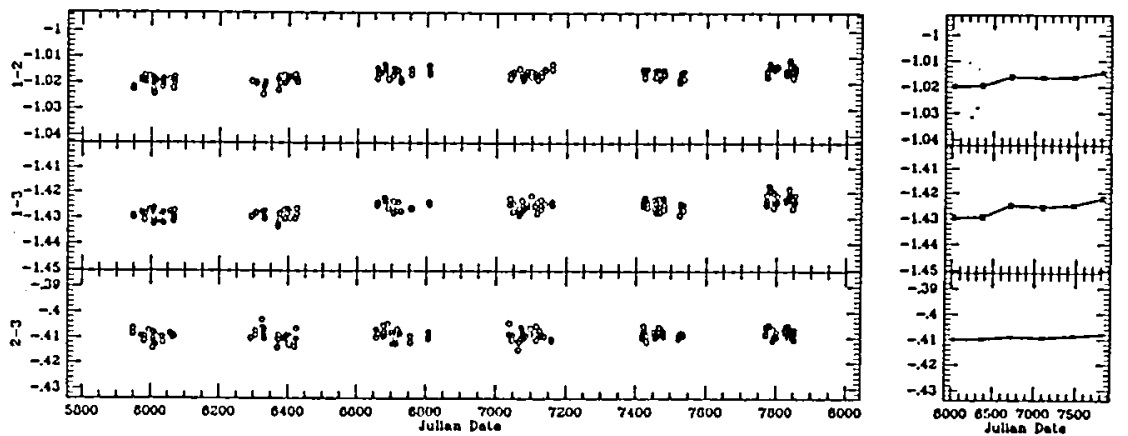

Figure 3. Differential light curves for a trio of stars comprising one program star and two comparison stars. The $b$ and $y$ magnitudes are averaged together. The left-hand panels show the individual nightly data points (two points per night, usually) for each of the pairwise combinations of stars; that is, star 1 minus star 2 , star 1 minus star 3, etc. The right-hand panels show the yearly mean magnitudes and their $95 \%$ confidence intervals. Star 1 is HD10476 (K1V), $\log \mathrm{R}^{\prime} \mathrm{HK}^{\prime}$ $=-4.87$, close to the Sun's value of -4.94 . This star has a 9-year HK activity cycle. The brightness has decreased by 0.0055 mag in $b$ and $0.0052 \mathrm{mag}$ in $y$ over 6 years. The comparison star pair (star 2-star 3 ) is unusually stable, with a range of $0.0026 \mathrm{mag}$ in $b$ and $0.0013 \mathrm{mag}$ in $y$. 

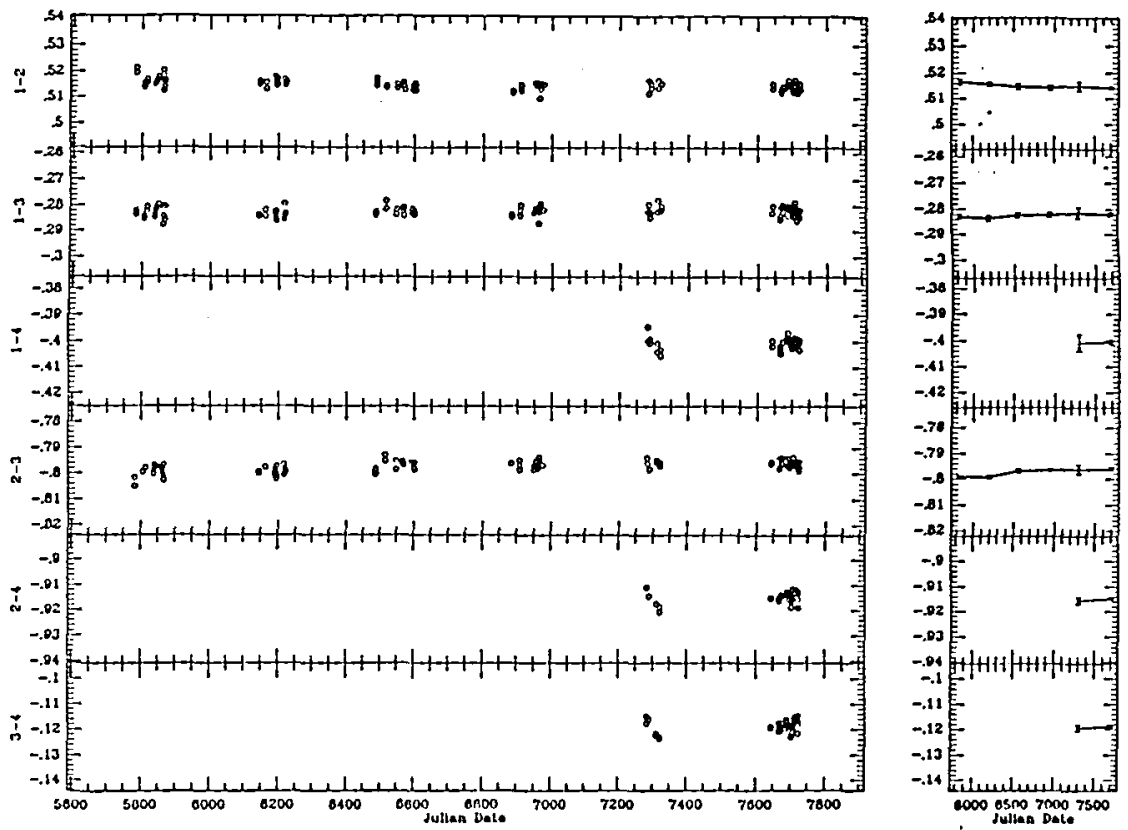

Figure 4. This group was observed as a trio for the first four seasons, but was promoted into a quartet by adding another comparison star when it became obvious that the identity of the "variable" star was unclear. Star 1 is HD143761 (G0V), a Mount Wilson HK standard and photometric standard star with a subsolar value of $\log \mathrm{R}^{\prime} \mathrm{HK}$. The observed range (star $1-\mathrm{star} 2$ ) is $0.0024 \mathrm{mag}$ in $b$ and $0.0021 \mathrm{mag}$ in $y$.
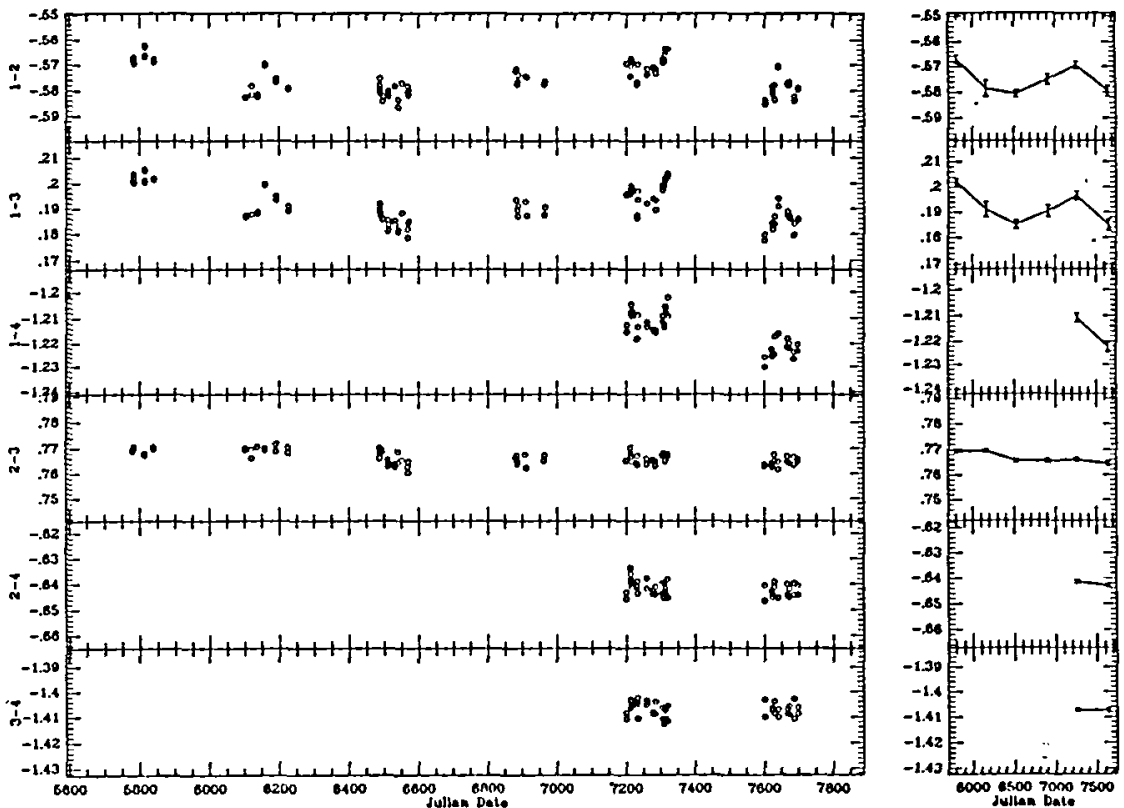

Figure 5. Star 1 is $H D 115383$ (GOV), $\log R^{\prime} H K=-4.43$, an active star. Although this star has long been used as a $u v b y$ standard, it shows a cyclic variation with an amplitude of $0.0119 \mathrm{mag}$ in $b$ and $0.0129 \mathrm{mag}$ in $y(1-2$ and 1-3). The range of the comparison star pair (2-3) is $0.0063 \mathrm{mag}$ in $b$ and $0.0041 \mathrm{mag}$ in $y$, large enough to indicate intrinsic variability, so we added another comparison star in the fifth season. We suspect star 2, a $\mathrm{K} 0$ giant, as the likely variable comparison star. 

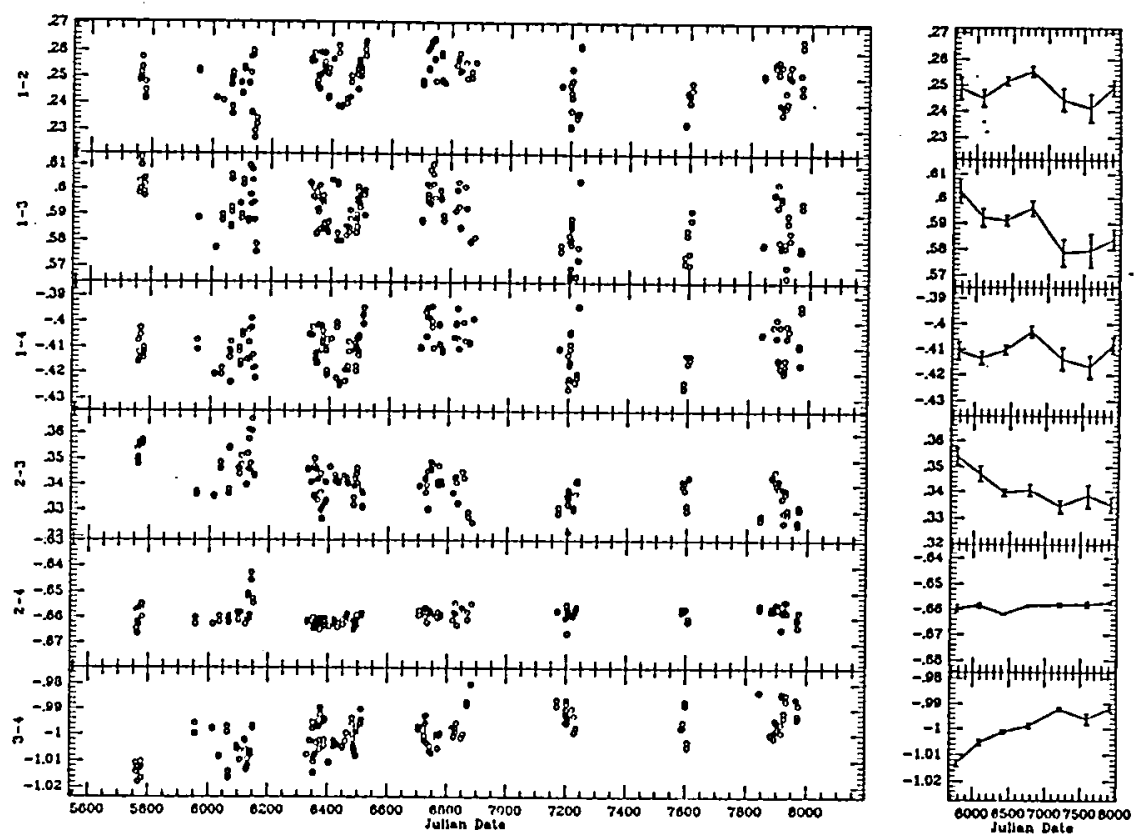

Figure 6. Star 1 is HD35296 (F8V), $\log \mathrm{R}^{\prime} \mathrm{HK}=-4.38$, an active star showing a trend in its HK index and cyclic brightness variation with an amplitude of 0.0165 in $b$ and 0.0116 in $y$. Star 3, HD39587 (GOV), $\log R^{\prime} \mathrm{HK}^{\prime}=-4.44$, displays a linear brightness decrease with a range of $0.0221 \mathrm{mag}$ in $b$ and 0.0204 mag in $y$. The comparison star pair (2-4) is fairly stable, with a range of about $0.004 \mathrm{mag}$.
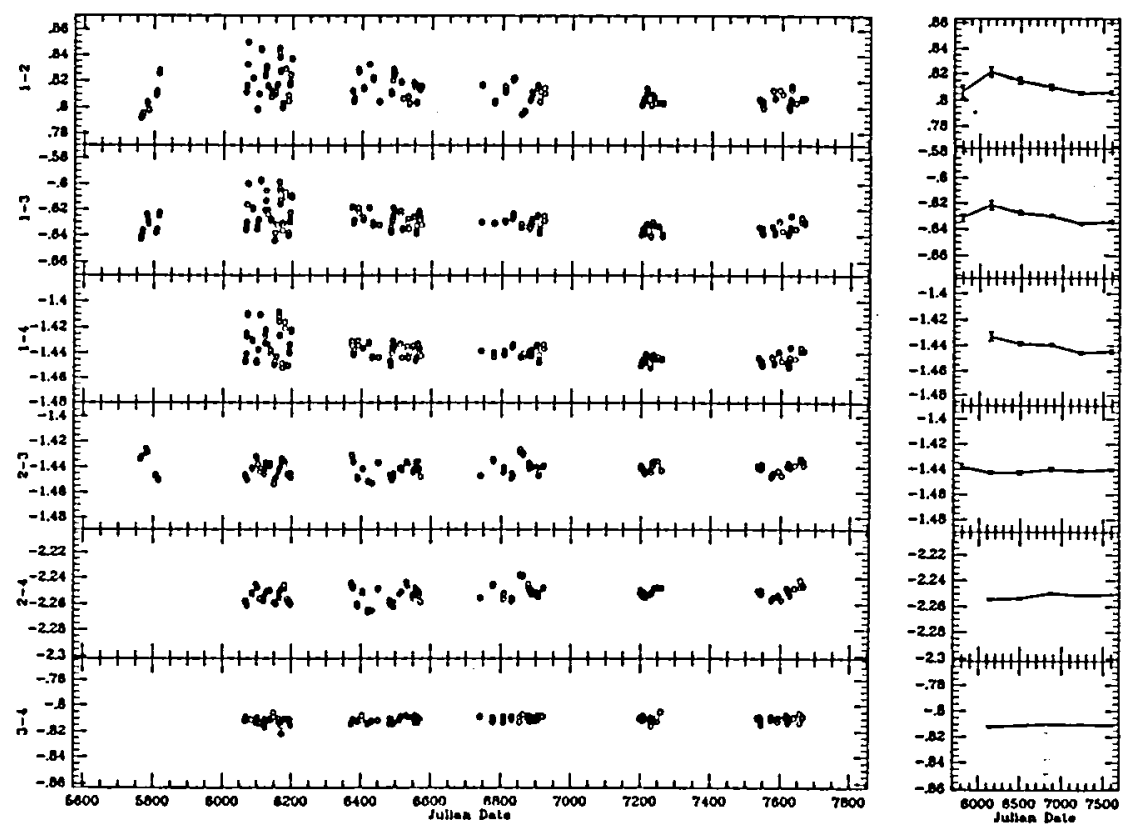

Figure 7. Star 1 is HD82885 (G8IV-V), another variable $u v b y$ standard star, with $\log \mathrm{R}^{\prime} \mathrm{HK}=-4.58$. The HK data showed a noisy trend with no apparent cycle. During the second observing season, the star was on average fainter by 0.01 mag than in the previous 13 years, during which we observed it as a $u v b y$ standard. During this season we determined a photometric rotation period of 18 days and an amplitude of 0.033 mag indicating the presence of large starspots. Star 2 in this group is HD82635 (G8.5III), another $u v b y$ standard that turned out to be slightly variable on rotational timescales. Over the long term it is quite steady. In the second season, it showed a rotational modulation with an amplitude of $0.012 \mathrm{mag}$ and a period of 40 days (16). Note that variability, when it occurs, scatters the points toward fainter magnitudes (upwards on the light curves) from an essentially unblemished level maximum. 


\title{
A PROGRAM OF PHOTOMETRIC MEASUREMENTS OF SOLAR IRRADIANCE FLUCTUATIONS FROM GROUND-BASED OBSERVATIONS
}

\author{
G.A. Chapman, A.D. Herzog, J.K. Lawrence, and S.R. Walton \\ San Fernando Observatory \\ Department of Physics and Astronomy \\ California State University, Northridge
}

\begin{abstract}
Photometric observations of the sun have been carried out at the San Fernando Observatory since early 1985 . Since 1986 , observations have been obtained at two wavelengths in order to separately measure the contributions of sunspots and bright facular to solar irradiance variations. We believe that the contributions of sunspots can be measured to an accuracy of about $\pm 30 \mathrm{ppm}$. The effect of faculae is much less certain, with uncertainties in the range of $\pm 300 \mathrm{ppm}$. The larger uncertainty for faculae reflects both the greater difficulty in measuring the facular area, due to their lower contrast compared to sunspots, and the greater uncertainty in their contrast variation with viewing angle on the solar disk. Recent results from two separate photometric telescopes will be compared with bolometric observations from the ACRIM that was on board the Solar Max satellite.
\end{abstract}

\section{INTRODUCTION}

High precision, two-dimensional photometric mapping of solar active regions was begun in 1982, partly in response to the accurate, spacebased measurements of the total solar irradiance. In the summer of 1982 , observations were carried out using a single linear diode array with the San Fernando Observatory $61 / 28 \mathrm{~cm}$ vacuum telescopes and vacuum spectroheliograph. Beginning in 1983, two linear diode arrays were operated, obtaining simultaneous photometric images in nearby wavelengths. These data have a pixel spacing of 0.94 arc-sec. Normally, data were obtained only for $512 \times 512$ pixel regions centered on specific active regions or predicted returns of active regions (Lawrence et $\mathrm{al}^{1}$ ). Occasionally, the disk of the sun was scanned in four swaths, each having a height of 512 pixels and a width of 2200 . Only a small part of the north and south poles were not scanned (Lawrence and Chapman'2).

To reduce the volume of data, to greatly simplify the observational procedure, and to make the observations less prone to bad weather, two new telescope/photometer systems were developed in 1984-1986. One of these systems, called the Cartesian Full Disk Telescope (CFDT) is designed to scan the full solar disk in about three minutes with a pixel spacing of 5 arc-sec. The CFDT produces a photometric image of the sun in one of several wavelengths with each pixel digitized to an accuracy of 12 bits. Since the sun's image is scanned by the earth's rotation, the image has virtually no distortion. The other photometric system is the Rotating Full Disk Photometer (RFDP). This system has a telescope with an aperture of $7.5 \mathrm{~cm}$ and a photometer that rotates about the disk center, producing an image with the disk center at pixel 1 and the solar limb near pixel 420. Due to limits on data rate, the data are averaged in real-time, $2 \times 2$, and written on magnetic tape. The effective pixel spacing is about 5 arc-sec. The time to acquire a complete image is approximately $2 \frac{1 / 2}{2}$ minutes. These two photometric instruments are complementary to each other in that they obtain photometric solar images that are, on the one hand undistorted near the disk center and, on the other hand, undistorted near the solar limb. These photometric systems are used by undergraduate and graduate students on a daily basis. In addition, full disk photographs are obtained, as a back-up, in $\mathrm{H} \alpha$ and in a $3840 \AA$ filter. In this report, we describe some of the preliminary results of these observations and some plans for the near future. 

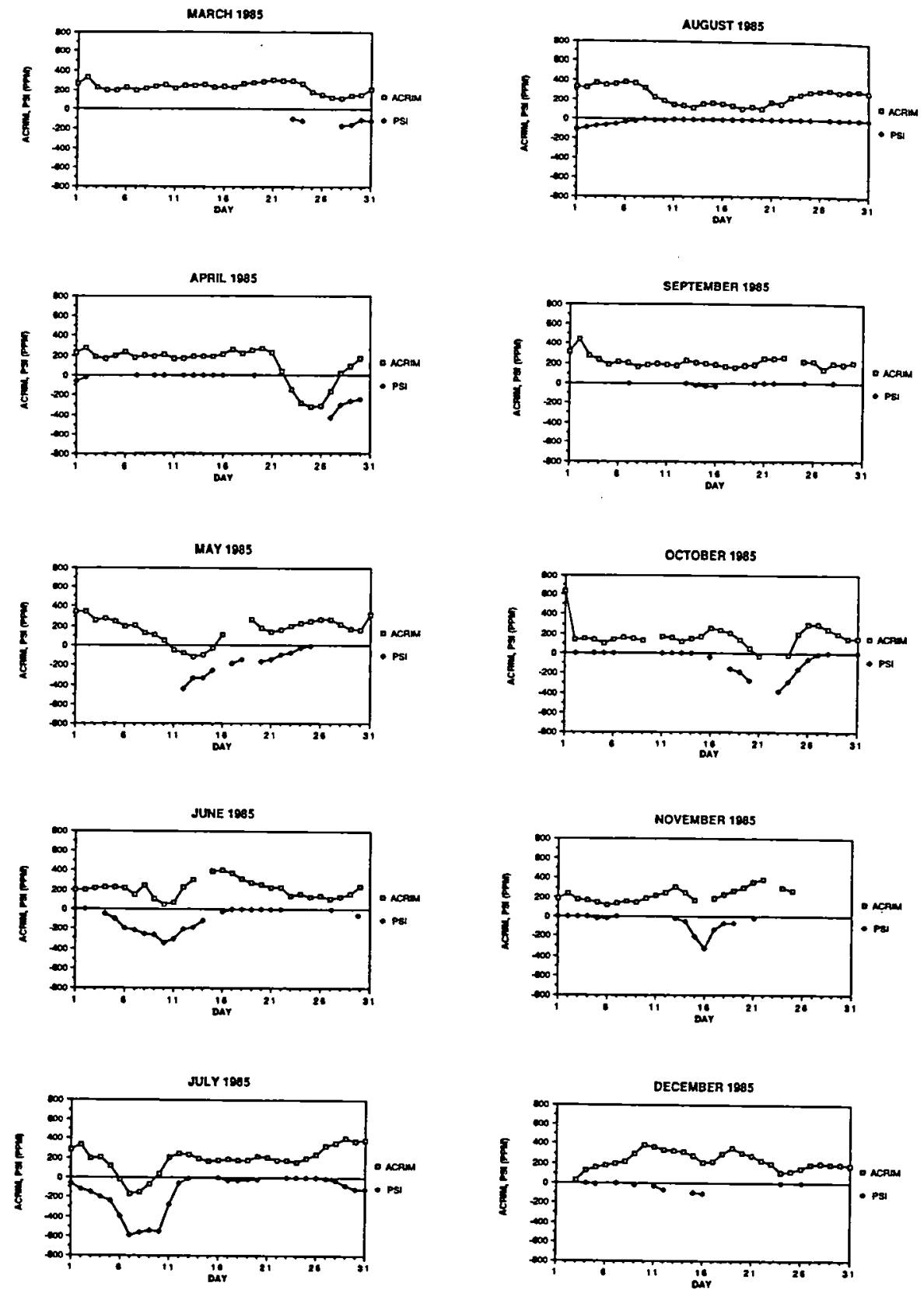

Fig. 1

Digital PSI determined from the CFDT during 1985 compared to the fluctuation in the ACRIM signal relative to $1367 \mathrm{~W} / \mathrm{m}^{2}$. The time difference between the ACRIM signal and the San Fernando Observatory PSI has not been removed. 


\section{SYNOPTIC FULL-DISK PHOTOMETRY}

The CFDT began routine observations using a red filter in the spring of 1985 (Chapman et al. ${ }^{3}$ ). Analysis of these data has resulted in a determination of sunspot irradiance deficits and areas (in units of millionths of a hemisphere) for 1985 (Chapman and Davis ${ }^{4}$ and Davis ${ }^{5}$ ). Beginning in 1986, an intermediate band filter at $3920 \AA$ was added. This filter, with a bandpass of $100 \AA$, was intended to detect faculae. In 1988 , a $10 \AA$ narrow band filter was added at a wavelength of $3934 \AA$. This filter was intended to detect faculae more effectively than the $3920 \AA$ filter. We present here some results for part of the summer of 1988 , as well as for most of the year 1985.

The RFDP was constructed and installed with the help of a NASA grant during 1984 and 1985. This photometer is fed by a $15 \mathrm{~cm}$ aperture achromat, stopped down to $7.5 \mathrm{~cm}$, with a focal length of $229 \mathrm{~cm}$. The data are obtained at a wavelength of $5319 \AA$ with a bandpass of $100 \AA$. The pointing of the RFDP is controlled by a separate guider. Because of the limitations in data storage rate, pixels are averaged, $2 \times 2$, to 5 arc-sec, before being written to magnetic tape. Thus, each image corresponds to 1320 records, each with a length of 256 pixels.

At the end of each record of a photometric image, an accurate measurement of the sky transparency is recorded. These data can be used later to remove the effect of changes in sky transparency that occur during the solar observation. These "exposure meter" observations are obtained at a wavelength of $0.53 \mu \mathrm{m}$.

\section{RECENT RESULTS}

The observations from the CFDT for the year 1985 have been processed (Chapman and Davis ${ }^{4}$ and Davis ${ }^{5}$ ). The Photometric Sunspot Index (PSI) is compared with fluctuations in the ACRIM signal in Fig. 1. The Photometric Sunspot Index is defined in Chapman and Meyer, ${ }^{6}$ based on the form first discussed in Willson et al. ${ }^{7}$ In our work, the corrected sunspot area is determined from the CFDT red image after removing the quiet sun limb darkening. This results in a smoothed contrast map that is searched for pixels that have a negative contrast that exceeds -8.5 percent. This criterion was

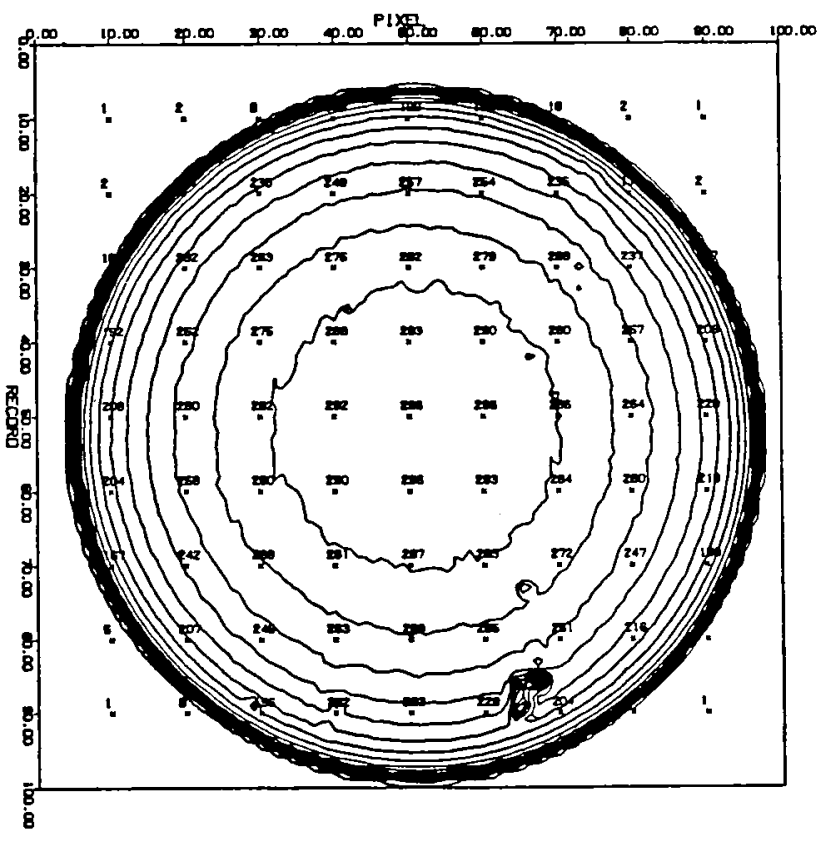

Fig. 2 A contour plot of the whole solar disk from the CFDT for 27 June 1988. Each pixel in this plot represents 20 arc-sec.

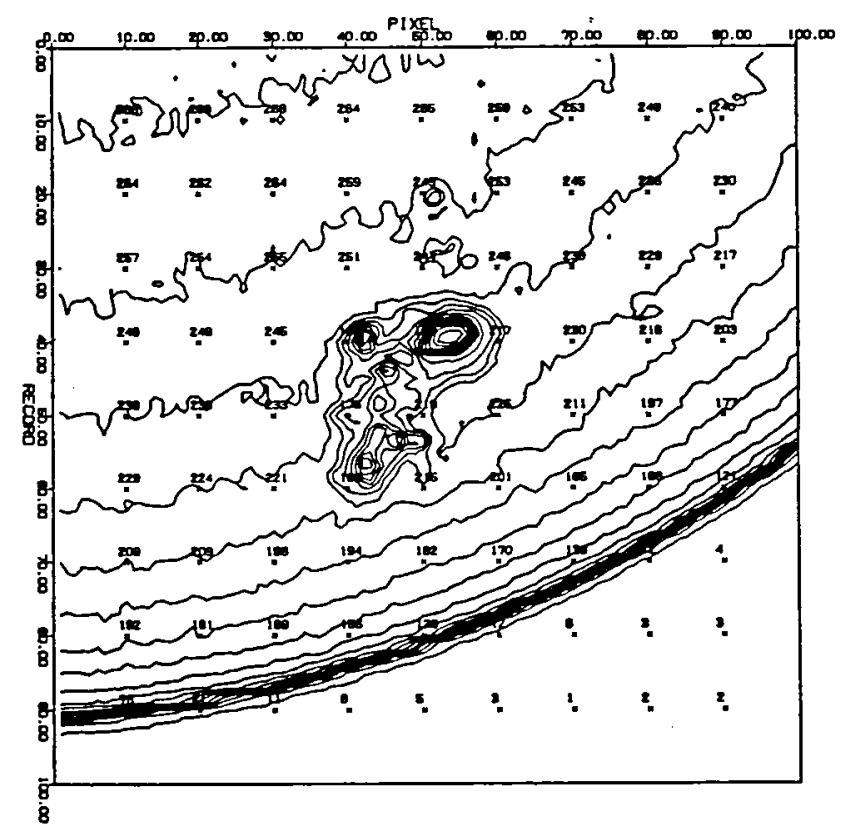

Fig. 3 A contour plot of the active region at the SE limb, from the image in Fig. 2, but at the full resolution, 5 arc-sec per pixel of the CFDT. 
determined by an examination of several sunspot images obtained with one arc-sec pixels using the $28 \mathrm{~cm}$ vacuum telescope and vacuum spectroheliograph. This same criterion is used to define a sunspot in the higher resolution RFDP data. We will see that there is a systematically smaller sunspot area from CFDT data compared with RFDP data that is probably due to the lower spatial resolution of the CFDT optical system (Chapman et al. ${ }^{3}$ and Herzog et al. ${ }^{8}$ ). A small adjustment in the contrast criterion for CFDT sunspots can bring these two systems into very close agreement. Corrected sunspot areas can also be determined from the CFDT K-line images by changing the sunspot contrast criterion from $8.5 \%$ to $-9.6 \%$ (Wilson ${ }^{9.10}$ ).

The Rotating Full Disk Photometer (RFDP) has a $7.5 \mathrm{~cm}$ objective and a 2.5 arc-sec pixel at the detector. Although the data are co-added, $2 \times 2$, in order to reduce the data rate, one expects to have larger sunspot signals from this system. This appears to be the case.

The appearance of the sun as seen by these two instruments is shown in Figs. 2-3. Fig. 2 shows the full disk seen by the CFDT at $6723 \AA$. In order to plot the full disk on one plot, the data have been averaged $4 \times 4$. Fig. 3 shows a sunspot group from the same day at the full resolution of the CFDT, approximately 5 arc-sec per pixel. Fig. 4 shows the same sunspot group as seen by the RFDP at a scale of 5 arc-sec per pixel. These data are most useful in studying active regions near the limb. An increasing geometric distortion that increases toward the disk center can be removed. Except for some distortion, the sunspot images in Figs. 3 and 4 look quite similar.

Fig. 5 shows the relation of corrected sunspot areas, in parts per million of the solar hemisphere, from the RFDP compared with sunspot areas published in the Solar Geophysical Bulletin. These data cover the time period from 9 May to 22 August 1988 (Herzog et al. ${ }^{8}$ ). Although the correlation coefficient is high, $r=$ 0.97 , there is a significant difference from unity in the slope of the relation. The published areas appear to underestimate the corrected area of sunspots, as measured by the RFDP, by approximately $30 \pm 2$ percent (one sigma error).

The effects of bright faculae are determined by searching a "flattened" K-line image for pixels

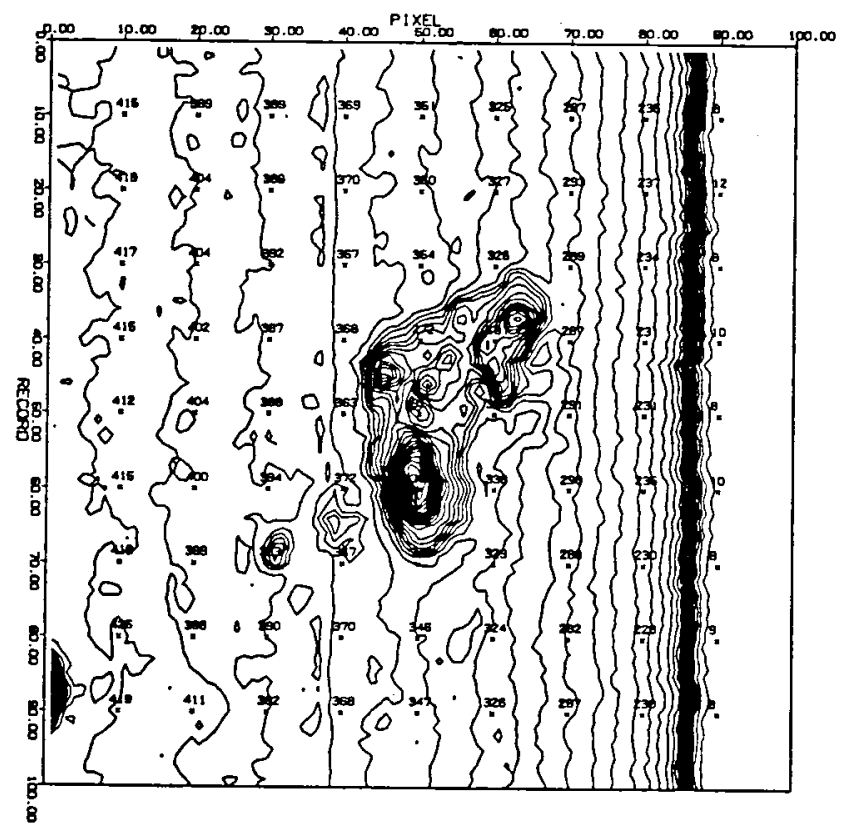

Fig. 4 A contour plot of the active region shown in Fig. 3 from the RFDP. The pixel size is nearly the same as for the CFDT.

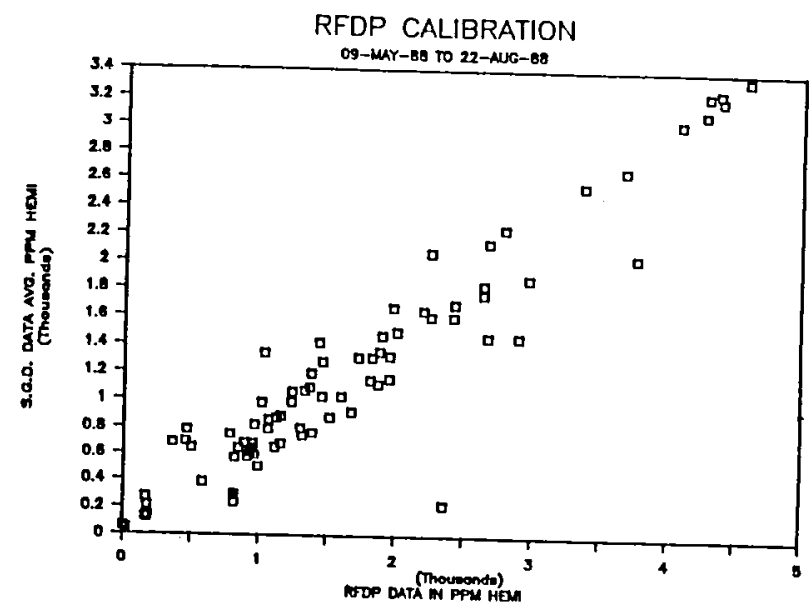

Fig. 5 A plot of corrected sunspot areas, in ppm, of the solar hemisphere determined from the RFDP and compared with those published in the SGD Bulletin. The values in the SGD represent averages from each station reporting. 
brighter than some criterion, usually $4 \%$ (about 2.5 sigma), and calculating a Photometric Facular Index (PFI), a measure of the irradiance excess caused by faculae.

The standard deviation in the Photometric Facular Index has been determined for several days when from 3 to 6 images have been obtained in the K-line filter. An analysis of these data suggests that the standard deviation in the PFI is about 200 millionths of the mean solar irradiance. If the mean solar irradiance is approximately 1367 $\mathrm{W} / \mathrm{m}^{2}$, then this photometric uncertainty corresponds to $0.27 \mathrm{~W} / \mathrm{m}^{2}$. This standard deviation includes noise from the possible evolution of active regions.

A preliminary analysis for 25 days between 10 June and 23 July 1988 shows that the CFDT and the RFDP corrected sunspot areas are highly correlated with a correlation coefficient of 0.990 . The slope is not unity and it appears that the larger aperture RFDP obtains sunspot areas that are 9 percent larger than those from the CFDT. There may be a 200 millionth zero point offset, although its value is not statistically significant (1 sigma). The analysis of more data will help to clarify this point (Herzog et al. ${ }^{8}$ ).

An analysis of ACRIM data and sunspot and facular photometry for a two-week period in June and July of 1988 shows that ground-based photometry can correlate with the spacecraft data with a multiple correlation coefficient of about 0.98 for 21 data points. The quiet sun irradiance, determined from these regressions, is approximately $1367.0 \mathrm{~W} / \mathrm{m}^{2}$. The details will be contained in a forthcoming paper (Chapman et al. ${ }^{11}$ ). The largest source of systematic noise appears to be the time difference between the ground-based data and the daily values of irradiance available from the ACRIM group. A good fit to the ACRIM data is obtained using the Photometric Irradiance Fluctuation (PIF) (see Chapman et al. $^{3}$ for further discussion of the PIF) to represent the sunspot signal but with a coefficient of 1.062. Basically, the PIF measures the photometric deficit of sunspot pixels relative to the mean solar irradiance. The facular signal used was based on the function given in Schatten et al., ${ }^{12}$ although the function given by Foukal ${ }^{13}$ gave nearly as good a fit. The Schatten et al.function required a coefficient of 2.035 . The squared partial regression coefficients for the
CFDT signal and the Schatten et al.-signal were 0.96 and 0.69 , respectively.

In summary, the ground-based program described here appears to be able to match accurate spacebased radiometry to within approximately 0.2 $\mathrm{W} / \mathrm{m}^{2}$, or in the range of 100 to 200 parts per million, at least for the two-week interval described above. With further refinements in the image processing and improved averaging of the space-based data, this noise can be further reduced. Extending such an analysis over a longer time base should lead to some interesting improvements in our understanding of the sun.

This research has been partly supported by NSF Grant ATM-8817634 and NASA Grant NAG-51219.

\section{REFERENCES}

1. Lawrence, J.K., Chapman, G.A., Herzog, A.D., and Shelton, J.C. 1985, Ap.J., 292, 297.

2. Lawrence, J.K. and Chapman, G.A. 1990, Ap.J., Oct. 1990, in press.

3. Chapman, G.A., Herzog, A.D., Laico, D.E., Lawrence, J.K, and Templer, M.S. 1989, Ap.J., 343, 547.

4. Chapman, G.A. and G.A. Davis, 1990, in preparation.

5. Davis, G.A. 1989 M.S. Thesis, CSUN.

6. Chapman, G.A. and Meyer, A.D. 1986, Solar Phys., 103, 21.

7. Willson, R.C., Gulkis, S. Janssen, M., Hudson, H.S. and Chapman, G.A. 1981, Science, 211, 700.

8. Herzog, A.D., Chapman, G.A., Gluszczak, M. and Waiton, S.R. 1990, BAAS, 21, 1110.

9. Walton, S.R., Wilson, $R$ and Chapman, G.A. 1990, BAAS, 21, 1179.

10. Wilson, R. 1990 M.S. Thesis, CSUN, in preparation.

11. Chapman, G.A., Herzog, A.D., Lawrence, J.K., Walton, S.R., Hudson, H.S. and Fisher, B., 1990, in preparation.

12. Schatten, K.H., Miller, N., Sofia, S., Endal, A.S., Chapman, G.A., and Hickey, J. 1985, Ap.J., 294, 689.

13. Foukal, P. 1981, in The Physics of Sunspots, L.E. Cram and J.H. Thomas, eds., Sunspot, NM, 391. 
SESSION 3: “THE MONSTER" IN THE SUN-EFFECTS OF INTERNAL FIELDS AND SOLAR ACTIVITY 



\title{
BASIC MECHANISMS OF SOLAR VARIABILITY
}

\author{
Sabatino Sofia \\ Center for Solar and Space Research \\ Yale University \\ New Haven, CT 06511
}

\section{INTRODUCTION}

Understanding and describing solar variability can be pursued in two different ways. One, the taxonomic approach, describes and catalogues the details of each of the manifestations of solar variability separately. The other, the global approach, considers the Sun as a system and deals with the entire ensemble of global solar properties, but with little detail. Whereas the first approach is preferred when information on the detailed behavior of one isolated phenomenon is required, (the rest of the system can be assumed to remain constant), the second approach is employed when completeness and selfconsistency of the entire set of phenomena are important, and little guidance on specific physical mechanisms is required. This paper will explore the global approach.

The crudest means of looking at solar variability in the global approach is by means of a general virial theorem which includes magnetic and rotational terms in addition to the usual kinetic and gravitational terms. This treatment is interesting in that it readily shows the inter-relationships between the various global solar parameters. In particular, for the case where rotational and magnetic energy is negligible, it shows that radius and luminosity changes are simply related. The virial approach, however, requires that the system be fully relaxed (including having reached thermal equilibrium), which only applies for timescales far in excess of the year-to-decades timescales important for climatically significant processes. Consequently, it is not very useful for our purposes. On the other extreme of sophistication, we may envision a complete, time-dependent model of the Sun including rotation and magnetic fields valid for timescales between months (the turnover timescale for the convection zone) and billions of years (the timescale for evolution). Such a model would clearly be able to describe the solar variability we are interested in. However, it does not yet exist,and it is not clear whether it could be constructed within the capability constraints of even the fastest computers currently available.

In order to simulate the behavior of a changing Sun in a realistic way, we have used a perturbation analysis (the results are summarized in Endal et al. 1985). In this approach, we use a standard solar model and then vary several of the model parameters to mimic the sudden or gradual change of some physical property within the Sun. The evolution following the perturbation is followed in a physically self-consistent way, that is, hydrostatic and thermal processes occur in their normal timescales. This treatment has allowed us to determine the sensitivity of the various global parameters to physical processes affecting the solar interior, and it has guided us in defining the least complex global solar model which can address the question of climatically significant variability. Not surprisingly, magnetic fields play the crucial role. In this paper, I discuss the current status of the model, what we have already learned from it, and the future prospects.

\section{THE STANDARD SOLAR MODEL}

All physical models involve approximations, and so the best model for a process is the least complex system which properly addresses it. In stellar evolution, the standard model assumes spherical symmetry, and ignores the effects of rotation and magnetic fields. This 
type of modelling has been the cornerstone of the field of stellar structure and evolution, and it has been remarkably successful in explaining the theoretical basis for the observations of field stars, globular clusters and open clusters as summarized by their H-R diagrams ( $c f$. ., Schwarzschild, 1958). When applied to the Sun, this model produces a remarkably constant star bent on increasing both its size and luminosity on timescales of billions of years. Of course, the "standard Sun" does not change on a short term, does not rotate, and it has no magnetic activity of any type.

On the other hand, the Sun does rotate, it has a magnetic activity cycle, and its total irradiance does change at least in concert with the activity cycle, as observations carried out by instruments on board SMM (Wilson et al., 1981), Nimbus 7 (Hickey et al., 1981), and ERBE (Lee et al., 1987) have demonstrated. In addition, there is evidence that the solar diameter may be also be changing, at least on timescales on the order of 90 years (Sofia et $a l ., 1985)$. Such a change would also affect the luminosity. In order to model these phenomena, the standard solar model is inadequate, and an upgraded model must be produced.

\section{THE UPGRADED SOLAR MODEL}

As we add complexity to our solar model, we must be mindful of following the "least complexity principle," namely, only incorporate into the model those elements that are essential to producing the desired behavior, which in this case is the short timescale variability. Clearly, magnetic effects must be included in order to produce the activity cycle. Moreover, since solar magnetic fields are thought to be produced by a dynamo mechanism, whose operation requires a differentially rotating magnetized flow, rotation must also be included. Finally, since the convective flow is not properly described by the mixing length theory of convection, a more realistic treatment of the convective flows is required. As stated earlier, these elements are in agreement with the results of the perturbation analysis of the standard solar model.

The above additions are extremely complex, but already substantial progress has been made in the implementation of some of the processes, individually. This implementation is described in some detail by Fox (1990), and it is based on research that our group has been carrying out over the past 15 years. For example, the rotating feature of the global code was developed by Endal and Sofia in the mid to late 70's (Endal and Sofia, 1976, 1978 and 1981), and this technique has been considerably extended recently at Yale University (cf., Pinsonneault et al., 1989).

The work on convection has also been carried out by Chan, Wolff and Sofia (Chan and Wolff, 1982; Chan, Sofia and Wolff, 1982, and Chan and Sofia, 1984, 1986, 1987 and 1989). This work has yielded a set of numerical relationships between the thermodynamic and dynamical properties of the flow, which are currently being used for modeling the solar convection zone. Finally, Fox and associates have developed a code to evolve non-reactive global magnetic fields in a differentially rotating solar model; Theobald, Fox, and Sofia (1990) have succeeded in modeling the small scale magnetic interaction with compressible convection.

\section{FUTURE ACTIVITIES}

All the elements have been developed, and they now must be joined together in a fully reactive, self-consistent upgraded solar model. The task is not trivial; besides the sheer size of the integrated code, a very broad range of time and space scales are considered at one 
time. Because of this, the task in its full generality is not likely to be overcome simply by the expanding capability of new computers. The most promising way to proceed is to study the properties of the microphysics (small scale flow, small scale convection, etc.) in order to obtain averaged relationships whose effects can be realistically incorporated in the global models without the requirement of a fine space and time mesh which makes their running impossible.

The expanded models must first be tested with a number of stars of different masses and ages to ascertain that the observational properties of star systems are properly reproduced by the new models. The next step is to refine the solar model by means of the large number of observational tools that will become available in the coming years. These tools include the helioseismological observations to be provided by the ground-based GONG project, by the SOHO satellite, and from the oscillation and diameter data to be provided by the SDS experiment both in its balloon-borne and satellite incarnations.

When the upgraded solar model can reproduce the variabilities that are known to occur, it will be run to follow the behavior of the solar luminosity over a period of a few hundred years. Finally, the luminosity variations produced by the model must be incorporated into climate models to determine the effect of solar variability on our climate. If this effect is properly validated with past data, it could be used for the purpose of forecasting future climate change. Whereas it is true that a lot of work remains to be carried out before the question of solar forcing of the terrestrial climate can be fully settled, it is also true that a comparable amount of progress has already been made. The importance of the results make it mandatory that we follow this task to the end of the road.

I would like to thank Peter Fox and T.J. Lydon for extensive conversations and considerable help. I would also like to acknowledge the NASA support, by means of grant NAG 5-736, which has allowed us to carry out the work outlined here. 


\section{REFERENCES}

Chan, K-L and Sofia, S., 1984, Astrophys.J., 282, 550.

Chan, K-L and Sofia, S., 1986, Astrophys.J., 307, 222.

Chan, K-L and Sofia, S., 1987, Science, 235, 465.

Chan, K-L and Sofia, S., 1989, Astrophys.J., 336, 1022.

Chan, K-L, Sofia, S., and Wolff, C.L., 1982, Astrophys.J., 263, 935.

Chan, K-L and Wolff, C.L., 1982, J. Comput. Phys., 47, 109.

Endal, A.S. and Sofia, S., 1976, Astrophys.J., 210, 184.

Endal, A.S. and Sofia, S., 1978, Astrophys.J., 220, 279.

Endal, A.S. and Sofia, S., 1981, Astrophys.J., 243, 625.

Endal, A.S., Sofia, S., and Twigg, L.W., 1985, Astrophys.J., 290, 748.

Fox, P.A., 1990, (this volume).

Hickey, J.R., Alton, B.M., Griffin, F.J., Jacobowitz, H., Pellegrino, P., Smith, E.A., VonderHaar, T.H., and Maschhoff, R.H., 1981, in Variations in the Solar

Constant, NASA Conference Proceedings Publication CP-2191, ed. S. Sofia, 59.

Lee, R.B., Barkstrom, B.R., and Cess, R.D., 1987, Applied Optics, 26, 3090.

Pinsonneault, M.H., Kawaler, S.D., Sofia, S., and Demarque, P., 1989, Astrophys.J., 338, 424.

Schwarzschild, M., 1958, Structure and Evolution of Stars (Princeton).

Sofia, S., Demarque, P., and Endal, A., 1985, American Scientist, 73, 326.

Theobald, M.L., Fox, P.A., and Sofia, S., 1990, BAAS, to appear.

Wilson, R.C., Gulkis, S., Janssen, M., Hudson, H.S., and Chapman, G.A., 1981, Science, 211, 700 . 


\title{
GLOBAL MODELS OF INTERMEDIATE TIMESCALE VARIABILITY ON THE SUN
}

\author{
Peter A. Fox \\ Center for Solar and Space Research, Yale University \\ P.O. Box 6666, New Haven, CT 06511
}

\begin{abstract}
In recent years a number of advances in both observation and theory have increased our understanding of the solar interior and how to model it. For climate studies, the timescale of interest for changes in the Sun ranges from decades to centuries. This paper attempts to highlight some of the theoretical advances that will contribute to the building of global models of the Sun's variability on intermediate timescales and describe what the current constraints on the important components are. Finally a short discussion presenting some implications for input to climate modeling will be presented.
\end{abstract}

\section{INTRODUCTION}

Standard models of the solar interior are constructed using the equations of stellar structure (Chandrasekhar ${ }^{1}$ ) and provide quite good agreement with the real Sun. They include a number of physical processes such as nuclear energy generation, radiative transfer, ionization, convection, etc. These physical processes are treated on a variety of timescales depending on their influence of the evolution of the Sun to the present epoch. Often approximations are used to establish the influence of processes shorter than the evolution timescale (of order millions to hundreds of millions of years). In addition, the accuracy of models can vary enormously depending on what features are being examined and what the corresponding observational limits are.

The very features that make current standard solar models suitable for studying stellar evolution are also the ones that prevent us from studying its variability on timescales of climatic significance. The key to upgrading current solar models for these studies is in identifying the components that are either missing or treated with inadequate approximations or invalid assumptions. In addition the numerical accuracy of models needs to be examined carefully.

In recent years, a number of advances in the understanding of solar interiors has taken place. Among these advances was the investigation of perturbations to solar models in both the convective efficiency and non-gas components to the pressure. These studies (e.g. Endal, Sofia and Twigg ${ }^{2}$ ) established guidelines for the type of disturbances that could be tolerated within the Sun, at what depth they were most and least effective, what the surface consequences for the solar radius and luminosity were and most importantly, what the timescale for the influence. Unfortunately they were difficult to constrain and true feedback or interaction between the physical processes could not be accommodated.

The emergence of helioseismology as a very tight constraint on solar models has improved the understanding of solar models in a number of areas, apart from the much higher numerical accuracy that is required. Improvements have been made in the treatment of the equation of state, nuclear reaction rates and cross sections, stellar opacities, surface boundary conditions and the solar chemical composition. In addition these studies have indicated the oscillation modes that sample the interface between the solar radiative and convective regions are particularly sensitive to structure changes and thus are an important constraint on models as will be discussed in later sections.

In addition, advancements in the understanding and modeling of stellar convection, stellar rotation and stellar magnetic fields (to be addressed in the following sections) are setting the scene for substantial improvements in solar and stellar models in the next few years. 


\section{INITIAL CONDITIONS}

As the components of solar models are improved toward self-consistency one important consideration is the availability of suitable initial conditions for the variability study. Specifically that means a carefully calibrated solar model that has details of internal rotation and large scale magnetic fields that are consistent with known observational constraints. In addition a high degree of numerical accuracy is required; fortunately this aspect is well under control even though the current treatment of certain processes may be uncertain at the 5 to $30 \%$ level.

At our present level of understanding, a unique initial model is very unlikely. Thus, it will be important to understand the influence of variations in the initial models. The solar-stellar connection (Sofia and Endal ${ }^{3}$ ) should provide invaluable information as it has done so far for recent studies of internal rotation (Pinsonneault et. al. $\left.{ }^{4}\right)$. Helioseismology will also play an important role in detecting changes in the internal structure of the initial models.

One aspect of solar variability that is particularly important when attempting to construct global models is the timescale over which certain types of variations occur. A number of empirical studies (such as some presented at this meeting) propose a range of timescales for influencing climate and also suggest particular solar physical features that may be responsible for the variations (in nearly all cases, the solar magnetic field as represented by the sunspot record for example). By including all standard physical phenomena into a global model the timescale of interest then determines what level of approximations may be valid for the models.

\section{ADVANCES IN CONVECTION MODELING}

Most of the important timescales for solar variability correspond to the range of timescales that exist in the solar convection zone (which occupies the outer $\approx 30 \%$ by radius and $\approx 2 \%$ by mass of the Sun). The current treatment of convection is by use of the mixing length theory (MLT). Although the MLT is adequate for evolutionary models of stars, it has limitations in the treatment of certain physical processes, such as rotation, magnetic fields and ionization which are probably vital to the global models to be constructed. In addition, many of the assumptions of MLT have been under scrunity for many years but without any firm conclusions or a proven replacement theory.

One significant recent advance on this area is due to Chan and Sofia. In a series of papers (Chan and Sofia ${ }^{5,6,7}$ ) they explored not only the validity of the MLT formalism but attempted to derive expressions for fluctuating quantities (such as temperature, velocity, etc.) in terms of local mean quantities just as the MLT does, but by using detailed models of fully compressible, deep and efficient convection (which applies over most of the solar convection zone).

For example, they found that, in agreement with MLT, that the mixing length does scale with the local pressure scale height and not the density scale height, and that in a region of efficient convection the total heat flux comprises two major components, the enthalpy flux and kinetic energy flux. The enthalpy flux can be roughly computed from the superadiabatic gradient and vice versa. However, in disagreement with MLT, the kinetic energy flux is downward directed for much of the convection zone and is not simply related to local quantities like other MLT relations. In addition the production of kinetic energy can be derived from local quantities but the dissipation of kinetic energy is non-local.

An example of the results from this study is given in Fig. 1 where the covariance of the vertical velocity and temperature fluctuation about the horizontal mean is shown versus the product of the mean vertical velocity and mean temperature. This is an important relationship that does not appear in MLT which assumes that the mean vertical velocity vanishes.

For solar cycle timescales an important variation in the total irradiance is due to the presence of sunspots and faculae on the solar disk. Although it is unlikely that this modulation of the irradiance produces direct changes 
in the earth's climate, the influence of redistributed heat flow through the solar convection zone on a long enough timescale could well have consequences for the solar structure (radius and luminosity) at the level that would produce secular changes.

The solar convection zone is a very complex physical system and only recently have detailed numerical models of variations in its heat transport been possible. Preliminary results from these studies (Fox, Sofia and $\mathrm{Chan}^{8}$ ) indicate that on small scales the heat flux that is diverted around an area of intense magnetic field does partially re-appear at the solar surface and does not seem to be stored within the convection zone over long timescales (more than tens of thousands of years). There are many uncertainties when considering solar magnetic fields, particularly their internal distribution and it is as yet unclear how important that distribution is for models of solar variability which should depend on more global properties like the total magnetic flux within the solar convection zone, for example.

Fig. 2 shows an example of one simulation from Fox, Sofia and $\mathrm{Chan}^{8}$. The horizontally averaged surface flux (in solar units) is shown as a function of time. Once the blocking begins a short relaxation time is evident after which the surface flux oscillates with an time integrated average quite close to the undisturbed value and certainly well above the value that would result from total blocking (represented by the dash line). This simulation is still very idealized and cannot reflect the true solar environment. Nevertheless, important information on the dynamics of diverted heat flow can be obtained.

In this regard it is important to understand what influence a time varying large scale internal magnetic field would have on heat transport (and thus structure) in the solar convection zone. A first principles effort to extend MLT is difficult but an extension to the existing attempt of using detailed numerical models seems straightforward.

The influence of small scale magnetic fields on compressible convection is also a field that is growing rapidly. The concentration of magnetic flux on the solar surface has been evident even in simpler models for quite a while, however more complex features such as magnetic reconnection, the influence of anisotropic magnetic resistivity and the production of Alfven and magneto-acoustic waves are now part of recent modeling efforts (Theobald, Fox and Sofia ${ }^{9}$ ). Fig. 3 shows two snapshots of a simulation involving magnetic fields, the vector potential in two dimensions just maps out the lines of magnetic force and the velocity vectors are overlayed.

This figure shows magnetic field concentration (where contour lines are close), expulsion (where lines are absent) and magnetic reconnection (compare the two frames and note where single line contours become closed loops).

A detailed understanding of small scale interaction of magnetic fields and convection should aid in our understanding of the important regenerative phase of the solar cycle (and thus the global) magnetic field.

It will be important to ascertain the influence of the latitude dependence of not only solar cycle phenomena but the solar magnetic field in general since the simplification to one dimensional models and thus uniform radius changes would ease the numerical burden on the global models.

\section{MAGNETIC FIELDS}

Magnetic fields are perhaps the key to understanding solar variability. The large scale component is certain important for secular changes whereas the small scale component (as manifested by the activity cycle field) seems only important for shorter timescales. However, it is the regenerate phase of the solar cycle, from small scale to large scale that could introduce some randomness into the Sun's global field. The irregular modulations of the solar activity cycle as shown by the sunspot record over two centuries provides some support to this point as does the evidence from other "solar-type" stars that have turned on and turned off their activity cycles over decade timescales (Baliunas ${ }^{10}$ ).

Magnetic fields are also important as part of the initial conditions for a study of solar variability, as mentioned 
above. Models have been produced which follow the evolution of various initial magnetic fields in the context of a rotating Sun $\left(\mathrm{Fox}^{11}\right)$. One feature of these calculations is the implied presence of a large scale magnetic field beneath the solar convection zone, see Fig. 4 (Fox and Bernstein ${ }^{12}$ Fox, Niznik and Bernstein ${ }^{13}$ ). One improvement to be added to the present calculation is the combined interaction of internal rotation and a large scale magnetic field in the radiative interior. There is strong evidence (observational and implied theoretical) for the existence of internal differential rotation and large scale magnetic fields in solar-type stars.

One constraint on interior magnetic fields that is yet to be fully developed is helioseismology. Except for very high field strengths (of order MGauss) the direct impact on oscillation mode frequencies is small. However changes in the solar structure (which may be position dependent) due to magnetic fields are likely to produce measureable changes in the oscillation frequencies. In addition there are, as yet still only suggestive, variations of oscillation frequencies (at particular wavelengths) over the solar cycle (Libbrecht and Woodard ${ }^{14}$ ).

\section{INTERNAL ROTATION}

The details of internal velocity motions are a secondary consideration to solar variability since they primarily contribute to other physical features (particularly magnetic fields). Their inclusion in global models is therefore essential for completeness. Fortunately this is one area that has made significant progress in recent years also. After the founding work of Endal and Sofia ${ }^{15}$, Pinsonneault et al. ${ }^{4,16}$ have provide an increasingly complete understanding of rotating stars (and specifically solar-type stars). These studies also provide a robust framework in which to develop and test the combined interaction of rotation and magnetic fields in solar-type stars. Many of the constraints utilized in these recent studies are also necessary for the study of variability.

One very important result of this study is the implication from more evolved stars that the Sun cannot be rotating as a solid body. Fig. 5 shows the computed periods of rotation for solar-type sub-giant stars, the upper curve is the theoretical prediction of periods if the stars at the solar age were rotating as solid bodies at the solar equatorial rate, the symbols represent observations (which are all upper limits) of those type of stars. The lower curve is the theoretical prediction that allows internal differential rotation, see Pinsonneault et. al. ${ }^{4}$ for more details. Recently this argument has become even more convincing from a study of horizontal branch stars (Pinsonneault et al. ${ }^{16}$ ).

Because the convection zone is an important component for our studies, the treatment of larger scale circulations will need to be improved. At present, for evolutionary purposes, the convection zone (not the interior) is assumed to rotate as a solid body and not have any latitude dependence. The level of detail that may be required in treating rotation, as with the solar dynamo itself, in the context of solar variability is still unclear.

\section{CLIMATE IMPLICATIONS}

For the global solar variability models to have a bearing on climate modeling they must have at least two properties; proven accuracy and predictability. Naturally, internal accuracy of the variations is a separate issue from agreement with solar observations. Even if we are limited to a 3 to 5 year predictability (as for empirical solar activity forecasting, Schatten and Sofia ${ }^{17}$, Layden et al $^{18}$ ) of solar luminosity variations, this should be a significant advance over the present situation for short term climate. For input to longer term climate predictions we must rely on the limited observational constraints that we presently have to calibrate the models.

Global models will primarily both be constrained by and predict changes in the solar radius (on climate timescales) that can be calibrated by luminosity variations. Additional constraints will results from helioseismology networks (because they use both intensity and doppler measurement) and combined irradiance and diameter measurements.

After suitable calibration, predictions can be envisioned once the appropriate global contributors and their timescales are identified. 


\section{CONCLUSIONS}

It is clear that global models of solar variability are complex, but necessarily so since the phenomena to be explained are beyond the capabilties of current standard models. However, we have identified the essential components that make up such a global model and in addition we are able to model all of these components at either the detailed (small length and time scales) level or the evolutionary level (long length and time scales). The important task of bridging the gap between the two scales is well underway and significant progress has been made in a number of areas. In addition, the development and integration of the model components are quite well constrained by detailed surface observations, the link between the Sun and Sun-like stars and helioseismology.

Of immediate interest are topics such as convection and magnetic field modeling, solar global magnetic fields (in both radiative and convective regions) and using helioseismology to constrain the initial models. We should expect to see substantial progress in these areas in the next few years.

\section{ACKNOWLEDGEMENTS}

This work is supported under grants from NASA (NAGW-777 and NAGW-778). My thanks go to Sabatino Sofia for comments on this paper and Marc Pinsonneault for permission to include one of his figures ${ }^{4}$ (Fig. 5).

\section{REFERENCES}

${ }^{1}$ Chandrasekhar, S., An Introduction to the Study of Stellar Structure, University of Chicago Press, Chicago (1939).

${ }^{2}$ Sofia, S. and Endal, A. S., The Solar-Stellar Connection: Solar Studies, Publ. Astron. Soc. Pacific, 99, 1241-1247 (1987).

${ }^{3}$ Endal, A. S., Sofia, S. and Twigg, L., Changes of Solar Luminosity and Radius Following Secular

Perturbations in the Convective Envelope, Astrophys. J., 290, 748-757 (1985).

${ }^{4}$ Pinsonneault, M. H., Kawaler, S. D., Sofia, S. and Demarque, P. R., Evolutionary Models of the Rotating Sun, Astrophys. J., 338, 424-452 (1989).

${ }^{5}$ Chan, K. L. and Sofia, S., Turbulent Compressible Convection in a Deep Atmosphere III. Tests on the Validity and Limitation of the Numerical Approach, Astrophys. J., 307, 222-241 (1986).

${ }^{6}$ Chan, K. L. and Sofia, S., Validity Tests of the Mixing Length Theory of Deep Convection, Science, 235, 465-467 (1987).

${ }^{7}$ Chan, K. L. and Sofia, S., Turbulent Compressible Convection in a Deep Atmosphere IV. Results of Three-Dimensional Computations, Astrophys. J., 336, 1022 (1989).

${ }^{8}$ Fox, P. A., Sofia, S. and Chan, K. L., Convective Flows around Sunspot-Like Objects, submitted to Solar Phys. (1990).

9 Theobald, M. L., Fox, P. A. and Sofia, S., Magnetic Interaction with Compressible Convection, BAAS, to appear (1990).

10 Baliunas, S., Studies of Solar Type Stars, these proceedings.

11 Fox, P. A., Large Scale Solar and Stellar Magnetic Fields, in Cool Stars, Stellar Systems and the Sun, Proceedings of the Fifth Cambridge Workshop on Cool Stars, Eds J. L. Linsky and R. E. Stencel, Springer-Verlag, Berlin, p 57 (1987).

12 Fox, P. A. and Bernstein, I. B., On the Generation of Magnetic Fields in the Sun, in The Internal Solar Angular Velocity, Proceedings of the 8th NSO Summer Symposium, Sunspot, NM Eds. B Durney and S Sofia, Reidel, Dordrecht, (1986).

13 Fox, P. A., Niznik, P. and Bernstein, I. B., Evolution of the Sun's Global Interior Magnetic Field, EOS, 69, 437 (1988).

${ }^{14}$ Libbrecht, K. G. and Woodard, M. F., Observations of Solar Cycle Variations in Solar P-Mode Frequencies and Splittings, BBSO preprint \#0306 (1989).

15 Endal, A. S. and Sofia, S., The Evolution of Rotating Stars. I. Method and Exploratory Calculations for a 7 $M_{\odot}$ Star, Astrophys. J., 210, 184-198 (1976).

${ }^{16}$ Pinsonneault, M. H., Deliyannis, C. P. and Demarque, P. R., Evolutionary Models of Halo Stars with

Rotation: I Evidence for Differential Rotation with Depth in Stars, Astrophys. J., submitted. 
${ }^{17}$ Schatten, K. H. and Sofia, S., Forecast of an Exceptionally Large Even Numbered Solar Cycle, J. Geophys. Res. Letters, 14, 632 (1987).

${ }^{18}$ Layden, A. C., Fox, P. A., Howard, J. M., Sarajedini, A., Schatten, K. H. and Sofia, S., A Scheme for Dynamo-based Empirical Forecasting of Solar Activity, J. Geophys. Res., submitted.

Fig. 1. Covariance of the temperature deviation and vertical velocity versus the product of the mean temperature and the mean vertical velocity. The different symbols represent variations in the character of the convective layer, such as depth, stratification, etc. so that the implied relation seems valid over a range of conditions.

Fig. 2. Time history of the solar surface output flux (normalized) comparing an undisturbed region with one in the presence of a flux blocking object (like a small sunspot). After an initial relaxation time of about one hour, the surface output flux is within about $1 \%$ of the solar value The dashed curve represent the output flux if total blocking had occurred. The oscillation in the disturbed output flux is due to heat diffusion within the flux blocking object, see Fox, Sofia and $\mathrm{Chan}^{8}$ for details.

Fig. 3. Two snapshots of the contours of the magnetic vector potential overlayed with the velocity vector field in a time dependent two-dimensional simulation of the interaction of convection and magnetic fields near the solar surface. The contour lines represent lines of magnetic force. Regions of intense field are found where the lines are closely spaced, and field free regions are those where the lines are widely spaced. Evidence of magnetic reconnection is also visible by comparing the two frames and can be seen toward the centers of the circulation patterns (closed contour lines).

Fig. 4 Illustration of a typical poloidal field (left hemisphere) and toroidal field (right hemisphere) configuration calculated from the global evolution of an initially dipolar magnetic field, under the influence of internal (differential) rotation, subject to a perfectly conducting outer boundary.

Fig. 5. Surface rotation period of subgiant stars as a function of effective temperature. compared with predictions of solar models including internal rotation (solid) and those assuming solid body rotation (dash). See Pinsonneault et al. ${ }^{4}$ for more details.

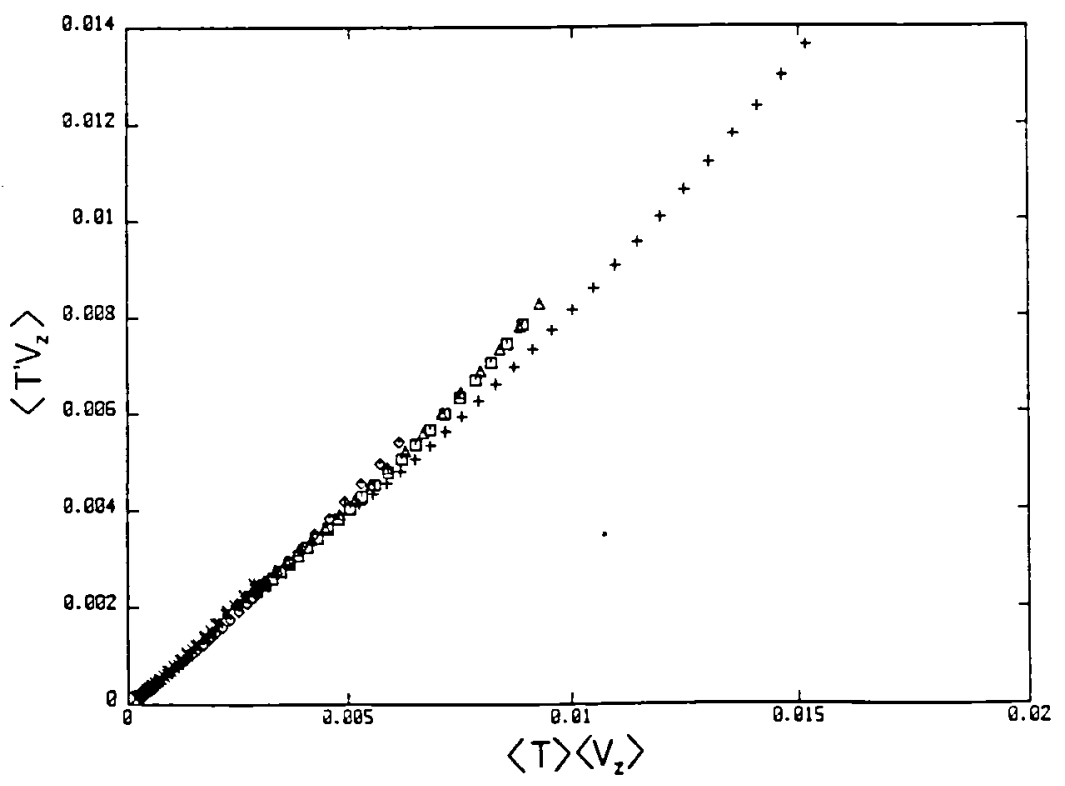

Fig.1 


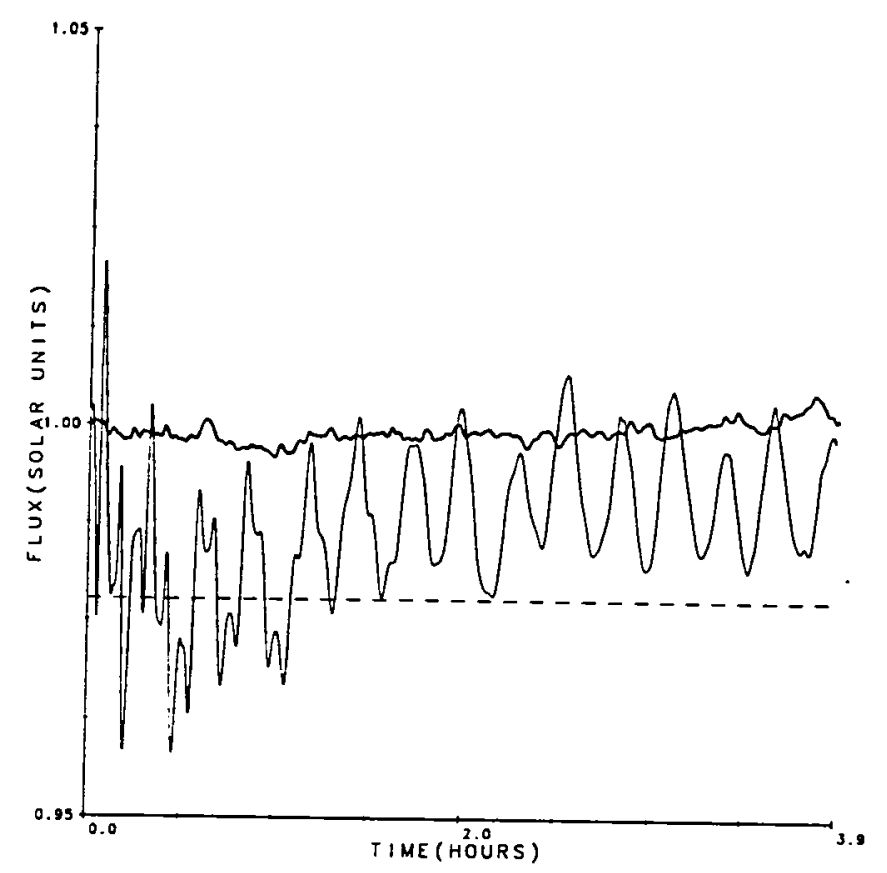

Fig.2

Vector Potential F:0001T: 1.84428

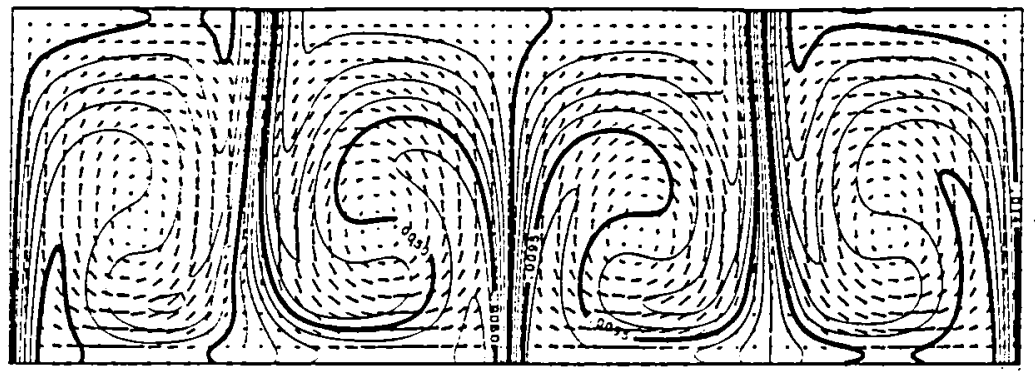
Vector Potentiol F:000iT:
$2.434 \overline{45}$

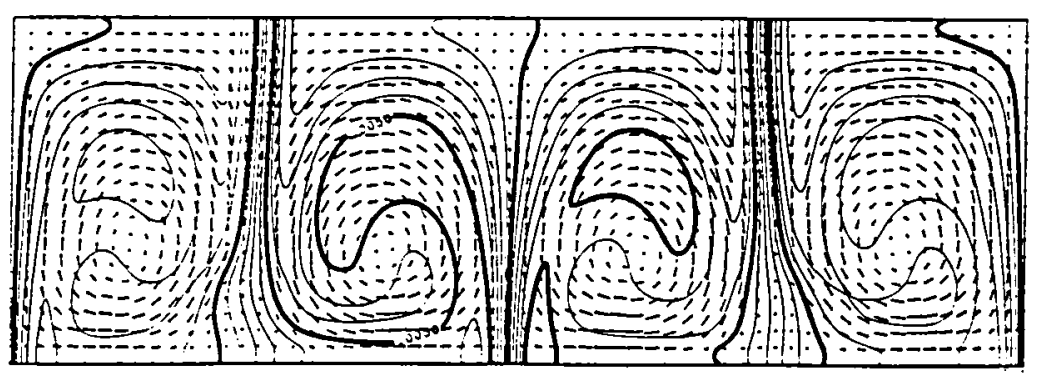

$\frac{\operatorname{Velocit}}{.33}$

Fig.3 
Fig.4

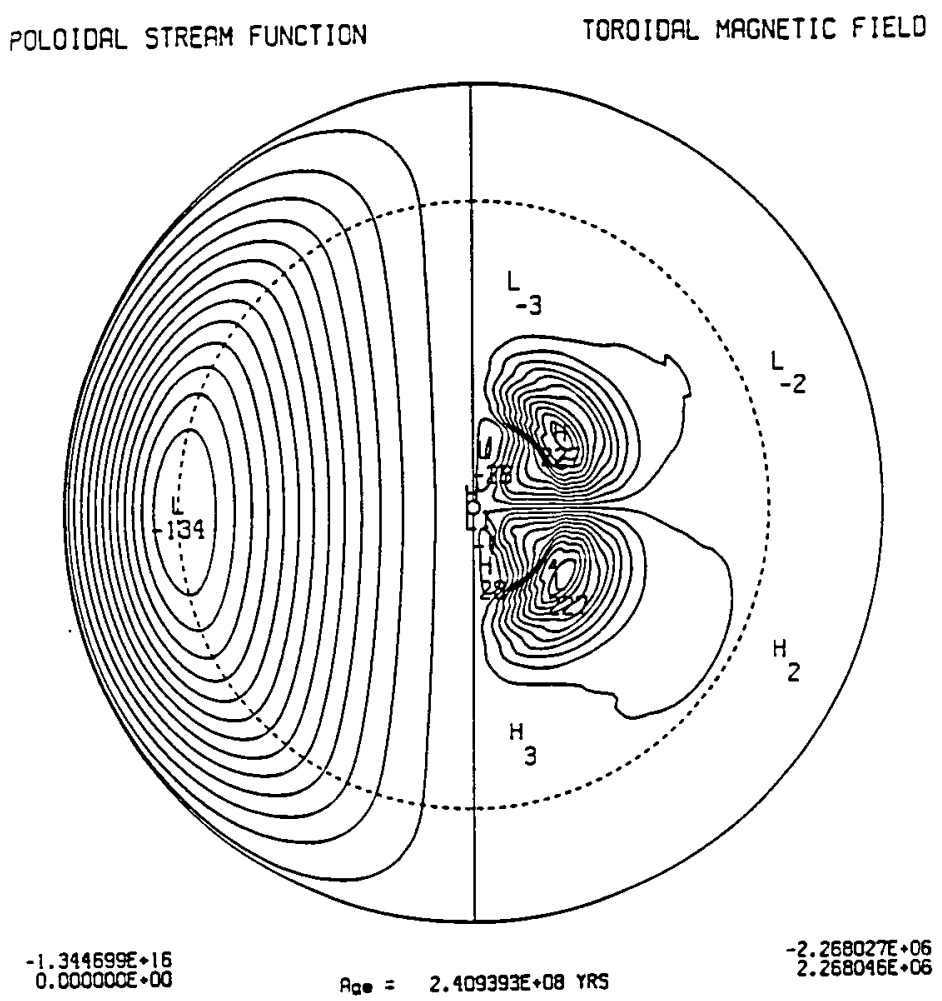

Fig.5

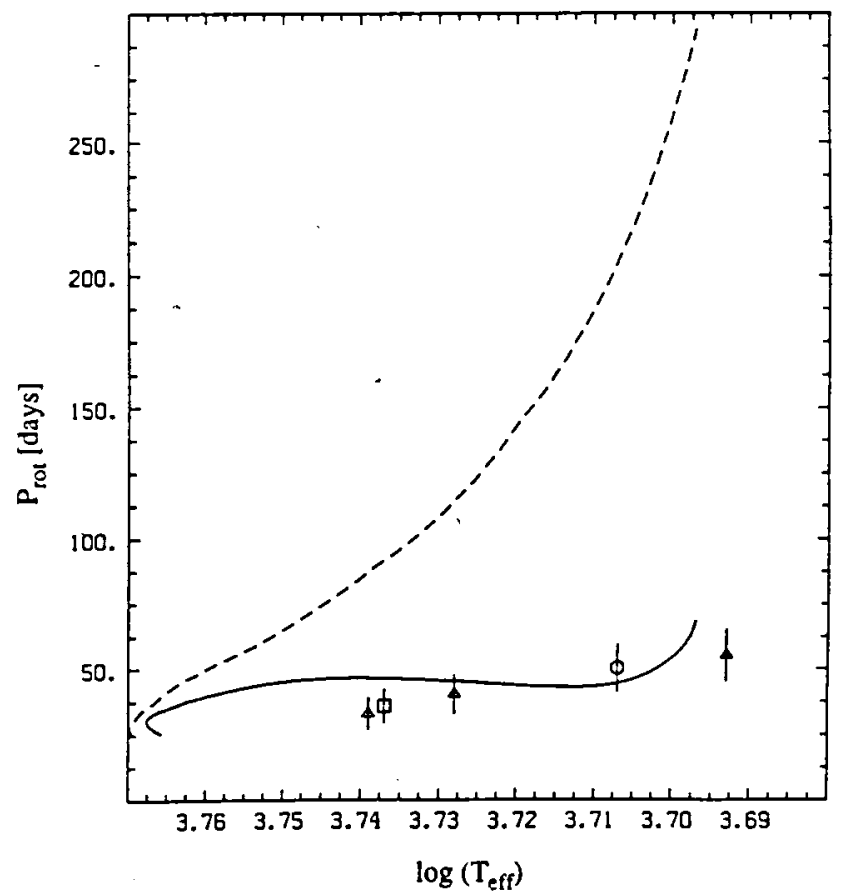




\title{
AH ESTIYATE OR CBANGES IN THE SUN'S TOTAL IRRADIANCE CAUSED BY UV IRRADIANCE VARIATIONS FROM 1874 TO 1988
}

\section{J. Lean}

E. O. Hulburt Center for Space Research, Naval Research Laboratory, Washington, DC 20375.

\begin{abstract}
Enhanced emission from bright solar faculae is a source of significant variation in the sun's total irradiance. Relative to the emission from the quiet sun, facular emission is known to be considerably greater at UV wavelengths than at visible wavelengths. Determining the spectral dependence of facular emission is of interest for the physical insight this may provide to the origin of the sun's irradiance variations. It is also of interest because solar radiation at $\lambda<300$ $\mathrm{nm}$ is almost totally absorbed in the earth's atmosphere. Depending on the magnitude of the UV irradiance variations, changes in the sun's irradiance that penetrates to the earth's surface may not be equivalent to total irradiance variations measured above the earth's atmosphere. Using an empirical model of total irradiance variations which accounts separately for changes caused by bright faculae from those associated with dark sunspots, the contribution of UV irradiance variations to changes in the sun's total irradiance is estimated during solar cycles 12 to 21 .
\end{abstract}

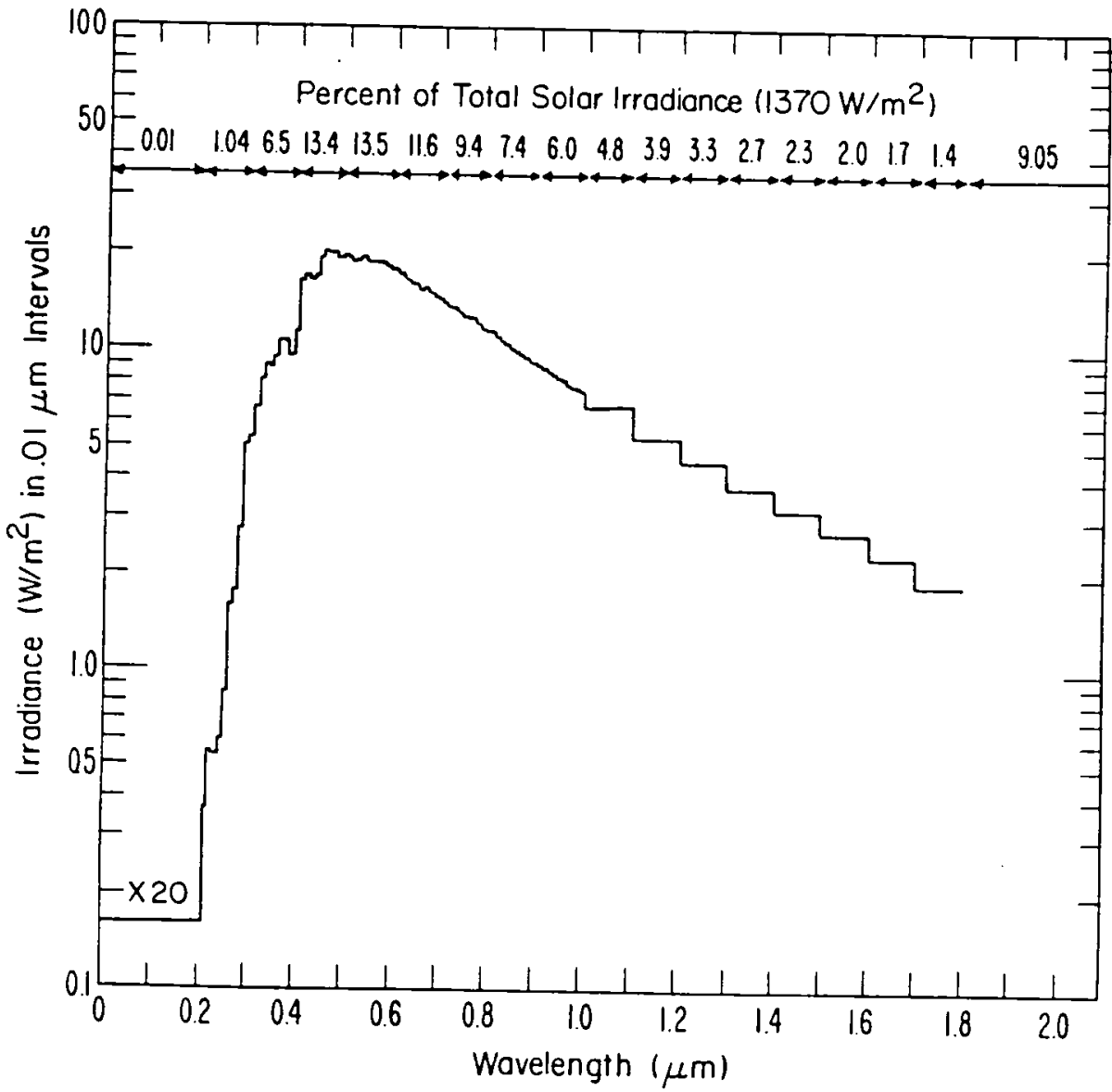

Fig. 1. Solar spectral irradiance, showing the percentage of the total irradiance emitted in specific wavelength intervals. 
As illustrated in Figure 1, approximately half of the sun's total irradiance is emitted at wavelengths between 400 and $800 \mathrm{~nm}$, with only $\sim 1 z$ of the irradiance emitted at $U V$ wavelengths less than $300 \mathrm{~nm}$. However, variations in the $U V$ portion of the sun's spectrum, which is formed higher in the sun's atmosphere than is the visible radiation, significantly exceed those at visible wavelengths. Estimates of UV irradiance variations during solar cycle 21 are shown in Figure 2. Like the total irradiance, the UV irradiances have their maximum values at times near maximum solar activity. However, the magnitude of the UV irradiance variations are more than an order of magnitude larger than the $\sim 0.082$ variation in the total solar irradiance that has been observed during solar cycle 21.1

Simultaneous observations during solar cycle 21 of the sun's total irradiance, $S$, and of the UV spectral irradiances at $205 \mathrm{~nm}$ and at $121.57 \mathrm{~nm}$ (HI La) are illustrated in Figure 3 . It has been shown recently, using data similar to that in Figure 3, that changes in the sun's energy at wavelengths from 200 to 300 $\mathrm{nm}$, although only 13 of the total radiative output, accounted for $19 z$ of the decrease in total irradiance from July 1981 to June $1985^{1}$. This is because at UV wavelengths the emission deficit in sunspots is negligible compared with enhanced emission from faculae, whereas at wavelengths between 400 and $800 \mathrm{~nm}$, where faculae contrast is much less than at UV wavelengths, the sunspot deficit and faculae enhancements are of the same order and therefore exert compensatory effects on the total irradiance.

Fig. 2. Solar UV spectral irradiances measured by the Solar U1traviolet Spectral Irradiance Monitor (SUSIM) ${ }^{2}$ on Spacelab-2 (upper panel), and an estimate of UV irradiance variability during solar cycle 21 (lower panel).
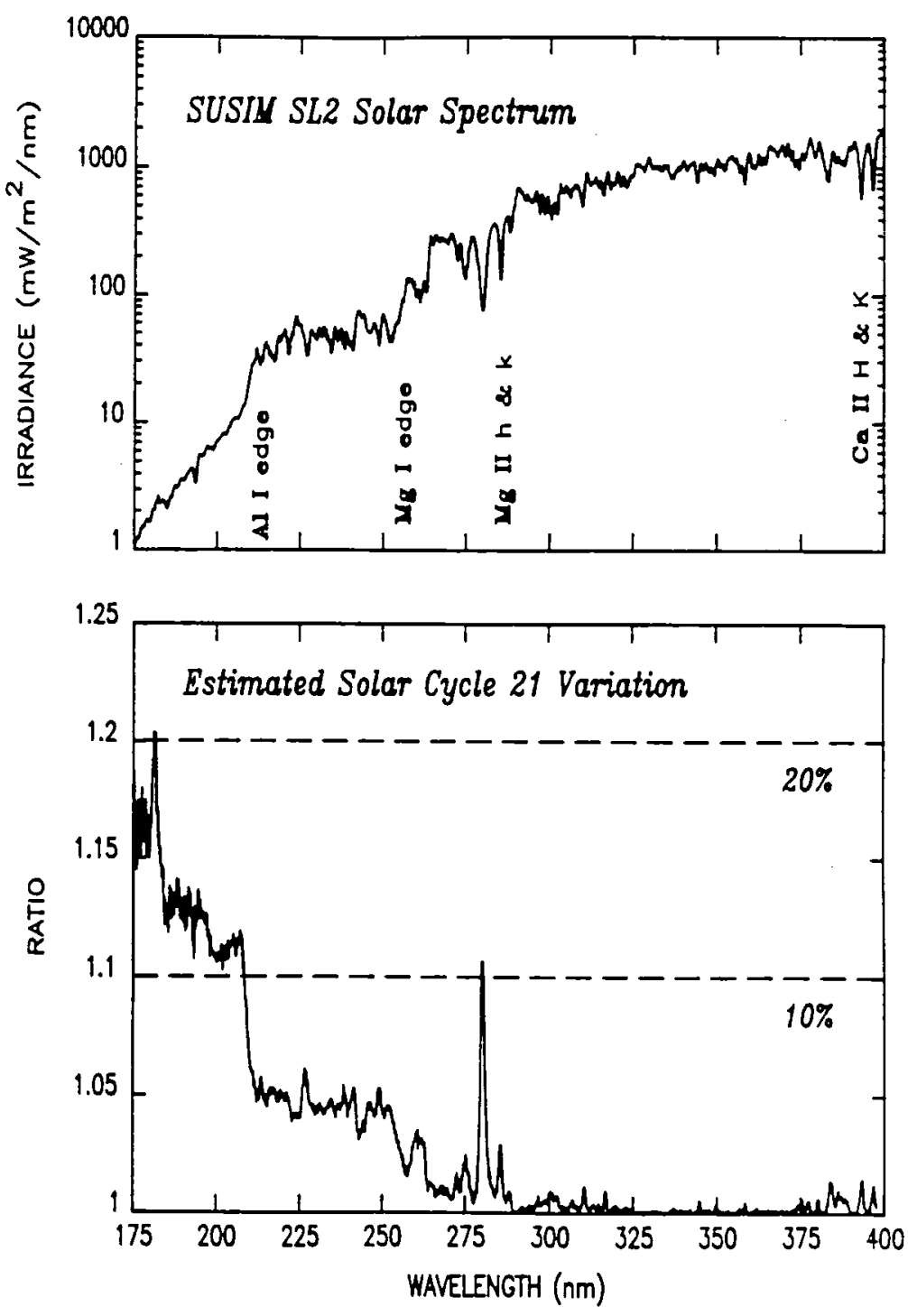


\section{EMPIRICAL MODEL OF TOTAL IRRADIANCE VARIATIONS}

Since the solar UV irradiances have been shown, during solar cycle 21 , to contribute significantly more to variations in the total irradiance than the 17 they contribute to the total irradiance itself, it is of interest to determine how changes in solar oV emissions have modulated total irradiance variations on historical time scales. This is investigated using an empirical model of the sun's total irradiance variations that accounts separately for the contribution of dark sunspots and bright faculae. ${ }^{3}$ In this model, which is described in detail elsewhere in these proceedings, 4 the sunspot blocking is determined directly from observations of the areas and locations of sunspots on the solar disc. Enhanced emission from bright faculae is estimated from a facular proxy via its correlation with a residual irradiance time series, S-Ps $-S_{0}$, calculated by subtracting the sunspot blocking from the measured total irradiances during times when both the proxy data and measurements of $S$ are available. To estimate bolometric facular emission during solar cycles prior to cycle $19,3,4$ monthly mean $R_{z}$ are used as the facular proxy.

Eig. 3. Variations during solar cycle 21 and the beginning of solar cycle 22 in a) the total solar irradiance, $S$, measured by the Active Cavity Radiometer (ACRIM) $^{5}$ on the Solar

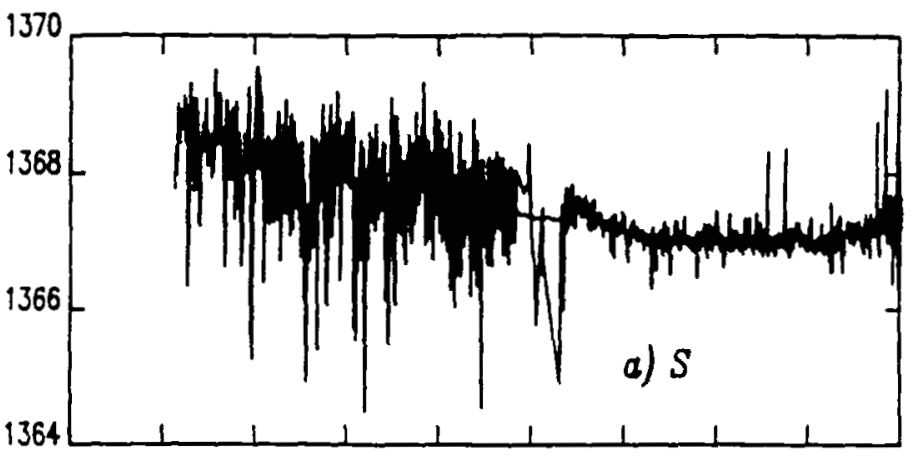
Maximum Mission satellite, b) the UV irradiance from 200 to $250 \mathrm{~nm}$, determined from the $\mathrm{Mg}$ Index empirical mode $1^{6}$ and $c$ ) the HI La irradiance at $121.57 \mathrm{~nm}$, measured by the solar Mesosphere Explorer (SME). ${ }^{7}$
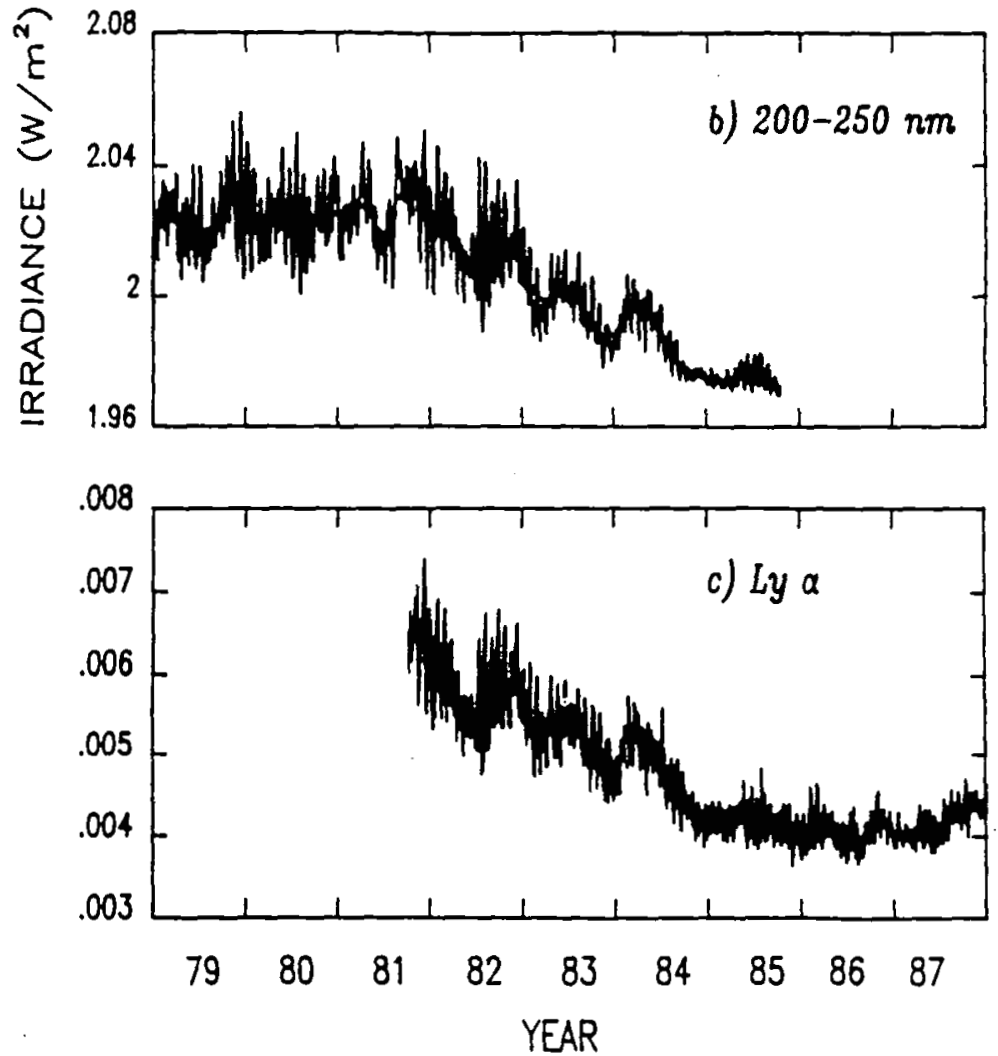
In order to partition the total facular brightness at all wavelengths into its UV and non-UV portions, it is necessary to establish the relationship between the two. Figure 4 illustrates that temporal variations in the measured irradiance residuals, $S-P_{S}-S_{O}$, are closely tracked by variations in the independently determined UV irradiances. This has been demonstrated elsewhere, over both solar rotation and solar cycle time scales, $1,3,4$ and is consistent with an understanding of the origin of the brightness source of total irradiance variations as being magnetic flux tubes; these carriers of solar activity extend from the photosphere, where the visible radiation is formed, to the top of the chromosphere, from where $L \alpha$ is emitted.
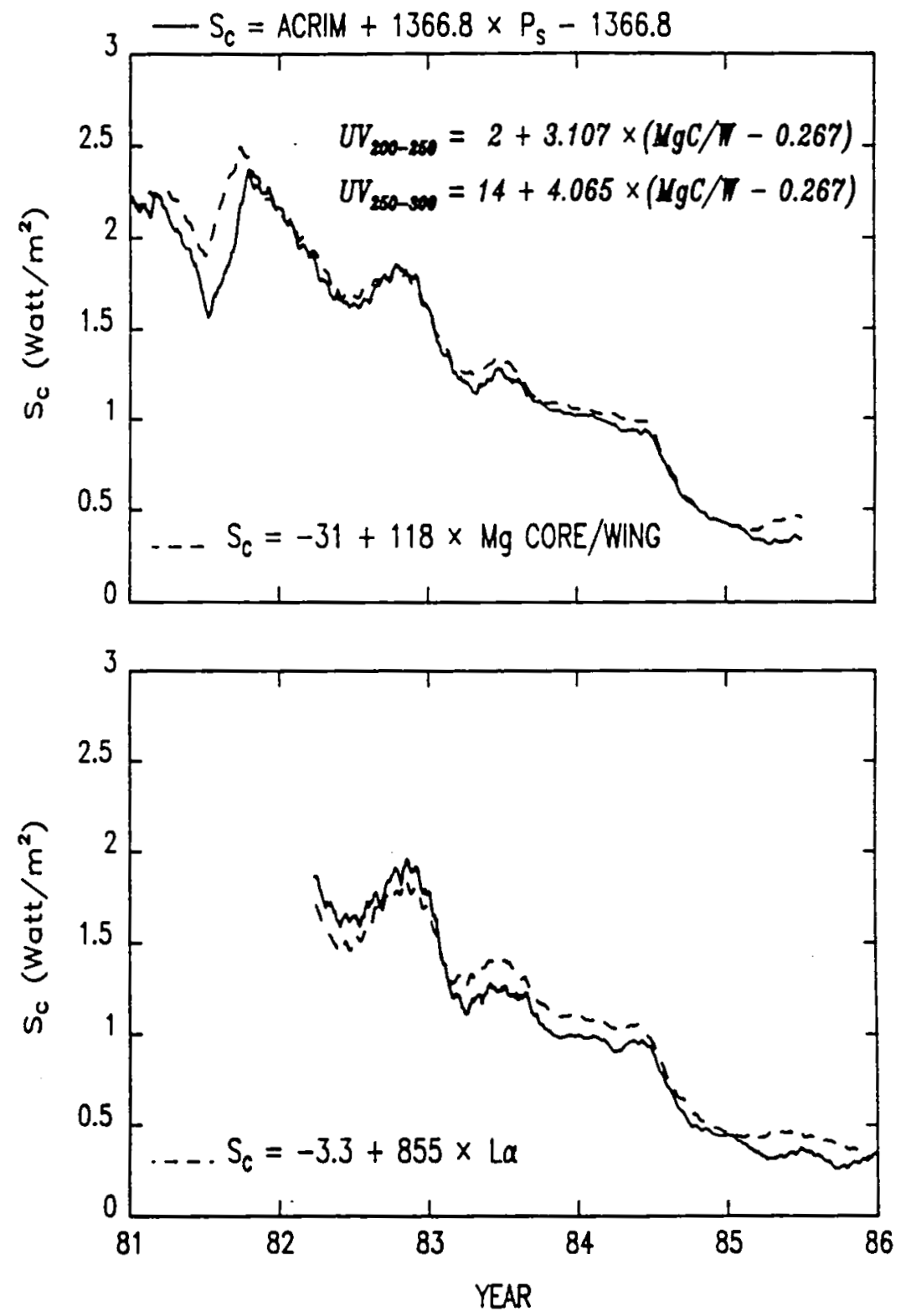

Fig. 4. Irradiance residuals, $\mathrm{S}-\mathrm{P}_{\mathrm{S}}-\mathrm{S}_{\mathrm{O}}$, derived from the total irradiance, $S$, measured by ACRIM, compared with a reconstruction of the residuals from the $\mathrm{Mg}$ Index (upper panel) and the La irradiance (lower panel). 


\section{ESTIMATED TOTAL IRRADIANCE VARIATIONS EXCLUDING ULTRAVIOLET IRRADIANCE VARIATIONS}

Because changes in the sun's UV emission are related, approximately linearly, to variations in the total irradiance facular emission, the UV portion of the enhanced facular brightness can be easily subtracted from the bolometric facular emission. The facular term, reduced by its UV component, $S-P_{S}-S_{O}-U V$, is then combined with $P_{S}$ to estimate variations in the sun's total irradiance, at wavelengths longward of $300 \mathrm{~nm}$. These variations are compared in Figure 5 with variations in the sun's total irradiance, determined from the same empirical model, but with the UV component of the facular emission retained. Figure 5 suggests that during solar cycles 12 to 21 , according to these calculations, the variation in the sun's total irradiance incident on the earth's surface was reduced, by as much as $20 \%$, from that at the top of the earth's atmosphere.

When the various solar activity time series, such as $R_{2}, F_{10.7}$ and the UV irradiances are studied closely, differences in their detailed temporal structures are evident. Because of this, the model calculation shown in Figure 5 must be considered as indicative only of the UVrelated component in the actual solar irradiance variations. Nevertheless, the results in Figure 5 suggest that in each cycle of solar activity since cycle 12, the UV emissions have contributed to the variability of the sun's total irradiance disproportionately to their fraction of the irradiance itself, and in such a way as to increase the amplitude in its 11 -year cycle.

Fig. 5. Percent changes in $S$, with and without the UV irradiance at 200 to $300 \mathrm{~nm}$ (upper panel), and the difference between them (lower pane1).
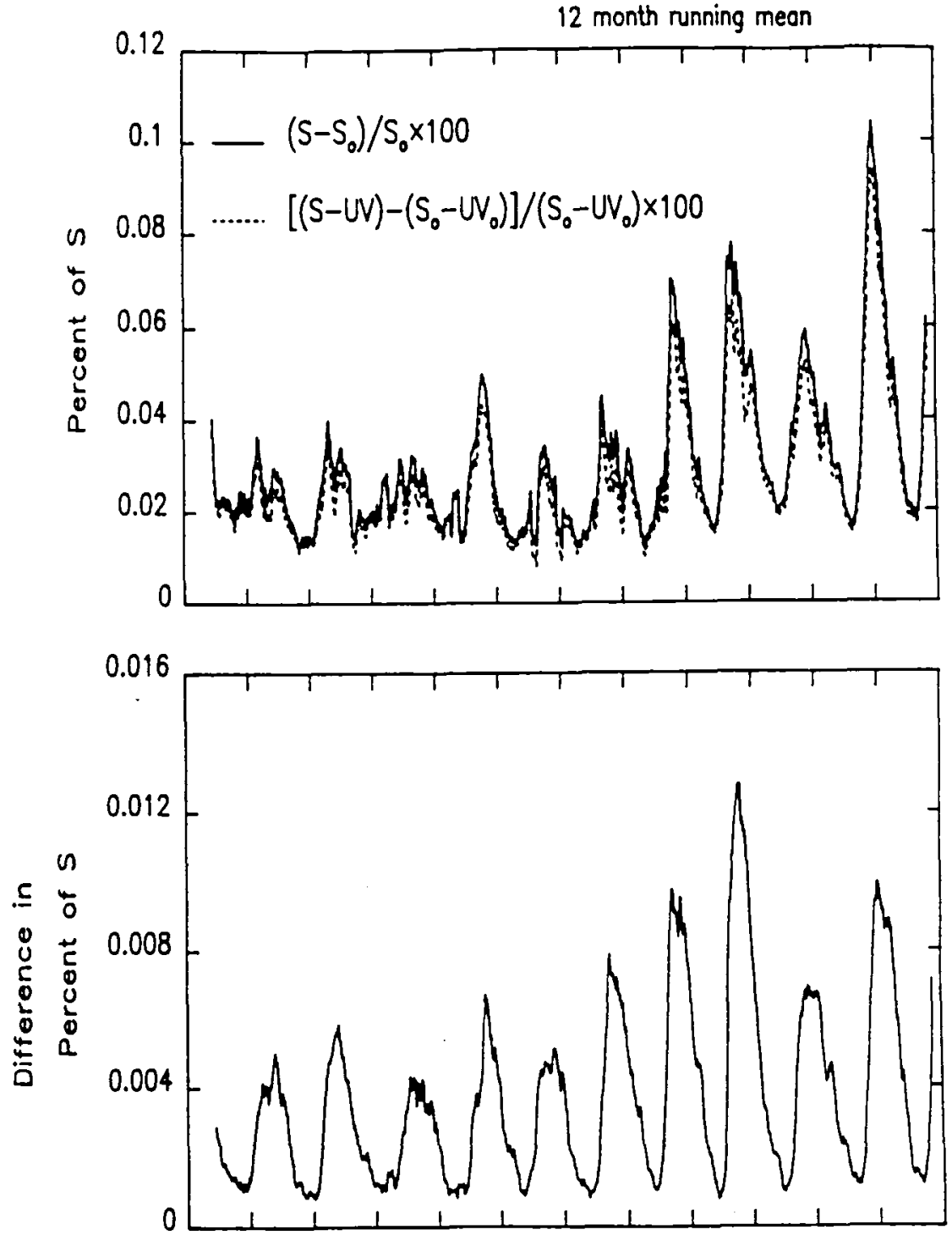

1870188018901900191019201930194019501960197019801990

YEAR 


\section{FUTURE MEASUREMENTS OF TOTAL AND UV SOLAR IRRADIANCES}

Understanding the role played by the sun's UV emission variations in the broader context of total irradiance variability will be improved when simultaneous observations of both the $U V$ and total irradiances are made during solar cycle 22. The reliability of future UV irradiance observations should exceed those made in solar cycle 21 which were hampered by wavelength dependent changes in instrument responsivity. It is planned, during solar cycle 22, to launch together on the Upper Atmosphere Research Satellite (UARS) three solar irradiance monitors, the Active Cavity Radiometer (ACRIM II), the Solar Ultraviolet Spectral Irradiance Monitor (SUSIM) and the Solar Stellar Irradiance Comparison Experiment (SOLSTICE). Figure 6 is a schematic of sUSIM. With multiple optical elements, the radiometric redundancy designed into the SUSIM experiment will allow detailed on-board monitoring of instrument responsivity. Data collected by these instruments will significantly improve our understanding of the magnitude and temporal variability of the sun's total irradiance, its UV spectral irradiance, and the interconnection between them.

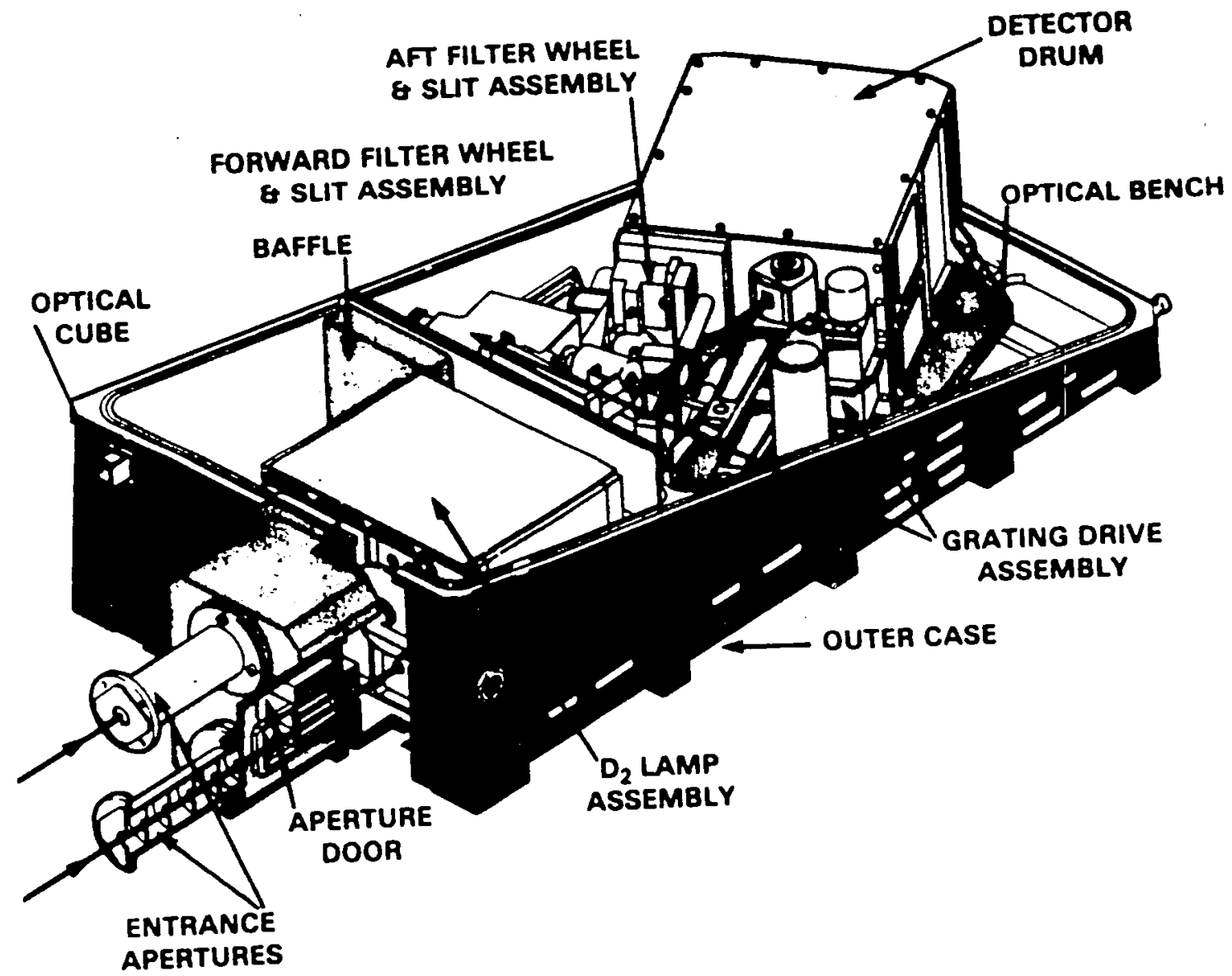

Fig. 6. NRL's SUSIM instrument, to be launched on the UARS satellite in mid 1991. 


\section{REFERENCES}

1. J. Lean, "Contribution of ultraviolet irradiance variations to changes in the sun's total Irradiance", Science, 244, 197, 1989.

2. M. E. VanHoosier, J.-D. F. Bartoe, G. E. Brueckner and D. K. Prinz, "Absolute solar spectral irradiance $120 \mathrm{~nm}-400 \mathrm{~nm}$ (Results from the Solar Ultraviolet Spectral Irradiance Monitor - SUSIM- experiment on board Spacelab 2", Astro. Lett. and Communications, 27, 163, 1988.

3. P. Foukal and $J$. Lean, "An empirical model of total solar irradiance variation between 1874 and 1988". Science, 247, 505, 1990.

4. J. Lean and P. Foukal, "Solar cycle modulation of total irradiance: An empirical model from 1874 to $1988^{\prime \prime}$, these proceedings.

5. R. C. Willson and H. S. Hudson, "Solar luminosity variations in solar cycle 21", Nature, 332, 810, 1988.

6. D. F. Heath and B. M. Schlesinger, "The Mg $280-\mathrm{nm}$ doublet as a monitor of changes in solar ultraviolet irradiance", J. Geophys. Res.. 91, 8672, 1986.

7. G. Rottman, "Results from space measurements of solar UV and EUV flux", in Solar Radiative Output Variations, Proceedings of a Workshop held Nov. 9-11, NCAR, Boulder, CO., edited by P. Foukal, p. 71, 1987. 
USING THE BOUNDARY CONDITIONS OF SUNSPOTS AS A TECHNIQUE FOR MONITORING SOLAR LUMINOSITY VARIATIONS

by

Douglas V. Hoyt

Research and Data Systems, Corp.

7855 Walker Drive, Suite 460

Greenbelt, MD 20770

Abstract. Recent satellite observations of the solar total irradiance confirm that it is varying with at least on the 11 year time scale. Both blocking by sunspots and re-emission by faculae are components in this variation, but changes in the temperature of the solar photosphere may also be a contributing component. The satellite observations are as yet of insufficient length to answer the question of whether the sun is varying in luminosity on time scales longer than the 11 year sunspot cycle. This paper examines proxy methods of re-constructing these longer term luminosity variations, with an examination of secular changes in sunspot structure as one tool. Solar rotation changes and solar diameter changes are other parameters which may reveal information about solar luminosity variations. All three variables give remarkablysimilar conclusions. Over the last century the Earth's surface temperatures and the structure of sunspots have varied. in a parallel manner. It is hypothesized that sunspots are embedded in a convective medium which itself is varying over long time periods. These variations in convective strength alter the boundary conditions on sunspots and hence cause their structure to vary. Simultaneous with the variations in convective strength, the solar luminosity will vary as well which, in turn, leads to changes in climate of the Earth. Variations in solar diameter and solar rotation support the hypothesis that solar luminosity has varied over the last century and reached a peak around 1925 to 1935. This evidence is reviewed along with a possible model of why sunspot structure may provide a good proxy measure of solar luminosity changes. 
". . . the whole surface [of the sun] seemed to be a flat [i.e., penumbra] of immense extent." William Herschel (Feb. 12, 1800)

"I am much inclined now to believe that openings[sunspots], ridges[faculae], nodules, and mottlings[granules] may assist us possibly to expect a copious emission of heat, and therefore mild seasons." William Herschel (Jan. 15, 1801)

\section{Introduction}

Sir William Herschel observed the sun from 1785 to 1837 , a period of 52 years, occasionally on nearly daily basis but at other times only after lapses of months or years. His notebooks from which the above two quotes are taken provide some of the most detailed observations of the sun in the period around 1800 . Although he is noted for hypothesizing that the solar radiant output varied and that it affected climate as the second quote testifies, he also noted that the structure of sunspots varied. Unfortunately his observations of sunspot structure are not sufficiently detailed to deduce if there was any long-term variations in their structure. Day to day variations however were often noted. His notebooks are sufficiently detailed that they indicate a prolonged solar minimum from about 1806 to 1811-12. Perhaps it just a coincidence that the period 1810-1816 appeared to be markedly cold in many regions of the Earth (e.g., see Groveman and Landsberg, 1979).

Does the solar total irradiance vary on time scales longer than 11 years? Does sunspot structure provide a method of deducing what these longer term variation might have been? Are these longer term changes connected to long term changes in the climate of the Earth? This paper will review the hypothesis that there is a such a connection and provide further supporting physical and statistical reasons why the hypothesis that sunspot structure gives a proxy measure of solar luminosity variations is reasonable.

\section{Review of the hypothesis}

Although only an eleven year cycle in total solar irradiance variations have been identified so far (e.g., Hoyt and Kyle, 1990), there remains the possibility that longer term variations may exist. In 1979 Hoyt hypothesized that such longer term variations did exist and could be reconstructed through the use of measurements of secular changes in sunspot structure. The basic hypothesis is as follows: As the strength of solar convection increases, the solar luminosity increases. Sunspots which are

embedded in the convective zone respond to the changes in convection with increased convection causing the penumbra of sunspots to be less extensive. Sunspot structure can be normalized by taking the ratio of the umbral areas to whole spot areas ( $u / w$ ) or the ratio of umbra areas to penumbral areas $(u / p)$. The time history of the variation of these ratios provides a time history of the solar convective strength and solar luminosity and therefore 
should be correlated with Earth temperatures. The original paper by Hoyt (1979) found a correlation to the Northern Hemisphere temperature time series of Budyko (1969) and Angell and Korshover (1975) of 0.57 . More details are provided in that paper.

U/W Ratio and De-trended Global Temps.

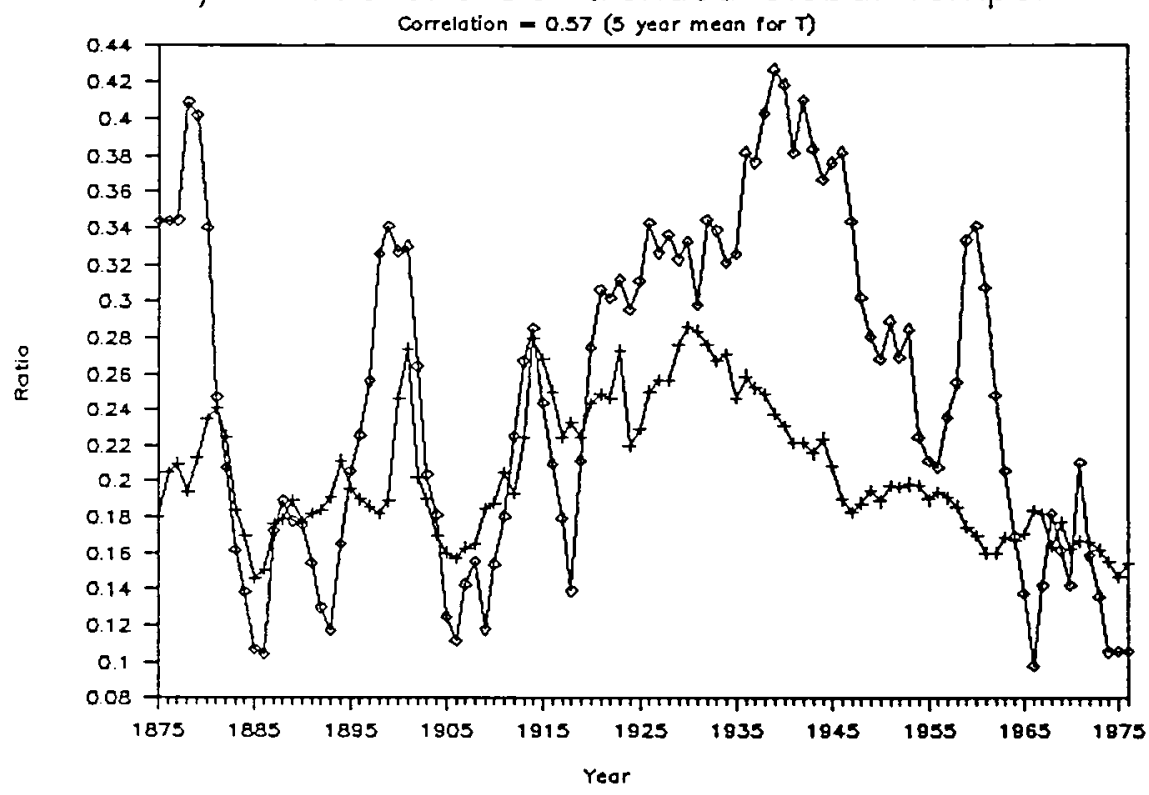

Figure 1. A plot of the Greenwich Observatory sunspot structure $(u / w)$ and the five year running mean smoothed Hansen and Lebedeff global temperatures with $0.6 \mathrm{C}$ added to place the two plots on the same scale. The correlation of the two curves is 0.57 .

Using the global temperature values of Hansen and Lebedeff (1987), the correlation to $u / w$ equals 0.26 . Spencer and Christy (1990) show that the Southern Hemisphere surface temperatures are not reliably measured so it is not surprising that the correlation would drop by going from Northern Hemisphere data set to a global data set. If the global temperature is de-trended to remove a trace gas and/or urban warming effects and five year running means of temperature are used (see Figure 1), the correlation is 0.57. The solar forcing hypothesis only explains variation in the temperature of a long-term nature. The short term variations are arising largely from internal chaotic behavior in the climate system such as El Ninos (e.g., see spencer and Christy) or interannualar cloud cover fluctuations (Hoyt, 1976).

The trends in sunspot structure shown in Figure 1 persist no matter what subset of the total sunspots used. Sunspots stratified by size, by type, or by Greenwich observer all have the same characteristic long-term variation shown in the figure. $A$ comparison of one Greenwich observer to another using one-way analysis of variance techniques shows that in all but one case out of 82 observers the Greenwich observers derive the same sunspot structure. A typical one standard deviation uncertainty in yearly 
mean $u / w$ values is about 0.003 whereas the mean values range from about 0.15 to 0.25 or 33 standard deviations. There is no reason to believe that sunspot structure does not change with time.

Although the theory of sunspot structure remains poorly explained, some idea of what is going on may be deduced from the sunspot model of Pizzo (1986). Figure 2 from that paper shows the contours of equal sunspot opacity and equal temperature. The two contours are nearly parallel so a small change in temperature, for example, would lead to a relative large change in sunspot structure. The location of the outer sunspot boundary is defined by the intersection of the optical depth equals one contour and a temperature contour. It would appear therefore that the change in sunspot structure is caused mostly by a change in the penumbras with little effect on the umbras. Thus, the outer boundary conditions on the sunspot apparently provide information on solar convection and luminosity. Each sunspot can be viewed somewhat as a thermometer embedded in the sun. Although no one sunspot provides a good measure of the convection, when large numbers of sunspots are used, the state of the convective strength can be monitored. That there do appear to be changes in the convective strength are supported by the works of Livingston (1982) using solar line bisectors and Kuhn et al. (1988) using limb brightness data.

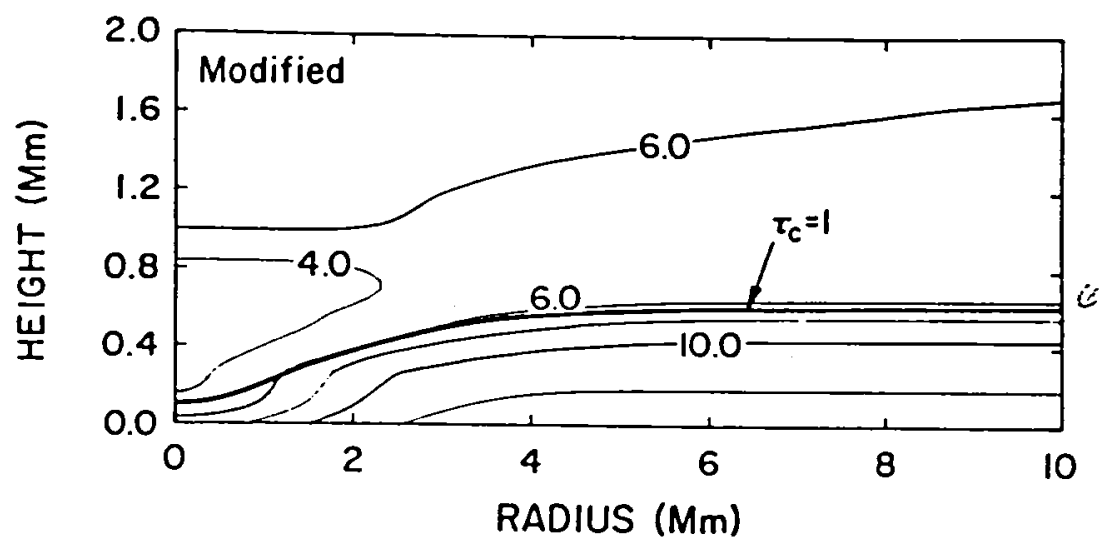

Figure 2. A model for sunspot structure from Pizzo (1986). The closely parallel contours of temperature and optical depth suggest a small change in photospheric temperature or solar luminosity will manifest itself as a large change in sunspot structure.

3. Variations in solar luminosity longer than 11 years?

There are several solar parameters which vary on the time scale of 70 to 90 years which may be associated with luminosity variations on the same time scale. Besides sunspot structure, there are variations in solar diameter (Gilliland, 1982) who deduced there was a maximum in solar luminosity in 1930 (close to the 1932 peak deduced from sunspot structure). In addition 
the solar rotation became most like a rigid body for the 1924-1935 solar cycle. The slowest equatorial rotation rates occurred in the 1912-24 solar cycle. The Gleissberg cycle of 80-90 years, the Maunder minimum (Eddy, 1976), and ice core oxygen isotope variations (e.g., Hibler and Johnsen, 1979) all suggest that solar luminosity variations longer than 11 years may be present.

Consider variations in solar rotation. Using single sunspots whose positions were measured at the Royal Greenwich observatory (RGO), the solar differential rotation can be calculated for each solar cycle and for each hemisphere of the sun. The results of this analysis are summarized in Table 1 . The equatorial

Table 1. The differential rotation of the sun in degrees per day assumed to be of the form $a+b * \cos$ (lat) for each solar cycle and for Northern Hemisphere and Southern Hemisphere single spots. $\mathrm{N}$ is the number of pairs of observations used.

\begin{tabular}{|c|c|c|c|c|c|c|c|c|c|c|}
\hline \multicolumn{7}{|c|}{ Northern Hemisphere } & \multicolumn{4}{|c|}{ Southern Hemisphere } \\
\hline & $\mathrm{N}$ & $\mathrm{a}$ & & b & s.d. & $\mathrm{N}$ & 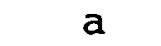 & s.d. & b & s.d. \\
\hline & 1286 & 533 & & -3.08 & 0.298 & 1625 & 14.514 & 0.022 & -2.88 & \\
\hline & 1545 & 563 & 023 & & & 1664 & & 23 & & \\
\hline & 97 & & & & & 992 & & & & \\
\hline $312-2$ & 1704 & & & & & 1574 & & & & \\
\hline 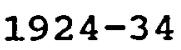 & 1610 & & 020 & & & 13 & & & 56 & \\
\hline & 1825 & & 17 & & & 18 & & & & \\
\hline & 2313 & & 17 & & & 22 & & & & \\
\hline & 2560 & & 0.014 & & & & & & -3.31 & \\
\hline & 2473 & & 0.017 & & & & & & & \\
\hline
\end{tabular}

Table 2. The equatorial rotation of the sun using single spots observed between +5 and -5 degrees solar latitude of the equator. The single spots are further selected to be within 60 degrees of longitude of the central meridian.

\begin{tabular}{crc} 
years & \multicolumn{1}{c}{$N$} & deg./day \\
$1879-1889$ & 673 & 14.586 \\
$1889-1902$ & 824 & 14.588 \\
$1902-1911$ & 728 & 14.511 \\
$1912-1924$ & 751 & 14.453 \\
$1924-1934$ & 715 & 14.482 \\
$1934-1944$ & 763 & 14.492 \\
$1944-1954$ & 1153 & 14.462 \\
$1954-1965$ & 1054 & 14.536 \\
$1965-1976$ & 1050 & 14.479
\end{tabular}

rotation rate in degrees per day shows long term variations with a minimum around 1924-34. The differential rotation also reached a minimum meaning the sun appeared to rotate more like a rigid body then at other times. The differential rotation is arising in some as yet not fully understood manner from the interaction of 
convection and viscosity. If the surface rotation is varying, the convection strength is probably also varying and therefore the luminosity would be varying as well. Using single sunspots within 5 degrees of the equator as tracers (Table 2), the equatorial solar rotation reached a minimum for the century one cycle earlier than the minimum in differential rate (i.e., 1912-24). Also within each 11 year cycle, the solar rotation appears more like a rigid body toward solar maximum which recent satellite observations indicate is time of maximum solar luminosity.

To show that the change in equatorial rotation was not an artifact of the fitting procedure, the single sunspots within 5 degrees of the equatorial were used to calculate the solar rotation rate. Table 2 shows that the same time history as the other analysis.

4. Predictions of the hypothesis and tests for its validity

This paper has presented some reasons why the hypothesis that variations in sunspot structure could provide a proxy measure of solar luminosity variations. If true, then other consequences will follow. For example, if one solar hemisphere is persistently more active then the other hemisphere for an extended time period, $u / w$ would be greater for that hemisphere. As another example, if one assumes the hypothesis is true, then for times of high solar luminosity, the penumbras would shrink relatively. As a consequence the fraction of large spots to all spots could be expected to decrease during these times (such as the early 1930's). Finally consider stars cooler than the sun such as $K$ and $M$ stars. Cool stars could be expected to have less convective pressure on sunspots then the sun, so these star-spots should have extended penumbras. For sufficiently cool stars, the whole surface may appear to be mostly penumbra with the consequent chaotic fields and absence of highly polarized light.

5. Concluding remarks

Variations in sunspot structure, solar diameter, solar rotation, and Earth surface temperatures have all shown a parallel behavior over the last century. One plausible connection between these time series is that the solar convective strength and hence solar luminosity is varying on these time scales as well. Indeed it would be hard to imagine how sunspot structure could change without any accompanying change in other solar parameters, particular luminosity. Kuhn et al., Livingston, and others have presented supporting evidence that there are changes in the convective strength of the sun.

A picture of long-term solar variations could be constructed as follows. Convective strength and solar luminosity increased from the late 1800's to a peak around 1930-35 and decreased steadily until at least 1976. Near the peak in solar luminosity, sunspots had their least extensive penumbras, the solar diameter was decreasing at its maximum rate, the sun was rotating more like 
a rigid body then at other times, and the equatorial rotation rate had just recently had its slowest rate of the century. Shortly thereafter the surface temperatures of the Earth reached a maximum.

Sunspot structure could be measured for sunspots after 1976 when the RGO stopped measurements. Older measurements may also provide a mechanism to extend the time history of sunspot structure. There are ample tests which could be performed to test the hypothesis that sunspot structure can be used as a proxy to measure long-term solar luminosity variations. Many of these tests were presented in the earlier paper (Hoyt, 1979) and as yet have not been tested.

\section{References:}

Angell, J.K. and J. Korshover, 1977. Estimate of the global change in temperature, surface to $100 \mathrm{mb}$ between 1958 and 1975. Mon. Wea. Rev., 105, 375-385.

Budyko, M. I., 1969. The effect of solar radiation variations on the climate of the Earth. Tellus, 21, 611-619.

Eddy, J.A., 1976. The Maunder minimum. Science, 192,1189-1200.

Gilliland, R.L., 1982. Solar, volcanic, and $\mathrm{CO} 2$ forcing of recent climatic changes. Climatic Change, 4, 111-132.

Groveman, B.S. and H.E. Landsberg, 1979. Simulated Northern Hemisphere temperature departures, 1579-1980. Geophys. Res. Lett., 6, 767-769.

Hansen, J., and S. Lebedeff, 1987. Global trends of measured surface air temperature. J.G.R., 92, 13345-13372.

Hibler, W.D. and S.J. Johnsen, 1979. The 20-yr cycle in Greenland ice core records. Nature, 280, 481-483.

Hoyt, D.V., 1976. Interannualar cloud cover variations over the contiguous United States. J. Appl. Meteor., 17, 354-357.

Hoyt, D.V., 1979. Variations in sunspot structure and climate. Climatic Change, 2, 79-92.

Hoyt, D.V., and H.L. Kyle, 1990. An alternative derivation of Nimbus 7 total solar irradiance variations. Conference on climate Impact of Solar Variability.

Kuhn, J.R., K.G. Libbrecht, and R.H. Dicke, 1988. The surface temperature of the sun and changes in the solar constant. Science, 242 , 908-910.

Livingston, W.C., 1982. Magnetic fields, convection and solar luminosity variability. Nature, 297, 208-209. 
Pizzo, V.J., 1986. Numerical solution of the magnetostatic equations for thick flux tubes, with application to sunspots, pores, and related structures. Ap. J., 302-785-808.

Spencer, R. and J.R. Christy, 1990. Precise monitoring of global temperature trends from satellites. Science, 247, 1558-1562. 


\title{
ENERGY TRANSPORT BELOW SUNSPOTS AND FACULAE
}

\author{
Kenneth H. Schatten and Hans G. Mayr, \\ Laboratory for Atmospheres \\ NASA/Goddard Space Flight Center \\ Greenbelt, MD 20771
}

\section{INTRODUCTION}

In the classical view of sunspot cooling, Biermann (1941) suggested that convection within sunspots is suppressed by the magnetic field. This would lead to a lower temperature in the umbra. Parker (1974) first noted a difficulty with this model, suggesting that the energy suppressed by the magnetic field should give rise to a bright ring around the sunspot, which is not observed. As an alternative to Biermann's field inhibition mechanism, Parker (1979) later examined the dynamical cooling for sunspots. He showed that in the Sun's upper convection zone, the "superadiabatic" environment is such that downflows with velocities of several $\mathrm{km} \mathrm{s}^{-1}$ could lower the temperature sufficiently to cool sunspots by $1000 \mathrm{~K}$. Such an inflow and downflow pattern had also been suggested by Meyer et. al. (1974) in order to resolve the problem with the physical stability of sunspots. These authors accepted the Biermann mechanism and proposed also an upflow pattern surrounding the sunspot, leading to its dissolution, but they did not examine the temperature structure. Recent support for a flow pattern has been found by Zirin and Wang (1989) who measured proper motions of $0.5 \mathrm{~km} / \mathrm{sec}$ from bright features seen in the penumbral region of sunspots, which then disappeared into the umbra.

The high velocity flows that Parker suggested were comparable to those observed by Frazier (1970) and Deubner (1976) within darkened solar features. However, more recently, these high velocities have been viewed as an observing artifact (Beckers, 1976; Miller, Foukal, and Keil, 1984; Cavallini, Ceppatelli and Righini, 1985), and it was shown that smaller vertical flows with speeds of order $10 \mathrm{~m} \mathrm{~s}^{-1}$ can be supported by the observations. Significant cooling from such low velocity flows was suggested by Schatten and Mayr (1985). The energetics of sunspots was compared to that of terrestrial hurricanes, in which latent energy is released in the transition of water vapor to liquid water. For sunspots it was suggested that the latent energy in the ionization of hydrogen could fuel the dynamical cooling. We found that dynamical cooling could occur with particle fluxes of $2 \times 10^{21}$ particles $\mathrm{cm}^{-2} \mathrm{~s}^{-1}$, corresponding to small downflow velocities of only $2 \mathrm{~m} \mathrm{~s}^{-1}$ at $2,000 \mathrm{~km}$ depth, thereby "rejuvenating" the Parker mechanism. In the present paper we shall reexamine this mechanism and adopt vertical velocities to compute the temperature distributions below sunspots and faculae.

In the flow model of Schatten and Mayr, the amount of material moving downwards below sunspots is replenished by material moving upwards in the surrounding active region, and the associated energy transport can power faculae. The vertical velocities are perceived to be part of a three dimensional circulation in which energy is essentially redistributed between sunspots and faculae: suppressing convection in sunspots, but aiding the heat transport to form faculae. The magnetic field plays the role of guiding these flow patterns. With this picture, the energy deficit in sunspots and the excess energy released in faculae would be in rough balance and indeed this is observed (e.g., Chapman et al., 1984).

Schatten and Mayr (1985) suggested that faculae could have a "hillock" (50 - 200km high) structure, formed as a result of the enhanced scale height and buoyancy of the hot gases below faculae. The suggested flow mechanism and the resulting hillock geometry of faculae are markedly different from the classical field inhibition mechanism in which faculae are perceived to form wells. In an attempt to distinguish between the two models, Schatten et al. (1986) concentrated on studying faculae. Faculae are virtually unobservable at disk center, but show generally an increasingly brighter contrast as they progress in their journey towards the limb. Very near the limb, their contrast may decrease slightly due to obscuration effects from the surrounding photospheric gases; however, they are still significantly brighter than the limb darkened gases. Sunspots on the other hand, with a dark appearance, are viewed most prominently at disk center.

With this picture, Schatten et al. then developed a conical "hillock" model against which facular contrasts could be compared. In this model, all the photospheric isotherms and isobars were vertically elevated into a $200 \mathrm{~km}$ conical hillock shape, ignoring 
the presumed small temperature changes at the surface that must be associated with the perceived expansion of the facular gas. This model is analogous to the addition of heat applied to a pot of boiling water. This will not significantly raise the temperature, but simply distort the otherwise flat surface. For the photosphere, the distorted geometry enhances the emission by increasing the gradient in temperature and density that the "pencils of radiation" must pass through in exiting the sun. This model provided reasonable agreement with observations at four widely differing continuum wavelengths from 4,000 to 10,000 $\AA$. Further, the disk central low contrast of faculae occurred naturally in this model, since a ray path exiting the facula normally would encounter the same density and temperature profile as a ray path exiting the photosphere normally. Recently, Schatten et al. (1989) also found a statistical relation between sunspot areas and facular plage size, in that faculae appear to "blanket" sunspot areas when they approach the Sun's limbs, consistent with the hillock geometry.

To begin with, in Section 2, we discuss the physical basis for our one dimensional "dynamical" model. The dynamics enters into the present calculations only through the energy equation. Magnetic fields have not been included for three reasons: 1) Static fields do not directly affect the energy balance. 2) Uniform fields, additionally, do not affect the vertical momentum balance. 3) To include fields would require a three-dimensional model, taking into account their complex interaction with a non-uniform convecting plasma, which is beyond the scope of our simplified model. In Section 3, three computer runs are discussed which illustrate the model's behavior. Our model requires some disturbance, and we consider field inhibition to initiate the motions. Thus in Section 4, Biermann's mechanism is considered to suggest that sunspot-field inhibition can complement dynamical cooling. We conclude with a discussion of our findings and suggest new observations that may serve to test the model. We shall emphasize that our one dimensional model cannot describe many of the details in the facular emission such as the brightness relationships discussed by Spruit and Zwaan (1980).

\section{DYNAMICAL ENVELOPE MODEL}

Stellar envelope models of the Sun (Endal and Twigg, 1982; Cox and Giuli, 1968; Paczynski, 1969) consider the global scale energy balance to describe the vertical temperature and density distributions. Assuming hydrostatic equilibrium, the transfer of radiation is balanced by diffusive (convective) heat transport, with the diffusion coefficients taken from mixing length theory.

For our analysis we adapt the envelope code of Endal and Twigg (1982), but additionally allow for energy advection or flow heating/cooling. In this section, we show how the energy equation applies to a superadiabatic atmosphere with transport occurring via flows, eddy diffusion, and radiation. We assume that these processes occur sufficiently fast so that equilibrium is established and the time dependence can be ignored. In the following, the equations are discussed for an ideal gas, but later, modifications will be made for the non-ideal gas of the real solar atmosphere. For the energy balance of an ideal gas, the change in internal energy can be written (Gill, 1982):

$$
\nabla \cdot \vec{F}^{\mathrm{rad}}+\nabla \cdot \rho E \vec{U}+p \nabla \cdot \vec{U}=-\frac{\partial(\rho E)}{\partial t} \approx 0
$$

where $E$ is the internal energy per unit mass; $\rho$ is the density, $p$ is the pressure; $F^{\text {rad }}$ is the radiative energy flux, and $U$ is the velocity. Neglecting the kinetic energy of the bulk motion $1 / 2 U^{2}$, the internal energy can be written as $C_{v} T$, where $T$ is the temperature.

Considering mass conservation, the perfect gas law and hydrostatic equilibrium, equation (2.1) can then be written as

$$
\nabla \cdot \vec{F}^{\mathrm{rad}}+\rho \mathrm{C}_{\mathrm{p}} \overrightarrow{\mathrm{U}} \cdot \overrightarrow{\mathbf{S}}=0
$$

Here, the specific heat at constant pressure is $C_{p}=C_{v}+R$, and the potential (superadiabatic) temperature gradient is $S=\nabla T+$ $\Gamma$, where $\Gamma=\mathrm{g} / \mathrm{C}_{\mathrm{p}}$. We write $\mathrm{U}=\mathrm{W}+\mathrm{V}$, introducing the vertical and horizontal velocity components, in the vertical, $\mathrm{r}$, and horizontal, $x$, directions, respectively. In a stratified atmosphere, the vertical variations are normally much larger than the horizontal variations $\partial / \partial r>\partial / \partial x$. Similarly, the horizontal velocities are usually much larger than the vertical velocities, so that $\partial W / \partial r \approx \partial V / \partial x$. Thus, horizontal as well as vertical energy advection should be important. The energy equation then can then be written in simplified form:

$$
\frac{\partial \mathrm{F}^{\mathrm{rad}}}{\partial \mathrm{r}}+\rho \mathrm{C}_{\mathrm{p}} \mathrm{WS}+\rho \mathrm{C}_{\mathrm{p}} \mathrm{V} \frac{\partial \mathrm{T}}{\partial \mathrm{x}}=0
$$

where $S=\partial T / \partial r+\Gamma$ is the vertical component of the vector $S$.

In Equation (2.3), the first term describes the net contribution from the radiative flux, the second term describes the 
contributions from the vertical advection, and the third term from the horizontal advection. The vertical and horizontal advections can dominate in different regions and can have opposite signs. Consider a downflow of moving material in the Sun's convection zone. In the upper portions of the convection zone, near the photosphere, the atmosphere is highly superadiabatic; thus, the downflow results in a large cooling (as cooler material from above replaces the warmer material below). This cooling diminishes with depth since the superadiabatic temperature gradient goes to zero there. For the horizontal advection we must consider the horizontal temperature gradient. As the material cools and descends, it is surrounded by hotter gases. Thus, the inflow of energy will produce heating. At higher altitudes near the photosphere, in the presence of a strong vertical magnetic field, this horizontal flow heating will be greatly diminished. Deeper down, however, where the vertical advection is small the horizontal advection may dominate.

In addition to the steady flow, we must also consider the usual eddy fluctuating component, w'. The latter enters in the expression:

$\overline{C_{p} \rho^{\prime} W^{\prime} S^{\prime}}=\frac{\partial\left(\rho C_{p} K S\right)}{\partial r}$

which contains the vertical eddy diffusion coefficient, $\mathrm{K}$. With (2.4), we can then describe the energy equation for localized disturbances within the Sun:

$$
\frac{\partial \mathrm{F}^{\mathrm{rad}}}{\partial \mathrm{r}}+\frac{\partial\left(\rho \mathrm{C}_{\mathrm{p}} \mathrm{KS}\right)}{\partial \mathrm{r}}+\rho \mathrm{C}_{\mathrm{p}} \mathrm{WS}+\rho \mathrm{C}_{\mathrm{p}} \mathrm{V} \frac{\partial \mathrm{T}}{\partial \mathrm{x}}=0
$$

Relating to standard envelope theory, we write equation (2.5) in the form:

$$
\frac{\partial F^{\mathrm{rad}}}{\partial \mathrm{r}}+\frac{\partial\left(\rho \mathrm{C}_{\mathrm{p}} \mathrm{KS}\right)}{\partial \mathrm{r}}+\mathrm{Q}_{\mathrm{eff}}=0
$$

where

$$
Q_{\text {eff }}=\underbrace{\rho C_{\mathrm{p}} W S}_{\begin{array}{c}
\text { vertical } \\
\text { advection }
\end{array}}+\underbrace{\rho C_{\mathrm{p}} V \frac{\partial T}{\partial x}}_{\begin{array}{c}
\text { horizontal } \\
\text { advection }
\end{array}}
$$

With $Q_{\text {eff }}$ we basically add the influence of flow (vertical and horizontal) to the standard envelope theory (in which $Q_{\text {eff }}=0$ ). This allows us to investigate the effects of flow on the vertical distributions of temperature, pressure, density and surface irradiance. For simplicity, a perfect gas was considered in the above equations. Variations in the specific heat associated with an imperfect gas, however, were incorporated into the computer code.

When a real gas is considered, the contribution from the latent energy becomes important for the energy equation. There are two ways to account for this effect. The astrophysical approach is to combine the changes in latent energy (release of ionization energy) with the specific heat and modify the adiabatic lapse rate accordingly. This approach is actually used in the envelope code presented in Section 3 (Endal and Twigg, 1982). Here, we provide a brief review of the equivalent but simpler meteorological approach which separately accounts for the changes in the latent energy (e.g.. water vaporization in the earth's atmosphere) and thermal energy.

The energy equation (2.1) is modified to include latent energy release for a terrestrial pseudo-adiabatic process in which the mass of air changes by precipitation. After (Gill, 1982), the additional term is:

$Q_{H}=-\frac{\rho L_{v}}{1-q_{w}} \frac{D q_{w}}{D t}$

where $L_{v}$ is the latent energy, and $q_{w}$ is the specific humidity. For hydrogen ionization, where $a=[H]^{+} /\left([H]^{+}+[H]\right)=$ $[\mathrm{H}]^{+} / \mathrm{H}_{\mathrm{T}}$ is the fraction ionization, and the ionization energy $\mathrm{I}=13.6 \mathrm{eV} /$ particle $=21.8 \times 10^{-12} \mathrm{erg} / \mathrm{particle}=1.31 \times 10^{13}$ $\mathrm{erg} /$ mole, equation (2.8) is modified, assuming that the reactants do not leave the volume element:

$\mathrm{Q}_{\mathrm{H}}=-\mathrm{I} \rho \frac{\mathrm{D} \alpha}{\mathrm{Dt}}=-\mathrm{I} \rho \mathrm{U} \cdot \nabla \alpha$

Temporal variations are ignored here. Following the earlier described procedure for the ideal gas, equation (2.6) is then modified 


$$
\frac{\partial \mathrm{F}^{\mathrm{rad}}}{\partial \mathrm{r}}+\frac{\partial\left(\rho \mathrm{C}_{\mathrm{p}} \mathrm{KS} \mathrm{S}_{\alpha}\right)}{\partial \mathrm{r}}+\mathrm{Q}_{\mathrm{eff}}=0
$$

where

$$
\mathrm{S}_{\alpha}=\frac{\partial \mathrm{T}}{\partial \mathrm{r}}+\Gamma+\frac{\mathrm{I}}{\mathrm{C}_{\mathrm{p}}} \frac{\partial \alpha}{\partial \mathrm{r}}
$$

is the superadiabatic temperature gradient with ionization. The adiabatic temperature distribution (lapse rate) for an ionized medium is equivalent to $S_{a}=0$, similar to the neutral, $S=0$, adiabatic temperature gradient.

As pointed out earlier, we adapt the computer code of Endal and Twigg (1982) and integrate the stellar envelope equations governing temperature, density, radius, and optical depth using the envelope mass, $M-M_{r}$, as the independent variable. This computer code accounts for the latent energy associated with ionization. We modify the energy equation by adding an effective heat source, $Q_{\text {eff }}$, which represents vertical and horizontal advection. We shall now construct models of sunspots and faculae to ascertain the cooling and heating necessary to form these photospheric features.

\section{SUNSPOT AND FACULAR MODELS}

In this section we utilize the above described stellar envelope code, which includes vertical and horizontal advection. We provide three model solutions to illustrate: 1) the effect of vertical advection; 2) the effect of horizontal advection; and 3) the significance of the depth at which these processes occur.

Sunspots, in addition to possessing a cooler effective temperature than the undisturbed photosphere, consist of a local geometrical depression of order $500 \mathrm{~km}$ (Allen, 1973). This depression was discovered by Alexander Wilson in 1774 from the observed extensive flattening of sunspot geometries as they approached the solar limb. The Wilson depression is generally viewed as a manifestation of the sunspot magnetic field as it excludes material in accordance with the horizontal pressure balance. In our model, we consider the vertical momentum equation and view the Wilson depression to be associated with the reduced buoyancy of the cooler, denser material below the sunspot umbra as well. Our envelope code attempts not only to model a cooler photosphere, but additionally a depressed surface for the sunspot umbra. For faculae, as discussed in the introduction, the buoyancy is thought to result in an uplifted surface with a minimal photospheric temperature enhancement. This appears capable of giving rise to the observed continuum contrast behavior.

Boundary Conditions, Our envelope code solves the second order energy equation and the first order equation of vertical momentum balance (hydrostatic equilibrium). Thus three boundary conditions are needed. We assume that the active region disturbance does not extend below a certain depth. Thus at the lower boundary at $12,000 \mathrm{~km}$ depth the temperature and density are assumed to be undisturbed and the energy flux there is equal to the Sun's irradiance. In practice, the equations are integrated downward, starting from the top of the photosphere, and a "shooting" technique is used to satisfy the lower boundary conditions. For this purpose, we choose the temperature (and equivalently the surface irradiance), the density, and the height of the radiating surface at an optical depth of $2 / 3$ which defines the top of the photosphere. We provide magnitudes for the vertical and horizontal advections to define $\mathrm{Q}_{\mathrm{eff}}$, and vary these rates until, for a given subsurface density, pressure, and temperature, we can match the 3 boundary conditions of the undisturbed convection zone at a depth of $12,000 \mathrm{~km}$.

Vertical Advection, Our first sunspot model 1, shown in Figure 1, describes the effects of vertical advection in a superadiabatic environment. The surface irradiance is lowered to 0.5 (in units of the Sun's normal surface irradiance), corresponding to a photospheric temperature of $4,836 \mathrm{~K}$, and a downflow velocity of $500 \mathrm{~m} \mathrm{~s}^{-1}$ is adopted. Numerous problems arise from this calculation. The temperature of the disturbed region remains lower than that of the background throughout the entire convection zone and, as a consequence, the densities and pressures are elevated above the background below $2000 \mathrm{~km}$ depth. In steady state, with vertical advection only, the flow heating cannot do both: lower the surface temperature to umbral values and return the temperature at deeper levels to background values. With vertical advection alone, our boundary conditions cannot be satisfied.

Vertical and Horizontal Advection. Considering both vertical and horizontal advection through an effective term, $Q_{\text {eff }}$, Figures 2-3 show our model 2 for sunspot and facula. Figure 2 presents the computed temperatures, optical depths, densities and pressures for models of a sunspot, a facula, and the undisturbed solar envelope in the top few thousand km. First, note that 
near the photosphere $(t=2 / 3)$, the three curves in each panel have similar shapes. They can be almost superposed by simply shifting them in altitudes. This supports the view that these active region features can be modelled simply by an altered geometry (e.g., hillock or well). Second, note that the sunspot cooling, together with hydrostatic equilibrium, provides for a reduced altitude of the umbral photosphere $(t=2 / 3)$, in accordance with the Wilson depression. Although the sunspot density in the upper layers is lower than the undisturbed material at these depths, the densities are larger for the corresponding optical depth. Unlike the previous case, at greater depths, the densities are minimally enhanced compared with the undisturbed material, and the pressures, densities, and other thermodynamic state variables are unchanged below a few thousand $\mathrm{km}$ depth. Figure 3 shows the normal radiative transport plus eddy heat conduction, basically the surface irradiance, $\mathrm{F}_{\mathrm{o}}$, plus the vertical and horizontal advection. The effective heating is equal to the sum of the last two terms. The additional horizontal advection enhances the superadiabatic gradient and allows a velocity of $50 \mathrm{~m} \mathrm{~s}^{-1}$, at $1,000 \mathrm{~km}$ depth, to offset the eddy heat conduction and lower the surface irradiance to umbral values. The return to background temperatures, densities and pressures satisfies hydrostatic balance associated with a reasonable sunspot depression.

Let us consider the magnitude of the advection terms to understand their relevance to the sunspot cooling problem. The chosen cooling associated with vertical advection peaked between 1,000 and $2,000 \mathrm{~km}$ depth. For densities of order $10^{-5} \mathrm{gm} \mathrm{cm}^{-3}$, one requires $50 \mathrm{~m} \mathrm{~s}^{-1}$ vertical velocities to achieve the peaked $800 \mathrm{gm} \mathrm{cm}^{-3} \mathrm{~s}^{-1} \mathrm{Q}_{\mathrm{eff}}$ cooling in this model. The horizontal advection is easily achieved with similar velocities. For example, one observes that at the same depth, e.g. 2,000 km, the sunspot is cooler than its unperturbed surroundings by $1000 \mathrm{~K}$. Even if we assume that the horizontal scale of this perturbation is $100,000 \mathrm{~km}$ wide, a horizontal temperature gradient of order $10^{-7} \mathrm{~K} \mathrm{~cm}^{-1}$ results. This allows horizontal velocities of $10 \mathrm{~m} \mathrm{~s}^{-1}$ to provide the heating necessary to return the cooled gases to their normal environment.

For faculae, the flow heating results in an uplifted photosphere related to the model of Schatten et al. (1986). Some important differences arise, too. For example, the isopicnic or equal density surfaces are spread out vertically near the photosphere, in addition to being uplifted. This will be the subject of a future paper. Our number 2 model is based on rough estimates of the photospheric boundary conditions, with the normal spot and facular surface irradiance and temperatures at $t=2 / 3$ being: $L_{n}=$ $1.0, \mathrm{~L}_{\mathrm{s}}=0.5, \mathrm{~L}_{\mathrm{f}}=1.1$ (in units of the Sun's normal surface irradiance); $\mathrm{T}_{n}=5,750, \mathrm{~T}_{\mathrm{s}}=4836$, and $\mathrm{T}_{\mathrm{f}}=5,897 \mathrm{~K}$, respectively. The flow velocities are near $50 \mathrm{~m} \mathrm{~s}^{-1}$ for downflow in the sunspot and near $20 \mathrm{~m} \mathrm{~s}^{-1}$ for upflow in the facula.

Surface elevation changes are associated with the depth of the cooling and heating terms, through the principle geophysicists refer to as "isostasy" - vertical hydrostatic equilibrium. A $600 \mathrm{~km}$ sunspot well and a $150 \mathrm{~km}$ facula hillock are calculated in this model. The sunspot value is close to the typically quoted $500 \mathrm{~km}$ Wilson depression (Allen, 1973). The value for the facula is comparable to the height required to explain facular contrasts in the Schatten et al. (1986) hillock model. The contrast modelling suggested a conical uplifted form with a base angle of $15^{\circ}$. The diameter for faculae is typically $1.6 "=1,200 \mathrm{~km}$ (Allen, 1973). This provides an altitude for the tip of the facular cone of $160 \mathrm{~km}$, comparable to the present value of $150 \mathrm{~km}$.

Advection at Greater Depth. Sunspot cooling has been discussed by Parker (1987) as occurring at a depth below $10,000 \mathrm{~km}$. The association of the cooling depth with the hydrogen ionization layer near $2,000 \mathrm{~km}$ is an important aspect of the Schatten and Mayr (1985) sunspot model. To illustrate the significance of the cooling/heating depth, we have run another model where we have supplied the effective heating near $6,000 \mathrm{~km}$ depth. The surface irradiances for sunspot and facula were kept the same. Figure 4 shows the same physical parameters as in Figure 2 plotted versus depth. For a cooling region located near 6,000 km, a Wilson depression of $2,800 \mathrm{~km}$ results which is much larger than observed. For the facula, an uplift of $700 \mathrm{~km}$ results. Cooling regions significantly deeper than the hydrogen ionization layer, if they offset a significant fraction of the surface irradiance, provide too Iarge a Wilson depression to be consistent with observations.

Because our computer model is one dimensional, a number of questions arise that bear on the three dimensional nature of the dynamics. For example, balancing only the vertical momentum equation leaves open what happens to the horizontal component. As our Figure 2 shows, there is no horizontal momentum balance, particularly near the upper levels. The spots have a reduced pressure and the faculae an enhanced pressure. Horizontal pressure gradients can be balanced by magnetic fields and/or flow motions. As pointed out by Meyer et al. (1974), an inflow pattern arising from reduced sunspot pressures can help stabilize sunspot fields against expansion.

For the faculae, the enhanced pressure would result in an outflow of material. The outflow would be greatest in the solar atmosphere above the photosphere, where pressure gradients are largest. There is observational evidence supporting this, in 
that plage have greater contrast in the chromosphere than faculae have in the photosphere. Additionally, the chromospheric manifestation of the facula (plage) covers a wider area than its photospheric manifestation, supporting the view of an outward expansion of the gases.

\section{FIELD INHIBITION AND FLOW}

Our model results show that appropriately chosen cooling and heating rates can reproduce the surface manifestations of sunspots (Wilson depression and $1000 \mathrm{~K}$ temperature reduction) and faculae $(150 \mathrm{~km}$ hillocks associated with small temperature enhancements). However, our model has the weakness that it is 1-D in the vertical, along field lines, where the field exerts no influence. In a more realistic, 3-D model, the magnetic field can play an important role for the sunspot development. Biermann's field inhibition mechanism has a very attractive feature: sunspots and faculae are observed to be associated with magnetic fields. Notwithstanding some of the difficulties with the Biermann mechanism we examine here its positive aspects which may complement the advection view.

The Biermann mechanism can serve to initiate the disturbance, and the flow circulation may be the consequence. Strong magnetic fields can disrupt the convection locally, effectively blocking the heat flux from the interior and thereby starving the sunspot umbra of energy. As a result, the temperature would decrease. With reduced temperature and concomitantly lower density and pressure compared to the surroundings, a circulation would be induced which transports the energy away from the sunspot to form faculae. This circulation can also carry along some magnetic field which is observed in facular regions. Moreover, the magnetic field inhibits cross field motions and thus can reduce the diffusion coefficient by breaking up/inhibiting the eddies. The energy flux would then be suppressed as in the Biermann mechanism. In contrast to the original Biermann mechanism, however, flows would carry away the blocked energy, thereby supplying the energy that goes into the faculae. Biermann's mechanism would also tend to reduce the calculated flow velocities. This in turn would slow down the motions required to offset the eddy heat transport, and smaller flow velocities would be needed than those computed in our 1-D model.

In our picture, faculae can then be viewed as evidence for the disbursement of heat below sunspots. The ejection and disbursement of faculae from the vicinity of sunspots into the surrounding active region can be understood as a result of the "aerodynamic lift force", $-\rho V \cdot \nabla V$. Considering downflows below the sunspot and weaker upflows surrounding the active region, this aerodynamic force is directed inward near the sunspot (stabilizing the spot) and outward in the facular regions (disbursing faculae) where the flow is upward.

How does this compare with the earlier views of these structures? Meyer et al. (1974) had downflows surrounding the sunspot in the early growth stages of sunspot development and upflow patterns in the decay stage. The flows were only utilized for stability, not cooling. In the present view, the upflows do not immediately surround the sunspot but rather occur on fibril fields "peeled off" sunspot structures moving to a considerable distance from the sunspot $(\approx 50,000 \mathrm{~km}$ away) in the region where faculae exist on the outskirts of the active region. Since faculae do follow sunspot development, we note that the Meyer et al. view is remarkably similar to our picture. However, these authors had not identified faculae with the upflow pattern which sheds the suppressed sunspot energy.

\section{DISCUSSION AND SUMMARY}

Sunspots and faculae have been modeled using a modified stellar envelope code. Downflow velocities of $50 \mathrm{~m} / \mathrm{sec}$ can achieve a 1,000 K drop in the surface temperature of the photosphere and reduce the surface irradiance to half its value. Concurrently, a $600 \mathrm{~km}$ Wilson depression forms associated with the enhanced density of the cooler gases. Similar upflow velocities provide for slightly enhanced temperatures and $150 \mathrm{~km}$ uplifted surfaces for faculae. The calculations show that, to first approximation, sunspot and facular structures (in density, temperature and pressure) can be obtained by simply vertically shifting the undisturbed photospheric material to form wells and hillock geometries, respectively. However, the chromospheric manifestations of these features can be quite different owing to the influence of the magnetic field and flow.

Schatten and Mayr calculated flow velocities of about $2 \mathrm{~m} / \mathrm{sec}$ and particle fluxes of $2 \times 10^{21}$ particles $\mathrm{cm}^{-2} \mathrm{~s}^{-1}$. In the present model, the required flow velocities are larger. However, the cooling in the present model occurs in a shallower layer, at 1,000 $\mathrm{km}$ depth, where the density is lower, providing a particle flux of $5 \times 10^{21}$ particle $\mathrm{cm}^{-2} \mathrm{~s}^{-1}$ which is comparable to the Schatten and Mayr value. We note that Zirin and Wang (1989) measured proper motions of $500 \mathrm{~m} / \mathrm{sec}$ from bright features seen in the penumbral region of sunspots, which also supports the inflow pattern. 
As pointed out before, or model is only 1-D and thus cannot include a magnetic field nor replicate the fluxtube size/brightness relationship discussed by Spruit and Zwaan (1980) and modeled by Spruit (1977) and others using the well model geometries. Thus the hillock model does not have some of the "richness" of support enjoyed by the well geometry. Nevertheless, the contrast behavior of faculae appears to fit the hillock geometry.

Biermann's magnetic field inhibition mechanism has an attractive feature: strong magnetic fields are always observed with sunspots. The fate of this energy in the Biermann mechanism, however, is still not clear. Allowing the eddy heat flux to be disbursed by subsurface magnetic fields into the surrounding faculae provides a way to connect faculae to sunspots. As the eddy heat flux is transported upward, it encounters the surrounding sheath of magnetic field structure associated with a sunspot. If this field acts like a conduit for energy transport along the field but as a barrier to heat transport across it, the eddy heat flux cannot enter the core of the sunspot but rather would be disbursed to the faculae surrounding a sunspot. The faculae would be connected to the material below sunspots by subsurface magnetic fields. This view may be referred to as a "field inhibition/transport" model as the field plays a dual role. It inhibits heat transport into the sunspot as in the Biermann mechanism, yet it augments heat transport into the surrounding faculae and plage structures through flow. Faculac thus appear essential for sunspot stability by allowing the Sun to shed the luminosity that would otherwise accumulate below the sunspot. The present modelling effort provides for uplifted facular structures - hillocks, as Schatten and Mayr (1985) suggested and as Schatten et al. (1986) utilized to model facular contrasts.

A crucial test for the flow model would be to observe Doppler shifts in the vicinity of active regions. Cyclonic motions (counterclockwise in the northern hemisphere) around sunspots are expected from the dynamical model. High resolution photographs of faculae may distinguish between the hillock and well geometries; a dark core representing the well bottom would support the well model and exclude the hillock geometry.

Acknowledgements: The authors appreciate helpful discussions with K. Chan, A. Endal, S. Sofia, and L. Twigg.

\section{REFERENCES}

Beckers, J. M. 1976, Ap. J. , 202, 739.

Biermann, L. 1941, Vieteljahresschrift Astr. Ges. 76, 194.

Cavallini, F., Ceppatelli, G. and Righini, A. 1985, Astr. Ap., $143,116$.

Chapman, G. A. 1987, Ann. Rev. of Astron. and Astrophys., 25, 633

Chapman, G. A., Herzog, A. D., Lawrence, J. V., and Shelton, J. C. 1984, Ap. J. Lett., 282, L99.

Chapman, G. A., and Gingell, T. W. 1984, Solar Phys., 91, 243.

Cox, J. P. and Giuli, R. T. 1968, Principles of Stellar Structure (New York, Gordon Breach).

Deinzer, W., Hensler, G., Schussler, M., and Weisshaar, E. 1984, Astron. and Astrophys., 139, 435.

Endal, A. S. and Twigg, L. W. 1982, Ap. J., 260, 342.

Gill, A. E. 1982, Atmosphere-Ocean Dynamics (New York Academic Press).

Howard, R. and LaBonte, B. J. 1980, Ap. J. Lett., 239, L33.

Hudson, H. S. 1988, Ann. Rev. Astron. Astrophys., 26, 473

Meyer, L., Schmidt, H. V., Weiss, N. O., and Wilson, P. R. 1974, M.N.R.A.S., $169,35$.

Miller, P., Foukal, P. and Keil, S. 1984, Solar Phys., 92, 33.

Paczynski, B. 1969, Acta Astronomica, 19, 1.

Parker, E. N. 1974, Solar Phys., 31, 249.

Parker, E. N. 1979, Cosmical Magnetic Fields, (Oxford: Clarendon).

Parker, E. N. 1987, Ap. J., 312, 868.

Schatten, K. H. and Mayr, H. G. 1985, Ap. J., 299, 1051.

Schatten, K. H., Mayr, H. G., Omidvar, K., and Maier, E. 1986, Ap. J., 311, 460.

Schatten, K. H., Orosz, J. A., Mayr, H. G. 1989, Ap. J., 347, 514.

Spruit, H. C. 1976, Solar Phys., 50, 269.

Spruit, H. C. 1977, Solar Phys., 55, 3.

Spruit, H. C. and Zwaan, C. 1980, Solar Phys. 70, 207.

Zirin, H. S., and Wang, H. 1989, Solar Phys., 119, 245. 


\title{
SOLAR IRRADIANCE OBSERVED FROM PVO \\ AND INFERRED SOLAR ROTATION
}

\author{
Charles L. Wolff and Walter R. Hoegy \\ NASA Goddard Space Flight Center \\ Greenbelt,MD 20771, U.S.A.
}

\begin{abstract}
Solar irradiance in the EUV has been monitored for 11 years by the Pioneer Venus Orbiter (PVO). Since the experiment moves around the Sun with the orbital rate of Venus rather than that of Earth, the measurement gives us a second viewing location from which to begin unravelling which irradiance variations are intrinsic to the Sun, and which are merely rotational modulations whose periods depend on the motion of the observer.
\end{abstract}

We confirm an earlier detection, made with only 8.6 years of data, that EUV irradiance is modulated by rotation rates of two families of global oscillation modes. One family is assumed to be $r$-modes occupying the convective envelope and sharing its rotation, while the other family (g-modes) lies in the radiative interior which has a slower rotation. Measured power in r-modes of low angular harmonic number $(\ell \leq 7)$ indicates that the Sun's envelope rotated about $0.7 \%$ faster near the last solar maximum (1979 thru 1982) than it did during the next rise to maximum (1986 thru 1989). No change was seen in the g-mode family of lines, as would be expected from the much greater rotational inertia of the radiative interior.

\section{THE EUV DATA}

The Langmuir probe on the Pioneer Venus Orbiter (PVO) measures a photoemission current which is a proxy for the solar extreme ultraviolet flux (EUV) when PVO is outside the ionosphere of Venus (see Brace et al., 1988). The measured flux covers the wavelength range from about 30 to $130 \mathrm{~nm}$. A measurement of the quantum efficiency of the probe surface material, Rhenium, performed at the National Institute of Standards and Technology, showed that the contributions to the measured photoelectron current are: $56 \%$ (from H Ly $\alpha, 121.6 \mathrm{~nm}$ ), $28 \%$ from the continuum between 30 and $110 \mathrm{~nm}$, $4 \%$ (from He II at $30.4 \mathrm{~nm}$ ), $2 \%$ (He I, $58.4 \mathrm{~nm}$ ), $2 \%(\mathrm{O} \mathrm{V}, 62.9 \mathrm{~nm}$ ), $4 \%$ (C III, $97.7 \mathrm{~nm}$ ), $\quad 2 \%(\mathrm{H} \mathrm{Lb}, 102.6 \mathrm{~nm}$ ), and $2 \%$ (O VI, $103.2 \mathrm{~nm}$ ). For details of the instrument see Krehbiel et al. (1980). Nearly 11 years of daily values of the flux were available, from day 343 of 1978 to day 328 of 1989 , except for times of superior conjunction when data transmission from Venus was not possible. Brace et al. (1988) have given translations of the Langmuir probe current ( $\mathrm{I}_{\mathrm{pe}}$, unit $10^{-9}$ amps) into EUV flux in photon $\mathrm{cm}^{-2} \mathrm{~s}^{-1}$ and also into the often used $10.7 \mathrm{~cm}$ flux value. They also discussed the utility of the Venus-based solar measurements for studies of how solar variability affects the ionosphere, atmosphere, and magnetosphere of Venus. A shorter segment of the EUV 
flux data was used in two earlier studies of solar periodicities: Hoegy and Wolff (1989), Wolff and Hoegy (1989, herein: "Paper I").

The upper panel of Figure 1 shows the 11 year record of solar EUV flux. To de-emphasize the larger fluctuations at solar maximum, we divided the data by its 400 day running mean to produce the more uniform sequence plotted below it. The lower panel is the data set that will be Fourier-analyzed.
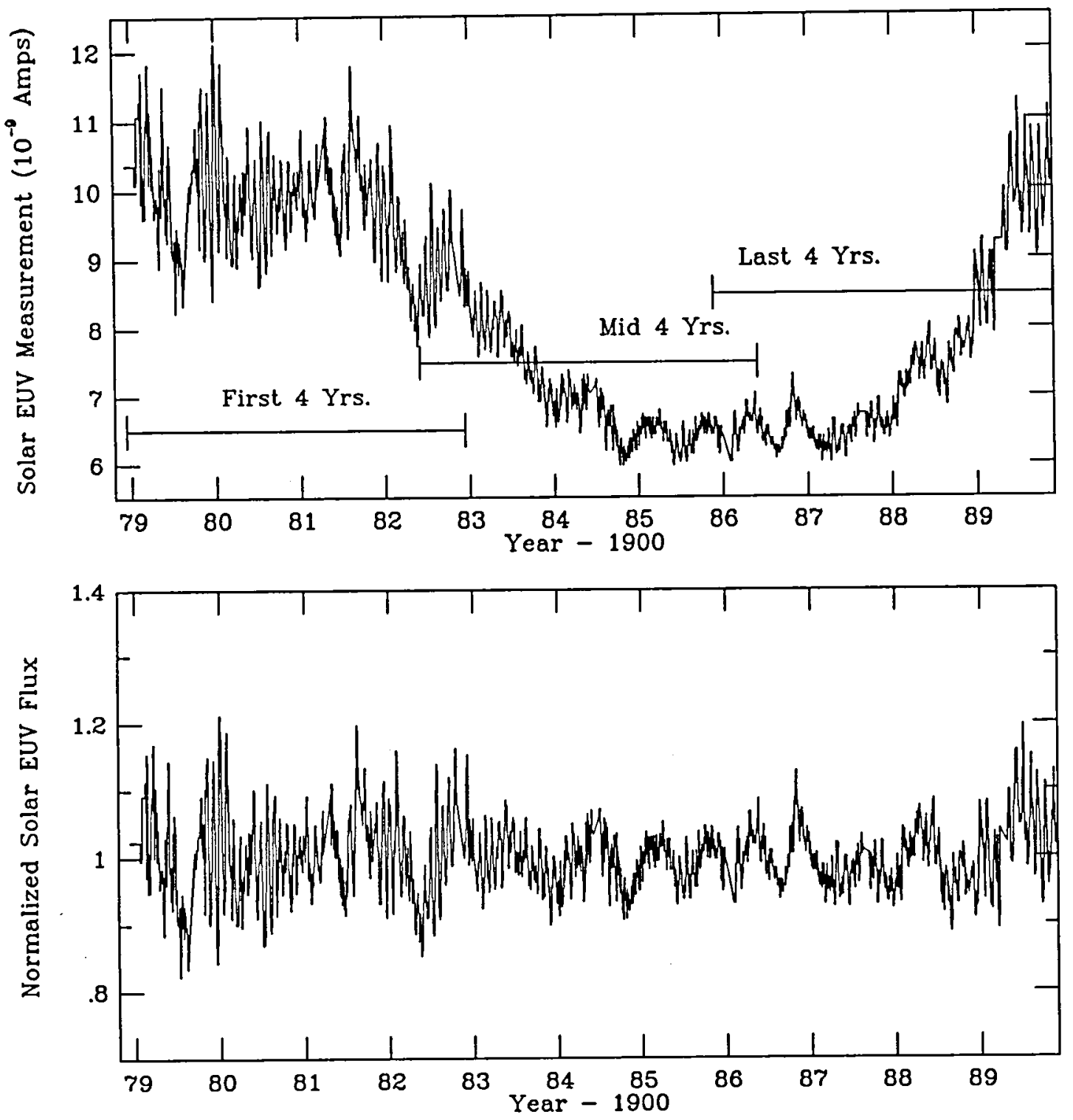

Figure 1--The upper panel shows the probe current which is proportional to solar EUV flux. It declines by about $35 \%$ after the maximum of the 11 year solar cycle. Dividing this data by its 400 -day running mean produces the data set in the lower panel. 


\section{FOURIER SPECTRUM}

Figure 2 shows a Fourier spectrum of the 11 year EUV data set. We plot amplitude (the square root of the power). The upper scales emphasize that a peak on this graph representing a solar rotation period will be seen at one period in the Venus experiment and at a shorter period if viewed from Earth, due to the orbital motions. However a peak caused by an intrinsic solar fluctuation happening at all longitudes, will be seen at the same period and frequency from any planet.

Four features stand out in the observed spectrum.

1. The broad cluster of peaks between 350 and $460 \mathrm{nHz}$ is caused, in part, by solar surface rotation rates. The two tallest peaks may be due to prominent, long-lasting features fixed to the solar surface. If so, they would be seen from Earth as 27.0 and 28.0 day periods.

2. Amplitudes near those two tall peaks appear to have a weak first harmonic; see cluster between 780 and $830 \mathrm{nHz}$.

3. There is a steady rise in amplitude as frequencies decrease below $130 \mathrm{nHz}$. This is caused by the dense collection of g-mode beat frequencies identified in the sunspot record by Wolff (1983). The measured amplitudes would increase all the way to the origin except that our analysis suppressed the lowest frequencies when dividing by the 400-day running mean.

4. As in Paper I, we attribute the very strong peaks near $105 \mathrm{nHz}$ and $105 / 2$ to the lowest harmonic r-mode $(l=1)$. This determines the mean sidereal rotation rate of that mode,

$$
v_{\mathrm{r} 1}=-1.0 \pm 1.5 \mathrm{nHz} \text {, }
$$

and thus updates the values, $106 \mathrm{nHz}$ and $-1.5 \pm 1.5 \mathrm{nHz}$, found in Paper I using a shorter time interval. A characteristic of the observed spectrum is the aliasing of many lines by $\pm 105 \mathrm{nHz}$ due to the nonlinear interaction between this r-mode and other oscillation modes.

Finally, lines in the spectrum tend to be split by a distracting fine structure; the average spacing between maximums is only $4.1 \mathrm{nHz}$. A considerably larger average spacing $\Leftrightarrow 10 \mathrm{nHz})$ was found in an earlier analysis of most of this data (Paper I). The fine structure is caused by aliasing between strong signals in the two solar maximum periods contained in the present data set, whereas the older analysis terminated in 1987 and contained only one solar maximum. The insert on Figure 2 shows how the fine structure distorts the signature of the $\ell=1 \mathrm{r}$-mode. Without the ripples, the cluster of power between 95 and $115 \mathrm{nHz}$ might be a fairly smooth peak with a maximum at $2 \mathrm{~F}$. The measured spectrum near $2 \mathrm{~F}$ is consistent with an $\mathrm{r}$-mode having two active longitudes per rotation (symmetry, $\mathrm{S}=2$ ) and having a larger amplitude near the 11 year solar maximums. We adopt this interpretation. The width of the cluster implies that the amplitudes are strong for roughly three years at a time and significantly weaker during the intervening solar minimum. The presence of power at $F$ too shows that the $S=1$ component is not at all negligible for this mode. 


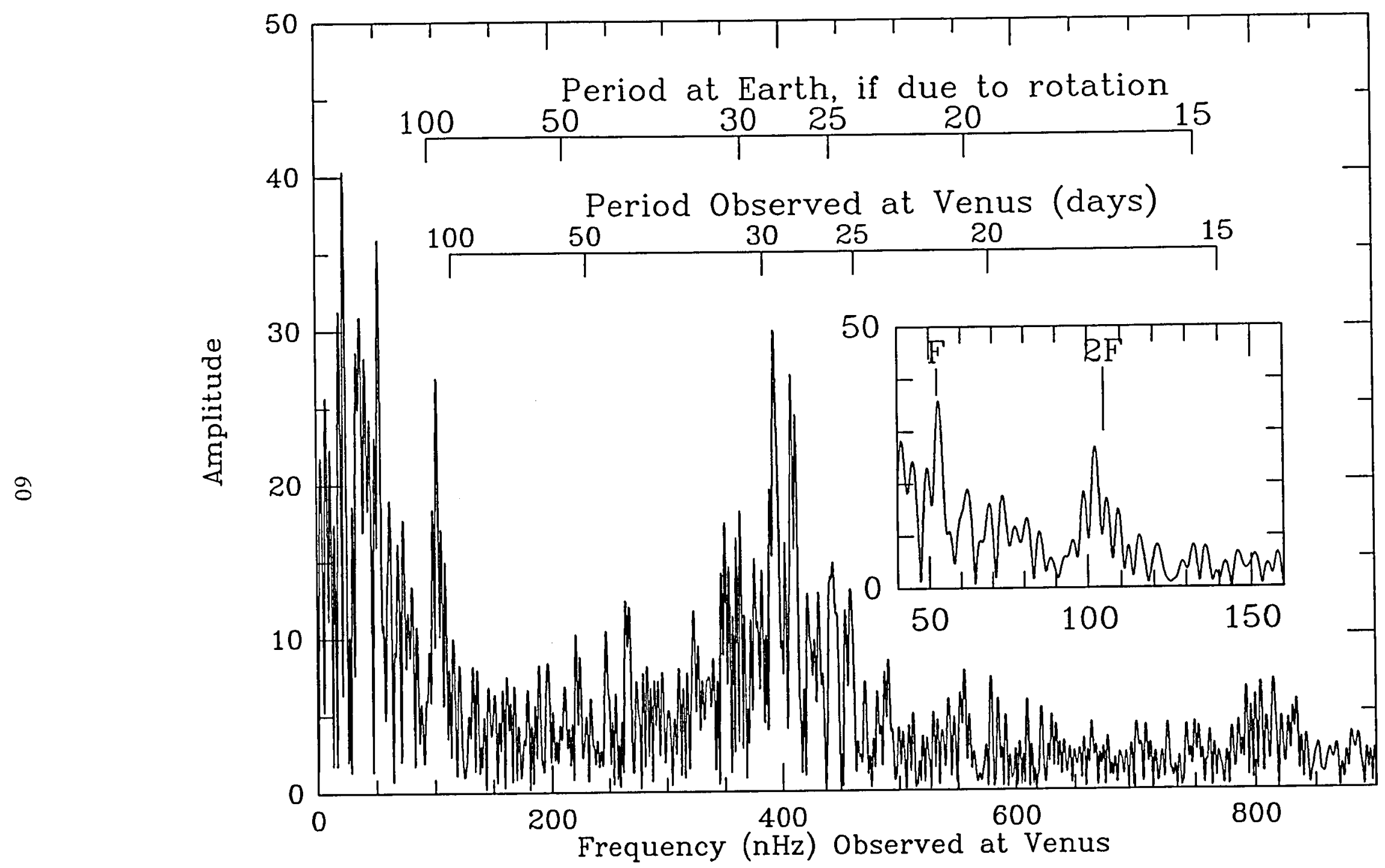

Figure 2 -- Fourier spectrum of the 11 years of normalized EUV flux shown on bottom panel of Figure 1. Below $130 \mathrm{nHz}$, the spectrum is influenced by g-mode beat frequencies and by the $\mathrm{r}$-mode for $\iota=1$ which causes very strong lines at $\mathrm{F}$ and $2 \mathrm{~F}$ (see insert). 
THE INTERIOR MODES (g-MODES)

Beat frequencies from the rotation of g-modes in the Sun's radiative interior seem to have influenced the sunspot record of the last two centuries. Wolff (1983) observed the frequencies and determined the sidereal rotation rate of each mode, multiplied by a symmetry factor $S$ whose value was either 1 or 2 . Since then, the symmetry $S=2$ has fit observations best and has been adopted. For $\ell>1$, Table 1 lists the sidereal rotations found in 1983 for all nonlinear g-modes which our EUV data is able to resolve. The value for $\ell=1$ is less certain and was suggested in Paper I based on most of the EUV data.

TABLE 1

Standing Solar g-modes

\begin{tabular}{|c|c|c|c|c|}
\hline \multirow[b]{2}{*}{$\ell$} & \multicolumn{2}{|c|}{ Sidereal Rotation } & \multicolumn{2}{|c|}{ Main EUV Fluctuation Expected } \\
\hline & $\begin{array}{l}\text { Frequency } \\
(\mathrm{nHz})\end{array}$ & $\begin{array}{l}\text { Period } \\
\text { (davs) }\end{array}$ & $\begin{array}{c}\text { Frequency } \\
(\mathrm{nHz})\end{array}$ & $\begin{array}{l}\text { Period } \\
\text { (days) }\end{array}$ \\
\hline 1 & 176. & 65.8 & 354. & 32.7 \\
\hline 2 & 314.7 & 36.8 & 631.3 & 18.33 \\
\hline 3 & 351.9 & 32.9 & 705.8 & 16.40 \\
\hline 4 & 362.0 & 32.0 & 725.9 & 15.94 \\
\hline 5 & 368.3 & 31.4 & 738.6 & 15.67 \\
\hline 6 & 371.9 & 31.1 & 745.9 & 15.52 \\
\hline $\mathrm{G}$ & 380.1 & 30.5 & 762.2 & 15.19 \\
\hline
\end{tabular}

As pointed out in Paper I, we do not expect to see g-mode rotation rates easily at the surface because their amplitude declines exponentially in the convective envelope. Rather, we expect to see the results of eruptions stimulated when their active longitudes near the base of the convection zone rotate past those of prominent global modes in the envelope. Since the $l=1$ mode in the envelope should be the most important for our experiment (which integrates over the full solar disk), the most detectible frequencies in the rotational range should be,

$$
v_{\mathrm{b}}=2\left(v_{\mathrm{g}}-v_{\mathrm{r} 1}\right)
$$

where $v_{g}$ is the g-mode rotation rate (second column of Table 1 ), and the factor 2 is the symmetry. Imposing equation (1), fixes the frequencies $v_{b}$. The right side of Table 1 lists them and corresponding periods, $v_{b}{ }^{-1}$. The seven frequencies plus their aliases at \pm 105 $\mathrm{nHz}$ make a set of 21 theoretical lines. We would expect to detect them all if there were not confusion from many other aliases and another family of modes in part of the same range ( $r$-modes, below). Even so, only 5 frequencies (marked $\mathrm{X}$ ) are not detected in the EUV spectrum, magnified on Figure 3. The detection criterion is that a theoretical line be closer to an obsereved maximum than to a minimum. The binomial expression gives probability of accidental agreemient as $<1 \%$ which confirms the detection of much higher statistical significance (Wolff, 1983) based on beat frequencies rather than rotation rates.

The three theoretical lines with frequencies $<500 \mathrm{nHz}$ are due to the g-mode for $l=1$. They cause strong observed lines which seem to be broader and, therefore, more conspicuously subdivided by the fine structure. Perhaps, their actual effect would be modelled better by smooth peaks, about $10 \mathrm{nHz}$ wide, fitted to the three clusters of observed power in the vicinity of each. Independent confirmation is needed that these are indeed due to the $\ell=1$ g-mode.

The periods in the right column of Table 1 should appear with identical values whether detected from Venus or Earth since they are intrinsic periods of the Sun. 
Figure 3--The degree of correspondence of g-mode frequencies (Table 1) with magnified portions of the EUV spectrum (Figure 2). Of the 21 lines, only 5 (marked $X)$ are not detected. The g-mode spectrum appears 3 times due to aliasing at $\pm 105 \mathrm{nHz}$ caused by interaction with the lowest harmonic r-mode.
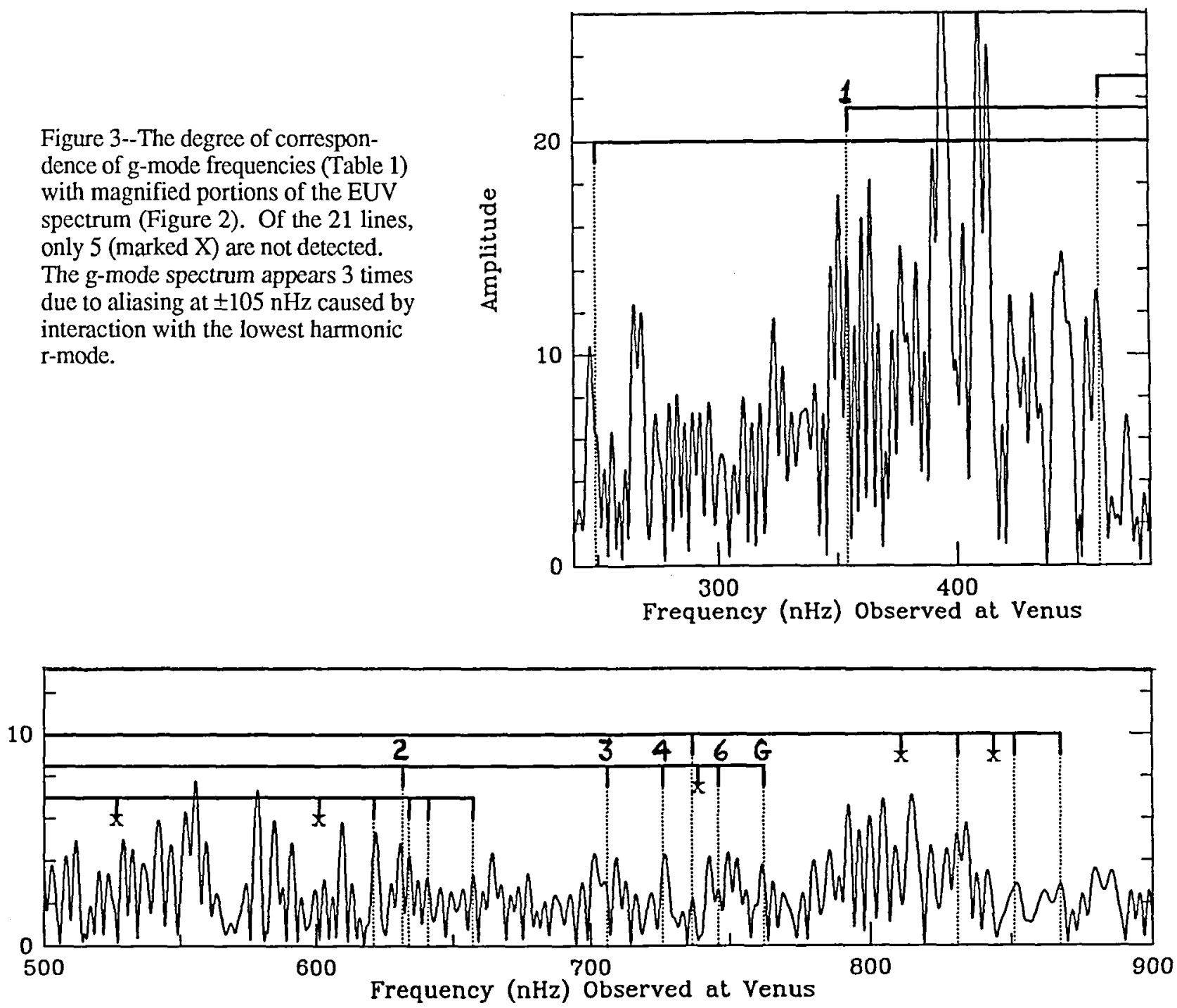

THE ENVELOPE MODES (r-MODES)

Many observers have reported changes in the rate of solar surface rotation at a given latitude. To see if the entire convective envelope might vary its mean rotation with time, we analyzed data from three, partly overlapping four year periods. Figure 4 shows the Fourier spectrum of each. The centroid of the high amplitude cluster near $400 \mathrm{nHz}$ seems to occur at lower frequencies during the last four years (bottom panel). This region is typical of solar surface rotation since a rotation period seen from Venus as $(400 \mathrm{nHz})^{-1}$ would be seen from Earth as 27.6 days. If some of this shift in the centroid is due to a slowing of the mean sidereal rotation, $v_{\infty}$, of the convective envelope, then the sequence of $r$-mode rotation rates,

$$
v_{\ell}=v_{\infty}[1-2 / \ell(\ell+1)]
$$

is reduced proportionally. The frequencies expected at Venus are twice the synodic rates,

$$
v_{\text {obs }}=2\left(v_{\ell}-51.5 \mathrm{nHz}\right)
$$


Figure 4--Fourier spectrums of the first, middle, and last 4 years of EUV data. They are quite different because 4 years is not nearly long enough to capture the full complexity of solar behavior. Typical solar surface rotation rates fall within the broad cluster of peaks near $400 \mathrm{nHz}$. The cluster is shifted to lower frequencies in the last four years, suggesting a slower average rotation during that interval.
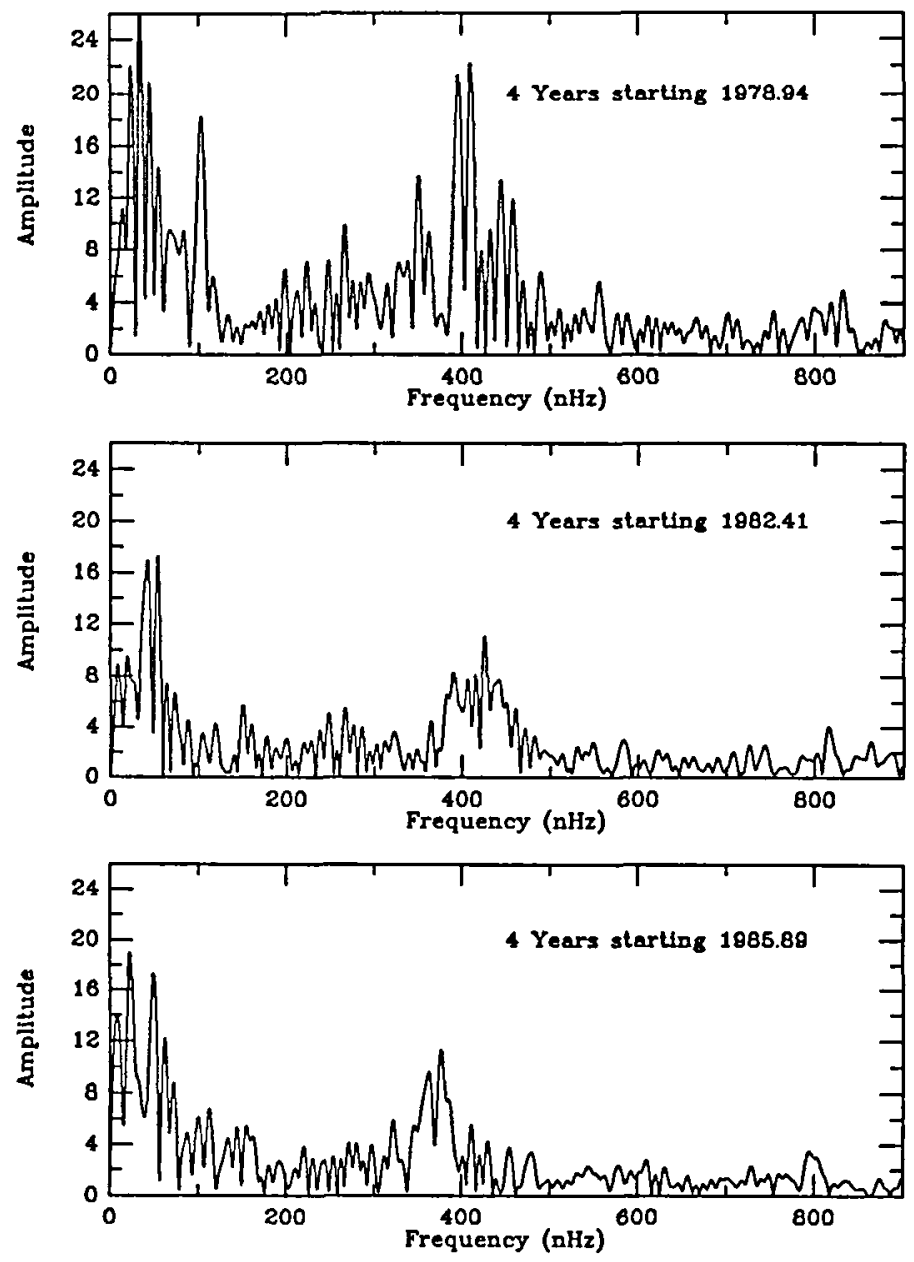

and also the aliases, $v_{\text {obs }} \pm 105 \mathrm{nHz}$. The sequence $2 \leq \ell \leq 7$ gives a group of 18 lines to test whether $v_{\infty}$ has changed. (Frequencies for $\ell>7$ are too close to be resolved by this four year data set and the $\ell=1$ mode is independent of a change in $v_{\infty}$ to first order.) Of the 18 lines, we detected respectively, 13 or 14 using the values of $v_{\infty}$ shown in Table 2 . They are listed as sidereal rates and then converted to synodic values.

Table 2

Mean Rotation of Convective Envelope

\begin{tabular}{|c|c|c|c|}
\hline \multirow[t]{2}{*}{ Observed Interval } & \multirow{2}{*}{$\begin{array}{l}\text { Sidereal Rate } \\
(\mathrm{nHz})\end{array}$} & \multicolumn{2}{|c|}{ Apparent from Earth } \\
\hline & & $\begin{array}{l}\text { Rate } \\
(\mathrm{nHz})\end{array}$ & $\begin{array}{l}\text { Period } \\
\text { (days) }\end{array}$ \\
\hline First 4 Years, $1978.9-1982.9$ & $460.0 \pm 2$ & 428.3 & 27.02 \\
\hline Last 4 Years, $1985.9-1989.9$ & $456.7 \pm 2$ & 425.0 & 27.23 \\
\hline
\end{tabular}


For an observer on Earth, the table shows that the mean rotation period of the convective envelope (as averaged by the r-modes) was 27.02 days during the first four years and 27.23 in the last four years. For the last four years, the deviation of each observed line from a predicted line is shown on Figure 5; vertical separations are for clarity. The four lines that failed our detection criterion are the two most extremely left or right. The very high degree of clustering argues for the reality of the fit since randomly chosen frequencies would appear with equal probability anywhere along the horizontal range of $11 \mathrm{nHz}$ which is the mean separation of maximums on Figure 4.

These fits are the best we found and the only ones close to 27 days. But, if it is conceivable that the Sun's convective envelope could change its speed by $4 \%$ or more during the 11 year interval, then several other solutions are possible. They are of lesser quality and more likely to be statistical accidents but we can't rule them out from the EUV data alone.

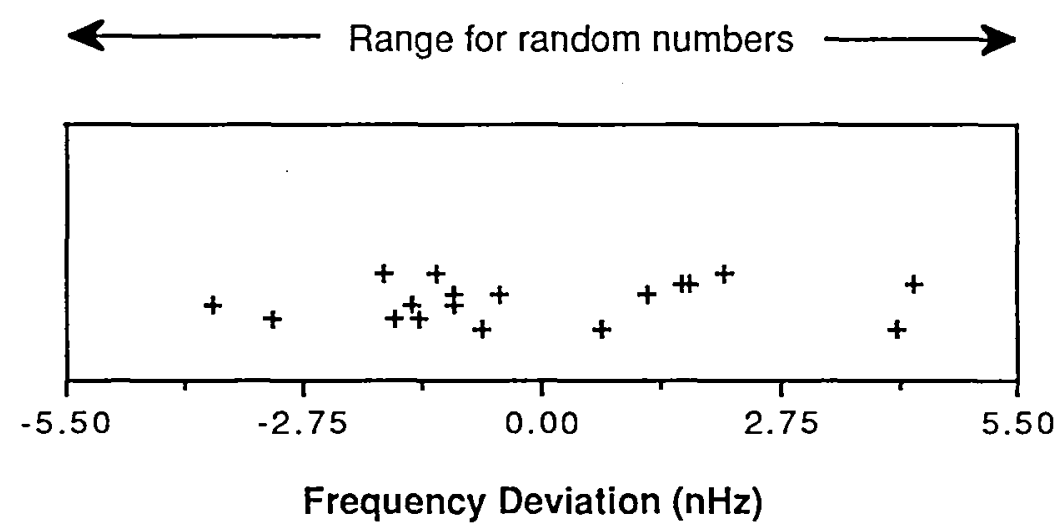

Figure 5--Deviation of peaks in the observed spectrum from the 18 frequencies caused by the rotation of solar r-modes. Concentration toward the central half of the plot confirms the r-mode interpretation since an irrelevant model, or random frequencies, would populate the entire horizontal range.

\section{REFERENCES}

Brace, L.H., Hoegy, W.R., and Theis, R.F., "Solar EUV Measurements at Venus Based on Photoelectron Emission from the Pioneer Venus Langmuir Probe", J. Geophys. Res., vol. 93, no. A7, 7282-7296, 1988.

Hoegy, W.R. and Wolff C.L., "A Seven Month Solar Cycle Observed with the Langmuir Probe on Pioneer Venus Orbiter", J. Geophys. Res., vol. 94, no. A7, 8663-8671, 1989.

Krehbiel, J.P., Brace, L.H., Theis, R.F., Cutler, J.R., Pinkus, W.H., Kaplan, R.B., "Pioneer Venus Orbiter Electron Temperature Probes", IEEE Trans. Geosci. Remote Sens., GE-18, 49, 1980.

Wolff, C. L., "The Rotational Spectrum of g-modes in the Sun", Astrophys. J., vol. 264, 667$676,1983$.

Wolff, C.L. and Hoegy, W.R., "Periodic Solar EUV Flux Monitored near Venus", Solar Phys., vol 123, 7-20, 1989, ("Paper I"). 


\title{
A CORRELATION BETWEEN CHANGES IN SOLAR LUMINOSITY AND DIFFERENTIAL RADIUS MEASUREMENTS
}

\author{
R. J. Kroll and H. A. Hill \\ Department of Physics, University of Arizona, Tucson, Arizona 85721 \\ B. J. Beardsley*
}

Photometrics Limited, Tucson. Arizona 85745

\begin{abstract}
Solar luminosity variations occurring during solar cycle 21 can be attributed in large part to the presence of sunspots and faculae. Nevertheless, there remains a residual portion of the luminosity variation distinctly unaccounted for by these phenomena of solar activity. At SCLERA (Santa Catalina Laboratory for Experimental Relativity by Astrometry), observations of the solar limb are capable of detecting changes in the solar limb darkening function by monitoring a quantity known as the differential radius. These observations are utilized in such a way that the effects of solar activity are minimized in order to reveal the more fundamental structure of the photosphere. The results of observations made during solar cycle 21 at various solar latitudes indicate that a measurable change did occur in the global photospheric limb darkening function. It is proposed that the residual luminosity change is associated in part with this change in limb darkening.

Measurements of the solar luminosity made by ACRIM and estimated contributions to the change in luminosity due to sunspots and faculae are given by Willson and Hudson. 1 From 1980 to 1986, the ratio of the fractional change in the residual luminosity to the fractional change in differential radius is $(\delta \mathrm{L} / \mathrm{L}) / \delta(\Delta \mathrm{r} / \mathrm{R}) \approx-7$. Because other phenomena may also contribute to the residual luminosity change, the magnitude of this ratio is taken to be the upper limit associated with limb darkening changes.

The feasibility of this hypothesized correlation is apparent when the luminosity change implied by the observed differential radius change from 1976 to 1980 is found to be within the limits allowed by the balloon, rocket, and satellite radiometric data. Additionally, it may explain the discrepancy between observation obtained by ACRIM and ERB and predictions based on some models of solar luminosity change from 1978 to 1981. Further, the residual luminosity change implied by the magnitude of the change in differential radius over the decade beginning in 1973 is great enough to possibly have consequences for the Earth's climate. The validity of this correlation would establish the differential radius as a quantity useful for investigating processes affecting the solar energy output independent of the solar cycle variations.
\end{abstract}

\section{INTRODUCTION}

Understanding the changes in solar luminosity has attained a pre-eminent role in current solar research. This is in part a consequence of the important influence that the solar energy output plays in determining the conditions of the Earth's climate. Important evidence that this connection has already been observed is the association of the Maunder Minimum in sunspot activity and the Little Ice Age of the eighteenth century. Further investigations into the characteristics of

\footnotetext{
${ }^{*}$ Burt Beardsley participated in this work as an Adjunct Assistant Research Scientist at the University of Arizona.
} 
solar variability and the Earth's environmental response may uncover how such a connection may arise and whether such events are likely to occur again. Recent improvements in the accuracy of solar luminosity measurements further stimulate the search for the causes of variability. The ability to measure long-term changes in total irradiance to fractions of a percent requires a deeper understanding not only of the phenomena known to be responsible for such changes, but also of other phenomena that may contribute to total irradiance variability. The challenges of explicating the causes of solar luminosity change and the effects of such changes on the Earth are formidable. yet promise substantial rewards.

During the 1980's, the ACRIM instrument aboard the SMM satellite contributed greatly to our ability to monitor the solar irradiance. It has provided measurements of unequaled precision over the long term. The observations indicate measurable changes in solar energy output over time scales ranging from days to years. Much of this variability is found to be associated with the presence of regions of solar activity. The irradiance is seen to diminish with the quantity of sunspots visible and is enhanced by the presence of faculae. In addition to the changes occurring over the lifetimes of active regions, a decreasing trend in the luminosity has been observed during the first two-thirds of the 1980's. This trend appears to be in phase with the solar activity cycle and may be found to have its cause in the features of solar activity. Initial explanations along these lines were found inadequate, which opened the possibility that phenomena other than solar activity are relevant to solar luminosity variability.

Among these alternatives are the possibilities that the energy flow out of the Sun was modulated by changes in the photospheric temperature and temperature gradient. Such causes would constitute changes in the global properties of the Sun and, specifically, a change in solar limb darkening. At SCLERA, observations of the solar limb are made and characteristics of the solar limb darkening function are quantified with a parameter known as the differential radius. During the time that the solar luminosity showed a downward trend, the differential radius values were also seen to change. Discussed in the following sections is the concept of the differential radius as a measure of limb darkening and its variation reflecting temperature gradient and solar luminosity changes. The possibility that the differential radius could serve as an indicator of global properties affecting solar luminosity is investigated.

\section{THE DIFFERENTIAL RADIUS}

The differential radius is based on a particular definition of the solar edge known as the finite Fourier transform definition, or FFTD. ${ }^{2}$ The FFTD locates the solar edge at a distance $\rho$ from the center of the sun such that

$$
\int_{-1 / 2}^{1 / 2} I(\rho+a \sin \pi s) \cos 2 \pi s d s=0 .
$$

where I is the observed solar intensity as a function of radius and a is the scan amplitude, a parameter indicating the amount of the solar limb being scanned. It has been shown ${ }^{2}$ that the FFTD has reduced sensitivity to atmospheric seeing when the parameter a is at least as large as the width of the seeing function, but that it is quite sensitive to the shape of the limb darkening function. It is also apparent that Eq. (1) is unchanged by any constant scaling of $I$ and is, therefore, insensitive to spatially large-scale changes in Earth's atmospheric transparency.

Equation (1) shows that the edge position $\rho$ and scan amplitude a are related through the limb darkening exhibited by $\mathrm{I}$. Therefore, for a particular limb intensity profile and latitude on the solar disk $\theta$,

$$
\rho=\rho(\mathrm{a}, \theta) \quad \text {. }
$$


This dependence of limb position on scan amplitude makes it possible to investigate the shape of the limb darkening function. Limb positions can be determined for different scan amplitudes $a_{i}$ and $a_{j}$, and the differential radius is defined as

$$
\Delta \mathrm{r}\left(\mathrm{a}_{\mathrm{i}}, \mathrm{a}_{\mathrm{j}}, \theta\right)=\rho\left(\mathrm{a}_{\mathrm{i}}, \theta\right)-\rho\left(\mathrm{a}_{\mathrm{j}}, \theta\right)
$$

When the solar limb profile is a step function, $\Delta r=0$. For $a_{i}<a_{j}$, the more limb darkening exhibited in an intensity profile, the greater is the differential radius value. Because of this dependence of $\Delta r$ on the limb darkening function, it can be used to monitor changes in the shape of the limb darkening function over time and, hence, to infer concurrent changes in the photospheric temperature gradient.

\section{THE OBSERVATIONS}

The limb darkening observations obtained at SCLERA are made by scanning an image of the Sun across three pairs of diametrically opposed slits. Two of the diameters are rotated by plus and minus one-eighth radian from the central diameter. This allows six limb profiles to be obtained near a chosen solar latitude. The scans are sinusoidal, taking 0.625 seconds each, during which 256 intensity measurements are made for each limb. The intensity of the continuum radiation at $5500 \AA$ (with a bandpass of $100 \AA$ ) is digitally recorded for each of the six positions. On days when observations are made, the procedure consists of the constant repetition of scans as the Sun is being tracked for as long as the weather or daylight permits. The entire detector assembly can be rotated so that a particular diameter can be followed dúring the day or so that limb profiles at various latitudes can be obtained. Since 1979, observations have been obtained in this manner. Prior to this time, the detector consisted of only one pair of slits.

Observations that have been used in earlier long-term variability studies $3,4,5$ were made in $1973,1979,1981,1983,1984,1986$, and through the first part of 1989 . This work includes data obtained in the later part of 1989 . Table 1 provides information about the data obtained in these years, including the scan amplitudes used to calculate $\rho(a)$. The data-reduction procedure results in a single differential radius value per year per observed latitude for the years prior to and including 1984 and two values per year for 1986 and later. For each solar latitude observed, a time series of differential radius values for each of the six limbs is calculated for given pairs of the four values of scan amplitude available. For the scan amplitude pairs of interest, values from the six differential radius time series at corresponding times are averaged, and any of the six values more than $1.5 \sigma$ from the mean are removed from the time series, since such variance is likely the effect of solar activity. The $1.5 \sigma$ cutoff value was found to be the optimum value for rejecting the data points judged bad by visual inspection from the small sample of six data points used. The remaining differential radius values at corresponding times are averaged to produce a time series of mean values for the day. The mean of this time series is calculated, and any single value greater than $2 \sigma$ from the mean is removed and a final daily mean is calculated. With daily averages of each observed solar latitude available, a seasonal or yearly value is determined for each latitude by averaging the daily values using $2 \sigma$ as the cutoff value in a two-cycle rejection procedure. This is done in order to eliminate days that are dominated by the effects of faculae or other transient surface phenomena.

As noted in Eq. (3), the differential radius is a function of the amplitude at which the solar image is scanned. As seen in Table 1, the scan amplitude for different years has varied and therefore must be taken into account in order to meaningfully compare differential radius values over the years. To do this, the differential radius is adjusted to standard scan amplitudes of 8 and 24 arcseconds. This adjustment is implemented by determining the dependence of the limb position on the scan amplitude using the mean limb darkening function found in 1987 and 1988 . Because for any year there are scan amplitudes available which differed by only a few arcseconds from the standard 8 and 24 arcsecond values, any errors acquired in this adjustment are estimated to be of 
TABLE 1

Summary of SCLERA Observations for Long-Term Variability

\begin{tabular}{lccc}
\hline Year & $\begin{array}{c}\text { Number } \\
\text { of Days }\end{array}$ & Latitude Angles & $\begin{array}{c}\text { Measured } \\
\text { Scan Amplitude } \\
\text { (arcseconds) }\end{array}$ \\
\hline 1973 & 7 & $\pm 15^{\circ}, \pm 105^{\circ}$ & $27.2,20.4,13.6,6.8$ \\
1979 & 16 & $0^{\circ}$ & $25.6,19.2,12.8,6.4$ \\
1981 & 13 & $\pm 13^{\circ}, \pm 39^{\circ}, \pm 64^{\circ}, \pm 90^{\circ}$ & $22.5,16.9,11.3,6.3$ \\
1983 & 59 & $0^{\circ}, \pm 45^{\circ}, \pm 90^{\circ}$ & $21.5,16.1,10.8,5.4$ \\
1984 & 21 & $0^{\circ}, \pm 45^{\circ}, \pm 90^{\circ}$ & $21.5,16.1,10.8,5.4$ \\
1986 & 65 & $0^{\circ}$ & $33.0,24.8,16.5,8.3$ \\
1987 & 82 & $0^{\circ}$ & $31.0,23.3,15.5,7.8$ \\
1988 & 59 & $0^{\circ}, \pm 45^{\circ}, \pm 90^{\circ}$ & $31.2,23.4,15.6,7.8$ \\
1989 & 42 & $0^{\circ}, \pm 45^{\circ}, \pm 90^{\circ}$ & $31.0,23.3,15.5,7.8$
\end{tabular}

the order of a few milliarcseconds. For a given scan amplitude, the actual amount of solar radius observed will vary throughout the year due to the change in the Earth-Sun distance. This variation can result in seasonal systematic trends if not corrected. Daily differential values are normalized to correspond to the amount of limb that would be scanned if the Sun were at aphelion in early July. This time is used because most of the observations were made near this time of year, so the corrections are minimized. The yearly and seasonal values determined as per the foregoing analysis are displayed in Fig. 1.

The other observations of interest are the total irradiance measurements made with the ACRIM instrument aboard the Solar Maximum Mission satellite. The satellite was launched in 1980 and relayed precision measurements of the solar luminosity until a failure in the pointing system in late 1980. Lower-quality data continued until its repair in March 1984 when precision data again resumed. The following analysis utilizes the results of Willson and Hudson ${ }^{1}$ who used the data from the periods when the satellite was in the "precision solar-pointed" mode.

\section{COMPARISON OF DIFFERENTIAL RADIUS AND ACRIM OBSERVATIONS}

In order to compare the changes in differential radius with the changes in solar luminosity. differential radius values available at or near the times of the ACRIM observations analyzed by Willson and Hudson ${ }^{1}$ are used. Differential radius values for 1979 and 1981 are combined in order to estimate a value for 1980, and values for 1984 and 1986 are combined and used as a measure of 1984 through 1986 values. These estimates can then approximate the changes in differential radius for comparison with the ACRIM observations.

Data obtained in 1979 and 1986 are different from that obtained in 1981 and 1984 in that the 1979 and 1986 observations were restricted to near the equator, while 1984 observations include the polar and $\pm 45^{\circ}$ regions in addition to the equator, and 1981 observations include the pole and latitudes at $25.71^{\circ}$ increments from the pole. Thus, by determining the yearly averages first, the combination of different kinds of data sets is facilitated. Whereas 1979 and 1986 observations contain information solely about the equatorial limb darkening, the 1981 and 1984 values reflect a globally averaged limb darkening function. This, it is felt, is a better indicator of conditions in the 


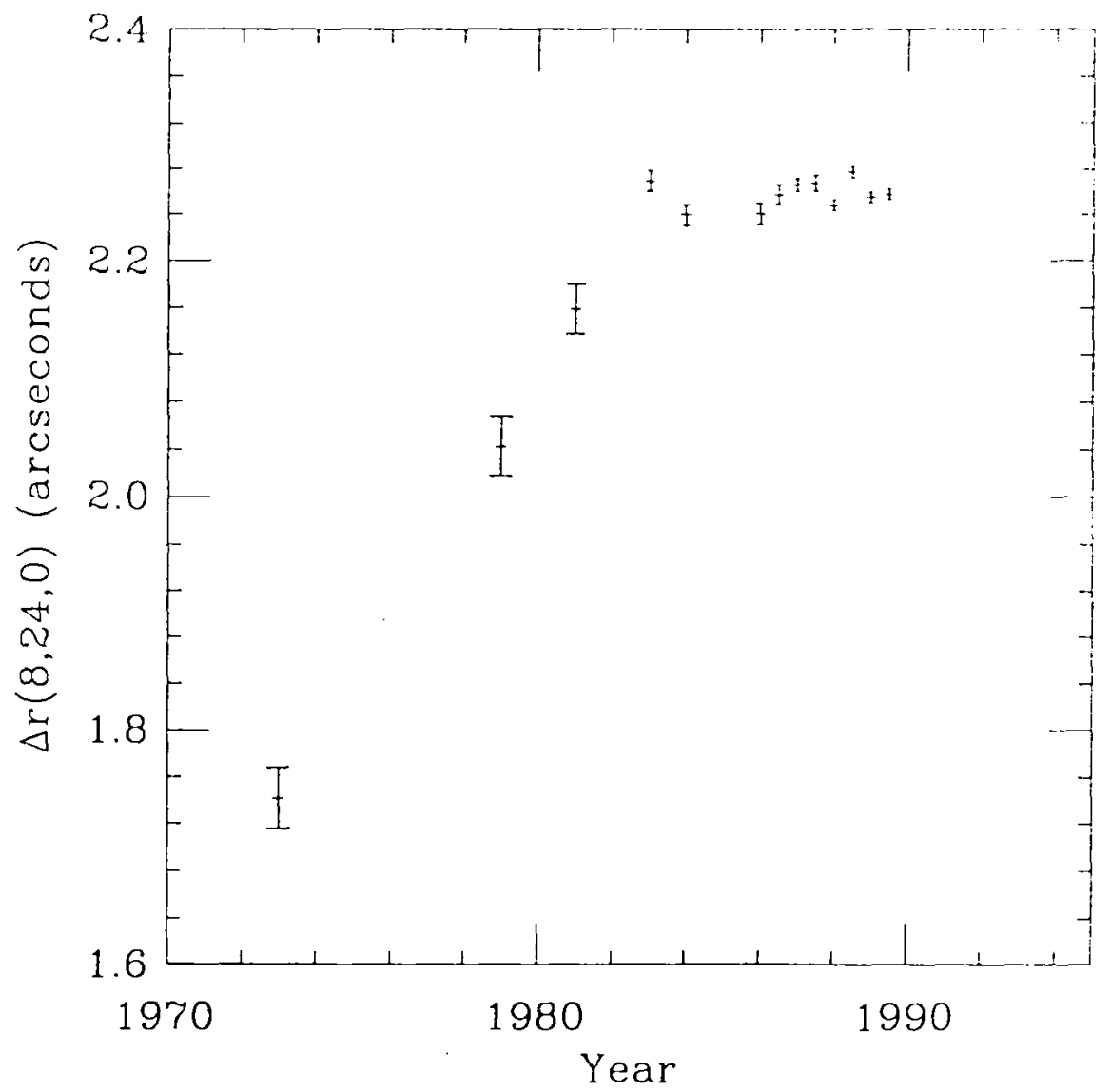

Figure 1. Differential radius results, corrected to standard scan amplitudes of 8 and 24 arcseconds, from observations made from 1973 to 1989.

photosphere than just the equatorial value when comparing to total irradiance. It should be noted, however, that even though differential radius values sometimes depend on the solar latitude, the equatorial values are a good indicator of values at other latitudes. In other words, differences between differential radius values at various latitudes are small compared to differential radius changes from year to year.

The ACRIM observations used here have been analyzed and presented by Willson and Hudson. ${ }^{1}$ As mentioned earlier, the data from 1980 and from mid-1984 to 1986 are used in their analysis. Briefly, their analysis attempts to account for the luminosity variation between the two time periods by calculating the contributions of sunspots and faculae. The sunspot deficit is quantified with the photometric sunspot index (PSI) calculated from the observed sunspot areas and brightnesses in terms of their positions on the solar disk. Likewise, the facular excess is calculated based on Ca-plage areas and their brightnesses as a function of position on the disk and designated the photometric facular index (PFI). Figure 3 of their paper shows the relationship between the sunspot-corrected observations (ACRIM+PSI) and the facular concentration as measured by the PFI. It is seen that the data concentrate in two groups -- the data from 1980 lying above the data from 1984-1986. The figure shows straight line fits to both groupings of data. Our analysis proceeds by extrapolating the linear fits to zero facular concentration. This effectively estimates the total irradiance for the two time periods corrected for both sunspot blocking and facular enhancement. The difference between the two intercept values reveals the residual change in solar luminosity during the time span in question. 
The residual change in total irradiance $\delta \mathrm{L}$ is found to be $-1.65 \mathrm{~W} / \mathrm{m}^{2}$. With the average irradiance $\mathrm{L}=1367.72 \mathrm{~W} / \mathrm{m}^{2}, \delta \mathrm{L} / \mathrm{L}=-1.2 \times 10^{-3}$. The change in differential radius $\delta(\Delta \mathrm{r})$ from the 1979-1981 time period to the 1984-1986 time period is found to be 0.14 arcseconds. Using $\mathrm{R}=945$ arcseconds as the aphelion value to which all of the differential radius values are normalized, $\delta(\Delta \mathrm{r}) / \mathrm{R}=1.6 \times 10^{-4}$. For the hypothesis that $100 \%$ of the residual change in total irradiance is associated with the observed change in differential radius, then the ratio of fractional change in irradiance to a correlated fractional change in differential radius is $(\delta L / L) / \delta(\Delta r / R) \approx-7$. This ratio is a quantity that can be monitored during the upcoming maximum of the solar cycle. Comparison of the most recent ACRIM observations with the latest differential radius values can test the degree to which the limb darkening, as measured by the differential radius, correlates with solar luminosity changes. It should be noted, however, that there is evidence which indicates that the correlation between the residual luminosity change and differential radius change is, in fact, less than $100 \%$.

One question regards how well the Ca-plage areas account for the contribution of faculae to the total irradiance. Willson and Hudson 1 suggest that much of the scatter in the ACRIM data introduced by applying the facular correction is due to the inadequacy of plage areas providing an accurate measure of facular contributions. Studies involving the variability of the CaK line indicate why this could be the case. With data obtained by White and Livingston, 6 Skumanich et al. ${ }^{7}$ show that it is not possible to model the variability in the $\mathrm{K} 1.0$ index with quiet sun (super granular cells and network) and plage components with realistic values of parameters characterizing these features. By introducing a third component, the "active network," which results when large active region faculae decay into unstructured remnants of magnetic flux, a much better accounting of $\mathrm{CaK}$ variability is found. Based on the correlation of $\mathrm{HeI}$ equivalent widths and full-disk CaK variations, Foukal and Lean ${ }^{8}$ find that the HeI line is a good indicator of the sunspot-corrected total irradiance, including the downward trend occurring between 1981 and 1984 . Livingston et al. ${ }^{9}$ suggest that a combination of the Mn5394 and CN3883 line strengths are even better indicators of irradiance variability over the entire solar cycle. In any case, there appear to be better parameters with which to account for the facular enhancement of the total irradiance than the plage index alone. The amount of residual irradiance change may, therefore, be somewhat smaller than that used in the preceding analysis, reducing the magnitude of the ratio $(\delta \mathrm{L} / \mathrm{L}) / \delta(\Delta r / \mathrm{R})$. The magnitude of the value stated above may well serve as an upper limit on the degree to which total irradiance variability is associated with changes in differential radius.

A second process which may account for a significant portion of the residual luminosity change is that of Kuhn et al. 10 This explanation proposes that changes in the energy transport result from variations of convective flow during the solar cycle. This causes the surface temperature and presumably the temperature gradient to vary with latitude and time, causing net changes in total irradiance.

One consequence of the hypothesis of $100 \%$ correlation is revealed when taking into account differential radius values obtained prior to 1980 . Differential radius values were calculated for the years 1973, 1979, and 1981. These values are consistent with a linear increase which continues into 1983-1984. By using a linear fit to the data from these five years, the change occurring from 1976 to 1980 , based on the $100 \%$ correlation hypothesis, implies $\delta \mathrm{L} / \mathrm{L} \approx-0.15 \%$. As indicated by Willson, $11 \pm 0.2 \%$ is the maximum long-term luminosity change that could have occurred during that time period, based on the results of rocket, balloon, and ACRIM experiments. Thus a $100 \%$ correlation hypothesis is not in conflict with the observations of total irradiance and differential radius prior to 1980 . On this basis, global changes in the photosphere may well have occurred over the decade beginning in 1976.

An important additional consequence of the observed differential radius change between 1973 and 1983 is that $\delta \mathrm{L} / \mathrm{L} \approx-0.4 \%$ during this time for the $100 \%$ correlation hypothesis. It is believed that this value is large enough to be considered climatically significant. It should be emphasized, however, that the magnitude of this value may be an upper limit. This also indicates 
that the differential radius measurements may have the sensitivity to measure changes in the photosphere which potentially change the solar luminosity to the degree that affects the conditions on Earth.

The possible contribution to the total irradiance change indicated by differential radius change may be relevant to the behavior of the satellite radiometers aboard both the SMM and Nimbus 7 satellites during the early years of their operation. Both instruments recorded definite decreases in the solar total irradiance since their respective launches until the minimum of solar cycle 21. In contrast to these observations, the modeling of the total irradiance modulation by Foukal and Lean, 8 based on the correlations with HeI 10830 equivalent width, suggest an increase in total irradiance over that time period until the maximum occurring in 1981. They suggest that instrumental degradation may explain this discrepancy, but allow that other processes not accounted for by their modeling may have influenced the total irradiance prior to 1981 . From 1978 to 1981 , the Foukal and Lean model indicates a luminosity increase of about $0.05 \%$ due to solar activity. Based on the linear fit to the differential radius data and a $100 \%$ correlation hypothesis between $\delta(\Delta \mathrm{R})$ and $\delta \mathrm{L}$, the solar output $\delta \mathrm{L}$, the residual change in luminosity, may have decreased approximately $0.1 \%$ during the same time period. The combined effect of the residual change and the change associated with solar activity suggests a net decrease in solar total irradiance, eliminating the conflict with the satellite observations.

The possible connection between the differential radius and the total irradiance demonstrates that the differential radius may serve, along with other observations, as a useful tool to probe the mechanisms operating in the solar atmosphere that affect the total irradiance. The illumination of these mechanisms could establish the differential radius as a quantity which can quantify changes in the solar luminosity that are of long-term consequence, as distinct from changes connected with the solar activity cycle. Indeed, such changes, as seen in Fig. 1, show little connection with the solar cycle, yet indicate that significant changes have occurred in the photosphere. The changes are of such magnitude that effects on the Earth's climate are possible. Solidifying the connection between differential radius and total irradiance will stand as both a scientific and practical achievement.

\section{ACKNOWLEDGMENTS}

This work was supported in part by the U.S. Department of Energy. 


\section{REFERENCES}

1. R. C. Willson and H. S. Hudson. "Solar Luminosity Variations in Solar Cycle 21," Nature 332, 810 (1988).

2. H. A. Hill, R. T. Stebbins, and J. R. Oleson, "The Finite Fourier Transform Definition of an Edge on the Solar Disk," Ap. J. 200, 484-498 (1975).

3. B. J. Beardsley, W. M. Czarnowski, R. J.Kroll, C. S. Cornuelle, L Yi, and H. A. Hill, "Variations in Solar Luminosity Since 1957 Using Differential Radius Observations," Bull. Am. Astron. Soc. 20, No. 4, 1011 (1988).

4. B. J. Beardsley, H. A. Hill, C. S. Cornuelle, and R. J. Kroll, "Solar Constant Variations Found from Secular and Latitudinal Changes in the Limb Darkening Function," paper presented at The Sun in Time Conference, March 1989, Tucson, Arizona.

5. R. J. Kroll, B. J. Beardsley, and H. A. Hill, "Recent Differential Radius Results at SCLERA," Bull. Am. Astron. Soc. 22, No. 1, 742 (1990).

6. O. R. White and W. C. Livingston, "Solar Luminosity Variation. III. Calcium K Variation from Solar Minimum to Maximum in Cycle 21," Ap. J. 249, 798 (1981).

7. A. Skumanich, J. L. Lean, O. R. White, and W. C. Livingston. "The Sun as a Star: Three-Component Analysis of Chromospheric Variability in the Calcium K Line," Ap. J. 282, 776 (1984).

8. P. Foukal and J. Lean, "Magnetic Modulation of Solar Luminosity, Ap. J. 328, 347 (1988).

9. W. C. Livingston, L. Wallace, O. R. White, "Spectrum Line Intensity as a Surrogate for Solar Irradiance Variations," Science 240, 1765 (1988).

10. J. R. Kuhn, K. G. Libbrecht, and R. H. Dicke, "The Surface Temperature of the Sun and Changes in the Solar Constant," Science 242, 908 (1988).

11. R. C. Willson, "Measurements of Solar Total Irradiance and its Variability," Space Science Reviews 38, 203 (1984). 


\title{
SOLAR RESPONSE TO LUMINOSITY VARIATIONS
}

\author{
S. Arendt \\ The University of Chicago \\ Laboratory for Astrophysics and Space Research \\ 933 East 56th Street \\ Chicago, IL 60637
}

The connection between solar luminosity and magnetic fields is now well-established. Magnetic fields under the guise of sunspots and faculae enhance or suppress heat transfer through the solar surface, leading to changes in the total solar luminosity. This raises the question of the effect that such surface heat transfer perturbations have on the internal structure of the sun. The problem has been considered previously by Foukal ${ }^{(1)}$ and Spruit ${ }^{(2,3)}$. We here generalize the calculation of Spruit, removing the assumption of a constant heat diffusivity coefficient by treating the full mixing length heat transfer expression. Further, we treat the surface conditions in a simpler manner, and show that the previous conclusions of Foukal and Spruit are unaffected by these modifications.

The model treats the solar convection zone as a plane parallel layer of perfect gas, denoting $H$ the heat flux, $P$ the pressure, and $T$ the temperature. We assume that the solar interior is unaffected by surface effects, and thus enforce a constant heat flux $H_{0}$ at the base of the convective layer. On the surface, we enforce a radiative boundary condition $H=\sigma T^{4}$, but allow $\sigma$ to vary with time to model the time variations of the effective emissivity (caused by sunspots and faculae). In general, the total height of the layer will vary in response to the variations in $\sigma$ while the total mass in the layer remains constant so that we define the location of the base and surface of the layer by the base and surface pressures $P_{0}$ and $P_{h}$. As mentioned, we neglect partial ionization effects by assuming a perfect gas equation of state, but leave $\gamma$ (the adiabatic index) unfixed to preserve generality. Finally, we assume a constant gravitational field.

The model allows us to study various plage/sunspot scenarios through the use of their effective emissivity variation $\sigma(t)$. For the present work, we use $\sigma(t)=\sigma_{0}+\delta \sigma u(t)$ where $u(t)$ is the unit step function, and $\delta \sigma$ is a small perturbation $\delta \sigma<<\sigma_{0}$. With this choice of $\sigma(t)$, we make the following observations. For $t<0$, we expect the fluid layer to be in a steady state with $H=H_{0}$ everywhere. At $t=0$, the surface luminosity will jump from $H_{0}$ to $H_{0}\left(1+\delta \sigma / \sigma_{0}\right)$. However, as $t \rightarrow \infty$ the layer will approach another steady state, with $H=H_{0}$ everywhere, so that the surface luminosity must relax from $H_{0}\left(1+\delta \sigma / \sigma_{0}\right)$ back to $H_{0}$. It is the timescale of this relaxation that we are interested in. In what follows, it will be shown that each steady state corresponds approximately to an adiabat, so that the $\sigma(t)$ variation forces the fluid layer from one adiabat to another.

To begin the full solution, consideration of Fig. 1 leads us to the following set of five equations, which, in order, are the continuity equation, the hydrostatic equation, conservation of energy, mixing length convective heat transport, and the equation of state:

$$
\frac{\partial \rho}{\partial t}+\frac{\partial}{\partial z}(\rho v)=0
$$




$$
\begin{gathered}
\frac{\partial P}{\partial z}+\rho g=0 \\
\frac{\partial H}{\partial z}+\frac{\partial}{\partial z}\left\{\frac{\gamma}{\gamma-1} P v+\rho g v z\right\}+\frac{\partial}{\partial t}\left\{\frac{1}{\gamma-1} P+\rho g z\right\}=0 \\
P=C_{P} \rho \sqrt{\frac{g}{T}} \frac{\ell^{2}}{4}\left\{\left(1-\frac{1}{\gamma}\right) \frac{T}{P} \frac{\partial P}{\partial z}-\frac{\partial T}{\partial z}\right\}^{\frac{3}{2}} \\
P=\left(\frac{k}{\mu}\right) \rho T
\end{gathered}
$$

where $z$ is the depth coordinate, $g$ is the solar gravitational constant, $v$ is the fluid velocity, $c_{P}$ is the specific heat per unit mass, $\ell$ is the mixing length and $\mu$ is the mass of an individual gas particle. We note that in equations (2) and (3) inertial terms second order in the perturbation have been neglected. In (4), we take the mixing length $\ell$ to be $\chi k T / \mu g$ where $k T / \mu g$ is the pressure scale height, and $\chi$ is a numerical factor of order unity.

To facilitate the application of the boundary conditions, we rewrite this set of equations using $P$ as an independent variable in place of $z$. Having done this, we find that equations (1)-(5) reduce to a single equation:

$$
\phi P^{2+\frac{1}{2 \gamma}}\left(\frac{\partial G}{\partial P}\right) G^{-2}\left\{\left(3-\frac{1}{2 \gamma}\right) \frac{1}{P} \frac{\partial G}{\partial P}+\frac{3}{2} \frac{\partial^{2} G}{\partial P^{2}}\right\}-\frac{\partial G}{\partial t}=0
$$

where

$$
\begin{gathered}
\phi=\frac{\sqrt{3} \chi^{2} g}{4} \\
G(P, z)=\left(\rho P^{-\frac{1}{\gamma}}\right)^{-\frac{1}{3}}
\end{gathered}
$$

To obtain the physical meaning of $G$, we note that $G$ can be shown to be proportional to $e^{K S}$ where $S$ is the entropy, and $K$ a constant.

In examining (6), we see that in steady state, the equation can be immediately solved to give:

$$
G(P)=C_{1}-C_{0} P^{-\left(1-\frac{1}{3 \gamma}\right)}
$$

where, for the boundary conditions on $H$ shown in Fig. 1, we find

$$
\begin{gathered}
C_{0}=\frac{1}{3}\left(\frac{4 H}{\chi^{2}} \frac{\gamma-1}{\gamma}\right)^{\frac{2}{3}} \frac{3 \gamma}{3 \gamma-1} \\
C_{1}=\left(\frac{k}{\mu}\right)^{\frac{1}{3}}\left(\frac{H}{\sigma}\right)^{\frac{1}{12}} P_{h}^{-\frac{1}{3}\left(1-\frac{1}{\gamma}\right)}+C_{0} P_{h}^{-\left(1-\frac{1}{3 \gamma}\right)}
\end{gathered}
$$

For solar-like conditions, one can show that the second term in (9) is important only near the surface of the layer, yielding immediately the fact that the steady-state solutions lie approximately on adiabats. 
We next use the steady state solutions (9) to linearize (6) as follows. For $t<0$ and for $t \rightarrow \infty$, the layer is in steady state, as mentioned previously. We denote the initial steady state as $G_{s s}^{i}(P)$ and the final steady state as $G_{s s}^{f}(P)$, where $G_{s s}^{i}$ and $G_{s s}^{f}$ are given by (9) with the proper values of $C_{0}$ and $C_{1}$. An examination of (10) and (11) shows that the two steady-state solutions differ only in their value of $C_{1}$ i.e. they lie on different adiabats. We next define $\Delta G(P, t)$ as:

$$
G(P, t)=G_{s s}^{f}(P)+\Delta G(P, t)
$$

where $\Delta G$ is considered small. We then use (12) in (6) and retaining only terms first order in $\Delta G$, we find:

$$
\theta P^{1+\frac{2}{3 \gamma}}\left(\frac{\partial^{2} \Delta G}{\partial P^{2}}+\frac{2-\frac{1}{3 \gamma}}{P} \frac{\partial \Delta G}{\partial P}\right)-\frac{\partial \Delta G}{\partial t}=0
$$

where

$$
\theta=\frac{3 \sqrt{3}}{8} \chi^{2} g \frac{\sqrt{C_{0}\left(1-\frac{1}{3 \gamma}\right)}}{C_{1}^{2}}
$$

The values of $C_{0}$ and $C_{1}$ in (12) are those appropriate for $G_{s s}^{f}(P)$. In deriving (13), we have neglected the pressure dependent term in $G_{s s}^{f}(P)$ since it is small, thereby assuming polytropic steady state solutions. The boundary conditions on $\Delta G(P, t)$ can be shown to be:

$$
\begin{gathered}
\left.\frac{\partial \Delta G}{\partial P}\right|_{P_{0}}=0 \\
\left.\Delta G\right|_{P_{h}}=\left.\psi \frac{\partial \Delta G}{\partial P}\right|_{P_{h}} \\
\Delta G(P, 0)=\kappa \\
\Delta G(P, t) \longrightarrow_{t \rightarrow \infty} 0
\end{gathered}
$$

where

$$
\begin{gathered}
\psi=\frac{1}{8}\left(\frac{k}{\mu}\right)^{\frac{1}{3}}\left(\frac{H}{\sigma}\right)^{\frac{1}{12}} \frac{P_{h}^{\frac{5}{3}}}{\left(1-\frac{1}{3 \gamma}\right) C_{0}} \\
\kappa=\left(\frac{k}{\mu}\right)^{\frac{1}{3}}\left(\frac{H}{\sigma}\right)^{\frac{1}{12}} P_{h}^{-\frac{1}{3}\left(1-\frac{1}{\gamma}\right)} \frac{1}{12} \frac{\delta \sigma}{\sigma}
\end{gathered}
$$

With (15)-(18), (13) is a well-defined boundary value problem whose solution can be written as an eigenfunction series as follows:

$$
\Delta G(P, t)=\sum_{m} A_{m} M_{m}(P) e^{-t / \tau_{m}}
$$

where

$$
\begin{gathered}
M_{m}(P)=P^{-\gamma \eta}\left\{J_{\nu}\left(\xi_{m} P^{\eta}\right)+B_{m} Y_{\nu}\left(\xi_{m} P^{\eta}\right)\right\} \\
B_{m}=\frac{-J_{\gamma+1}\left(\xi_{m} P_{0}^{\eta}\right)}{Y_{\gamma+1}\left(\xi_{m} P_{0}^{\eta}\right)}
\end{gathered}
$$




$$
\begin{gathered}
A_{m}=\frac{-(\delta \kappa / \eta \psi) P_{h} M_{m}\left(P_{h}\right)}{M_{m}^{2}\left(P_{h}\right)\left\{\eta_{m}^{2} P_{h}^{2 \eta}+\frac{2 \nu}{\eta} \frac{P_{h}}{\psi}+\left(\frac{P_{h}}{\psi \eta}\right)^{2}\right\}-\left(\frac{2}{\pi}\right)^{2} P_{h}^{-2 \nu \eta} / Y_{\nu+1}^{2}\left(\xi_{m} P_{0}^{\eta}\right)} \\
\nu=\frac{3 \gamma-1}{3 \gamma-2} \\
\eta=\frac{3 \gamma-2}{6 \gamma} \\
\xi_{m}=\frac{1}{\eta} \frac{1}{\sqrt{\theta \tau_{m}}}
\end{gathered}
$$

The characteristic times $\tau_{m}$ are solutions to the eigenvalue equation:

$$
\frac{P_{h}^{1-\eta}}{\psi \eta \xi_{m}}\left[J_{\nu}\left(\xi_{m} P_{h}^{\eta}\right)+B_{m} Y_{\nu}\left(\xi_{m} P_{h}^{\eta}\right)\right]+\left[J_{\nu+1}\left(\xi_{m} P_{h}^{\eta}\right)+B_{m} Y_{\nu+1}\left(\xi_{m} P_{h}^{\eta}\right)\right]=0
$$

Having the solution for $\Delta G(P, t)$, we note the following connection to the heat flux.

$$
\begin{aligned}
H(P, t) & =H_{0}+\delta H(P, t) \\
\delta H(P, t) & =\frac{3}{4} \frac{H_{o} P^{1-\eta \gamma}}{\eta \nu C_{0}} \frac{\partial \Delta G}{\partial P} \equiv H_{0}\left(\frac{\delta \sigma}{\sigma_{0}}\right) \sum D_{m} e^{-t / \tau_{m}}
\end{aligned}
$$

The surface luminosity is then given by (29) evaluated at $P=P_{h}$.

As noted by Spruit, the solution (29) exhibits some general characteristics. The luminosity is shown to relax on two different timescales: one on the order of $10^{5}$ years, and the other on the order of 50 days. The eigenvalue equation can be solved to yield an expression for the long timescale as follows:

$$
\tau_{0}=\frac{4}{9} \frac{\psi \eta \nu C_{0} C_{1}^{2}}{g H\left(\eta^{2}-\frac{1}{36}\right)} \frac{P_{0}^{3 \eta+\frac{1}{2}}}{P_{h}^{\eta+\frac{3}{2}}}
$$

The next longest timescale is found from the first zero of

$$
J_{\gamma+1}\left(\frac{P_{0}^{\eta}}{\eta \sqrt{\theta \tau_{1}}}\right)=0
$$

Finally, we find $D_{0}$, the amplitude of the long timescale mode, to be::

$$
D_{0}=\left(1+\frac{3 \gamma}{3 \gamma-1} \frac{P_{h}}{\psi}\right)^{-1}
$$

For typical values of $P_{h}=10^{6}$ dynes, $P_{0}=5 \times 10^{13}$ dynes, $\gamma=\frac{3}{2}$ and, $\chi=1$, we find $\tau_{0}=350,000$ years, $\tau_{1}=51$ days, $D_{0}=.549$. 
In summary, the model shows that following the application of a step function emissivity change, a fraction $1-D_{0}$ of the luminosity change relaxes away after $\sim 50$ days. This corresponds to the thermal diffusion time across the convection zone, adjusting the difference of the adiabatic temperature gradient and the actual temperature gradient to a value in correspondence with the surface change. In other words, the whole convection zone "feels" the perturbation on this timescale. The remaining fraction relaxes away on a timescale of $10^{5}$ years, corresponding to the convective layer radiating away enough energy so that it can adjust to its new adiabat. These are the same results arrived at by Spruit and Foukal.

For variations of $\sigma$ on timescales of 10-200 years, then, the only important relaxation is the 50 day one. If the amplitude of this relaxation is small, the luminosity follows the $\sigma$ variation.

The author would like to thank Dr. P. Foukal for his useful comments on this work.

\section{References}

1. Foukal, P., Fowler, L.A., and Livshits, M. 1983, Ap. J. 267, 863.

2. Spruit, H.C. 1982, Astr. Ap. 108, 348.

3. Spruit, H.C. 1982, Astr. Ap. 108, 356. 

SESSION 4: LONG-TERM SOLAR VARIABILITY AND CLIMATE 


\title{
TESTING THE SUN-CLIMATE CONNECTION WITH PALEOCLIMATE DATA
}

\author{
Thomas J. Crowley and Matthew K. Howard \\ Applied Research Corporation \\ 305 Arguello Drive \\ College Station, TX 77840
}

\begin{abstract}
If there is a significant sun-climate connection, it should be detectable in high-resolution paleoclimate records. Of particular interest is the last few thousand years, where we have both indices of solar variability $\left({ }^{14} \mathrm{C}\right.$ and ${ }^{10} \mathrm{Be}$ ) and climate variations (alpine glaciers, tree rings, ice cores, corals, etc.). Although there are a few exceptions, statistical analyses of solar and climate records generally indicates a "flickering" relationship between the two -sometimes it seems to be present, sometimes not. The most repeatable solar climate periods occur at $\sim 120$ and $\sim 56$ yrs, although there is also evidence for $\sim 420$ and $\sim 200$ yr. power in some records. However, coherence between solar and climate spectra is usually low, and occurrence of solar spectra in climate records is sometimes dependent on choice of analysis program. These results suggest in general a relatively weak sun-climate link on time scales of decades to centuries. This conclusion is consistent with previous studies and with the observation that inferred climate fluctuations of $1.0-1.5^{\circ} \mathrm{C}$ on this time scale would require solar constant variations of approximately $0.5-1.0 \%$. This change in forcing is almost an order of magnitude greater than observed changes over the last solar cycle and appears to be on the far-outer limit of acceptable changes for a Maunder Minimum-type event.
\end{abstract}

\section{INTRODUCTION}

The reality of a future greenhouse warming has been the topic of much heated debate. Many of the objections are based on two major points: (1) the warming is not as great as predicted by most climate models; and (2) the temperature is not uniformly increasing, in that there are significant decadal-scale fluctuations having a magnitude of about $0.4^{\circ} \mathrm{C}$ that significantly modify the global average temperature curve of the last century.

In order to better evaluate the significance of a greenhouse warming, it is necessary to understand the origin of decadal-scale fluctuations in global temperature that are due to "natural variability" in the climate system. At present there are three main hypotheses for decadal- to centennial-scale fluctuations due to factors other than $\mathrm{CO}_{2}$ (see full discussion 
in ref. 1) -- solar variability ${ }^{2}$, volcanism ${ }^{3}$, and nonlinear interactions in the ocean atmosphere system ${ }^{4,5}$.

The number of oscillations in the last century are too few to rigorously test different hypotheses as to the origin of climate fluctuation in the present century. This is where paleoclimate data come in, for there are many different realizations of such climate fluctuations over the last few thousand years (e.g., Fig. 1). These oscillations occur prior to the time of $\mathrm{CO}_{2}$ buildup (about 1850) and therefore serve as an ideal testing ground for different mechanisms.

In this study we will examine one of the mechanisms proposed to explain decadal- to centennial-scale climate fluctuations -- solar variability. We compare an index of solar variability $\left({ }^{14} \mathrm{C}\right)$ with a number of different climate records of the last few thousand years.

\section{METHODS}

Solar Records Our sources of cosmogenic data are measurements of ${ }^{14} \mathrm{C}$ from tree rings ${ }^{6}$. Comparison of ${ }^{14} \mathrm{C}$ and ${ }^{10} \mathrm{Be}$ records, which have significantly different geochemical cycles, indicate that a common signal, suggestive of cosmogenic origin, can be extracted from both records 7 . A previous study of this record indicates a fundamental mode of solar response at a period of $\sim 420 \mathrm{yr}$, with harmonics at $220,140,89,67,57,52$, and 45 years ${ }^{8}$.

Climate Records Our terrestrial records come from a number of sources:

(1) a record of alpine glacial moraine variations for the last 5000 years developed by Röthlisberger 9 . This record, although small in number, is from a wide variety of locations (Alaska, Scandinavia, Alps, South America, Himalayas, Sierras, and New Zealand). The record was originally analyzed by Stuiver and Braziunas ${ }^{8}$ for level of agreement with the ${ }^{14} \mathrm{C}$ record. Results suggest a possible solar-terrestrial link in the $\sim 110$ period ${ }^{8}$. We extend their study by compositing the record in order to remove sources of regional noise.

(2) ice core records from three widely distributed locations -- Greenland, Peru, and Antarctica $10,11,12$.

(3) tree ring records from two locations far removed from the ice cores -- the Sierras and western China ${ }^{13,14 .}$

Statistical procedures The data were regularly spaced in time with a cubic spline used to interpolate the data to a regular 5 year grid. In most cases this sampling interval was shorter than the average of the irregular spacing of the original data $(\sim 20-40 \mathrm{yr})$. The interpolated curves were compared to the original curves, and interpolated values were adjusted manually at those locations where the cubic spline performed poorly. The ${ }^{14} \mathrm{C}$ record contained more high frequency energy than the other records so this record was smoothed using running averages until both records appeared to have similar high frequency characteristics. Finally, all data were normalized. 
A statistical analysis program based on Newton ${ }^{15}$ was used to analyze the data pairs. The package calculates the raw periodogram, smoothed spectral and cospectral estimates, and correlation coefficients. Preprocessing removes the mean and detrends the individual data series prior to computing the spectra. Spectral estimates are obtained by the Fourier transformation of the covariance functions. Smoothed estimates are formed by applying a Parzen window to the covariance function prior to transformation. Three window widths were used, 1/5,1/3 and $2 / 5$ of the number of data points. The amount of smoothing is inversely proportional to the window width.

\section{RESULTS}

Figure 2 compares the ${ }^{14} \mathrm{C}$ record with various climate indices. Although in general there is not a strong visual correlation between solar and climate records, comparison of their individual spectra yields some indication of a solar-terrestrial connection (Table 1). In particular, the western Chinese tree ring record has quite a strong correlation with ${ }^{14} \mathrm{C}$ $(r=-0.54)$. The South Pole ${ }^{18} \mathrm{O}$ record has the next strongest solar correlation $(0.37)$. If $r$ is relaxed to search for maximum correlation, there is a significant increase in the Greenland ${ }^{18} \mathrm{O}$ correlation.

The above discussion and Table 1 suggest that, if the solar-climate connection is real, the regional response can vary considerably, from warm periods correlating with sunspot maxima (Greenland and South Pole) to cold periods correlating with sunspot maxima (China). The large lag offsets between different regions could conceivably reflect displacements of the atmospheric circulation, a subject discussed more fully in ref. 1 . Our study also suggests that western China might be the most sensitive region to respond to solar variations.

The most consistently occurring solar peaks are at periods of $\sim 120$ and $\sim 56$ years. Peaks at $\sim 200$ and $\sim 120$ yr. support previous findings ${ }^{8,16}$; peaks at $\sim 420$ and $\sim 56$ yrs appear to represent new findings. Although solar spectra can be found in some of these climate records, in most cases there are a number of significant differences between the solar peaks

\begin{tabular}{|c|c|c|c|c|c|c|c|}
\hline \multicolumn{8}{|c|}{ SOLAR SPECTRA IN CLIMATE RECORDS } \\
\hline PERIOD & $\sim 420$ & $\sim 200$ & $\sim 120$ & $\sim 87$ & $\sim 56$ & $\mathbf{r}$ & $\mathrm{r}_{\max }$ \\
\hline GLACIERS & $\mathrm{x}$ & & $\mathbf{X}$ & $\mathbf{X}$ & & -0.11 & $(330)$ \\
\hline SIERRA TR & & & $\mathbf{X}$ & & $\mathbf{X}^{*}$ & -0.10 & $(70)$ \\
\hline CHINA TR & & $\mathbf{X}^{*}$ & $\mathrm{x}$ & $\mathrm{x}$ & $\mathrm{x}$ & -0.54 & $-54(0)$ \\
\hline GRN O18 & $\mathbf{X}$ & & $\mathbf{X}$ & & $\mathbf{X}^{*}$ & -0.01 & (45) \\
\hline PERU O18 & & & $\mathrm{x}$ & $\mathrm{x}$ & $\mathbf{X}$ & 0.05 & (15) \\
\hline SPOLE O18 & & $\mathbf{X}^{*}$ & $\mathrm{x}$ & $\mathrm{x}$ & $\mathrm{x}$ & 0.37 & $.42 \quad(-20)$ \\
\hline
\end{tabular}

Table 1. Correlations between solar spectra in climate records. A small "x" indicates that solar periods occur but not consistently (as defined by variations in the amount of record smoothing). Bold $X^{\prime}$ 's refer to a more consistent occurrence, and bold $\mathrm{X}^{*}$ s refers to records that have coherences $>0.6$. Parentheses after $r_{\max }$ refers to number of years the climate time series leads or lags at maximum correlation (minus sign indicates climate leads ${ }^{14} \mathrm{C}$ ). 
and peaks that, for example, occur in Pleistocene time series. In the latter, many different climate spectra have been compared to orbital forcing (the Milankovitch effect), with conclusions often being that, regardless of technique used in the analysis, orbital periods frequently occur in climate records of the Pleistocene ${ }^{17}$. Furthermore, coherence between climate and orbital spectra are often quite high $(>0.8)$.

As opposed to our experience with Pleistocene records, analysis of time series covering the last few thousand years indicates that the solar spectra are not nearly so robust - they appear in some records but not others, and often disappear with slight changes in smoothing. Furthermore, coherences are often quite low $(<0.5)$, although in a few cases (marked by bold $X^{*}$ in Table 1 ) coherences are significantly higher $(>0.6)$.

\section{DISCUSSION AND CONCLUSION}

The above results provide some evidence for a sun-climate link. However, the results also suggest that a sun-climate connection, if present, is relatively weak. These results are consistent with some earlier sun-climate comparisons, which suggest that $<10 \%$ of the climate record can be explained by solar variability $18,19,20$. It is possible that other more sophisticated statistical procedures could elicit a more significant relationship, so we do not claim our results represent a final assessment of the problem. What we can say is that in general the connection certainly does not leap out at us, and that in itself suggests that even if one can find a statistically significant correlation in the future, it is unlikely to explain a great deal of the variance. This conclusion is different than an earlier study of possible sun-climate connection which suggested the correlation might be higher ${ }^{21}$.

The above conclusion is also consistent with another line of reasoning. As summarized in Crowley and North ${ }^{1}$, most centennial-scale climate fluctuations of the last 1000 years were on the order of $1.0-1.5^{\circ} \mathrm{C}$ in magnitude. If these changes were entirely due to solar variations, they would require equivalent variations in the solar constant of perhaps 0.5 $1.0 \%$, with the magnitude of solar variation depending on the sensitivity of the climate model. This estimate is approximately an order of magnitude greater than observed fluctuations over the last solar cycle 22 . Although it is not inconceivable that more extreme fluctuations such as the Maunder Minimum could be associated with larger variations in solar activity, the required numbers (assuming our climate models have the proper sensitivity) appear to be on the far-outer limit of the possible range.

Finally, we note that our results have some implications for possible future modulation of a greenhouse warming by solar activity 23 . The very low correlations in the past indicate that such a modulation is unlikely to be greatly significant (perhaps $0.1-0.2^{\circ} \mathrm{C}$ change in global mean temperature). Other sources of natural variability may still be important, however, in such a modulation.

\section{ACKNOWLEDGEMENTS}

We thank A. Arking and K. Schatten for an invitation to this meeting and W Hyde and G. North for comments. This work was supported by NSF Grant ATM-8722145 and U.S. Department of Energy contract B-PNL 017113 AB-1. 


\section{REFERENCES}

1. Crowley, T. J., and G. R. North, Paleoclimatology, Oxford Univ. Press, New York, 1990 (in press).

2. Eddy, J. A., "The Maunder Minimum," Science 192:1189-1202, 1976.

3. Hammer, C. U., H. B. Clausen, and W. Dansgaard, "Greenland ice sheet evidence of post-glacial volcanism and its climatic impact," Nature 288:230-235, 1980.

4. Hasselmann, K., "Stochastic climate models, I, Theory," Tellus 28:473-484, 1976.

5. Wigley, T. M. L., and S. C. B. Raper, "Natural variability of the climate system and detection of the greenhouse effect," Nature 344:324-327, 1990

6. Stuiver, M., and T. F. Braziunas, "The solar component of the atmospheric ${ }^{14} \mathrm{C}$ record," in Secular Solar and Geomagnetic Variations in the Last 10,000 Years, F. R. Stephenson and A. W. Wolfendale (Eds.), pp. 245-266, Kluwer, Dordrecht, Netherlands, 1988.

7. Beer, J., et al., "Information on past solar activity and geomagnetism from ${ }^{10} \mathrm{Be}$ in the Camp Century ice core," Nature 331:675-680, 1988.

8. Stuiver, M., and T. F. Braziunas, "Atmospheric ${ }^{14} \mathrm{C}$ and century-scale solar oscillations," Nature 338:405-408, 1989.

9. Röthlisberger, F., 10,000 Jahre Gletschergeschichte der Erde Aarau, Verlag, Sauerländer, 1986.

10. Johnsen, S. J., W. Dansgaard, H. B. Clausen, and C. C. Langway, "Climatic oscillations 1200-2000 A.D.," Nature 227:482-483, 1970.

11. Thompson, L. G., E. Mosley-Thompson, W. Dansgaard, and P. M. Grootes, "The Little Ice Age as recorded in the stratigraphy of the tropical Quelccaya Ice Cap," Science 234:361-264, 1986.

12. Mosley-Thompson, E., L. G. Thompson, P. M. Grootes, and N. Gundestrup, "Little Ice Age (Neoglacial) paleoenvironmental conditions at Siple Station, Antarctica," Annals of Glaciology, 1990 (in press).

13. LaMarche, V. C., "Paleoclimatic inferences from long tree-ring records," Science 183:1043-1048, 1974.

14. Wang, Y., L. Guangyuan, X. Zhang, and C. Li, "The relationships between tree rings of Qilianshan juniper and climatic change and glacial activity during the past 1000 years in China," Kexue Tongbao 28:1647-1652, 1983.

15. Newton, J. H., TIMESLAB: A Time Series Analysis Laboratory, 623 pp., Wadsworth \& Brooks/Cole, Pacific Grove, Calif., 1988.

16. Sonett, C. P., and H. E. Suess, "Correlation of bristlecone pine ring widths with atmospheric ${ }^{14} \mathrm{C}$ variations: A climate-sun relation," Nature 307:141-143, 1984.

17. Berger, A. L., J. Imbrie, J. D. Hays, G. J. Kukla and B. Saltzman (Eds.), Milankovitch and Climate, 895 pp., D. Reidel, Dordrecht, Netherlands, 1984.

18. Mitchell, J. M., C. W. Stockton, and D. M. Meko, "Evidence of a 22-year rhythm of drought in the western United States related to the Hale solar cycle since the 17th century," in: Solar-Terrestrial Influences on Weather and Climate B. M. McCormac and T. A. Seliga (Eds.), D. Reidel, Hingham, Mass., pp.125-144, 1979.

19. Stuiver, M., "Solar variability and climatic change during the current millennium," Nature 286:868-871, 1980.

20. Jacoby, G. C., and E. R. Cook, "Past temperature variations inferred from a 400year tree-ring chronology from Yukon territory, Canada," Arc. Alp. Res. 13:409418, 1981.

21. Wigley, T. M. L., "The climate of the past 10,000 years and the role of the sun," in: Secular Solar and Geomagnetic Variations in the Last 10,000 years, F. R. 
Stephenson and A.W. Wolfendale (Eds.), pp. 209-224, Kluwer, Dordrecht, Netherlands, 1988.

22. Kyle, H. L., P. E. Ardanuy, and E. J. Hurley, "The status of the Nimbus-7 earthradiation-budget data set," Bull. Am. Met. Soc. 66:1378-1388, 1985.

23. George C. Marshall Institute, Scientific Perspectives on the Greenhouse Problem, Washington, D.C., 37 pp., 1989.

24. Zhu, K. (Chu, K.), "A preliminary study on the climatic fluctuations during the last 5,000 years in China," Scientia Sinica 16:226-256, 1973.

25. Zhang, D., "Winter temperature variation during the last 500 years in southern China," Kexue Tongbao 25:497-500, 1980.

26. State Meteorological Administration (SMA), Annals of 510 Years' Precipitation Record in China (in Chinese), The Meteorological Research Institute, Beijing, 1981.

27. Zhang, D., "Synoptic-climatic studies of dust fall in China since historic times," Scientia Sinica (Ser.B) 27:825-836, 1984.

28. Zhang, J., and T. J. Crowley, "Historical climate records in China and reconstruction of past climates," Jour. Clim. 2:833-849, 1989.

29. Ren, Z., "The abnormal periods of climate in China over the past 5000 years and their causes. Adv. Atmos. Sci. 4:210-217, 1987. 


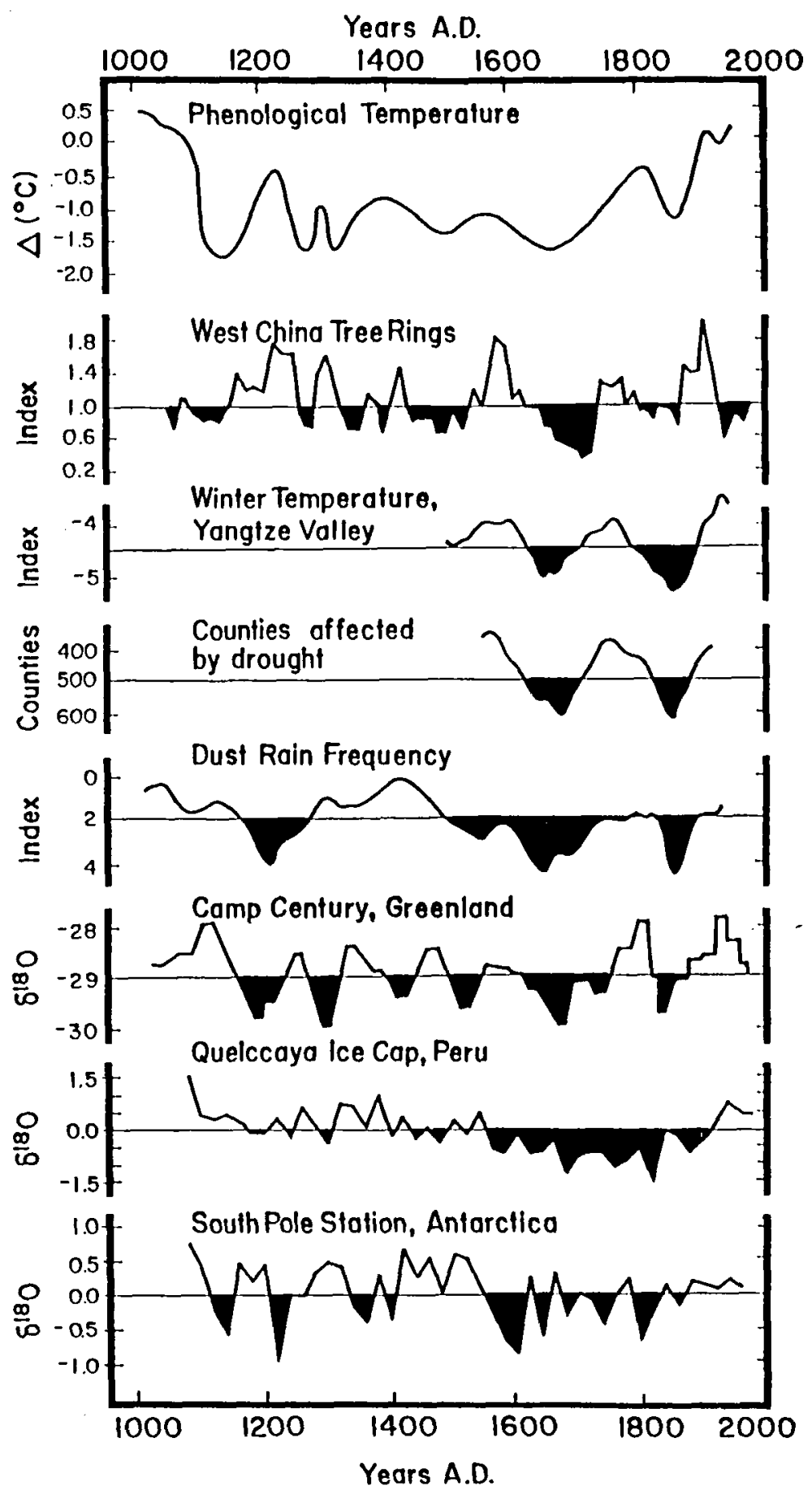

Fig. 1 Examples of decadal- and centennial-scale climate fluctuations over the last 1000 years: (a) The phenological temperature in China ${ }^{24}$. This index is based on timing of recurrent weather-dependent phenomena, such as dates of flowering of shrubs or arrivals of migrant birds, or distribution of climatically sensitive organisms; (b) The growth ring index of a juniper tree from western China ${ }^{14}$; (c) The winter temperature index in the lower reaches of the Yangtze River ${ }^{25}$; (d) The number of counties affected by drought in China ${ }^{26}$; (e) The frequency curve of dust rains in China ${ }^{27}$; (f) The $\delta^{18} \mathrm{O}$ record from Camp Century, Greenland ${ }^{10}$; (g) The $\delta^{18} \mathrm{O}$ record from a Peru ice core ${ }^{11}$; (h) The $\delta^{18} \mathrm{O}$ record from the South Pole ${ }^{12}$. Shading equals cool intervals. [Courtesy E. Mosley-Thompson; modified from ${ }^{28,} 29$ ] 

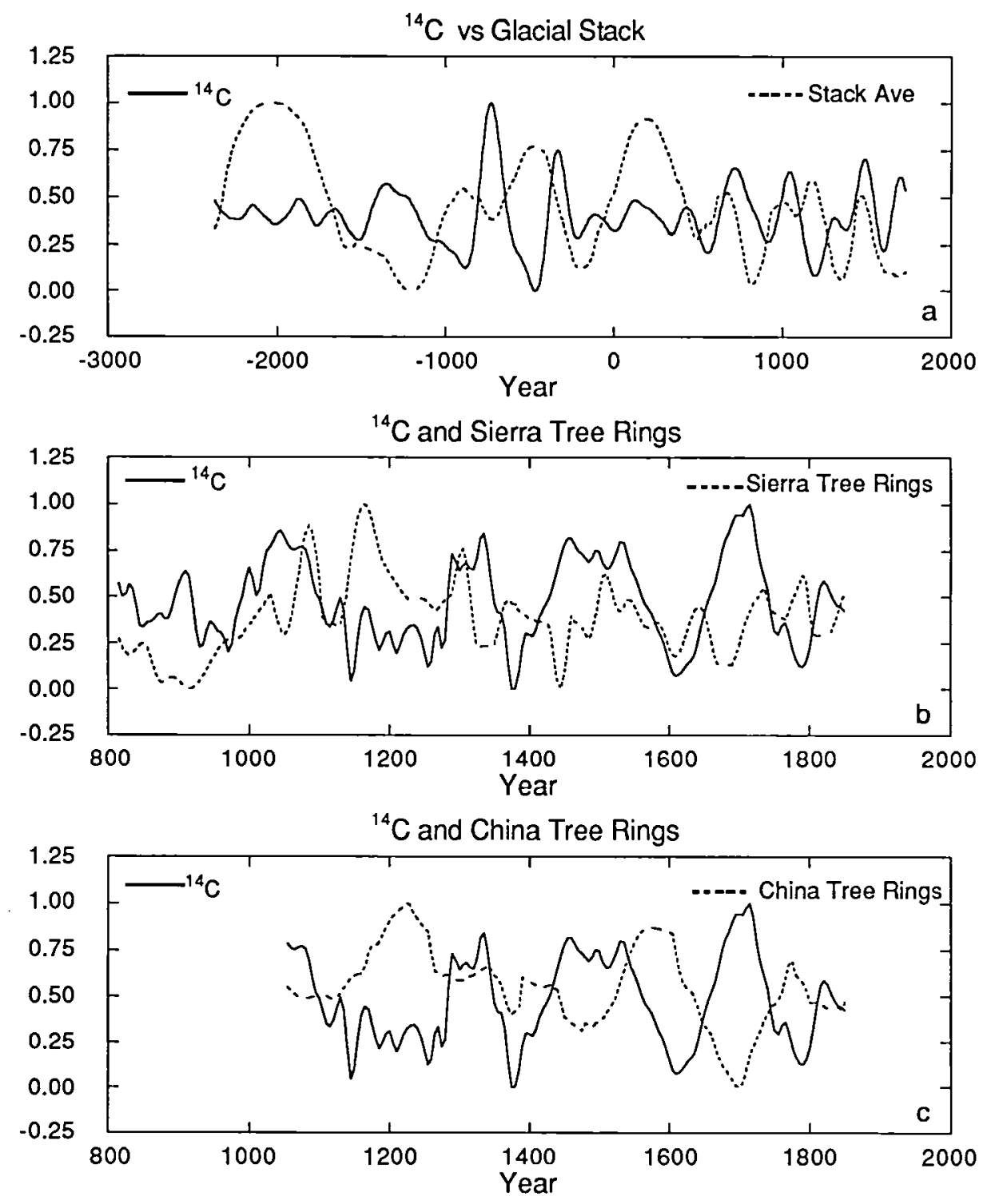

${ }^{14} \mathrm{C}$ and Greenland $\delta^{18} \mathrm{O}$

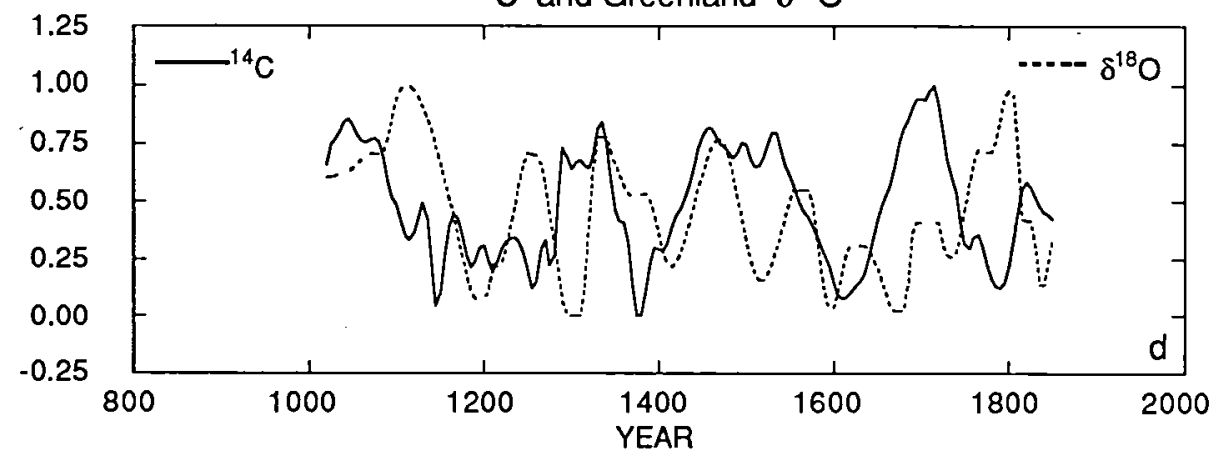



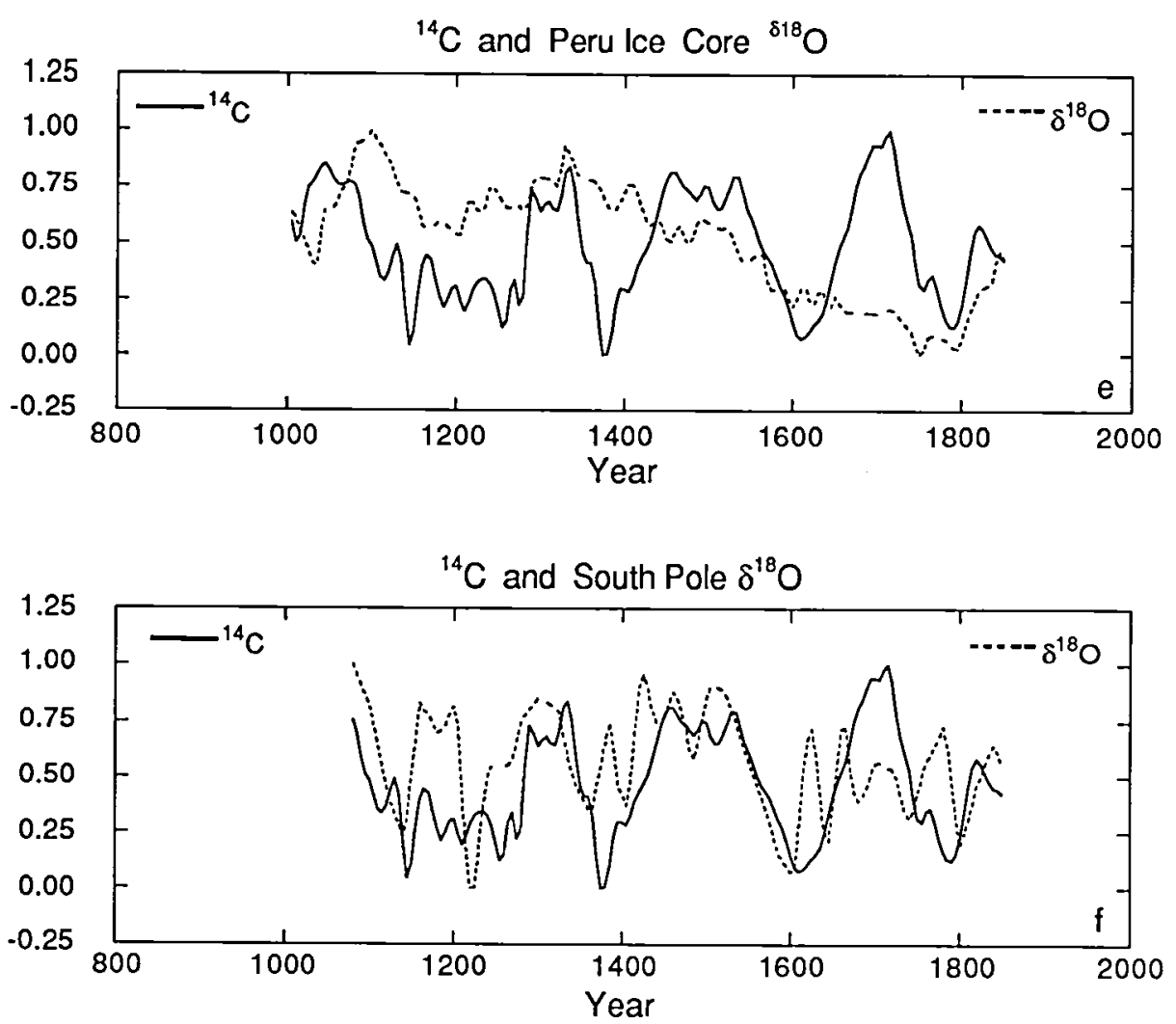

Fig. 2 Comparison of ${ }^{14} \mathrm{C}$ record of solar variability with five different indices of climate change: (a) a composite record of alpine glacial advances ${ }^{9}$; (b) and (c) tree rings from the Sierras and western China ${ }^{13}, 14 ;$ and (d-f) $\delta^{18} \mathrm{O}$ records from Greenland, Peru, and Antarctica $10,11,12$. 


\title{
SENSITIVITY OF THE OCEANIC TURBULENT BOUNDARY LAYER TO CYCLIC INSOLATION CHANGE WITH RESPONSE PERIODS OF 23 TO $2.5 \mathrm{KY}$ : AN EQUATORIAL ATLANTIC RECORD FOR THE LAST 200KA.
}

\author{
Andrew McIntyre \\ Lamont-Doherty Geological Observatory of Columbia University, Palisades, N.Y. 10964 and \\ Dept of Geology, Queens College of the City University of New York, Flushing, N.Y. 11367.
}

\section{ABSTRACT}

Time series of sea-surface temperature in cores sited beneath the region of maximum divergence centered on $10^{\circ} \mathrm{W}$ are characterized by two sets of periodic signals. The dominant signal is centered on a period of $23 \mathrm{Ky}$ and is coherent with and lags, $\sim 2.5 \mathrm{Ky}$, the precessional component of orbitally controlled insolation. The subdominant periods occur between 4.0 and $2.5 \mathrm{Ky}$. Both sets of signals record variation in the seasonal intensity of oceanic divergence modulated by variation in tropical easterly intensity. The longer periods are a response to precessional forcing. The forcing responsible for the shorter periods is unknown.

\section{INTRODUCTION}

Direct response of climate to insolation forcing is well documented for the annual cycle (1). Evidence from the geologic record indicates that orbital variations of insolation control climate at the primary periods of eccentricity, obliquity, and precession (2). Between the annual cycle and orbital cycles there is an interval where evidence and hypotheses for solar control are uncommon yet tantalizing. Historical evidence, the Maunder and Sporer minimums in sun-spot number, indicates the inconstancy of the solar constant (3) but is too short a record to establish cyclicity. The geologic record contains cycles that fall within the interval, e.g. oscillations in alpine glacier extent (4) and ice accumulation on Greenland (5) both with estimated periods around $2.5 \mathrm{Ky}$. To date, there is neither acceptable cause nor mechanism to explain the signals in these short continental records. Signals from long continuous records in ocean sediments have supplied the best proofs of orbital control of climate $(6,7,8)$. In these long records cycles with periods lower than the orbitals have been discerned (9). This paper describes the signals generated by a known oceanographic response to climatic forcing over an interval of $200 \mathrm{Ka}$.

\section{DATA BASE}

When ignorant, one must work with the best known and most responsive system available. The climate/ocean system response to annual insolation forcing for the turbulent boundary layer of the Equatorial Atlantic is well documented. Asymmetry of continent and ocean configures the tropospheric structure such that the southern hemisphere tropical easterly winds extend into the northern hemisphere for much of the year (10). Sea surface temperature, SST, is cool due to upwelling/divergence at and south of the equator. This diminishes cloud cover and enhances radiative heat gain in the south Equatorial Current, SEC, (11). The strong seasonal variation in the forcing winds, the tropical easterlies, produces a fluctuating equatorial system $(12,13,14)$. In boreal summer, June-September, strong southern tropical easterlies invade the northern hemisphere and the Inter Tropical Convergence Zone is furthest from the equator. Along the equator, divergence, SEC speed, and thermocline slope are all at their maximum, SST in the eastern equatorial Atlantic is at its minimum, and the sea surface slopes upward to the west $(14,15,16)$. The water transiting the SEC warms $(17,18)$ and forms the heat reservoir of the western Atlantic/Caribbean.

In boreal winter (December-March), the southern hemisphere tropical easterlies are weak and part of the equatorial surface water piled up in the west flows back as countercurrents (19). 
Divergence, thermocline slope, and SEC speed are minimal, and SST is at its annual maximum. Thus the SEC and its attendant features have two quite different seasonal aspects. In terms of the TBL and attendant biota, the system is forced by changes in annual insolation which controls tropical easterly wind zonality and Ekman divergence. These, in turn, produce marked variations in the planktonic community whose remnants provide the proxy of climate/oceanographic change.

Statistical-dynamic models developed for and applied to equatorial oceanography $(20,21,22,23,24,25,26,27,28)$ support observational data with respect to the seasonal response of the equatorial Atlantic. The westward-flowing SEC is tropical easterly forced and responds essentially in phase with tropical easterly variations. The maximum change in the TBL is centered on $10^{\circ} \mathrm{W}$.

The annual response of the TBL to tropical easterly control is a model applicable to orbitally forced variations. The stronger the Hadley circulation, the more intense the zonal velocity of the tropical easterly winds. This leads to greater Eckman drift and increased equatorial divergence. For times of maximum Hadley development, there is a decrease in the meridional wind vector, with a concomitant increase in aridity in Africa (10). This scenario has been documented for the last glacial maximum by CLIMAP (29). Global climate models have been used to simulate the atmosphere of the last glacial maximum $(30,31,32,33,34)$. All show tropical easterly wind zonality as strong or stronger than today, a time when perihelion is aligned with boreal winter. A corollary to this exists in the modeling of the youngest interval when perihelion was aligned with boreal summer. COHMAP Members (35) show in their simulation for $9 \mathrm{ka}$ that the meridional component was stronger while the zonal component was weaker relative to both today and the last glacial maximum at $18 \mathrm{Ka}$. This circumstantial evidence supports tropical easterly modulation of divergence.

When perihelion is aligned with boreal summer, summer insolation is at a maximum over North Africa and the monsoon dominates. The result, at the equator, is a time of minimal divergence, productivity, and seasonality, with the warmest equatorial SST. This is depicted in cartoon form in Figure 1.

When perihelion is aligned with boreal winter, the southern hemisphere tropical easterlies dominate. The result, at the equator, is a time of maximum divergence, productivity, and seasonality, with the coolest SST (Fig. 1) .

Three deep-sea cores, RC24-07 ( $\left.1^{\circ} 20.5^{\prime} \mathrm{S}, 11^{\circ} 53.3^{\prime} \mathrm{W}\right)$ and RC24-16 $\left(5^{\circ} 2.3^{\prime} \mathrm{S}, 10^{\circ} 11.5^{\prime} \mathrm{W}\right)$, sited beneath the zone of maximum variation centered on $10^{\circ} \mathrm{W}$ and $\mathrm{V} 30-40\left(0^{\circ} 12.0^{\circ} \mathrm{S}, 23^{\circ}\right.$ $09.0^{\prime} \mathrm{W}$ ) west of, but still within, the region of maximum divergence document the orbital control of equatorial oceanography $(7,8)$. The signals of planktonic organisms that inhabit the TBL and presented here as estimated SST show marked cyclic character (Fig. 2). Spectra of these signals are dominated by the precessional band centered on $23 \mathrm{Ky}$ which accounts for approximately $49 \%$ of the total variance (Fig. 2). The signal indicates that, with increasing zonality of the tropical easterly winds, divergence and thermocline shallowing intensify (lower SST) to reach maxima when perihelion is centered on boreal winter. When perihelion is centered on boreal summer tropical easterlies have minimum zonality and the opposite conditions occur. These signals are coherent and nearly in phase with both the precessional component of orbital variation and boreal summer insolation $(6,7)$.

The presence and dominance of precessional periods is unequivocal; it can be both seen in and quantified from these time-series (Fig 2). In addition, there are variations of lower amplitude and shorter period (higher frequency) but these are overshadowed by the precessional signal. They are particularly evident in the time, $0-100 \mathrm{Ka}$, when eccentricity modulated precessional forcing is minimal. Are these periodic or aperiodic? 


\section{SHORT PERIOD RESPONSE}

Eccentricity modulation of the precessional component of orbital forcing is at it lowest over the last $330 \mathrm{Ka}$ between approximately 100 and $0 \mathrm{Ka} \mathrm{(36).} \mathrm{This} \mathrm{interval} \mathrm{has} \mathrm{been} \mathrm{extracted} \mathrm{from} \mathrm{the} \mathrm{two}$ cores with the best chronologic control for analysis of short period response. Both V30-40 and RC24-16 contain well defined short period signals within this interval of time (Fig. 3). The time span between peaks was computed and is presented as a stem and leaf display (Fig. 3). The mean value for this computation is $3.0 \mathrm{Ky}$ for V30-40 and $2.8 \mathrm{Ky}$ for RC24-16. Both the signals and the periods are intriguing but are they real or merely an artifact of sample interval and/or data acquisition?

The sampling interval has a mean value of $0.8 \mathrm{Ky}$ and a range of 1.6 to $0.4 \mathrm{Ky}$ based upon the chronology applied to convert a depth series into a time series (see 7 for details of method). Added to this is the age error of $+/-1.5 \mathrm{Ky}$ involved in the chronologic control (2). The combined error on any one point could produce extreme periods $>9 \mathrm{Ky}$ between two adjacent highs or lows. The fact that the periods have the form of a gaussian distribution and half the possible range indicates this is not a valid explanation for the signal (Fig. 3).

These cores were counted by one individual sequentially from youngest to oldest sample. Many of these highs are defined by single data points (7). Is the periodic response a result of counting error in the data? Two pieces of evidence deny this idea. First, many of these levels, chosen randomly, were recounted to determine if counting error alone could produce these short period signals. In all cases the recounted samples agreed within $2 \%$ of the original value and without systematic error in time. This is approximately a 5\% error in SST estimation and is insufficient to alter signal shape. Second, signal regularity is high, e.g $88 \%$ of V30-40 and $80 \%$ of RC24-16 signal is regular in terms of high - low - high values within one standard deviation of the mean. Random error as a cause of these signals would have values grouped around $50 \%$. The short period signals are considered real.

The Tukey method of spectral analysis (37), is used for oceanic records because it has proven reliable when applied to records of variable interval (6). However, its output is influenced by strong amplitude. Prewhitening cannot remove, only suppress, the longer periods whose amplitudes are high (like precession) in these cores. Nevertheless, prewhitening does show that there are higher frequencies common to signals intra and intercore. The most significant are found between 4.0 and $2.5 \mathrm{Ky}$. There is an alternative way to examine the shorter and lower amplitude periods. Computing the first derivative of the time series emphasizes the cyclics of change while minimizing biasing by amplitude. Spectral analysis of the times series of the first derivative give weight to the regularity of response rather than the amplitude, i.e. significance depends on the periodic dominance of a period (Fig. 4). Spectral analyses of the proxy times series and the derivative time series document the presence of these short periods in both data. The periods between 3.5 and 2.5 are significant.

\section{CONCLUSIONS}

There are at least 2 sets of significant periods recorded in these cores. Those correlated with the primary orbital period of precession dominate the signals and indicate that the equatorial Atlantic responds to insolation forcing by the precessional component of orbital variation. They can be explained by the intermediary mechanism of the tropical easterly control of TBL dynamics described here and in McIntyre et al (7). A second set of periods, subdominant in terms of time and amplitude, indicates that this sensitive region of the ocean oscillates at much shorter periods that fall between the primary orbital and the annual periods. The character of these short periods indicates they are a response to the same type of TBL dynamics as are the longer periods, i.e. tropical easterly modulation. The force that produces these short periods remains a mystery. 


\section{ACKNOWLEDGEMENTS}

This research was supported by the National Science Foundation, Marine Geology and Geophysics grants OCE 85-16133 and OCE86-08328 and Office of Climate Dynamics grant ATM88-12637. This paper is Lamont-Doherty Geological Observatory contribution number $\mathrm{xxxx}$.

refs. in numerical order

\section{REFERENCES}

1. Halley, E., "A discourse concerning the proportional heat of the sun in all latitudes", Philos. Trans., 16, 366-370, 1693.

2. Imbrie, J., J. D. Hays, D. G. Martinson, A. McIntyre, A. C. Mix, J. J. Morley, N. G. Pisias, W. L. Prell, and N. J. Shackleton, "The orbital theory of Pleistocene climate: Support from a revised chronology of the marine $18 \mathrm{O}$ record", in Milankovitch and Climate, Part I, edited by A. L. Berger et al., p. 508, Hingham, Mass., 1984.

3. Eddy, J. A.," An historical review of solar variability, weather and climate", in Weather and Climate Response to Solar Variation, ed. B.M. McCormac, pp, 1-15, Colorado Associations University Press, Boulder, Colo., 1983.

4. Denton, G.H. and Karlen W., Holocene climatic variations-their pattern and possible causes", Jour. Quat. Res., 3, 155-205, 1973.

5. Dansgaard, W.and Oeschger, H.," Past environmental long-term records from the Arctic, in The environmental record in glaciers and ice sheets", eds. H. Oeschger and C.C. Langway Jr, 287-318, John Wiley and Sons, 1989.

6. Imbrie, J., A. McIntyre, A. Mix, "Oceanic Response to Orbital Forcing in the Late Quaternary: Observational and Experimental Strategies", in Climate and Geosciences, eds A. Berger, J. C. Duplessey, S. Schneider, 121-164, Kluwer Acad. Pub.,Dordrecht, 1989.

7. McIntyre, A., Ruddiman, W.F., Karlin, K., Mix, A.C., "Surface water response of the equatorial Atlantic Ocean to orbital forcing", Paleoceanography, 4, 19-55, 1989.

8. Karlin, K., Ruddiman, W.F., McIntyre, A.," Comparison of Late Pliocene and Late Pleistocene sea-surfae temperatures of the equatorial Atlantic divergence", Proc. of $O D P$, 108, 187-210, 1989.

9. Pestiaux, P., Duplessey, J.C., Berger, A., "Paleoclimatic variability at frequencies ranging from $10^{4}$ cycles per year to $10^{3}$ cycles per year - evidence for nonlinear behavior of the climate system", in CLIMATE History, Periodicity, and Predictability, eds. M.R, Rampino, J.E. Sanders, W.S. Newman, L.K. Konigsson, 285-299, Van Nostrand Reinhold Co. N.Y., 1988.

10. Riehl, H., Climate and Weather in the Tropics, 611 pp., Academic, San Diego, Calif., 1979.

11. Hastenrath, S., "Relative role of atmosphere and ocean in the global heat budget: tropical Atlantic and eastern Pacific", Q. Jo. Ro. Meteorol. Soc., 103, 519-526, 1977.

12. Philander, S. G. H., "Variability of the tropical oceans", Dyn. Atmos. Oceans, 3, 191-208, 1979.

13. Reverdin, G., "Heat budget of the tropical Atlantic Ocean -- seasonal upwelling", Deep Sea Res., 32, 363-368, 1985.

14. Servain, J., and D.M. Legler, "Empirical orthogonal analyses of tropical Atlantic sea surface termperatures and wind stress: 1964-1979", J. Geophys. Res., 91, 14,181-14,191, 1986.

15. Katz, E. J., and S. L. Garzoli, "Response of the western equatorial Atlantic Ocean to an annual wind cycle", Jo. Mar. Res., 40, 307-327, 1982.

16. Katz, E. J., and S. L. Garzoli, "Thermocline displacement across the Atlantic north equatorial counter current during 1983", Geophys. Res. Lett., 11, 737-740, 1984. 
17. Molinari, R. L.,"Observations of near-surface currents and temperature in the central and western tropical Atlantic Ocean", J. Geophys. Res., 88, 4423-4438, 1983.

18. Bunker, A. F., and L. V. Worthington, "Energy exchange charts of the North Atlantic Ocean", Bull. Am. Meteorol. Soc., 52, 670-678, 1976.

19. Richardson, P. L., and G. Reverdin, "Seasonal cycle of velocity in the Atlantic north equatorial countercurrent as measured by surface drifters, current meters, and ship drifts", J. Geophys. Res., 92, 3691-3708, 1987.

20. Cane, M., "The response of an equatorial ocean to simple wind-stress patterns", Jo. Mar. Res., 37, 233-252, 1979.

21. Cane, M. A. and E. Sarachik, "Forced baroclinic ocean motion, I, The linear equatorial unbounded case", Jo. Mar. Res., 35, 629-655, 1976.

22. Cane, M. A., and E. Sarachik, "Forced baroclinic ocean motion, II, The linear equatorial bounded case", J. Mar. Res.,36, 395-437, , 1977.

23. Cane, M. A., and E. Sarachik, "Forced baroclinic ocean motion, III, The linear equatorial basin case", J. Mar. Res., 37, 355-398, 1979.

24. Moore, N. W., and S. G. H. Philander, "Modeling of the tropical oceanic circulation", in The Sea, vol. 6, pp. 319-361, Wiley Interscience, New York, 1977.

25. Philander, S. G. H., and R. C. Pacanowski, "The generation of equatorial currents", $J$. Geophys. Res., 85, 1123-1136, 1980.

26. Philander, S. G. H., and R .C. Pacanowski, "The oceanic response to cross-equatorial winds (with application to coastal upwelling in low latitudes)", Tellus, 33, 201-210, 1981.

27. Philander, S. G. H., and R. C. Pacanowski, "A model of the seasonal cycle in the tropical Atlantic Ocean", J. Geophys. Res., 91, 14,192-14,206, 1986a.

28. Philander, S. G. H., and R. C. Pacanowski, "The mass and heat budget in a model of the tropical Atlantic Ocean", J. Geophys. Res., 91, 14,212-14,220, 1986 b.

29. CLIMAP Project Members, A. McIntyre comp., "Seasonal reconstructions of the Earth's surface at the last glacial maximum", Geol. Soc. Am. Map Chart Ser., MC-36, 1981.

30. Gates, W. L., "The numerical simulation of Ice-Age climate with a global general circulation model", J. Atmos. Sci., 33, 1844-1873, 1976.

31. Kutzbach, J. E., and P. J. Guetter, "The influence of changing orbital parameters and surface boundary conditions on climate simulations for the past 18,000 years", Jo. Atmos. Sci., 43, 1726-1759, 1986.

32. Manabe, S., and D. G. Hahn, "Simulation of the tropical climate of an ice age", J. Geophys. Res., 82, 3889-3911, 1977.

33. Manabe, S., and A. J. Broccoli, "The influence of continental ice sheets on the climate of an ice age", J. Geophys. Res., 90, 2167-2190, 1985.

34. Schneider, S. H., and S. L. Thompson, "Ice ages and orbital variations: Some simple theory and modeling", Quat. Res. 12, 188-203, 1979.

35. COHMAP Members, "Climatic changes of the last 18,000 years: observations and model simulations", Science, 241, 1043-1052, 1988.

36. Berger, A., "Long-term variations of daily insolation and Quaternary climatic changes", Jo. Atmos. Sci., 35, 2362-2367, 1978.

37. Blackman, R. B., and J. W. Tukey, The Measurement of Power Spectra From the Point of Communications Engineering, Dover, Mineola, N. Y., 1958. 


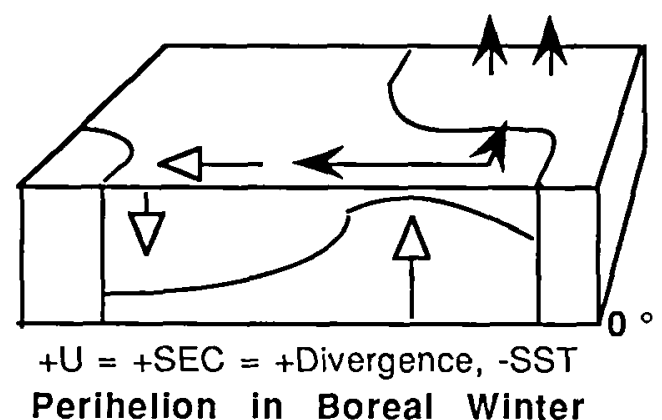

Perihelion in Boreal Winter

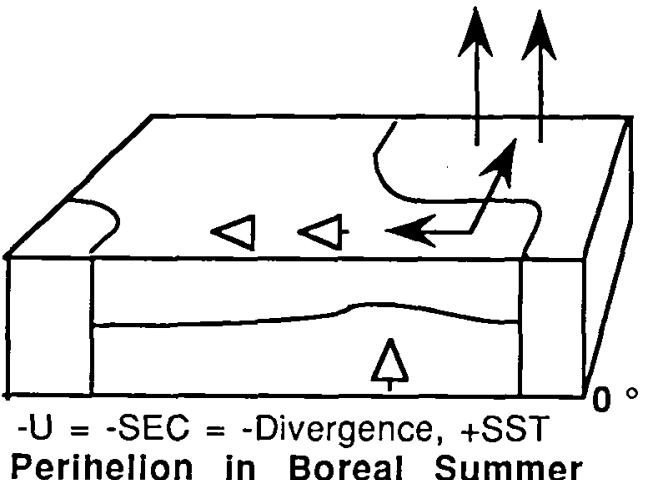

Perihelion in Boreal Summer

Figure 1. Cartoon of the response of the turbulent boundary layer, TBL, of the equatorial Atlantic to the annual extremes (boreal summer and boreal winter) of precessional forcing. The front of the cartoon represents a cross section of the TBL along the equator and the curved line indicates the position of the thermocline. Solid arrows are wind, open arrows are ocean motion. When perihelion is centered on boreal summer, the zonal, $U$, component of the southern hemisphere tropical easterly is decreased relative to the meridional. The result is a decrease in SEC velocity, seasonal divergence, and an increase in SST. Aphelion in boreal summer yields the opposite effect.

\section{V30-40 RC24-16}

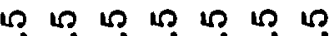

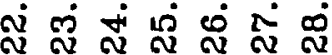
ת

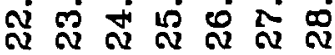
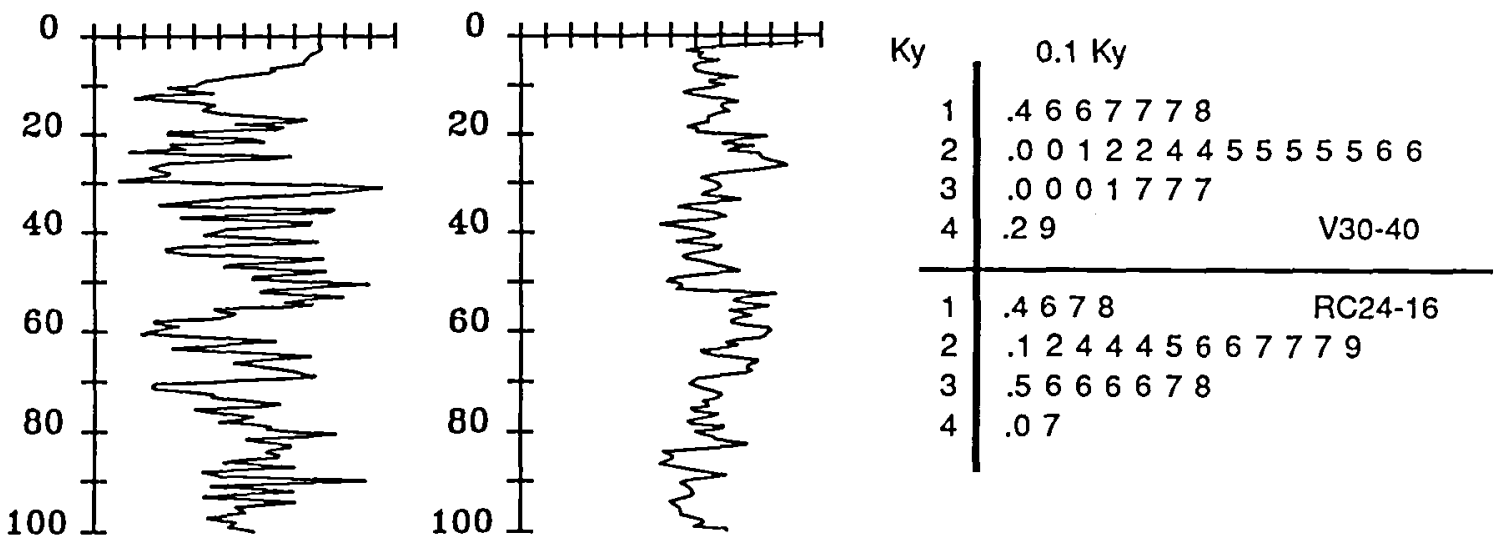

Figure 3. Time series for cores V30-40 and RC24-16 over the interval of time where eccentricity modulation of precession is minimal. In this time interval high-amplitude shortperiod response is not masked by the strong precessional signal. The time interval between prominent peaks has been measured and is displayed in the stem and leaf display below the time series. The mean values for cores V30-40 and RC24-16 is 3.0 and $2.8 \mathrm{Ky}$ respectively. 
V30-40 SSTc

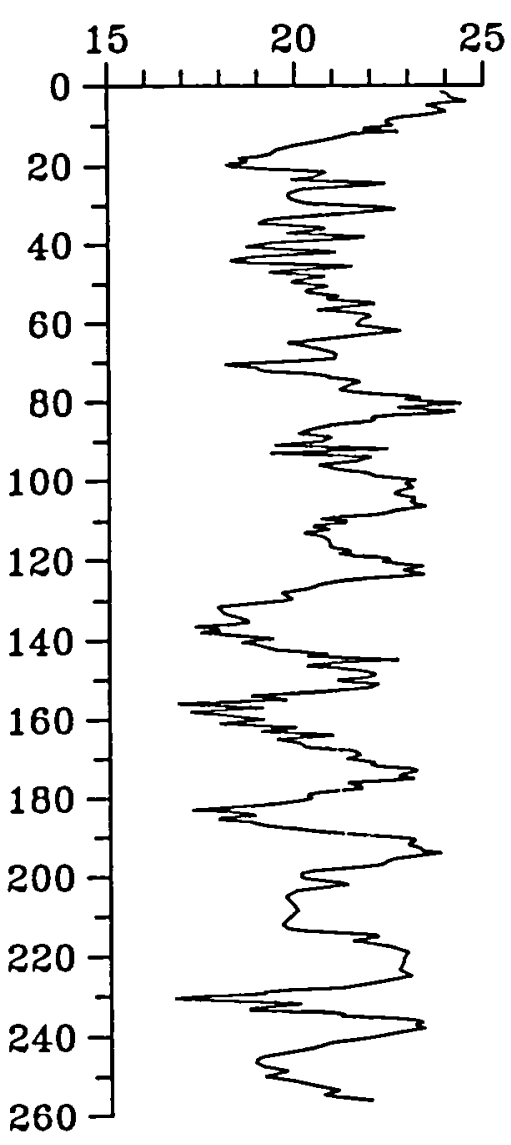

RC24-7 SSTc
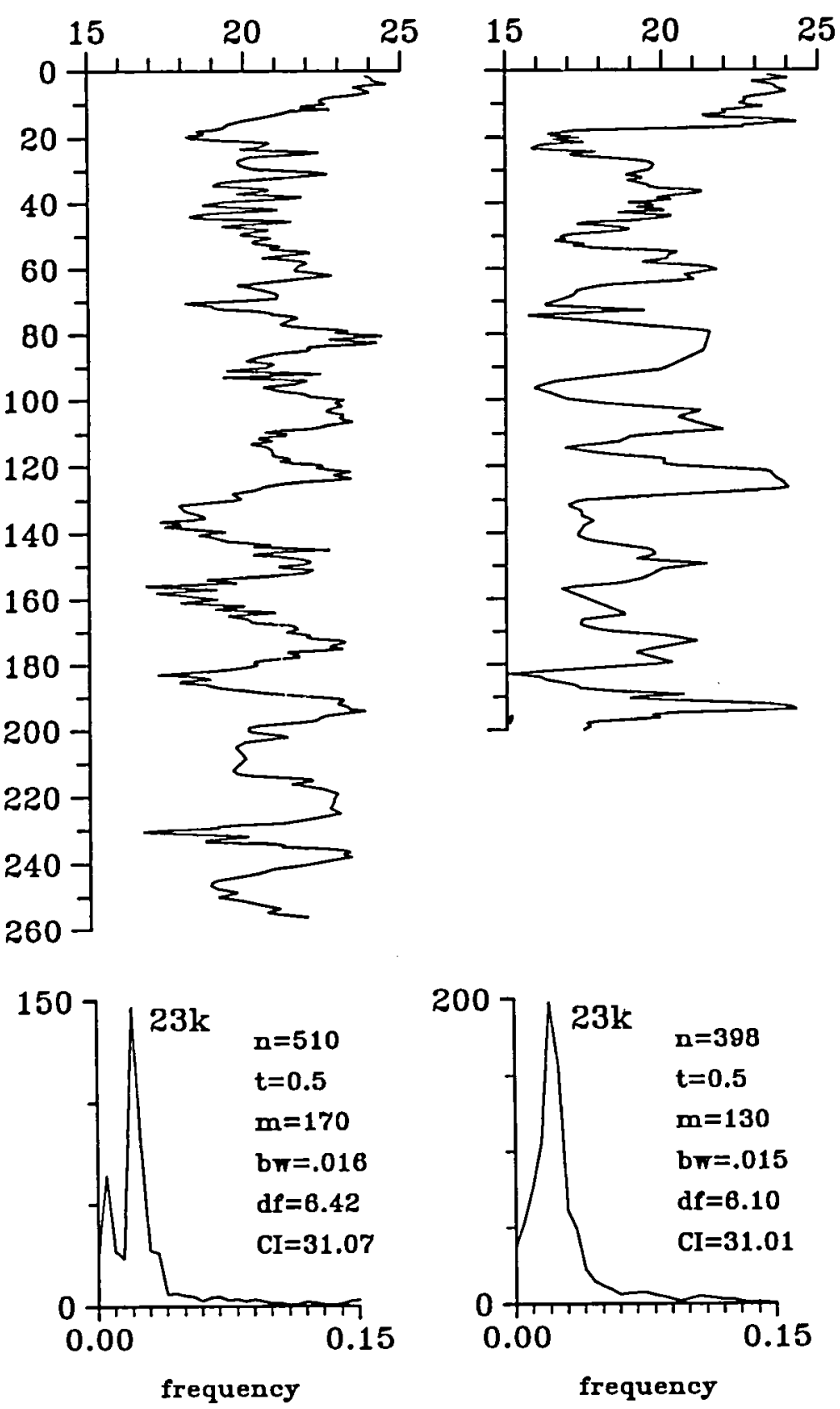

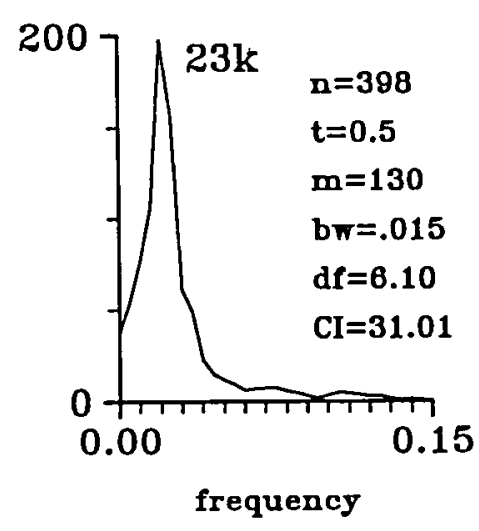

RC24-16 SSTc
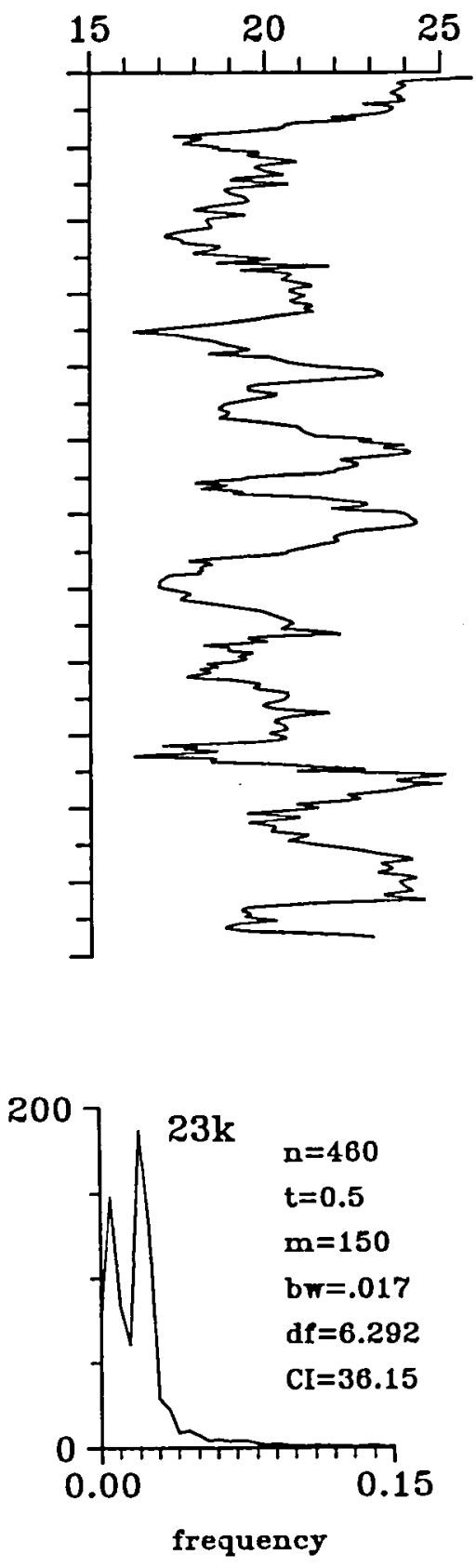

Figure 2. (a) Time series of estimated SST in three deep-sea cores from the maximum divergence region of the equatorial Atlantic (all data published in 7 and 8). The $X$ axes are ${ }^{\circ}$ Celcius and the y axes in Ka.(kiloyears before present). (b) variance spectra of SST. Dominant periods in Ky (kiloyears) are indicated. Abbreviations are: n, number of points; int., time interval between points; $\mathrm{m}$, lag (the number of points lagged in the Fourier analysis); BW, bandwidth; df, degrees of freedom; CI, confidence interval for the lower limit. These abbreviation apply to figure 4 as well. 

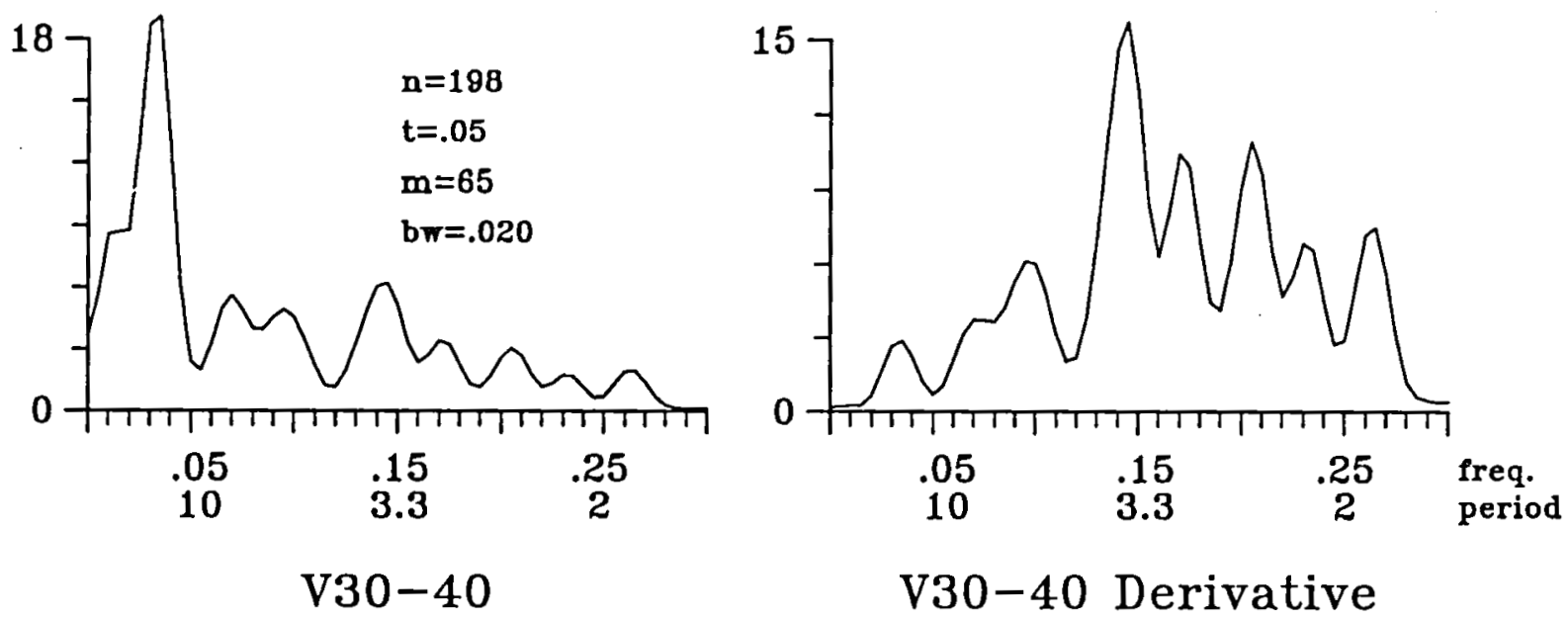

V30-40 Derivative

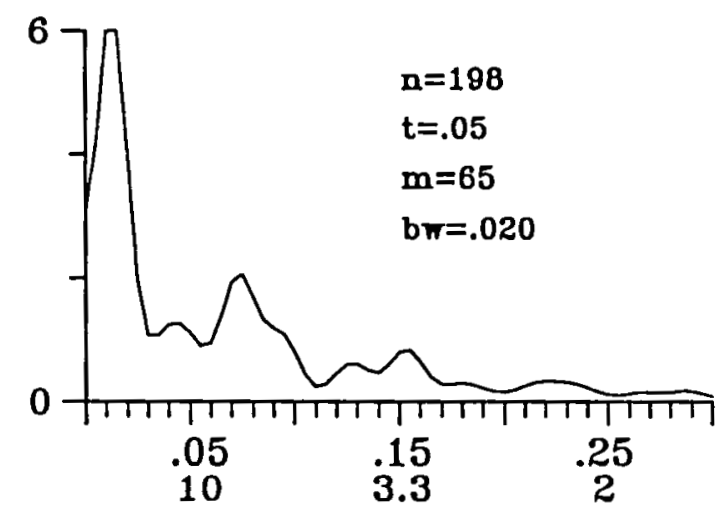

RC24-16

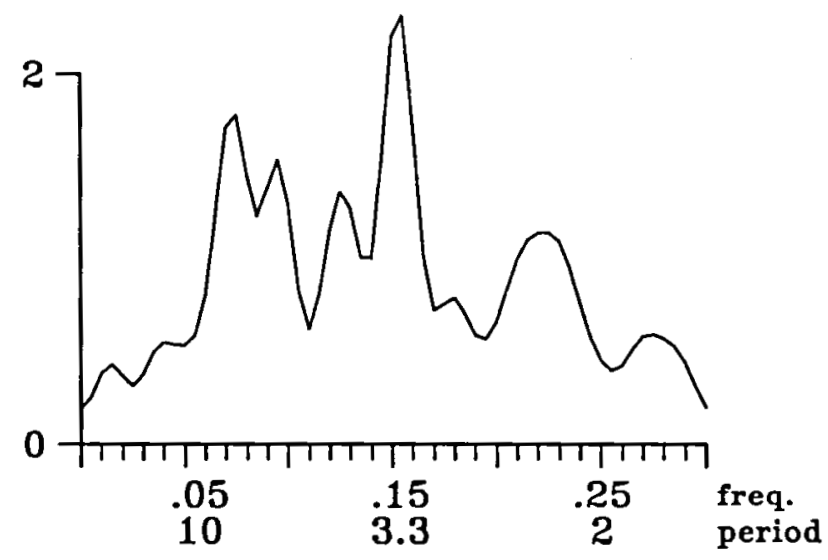

RC24-16 Derivative

Figure 4. Variance spectra of estimated SST (left) and the first derivative of estimated SST (right) for the interval 0-100Ka from V30-40 and RC24-16. The first derivative enhances the shorter period spectrum by essentially prewhitening the entire series giving a better definition of these short period oscillations. 


\title{
A PROBABLE 2400 YEAR SOLAR QUASI-CYCLE IN ATMOSPHERIC $\Delta^{14} \mathrm{C}$
}

\author{
L. L. Hood and J. L. Jirikowic \\ Lunar and Planetary Laboratory, University of Arizona \\ Tucson, Arizona 85721 USA
}

\begin{abstract}
A 2200-2600 year quasi-periodicity is present in atmospheric $\Delta^{14} \mathrm{C}$ records after removal of long-term trends due to the geomagnetic dipole amplitude variation. This periodicity consists of both a long-term variation of the mean and a superposed, approximately recurring pattern of century-scale variations. The strongest of these latter variations occur near maxima of the $\sim 2400$ year $\Delta^{14} \mathrm{C}$ cycles. The residual record can be modeled to first order as an amplitude modulation of a century-scale periodic forcing function by a $\sim 2400$ year periodic forcing function. During the last millennium; the largest centuryscale variations (occurring near the most recent 2400 year $\Delta^{\mathbf{1 4}} \mathrm{C}$ maximum) are known to be mainly a consequence of the pronounced Maunder, Spörer, and Wolf solar activity minima, as verified by independent proxy solar activity records. Therefore, during this period, amplitude modulation has been occurring primarily in the sun and not in the terrestrial radiocarbon system. It is therefore inferred that the $\sim 2400$ year forcing function is mainly solar although some secondary terrestrial feedback into the $\Delta^{14} \mathrm{C}$ record is likely. This conclusion has implications for the predictability of future pronounced solar activity minima and for the interpretation of certain minor Holocene climatic variations.
\end{abstract}

\section{INTRODUCTION}

Deviations of atmospheric ${ }^{14} \mathrm{C}$ versus time $\left(\Delta^{14} \mathrm{C}\right)$ are produced in part by solarinduced changes in galactic cosmic ray flux which in turn modulate the radiocarbon production rate [Stuiver, 1961; Stuiver and Quay, 1980; Sonett, 1984; Damon, 1988; and references therein]. Directly dated tree ring $\Delta^{14} \mathrm{C}$ records covering the last $\sim 8000$ years therefore represent one of the best available proxy measures of solar magnetic variability. However, an important problem in the interpretation of such records is the separation of solar variability contributions from other potential terrestrial sources of radiocarbon variability. In this paper, empirical methods are used to investigate whether millennium-scale variations in the radiocarbon record are primarily solar or terrestrial in origin.

\section{ANALYSIS}

Figure 1 shows a high-precision $\Delta^{14} \mathrm{C}$ record resulting from the 12 th International Radiocarbon Conference [Stuiver and Kra, 1986]. A long-term trend is present that has been shown to be largely consistent with a modeled response to the $\sim 11,000$ year geomagnetic dipole moment amplitude variation as derived from archeomagnetic records [Sternberg and Damon, 1983; Damon, 1988]. Figure 2 shows the same record after detrending by removal of a least-squares-fitted cubic polynomial representing long-term changes in geomagnetic dipole moment intensity. Note that the residual radiocarbon variations are now plotted with an inverse scale so that decreases in $\Delta^{14} \mathrm{C}$ are upward and correspond to increases in 


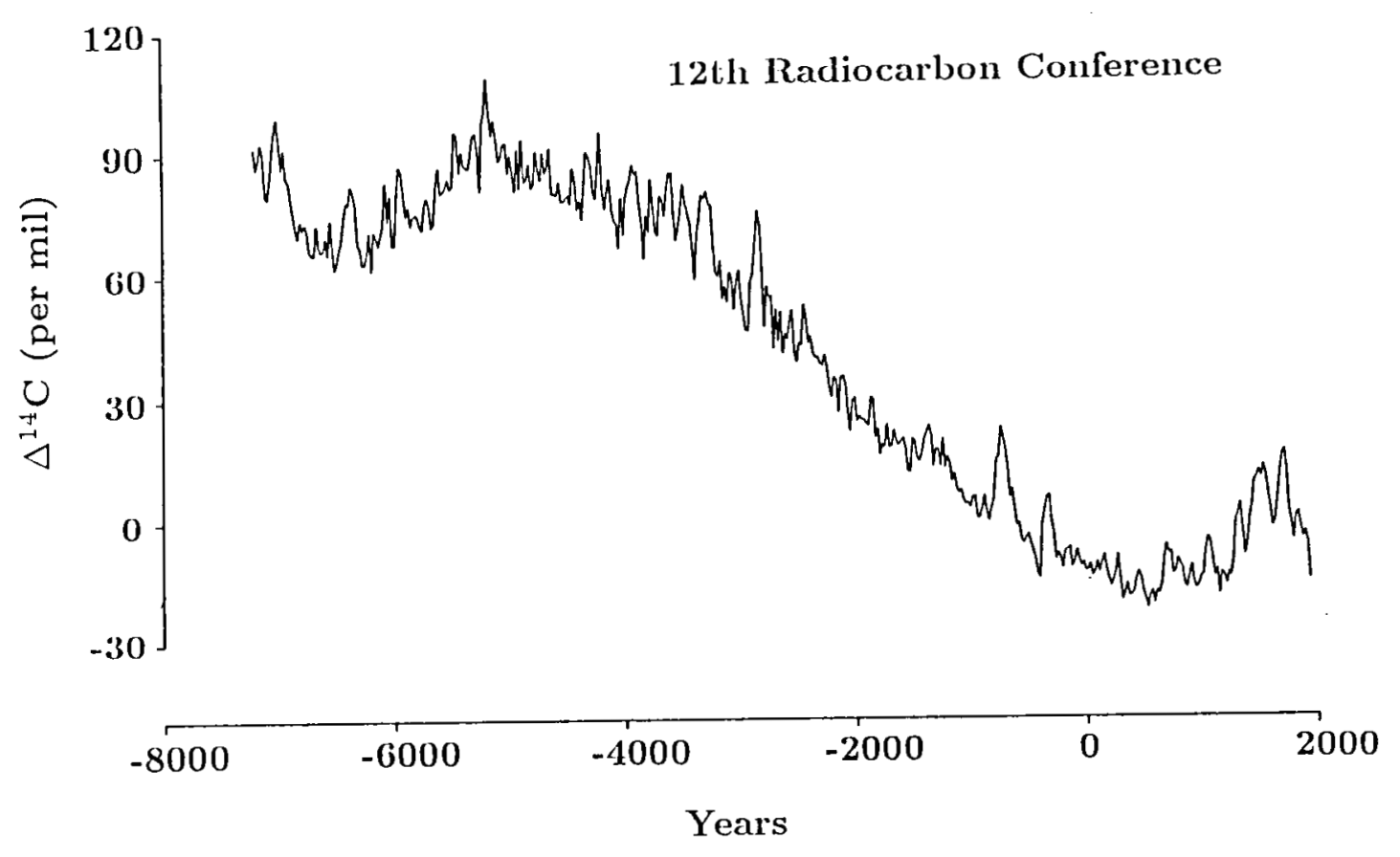

Figure 1. 12th International Radiocarbon Conference calibration record produced by combining high-precision records from a series of laboratories [Stuiver and Kra, 1986]. (Data courtesy of M. Stuiver and R. Kra)

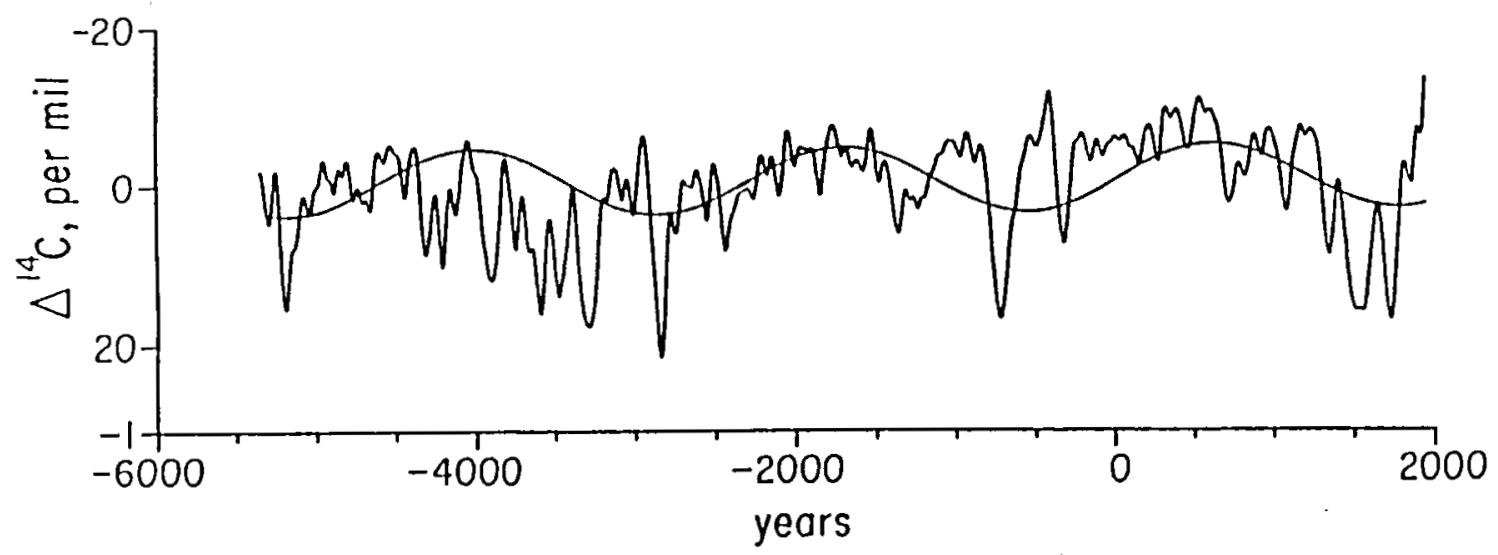

Figure 2. Residual Conference $\Delta^{14} \mathrm{C}$ record after detrending to remove the long-term geomagnetic variation and smoothing to minimize variations with periods less than approximately 100 years. Note that the vertical scale has been inverted so that $\Delta^{14} \mathrm{C}$ maxima map solar activity minima. A least-squares-fitted sinusoid is superposed to indicate the approximate locations of maxima and minima of the $\sim 2400$ year quasi-cycle. 
solar activity.

The residual variations of Figure 2 exhibit a 2200-2600 year quasi-periodicity that has been recognized in power spectral analyses and visual examinations of various radiocarbon records [Houtermans, 1971; Denton and Karlén, 1973; Suess, 1980; Sonett and Finney, 1989]. To indicate the approximate locations of maxima and minima of this quasi-cycle, a sinusoid with a $\sim 2400$ year period was least-squares-fitted to the residual record (fitted parameters: period $=2310$ years; amplitude 4.08 per mil) and is superposed on the record in Figure 2. A geomagnetic origin for this quasi-periodicity in $\Delta^{14} \mathrm{C}$ has been considered unlikely since there is no dipole moment amplitude variation near a period of 2400 years [Damon and Sonett, 1990]. However, there is a well-known climatic period near 2500 years [Mitchell, 1976; Pestiaux et al., 1987]. Several earlier investigators have reported that this $\sim 2500$ year climate variation has been nearly in phase with the inverted $\Delta^{14} \mathrm{C}$ record during the last 8000 years [Bray, 1968; 1970; Denton and Karlén, 1973]. Figure 3 compares the residual $\Delta^{14} \mathrm{C}$ record of Figure 2 to well-dated proxy northern hemispheric climate indicators including $\delta^{18} \mathrm{O}$ from the Camp Century Greenland ice core [Dansgaard et al., 1984] and the Devon Island Canada ice core [Fisher, 1982] as well as to the temperaturesensitive Campito Mountain bristlecone pine tree ring width record of LaMarche [1974]. A tendency for climatic minima to be associated with $\Delta^{14} \mathrm{C}$ maxima is evident. The last of these climatic minima was the Little Ice Age [Eddy, 1977].

There are two "end-member" candidate sources of the $\sim 2400$ year quasi-periodicity in atmospheric $\Delta^{14} \mathrm{C}$. First, the $\Delta^{14} \mathrm{C}$ could be responding entirely to a terrestrial climate cycle of uncertain origin through a redistribution of ${ }^{14} \mathrm{C}$ between the atmospheric and oceanic reservoirs. Second, the $\Delta^{14} \mathrm{C}$ could be responding entirely to a solar quasi-cycle of the same period and the $\sim 2500$ year climate period could be independently driven by solar variability. In order to distinguish between these possibilities, it is helpful to consider the detailed characteristics of the radiocarbon record of Figure 1 together with independent proxy solar activity records covering the last 1000 years.

Century-scale variations in the $\Delta^{14} \mathrm{C}$ record are believed to be dominantly of solar origin as evidenced by their correlation with the independently verified Maunder and Spörer solar activity minima of the last millennium [Eddy, 1976; 1977; Stuiver and Quay, 1980; Stuiver and Braziunas, 1989]. As can be seen in Figure 2, the strongest century-scale variations tend to occur near successive maxima of the $\sim 2400$ year $\Delta^{14} \mathrm{C}$ cycles. In addition, it has been shown that century-scale variations in the most recent $\sim 2400$ year cycle in $\Delta^{14} \mathrm{C}$ are positively correlated with similar short-term variations in each of the two previous cycles [Hood and Jirikowic, 1990]. Thus, the quasi-periodicity consists of both a long-term variation of the mean and a superposed, approximately recurring pattern of century-scale variations.

The behavior of the residual $\Delta^{14} \mathrm{C}$ record can be interpreted in terms of amplitude modulation of a century-scale solar forcing function by a longer-term forcing function [Sonett, 1984]. Figure 4 shows a simplified example of amplitude modulation in which a 200-year sinusoid is modulated by a 2400-year sinusoid. Pronounced short-term maxima (note the inverted scale) occur in the resulting time series (Figure 4c) at intervals of the longer period. If the 2400-year forcing function (represented in Figure 4b) were dominantly terrestrial in origin, then we would expect to be able to observe 


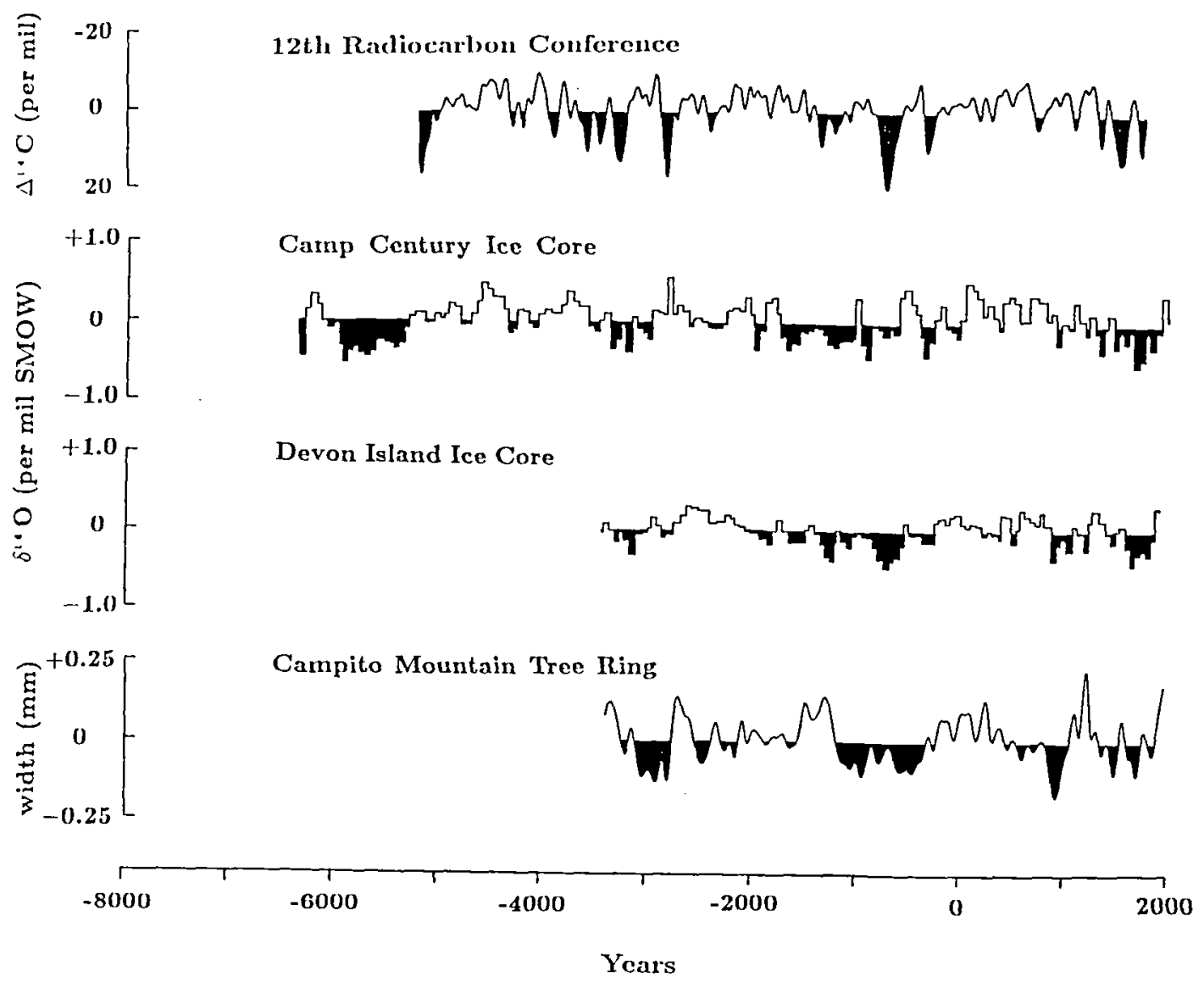

Figure 3. Comparison between the residual Conference $\Delta^{14} \mathrm{C}$ record and several well-dated proxy northern hemispheric climatic indicators for the last 8000 years (see the text). 


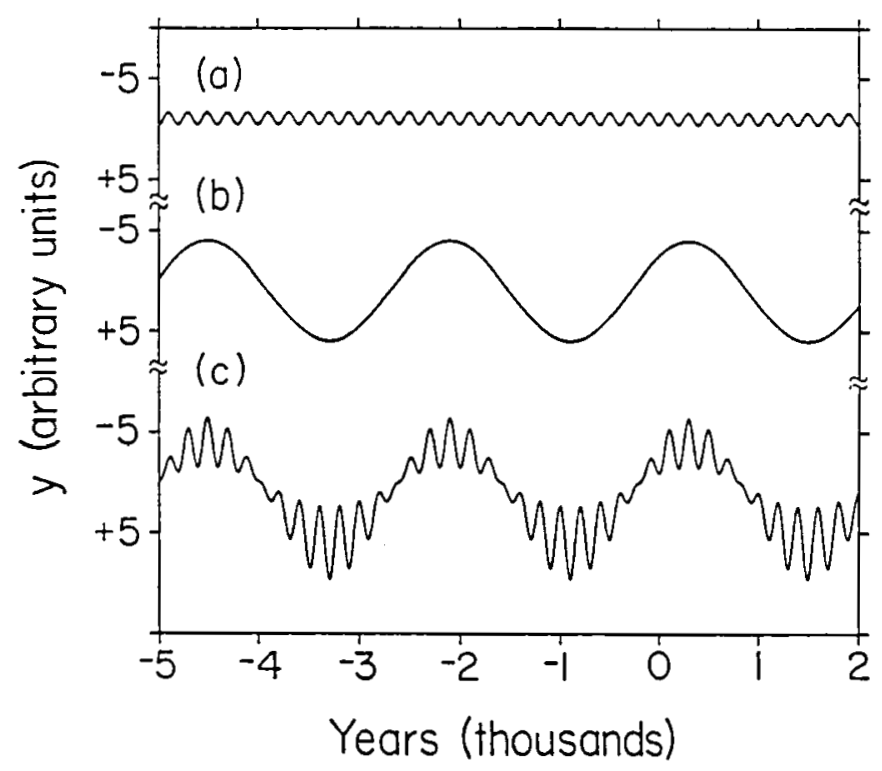

$$
y=\left(1+A \cos \omega_{1} t\right)\left(1+B \cos \omega_{2} t\right)
$$

where $A=5, B=0.6, \omega_{1}=2 \pi / 2400 \mathrm{yr}^{-1}, \omega_{2}=$ $2 \pi / 200 \mathrm{yr}^{-1}$, and $t$ is the time in years.

Figure 4. Simple model of amplitude modulation of a 200 year sinusoid (a) by a 2400 year sinusoid (b). The product is shown in (c) and has characteristics that are qualitatively similar to those of the residual $\Delta^{14} \mathrm{C}$ record.

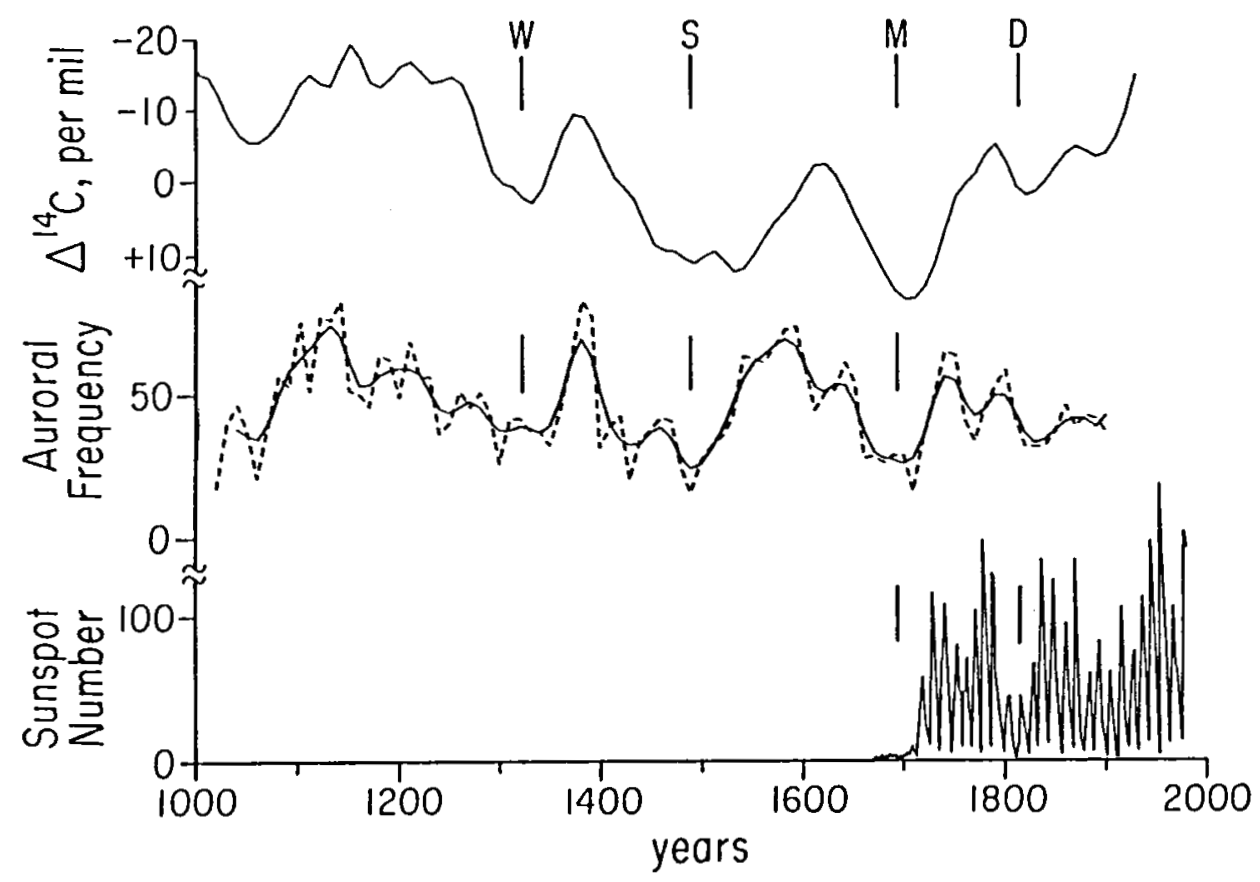

Figure 5. A comparison between the last 1000 years of the residual Conference $\Delta^{14} \mathrm{C}$ series and two independent proxy solar activity indicators (see the text). 
the unmodulated solar signal (represented in Figure 4a) in independent proxy solar records covering the last 1000 years. However, the latter records show the occurrence of pronounced minima in the form of the Wolf, Spörer, and Maunder solar activity minima.

Figure 5 compares the residual $\Delta^{14} \mathrm{C}$ record for the current millennium with the auroral frequency record compiled by Schove [1987] and with mean annual sunspot number since 1670 [Waldmeier, 1961; Eddy, 1976]. Within the recent interval covered by the sunspot record, both the Dalton and Maunder sunspot minima are approximately reflected in the residual inverted $\Delta^{14} \mathrm{C}$ record. At earlier times, the auroral record remains positively correlated with the inverted $\Delta^{14} \mathrm{C}$ time series $(R=0.55$ at 20 years lag versus the smoothed auroral record for the entire series) and exhibits minima that can be identified with the Maunder, Spörer, and Wolf minima in the $\Delta^{14} \mathrm{C}$ time series. A broad maximum centered on A.D. 1150, known as the medieval maximum, is also evident in both records. From the existence of pronounced minima in these independent proxy solar records near the last $\Delta^{14} \mathrm{C}$ maximum, it is clear that amplitude modulation of centuryscale solar variations has been occurring in the sun during the last 1000 years and not just in the terrestrial radiocarbon system. By inference, the earlier strong variations occurring at $\sim 2400$ year intervals are most probably produced mainly by solar changes although some secondary climatic feedback effects on $\Delta^{14} \mathrm{C}$ can not be excluded. On this basis, the $\sim 2400$ year forcing function that is modulating the atmospheric $\Delta^{14} \mathrm{C}$ record is suggested to be primarily solar.

\section{IMPLICATIONS}

Clearly, the construction of more complete quantitative models for $\Delta^{14} \mathrm{C}$ production and exchange appropriate for millennium scale variations would be valuable and may elucidate further the relative roles of terrestrial and solar forcing mechanisms. However, the empirical evidence discussed here for a dominantly solar origin of both the century-scale and longer-term $(\sim 2400$ year $)$ residual variations in the Conference $\Delta^{14} \mathrm{C}$ record does allow several provisional implications to be stated. The first of these relates to the predictability of future long-term activity changes. Although the Babcock-Leighton models for the Hale magnetic cycle (involving distortion of an initially poloidal field by differential rotation in the convection zone) have been developed, these models along with dynamo theories for the origin of the magnetic field itself are not yet sufficiently advanced to allow extensions to longer time scales [e.g., Gough, 1977]. From a purely empirical standpoint, however, it is reasonable to expect that the inferred quasi-cyclicity will persist in the future. In particular, it can be projected that large Maunder- and Spörer-type minima are not likely to occur again until ca. A.D. 4000 and that these will be preceded by a lengthy period of relatively high solar activity. In addition, the above analysis (indicating that the $\sim 2400$ year $\Delta^{14} \mathrm{C}$ periodicity is dominantly of solar origin) supports a possible solar origin for the Holocene climatic cycle of the same approximate period (Figure 3).

Acknowledgments. M. Stuiver and R. Kra kindly provided a digital form of the 12th International Radiocarbon Conference calibration record. We thank C. P. Sonett, S. A. Finney, and P. E. Damon for helpful discussions. 


\section{References}

Bray, J. R., Glaciation and solar activity since the Fifth Century BC and the solar cycle, Nature, 220, 672-674, 1968.

Bray, J. R., Temporal patterning of post-Pleistocene glaciation, Nature, 228, 353, 1970.

Damon, P. E., Production and decay of radiocarbon and its modulation by geomagnetic field-solar activity changes with possible implications for global environment, in Secular Solar and Geomagnetic Variations in the Last 10,000 Years, edited by F. R. Stephenson and A. W. Wolfendale, Kluwer Academic Publishers, Dordrecht, 1988.

Damon, P. E., and C. P. Sonett, Solar and terrestrial components of the atmospheric ${ }^{14} \mathrm{C}$ variation spectrum, in The Sun Through Time, edited by C. P. Sonett, M. S. Giampapa, and M. S. Mathews, University of Arizona Press, Tucson, 1990, in press.

Dansgaard, W., S. J. Johnsen, H. B. Clausen, D. Dahl-Jensen, N. Gunderstrup, C. Hammer, and $\mathrm{H}$ Oeschger, North Atlantic oscillations revealed by deep Greenland ice cores, in Climate Processes and Climate Sensitivity, edited by J. E. Hansen and T. Takahashi, Geophysical Monograph 29, AGU Maurice Ewing Series, v. 5, pp. 288-298, Washington, 1984.

Denton, G. H. and W. Karlén, Holocene climatic variations-their pattern and possible cause, Quaternary Research, 3, 155-205, 1973.

Eddy, J. A., The Maunder Minimum, Science, 192, 1189-1202, 1976.

Eddy, J. A., Climate and the Changing Sun, Climate Change, 1, 173-190, 1977.

Fisher, D. A., Carbon-14 production compared to oxygen isotope records from Camp Century, Greenland and Devon Island, Canada, Climate Change, 4, 419-426, 1982.

Gough, D. O., Theoretical predictions of variations in the solar output, in The Solar Output and its Variation, edited by O. R. White, 451-474, Colorado Associated University Press, Boulder, 1977.

Hood, L. L: and J. L. Jirikowic, Recurring variations of probable solar origin in the atmospheric $\Delta^{14} \mathrm{C}$ time record, Geophys. Res. Lett., 17, 85-88, 1990.

Houtermans, J. C., Geophysical interpretation of bristlecone pine radiocarbon measurements using a method of Fourier analysis of unequally spaced data, Ph.D. thesis, University of Bern, 1971.

LaMarche, V. C. Jr., Paleoclimatic inferences from long tree-ring records, Science, 183, 1043-1048, 1974.

Mitchell, J. R. Jr., An overview of climatic variability and its causal mechanisms, Quat. 
Res., 6, 481-493, 1976.

Pestiaux, P., J. C. Duplessy, and A. Berger, Paleoclimatic variability at frequencies ranging form $10^{-4}$ cycle per year to $10^{-3}$ cycle per year-evidence for nonlinear behavior of the climatic system, in Climate: History, Periodicity, and Predictability, edited by M. R. Rampino, J. E. Sanders, W. S. Newman, and L. K. Kon̈igsson, 285-299, Van Nostrand Reinhold Company, New York, 1987.

Schove, D. J., Sunspot cycles and weather history, in Climate: History, Periodicity, and Predictability, edited by M. R. Rampino, J. E. Sanders, W. S. Newman, and L. K. Kon̈igsson, 355-377, Van Nostrand Reinhold Company, New York, 1987.

Sonett, C. P., Very long periods and the radiocarbon record, Rev. Geophys. Space Phys., 22, 239-254, 1984.

Sonett, C. P., and S. A. Finney, The spectrum of radiocarbon, Phil. Trans. Royal Soc. London, 1989 (in press).

Stuiver, M., Variations in radiocarbon concentration and sunspot activity, J. Geophys. Res., 66, 273-276, 1961.

Stuiver, M., and T. F. Braziunas, Atmospheric ${ }^{14} \mathrm{C}$ and century-scale solar oscillations, Nature, 398, 405-408, 1989.

Stuiver, M., and R. Kra, editors, Twelfth Radiocarbon Conference Proceedings, Radiocarbon, 28, 1986.

Stuiver, M., and P. D. Quay, Changes in atmospheric carbon-14 attributed to a variable Sun, Science, 207, 11-19, 1980.

Sternberg, R. S., and P. A. Damon, Atmospheric radiocarbon: Implications for the geomagnetic dipole moment, Radiocarbon, 25, 239-248, 1983.

Suess, H. E., The radiocarbon record in tree rings of the last 8000 years, Radiocarbon, 22, 200-209, 1980.

Waldmeier, M., The Sunspot-Activity in the Years 1610-1960, Schulthess, Zurich, 1961. 
IS THE SUN A LONG PERIOD VARIABLE?

\author{
Charles P. Sonett \\ Dept. of Planetary Sciences and Lunar and Planetary Laboratory \\ University of Arizona, Tucson, Arizona 85721
}

\begin{abstract}
The inventory of atmospheric radiocarbon exhibits quasi-periodic variations of mean period of $\bar{\Lambda}_{m}=269$ years over the entire 9000 year record. But the period is inconstant and subject to random variability $\left(\sigma_{m}^{1 / 2}=119\right.$ years $)$. The radiocarbon maxima correspond to the quasiperiodic extension of the Maunder minimum throughout the Holocene and resolve the long-standing issue of Maunder cyclicity. The radiocarbon maxima are amplitude modulated by the 2300 year period and thus vary significantly in peak value. The $\sim 2300$ year period in turn appears to not be modulated by the secular geomagnetic variation. Detection of a Maunder-like sequence of minima in tree ring growth of Bristlecone pine and its correlation with the Maunder $[1890,1922]$ cyclicity in the radiocarbon record supports the inference that solar forcing of the radiocarbon record is accompanied by a corresponding forcing of growth of timberline Bristlecone pine. Because of the random component of the Maunder period, prediction of climate if tied to the Maunder cycle, other than probabilistically, is significantly hindered. For the mean Maunder period of 269 years the probability is $67 \%$ that a given climatic maximum lies anywhere between 150 and 388 years.
\end{abstract}

\title{
INTRODUCTION
}

We have analyzed a composite set of radiocarbon $\left({ }^{14} \mathrm{C}\right)$ records comprising a sequence extending from 7202 $\mathrm{BC}$ to $1900 \mathrm{AD}$ together with a reappraisal of the Campito Mt. Bristlecone pine (Pinus longaeva) record of tree ring growth [La Marche and Harlan 1973]. The Bristlecone record has previously been reported to contain spectral features particularly at $\sim 2000$ and $\sim 200$ years correlated with the La Jolla radiocarbon sequence [Sonett and Suess, 1984]. Moreover, the cross-MEM spectrum of the two records shows non-zero coherence at these periods with the tree record leading by some 70-80 years [Sonett 1988]. Because this lag is in gross disagreement with the bomb-derived value between production and inventory of radiocarbon, we had not in the past strongly emphasized these results since the publication of Sonett and Suess. As it turns out this lag appears to be consistent with the negative correlation between Maunder minima recorded by radiocarbon and the Bristlecone pine. That the Bristlecone record shows a cyclicity record with some similarity to the 'Maunder cycles' of the radiocarbon record but correlated negatively with the radiocarbon record suggests, presupposing tree growth to vary directly with solar irradiance, that the Sun's long period variability is reflected into a climatic replication of the Maunder cycle. The Maunder cycle contains a strong stochastic (or chaotic) element which explains some of the difficulties encountered in past searches for simple harmonically related periods in the radiocarbon record.

\section{THE DELTA RADIOCARBON SEQUENCE}

The radiocarbon sequence reported here is composed of segments, sometimes overlapping, from records from Pearson? et [1986], Linick et al [1986], Kromer et al [1986], and Suess and Linick [unpublished]. To make up a continuous sequence we interleaved all records in ascending time. The composite sequence extends from 7202 BC-1900 AD. 


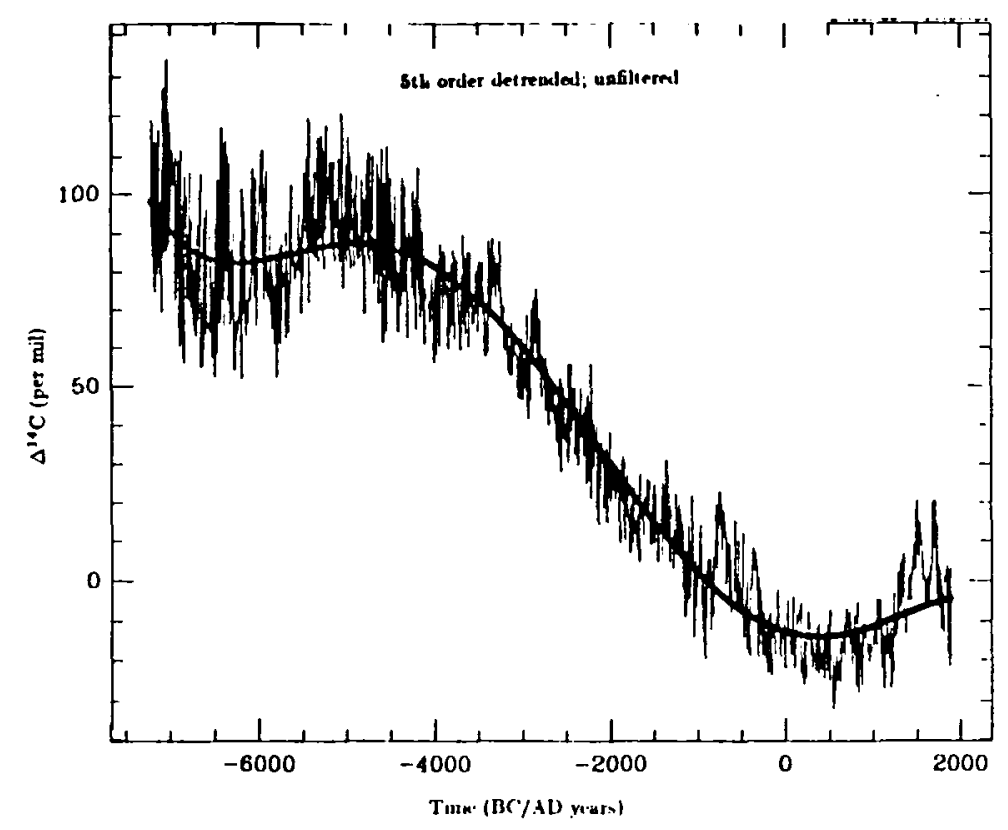

Fig. 1. Composite $\Delta^{14} \mathrm{C}$ record (detailed in Table 1) with model 5th order detrend polynomial.

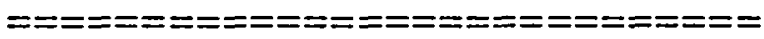

The major trend shown in Fig. 1 is generally attributed to a nearly secular increase in the Earth's magnetic field [Buchta 1970; Barton et al 1979; McElhinney and Senenyake 1982; Creer 1988] (and has often been removed using a sinusoidal function). The recent extension of the chronology clearly shows the secondary minimum centered at about $6000 \mathrm{BC}$. This new addition requires a high order (5th) detrender fpr computation of the spectrum. The filtered, splined, and equally spaced $\Delta^{14} \mathrm{C}$ sequence contains the complete spectrum of variations, including a very long period component identified with the $\Lambda_{c} \sim 2300$ year line.

For the primary matter at hand involving narrow banding the sequence, a numerical filter with Pascal distributed weights (filtering to $\sim 100$ years) is used. This is followed by subtraction of a low frequency version of the sequence obtained by passing the sequence through 99 point Pascal weights with cutoff $\sim 1000$ years. This yields the multi-hundred year response sequence (Fig. 2) which displays 34 maxima of varying amplitude; amplitude modulation of the signal has a period of approximately 2300 years, consistent with the low frequency line in the periodogram. There are a total of 34 radiocarbon maxima in Fig. 2 measured between positive peaks. Tests using varying thresholds disclosed that essentially all minima were incorporated into the search routine for the choice used. The maxima are taken to correspond to solar activity (Maunder) minima [Stuiver and Quay 1981]. Variable amplitudes are attributable to amplitude modulation (AM) of the signal by the $\Lambda_{c} \sim 2300$ year period. Maxima during the last millennium are much smaller than at 1300 AD. During the historical sunspot Maunder minimum, sunspot activity was zero or very small. If the $1300 \mathrm{AD}$ radiocarbon maximum also infers a sunspot "zero" then the additional increment of radiocarbon, over and above what is necessary to explain a sunspot minimum, requires a modification of the atmospheric inventory with an increase in total carbon dioxide. In short, for radiocarbon increases over and above that associated with the historical Maunder period a climatically forced atmospheric $\mathrm{CO}_{2}$ inventory change is inferred with radiocarbon a tracer of total carbon though most of this variability appears to be associated with the $\sim 2300$ year periodicity. 


\section{BRISTLECONE PINE SEQUENCE AND STATISTICS}

The Campito Mt. Bristlecone record (3435 BC-1970 AD) [La Marche and Harlan 1973] from timberline in the White Mts. provides an absolute chronology of growth ring spacing [La Marche and Harlan 1973; La Marche 1974]. It is too long for efficient computation involving multihundred year periods, so we filtered it first by a 9 weight Pascal filter followed by narrow banding as for the radiocarbon. This sequence shows the same general properties as $\Delta^{14} \mathrm{C}$. Tables $1 \mathrm{a}$ and $\mathrm{b}$ contain the statistics of the Maunder cycles for both radiocarbon and Bristlecone.

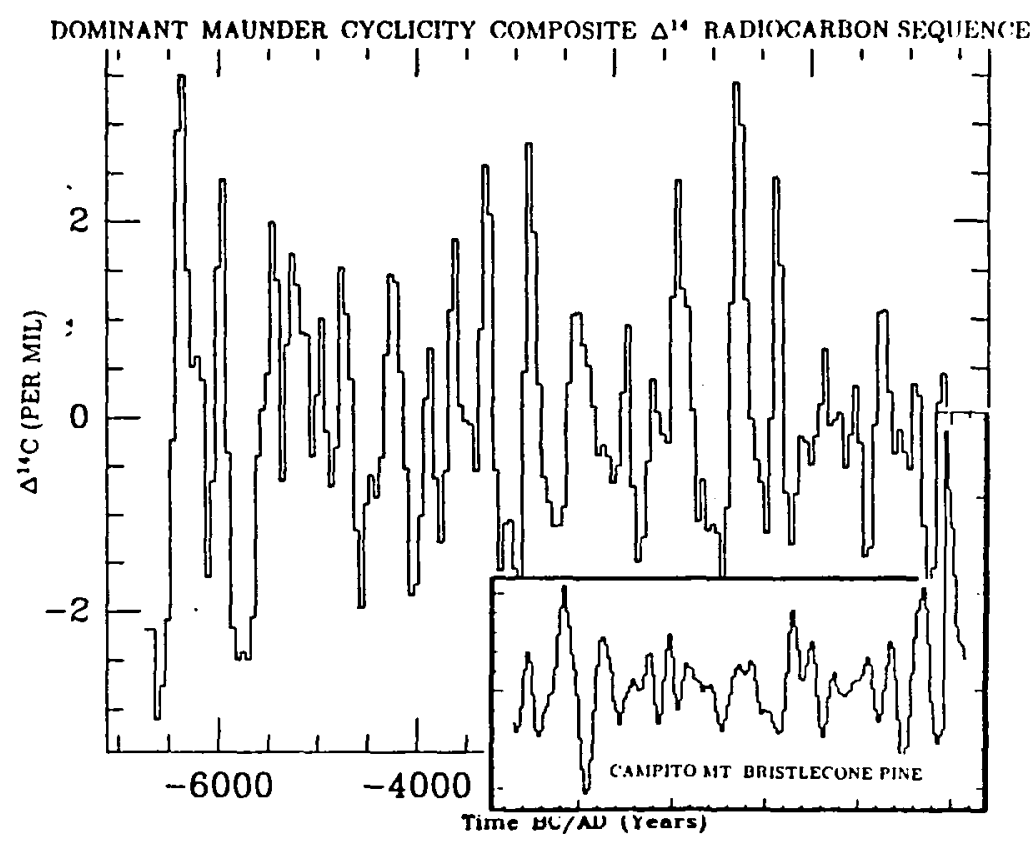

Fig. 2. $\Delta^{14} \mathrm{C}$ narrow banded to emphasize the dominant period characterized by $\Lambda_{m}=269$ years. 2300 year variability can be seen as an amplitude modulation of the sequence peaks; vestigial suggestion of $\sim 1000$ years is also present; (b) Inset: time sequence for Campito Mt. Bristlecone pine with narrow banding corresponding to the radiocarbon record of Fig. 1.

$=========================$

THE RADIOCARBON AND TREE RING SPECTRA

Detrended periodograms disclose complex spectra in the multi-hundred year and millennial range of periods for both sequences. Estimates of spectral line feature periods have been made using the Bayesian estimator of Bretthorst [1988]. Apparently robust periods are present in the same approximate neighborhood ( 200 and $\sim 2300$ years) for both sequences; however the cross MEM spectrum (Sonett and Suess 1984) shows a more convincing relation between the two sequences. 
Table 1a. Radiocarbon derived Maunder statistics maxima spacing statistics (years) ${ }^{1}$

\begin{tabular}{ccc}
\hline Moment (years) & Value (a) & Value (b) \\
\hline Mean period & 269 \\
Variance & 119 \\
No. maxima & 32 \\
\hline
\end{tabular}

${ }^{1}$ Based upon a lower search bound of -1 per mil of the heavily filtered data (See Fig. 2)

Table 1b. Bristlecone-derived Maunder statistics

$\begin{array}{lc}\text { Mean period } & 166 \\ \text { Variance } & 88 \\ \text { No. maxima } & 19\end{array}$

THE VERY LONG RADIOCARBON PERIOD

Both ${ }^{10} \mathrm{Be}$ and ${ }^{14} \mathrm{C}$ are produced by the $\mathrm{CR}$ flux, though production of ${ }^{10} \mathrm{Be}$ is via atmospheric spallations and is scavenged from the atmosphere in 2-3 years by attachment to aerosols [Raisbeck et al, 1981. Since production of ${ }^{14} \mathrm{C}$ and ${ }^{10} \mathrm{Be}$ are coeval and ${ }^{10} \mathrm{Be}$ is rapidly stored it serves as an important $\mathrm{CR}$ proxy to radiocarbon. ${ }^{10} \mathrm{Be}$ exhibits $9-11.4$ year variations as well as variability during the Maunder minimum. The Camp Century/Dome $\mathrm{C}$ composite ${ }^{10} \mathrm{Be}$ ice core record shows a remarkable though imperfect correlation inferring that the Maunder cycle is also present in the Be record [Beer and Raisbeck, in press]. The record of ${ }^{10} \mathrm{Be}$ is imperfect; evidence for a 2300 year period in the record of this isotope is uncertain at best and generally lacking. This supports the view that the 2300 year radiocarbon line is of terrestrial origin.

The periodicity of ca. 2300 years is also found in the $\delta^{18} \mathrm{O}$ record in ice cores and foraminifera from ocean cores (Pestiaux et al, 1987; 1988). Glaciation shows this period as does the Middle Europe oak dendroclimatic record and Dansgaard et al (1984) report the most prominent period in their Camp Century $\delta^{18} \mathrm{O}$ core to be a line at 2550 years. Early publications of Dansgaard's group discuss periodicities in the $\delta^{18} \mathrm{O}$ record for the time interval from 1200-2000 AD (Johnsen et al. 1970; Dansgaard et al., 1971). Their chronology was based on the assumed average accumulation rate of ice, and their power spectrum for this time interval shows prominent periods at 78 and 181 years. They associate the 78 year period with the Gleissberg sunspot periodicity. In a longer section of the core going back to about 10,000 years, they obtain a period of 350 years, again using the ice accumulation time scale (ibid., 1971), and at about 45,000 BP ice accumulation rate years, they find a persistent oscillation with period of $\sim 2000$ years.

Can the source of the 2300 year period be isolated? It appears to be free of modulation by the $\sim 10,000$ year secular trend. If the 2300 year source were extraterrestrial, it should interact non-linearly with the secular trend since the they are related through $Q \sim B^{-0.52}$ [Elsasser et al 1954]. No such effect has so far been detected. Moreover an extraterrestrial mechanism should have to be non-linear mechanism to account for its modulation of the 269 year period. This would not likely be seated in the solar wind which is adiabatic to both periods. Therefore the solar atmosphere becomes a prime candidate.

On the other hand characteristic deep water residence times for the global oceans range from 1,000 years for the Atlantic to 2,000 years for the full Atlantic-Pacific circulation [Broecker and Peng 1970; Broecker and $\mathrm{Li} \mathrm{1970].} \mathrm{In} \mathrm{themselves} \mathrm{these} \mathrm{delay} \mathrm{times} \mathrm{by} \mathrm{themselves} \mathrm{cannot} \mathrm{explain} \mathrm{the} \mathrm{global} \mathrm{ocean-atmosphere}$ 
resonance required to drive a 2300 year total $\mathrm{CO}_{2}$ cycle, but the times are consistent with such a resonance. Yiou et al [in press] report periods, primarily Milankovich-derived from deuterium analysis of the Vostok ice core. It is noteworthy that periods at 4.4, 3.5, 2.7, and 2.4 kyears are detected. Their assignment of these is to harmonics arising from orbit-climate interactions. Sonett [1985], Sonett and Finney [in press], and Damon and Sonett [in press] find a 2.3 kyear period in radiocarbon (Damon and Sonett also report vestigial evidence of an $\sim 4$ kyear period using the Bretthorst algorithm). It may never be possible to isolate the 2.3 kyear forcing with confidence. If due to orbit-insolation it may still drive global $\mathrm{CO}_{2}$ and thus dilution of radiocarbon. If so a search for an ocean-atmosphere resonance independently of orbit- insolation may be meaningless.

\section{CLIMATE AND THE PARTITION OF CARBON}

Assuming that the Bristlecone record can be duplicated elsewhere a role for the Sun in climate is inferred; but it is complicated by the lack of understanding of how correlated bolometric and hydromagnetic mechanisms can coexist on the Sun. Moreover the bolometric component forces modification of the atmospheric $\mathrm{CO}_{2}$ inventory through mediation of the ocean surface temperature and possibly other effects. The Natl. Academy [1975] report settles upon a $2.50 \mathrm{C}$ temperature change per doubling of the $\mathrm{CO}_{2}$ atmospheric inventory. Sensitivity studies of temperature vs. $\mathrm{CO}_{2}$ are reported, e.g. by Hansen et al [1981] and Lal [1985] and point out the complications arising from sensitivity of the concentration to reservoir content. The atmospheric $\mathrm{CO}_{2}$ inventory and radiocarbon concentration are jointly determined by CR flux and the terrestrial environment; partitioning of forcing of the net radiocarbon level between atmospheric dilution and CR production is uncertain because both the $\mathrm{CR}$ flux and terrestrial reservoir exchange are inconstant. A number of the spectral features found in the radiocarbon model have amplitudes of $(O) 2.5$ per mil. Using the Houtermans et al [1973] model for production and reservoir concentration values to establish a qualitative approximation, a 200 year period in production rate results in an attenuation in atmospheric disturbance $\sim 20$ times, depending upon transfer coefficients. Thus the interplanetary peak-peak variation is very approximately 2.5 \%. For the 2300 year cycle attenuation is nil. For a steady state (CR addition just equal to decay), the peak-peak radiocarbon variation is due to dilution; the global $\mathrm{CO}_{2}$ variation is $0.5 \%$.

Growth of trees measured by ring width can aid in establishing the thermal chronology for much of the Holocene but is a complicated function of moisture and air temperature. At high altitude tree growth may even be directly dependent upon solar irradiance rather than indirectly through air temperature [Fritts 1976]. The Campito Mt. record is from the timberline band which progresses and regresses with time. La Marche and Mooney [1967] find a timberline retreat of about 120-150 meters in timberline altitude from the altithermal till now. La Marche [1973] finds a retreat from $2500 \mathrm{BC}$ till $1700 \mathrm{AD}$ at Sheep Mt. (White Mts.) of about 200 meters suggesting a temperature variation of about $2.1^{\circ} \mathrm{C}$ based on lapse rate. In support of this La Marche [1974] finds a significant correlation between the combined central England [Lamb 1980] and Northern hemisphere [Mitchell 1961] temperature record and White Mt. timberline tree ring width from 800 to $1960 \mathrm{AD} . \Delta T \sim 1.5^{\circ} \mathrm{C}$ vs. $\Delta W \sim 0.5$ The establishment of a true dendrochronological timberline thermometer is still to be made, but changes in the timberline Bristlecone ring growth record support a response primarily seated in temperature. La Marche [1974] discussed temperature-moisture stressing upon tree physiology, suggesting that the Campito Mt. Bristlecone pine timberline sequence records primarily thermal stressing.

Although the $\Delta^{14} \mathrm{C}$ Maunder peaks (Fig. 2) tends to occur at Bristlecone pine minima detailed correspondence is not always good. The tree response is expected to follow solar irradiance closely while the atmospheric radiocarbon inventory varies approximately as the integrated CR flux which is suppressed by several decades and is noisy. The radiocarbon record also is likely subject to other forcings which do not correlate with tree growth. But from an average standpoint, as the radiocarbon peaks correspond to solar activity minima, their inverse (solar activity maxima) should correlate positively with tree ring thickness maxima, which is the case. This supports the inference that the trees are responding to aerial temperature rather than to some exotic forcing and that atmospheric temperature is indeed correlated with long period 
solar activity. Extraction of a time-temperature record for the White Mts. from the tree record is difficult for two reasons: the uncertain physiology of the Bristlecone, their extreme antiquity, and the ever possibility that their growth is stressed by other factors than air temperature. The $\sim 1000$ year "half cycle' in the Bristlecone pine record reported by La Marche [1974] (his Fig. 4) also appears in the analysis of this paper. It corresponds about to the time from the Medieval warm epoch to the present [Lamb, 1982].

\section{SOLAR VARIABILITY}

Gough [1988] has reviewed characteristic times for the Sun but no specifically multi-hundred year periods are identified. It is possible to place global hydromagnetic modes conceptually within a solar context if a core magnetic field of (O) 1 gauss is admitted [Cowling 1945; Sonett 1982; Levy and Boyer 1982; Moss 1987]. Global solar Alfven wave transit times vary only by about a factor of 10; though estimates are only qualitative they do suggest the possibility of eigenmodes corresponding to periods of perhaps 100-1000 years. However the inference that the Maunder cycle is a manifestation of chaotic behavior in the solar dynamo seems more plausible, e.g. Weiss and Cataneo 1984], though for either source model for the variability the theoretical foundation is sketchy. Such modes would have to exhibit a significant bolometric connection to account for the combined radiocarbon and Bristlecone variability. It is hardly a hypothesis but the well-known deficit in the high energy solar neutrino flux (See Wolfsberg and Kocharov [in press] for review.) does suggest that understanding of the solar core is still uncertain. Sporadic reports of solar radius variability infer a changes in solar diameter. Gilliland's [1981] work shows a maximum in solar diameter ca. 1910 corresponding to a minimum in solar activity. Moreover his results show a return to maximum in about 70-80 years suggesting a relation to the Gleissberg period. Ribes et al [in press] review the field including 18th and 19th century solar observations.

\section{SUMMARY}

The Maunder cycle appears throughout the entire 9000 year record of $\Delta^{14} \mathrm{C}$ as 34 episodes of increases in delta radiocarbon corresponding to decreases in interplanetary modulation. The mean period, $\bar{\Lambda}_{m}=269$ years but the variability is large $(\sigma=129$ years). Corresponding cyclicity exists in the record of tree ring growth of Bristlecone pines from timberline in the White Mts. of Eastern California over the shorter period of $\sim 5000$ years. The tree variations are thought to arise from variability of air temperature though a direct dependence of growth upon solar irradiance has not been ruled out. The stochastic (or chaotic?) component in the Maunder period is large, making predictions of a related climate variability probabilistically and highly uncertain. Though the mean Maunder period is 269 years, the probability is $67 \%$ that a given climatic maximum lies anywhere between 150 and 388 years. But the maxima are mediated by the 2300 year period which has a very different phase relation between inventory and source function than does the 269 year period. Indeed it may not be possible to assess phase in a fully meaningful way because of the extreme jitter in the Maunder cycle. Since both hydromagnetic (CR modulation by the solar wind) and bolometric (irradiance) variability may be correlated, our conjecture is that the solar dynamo displays chaotic behavior, though whether this will be supported by further work is uncertaion.

\section{ACKNOWLEDGEMENTS}

I thank H.C. Fritts and M.K. Hughes for bringing to my attention a number of works on the Bristlecone record, C.R. Stockton for supplying the Campito Mt. Bristlecone record, P.E. Damon for pointing out many nuances in the radiocarbon record, and finally H.E. Suess for originally bringing my attention to the reality and importance of the 200 year line in the radiocarbon spectrum. 


\section{REFERENCES}

Barton, C.E., R.T. Merrill, and M. Barbetti, Intensity of the earth's magnetic field over the last 10,000 years, Phys. Earth Plan. Int. 20, 96 [1979].

Beer, J., M. Andree, H. Oeschger, B. Stauffer, R. Balzer, G. Bonani, C. Stoller, M. Suter, W. Wölfli, and R.C. Finkel, Temporal ${ }^{10} \mathrm{Be}$ variations in ice, Radiocarbon, 25, 269-278 [1983].

Beer, J. and G.M. Raisbeck, in "The Sun in Time", eds. C.P. Sonett, M.S. Giampapa, and M.S. Matthews, Univ. Arizona press [in press].

Bretthorst, G.L., Bayesian spectral analysis and parameter estimation, vol. 48, "Lecture Notes in Statistics", eds. J. Berger, S. Fienberg, J. Gani, K. Krickeberg, and B. Singer, Springer-Verlag [1988].

Broecker, W.S. and Y.-H. Li, Interchange of water between the major oceans, J. Geophys. Res. 75, 3545-3552 [1970].

Broecker, W.S. and T.-H. Peng, "Tracers in the Sea", Lamont-Doherty Geological Obs., New York [1970].

Buchta, V., Influence of the earth's magnetic field on radiocarbon dating, in Radiocarbon variations and absolute chronology, Nobel Symposium 12, 595 [1970].

Cowling, T.G., On the Sun's general magnetic field, Mon. Not.R. astr. Soc., 105, 166-174 [1945].

Creer, K.M., Geomagnetic field and radiocarbon activity through Holocene time, in "NATO Advanced Research Workshop on Secular, Solar, and Geomagnetic variations through the last 10,000 years", eds. F.R. Stephenson and A.W. Wolfendale, NATO ARW series, Kluwer Acad. Publ. [1988].

Damon, P.E. and C.P. Sonett, Solar and Terrestrial components of the ${ }^{14} \mathrm{C}$ variation spectrum, in "The Sun in Time", eds. C.P. Sonett, M.S. Giampapa, and M.S. Matthews, Univ. Ariz. Press [in press].

Elsasser, W., E.P. Ney, and J.R. Winckler, 1956 Cosmic ray intensity and geomagnetism, Nature 178, 1226 [1956].

Fritts, H.C. "Tree Rings and Climate", Academic Press [1976].

Gilliland, R.L., Solar radius variations over the past 265 years, Ap.J., 248, 1144-1155 [1981].

Gleissberg, W., A table of secular variations of the solar cycle, Terr. Mag. and Atm. Elect., 49, 243-244, [1944].

Gleissberg, W., Ascent and descent in the eight year cycle of solar activity, J. Brit. Astron. Assoc., 76, 265-268 [1966]. 
Gough, D., Theory of solar variation, Il Nuovo Cimento, 90-132 [1988].

Hansen, J., D. Johnson, A. Lacis, S. Lebedeff, P. Lee, D. Rind, and G. Russell, Climate impact of increasing atmospheric carbon dioxide, Science, 213, 957-906 [1981].

Houtermans, J.C., H.E. Suess, and H. Oeschger, Reservoir models and production rate variations of natural radiocarbon, J. Geophys. Res., 78, 1897-1908 [1973].

Kromer, B., M. Rhein, M. Bruns, H. Schoch-Fischer, K.O. Münnich, M. Stuiver, amd B. Becker, Radiocarbon dating for the 6 th to the 8 th millennia, Radiocarbon $28,954-960$ [1986].

Lal, D., The Carbon Cycle and Atmospheric $\mathrm{CO}_{2}$ : Natural Variations Archean to Present, Geophys. Mono. 32, eds. E.T. Sundquist and W.S. Broecker, American Geophys. Union, Washington, D.C. [1985].

Lamb, H.H., "The Changing Climate", Methuen, London [1982].

La Marche, Jr., V.C., Holocene climatic variations inferred from treeline fluctuations in the White Mts., California, Quaternary Res. 3, 632-660 [1973].

La Marche, V.C. and H.A. Mooney, Altithermal timberline advance in western United States, Nature 213, 980-982 [1967]

La Marche, V.C., Paleoclimate inferences from long tree-ring records, Science 183, 1043-1048 [1974].

La Marche, V.C. and T.P. Harlan, Accuracy of tree ring dating of Bristlecone pine for calibration of the radiocarbon time scale, J. Geophys. Res., 78, 8849-8857 [1973]

Levy, E.H. and D. Boyer, Oscillating dynamo in the presence of a fossil magnetic field. The solar cycle, Ap.J., 254, L19-L22 [1982].

Linick,T.W., A. Long, P.E. Damon, and C.W. Ferguson, High-precision radiocarbon dating of Bristlecone pine from 6554 to $5350 \mathrm{BC}$, Radiocarbon 28, 943-953 [1986].

Maunder, E.W., The prolonged sunspot minimum, 1645-1715, Brit. Atron. Assoc. J., 32, 140-145 [1922].

Maunder, E.W., Mon. Not. Roy. Astron. Soc.,1, 251 [1890].

McElhinny, M.W. and W.E. Senanyake, Variations in the geomagnetic dipole: the past 50,000 years, Geomag. Geoelect 34, p.39 [1982].

Mitchell, J.M., Recent secular changes of global temperature, New York Acad. Sciences 95, 235-250 [1961].

Moss, D., The internal magnetic field of the Sun, Mon.Not. R. astr. Soc., 224, 1019-1029 [1987].

Natl. Acad. of Sciences, Understanding Climate Change, Washington, D.C. [1975]. 
?Pearson, G.W., J.R. Pilcher, M.G.L. Baillie, D.M. Corbett, and F. Qua, High precision ${ }^{14} \mathrm{C}$ measurement of Irish oaks to show the natural ${ }^{14} \mathrm{C}$ variations from AD 1840 to $5210 \mathrm{BC}$, Radiocarbon 28,911 [1986].

Raisbeck, G.M. and F. Yiou, Cosmogenic ${ }^{10} \mathrm{Be} /{ }^{7} \mathrm{Be}$ as a probe of atmospheric transport processes, Geophys. Res. Lett. 8, 1015-1018 [1981].

Ribes, E., B. Beardsley, T.M. Brown, P. Delache, J.R. Kuhn, F. Leclare, and N.V. Leister, The variability of the solar diameter, in "The Sun in Time". eds. C.P. Sonett, M.S. Giampapa, and M.S. Matthews, Univ. Arizona Press [in press].

Sonett, C.P., Sunspot index from square law modulation of the Hale cycle, Geophys. Res. Lett, 9, 1313 [1982].

Sonett, C.P. and H.E. Suess, Correlation of bristlecone pine ring widths with atmospheric ${ }^{14} \mathrm{C}$ variations: a climate-Sun relation, Nature 307, 141 [1984].

Sonett, C.P., Very long solar periods and the radiocarbon record, Rev, Geophys. Spa. Phys., 22, 239-254 [1985].

Sonett, C.P., Time series', radiocarbon, and varves, Proc. Enrico Fermi School of Physics, XCV course in Solar-Terrestrial Relationships and the Earth Environment in the last Millennia, Il Nuovo Cimento 274-311 [1988].

Sonett, C.P. and S.A. Finney, The spectrum of radiocarbon, Phil. Trans. Roy. Soc. [in press].

Stuiver, M., and P.D. Quay, Atmospheric ${ }^{14} \mathrm{C}$ changes resulting from fossil fuel $\mathrm{CO}_{2}$ and cosmic ray variability, Earth and Plan. Sci. Lett., 53, 349-362 [1981].

Suess, H.E., The radiocarbon record in tree rings of the last 8000 years, Radiocarbon, 20, 1 [1978].

Weiss, N.O. and F. Cattaneo, Periodic and aperiodic dynamo waves. Geophys. and Astrophys. Fluid Dynamics, 30, 305-341 [1984].

Wolfsberg, and G.E. Kocharov, in "The Sun in Time", eds. C.P. Sonett, M.S. Giampapa, and M.S. Matthews, Univ. Arizona Press [in press].

Yiou, P., C. Genthon, J. Jouozel, M. Ghil, H. Le Treut, J.M. Barnola, C. Lorius, and Y.N. Korotkevitch, High-frequency paleovariability in climate and in $\mathrm{CO}_{2}$ levels from Vostok ice- core records, in "Interaction of the Global Carbon and Climatic Systems", ed. R. Keir, Electr. Power Res. Inst. [in press]. 


\title{
TRENDS IN SOLAR VARIABILITY
}

\author{
Robert Jastrow \\ Dartmouth College
}

and

\section{Sallie Baliunas}

Harvard-Smithsonian Center for Astrophysics and Dartmouth College

Radiocarbon $(\Delta \mathrm{C}$-14) records suggest a 200 -year periodicity in solar activity in the last millennium. We have examined the Carbon-14 record going back 8 millennia for this and other periodicities. The computation differs from that in most previous work in its use of techniques developed for unevenly spaced sampling without rebinning the data to equally spaced intervals. A variation with a 200-year period is a strong feature of the Carbon-14 record going back several millennia. Periodicities that appear to be significant will be listed and their physical interpretation discussed. 
THE PROGRAM ON RADIATIVE INPUTS OF THE SUN TO THE EARTH (RISE)

Peter V. Foukal

Cambridge Res. and Inst. Inc.

21 Erie St.

Cambridge, MA 02139

General concerns about changes in the Earth's climate and in the ozone layer have increased the importance of measuring and understanding variations in the sun's radiative outputs. These outputs appear, for instance, at the top of the list of global change forcing agents in the recent FY90 document on the U.S. Global Change Program. Significant advances have been made over the past decade in radiometry of the total solar irradiance. Photometry of light variations in stars similar to the sun, but much younger, is providing new insights into the sun's variations in luminosity and UV radiation at previous epochs of interest to paleoclimate studies. Measurement of the sun's 11-year output variability in the ultraviolet and extreme ultraviolet still posses a challenge of great importance. All of these topics are addressed by the Program on Radiative Inputs of the Sun to Earth.

RISE is a 5-year program of observations, data analysis, and theory, that has been defined at two workshops held in Boulder, Colorado in November 1987, and in Tuczon, Arizona in October 1989. These meetings involved about 60 solar and atmospheric physicists, and stellar astronomers. A proceedings of the 1987 workshop was issued in early 1988. A substantial component of the program, consisting of ground-based observations, data analysis, and theory, is directed at the National Science Foundation and the program is seeking a FY92 funding start at the NSF. RISE also makes recommendations on measurements required from NASA and NOAA satellites. A document describing the RISE program background and recommendations is now available from the chairman of the steering committee, P. Foukal. Questions from prospective participants are encouraged. 


\title{
SOLAR ASTRONOMY DATA BASE: Packaged Information on Diskette
}

\author{
John A. McKinnon \\ National Geophysical Data Center \\ Boulder, Colorado 80303 USA
}

In its role as a library, the National Geophysical Data Center has transferred to diskette a collection of small, digital files of routinely measured solar indices for use on an IBM-compatible desktop computer. Recording these observations on diskette, allows us to distribute specialized information to researchers with a wide range of expertise in computer science and solar astronomy.
We made every data set self-contained by including formats, extraction utilities, and plain-language descriptive text. Moreover, for several archives, two versions of the observations are provided-one suitable for display, the other for analysis with popular software packages. Since the files contain no control characters, each one can be modified with any text editor.

Table 1. Selected solar and geophysical data on diskette. Nearly all tabulated values published monthly in Solar-Geophysical Data are available on sets of IBM-compatible diskettes. We partitioned each archive into a family of files that contain short, chronologically sorted records for either 1 month or a year. Except in two cases, these files are not images of tables published earlier.

\begin{tabular}{|c|c|c|c|c|c|}
\hline Data Set Description & Observat & fion Period & Gaps* & (MB) & Disks \\
\hline $\begin{array}{l}\text { IMF Sector Boundaries } \\
\text { Mean Solar Magnetic Field } \\
\text { Geomagnetic Indices (Kp, Ap, Cp, C9) } \\
\text { Neutron Monitors Climax/Huancayo }\end{array}$ & $\begin{array}{ll}01 & 1947 \\
03 & 1968 \\
01 & 1932 \\
01 & 1953\end{array}$ & $\begin{array}{l}121989 \\
121989 \\
091989 \\
101989\end{array}$ & & $\begin{array}{r}0.3 \\
0.3 \\
1.5 \\
39.2\end{array}$ & $\begin{array}{l}1 \\
1 \\
1 \\
8\end{array}$ \\
\hline $\begin{array}{l}\text { Flare Patrol Intervals } \\
\text { Flare Reports (not grouped) } \\
\text { Coronal Index of Solar Activity }\end{array}$ & $\begin{array}{l}011955 \\
041938 \\
011964\end{array}$ & $\begin{array}{l}101989 \\
101989 \\
121986\end{array}$ & $65,66,67$ & $\begin{array}{r}20.5 \\
33.8 \\
0.2\end{array}$ & $\begin{array}{r}5 \\
10 \\
1\end{array}$ \\
\hline $\begin{array}{l}\text { Total Solar Irradiance } \\
\text { Solar Lyman-Alpha Flux } \\
\text { Solar Radio Flux Ottawa (10.7 cm) } \\
\text { Solar Radio Bursts-Fixed Frequency }\end{array}$ & $\begin{array}{l}111978 \\
011982 \\
021947 \\
011960\end{array}$ & $\begin{array}{l}071989 \\
031989 \\
011990 \\
101989\end{array}$ & 60 & $\begin{array}{l}0.3 \\
0.1 \\
0.5 \\
31.8\end{array}$ & $\begin{array}{l}1 . \\
1 \\
9\end{array}$ \\
\hline $\begin{array}{l}\text { Sunspot Count Zurich/International } \\
\text { Sunspot Groups Greenwich } \\
\text { Sunspot Groups USAF/Mt. Wilson } \\
\text { Calcium Plage Regions } \\
\text { Regions of Solar Activity }\end{array}$ & $\begin{array}{l}011749 \\
051874 \\
011962 \\
011962 \\
011969\end{array}$ & $\begin{array}{l}101989 \\
111981 \\
071989 \\
121986 \\
081982\end{array}$ & & $\begin{array}{r}0.7 \\
13.7 \\
5.9 \\
6.3 \\
5.7\end{array}$ & $\begin{array}{l}1 \\
5 \\
2 \\
2 \\
2\end{array}$ \\
\hline
\end{tabular}

*Notation under "Gaps" heading: bold = preliminary; underline = partial; plain=missing.

Diskette versions of familiar solar data sets cannot banish all the computer demons of the past. But for small archives they do offer more than a 100-fold increase in convenience over magnetic tapes. To obtain more information about our products, contact the National Geophysical Data Center, Mail Code E/GC2, 325 Broadway, Boulder, Colorado 80303 USA. Phone (303) 497-6346, FAX (303) 497-6513, SPAN 9555::HCOFFEY. 


\title{
NOAA/TIROS AND GOES OBSERVATIONS OF GALACTIC AND SOLAR COSMIC RAYS OVER A SOLAR CYCLE
}

\author{
Herbert H. Sauer \\ NOAA Space Environment Laboratory \\ Boulder Colorado 80303
}

\begin{abstract}
Correlations between solar activity and atmospheric processes have been investigated for more than 30 years, and the reality of the strong correlations found has been well accepted. However, it remains problematic to establish mechanisms capable of coupling the physical manifestations of solar variability to the lower atmosphere. There has been to date a lack of long-term satellite measurements of particles of sufficient energy to penetrate the Earth's atmosphere. The NOAA Space Environment Laboratory has maintained energetic particle detectors on board both the NOAA/ TIROS and GOES series of satellites. These instruments monitor the flux of energetic protons to energies greater than about $800 \mathrm{MeV}$. Measurement of particles above $350 \mathrm{MeV}$ began October 1978 , and continue to date. The instruments are briefly described, along with the current program to reduce the data. The goal is to provide a data base that will help to assess the significance of variations of atmospheric and ionospheric properties due to energetic particle precipitation.
\end{abstract}

\section{INTRODUCTION}

Many authors have proposed cosmic rays and their variations as the mediator or carrier of solar variability since Ney ${ }^{1}$ examined the relationship between cosmic rays and the weather in 1959 . The suggestion is attractive because there have been established correlations of atmospheric phenomena and cosmic ray variations on time scales ranging from days, through solar cycles, to centuries. 2,3 Recent studies by Labitzke ${ }^{4}$ and Labitzke and van Loon, ${ }^{5}$ demonstrating the solar cycle relationship of northern hemisphere stratospheric temperatures and pressures that depend on the phase of quasi-biennial oscillation of the equatorial stratospheric winds, have served to stimulate inquiry in this area. In a recent paper, Tinsley et al. ${ }^{6}$ address the hypothesis that cosmic rays and solar energetic particles of energy 100 to $1000 \mathrm{MeV}$ are the principal mediators of solar variability. They conclude that the hypothesis is supported on at least two grounds: first, the mentioned close correlations on all three time scales (days, solar cycle, and century), and secondly, that opposite tropospheric responses are observed for Forbush decreases as for solar flare responses. They further note the lack of appropriate long-term measurements over this energy range. It is the purpose of this note to present the development of a data base of observations over this energy range extending back to 1978 .

\section{DATA RESOURCES}

The Space Environment Laboratory (SEL) of the National Oceanic and Atmospheric Administration (NOAA) maintains monitoring instruments aboard both the GOES series of geosynchronous orbiting satellites, and the NOAA/TIROS series of low-altitude, Sun-synchronous, polar-orbiting satellites.

The Space Environment Monitor (SEM) package on the GOES satellites is comprised of three sensor assemblies: the X-Ray Sensor (XRS) which measures the whole-Sun X-Ray emission between .5 and 8 Angstrom, a Magnetometer assembly which measures the local vector magnetic field, and 
the Energetic Particle Sensor (EPS) which measures the energetic particle population at geostationary orbit. The EPS consists of several silicon detector assemblies which initially covered the range from .6 MeV to several hundred MeV. Beginning with GOES -4 in 1982, a Cerenkov Telescope called the High Energy Proton and Alpha Detector (HEPAD), was added. However, satellite and instrument failures prevented HEPAD data acquisition until the launch of GOES-6 in 1983. The HEPAD measures protons in an energy range from about 370 to greater than $800 \mathrm{MeV}$, and with less certainty, alpha particles in the range from about 600 to greater than $850 \mathrm{MeV} /$ nucleon. The half angle acceptance cone of the HEPAD is $34^{\circ}$, with a geometric factor of about $.9 \mathrm{~cm}^{2} \mathrm{sr}$. The GOES spacecraft are spinning at about $100 \mathrm{rpm}$, with their rotation axis approximately parallel to the Earth'. Since the HEPAD looks out orthoganally to the rotation axis and the spin rate is much higher than the data accumulation rate (4 s.), it represents a reasonably omnidirectional detector, covering the equatorial half of the unit sphere.

The energetic particle instruments of the NOAA/TIROS series also consist of several detector assemblies: the Total Energy Detector (TED) measures the differential and total energy flux of .3 to $20 \mathrm{keV}$ electrons and protons, the Energetic Particle Sensor (EPS) assembly contains independent particle telescopes separately covering the range from $>30$ to $>300 \mathrm{keV}$ for electrons and the range of $30 \mathrm{keV}$ to $>2.5 \mathrm{MeV}$ for protons, and a set of large aperture silicon detectors for the energy range of $>16 \mathrm{MeV}$ to $>80 \mathrm{MeV}$. Further, the TIROS-N and NOAA-6 satellites EPS also contain a HEPAD that is nominally identical to that of the GOES (EPS), except that it looks radially outward from the Earth. These instruments have been described by Seale and Bushnell 7 . A brief summary of the data coverage of these instruments is given in Table I.

Data from these instruments are received by the SEL's Space Environment Services Center in real or near-real time, and a synopsis of some of the data is provided in the SESC weekly publication, Preliminary Report and Forecast of Solar Geophysical Data. More complete data sets are archived

Table 1

Summary Energetic Particle Data Description

\section{GOES EPS}

Protons: $\quad 7$ Differential energy channels

Alphas: $\quad 6$ Differential energy channels

Electrons:

$$
\begin{aligned}
& 0.6<\mathrm{E}<500 \mathrm{MeV} \\
& 3.8<\mathrm{E}<500 \mathrm{MeV} \\
& \mathrm{E}>2 \mathrm{MeV}
\end{aligned}
$$

\section{NOAA/TIROS TED}

$\begin{array}{ll}\text { Protons: } & 11 \text { Differential energy channels } \\ \text { Electrons: } & 3 \text { Integral electron channels }\end{array}$

\section{NOAA/TIROS MEPED}

$\begin{array}{ll}\text { Protons: } & 8 \text { Differential energy channels } \\ \text { Electrons: } & 11 \text { Differential energy channels }\end{array}$
$.30<\mathrm{E}<20 \mathrm{keV}$

$.30<\mathrm{E}<20 \mathrm{keV}$

HEPAD (Common to the GOES-6, TIROS-N, and NOAA-6 satellites)

$\begin{array}{lll}\text { Protons: } & 3 \text { Differential channels } & 370<\mathrm{E}<800 \mathrm{MeV} \\ & \text { 1 Integral channel } & \mathrm{E}>800 \mathrm{MeV} \\ \text { Alphas: } & \text { 1 Differential channel } & 600<\mathrm{E}<800 \mathrm{MeV} / \mathrm{n} \\ & 1 \text { Integral channel } & \mathrm{E}>800 \mathrm{MeV} / \mathrm{n}\end{array}$

$.03<\mathrm{E}<215 \mathrm{MeV}$

$>30,>100,>300 \mathrm{keV}$ 
with the National Geophysical Data Center, Boulder, Colorado for dissemination to the scientific community.

Satellite observation of energetic protons up to the order of $100 \mathrm{MeV}$ have been available from the GOES sensors and from a number of other satellite data sets for a number of years. For example.

Armstrong et al. ${ }^{8}$ have presented a useful survey of interplanetary cosmic ray integral fluxes for the ycars 1963-1982, up to $\mathrm{E}>60 \mathrm{MeV}$. But corresponding long-term observations in the energy range encompassing those of solar cosmic ray ground-level events has been lacking. The HEPAD data emphasized here had not previously been available, and represent an extended data source in an energy regime of meteorological significance. Figure 1 illustrates the principal proton data availability from the sensors aboard the GOES and NOAA/TIROS satellites since the beginning of HEPAD data acquisition in November 1978.

\section{DISCUSSION}

Most studies concerning the relationship of galactic and solar energetic particles to atmospheric dynamics, chemistry, and metcorological phenomena have relied primarily upon data from Neutron Monitors. Such detectors have provided the only long-term monitoring of the Galactic Cosmic Ray flux, and its sporadic admixture of energetic solar particles. Unfortunately, Neutron Monitors principally monitor the energy range between 1 and $10 \mathrm{GeV}$ and further represent relatively small angle detectors because of atmospheric collimation and geomagnetic field effects. Their calculated asymptotic cones of acceptance can be as small as $10^{\circ} .{ }^{9}$ Neutron Monitor measurements represent a surrogate for the near-Earth energetic particle populations and their spectra in the energy range of $100-1000 \mathrm{MeV}$, the energy range most responsible for the ionization and its variation in the lower stratosphere and troposphere. ${ }^{10}$ The GOES and NOAA/TIROS, for the first time, provides a consistent data base over this important energy range for the order of a solar cycle.

GOES-6 data for the geophysically active period from July through December 1989 have been reduced. In Figure 2, the hourly averages of the GOES-6 measurements of the integral fluxes $>370$ $\mathrm{MeV}$ and $>800 \mathrm{MeV}$ are compared to hourly pressure-corrected neutron monitor relative count rates from Deep River (Lat. $46.1^{\circ}$; W. Long. $77.5^{\circ}$; Cutoff $=.6 \mathrm{GV}$ ) (National Geophysical Data Center, 1990), and Apatity (Lat. $67.3^{\circ}$; E. Long. $33.2^{\circ}$; Cutoff $=1.02 \mathrm{GV}$ ) (personal communication: M.A. Shea, 1990), for the period July 1 to December 31, 1990. Note that factor of 2-3 increases in the $>370 \mathrm{MeV}$ fluxes occurring on April 25 (Day 206), August 13 (Day 225), and November 15 (Day 319) are just discernable at Deep River or Apatity. These events represent a significant variation to tropospheric ionization rates. The hardest event of this period occurred on September 29 and 30 (Days 272,3), and it was reported 11 that this was the first time the red warning light of the Concorde SST dose-rate monitor had lit, indicating a rate in excess of $10 \mathrm{mrem} / \mathrm{hr}$. There were seven Concorde flights on that day between Paris ${ }^{11}$ or London ${ }^{12}$ and the U.S. all of which reported total doses in excess of background levels.

Figure 3 compares the hourly neutron monitor rates as before for Deep River and Apatity with 5 -minute averaged values of the $>370 \mathrm{MeV}$ and $>800 \mathrm{MeV}$ integral fluxes measured by GOES- 6 on September 29 and 30, 1989. Additionally, the heavy dots on the lower panel ( $>800 \mathrm{MeV}$ ) denote the total doses reported from the dosimeters carried on the above-referenced Concorde flights on September 29. Their nominal flight altitude is about $59,000 \mathrm{ft}(18 \mathrm{~km}$, the upper troposphere). While it is fortuitous that the magnitude of the dosages approximate the $>800 \mathrm{MeV}$ flux, it is not fortuitous that the time profiles should be reasonably congruent. As noted previously, the ionization and its variation at tropospheric heights is dominated by the particle fluxes in the $100-1000$ $\mathrm{MeV}$ energy range. It is concluded that the GOES and NOAA/TIROS instruments, particularly with the inclusion of the HEPAD, for the first time provides an appropriate record of galactic and 
solar energetic particle fluxes and their variation to more critically examine the variation of ionization of the lower atmosphere, and its relationship to the chemical, electrical and meteorological dynamics of Earth's atmosphere.

\section{REFERENCES}

1. Ney, E.P., "Cosmic Radiation and the Weather," Nature, 183, pp. 451-452, 1959.

2. Herman, J.R. and R.A. Goldberg, "Sun, Weather and Climate," NASA Spec. Pub. SP426, 1978.

3. Eddy, J.A., "The Maunder Minimum," Science, 192, pp. 1189, 1976.

4. Labitzke, K., "Sunspots the QBO, and the Stratospheric Temperature in the North Pole Region," Geophys. Res. Ltrs., 14, pp. 535-537, 1987.

5. Labitzke, K. and H. van Loon, "Associations Between the 11-Year Solar Cycle, the QBO and the Atmosphere," I. The Troposphere and the Stratosphere of the Northern Hemisphere in Winter, J. Atmos. Terr. Phys., 50, pp. 197-206.

6. Tinsley, B.A., G.M. Brown, and P.H. Scherer, "Solar Variability Influences on Weather and Climate: Possible Connections Through Cosmic Ray Fluxes and Storm Intensification," J. Geophys. Res. 94, pp. 14,783-14,786, 1989.

7. Seale, R.A., and R.H. Bushnell, The TIROS-N/NOAA A-J Space Environment Subsystem, NOAA Tech. Mem., ERL SEL-75, April 1987.

8. Armstrong, T.A., C. Brungardt, and J.E. Meyer, "Satellite Observations of Interplanetary and Polar Cap Particle Fluxes from 1963 to the Present," Weather and Climate Responses to Solar Variations, ed. B.M. McCormac, pp. 71-79, Colorado Assoc. Univ. Press, Boulder, Colo., 1983.

9. Smart, D.F., and M.A. Shea, "Solar Proton Events During the Past Three Solar Cycles," J. Spacecraft, 26 (6), pp. 403-415, 1989.

10. Ely, J.T.A., "Solar Activity and Terrestrial Weather: The Magnetic Coupling Model," NASA Conf. Publ. 2098, 1979.

11. Reported by A. Vaillant, Air France, Charles deGaulle Airport, Paris, France, October 1989.

12. Reported by Capt. Peter Horton, Concorde Flight Technical Manager, London Heathrow Airport, Hounslow, England, October 1989. 


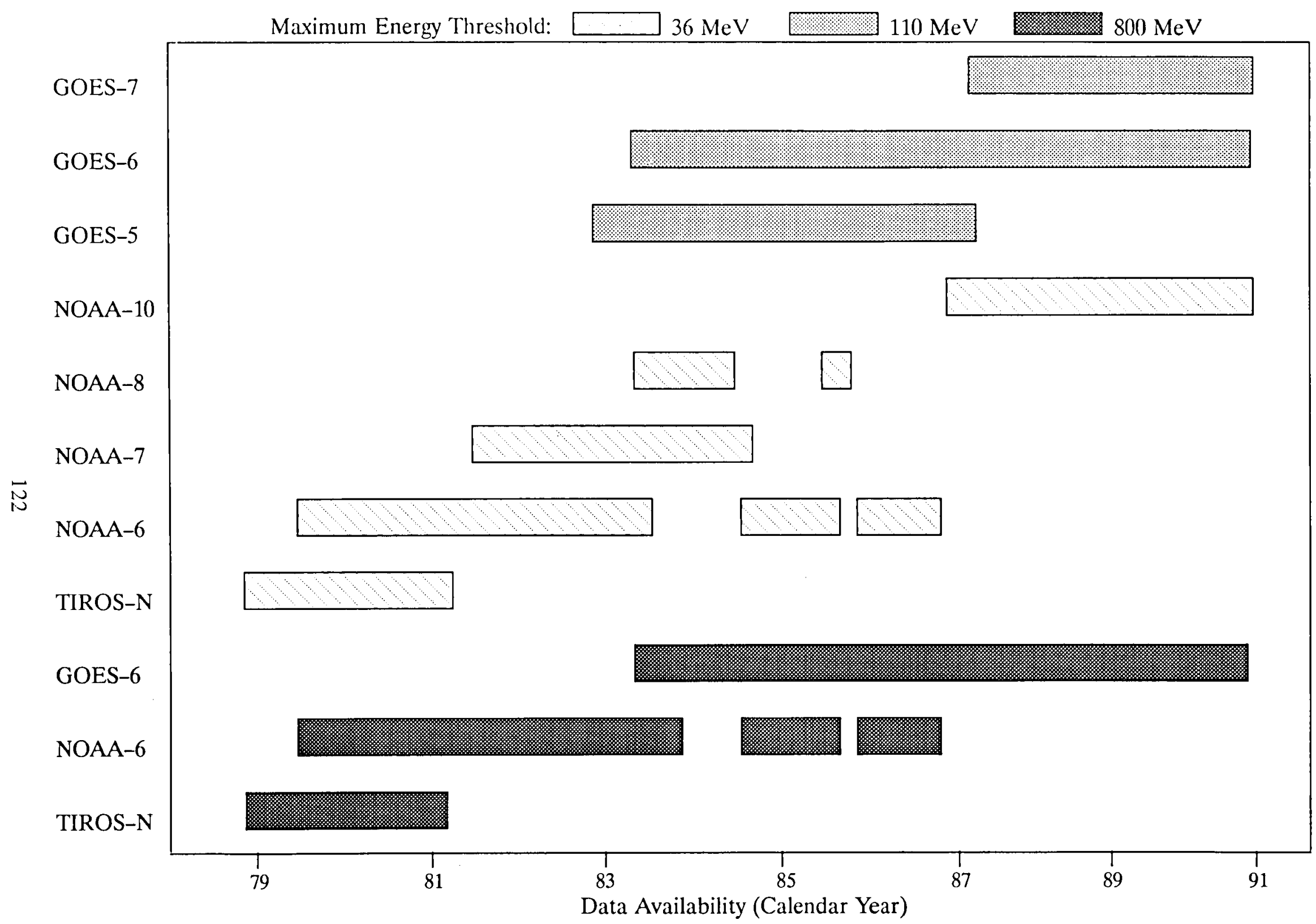

Figure 1. Bar graph of principal proton data availability from the detectors aboard the GOES and NOAA/TIROS satellites since October 1978, when HEPAD data collection began. 

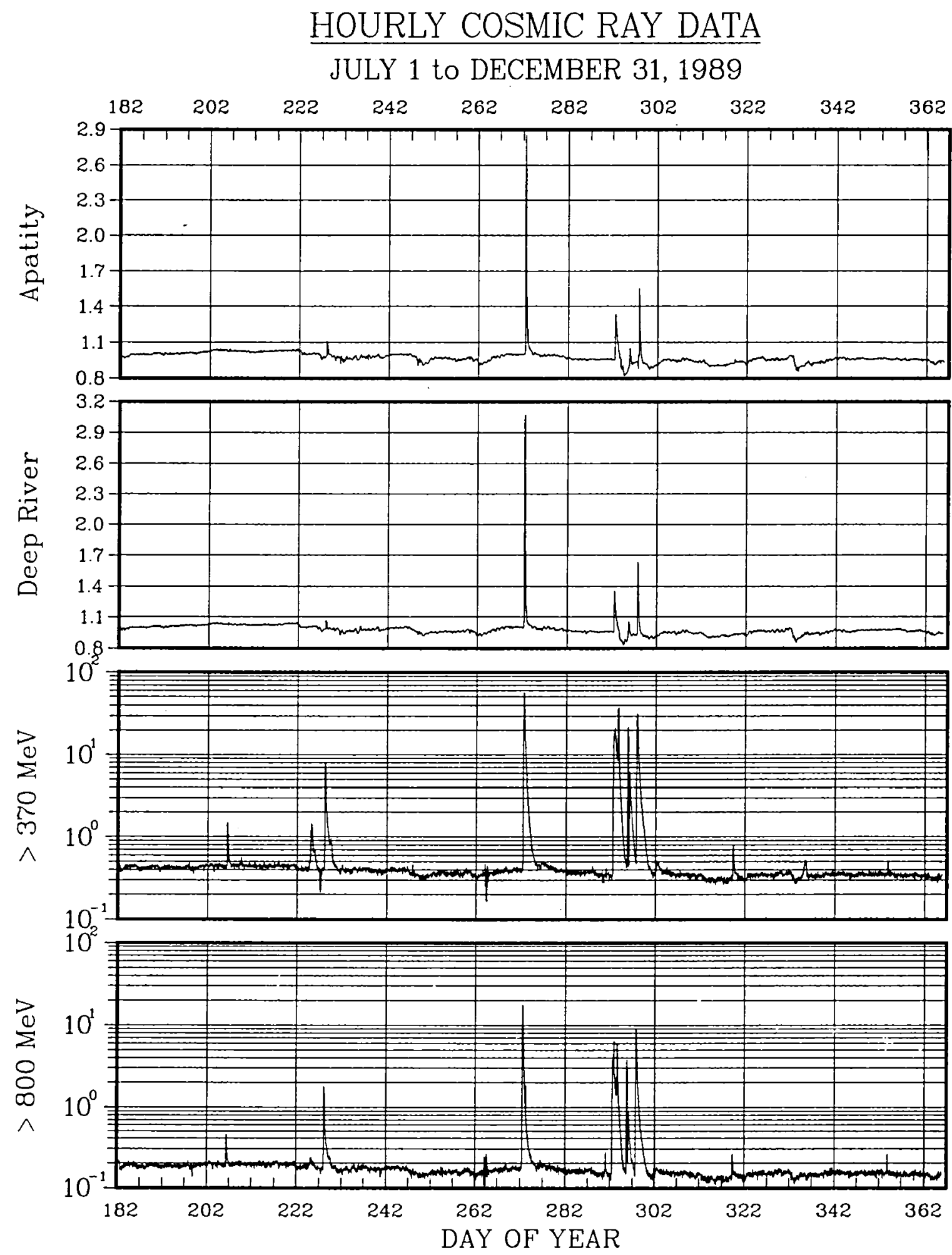

Figure 2. Hourly normalized data averages of the Deep River and Apatity Neutron Monitors with the corresponding hourly averages of the $>370 \mathrm{MeV}$ and $>800 \mathrm{MeV}$ integral proton fuxes (protons $/ \mathrm{cm}^{2} \mathrm{~s}$ sr) measured by the GOES-6 HEPAD for the period July 1 to December 31, 1989. 


\section{MINUTE COSMIC RAY DATA}

\section{SEPTEMBER 29 and 30, 1989}
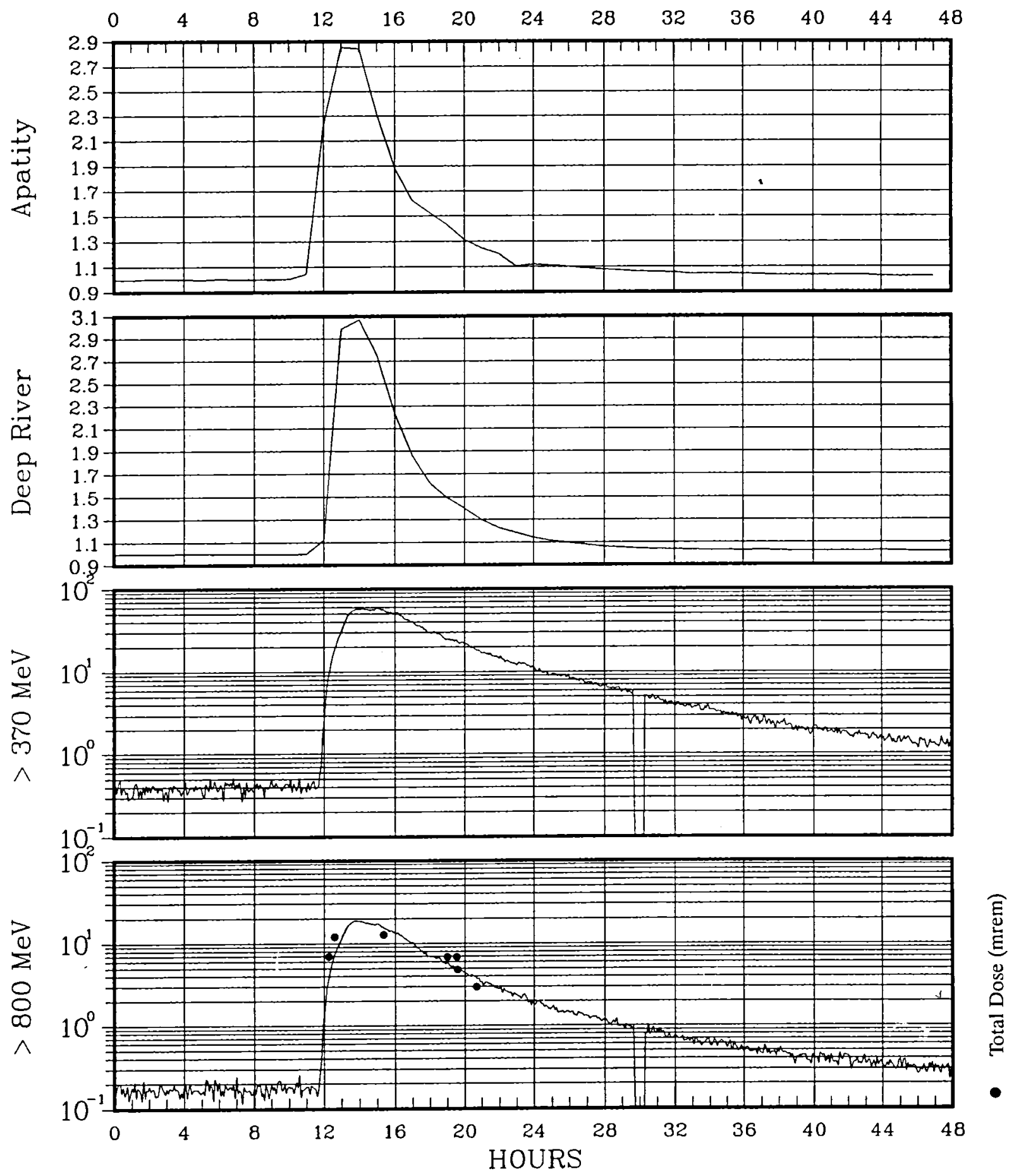

Figure 3. Hourly normalized data averages of the Deep River and Apatity Neutron Monitors with the corresponding 5-minute averages of the $>370 \mathrm{MeV}$ and $>800 \mathrm{MeV}$ integral proton fluxes (protons $/ \mathrm{cm}^{2} \mathrm{~s}$ sr) measured by the GOES-6 HEPAD for the solar cosmic ray event of September 29 and 30, 1989. The heavy dots in the figure indicate the total dose (mrem) measured by the Concorde SST's flights on September 29 (see text). 


\author{
S.D. Bouwer, J. Pap, R.F. Donnelly \\ CIRES, University of Colorado / NOAA, Space Environment Laboratory \\ Mail code R/E/SE, 325 Broadway, Boulder CO 80303, USA
}

\begin{abstract}
An important aspect in the power spectral analysis of solar variability is the quasistationary and quasiperiodic nature of solar periodicities. In other words, the frequency, phase, and amplitude of solar periodicities vary on time scales ranging from active region lifetimes to solar cycle time scales. In this study we employ a dynamic, or running, power spectral density analysis to determine many periodicities and their time-varying nature in the projected area of active sunspot groups $\left(S_{a c t}\right)$, the SMM/ACRIM total solar irradiance $(S)$, the Nimbus-7 MgII center-to-wing ratio $\left(R\left(\mathrm{MgII}_{\mathrm{c} / \mathrm{w}}\right)\right)$, the Ottawa $10.7 \mathrm{~cm}$ flux $\left(F_{10.7}\right)$, and the GOES background X-ray flux $\left(X_{b}\right)$ for the maximum, descending, and minimum portions of solar cycle 21 (i.e., 1980-1986). This technique dramatically illustrates several previously unrecognized periodicities. For example, a relatively stable period at about 51 days has been found in those indices which are related to emerging magnetic fields. The majority of solar periodicities, particularly around 27,150 and 300 days, are quasiperiodic because they vary in amplitude and frequency throughout the solar cycle. Finally, it is shown that there are clear differences between the power spectral densities of solar measurements from photospheric, chromospheric, and coronal sources.
\end{abstract}

\title{
INTRODUCTION
}

A frequently overlooked aspect in the time series analysis of solar data is that the data are not stationary. A process is generally considered nonstationary if the mean and autocorrelation suddenly changes over time. Bouwer ${ }^{1}$ has shown that nonstationarity is particularly true of coronal measurements. As active regions emerge and decay, they generally persist with strong emissions for as long as eight solar rotations. Donnelly et al. ${ }^{2,3}$ have shown that chromospheric emissions persist longer than coronal emissions. The formation of active regions may occur at different solar latitudes and longitudes which affect both the observed phases and rotation rates. At best, solar time series can be considered quasistationary; i.e., the mean and autocorrelation are either slowly varying, or suddenly changing at the beginning of a new episode of major active region development and remaining relatively constant for the lifetime of the active region complex. The net result in solar time series measurements of these non-random, sudden changes in the frequency and amplitude of solar periodicities is a quasiperiodic time series, i.e., apparent periodicities whose amplitudes come and go over active region lifetimes, and which are modulated in frequency.

The emphasis here in the power spectral analysis is on the quasiperiodic nature of solar variations; consequently we employ a rumning power spectral density analysis discussed by Bath ${ }^{4}$, referred to here simply as a dynamic power spectra. By moving a power spectral density transform through the autocorrelation of the time series data, we illustrate the time-varying characteristics of photospheric, chromospheric, and coronal periodicities on short and intermediate time scales from about twelve days to about a year. 
In an analysis technique introduced by Lean and Repoff ${ }^{5}$, the basic power spectrum employed here is done without interpolating the original data. Briefly, the power spectrum is calculated by first detrending the time series using an $\mathrm{nt}^{\text {th }}$ - order polynomial to remove the solar cycle. Next the autocorrelation is calculated, tapering the tail of the autocorrelated clata to remove spurious sidelobes. The tapering is done in this analysis using a Tukey-Hanning window (a cosine weighing function) starting at lag 205 days. The power spectral density is then calculated by performing a fast Fourier transform on the autocorrelated data. In the figures that follow, the power spectrum is reported as the percent of total power at each frequency.

A dynamic power spectra of solar time series data is calculated here by computing the power spectral density for a relatively short time window (e.g., 1024 days), then successively stepping the window through time (e.g., 27 days) on the autocorrelated data. By then contouring the power found as a function of frequency and time using a triangulation algorithm, an overall picture of how the power spectral density changes over time can be clearly visualized. Because this form of spectral analysis is exploratory, i.e., looking for overall patterns in solar variability, estimates of statistical significance are not calculated. Test cases of known spectral characteristics were used to confirm the overall correctness of the analysis procedures, determine noise estimates, and to find the approximate temporal resolution of the running transform. The running transform is capable of detecting a change in frequency or amplitude within a lag of about 250 days, depending on the relative amplitude of the cliange. Since an $4^{\text {th }}$ - order polynomial is used to detrend the data for the solar cycle variations and because of the relatively short time windows used in the Fourier transforms, periods in excess of 256 days are not well determined. Furthermore, because the frequency resolution of a Fourier transform is determined by the length of the time series, the frequency resolution at short-term periods (i.e., 16 to 48 days) corresponds to slightly less than a one day difference in period.

Photospheric solar variability is represented in this study with both $S$ and $S_{a c t}$, although Smith and Gottlieb ${ }^{6}$ have shown that roughly one-third the total solar irradiance variation comes from UV sources primarily chromospheric in origin, and the majority of the remainder from photospheric sources. Projected areas of active sunspot groups, which are defined by $\mathrm{Pap}^{7}$ as being associated with emerging magnetic fields and are classified as newly formed and developing sunspot groups with gamma or delta magnetic configurations, are considered a better photospheric index than $S$ in this study.

Chromospheric solar variability is represented here using $R\left(\mathrm{MgII}_{\mathrm{c} / \mathrm{w}}\right)$. Based on Nimbus-7 satellite UV measurements, the $R\left(\mathrm{MgII}_{\mathrm{c} / \mathrm{w}}\right)$ index was developed by Heath and Schlesinger ${ }^{8}$ as a relative photometric measure, and as a result it is relatively insensitive to long-term instrumental degradation problems. Tobiska and Bouwer ${ }^{9}$ demonstrate that $F_{10.7}$ shows both chromospheric and coronal components in its total flux. Wagner ${ }^{10}$ presents $X_{b}$ flux that represents coronal sources above $3 \times 10^{6}{ }^{\circ} \mathrm{K}$, where the sources come from the closed magnetic flux loops associated with active regions. All data reported here are from a terrestrial viewpoint (resulting in synodic rotation rates) at one astronomical unit ( $\mathrm{AU})$.

\section{RESULTS}

In Figure 1 we show the five measurements representing: (1) The hot corona using a background $\mathrm{X}$-ray index (i.e., effects of flares are reduced). (2) A combination of the chromosphere, transition region, and corona using the Ottawa $10.7 \mathrm{~cm}$ flux $F_{10.7},(3)$ Chromospheric flux using the Nimbus- 
$7 \mathrm{UV}$ index $R\left(\mathrm{MgII}_{\mathrm{c} / \mathrm{w}}\right)$, (4) Mostly photospheric irradiance using SMM/ACRIM total irradiance $S$. and (5) Emerging magnetic flux using the projected area of active sunspots $S_{\text {act }}$.

Also shown in the Figure 1 time series as a dashed line is the $4^{\text {th }}$-order polynomial used to remove the long-term solar cycle variation before calculating the power spectrum. To a first-order approximation, the five time series show similar solar cycle and intermediate-term characteristics. Most important are the intermediate-term variations; all the time series show major episodes of active region evolution lasting about 6-12 solar rotations that have nearly concurrent local minima, particularly during 1981 to 1984 . However, the relative persistence of active regions and solar rotational effects differ significantly between the time series, and since the amplitude of these variations are generally about as large as the intermediate variations, a new analysis that accounts for the quasiperiodic nature of the time series is needed to meaningfully distinguish the differences.
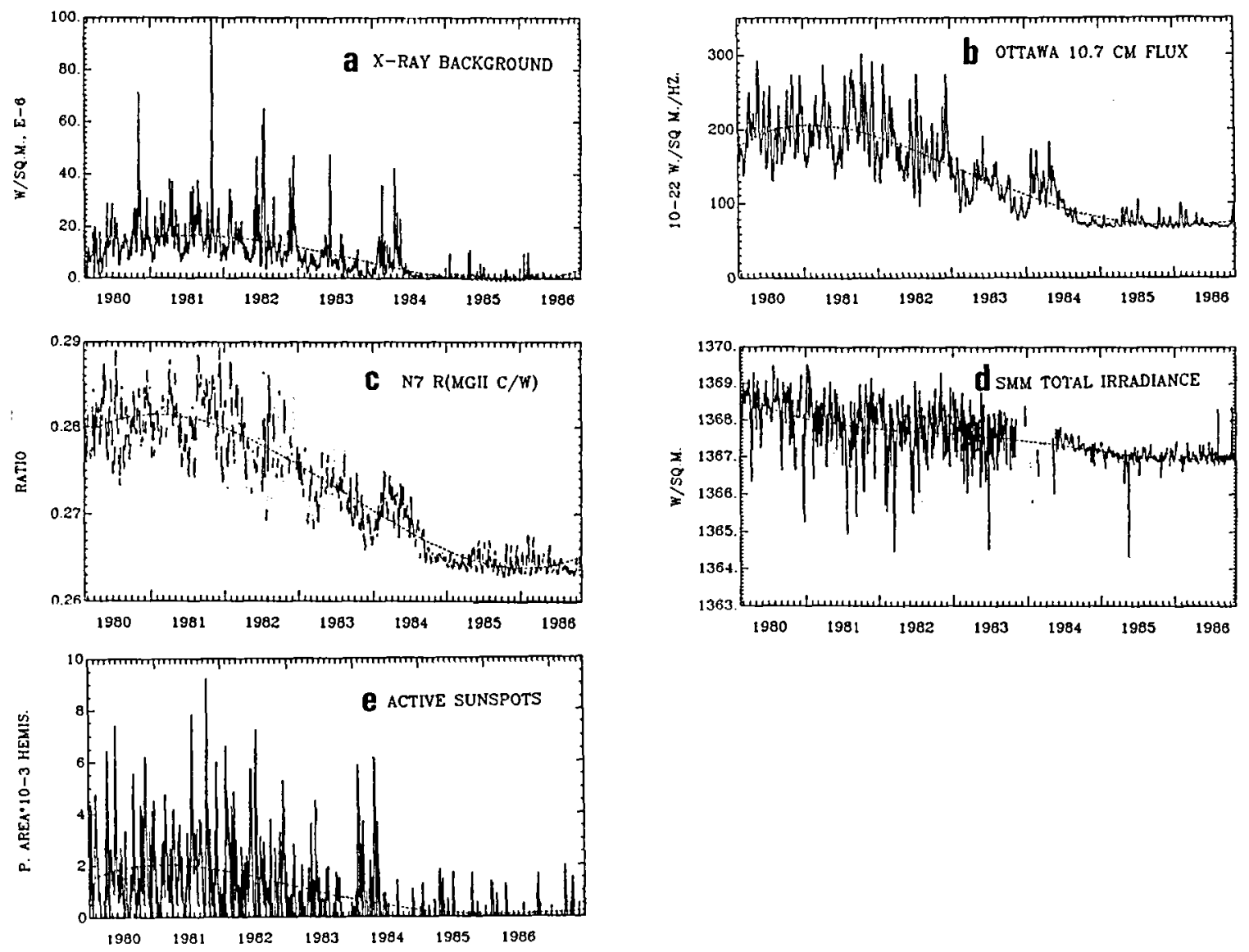

Figure 1. Time series from: (a) GOES Background flux $X_{b}$, representing coronal sources, (b) Ottawa $10.7 \mathrm{~cm}$ flux $F_{10.7}$ representing both chromospheric and coronal sources (c) Nimbus- $7 R\left(\mathrm{MgII}_{c / \mathrm{w}}\right)$ representing chromospheric sources, (d) SMM Total solar irradiance $S$ representing mostly photospheric sources, and (e) Projected area of active sunspots $S a$, representing emerging magnetic fields. All time series are from a terrestrial viewpoint at 1 $\mathrm{AU}$. Also shown as a dashed line is the $4^{\text {th }}$ - order polynomial used to detrend the time series in the subsequent spectral analysis.

The dynamic power spectra calculated from the time series in Figure 1 are shown in Figure 2 as contour diagrams. As the transform is stepped through the autocorrelated data, the date at the center of the transform is calculated and displayed on the left axis. The ordinate is shortened to the length of the time series in which the running transform can detect a change. The synodic 
period in days is shown on the bottom axis, and the equivalent frequency in microhertz is shown on the top axis. Fach horizontal slice represents a simple power spectrum of power as a funclion of frequency. In effect, each plot of power as a function of time and frequency is visually much like the elevation data of a mountainous contour map. The highest "peaks" of the mountains in Figure 2 have been truncated by about $0.5 \%$ (except $\mathrm{S}$, which was truncated $0.3 \%$ from the maximum) below the maximum to make the figure more readable by highlighting the peaks. It is important to note that much of the fine details in the contour diagrams are due to the combined effects of "noisy" data and the limitations of the power spectral analysis and contouring algorithm. Nevertheless, the overall patterns are accurate and are the important results of this study.
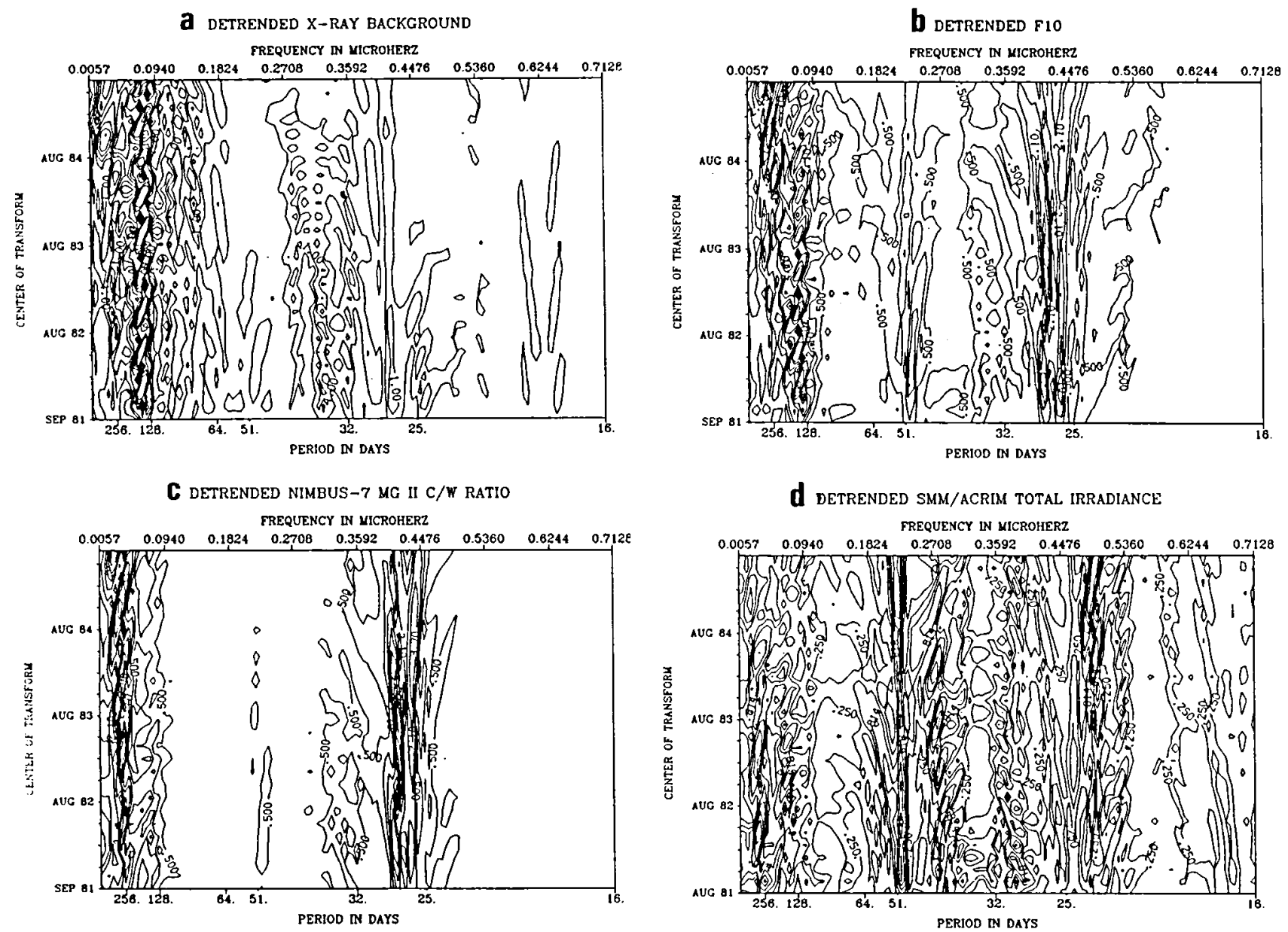

Figure 2. Dynamic power spectra of: (a) Background X-ray $X_{b}$, (b) Ottawa $10.7 \mathrm{~cm}$ flux $F_{10.7}$, (c) Nimbus-7 $R\left(\mathrm{MgII}_{\mathrm{c} / \mathrm{w}}\right)$, and the SMM total solar irradiance $S$. The ordinate shows the center date of the running time window, which can detect a change in the time series to within about a 250-day lag. Power is indicated as the percent of total power, and spectral noise is estimated to be $0.25 \%$ of total power. For (a), the contour lines start at 0.50 and proceed to a 4.1 maximum, in 0.60 intervals. For (b), the contour lines start at 0.50 and proceed to a 6.5 maximum in 1.0 intervals. For (c) the contour lines start at 0.50 and proceed to a 5.3 maximum in 0.8 intervals. Finally, for (d) the contours start at 0.25 and proceed to 1.66 in 0.28 intervals. To increase readability by highlighting peaks, power above the maximum minus 0.5 was truncated, except for (d) in which 0.3 was used to truncate the higher peaks. The time window step for (a) was 28 days, for (b) and (c) 27 days, and for (d) 25 days.

An important consideration in dynamic power spectra is how to determine the step to move the window through the time series. For the autocorrelation algorithm used in this study, which was 
selected for its ability to ignore missing data, moving the running window at a time step other than the period of interest (or it's harmonics and subharmonics) will cause the power spectral rensity to show a periodicity of varying frequency. 'This effect is due to the loss of phase information in the autocorrelation algorithm employed here. An example of this can be seen in $X_{b}$ of Figure 2, in which the 153-day period shows "islands" of power with a slope from the vertical. If the rumning window is stepped at 153 days, the contour diagram will show a single vertical island of power in the contour diagram. To reduce this effect, the running window was stepped in time at the number of days most near the rotational period that produced the maximum frequency stability. Hence the step sizes range from 25-28 days. However, there are a number of examples (some not shown here) in which no window step choice will straighten out a particular periodicity. In Figure 2, the weak periods between 16 to 18 days in $S$ are such a case: the slope changes too much to be due to the spectral analysis. Other examples can be found near 27 days in $R\left(\mathrm{MgII}_{\mathrm{c} / \mathrm{w}}\right)$ and $F_{10.7}$, in which there appears to be a gradual frequency modulation at sidebands of the 27-day periods. To better determine the frequency stability, an alternative technique should be employed.

A curious result of changing the step size of the running window to achieve maximum frequency stability is the resultant variation in the window step as a function of solar height. If we assume that the apparent synodic rotation rate corresponds to the running window step of maximum frequency stability, then the magnetic and photospheric sources $S_{a c t}$ and $S$ rotate at 25 days, the chromospheric $R\left(\mathrm{MgII}_{\mathrm{c} / \mathrm{w}}\right)$ and $F_{10.7}$ at 27 days, and the coronal $X_{b}$ at 28 days. This result is consistent with numerous earlier results (see for example El-Raey and Scherrer ${ }^{11}$, Gilman ${ }^{12}$, and Howard $\left.{ }^{13}\right)$.

The contour diagrams of the dynamic power spectra in Figure 2 demonstrate the quasiperiodic nature of solar variability. This is seen by observing that in Figure 2 many periodicities have amplitudes that are present for only a portion of the time series. For example, the roughly 300 day period in $S$ almost completely disappears near solar minimum, and the 51-day period in $X_{b}$, $F_{10.7}$, and $R\left(\mathrm{MgII}_{\mathrm{c} / \mathrm{w}}\right)$ is only present during solar maximum. Close inspection will show other instances. In a separate study by Pap et al. ${ }^{14}$, it is also shown that there are dramatic differences in the power spectra of all solar indices determined during the ascending and descending portions of the solar cycle.

An interesting feature in Figure 2 is the wide range of periodicities in $X_{b}$ from 25 to about 36 days, and to a lesser extent the 16 to 25-day periods. Because of the square waveform shape of an $\mathrm{X}$-ray source caused by the rotation of the major sources across the visible disk in an optically thin solar atmosphere (see for example, Donnelly ${ }^{15}$ ), a broad fundamental frequency and many of its harmonics and subharmonics will appear in the power spectra. This effect of a non-sinusoidal waveform shape due to the rotation of a source, terrestrial viewing angle, and limb darkening effects can also clearly be seen in $S$ and $S_{\text {act }}$, and to a lesser extent $F_{10.7}$. Since the rotational amplitude in $R\left(\mathrm{MgII}_{\mathrm{c} / \mathrm{w}}\right)$ in more sinusoidal in its waveform sliape due to the center-to-limb variation, the odd harmonics of the 27 -day period are not visible in Figure 2 . A second effect that may cause a broad range of periodicities around 36 days may be due to some as yet undetermined physical cause.

Another interesting feature in Figure 2 is the 150 to 155 -day period that is dominant in $X_{b}$ for the duration of the time series. This period is also strong in $F_{10.7}$ during the descending portion of the cycle, but is replaced by a 300-day period during solar minimum. Note that the 150 to 
155-day period is only weakly present in $S$ during solar maximum and nearly non-existent in $R\left(\mathrm{MgII}_{\mathrm{c} / \mathrm{w}}\right)$. In contrast, consider the relatively simple dynamic spectra in $R\left(\mathrm{MgII}_{\mathrm{c} / \mathrm{w}}\right):$ only the 27-day and 300 to 310 -day periods dominate the contour diagram. Also note how $F_{10.7}$ show characteristics of both $X_{b}$ and $R\left(\mathrm{MgII}_{\mathrm{c} / \mathrm{w}}\right)$.

$S$ produces ill-resolved power mostly around a 300 -day period, with a broad range of relatively weak power from about 36 to 64 days, and from about 80 to 150 days. There are a number of stable periods very near 51 days, and to a lesser extent near 25 days. Actually, the power seems to appear in $S$ at narrow sidebands of the 51 and 25-day periods, and the nearly vertical lines in the contour plots suggest constant frequency stability. Note that since power is calculated in Figure 2 as the percent of total power at a particular frequency, the result is that power near 51 and 25 days is enhanced with respect to the intermediate-term variations during solar minimum. Also recall sunspot blocking effects and faculae are competing effects on $S$, resulting in complicated power at sidebands of the 27-days rotational period, as discussed by Foukal and Lean ${ }^{16}$. The somewhat random longitudinal appearance of sunspot blocking effects and lack of persistence of these effects (typically less than two solar rotations), could also produce unusual periodicities in $S$.

\section{DETRENDED ACTIVE SUNSPOT GROUPS, PROJECTED AREAS}

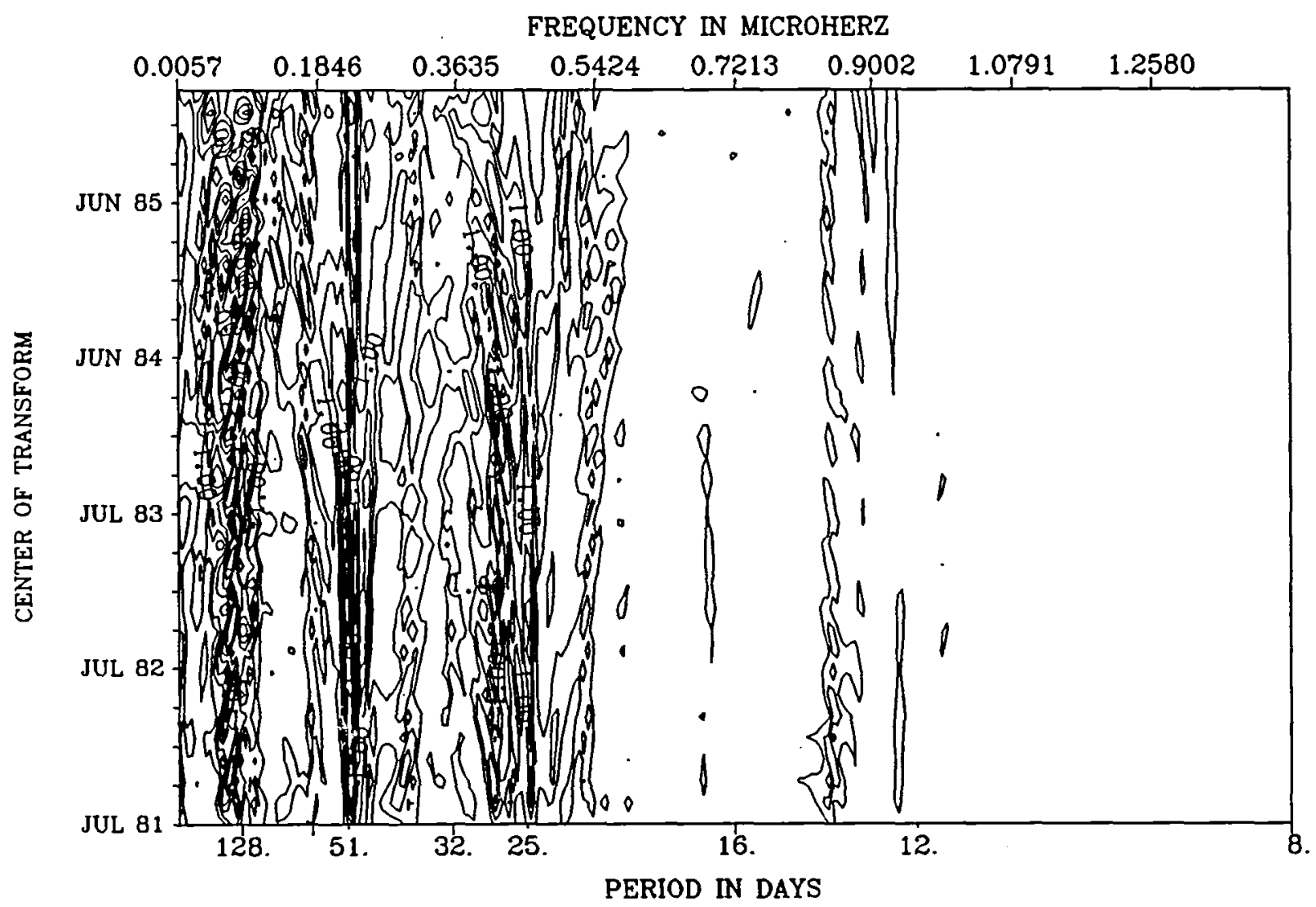

Figure 3. Enlarged dynamic power spectra of active sunspots $S a$ using a running 1024-day window, stepped by every 25 days, and enlarging the bottom scale to more clearly show shorter periods. Contours start at 0.50 and proceed to a maximum of 3.00 in 0.50 intervals, and power above 0.5 of the maximum was truncated. 
In order to more closely examine the source of the 51 -day period and it's harmonics, $S_{a c t}$ is shown in Figure 3 for periods down to 8 days, and for a slightly longer time series than indicated in Figure 1. By using an active sunspot index, we are in effect looking down the magnetic field lines of emerging magnetic flux to deep sub-photospheric layers. Clearly, the 51-day period persists with the most power and stability for the duration of the time series. The 25-clay period is strong, but weakens in power during solar minimum when only a few sunspots are formed. The 12.5-day period is present, but with weak amplitude. The unstable periodicities around 150 days slowly show more power near 300 days during solar minimum. Note the periods near 13.7 days: these are not harmonics of the 27-day period, but represent the fact that active regions tend to be distributed about 180 degrees in solar longitude, as suggested by Donnelly et al.. ${ }^{17}$ It is important to note that a similar analysis of the projected area of passive sunspot groups (not shown here) yields a distinctly different dynamic power spectra: The majority of power can be found in passive sunspot periodicities around 27,32-36, and 150-300 days. Clearly $S_{a c t}$ and $S$ demonstrate that emerging magnetic fields dominate photospheric time series measurements, and that a 51-day period of stable frequency is the fundamental period.

\section{CONCLUSIONS}

Power spectral density as a function of the solar cycle phase shows major differences between the solar source regions described here as photospheric, chromospheric, and coronal. There is compelling evidence to suggest the quasiperiodic nature of solar periodicities at solar rotation and active region evolution time scales. Remarkable is the stability in frequency of periodicities related to new emerging magnetic structures.

The period found in the data analyzed in this study that is most stationary in frequency and amplitude is the 51-day period in $S_{a c t}$ and $S$. This is a most unexpected and unexplained result: When one considers that at any particular time some number of sunspots distributed in solar longitude and at various stages of evolution all exhibit roughly the same characteristics of amplitude and frequency, then one is inclined to at least suggest that some process is affecting all new sunspots in exactly the same fashion. Since the 51-day period has more power in $S$ and $S_{\text {act }}$ than short or intermediate periodicities, and because the 51-day period is sometimes present when 150-300 day periods are not, we know that it is not simply a sub-harmonic of solar rotation or a harmonic of intermediate-term periodicities. Part of the reason the 51-day period is so well resolved is because it lies in a portion of the frequency grid that is well isolated from the interfering effects of the adjacent 27-day and 150-day periods. If the 51-day period were intermittently present in the time series, then it's relative power would be severely diminished and it's spectral peak very broad in frequency, and this is not the case in the measurements except during solar minimum. While a number of authors have reported the 51-day period before, Bai ${ }^{18}$ empliasized its importance in major flare occurrences, which are related to emerging magnetic structures.

The chromospheric observations seem to be dominated by an undetermined convective process on the order of 300 days modulating UV flux. The high-temperature portions of the corona are dominated by an approximately 150-day period, and emerging magnetic fields show equally strong periods at 51 and 150 days. Lean and Brueckner ${ }^{19}$ discuss the characteristics of magnetic flux with associated with the 155-day periods, observing the period in sunspot blocking but not seeing it in plages. The dynamic spectral analysis in this study with respect to the 51-day period suggests that emerging magnetic flux has a major effect on photospheric flux, barely effects chromospheric 
flux, and has a moderate effect on coronal flux at high temperatures (e.g., $3 \times 10^{6}{ }^{\circ} \mathrm{K}$ ).

Finally, the effectiveness of dynamic spectral analysis in describing solar variability sheds a new light on more traditional periodicity analysis techniques. The sun is far too complex of an oscillator to attempt to characterize it's variability with a single index or analysis technique. The fundamental assumption that needs to be challenged in many forms of statistical analysis of solar variability is the stationary presupposition: the mean, standard deviation, and autocorrelation of solar data often are not constant in time, and this may effect the interpretation of results derived from linear statistical techniques that assume stationarity.

\section{ACKNOWLEDGEMENTS}

We express our gratitude to Dr. R. C. Willson for providing the SMM/ACRIM data, Dr. D. Heath for providing and Nimbus-7 SBUV data, and Dr. W. Wagner for providing the GOES $\mathrm{X}$-ray data. This research was supported by a grant of the NOAA/Sun-Climate Staff.

\section{REFERENCES}

1. Rouwer, S.D., "Intermediate-term epochs in solar soft X-ray emission", J. Geophys. Res., 88, 7823-7830, 1983.

2. Donnelly, R. F., Heath, D. F., Lean, J. L., "Active-region evolution and solar-rotation variations in solar UV irradiance, total solar irradiance, and soft X rays", J. Geophys. Res., 82, 10,318-10,324, 1982.

3. Donnelly, R. F., "Temporal trends of solar EUV and UV full-disk fluxes", Solar Phys., 109, 37-58, 1987.

4. Bath, M., Spectral Analysis in Geophysics, p.125, Elsevier Sci., 1974.

5. Lean, J.L., and Repoff, T.P., "A statistical analysis of solar flux variations over time scales of solar rotation: 1978-1982", J. Geophys. Res., 92, 5555-5563, 1987.

6. Smith, E.V.P., Gottlieb, D.M., "Solar flux and its variations", Space Science Reviews., 16, 771-802, 1974.

7. Pap, J., "Variation of the solar constant during the solar cycle", Astrophys. Space Sci. $127,55,1986$.

8. Heath, D.F., Schlesinger, B.M., "The Mg 280-nm doublet as a monitor of changes in solar ultraviolet irradiance", J. Geophys. Res., 91, 8672-8682, 1986.

9. Tobiska,W. K., Bouwer, S. D., "Intermediate-term variations of chromospheric and coronal solar flux during high solar cycle 21 activity", Geophys. Res. Lett., 8 , 779-782, 1989.

10. Wagner, W.J., "Observations of 1-8 $\AA$ solar X-ray variability during solar cycle 21", Paper 12.2.2, Helsinki COSPAR meeting, July 1988.

11. El-Raey, M., Scherrer, P.H., "Differential Rotation in the solar atmosphere inferred from optical, radio, and interplanetary data", Solar Phys., 26, 15-20, 1972

12. Gilman, P.A., "Solar rotation", Annual Review of Astro. \& Astro., 12 , 47-70, 1974.

13. Howard, R., "The rotation of the sun", Rev. Geophys.\& Space Phy., 16, 4, 721-732, 1978.

14. Pap, J, Tobiska, W.K., Bouwer, S.D., "Periodicities of solar irradiance and solar activity indices I", accepted by Solar Physics, Apr. 1990.

15. Donnelly, R.F., "Comparison of nonflare solar soft X ray flux with $10.7 \mathrm{~cm}$ flux", J. Geophys. Res., 87, 6331-6334, 1982.

16. Foukal, P., Lean, J., "The influence of faculae on total solar irradiance and luminosity", Astrophys. J., 265, 1056, 1983.

17. Donnelly, R. F., J.W. Harvey, D.F. Heath, T.P. Repoff, "Temporal characteristics of the Solar UV Flux and He I line at 1083 nm", J. Geophys. Res., 90, 6267-6273, 1985.

18. Bai, T., "Periodicities of the flare occurance rate in solar cycle 19", Astrophys. J., 318, L85-L91, 1987. 
SESSION 5: CLIMATE CONNECTIONS I 
- 


\title{
COMPARISON OF SOLAR AND OTHER INFLUENCES ON LONG-TERM CLIMATE*
}

\author{
James E. Hansen, Andrew A. Lacis and Reto A. Ruedy \\ Goddard Institute for Space Studies, 2880 Broadway, New York, NY 10025
}

\begin{abstract}
In this paper I first show examples of climate variability and discuss unforced climate fluctuations, as evidenced in both model simulations and observations. I then compare different global climate forcings, a comparison which by itself has significant implications. Finally, I discuss a new climate simulation for the 1980s and 1990s which incorporates the principal known global climate forcings. The results indicate a likelihood of rapid global warming in the early 1990 s.
\end{abstract}

\section{INTRODUCTION}

I got a little worried yesterday afternoon when I started to think about what talk I could give today. I had brought a pile of viewgraphs from my office, thinking that I would be able to select several to make a decent talk - with the idea of comparing possible solar-forced climate change with climate change due to other forcings, and comparing all of these with unforced climate fluctuations. That may sound o.k., but when I looked at my viewgraphs, they seemed pretty dull to me.

However, after thinking about them awhile, I realized that they lead to a remarkable conclusion, one with political and social implications. And, surprisingly, the result derives, in part, from the little one-tenth of one percent change in the solar irradiance measured by Dick Willson, which is usually dismissed as of no climatic importance.

So if you stick it through, you may find the conclusion interesting. I can't guarantee that I will convince you of the conclusion. But I'm pretty sure that it is right. In fact, if you disagree with it, I would be happy to make a friendly little wager-one which much of the community apparently believes to be very improbable, so perhaps it's a good chance for you to take my money.

\section{OBSERVED CLIMATE VARIABILITY}

The first viewgraph (Fig. 1) shows temperature variations over the past 160,000 years, as inferred from isotopes in an ice core from Antarctica. The temperature thus refers to this specific region, at the level in the atmosphere where the snow formed.

A smoothed estimate of global temperature over the same period is shown in Figure 2. Global temperature fluctuates on this time scale by about $5 \mathrm{C}$

*This paper is the talk given by one of us (JEH) at the conference Climate Impact of Solar Variability, NASA Goddard Space Flight Center, Greenbelt, Maryland, April 25, 1990, with two added explanatory notes.

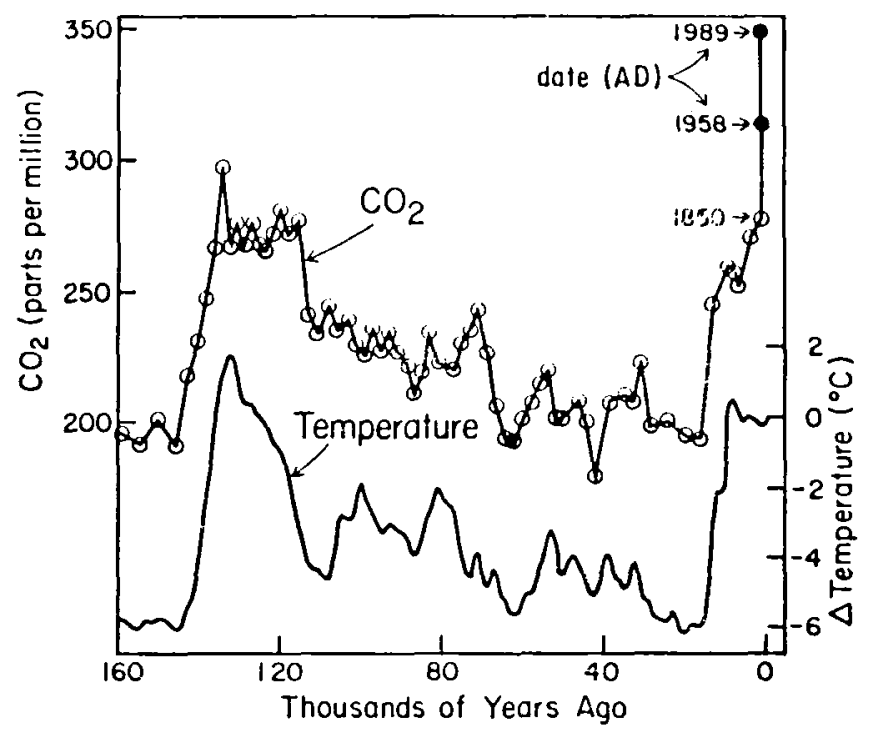

Fig. 1. Atmospheric $\mathrm{CO}_{2}$ and temperature change in the past 160,000 years as inferred from Soviet/French Vostok Antarctic ice core. Twentieth century data points are based on Keeling's measurements.

between the interglacial periods and the depths of the glacial periods. Similar oscillations of global temperature have occurred many times over the past few million years.

These glacial to interglacial climate fluctuations appear to be related to a certain type of solar variation - the seasonal and geographical redistribution of insolation caused by changes in the Earth's orbital elements (the eccentricity of the orbit, the tilt of the spin axis, and the season of periapsis). Specifically, a high correlation is found between the climate changes and the changes of the Earth orbital elements, and thus the latter are called the "pacemakers of the ice ages."

However, the orbital changes by themselves cause very little net heating or cooling averaged over the year and over the planet. The mechanisms which maintain the global temperature can be investigated by examining the glacial to interglacial change of the 


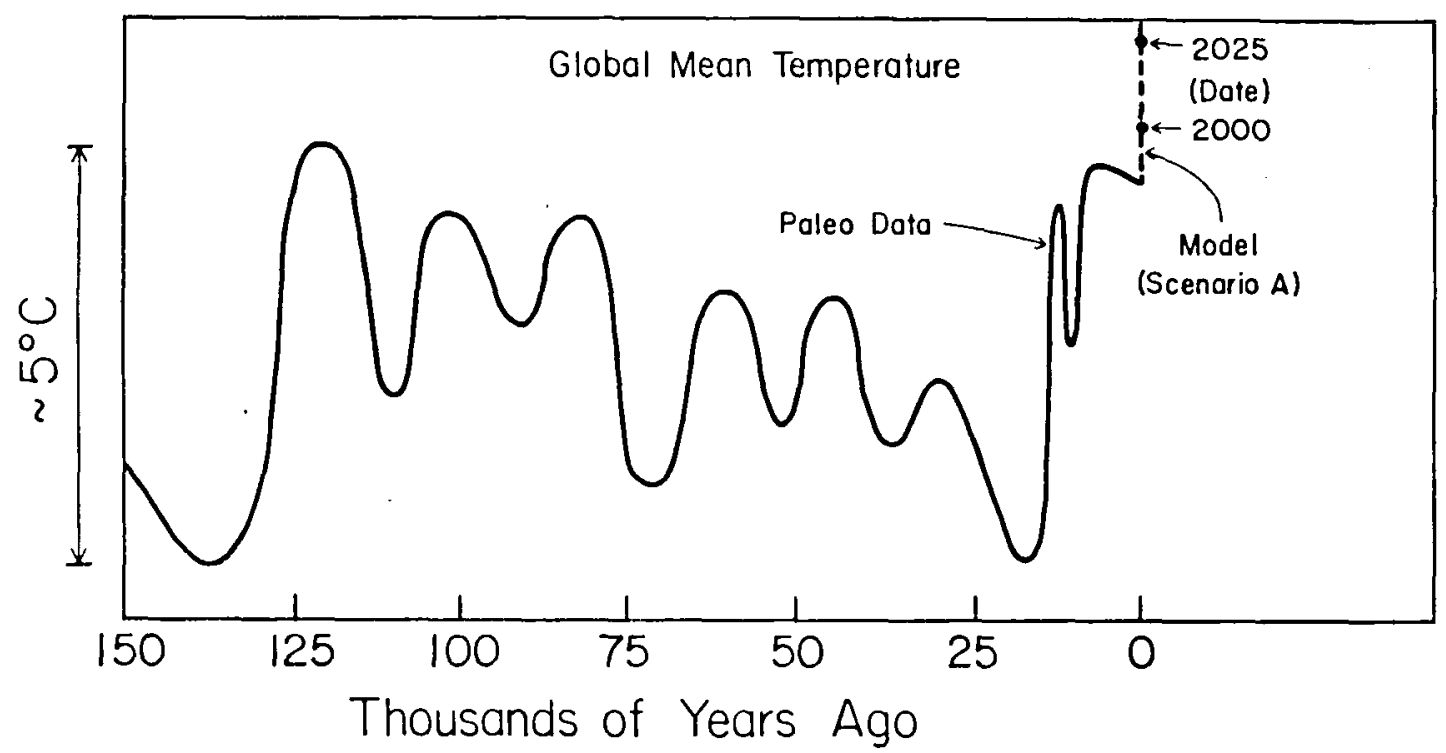

Fig. 2. Smoothed estimate of global temperature change over the past 150,000 years, and a projection assuming continued rapid growth of greenhouse gases.

planetary radiation balance. It turns out that the principal factors maintaining the ice age cold were, (1) the increased area of ice sheets and snow, which reflected sunlight to space, (2) decreased amounts of atmospheric carbon dioxide and methane, which reduced the greenhouse effect, and (3) increased amounts of aerosols and dust, which also reflected sunlight.

Most likely, these mechanisms were feedbacks, that is processes which amplified a tendency for climate change driven by the earth orbital elements or other factors. Indeed, although the fluctuations appear to be almost synchronous (Fig. 1), the carbon dioxide change usually lags slightly behind the temperature change, as expected for a feedback.

Figure 3 shows global temperature on the 100 year time scale, based on the network of meteorological stations. There are several issues about this record, especially (1) whether the station coverage is adequate to estimate global trends, and (2) whether the station records contain systematic biases, such as urban warming. The error due to incomplete spatial coverage can be estimated quite well from knowledge of spatial and temporal variability of temperature and has been shown to be reasonably small, as indicated by the error bars (Fig. 3). Urban effects on the global temperature change have been estimated in several different ways and found to be not larger than $0.1-0.2 \mathrm{C}$.

It is apparent that over the full century there is a substantial warming trend. However, within that period there are intervals of cooling, most notably the cooling trend from about 1940 to 1970 . There are also year to year fluctuations as large as a few tenths of a degree. Within the 1980s the maxima in 1983 and 1987-88 are associated with major El Niños, as evidenced by the spatial pattern of the warming.

Note that there is no significant trend of global temperature within the 1980s. Recently great publicity was given to an apparently surprising result: "satellites find no warming in the 1980s." This statement was bound to deceive the public, because of all the prior publicity about the 1980s

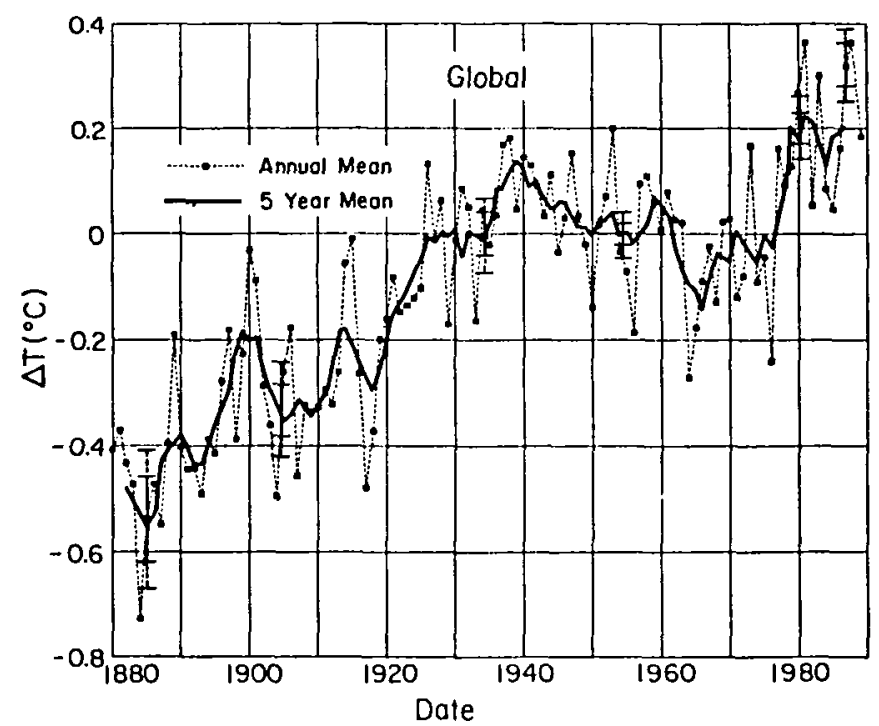

Fig. 3. Global temperature change of the past century based on measurements at meteorological stations. 


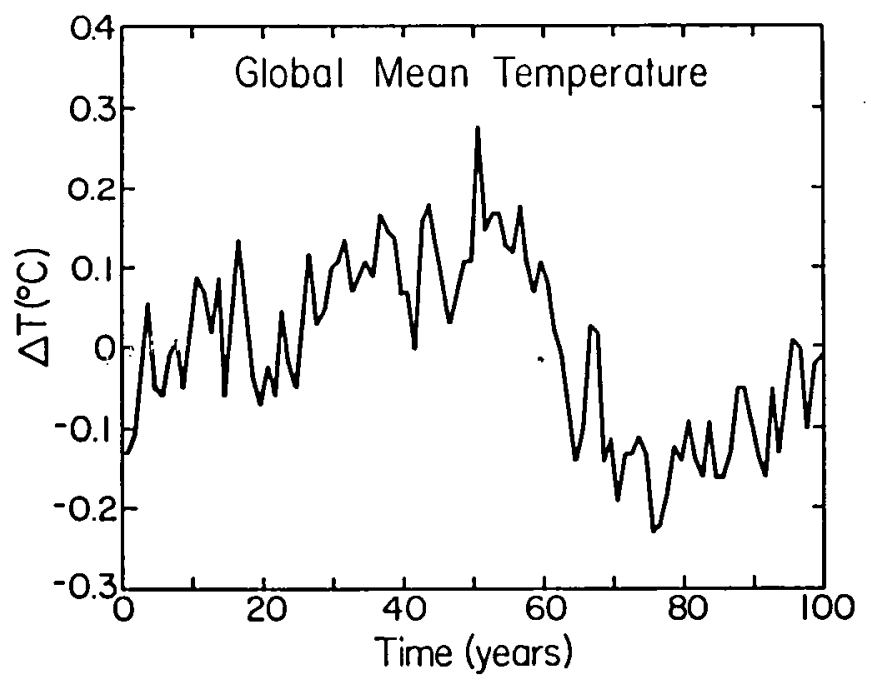

Fig. 4. Global temperature in 100 year run of a climate model with no variations of climate forcing.

being the warmest decade of the century with the several warmest years all in the 1980s. In reality, of course, there was no contradiction with the previous findings; the satellite record was simply too brief to be used for studying long term trends.

Also note that even the investigators' optimistic estimate of the satellite error as being $0.005 \mathrm{C} /$ year corresponds to $0.5 \mathrm{C} /$ century, an error which would mask the trend of the past century. This large error does not mean that the satellite data are of no value for study of long term climate change. On the contrary, an improved observing system would involve a combination of satellite data, to provide nearly global coverage, and a continuation of surface and upper air (radiosonde) measurements at meteorological stations, to provide absolute calibration and a continuation of long term records.

\section{UNFORCED CLIMATE FLUCTUATIONS}

Climate fluctuates without any change of climate forcing. The climate fluctuations arise because the coupled non-linear equations describing atmospheric structure and motion are unstable to small perturbations. In effect, the atmosphere and ocean do a lot of sloshing around. Some of the sloshing, for example, the El Niño/Southern Oscillation phenomena, may be predictable on a limited time scale, but most of it is of a chaotic nature for which long term prediction is only possible in a statistical sense.

Unforced climate variability can be studied to a degree with global climate models, even if the ocean dynamics is fixed. Figure 4 shows the global mean temperature simulated in a 100 year run of our GCM

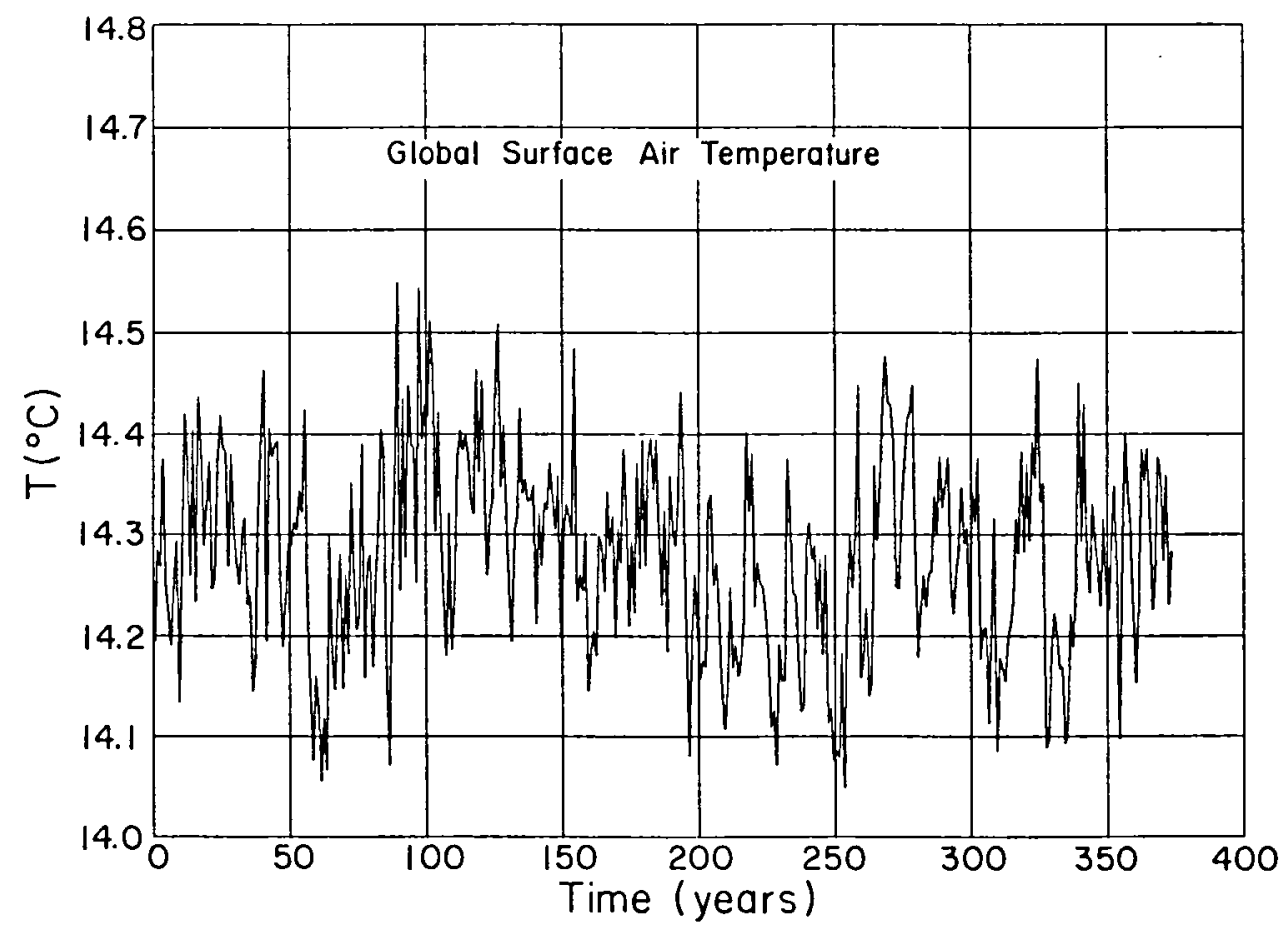

Fig. 5. Global temperature in a long run of a climate model (see Endnote 1) with no variations of climate forcing. 


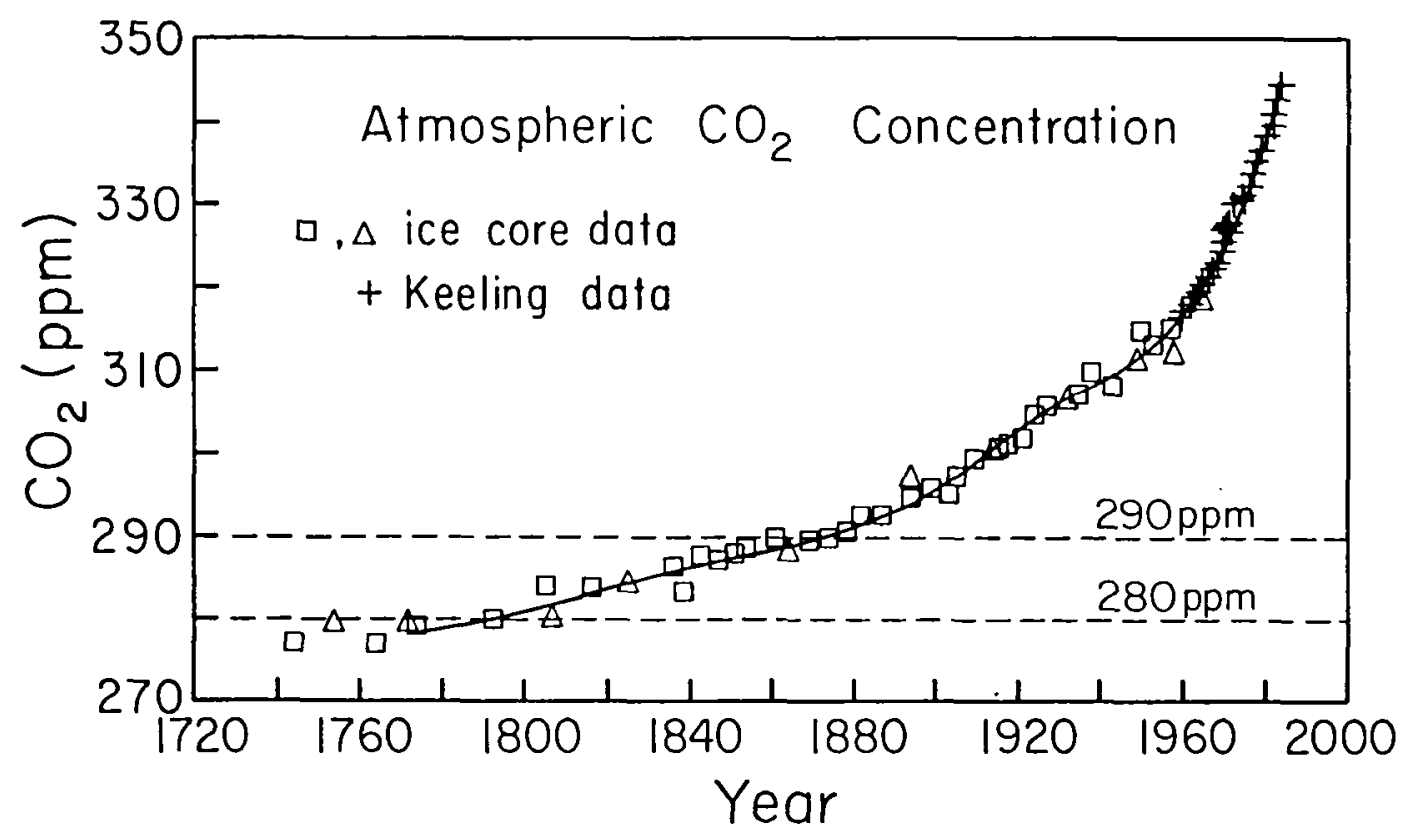

Fig. 6. Atmospheric $\mathrm{CO}_{2}$ concentration based on ice core data (triangles and squares) and data from Mauna Loa (crosses).

with the solar irradiance and atmospheric composition (except water vapor) fixed, but with ocean temperature computed. Several fast feedback processes, such as atmospheric water vapor amount, cloud cover, and sea ice distribution were free to vary, but the solar irradiance, atmospheric $\mathrm{CO}_{2}$ and other trace gases, and ice sheet area were fixed. The global mean temperature in this 100 year control run, without climate forcings, varied by as much as several tenths of a degree Celsius, with fluctuations from year to year as well as trends over periods of decades.

We have also made a longer control run, without any change of climate forcings, with a slightly modified version of our GCM (see Note 1). The global mean temperature in this longer run (Fig. 5) has year to year and decadal variations, as in the 100 year run (note that the compressed horizontal scale in Fig. 5 makes the variations appear steeper), but it also shows trends on longer time scales, such as the cooling from year 100 to year 250 .

It's instructive to consider how these unforced climate fluctuations would differ if we allowed additional feedbacks to operate, particularly feedbacks which are expected to be significant on paleoclimate time scales. Empirical data indicate that on long time scales ice sheet area tends to decrease with increasing global temperature, and the abundance of the greenhouse gases $\mathrm{CO}_{2}$ and $\mathrm{CH}_{4}$ tends to increase with increasing global temperature, so both mechanisms are probably positive feedbacks on paleoclimate time scales. Changes of vegetation cover and atmospheric aerosol amount are more variable, and do not appear to have as large a global impact. Overall, it appears that long time scale feedbacks could make unforced climate fluctuations even much larger than those in Figs. 4 and 5. It is difficult to model the variations of these factors, because we do not understand the mechanisms well enough. But it is likely that unforced climate fluctuations are quite large on paleoclimate time scales.

Unforced climate fluctuations complicate the search for any forced climate change such as may arise from changes of solar irradiance and anthropogenic greenhouse gases. Changes of ice sheet area can be ignored on decadal time scales, and changes of $\mathrm{CO}_{2}$ and $\mathrm{CH}_{4}$, although they may be in part a result of climate feedbacks, are known accurately from observations. But one variable ignored in our climate simulations, fluctuations of ocean heat transports, is clearly a significant contributor to global temperature fluctuations.

For example, in the record of observed global temperature for the past century (Fig. 3) the two major El Niños of the past decade show up as relative maxima in 1983 and 1987-88. Ocean transport variability also probably contributes to some of the longer time scale variability in this global temperature record, but we have no proof of that. The standard deviation of global annual mean temperature in our GCM without forcing and with fixed ocean heat transports is about 0.1C. As expected, the standard deviation for the observations is larger, being about $0.15 \mathrm{C}$ even if the long term 
trend in the data is removed. At least in part, the larger variability in the observations is probably due to interannual fluctuations in ocean transports.

\section{CLIMATE FORCINGS}

A climate forcing, natural or anthropogenic, is a change imposed on the climate system which modif ies the planetary radiation balance, thus affecting the planetary temperature. The natural forcings which appear to most significant, based on systematic comparison of radiative effects, are changes of stratospheric aerosols due to large volcanoes and changes of solar irradiance. The largest anthropogenic forcings appear to be increasing infraredabsorbing (greenhouse) gases, man-made tropospheric aerosols, and perhaps changes of surface reflectivity due to desertification and deforestation.

Most of the greenhouse gas changes are known rather well. For example, the $\mathrm{CO}_{2}$ changes over the past 250 years are shown in Fig. 6 . Changes of the other major greenhouse forcings, chlorofluorocarbons, methane, and nitrous oxide, are known reasonably well also.

The net climate forcing by $\mathrm{CO}_{2}, \mathrm{CFCs}, \mathrm{CH}_{4}$ and $\mathrm{N}_{2} \mathrm{O}$ for the period from 1958 (the International Geophysical Year, when Keeling began his measurements) to the present is more than $1 \mathrm{~W} / \mathrm{m}^{2}$ (Fig. 7). This is the rate of heating of the Earth's troposphere as computed with a simple (one-dimensional radiative-convective) or a more sophisticated (three-

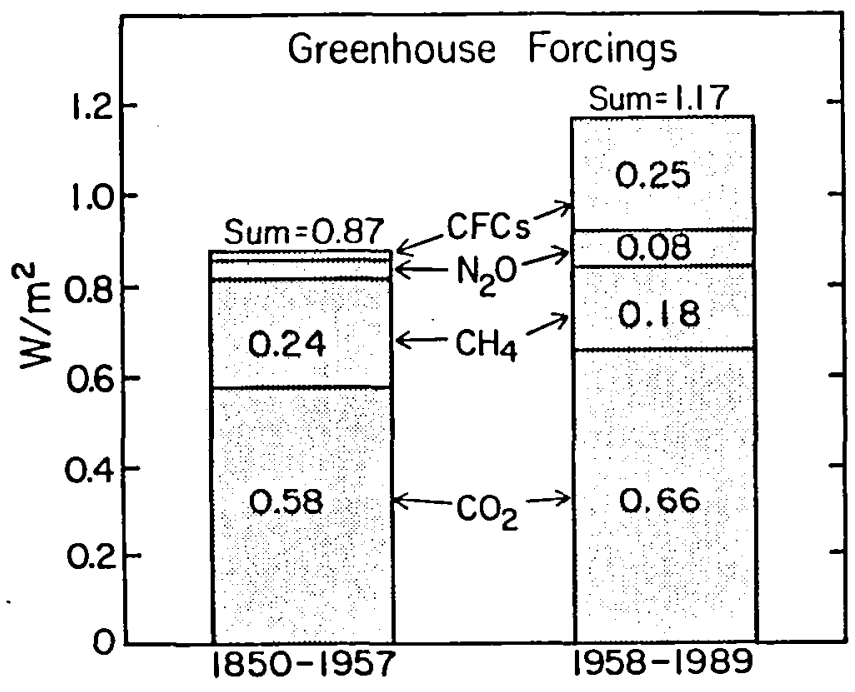

Fig. 7. Added greenhouse climate forcings for the periods 1850-1957 and 1958-1989. dimensional) climate model for the indicated changes of these gases. The increase of greenhouse forcing between 1850 and the present is more than $2 \mathrm{~W} / \mathrm{m}^{2}$ (Fig. 7). The uncertainty in the radiation calculations is perhaps $10-20 \%$. The uncertainty due to other, unmeasured greenhouse gases, particularly upper tropospheric ozone (which could cause either a heating or cooling) or stratospheric water vapor (which is suspected of increasing due to oxidation of increasing methane), is perhaps another $10-20 \%$. We conclude that there has been a steady increase in the anthropogenic greenhouse gas climate forcing, which has now reached a level of about $2-2.5 \mathrm{~W} / \mathrm{m}^{2}$.

Unfortunately, the anthropogenic aerosol forcing is much more uncertain, and its present trend is unknown. Perhaps as much as $25-50 \%$ of present tropospheric aerosols are anthropogenic, which would be a global climate forcing between -0.5 $\mathrm{W} / \mathrm{m}^{2}$ and $-1 \mathrm{~W} / \mathrm{m}^{2}$, i.e., a cooling which would balance a significant fraction of the greenhouse warming. Moreover, these aerosols are believed to increase cloud reflectivity, causing further, but very uncertain, negative climate forcing. But aerosol observations are too sparse to define the net aerosol climate forcing. Based on the fact that most anthropogenic aerosols are in the Northern Hemisphere and observations show comparable warming trends in both hemispheres over the past century, it has been argued that the net aerosol forcing must be significantly less than the greenhouse forcing. But clearly we need better global tropospheric aerosol measurements.

Other important climate forcings include changes of stratospheric aerosols and solar irradiance, as illustrated in Fig. 8 for much of the past decade. A solar irradiance change of $0.1 \%$ yields a climate forcing equivalent to about a $15 \mathrm{ppm} \mathrm{CO}_{2}$ change, while at its maximum the global climate forcing of El Chichón aerosols was equivalent to a more than $100 \mathrm{ppm} \mathrm{CO} 2$ change. Of course greenhouse, aerosol and solar forcings of equal global magnitude would not yield identical climate changes. But tests with our GCM using equivalent forcings, namely $2 \%$ solar constant change, doubled $\mathrm{CO}_{2}$, and a change of stratospheric aerosol optical depth of 0.15 , yielded generally similar global climate changes (with added aerosols causing cooling). Although climate sensitivity is uncertain by perhaps a factor of three, this uncertainty applies equally to all of the forcings.

Solar and greenhouse forcings during their periods of precise monitoring are contrasted in Fig. 9. Over the common period of accurate solar and greenhouse data the changing sun significantly modulates the net climate forcing, but it does not alter the overall trend. ${ }^{*}$ [The upper two solar curves 
in Fig. 9 use the annual mean and monthly mean of the present official results of the Nimbus 7 ERB, as available from the National Space Science Data Center and described by Hickey et al., Space Sci. Rev., 48, 321, 1988. The lower curve is an alternate preliminary reduction of the Nimbus 7 data which includes some modifications aimed chiefly at correcting for variations in telescope pointing, as described by D.V. Hoyt and H.L. Kyle (private communication) in a manuscript which will be submitted for publication. Uncertainties in data reduction and calibration, as well as significant differences between Nimbus 7, SMM and ERBE solar irradiance data, highlight the difficulty in achieving the high accuracy needed for climate studies. Adequate monitoring of long term solar change requires having two well-calibrated radiometers in space simultaneously with frequent solar observations, as discussed below. Observing capability during the period 1978-1989 was sufficient to conclude with a high degree of confidence that there was a decline of total solar irradiance of the order of $0.1 \%$ during the first several years of this period and at least a partial recovery in the late 1980s.]

Stratospheric aerosol and greenhouse forcings are contrasted in Fig. 10. The aerosol forcing is based mainly on estimates of atmospheric transparency obtained at astronomical observatories. This stratospheric aerosol forcing, which is mostly a result of volcanic eruptions, at times rivals or exceeds the greenhouse forcing, but the latter clearly dominates the long term trend.

Comparison of climate forcings, as in Figures 9 and 10, exaggerates the importance of high frequency variability. Because of the inertia of the climate system, brief forcings have much less impact than those maintained for several decades. Nevertheless, it is apparent that both solar and volcanic aerosol variations potentially are significant causes of climate variability.

Ground-based measurements of solar irradiance and solar diameter during the past 200 years have
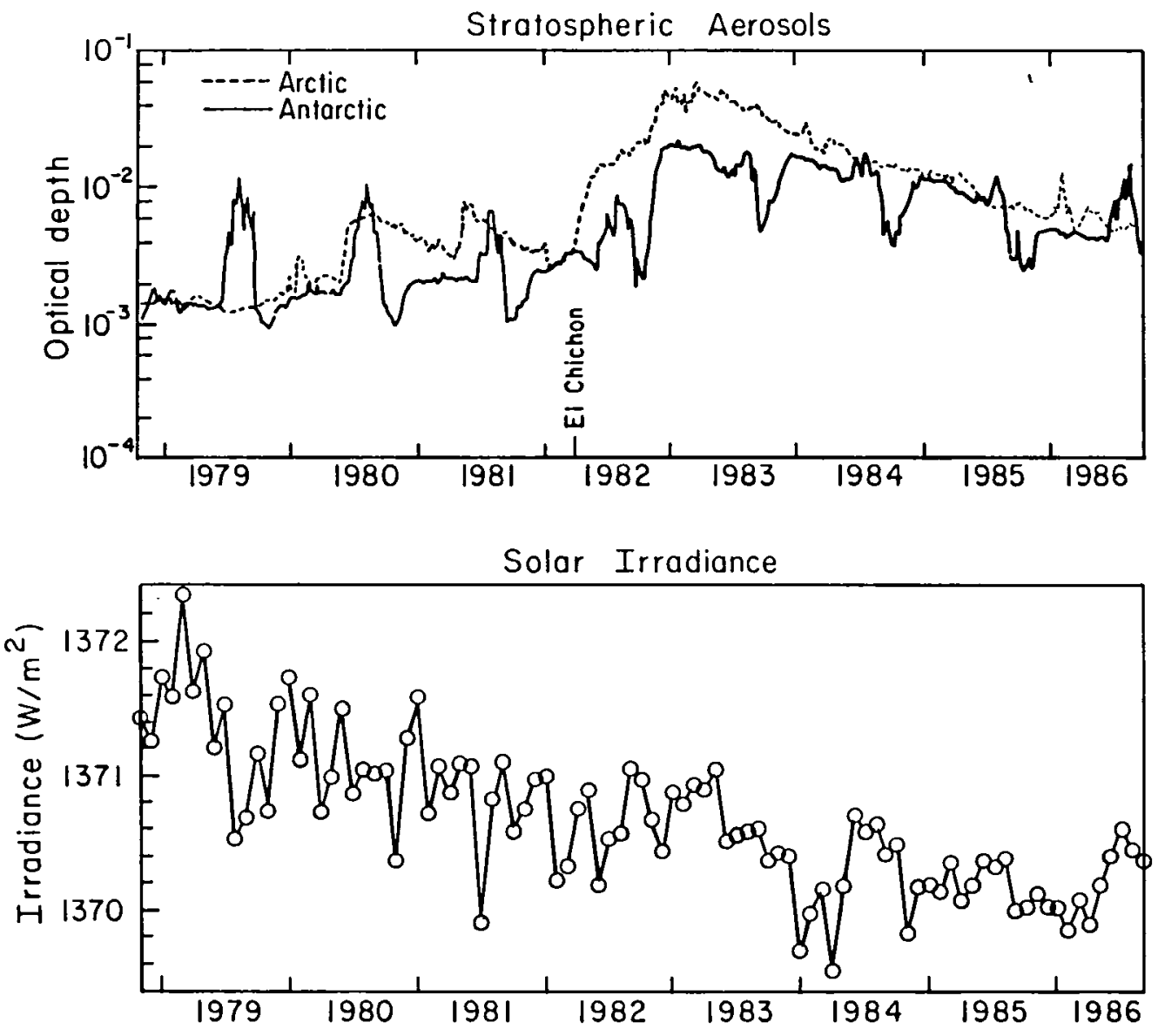

Fig. 8. Stratospheric aerosol optical depth measured by the SAM II instrument and solar irradiance measured by the ERB instrument on the Nimbus 7 spacecraft (SAM II data from P. McCormick; ERB data from J. Hickey, B. Alton, H.L. Kyle and D. Hoyt, as described in Space Science Reviews, 48, 321-342, 1988). 


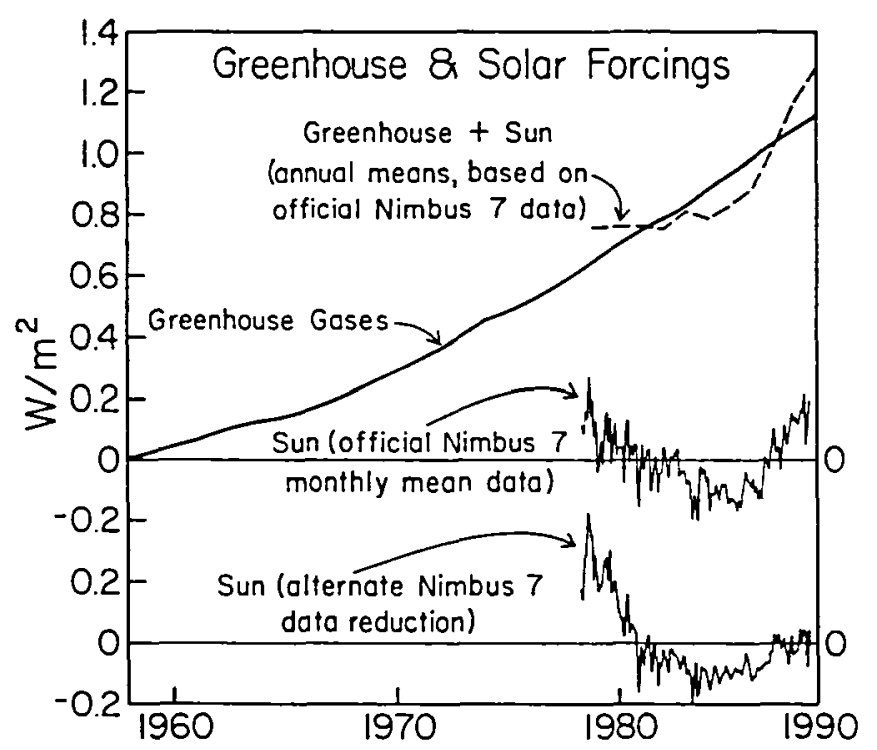

Fig. 9. Greenhouse and solar climate forcings in the past three decades. Solar forcing is based on Nimbus 7 ERB data (see parenthetical comment* in text).

placed an upper limit of about $0.3 \%$ on solar variability during that period. However, that degree of variation is sufficient to leave the sun as a candidate for causing the 1940-1970 global cooling, if indeed that was a forced climate change. Moreover, a solar decline of $0.3 \%$, if maintained on a century time scale, would cause a cooling of about $0.5 \mathrm{C}$, if climate sensitivity is $3-4 \mathrm{C}$ for doubled $\mathrm{CO}_{2}$. Since $0.5 \mathrm{C}$ is about the magnitude of estimated global cooling during the Little Ice Age, the sun is a viable candidate for forcing that climate fluctuation.

We conclude that solar variability may be a significant climate forcing mechanism, so it is important to maintain accurate solar monitoring. Unfortunately, the most precise solar irradiance data terminated as the Solar Maximum Mission was brought down by atmospheric drag in 1989. Hopefully the solar instruments on Nimbus 7 and the Earth Radiation Budget satellites will continue to function until the planned 1991 launch of the Upper Atmospheric Research Satellite, with its active cavity radiometer. The Nimbus 7 instrument has been a remarkable workhorse over more than 11 years, but it can not measure long term degradation of its sensor. The SMM experiment carried three sensors with two of them normally shuttered, thus allowing occasional calibration of solar-induced degradation of the sensitivity of the primary sensor. A degradation of $0.05 \%$ was measured over the $9 \frac{1}{2}$ year lifetime of SMM, and this instrument's selfcalibrated irradiance was useful for comparison with the simultaneously operating Nimbus 7 instrument.
The ERBE instruments presently provide a crosscheck on Nimbus 7 , but their data have a higher noise level because of a low frequency of solar viewing and other factors. We need to strive to have two well-calibrated instruments simultaneously in orbit, to provide a cross-check and continuity of calibration when an instrument ceases operation.

Finally, I mention one other conclusion which follows from comparison of the above climate forcings. The opinion has been expressed, in a report of the Marshall Institute, that greenhouse warming may be beneficial because it might just cancel cooling in the 21 st century due to a declining solar irradiance. But it is apparent that the equivalent of doubled $\mathrm{CO}_{2}$, which is expected by the middle of next century if there are no reductions in greenhouse gas emissions, would require that solar irradiance decline by $2 \%$ to counter the greenhouse climate forcing. While such a solar decline is not strictly impossible, it is much larger than existing indications of solar variability. Given available scientific evidence, it would be foolish to base greenhouse policy on the hope that solar variability will somehow counter greenhouse warming.

\section{CLIMATE SIMULATION}

We carried out one new climate simulation for this conference. This calculation focused on the past decade and the next few years, for the purpose of

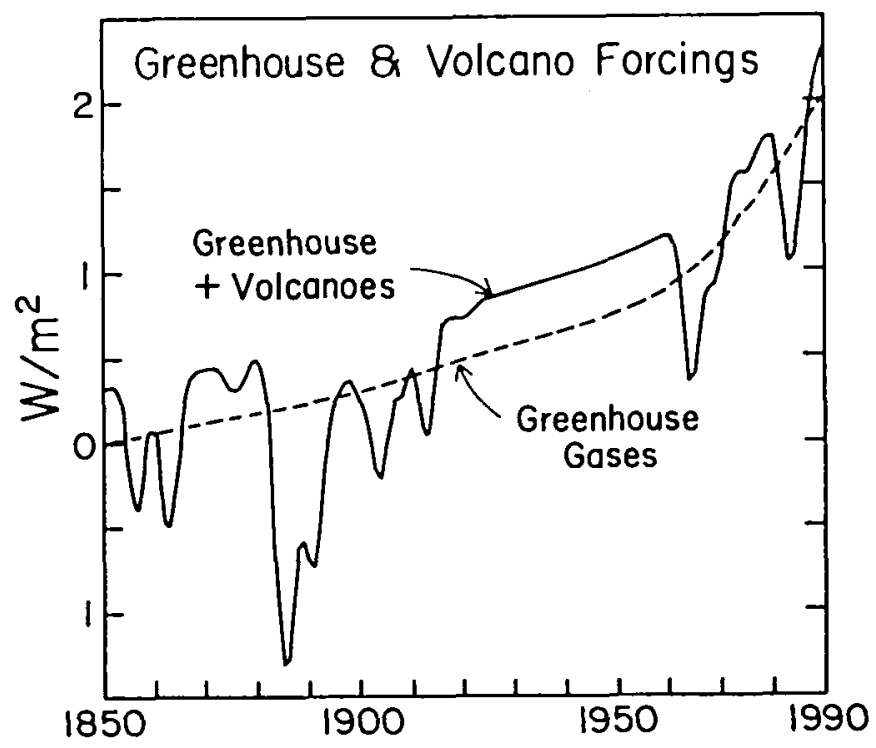

Fig. 10. Greenhouse and stratospheric aerosol climate forcings in the past century. Aerosol optical depth is based mainly on atmospheric transmission measurements at astronomical observatories and lunar eclipses. Zero point of aerosol forcing is the 1850-1989 mean. 
examining the climate implications of recent information on changes of solar irradiance and other climate forcings during the 1980 s. The new calculation builds on a base of earlier calculations (J. Geophys. Res. 93, 9341, 1988) and uses the same climate model. The previous simulations used a range of scenarios for greenhouse gas changes (A, B and $C$ ) and a rough estimate of stratospheric aerosol optical depth following El Chichon, which erupted less than a year before the simulation was started. Greenhouse gases and stratospheric aerosols were the only changing climate forcings in the earlier simulations.

The greenhouse gas forcing in the new simulation, labeled $B_{n}$, is based on our recent calculation (J.Geophys. Res. 94, 16417, 1989) of the combined forcing by $\mathrm{CO}_{2}, \mathrm{CFCs}, \mathrm{CH}_{4}$ and $\mathrm{N}_{2} \mathrm{O}$, and is almost identical to scenario $\mathrm{B}$ of the previous study. Both $B$ and $B_{n}$ assume linear growth of greenhouse forcing in the future, as opposed to the exponential growth of scenario A ("business as usual") and the eventual no-growth of scenario $C$ ("draconian emission cuts"). Scenarios B and $B_{n}$ appear to be more realistic than scenarios $A$ and $C$ for recent and near future changes of greenhouse gases.
The solar irradiance was constant in scenario $B$, i.e., there was no solar forcing. In scenario $B_{n}$ we used an analytic approximation for satellite measurements of solar irradiance as suggested to us by Dick Willson: a cosine function with full amplitude $0.1 \%$, period 10.95 years, and maximum at 1980.82. Good data through the present solar maximum may allow an improved representation, but this approximation should be sufficient for our present purposes.

The stratospheric aerosols in scenario $B$ are described in our 1988 paper. Scenario $B_{n}$ uses aerosol opacities derived from approximately annual lunar eclipse data of Richard Keen (Science, 222, 1011,1983 and private communication). The $B_{n}$ aerosols have a larger maximum optical depth $(0.12)$ than the $B$ aerosols and do not decrease quite as rapidly, so the $B_{n}$ aerosols tend to give somewhat more cooling in the middle 1980s. For aerosols, we are not certain whether $B$ or $B_{n}$ is more accurate. Presently we are working with Jim Pollack and Pat McCormick to use a number of data sources to try to define the aerosol forcing more precisely. The net impact of the changes of climate forcing in $B_{n}$, as compared to $\mathrm{B}$, is a slightly increased forcing around 1980 , a decrease in the middle 1980s, and an increase

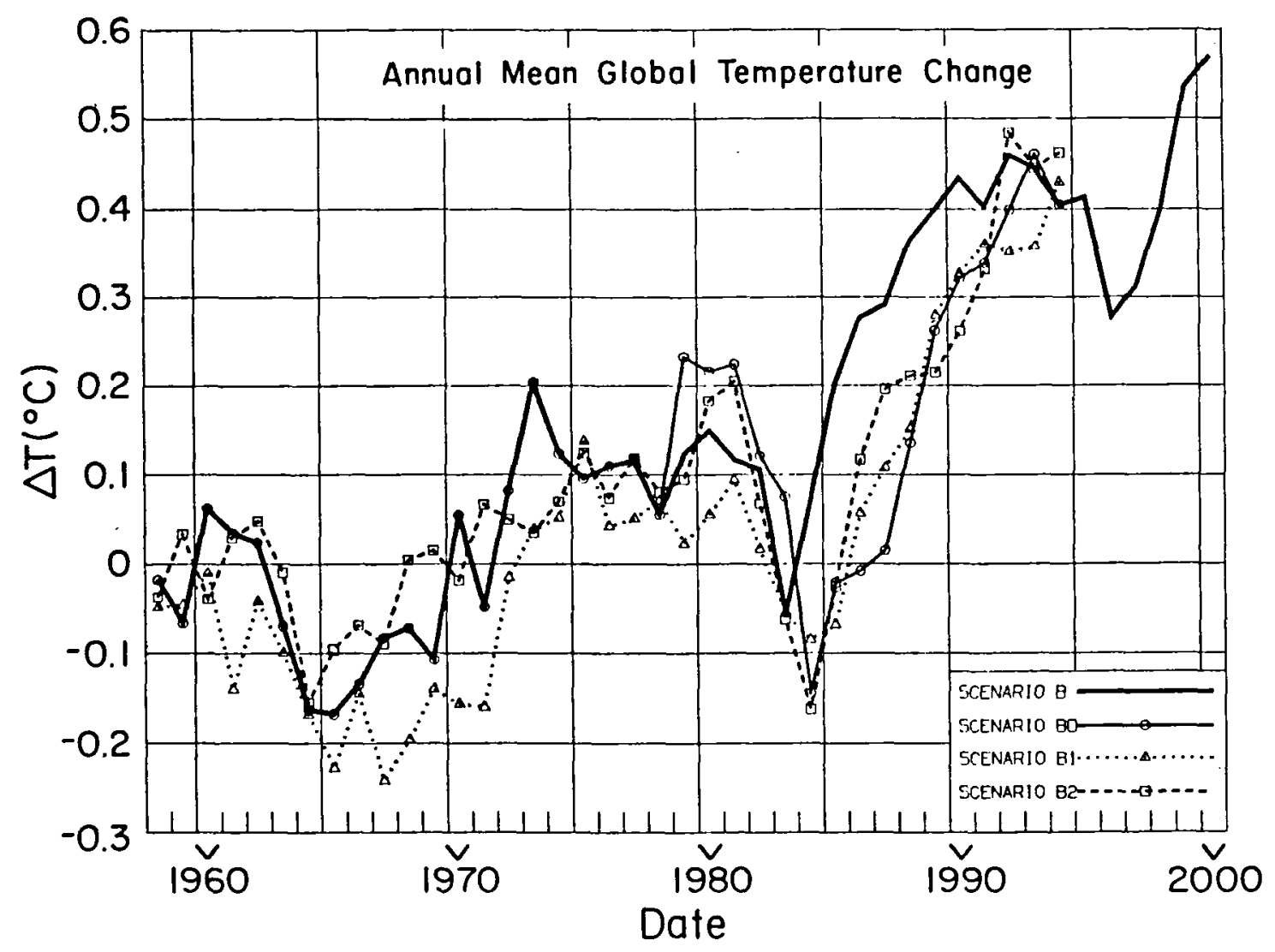

Fig. 11. Global temperatures for three new runs $\left(B_{n}\right)$ of model II compared to previously published scenario $B$. 
at the end of the 1980s and early 1990s.

One point we want to stress is the "butterfly effect." If a climate model simulation is repeated, with identical climate forcing but with some initial atmospheric parameter (temperature, wind, etc.) changed ever so slightly (a flap of a butterfly's wings in Buenos Aires), the simulations follow different chaotic paths. Thus we must make several runs of the model, if we wish to draw any conclusions about climate change on time scales of several years.

We illustrate the global temperature for three new runs of the model $\left(B_{0}, B_{1}\right.$ and $\left.B_{2}\right)$ in Figure 11 , along with the published run, scenario $B$. The $B_{1}$ and $B_{2}$ runs begin on January 1,1958 with the same initial conditions as run $B$ except for a slight noise added to atmospheric temperatures. Since the forcings for $B$ and $B_{n}$ are almost identical for the period 1958-1978, the results for $\mathrm{B}, \mathrm{B}_{1}$ and $\mathrm{B}_{2}$ in that 20 year period give an indication of the range of chaotic behavior of the model. Run $B_{0}$ starts in 1978 and uses $B_{n}$ climate forcing (its initial state is identical to run B on January 1, 1978).

Note that all the simulations show some response (cooling) to the large short-term negative climate forcings caused by the large volcanoes in 1963 (Agung) and 1982 (El Chichon). Of course the model does not contain the variability associated with fluctuations in ocean heat transports, such as El Niños. Thus, although the real world (Fig. 3) shows clear evidence of cooling after Agung, apparent cooling in the 1982-1985 time frame is interrupted by an intense El Niño warming in 1983.

The dominant feature of the new simulations (runs $\mathrm{B}_{0}, \mathrm{~B}_{1}$ and $\mathrm{B}_{2}$ ) is the very strong warming in the late 1980s to a level in the early 1990s clearly above any earlier global temperature. The magnitude of the warming exceeds the noise level of the model. The principal change in scenarios $B_{n}$, as compared to $\mathrm{B}$, is a pushing back of the warming by about 2 years to the end of the 1980s. However, both $B$ and $B_{n}$ reach very high global temperatures, about $0.4 \mathrm{C}$ above the level of the 1950 s, in the early 1990s.

What assumptions does this result depend upon? First, model sensitivity: our model has a sensitivity $4 \mathrm{C}$ for doubled $\mathrm{CO}_{2}$. Any sensitivity in the range 2 to $5 \mathrm{C}$ is consistent with empirical data, such as that provided by paleoclimate studies. A sensitivity toward the lower end of this range would reduce the predicted warming, but not by so much as the ratio of sensitivities. Calculations should be carried out with a lower sensitivity, but we do not expect the qualitative prediction to change. Second, neglect of other unknown forcings: there are a number of poorly known forcings, such as anthropogenic tropospheric aerosols, sulfate alterations of cloud properties, and aircraft contrails, for example. But the evidence, as we have discussed, suggests that the anthropogenic greenhouse is the dominant forcing. A large volcano could change this picture, but their frequency, several per century, makes an occurrence unlikely in the next year or two. Third, neglect of unforced natural cooling. Actually our "butterfly" range of experiments accounts for much unforced variability. The El Niño cycle is probably the main unaccounted unforced variability, and the Earth has just come through the cool ( $\mathrm{La} \mathrm{Niña)} \mathrm{phase} \mathrm{of} \mathrm{that}$ cycle, so, if anything, the El Niño is likely to add to warming in the next 1-3 years. The possibility of a sudden "flip" of ocean circulation, as discussed by Broecker, seems slim on the time frame of the next 1-3 years, and there is little reason to believe such an occurrence would lower global mean temperature.

\section{DISCUSSION}

My conclusion is that we are likely to set a modern global temperature record in the next 1-3 years, measurably exceeding the already high levels of the 1980s. I described this, in the introduction, as a remarkable conclusion because it is even more immediate and specific than our previous conclusion that the 1990s would see a record temperature level. And other scientists, although most are in agreement that the greenhouse effect will eventually cause global warming, have been unwilling to conclude even that we should expect record warmth in the 1990s. This reluctance is usually based on the observation that natural fluctuations are large in decadal periods, and the fluctuations are toward cooling as often as toward warming. What is overlooked, I believe, is the fact that the present climate is out of equilibrium with current atmospheric composition. Because of that, it is difficult for global temperature to maintain a large "fluctuation" in the direction of lower temperature. Indeed, that point is illustrated by our "butterfly" experiments. And even if we insert a volcano of El Chichón magnitude in 1995, as we did in our 1988 paper, the 1990s are warmer than the 1980s in the model.

Are there political and social implications to be drawn from this conclusion, without stretching it too far? The results suggest that a candidate for election in 1992 may be making a serious mistake if he argues that this is a time only for research and not to take action to slow emissions - because there is a very good chance that he would get burned before the 1992 election, burned by empirical evidence of a 
warming world. To be burned badly would require some large regional climate impacts as well, but the chance of that happening will rise with increasing global temperature. A social implication follows from the observation that serous attempts to control greenhouse gas emissions will be strongly resisted, and thus hypothetical climate change next century is unlikely to spur much action. So clear-cut global warming in the next $1-3$ years could provide a push needed to help move us toward the changes which will be required to bring down greenhouse gas emissions.

That brings me to my friendly wager. The conclusion about expected near term warming is based on simulations with a global climate model the tool which has been thoroughly condemned as being unreliable - condemned by many scientists as well as bureaucrats. Also the statement I have made, that the world is getting warmer and that it is probably due to the greenhouse effect, has been widely criticized as being unjustified. If those criticisms are really believed, then surely someone would be willing to accept the following wager:

I claim that at least one year in the period 1990-1992 will be warmer than any year in the previous century. I win only if it is true in all of the main long term data sets: the GISS analysis of meteorological stations, the East Anglia analysis including ships, and Angell's upper air (radiosonde) data set. I get three years and you get 100 years. Someone mentioned that there will probably be an El Niño in the period 1990-1992. That may be so, but there were probably 20 or $30 \mathrm{El} \mathrm{Niños} \mathrm{in} \mathrm{the} \mathrm{previous}$ century, including two very intense EI Niños in the 1980 s, which was the warmest decade. So there should be plenty of souls willing to make this wager. The offer remains open, so please contact me.

Can we learn anything about long term climate from temperature and other data for just the next three years? I think we can. If nature cooperates by holding off any large volcanoes, and if there is significant global warming consistent with the model calculations, it will improve our confidence in our understanding of the climate system's response to a sustained global forcing. On the other hand, if there is no warming or a cooling, it will suggest that we have overestimated climate sensitivity, overlooked other important climate forcings, or underestimated fluctuations in ocean transports.

\section{ENDNOTES}

\section{Wonderland Model}

The climate model used for the several hundred year simulation (Fig. 5) is a modification of the GISS model II. The physics is the same as in model II, except that the fundamental equations include a more accurate representation of the impact of water vapor on surface pressure and atmospheric thermodynamic properties, for the purpose of allowing simulations over a greater range of climate states. The primary difference compared to model II is the geography (Fig. 12), which, borrowing from an idea of Suki Manabe, covers only 120 degrees of longitude in the wonderland model, with cyclic repetition to fill out spherical geometry. The amount of land as a function of latitude is the same as in the real world, and the zonal mean climate simulated by the wonderland model is almost the same as for model II.

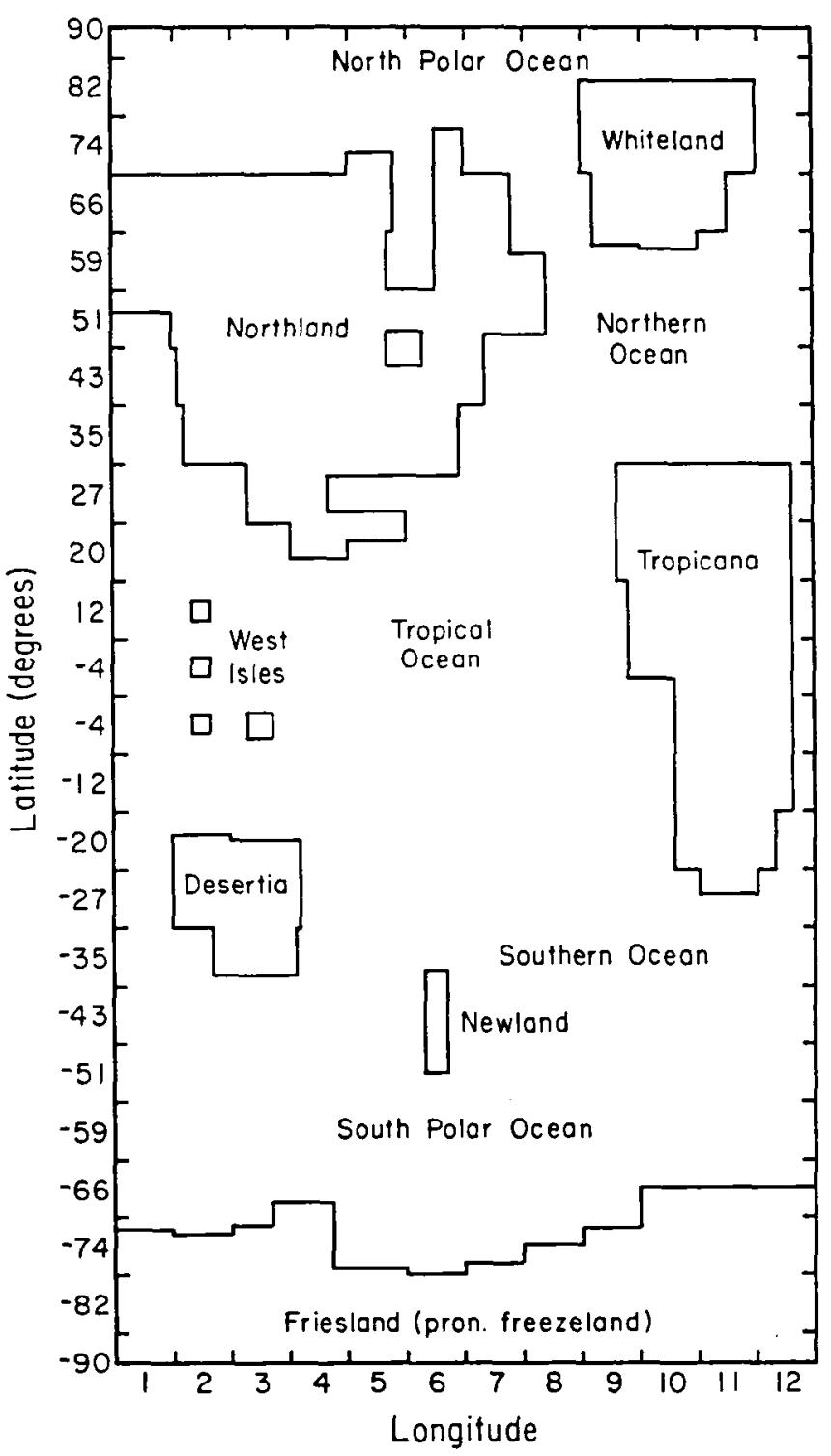

Fig. 12. Wonderland climate model. 
The main advantage of the wonderland model is that it is three times faster than model II, thus allowing long simulations to be carried out on a computer of modest capabilities. A secondary advantage is the fictitious geography, which emphasizes the fact that the model is not the real world, thus helping the user focus on the climate processes simulated by the model.

\section{Rationale for a wager}

It has been pointed out to me that proposing a wager, especially since it was reported in a scientific journal (Science May 4, 1990), appears to be a bit unprofessional. On the other hand, it can be argued that it is worthwhile to draw attention to the issue of whether climate change during just the next few years has implications for longer term climate change.

However, the original rationale for the wager had a slightly different purpose. I concocted the proposed bet before the AGU meeting last December, under the impression that I would be in a panel discussion with Dick Lindzen. He has left the impression with the public that he disagrees with my assertion that the world is getting warmer and that the warming is probably due to the greenhouse effect; also, he argues that climate sensitivity is much less than indicated by the models and that the anthropogenic greenhouse warming may be small compared to natural variability. It seemed to me that given his position, he had to either accept the bet or do a fancy shindig which would undermine the extreme view credited to him. As it turned out, there was no panel discussion following our talks, so
I had to save the proposition for the next conference. I had hoped that Bob Jastrow would bite on it, but he demurred on the grounds that the sun is increasingly warming the earth! That is interesting, since he has long argued that the Earth is already heading into an ice age. Also, the satellite data do not indicate a greater irradiance this solar cycle than in the previous one.

Wagers aside, it is interesting to look at data for the first few months of 1990 . Preliminary numbers from our (Hansen and Lebedeff) analysis of surface air reports from meteorological stations show anomalies (relative to $1951-1980$ ) of $+0.5,+0.4,+1.5$, $+0.9 \mathrm{C}$ for the Northern Hemisphere and $+0.1,-0.2$, $+0.2,+0.2 \mathrm{C}$ for the Southern Hemisphere for the first four months of 1990 . The March anomaly is the largest for any month in our record, and, if the mean for the first four months held for the next eight months, 1990 would be the warmest year in our record. However, the largest anomalies almost always occur in Northern Hemisphere winter and early spring, and throughout the warm decade of the 1980 s the first half of the year was considerably warmer than the second half. Thus, for 1990 to rank as the warmest year will require that the remaining months be substantially warmer than they were in the 1980s.

Acknowledgements. We thank Richard Keen, H. Lee Kyle and Richard Willson for providing observational data. This research was supported by the NASA Climate Program Office and the Environmental Protection Agency. 


\title{
Solar Activity and the Sea-Surface Temperature Record - Evidence of a Long-Period Variation in Solar Total Irradiance?
}

\author{
George C. Reid \\ Aeronomy Laboratory, NOAA, Boulder, Colorado
}

There have been many suggestions over the years of a connection between solar activity and the earth's climate on time scales long compared to the 11-year sunspot cycle. They have remained little more than suggestions largely because of the major uncertainties in the climate record itself, and the difficulty in trying to compile a global average from an assembly of measurements that are uneven in both quality and distribution. Different climate time series have in fact been used to draw conflicting conclusions about the existence, or even the sense, of a presumed response to solar activity, some suggesting a positive correlation, some a negative correlation, and some no correlation at all. The only excuse for making yet another such suggestion is that much effort has been devoted in recent years to compiling climate records for the past century or more that are internally consistent and believable, and that a decadal-scale record of solar total irradiance is emerging from spacecraft measurements, and can be used to set limits on the variation that is likely to have occurred on these time scales.

The work described here was originally inspired by the observation that the time series of globally averaged sea-surface temperatures over the past 120 years or so, as compiled by the British Meteorological Office group (Folland and Kates, 1984), bore a reasonable similarity to the long-term average sunspot number, which is an indicator of the secular variability of solar activity. The two time series are shown in Figure 1, where the sunspot number is shown as the 135-month running mean, and the SST variation is shown as the departure from an arbitrary average value.The simplest explanation of the similarity, if one accepts it as other than coincidental, is that the sun's luminosity may have been varying more or less in step with the level of solar activity, or in other words that there is a close coupling between the sun's magnetic condition and its radiative output on time scales longer than the 11-year cycle. Such an idea is not new, and in fact the time series shown in Figure 1 can be regarded as a modern extension of the proposal put forward by Eddy (1977) to explain the covariance between various global climate indicators and solar activity as revealed by the $\mathrm{C}^{14}$ record over the past millenium.

Spacecraft measurements of the sun's total irradiance have only become available since about 1979, and they have indeed shown a long-term variation that appears to be positively linked to the sunspot number. Whether the variation is simply related to the 11-year cycle, or whether it contains longer-period components, remains unresolved, but Fröhlich (1987) has pointed out that earlier individual measurements from balloons, rockets, and spacecraft can be taken as indicating the existence of a longer term variation. Figure 2 is based on the data compiled by Fröhlich (1987), and shows measurements made between 1967 and 1985 with instruments similar to those used in the more recent spacecraft measurements. While the earlier measurements show a considerable scatter, only two of them are as high as the later sequence, and there is an average difference of about $4 \mathrm{~W} \mathrm{~m}^{-2}$ between the values obtained around the peak of solar cycle 20 in 1969 and those obtained ncar the peak of solar cycle 21 in 1980. Fröhlich (1987) suggested that this might be evidence for a 22 -year cycle in irradiance, but it might equally well be taken as evidence for the kind of long-term relationship between solar activity and solar irradiance inferred above.

Making the very simple assumption that the long-term component of the irradiance is linearly proportional to the envelope of the sunspot number time series shown in Figure 3, we can use the difference of $4 \mathrm{~W}$ $\mathrm{m}^{-2}$ between the maxima of cycles 20 and 21 to calibrate the relationship. The empirical relation derived in this way is

$$
\mathrm{S}\left(\mathrm{W} \mathrm{m}^{-2}\right)=1353.6+.089 \mathrm{~N}
$$

where $S$ is the total irradiance and $N$ is the sunspot number envelope. Figure 4 shows a scatter plot of the annual values of globally averaged SST and the sunspot-number envelope. The correlation coefficient is 0.71 , maximizing at zero lag, and a calculation of the number of degrees of freedom taking autocorrelation into account indicates that the correlation is significant at a level between $95 \%$ and $99 \%$. 
structure of the ocean developed by Hoffert et al. (1980), and the resultant variation of sea-surface temperature has been computed. The upper mixed layer of the ocean is assumed to have a uniform temperature, determined by solar radiation from above and by loss of heat by radiation to space, by downward diffusion into the decp ocean, and by upward advection of cold water forming the global thermohaline circulation. The calculation was stanted during the Maunder Minimum in 1660, when the temperature was assumed to be at its equilibrium value for a sunspot-envelope number of 5, and ended in 1990, where the sunspot envelope was estimated on the basis of the progress of solar cycle 22 through 1989. The time step used was 6 months, and the calculations were carried to the bottom of the 4000-meter deep ocean in steps of $100 \mathrm{~m}$.

The result is shown in Figure 5, where the solid line shows the model output, and the smoothed measurements of globally averaged SST (C.K. Folland, private communication, 1987) have been superimposed. The SST values are shown as departures from an arbitrary mean, and have been positioned so as to overlay the model curve. The two scales, however, have the same dimensions.

Until we know more about how the sun's total irradiance varies on long time scales, the picture presented here must remain speculative. Taken together with the evidence presented earlier by Eddy (1977), however, the possibility remains that solar irradiance variations keyed to the long-term changes in solar activity have played a major role in influencing terrestrial climate over the past millennium.

\section{References}

Eddy, J.A., Climate and the changing sun, Clim. Change 1, 173-190 (1977).

Folland, C.K., and F. Kates, Changes in decadally averaged sea surface temperature over the world 1861-1980, in Milankovitch and Climate, Part 2, ed. A.L. Berger et al., Reidel Publishing Co., 721 -727 (1984).

Fröhlich, C., Variability of the solar "constant" on time scales of minutes to years, J. Geophys. Res. 92, 796-800 (1987).

Hoffert, M.I., A.J. Callegari, and C.-T. Hsieh, The role of deep sea heat storage in the secular response to climate forcing, J. Geophys. Res. 85, 6667-6679 (1980).

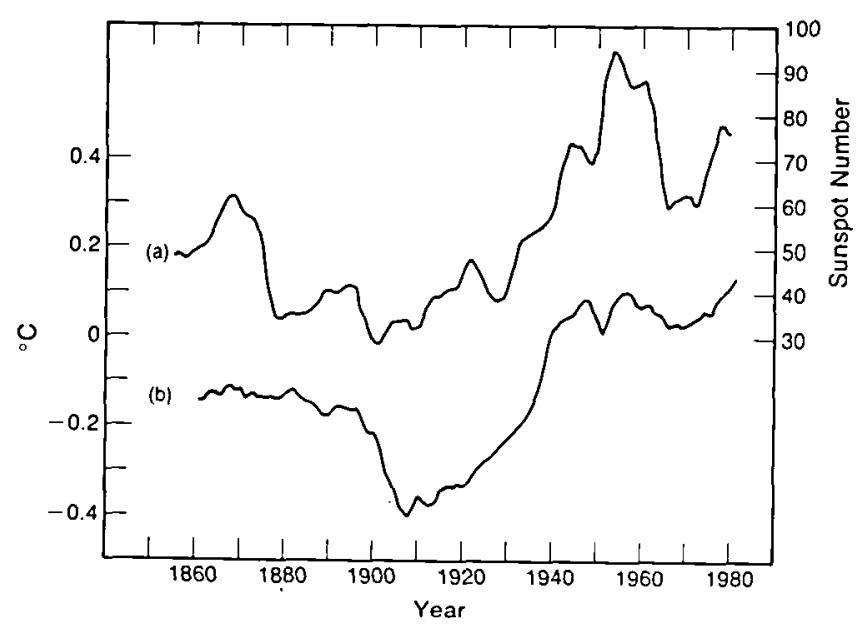

Fig. 1. (a) 135-month running mean sunspot number; (b) global average sea-surface temperature.

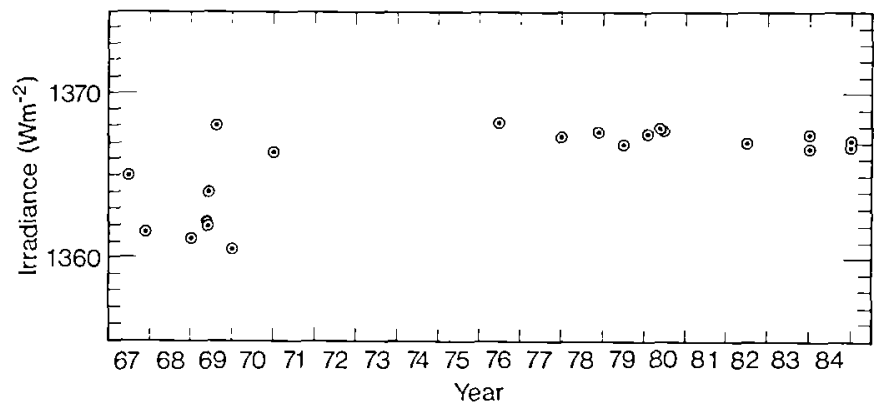

Fig. 2. Measurements of the sun's total irradiance made from balloons, rockets and spacecraft (redrawn from Fröhlich [1987]). 


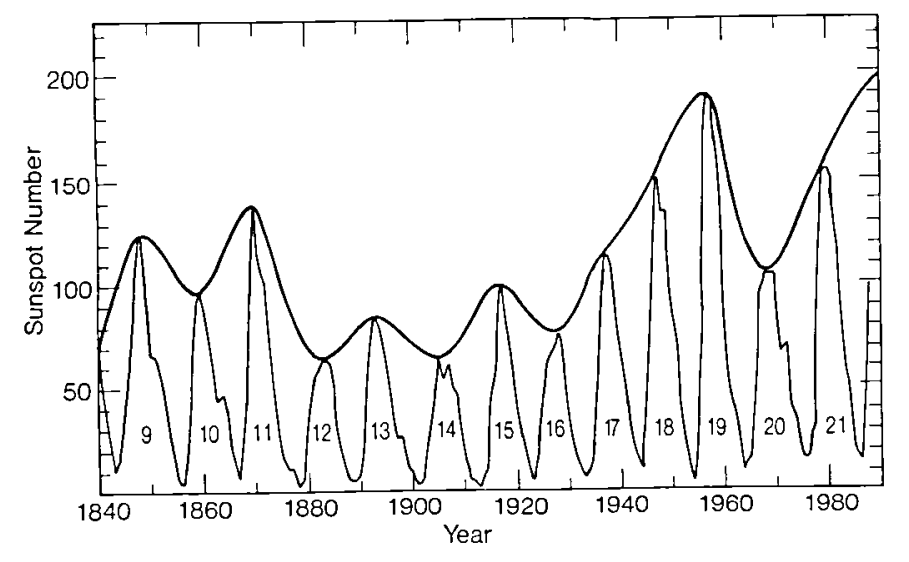

Fig. 3. Sunspot number 1840-1988 and its envelope.

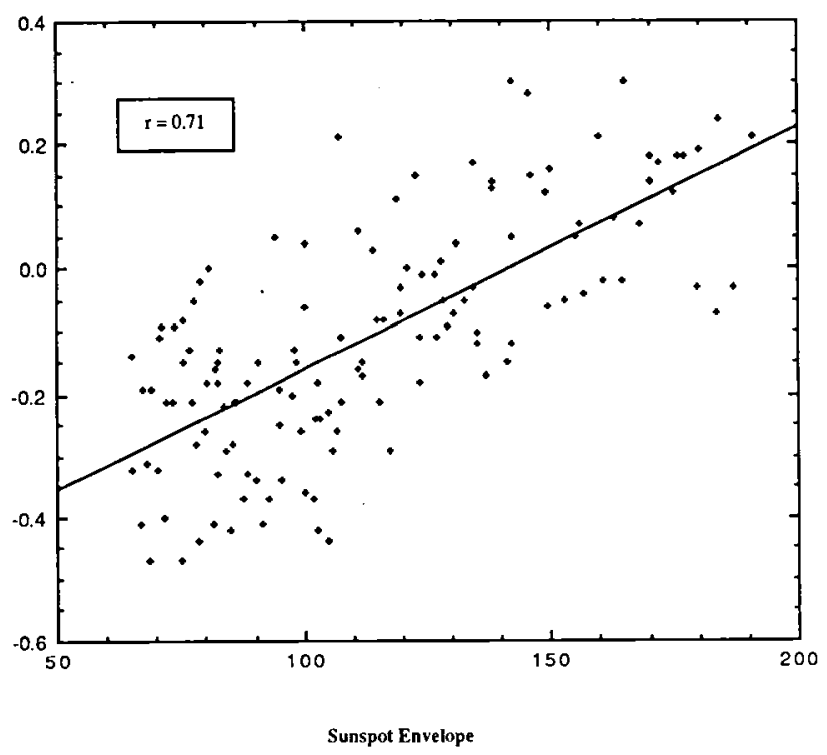

Fig. 4. Annual values of globally averaged SST versus sunspot-number envelope.

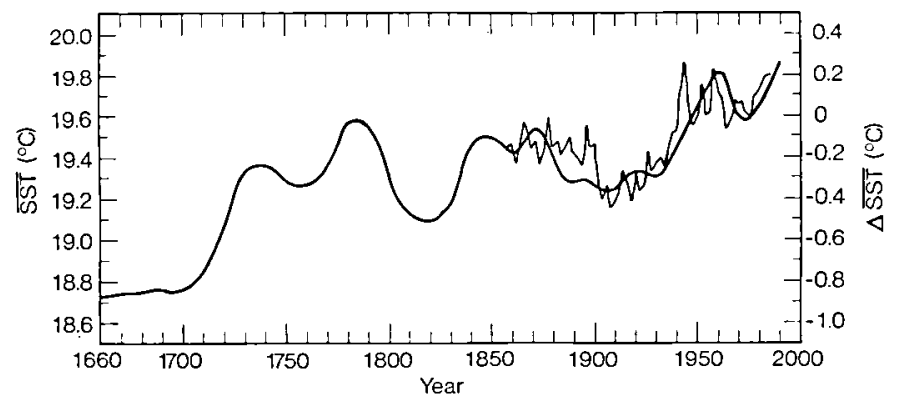

Fig. 5. SST predicted by the model (LH scale), and the smoothed globally averaged observed SST, expressed as the departure from an arbitrary mean. 
JANUARY - FEBRUARY TROPOSPHERIC CLIMATE FOR THE

NORTHERN HEMISPHERE AND THE 11-YEAR SOLAR CYCLE, THE QBO AND THE SOUTHERN OSCILLATION

Anthony G. Barnston and Robert E. Livezey

Climate Analysis Center, Washington, DC

\section{INTRODUCTION}

This study examines a recently discovered association between the 11-year solar cycle and the atmosphere that is most easily detectable when the two phases of the Quasi-biennial Oscillation (QBO) are considered individually rather than pooled. The influence of the Southern 0scillation (SO) for either of the two QBO phases is then combined with that of the solar cycle in the form of two-predictor multiple regression.

Documentation of a solar cycle-QBO-atmosphere relationship in Northern Hemisphere winter began for the stratosphere near the North Pole (Labitzke 1987) and was subsequently extended to cover the extratropical Northern Hemisphere for the lower stratosphere and middle troposphere (Labitzke and van Loon 1988), and finally for the lower troposphere and the surface (van Loon and Labitzke 1988). The last paper reported statistically highly significant QBO-stratified solar cycle-atmosphere relationships at a number of locations; these involved surface air temperature, sea level pressure, and geopotential height. Further study indicated effects during other seasons and in the Southern Hemisphere (Labitzke and van Loon 1989). The field significance (Livezey and Chen 1983) of the solaratmosphere correlation fields reported in van Loon and Labitzke (1988) was evaluated in Barnston and Livezey (1989)--to be called BL89--and found to be quite strong for both east and west QBO phases for the January-February period.

In January-February of 1989, however (which was not included in the analyses in BL89) the climate pattern strongly opposed that expected for the solar/QBO conditions. This exception was attributed to the strong SO situation: a cold tropical Pacific ocean and a high so index. In fact, there had never been even a moderately strong high SO episode coincident with a solar maximum during the west QBO phase since QBO records first became available in 1951. The need to stratify by $Q B O$ phase results in sample sizes of only about 20 years per phase, leaving some very noticeable vacant sectors in the two-dimensional sample space of solar flux and so.

The 1989 event helped provide evidence that the So needs to be considered as well as the solar cycle and QBO information in developing predictive relationships with the climate. This study incorporates both the solar and so factors in predicting the Northern Hemisphere $700 \mathrm{mb}$ height anomalies for the JanuaryFebruary period, separately for west and for east $Q B O$ phases. The study is also extended to United States surface temperature in certain analyses.

\section{DATA AND ANALYSIS METHODS}

The data used in this study are essentially identical to those described and used in BL89, extended to include 1989 and the SO. They include January-February means for 1951-89 of $10.7 \mathrm{~cm}$ wavelength solar flux, surface temperatures at 92 continental U.S. stations, and $700 \mathrm{mb}$ heights at 358 approximately equal area Northern Hemispheric grid points from $20^{\circ} \mathrm{N}$ northward. January mean values of the $45 \mathrm{mb}$ u-component of the equatorial wind over the equator led to a dichotomous 
definition of the QBO phase - west or east - for this same period. Further details about these data are available in BL89 and references thereof. A Southern oscillation Index (SOI) was computed from two equally weighted components: the standardized anomaly of Tahiti minus Darwin sea level pressure (SLP) and that of the average sea surface temperature (SST; multiplied by -1) in the highly associated east-central tropical Pacific area of $10^{\circ} \mathrm{S}-10^{\circ} \mathrm{N}, 120-160^{\circ} \mathrm{W}$. The JanuaryFebruary averaging period was used for SST, but some noise filtering was applied to SLP by using a weighted average of $(\mathrm{Jan}+\mathrm{Feb}) / 3+(\mathrm{Dec}+\mathrm{Mar}) / 6$. The SST and SLP were combined to capture a more balanced, stable and complete index of the SOI than either provides alone.

Many of the 2-variable relationships discussed in this paper, described by spatial fields of correlation coefficient, are statistically tested for field significance with Monte Carlo techniques that are detailed in BL89. The major considerations in these statistical tests are the temporal autocorrelations of the two variables (e.g. the solar flux has a strong autocorrelation associated with its 11-year cycle, and any variable that is well correlated with the flux probably does also) and the spatial correlation among neighboring grid points of the field variables. Both factors reduce the degrees of freedom in the timespace system and make chance occurrences of high amplitude correlation patterns more likely. As in BL89, the integrated t-statistic, or average of the $t$-values over all grid points of the correlation field, is used as the test statistic. A Monte Carlo procedure that randomly shuffles an appropriate variable is used to generate a distribution of such statistics that would be expected by chance, with which to compare the observed result. The significance level, or probability that the observed relationship is of a strength that could have occurred by chance, is then obtained. This method is a refined version of that of Livezey and Chen (1983) in that it represents the integrated significance level using the local $t$-values themselves rather than a count of how many of them exceed a given local significance threshold, as was done in Livezey and Chen (1983). Using the $t$-values themselves allows for more accurate evaluations of irregularly shaped distributions of local significance, should they happen to occur.

QBO phase-stratified bivariate multiple linear regression models are developed to predict grid point values of $700 \mathrm{mb}$ height on the basis of the SOI and the solar flux. In the presentation of results the regression coefficients are normalized as partial correlations between either of the two predictors and the predictand, accounting for the effect of the other predictor in the equation.

\section{IMPACT OF 1989 ON SOLAR FLUX-CLIMATE RELATIONS FOR WEST QBO PHASE}

The January-February correlation field between $10.7 \mathrm{~cm}$ solar flux and the Northern Hemisphere $700 \mathrm{mb}$ height is shown in Fig. 1, for the 21 west QBO phase years for the 1951-88 period. The field significances are strong, as shown at the top of Table 1. (The QBO phase assignment shuffle Monte Carlo significance test and the alternative option of a solar flux phase/shape shuffle test are described in BL89 on pages 1298 and 1304, respectively. The QBO phase assignment shuffle is random and does not restrict the phase sequences to the observed alternating patterns; this produces a maximally stringent significance test.) The strong dipole with centers in Canada and the western Atlantic near $30^{\circ} \mathrm{N}$ and the associated anomalous circulation pattern would be a helpful index for developing long-range forecasts for west QBO phase years for January-February in North America.

In January-February 1989 the QBO phase was west and the flux anomaly was high, but the North American climate strongly opposed that expected from the 
1951-88 relationship (Fig. 2). This weakened the west phase flux-climate correlation fields considerably (Fig. 3) as well as the associated field significances (bottom of Table 1). Note that the significance for east QBO phase relationships also changed slightly with the inclusion of 1989 . This is because the near-record high amplitude 1989 pattern became available for random selection in the Monte Carlo background distribution-generating QBO phase shuffle process, changing the relative extremeness of the observed patterns. A possible clue to the cause of the strong 1989 exception is found in the January-February 39-year (1951-89) correlation between the SOI (where low SOI values correspond to El Niños or warm episodes) and $700 \mathrm{mb}$ height (Fig. 4). Note that over North America and the eastern Pacific this pattern is quite similar (but of opposite sign) to that of the flux-height correlation field for west QBO phase (Fig. 4), al though elsewhere in the Pacific they differ. One explanation for the 1989 exception is that the cold event of 1988-89 superseded the solar/QBO situation in forcing the January-February North American climate pattern. Hence, the patterns of Figs. 2 and 4 are relatively similar, and Fig. 4 did not change very much as a result of 1989. Inspection of the data reveals that for west QBO phase years in the 195188 period there was a weak negative correlation $(-0.26)$ between the SOI and the flux, and that no year had flux and SOI values both at least 0.5 standard deviations above their means (in 1989 they were above by 1.80 and 1.24 standard deviations, respectively). Before 1989, warm events tended to occur slightly more frequently during high than low flux winters, perhaps bolstering the apparent association between flux and North American climate. After 1989 the S0-flux correlation is largely neutralized $(-0.11)$, presumably having been due to chance.

The global significance of the Fig. 4 SOI versus height correlation field was tested by systematically shifting the phase of the SOI by one month increments to generate a null (or no-effect) distribution. This phase shuffling approach (which generates 468 trials) is designed to preserve most of the autocorrelational features of the SOI (which are weak interannually) in generating the null distribution of integrated t-statistics (Sec. 2) against which to compare the observed integrated $t$ to estimate the field significance. The resulting p-value is 0.004 for the correlation with Northern Hemisphere $700 \mathrm{mb}$ height, providing evidence of stability of the Fig. 4 pattern.

\section{MULTIPLE REGRESSION PREDICTING CLIMATE FROM FLUX AND SOI, FOR WEST QBO PHASE}

In Section 3 it was suggested that during west QBO phase January-Februarys the solar flux and the SOI both help to determine the North American climate, and can either work in the same direction or oppose one another. Their near-independence makes them suitable for a two predictor multiple linear regression model predicting $700 \mathrm{mb}$ height or surface temperature at a given location or over a relevant area.

The result of such a multiple regression is shown in Fig. 5 for JanuaryFebruary $700 \mathrm{mb}$ height and Fig. 6 for U.S. surface temperature, with only the significant ( $p<0.05$ ) SOI (flux) regression coefficients indicated by solid (dashed) contours. The coefficients are normalized; i.e., they are partial correlations with the predictand. Asterisks denote statistically insignificant individual predictor coefficients but a significant regression equation, implying some participation on the parts of both predictors. The stippled areas have $R^{2}$ values of 0.5 or higher.

The correlations with height (Fig. 5) suggest that the SOI has overall a more pervasive effect on the climate, but the solar effect is strong in several 
locations and thus worthy of consideration. Areas that appear to be affected significantly by both factors are (1) the western Atlantic near $30^{\circ} \mathrm{N}$, (2) Canada and (3) North Africa. The correlations with U.S. surface temperature (Fig. 6) indicate significant effects from both SOI and flux in the southeastern U.S. despite the relative domination there by SOI in the heights. It is found that the 22-year west QBO phase sample correlation between the SOI and $700 \mathrm{mb}$ heights (which enters into the present multiple regression) is similar to Fig. 4 (a)l 39 years) except that the southeast U.S. positive center is emphasized at the expense of the Canadian negative center; the correlations with U.S. temperature reflect this feature.

On the basis of the regression results, a general SO/flux-related regional index over North America is defined for illustrative purposes for the west QBO phase years. One "pole" of the index is over Canada, comprising the area 35$120^{\circ} \mathrm{W}, 55-65^{\circ} \mathrm{N}$, and the other is in the southeast U.S./western Atlantic, with area $45-100^{\circ} \mathrm{W}, 25-35^{\circ} \mathrm{N}$. The corners of these areas are shown in Fig. 5 . Within the area of either pole each of the two predictors acts (significantly or subsignificantly) in the same direction, and at some grid points one predictor may overshadow the other. The Aleutian Islands area is not used as a third component of the index because the two predictors combine differently there (the flux affects the heights in the same sense as in Canada, whereas the SOI affects this area oppositely from Canada). The North African area is technically eligible for inclusion in the index, but is ignored in order to concentrate on North America. An appropriate index for the Atlantic-Canadian dipole, based on the corresponding seesaw feature in the relationships of both the SOI and the flux with the heights, is the $700 \mathrm{mb}$ height difference between the Atlantic and the Canadian areas. Either a high flux or a low SOI (warm event) would tend to produce negative difference anomalies (i.e., positive height anomalies in Canada and negative anomalies in the Atlantic), and vise versa.

When the multiple regression model is applied to the Atlantic-Canadian difference index itself, the relationship is of approximately the same strength as near the center of one of the dipole areas. Specifically, $R^{2}=0.43$, the SOI coefficient is 0.46 , the flux coefficient is -0.42 , and the significance p-values for the SOI, the flux, and the regression equation as a whole are $0.008,0.026$ and 0.005 , respectively. The strength of the relationship between the dipole difference index and each of the two predictors is shown in Fig. 10, where the abscissa is the standardized SOI, the ordinate the standardized solar flux, and the body of the figure contains individual year values of the standardized $(X 100)$ dipole height difference index. Values greater than +1 standard deviation are encircled (implying subnormal Canadian heights and/or above-normal southeast U.S./Atlantic heights), and those more negative than -1 standard deviation are enclosed in squares. Dotted enclosures denote greater than three-quarters standard deviation departures. Some broad clustering is apparent, with years having high positive height differences tending toward the lower right portion of the domain. The 1989 value defies this, being in the upper right portion. While the 1989 predictand value might be expected to be much weaker (being forced oppositely by the flux and the SOI), its high positive value can at least be explained by the high SOI value, leaving the suggested relationship with the flux much less damaged than it would be in a univariate regression model based on the flux alone. Note the weak negative correlation between flux and SOI when the 1989 point is removed, and the associated lack of points with low values for both predictors (which still remains after including 1989). 
The $700 \mathrm{mb}$ height difference index should be regarded as an a posteriori predictand in the sense that it was defined on the basis of an exhaustive series of multiple regression experiments using each of 358 grid points of $700 \mathrm{mb}$ height as a predictand.

\section{MULTIPLE REGRESSION PREDICTING CLIMATE FROM FLUX AND SOI, FOR EAST QBO PHASE}

The exercise described in the previous section is now applied to the 17 years of mean January-February data for the east phase of the QBO, using the SOI and the solar flux as predictors for the Northern Hemisphere $700 \mathrm{mb}$ height at each of 358 grid points and the U.S. surface temperature at each of 92 stations. The east phase zero-order correlations between flux and $700 \mathrm{mb}$ height, shown in Fig. 8, emphasize the Pacific 0cean, Arabia, Europe and Mexico rather than the heart of North America as was noted for west phase years. The strength of the currently sampled flux versus height correlation field is stronger than that for west phase, as reflected in the significance levels given in the bottom half of Table 1. The flux versus U.S. temperature correlation field (not shown) is generally weak except for a smal1, intense $(>0.60)$ area in the southern Rockies.

Results of the east phase multiple regression are shown for $700 \mathrm{mb}$ height in Fig. 9, in which we note a significant influence from both predictors in the Kamchatka/Bering Sea area and, to a lesser degree, in the subtropical Pacific just west of the date 1 ine. No regions of the continental U.S. have overlapping significant surface temperature predictability (not shown); moreover, even areas of significant single predictor influence are limited.

A SO/flux-related dipole was defined for the east QBO phase regression results analogous to that for the west phase described in Sec. 4 . One pole is in the Kamchatka/Bering Sea area defined by $150^{\circ} \mathrm{E}-155^{\circ} \mathrm{W}, 45-70^{\circ} \mathrm{N}$, and the other in the subtropical Pacific at $155^{\circ} \mathrm{E}-160^{\circ} \mathrm{W}, 20-25^{\circ} \mathrm{N}$. The corners of these areas are shown in Fig. 9. To the west of both the northern and southern areas there is more area strongly associated with the S0, but this is excluded from the dipole index because of an insufficient strength of association with the flux. The dipole index in this case is the subtropical Pacific area average $700 \mathrm{mb}$ height minus that in the northern area; either a low SOI (an El Niño or warm episode) or a low flux will tend to create high index values during east QBO phase JanuaryFebruary periods.

Application of the multiple regression to the dipole difference index itself results in a relationship of strength comparable to the average of that found near the center of the two poles, which is high in the east phase case. The $R^{2}$ is 0.77 , the SOI and flux normalized coefficients (followed by p-values) are $0.60(0.0004)$ and $-0.62(0.0003)$, respectively, with an overall equation $p$-value of less than 0.0001 . It must be stressed once again that the dipole difference index and its multiple regression significance are highly a posteriori in character and are used for illustrative rather than statistical evaluative purposes. The 3-way multiple regression scatterplot (analogous to Fig. 7) for east phase years, shown in Fig. 10, exhibits a marked tendency for clustering of negative predictand values in the upper right sector (high SOI, high flux) and positive values in the opposite corner. Although the correlation between flux and SOI is 0.02 which quells suspicions that one of the predictors may be redundant with the other, there are relative "holes" in the two high flux corners of the flux-SOI sample space. This leaves the presently sampled relationships subject to some reordering should nonconforming future predictand values appear in the vacant sectors of the predictor plane. 
6. MORE GENERAL STATISTICAL ASSOCIATIONS AMONG FLUX, QBO, SO AND THE CLIMATE.

In this section the statistical relationships among the climate and the three potential predictors (flux, QBO, SO) are examined in a more general way in order to place the regression results of the previous section in an appropriate perspective.

\section{a. Modulation of the effect of the SO on climate by flux and QBO phase}

The effect of the SO on the January-February Northern Hemisphere 700mb height (and, by implication, US surface temperature), illustrated in Fig. 4, was found to be highly statistically significant for height (Sec. 3). We ask whether the solar flux or the $\mathrm{QBO}$ appear to condition the SO-height relation to an extent beyond that of the expected sampling variability. When the hemispheric SOI versus $700 \mathrm{mb}$ height correlation field is recomputed separately for the cases of above and below average solar flux (regardless of QBO phase), differences from the 39-year correlations are statistically insignificant.

When the SOI and $700 \mathrm{mb}$ height data are correlated separately for west and east QBO phase years, results are roughly similar to those of the pooled 39-year analysis of Fig. 4. However, the individual QBO phase results (shown indirectly in Figs. 5 and 9) differ sufficiently for the west phase pattern to resemble the Pacific/North American (PNA) rotated principal component pattern markedly more than the Tropical/Northern Hemisphere (TNH) pattern and the east phase pattern to most strongly resemble the TNH and West Pacific Oscillation (WPO) patterns (Barnston and Livezey 1987). Statistical tests suggest that the QBO may indeed modulate the influence of the SO on the climate. Because the solar flux is not directly involved in this three-way association, details of the latter will not be presented here but are found in Barnston et al (1990).

\section{b. Modulation of flux-climate relationships by so}

The Northern Hemispheric field of correlation between the solar flux and $700 \mathrm{mb}$ height using all 39 years in the 1951-89 period is fairly weak and produces insignificant $p$-values in Monte Carlo significance tests. Results here are in agreement with van Loon and Labitzke (1988) who stated that flux-climate relationships in the troposphere are detectable only for QBO phase-stratified data. It has been shown that stratification of the flux and climate data by QBO phase leads to stronger relationships.

The relationship between QBO phase itself and $700 \mathrm{mb}$ height (regardless of the flux or the SOI), depicted by a composite height anomaly field for either phase, is a globally insignificant, fairly weak and partly fragmented but recognizable version of the vigorous zonally symmetric pattern (with a negative anomaly over the pole for west phase) found by Holton and Tan (1980) in a similar analysis for the stratosphere.

When stratification of the flux-climate data by low versus high SOI is carried out, resulting correlation fields are fairly similar to the overall field and differences between the two are statistically insignificant.

The final idea explored is that of the SO as a mediator within the QBO phasestratified relationships between flux and hemispheric $700 \mathrm{mb}$ height. The west and east QBO phase data were further stratified into 7-member samples of highest versus lowest SOI, and flux-height correlation fields were computed for each of 
the four groups. The flux ranges for each of the groups are minimally adequate (there is never a lack of low or high flux values) but poorly sampled. A systematic mediating role of the SOI in QBO phase-stratified flux-climate associations is not apparent from either of the two sets of small sample comparisons.

\section{SUMMARY AND DISCUSSION}

The strong and well-defined relationship between the 11 -year $10.7 \mathrm{~cm}$ solar flux cycle and the lower troposphere Northern Hemisphere January-February climate for QBO phase-stratified samples (van Loon and Labitzke 1988, Barnston and Livezey 1989) failed for the west QBO phase in 1989. In this report the opposing 1989 event is explained, at least in part, on the basis of the phase of the so (the cold tropical Pacific SST event of 1988-89). It is demonstrated that both the SO and the solar flux have moderate and quasi-independent correlations with the climate over certain regions, and where there is strong overlap they can work either in harmony or in opposition. In 1989 in North America the influences of the SO and the flux conflicted to an unprecedented extent, and the SO was the controlling influence in most regions of the continent (western Canada being one exception). The 1989 event draws attention to the smallness of the QBO phasestratified samples and the still more serious "holes" in the two-dimensional sample space of flux and SO when both factors are viewed as predictors within one QBO phase.

Bivariate linear regression equations were developed separately for each QBO phase using the SOI and the solar flux as predictors, and each of several hundred grid point values of $700 \mathrm{mb}$ height and 92 stations of U.S. surface temperature as predictands. An a posteriori-developed height dipole difference index was also used as a predictand. Within each of the two QBO phase conditions, the flux and the SOI are approximately independent. The spatial distributions of the numerous significant regression coefficients for the flux and so predictors in the prediction of height or temperature help indicate where each predictor is of value and should enable more skillful overall forecasts to be made than would be possible using onty one of the predictors. These spatial patterns may aid in the search for physical underpinnings for the solar effect with its currently unexplainable dependence on the phase of the $\mathrm{QBO}$. A more general exploration of statistical associations among the solar flux, the $\mathrm{QBO}$, the $\mathrm{SO}$ and the climate was pursued to help complete the description created by the regression equations. No noteworthy relationships were uncovered except for a suggestion of a preference for a PNA (TNH) $700 \mathrm{mb}$ circulation pattern in response to the SO in the west (east) QBO phase, the details of which are forthcoming in a separate paper by the present authors. The effect of the SO on the climate was found to remain broadly the same regardless of the flux level.

A major question underlying this study is that of the authenticity of the influence of the solar cycle on the climate. The west phase failure of 1989 can be explained partly by the So situation. The part that cannot be readily explained is why the effects of the So were so prominent and those of the solar situation so weak. The solar effects in the stratosphere in January-February 1989 were much more in keeping with the previously established relationship than in the tropos-phere ( $K$. Labitzke, personal communication; Fig. 10 of van Loon and Labitzke 1990). Perhaps this suggests that the solar effects work initially or most directly in the stratosphere and secondarily or indirectly in the troposphere where So effects would dominate when forced strongly. If the solar-climate effects are nonlinear, then the present regression approach would underestimate the strength of the association. 
The most prohibitive barrier to establishing the strength of a solar-climate association is the severe sampling problem brought about by (1) the need to stratify by QBO phase, effectively halving the sample sizes, (2) the need to use two predictors rather than one (as demonstrated by the strong effect of the SO in 1989), and (3) the somewhat asymmetric distribution of the flux with its relatively infrequent but large excursions above its mean. Monte Carlo statistical testing can largely overcome the spatial and temporal dependence problems that violate the assumptions of classical statistical assessment tools, but it cannot help at all with insufficient sampling. Because of the small sample size, the truth (i.e., population statistic) about the solar-climate association must remain unknown except for the annual updates of sample associations with fairly wide error bars. In 40 years there will be a doubling of the present sample sizes and a clearer picture of the relationships, coupled with a hopefully greater understanding of the physical mechanisms through which the solar flux may affect the tropospheric climate.

In the meantime, the apparent effects may be cautiously accepted and used in a statistical mode to help develop operational winter long-range forecast tools at the $\mathrm{Cl}$ imate Analysis Center (CAC). Such efforts are already in progress, both in an analog system (Livezey and Barnston 1988, Barnston and Livezey 1989) and in a regression scheme as shown here.

\section{REFERENCES}

Barnston, A. G. and R. E. Livezey, 1987: Classification, seasonality and persistence of $10 w$-frequency atmospheric circulation patterns. Mon. Wea. Rev., $115,1083-1126$.

Barnston, A. G. and R. E. Livezey, 1989: An operational multifield analog prediction system for United States seasonal temperatures. Part II: Spring, summer, fall and intermediate three-month period experiments. J. climate, 2 , 513-541.

Barnston, A. G. and R. E. Livezey, 1989: A closer look at the effect of the 11year solar cycle and the quasi-biennial oscillation on Northern Hemisphere 700 $\mathrm{mb}$ height and extratropical North American surface temperature. J. Climate, 2, $1295-1313$.

Barnston, A. G., R. E. Livezey and M. S. Halpert, 1990: Modulation of Southern Oscillation-Northern Hemisphere mid-winter climate relationships by the QBO. J. Climate, $\underline{3}$, submitted.

Holton, J.R. and H. Tan, 1980: The influence of the equatorial Quasi-Biennial Oscillation on the global circulation at $50 \mathrm{mb}$. J. Atmos. Sci., 37, 22002208.

Labitzke, K., 1987: Sunspots, the QBO, and the stratospheric temperature in the north polar region. Geophys. Res. Lett., 14, 535-537. , and H. van Loon, 1988: Association between the 11-year solar cycle, the $Q B O$, and the atmosphere. Part I: The troposphere and stratosphere on the Northern Hemisphere winter. J. Atmos. Ter. Phys., 50, 197-206. , and 1989: Association between the 11-year solar cycle, the QBO, and the atmosphere. Part III: Aspects of the association. Climate, 2, 554-565.

Livezey, R. E., and W. Y. Chen, 1983: Statistical field significance and its determination by Monte Carlo techniques. Mon. Wea. Rev., 111, 4659.

Livezey, R. E. and A. G. Barnston, 1988: An operational multifield analog prediction system for Unites States seasonal temperatures. Part I: System design and winter experiments. J. Geophys. Res., 93, 10,953-10,974. 
van Loon, H., and K. Labitzke, 1988: Association between the 11-year solar cycle, the QBO, and the atmosphere. Part II: Surface and $700 \mathrm{mb}$ in the Northern Hemisphere in winter. J. Climate, 1, 905-920.

van Loon, H., and K. Labitzke, 1990: Association between the 11-year solar cycle and the atmosphere. Part IV: The stratosphere, not grouped by the phase of the QBO. J. Climate, $\underline{3}$, In Press.

\begin{tabular}{|c|c|c|c|c|}
\hline \multicolumn{5}{|c|}{$1951-88$} \\
\hline & \multicolumn{2}{|c|}{$700 \mathrm{mb}$ hejaht } & \multicolumn{2}{|c|}{ U.S. Surface Temperature } \\
\hline $0 \mathrm{BO}$ Phase & $\frac{\text { QBO }}{\text { Shuffle }}$ & Flux Phase/Shape & $\frac{\text { QBO }}{\text { Shuffle }}$ & Flux Phase/Shape \\
\hline & & & & \\
\hline West $(N=21)$ & .003 & .12 & .07 & .018 \\
\hline $\begin{array}{l}\text { East }(N=17) \\
\text { Joint }(21,17)\end{array}$ & .025 & .019 & .41 & .07 \\
\hline $\begin{array}{l}\text { Joint }(21,11) \\
\text { Pooled }(N=38)\end{array}$ & .001 & $\overline{.52}$ & .033 & $\overline{.26}$ \\
\hline
\end{tabular}

1951-89

\section{QBO Phase}

West $(\mathrm{N}=22)$

East $(N=17)$

Joint $(22,17)$

Pooled $(N=39)$
$700 \mathrm{mb}$ height

$\frac{\text { QBO }}{\text { Shuffle }} \frac{\text { Phase }}{\text { Flux }} \frac{\text { Phase/Shape }}{\text { Shuffle }}$

$\begin{array}{ll}.14 & .23 \\ .053 & .019 \\ .055 & .41\end{array}$

U.S. Surface Temperature

$\frac{\text { QBo }}{\text { Shuffle }} \frac{\text { Fhase }}{\text { Shuffle }}$

.12
.28
.044
-
.39

.07

.45

Table 1. Comparison of field significance with and without 1989 for January-February correlation fields for solar flux versus Northern Hemisphere $700 \mathrm{mb}$ height or U.S. surface temperature, using both a QBO phase assignment shuffle and a solar fiux phase/shape shuffle Monte Carlo significance test. Results are for west and east QBO phases, for the joint result of the set of two individual phase results, and for pooled (unstratified) samples. 


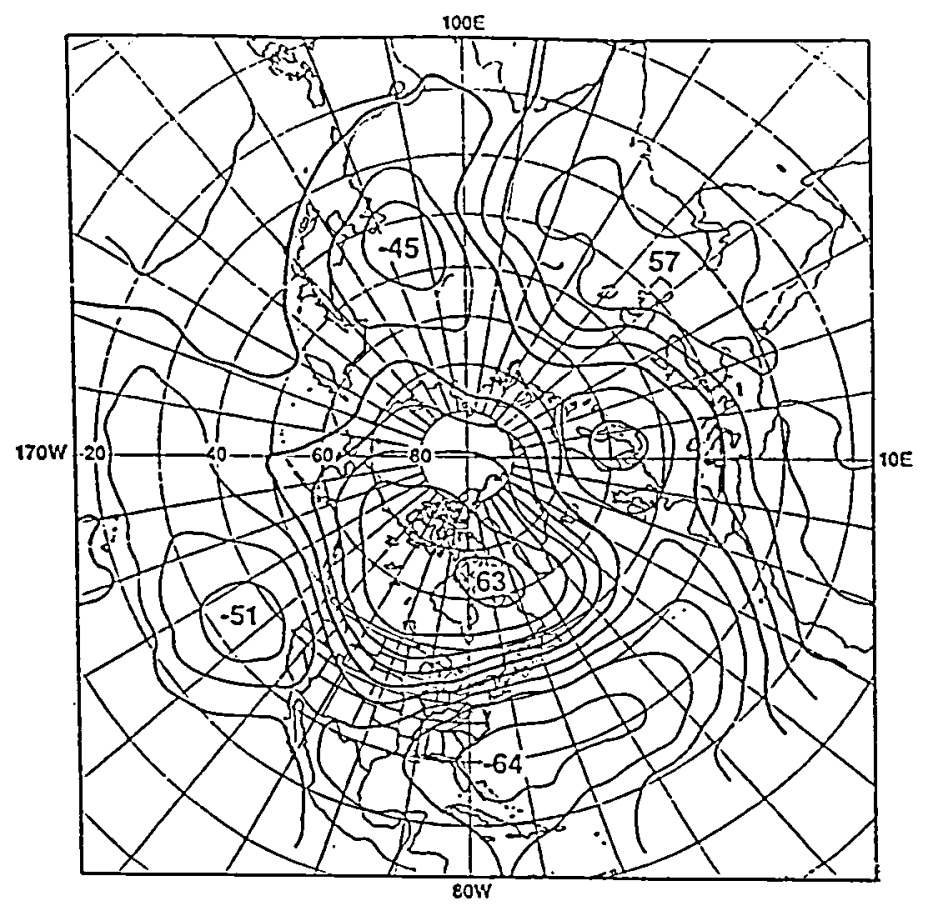

FIG. 1. Correlation $(X 100)$ between $10.7 \mathrm{~cm}$ solar flux and $700 \mathrm{mb}$ height for the west QBO phase for the January-February period, 1951-88 (21 years). Contour interval 15.

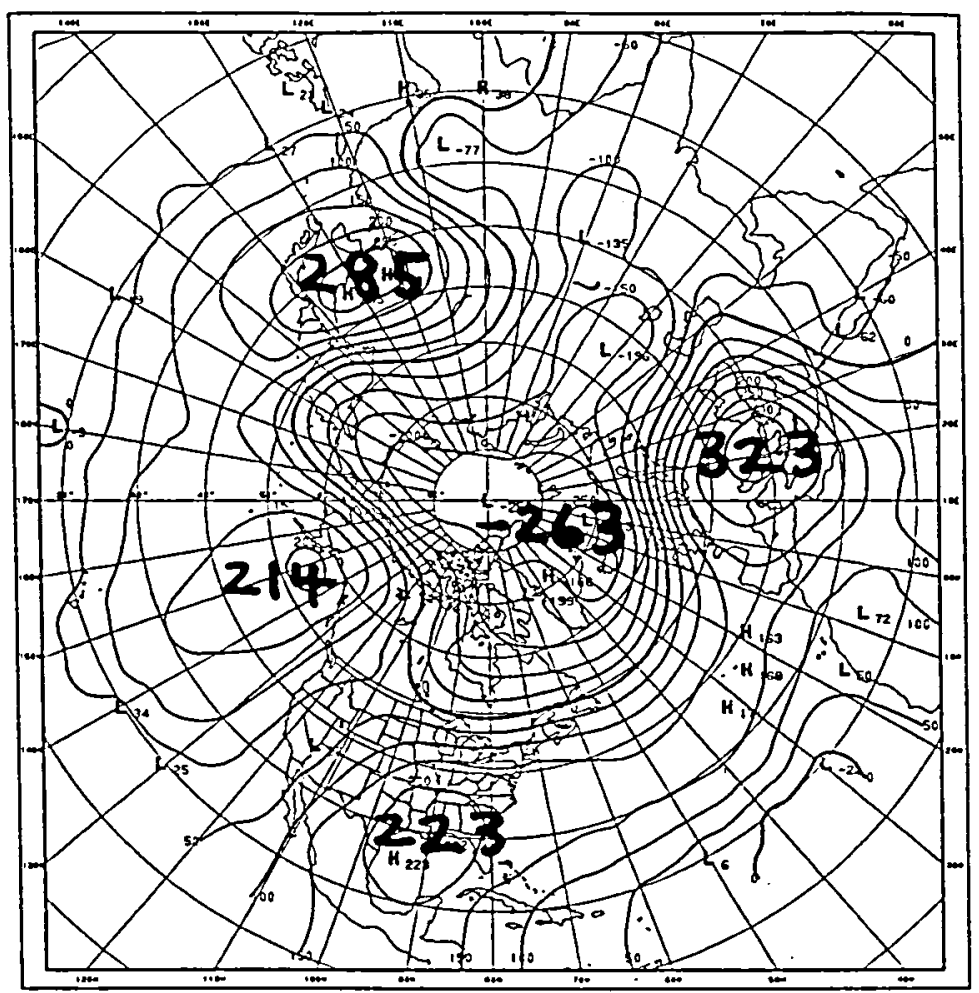

FIG. 2. Standardized anomalies (X100) of the $700 \mathrm{mb}$ height for January-February 1989 


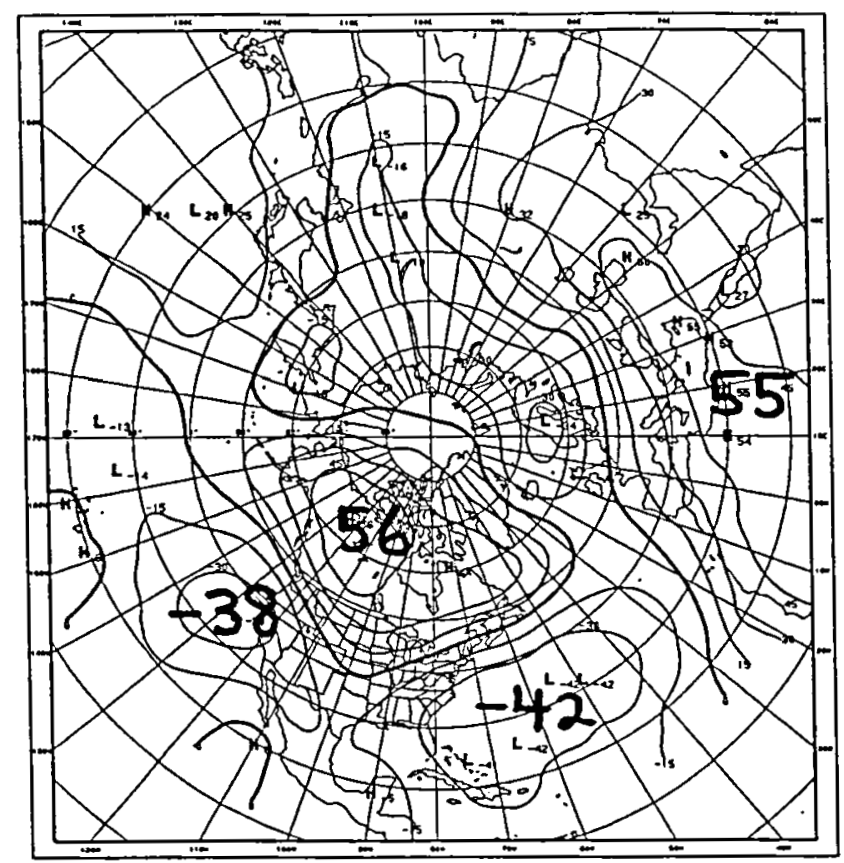

FIG. 3. As in Fig. 2 (west QBO phase), except for 1951-89 (22 years).

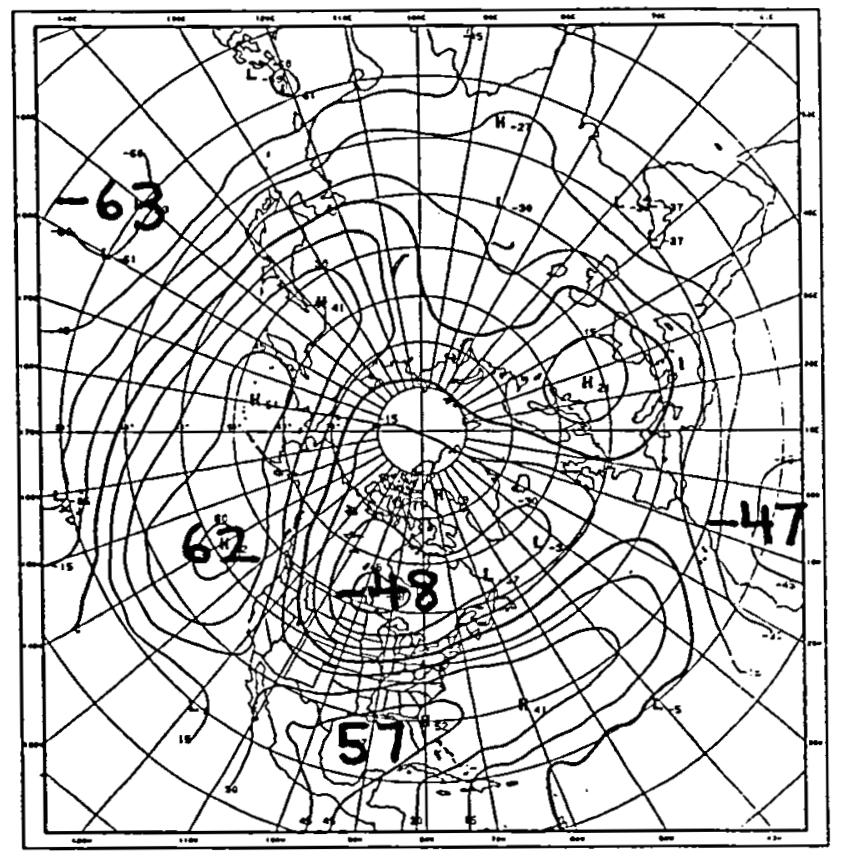

FIG. 4. Correlation (X100) between a Southern Oscillation Index (SOI; based on both Tahiti minus Darwin SLP and on SST in area $120-160^{\circ} \mathrm{W}, 10^{\circ} \mathrm{N}-10^{\circ} \mathrm{S}$ ) and 700mb height for the January-February period, 1951-89 (39 years). 


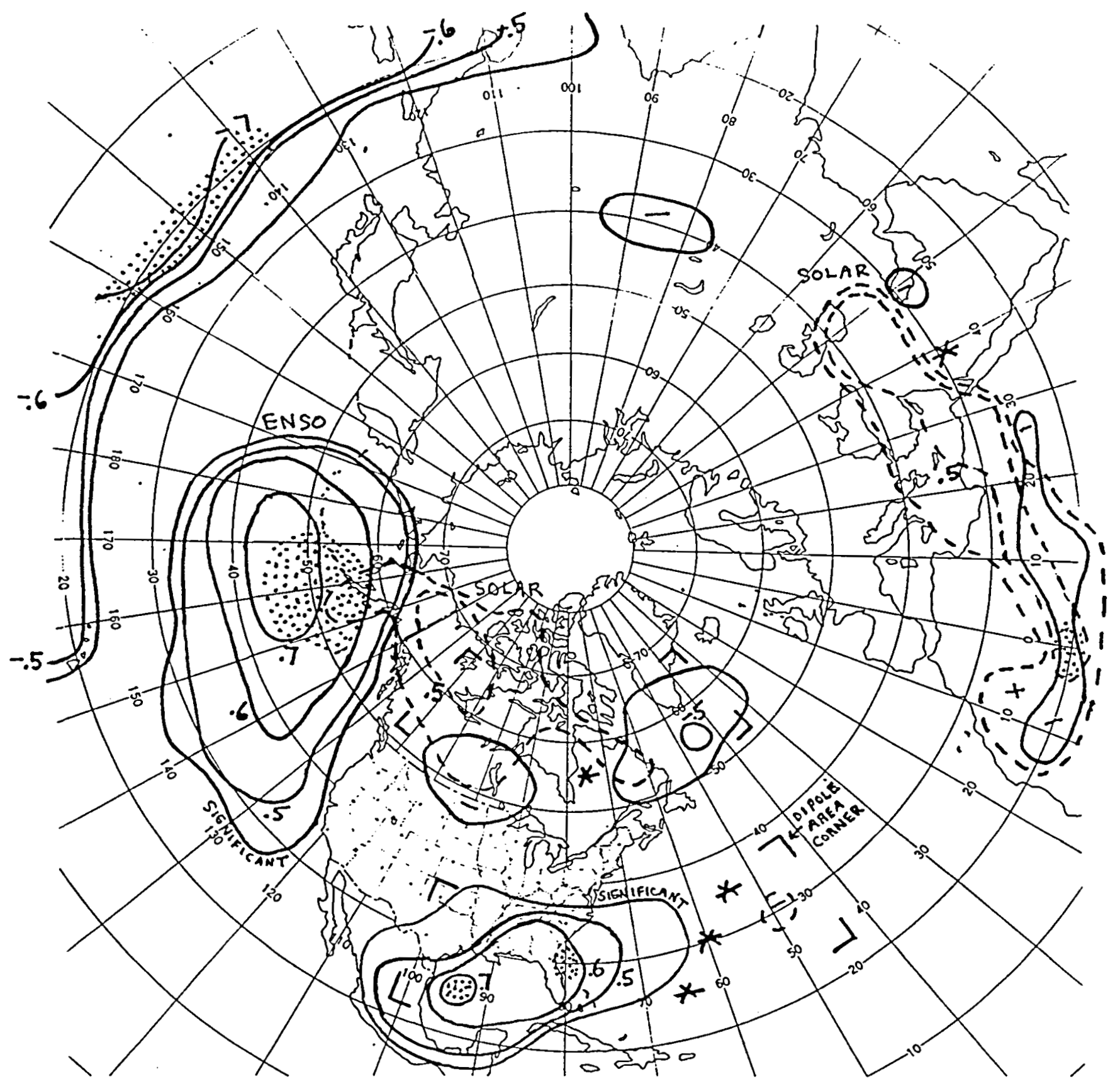

FIG. 5. Two predictor multiple linear regression predicting January-February $700 \mathrm{mb}$ height at 358 grid points from (1) a Southern 0scillation Index (SOI) and (2) $10.7 \mathrm{~cm}$ solar flux, for the 22 west QBO phase years from 1951 to 1989. Normalized regression coefficients (i.e., partial correlations) are plotted only if significant $(p<.05)$; solid contours are for SOI, dashed contours for flux. Stippling denotes $R^{2}$ values of 0.5 or higher, and the asterisk indicates that the individual predictor coefficients are insignificant but the equation is significant. The corners of the two rectangular areal poles of an SOI/flux-related dipole (see text) are shown. 


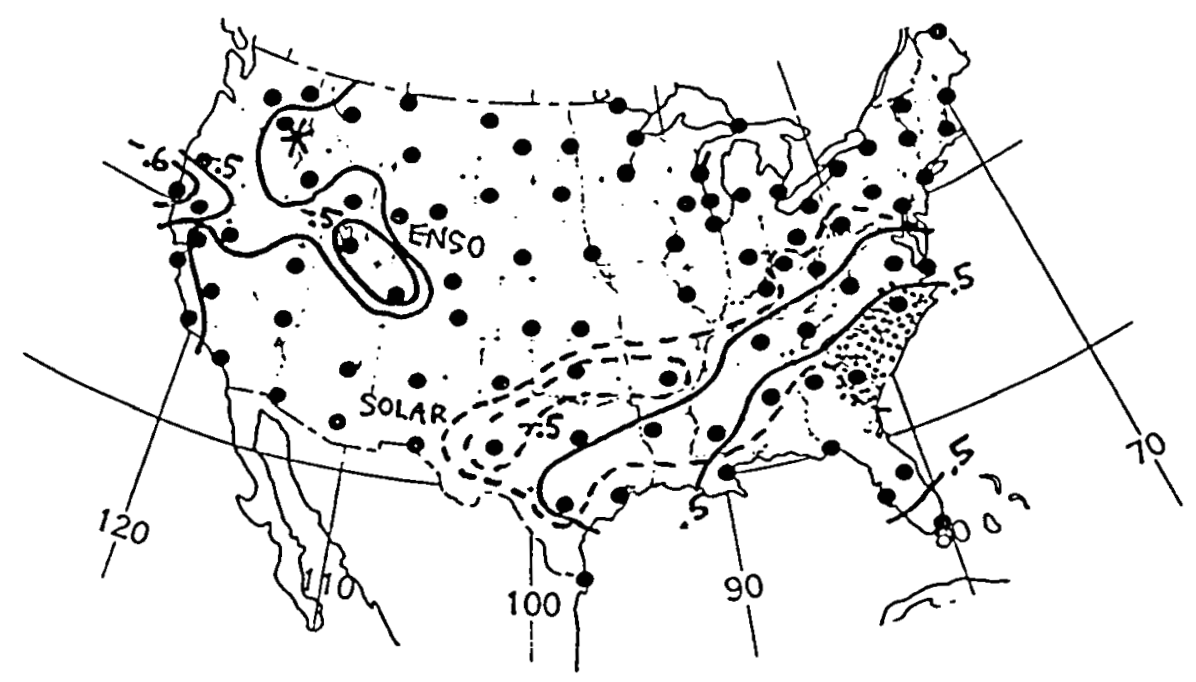

FIG. 6. As in Fig. 5 (west QBO phase multiple regression) except for 92 stations of U.S. temperature (22 years).

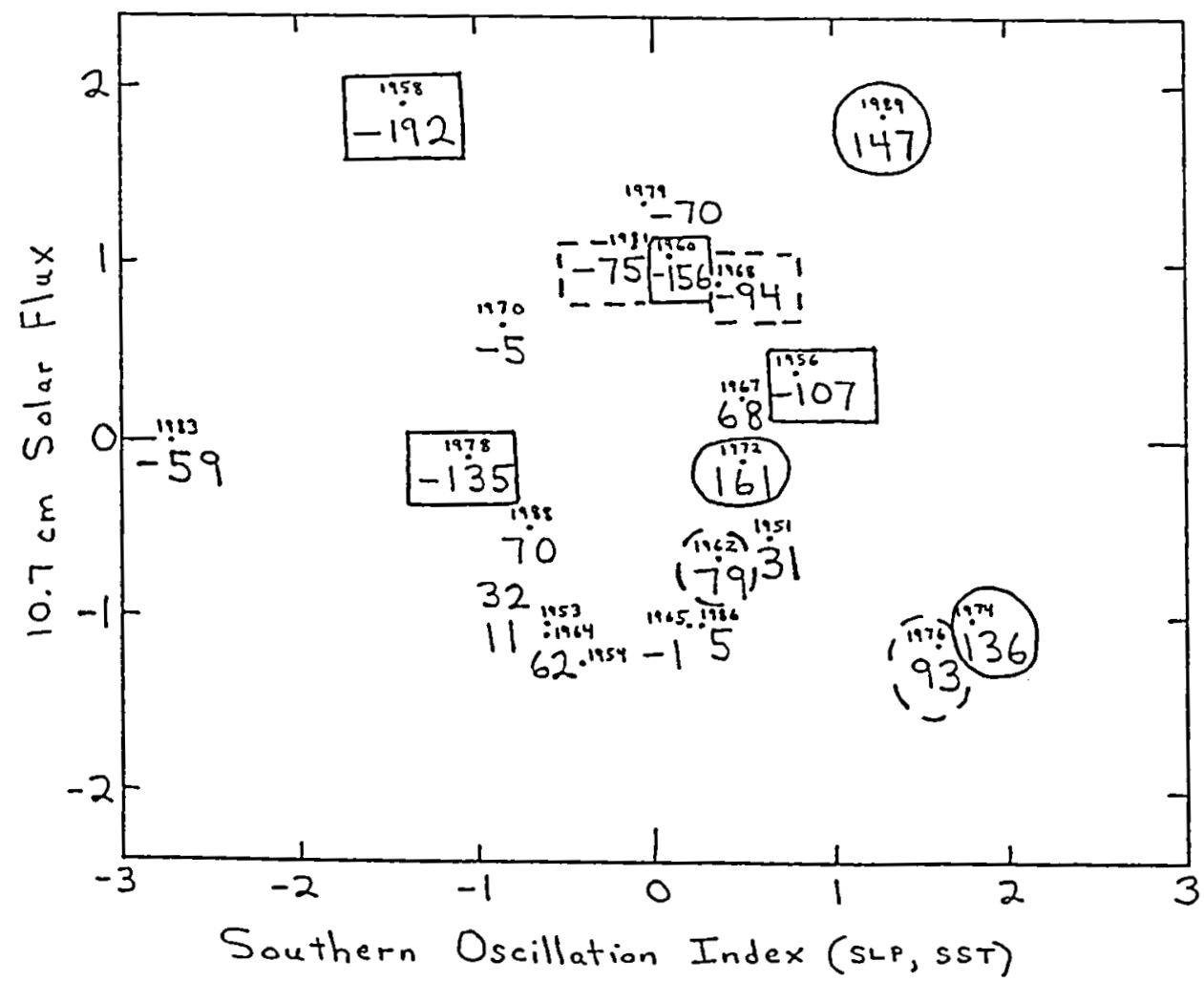

FIG. 7. Three-way individual year scatter plot for west QBO phase multiple regression predicting the $700 \mathrm{mb}$ height dipole difference anomaly (southeast U.S./ western Atlantic minus Canada), shown by printed value in body of figure (with year shown) using as predictors the SOI (abscissa) and the solar flux (ordinate). All variables are standardized; predictand values are also multiplied by 100 . 


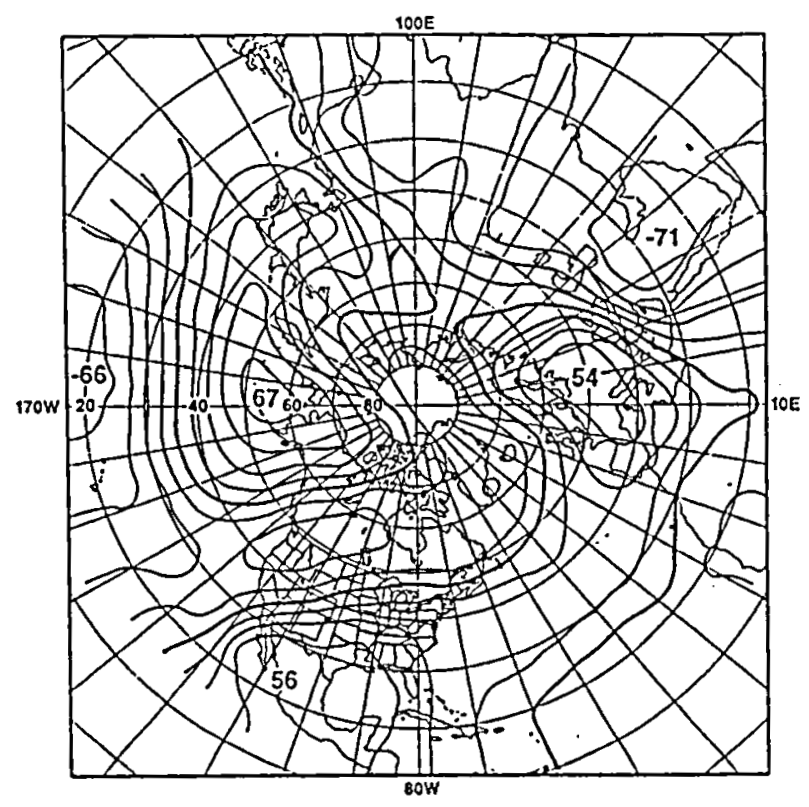

FIG. 8. As in Fig. 1 except for the east QBO phase for 1951-89 (17 years). (FIG. 9 is on following page.)

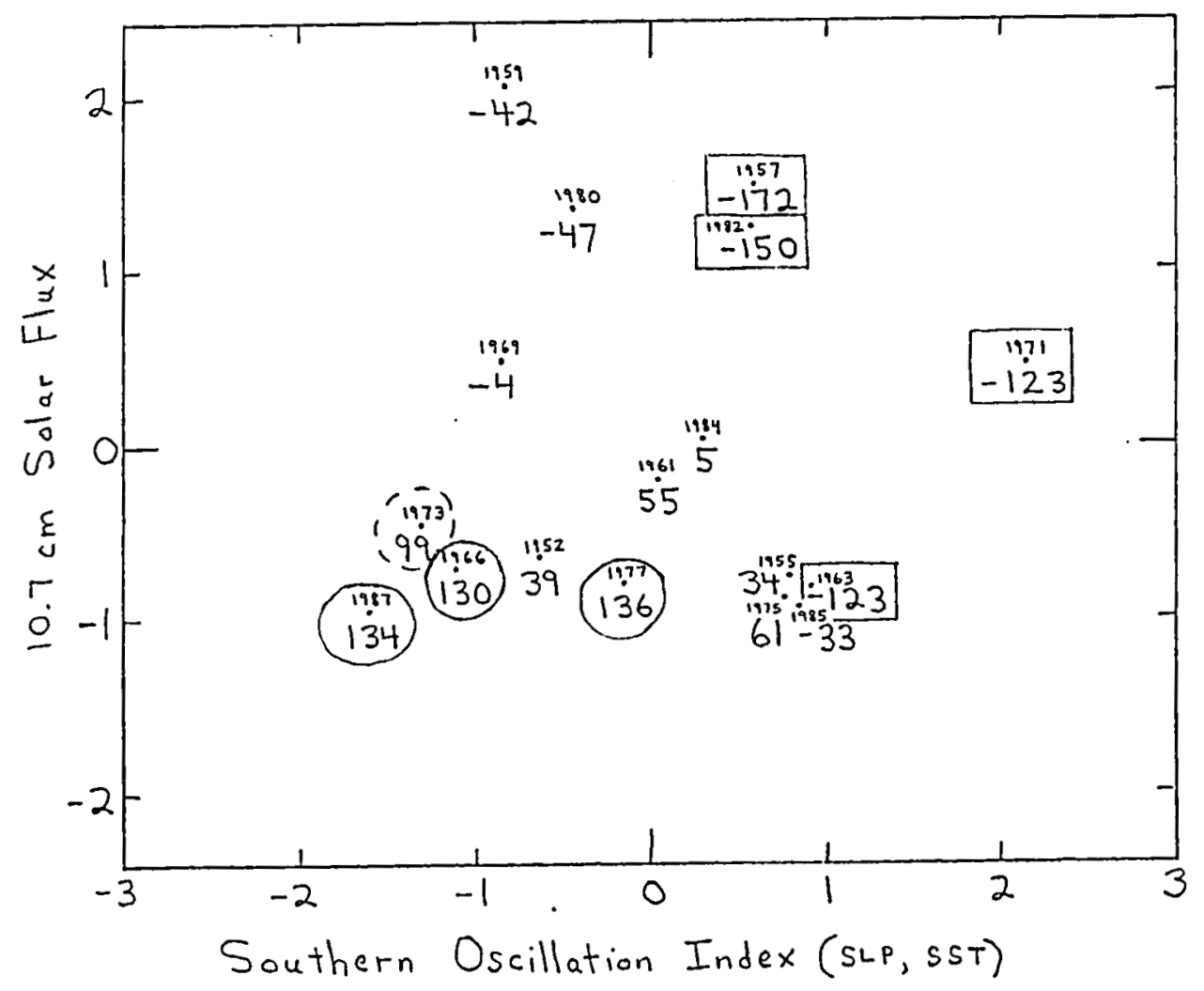

FIG. 10. As in Fig. 7 except for the east QBO phase with its $700 \mathrm{mb}$ height dipole difference anomaly (subtropical Pacific minus Kamchatka/Bering Sea).

(Fig. 9 is on following page.) 


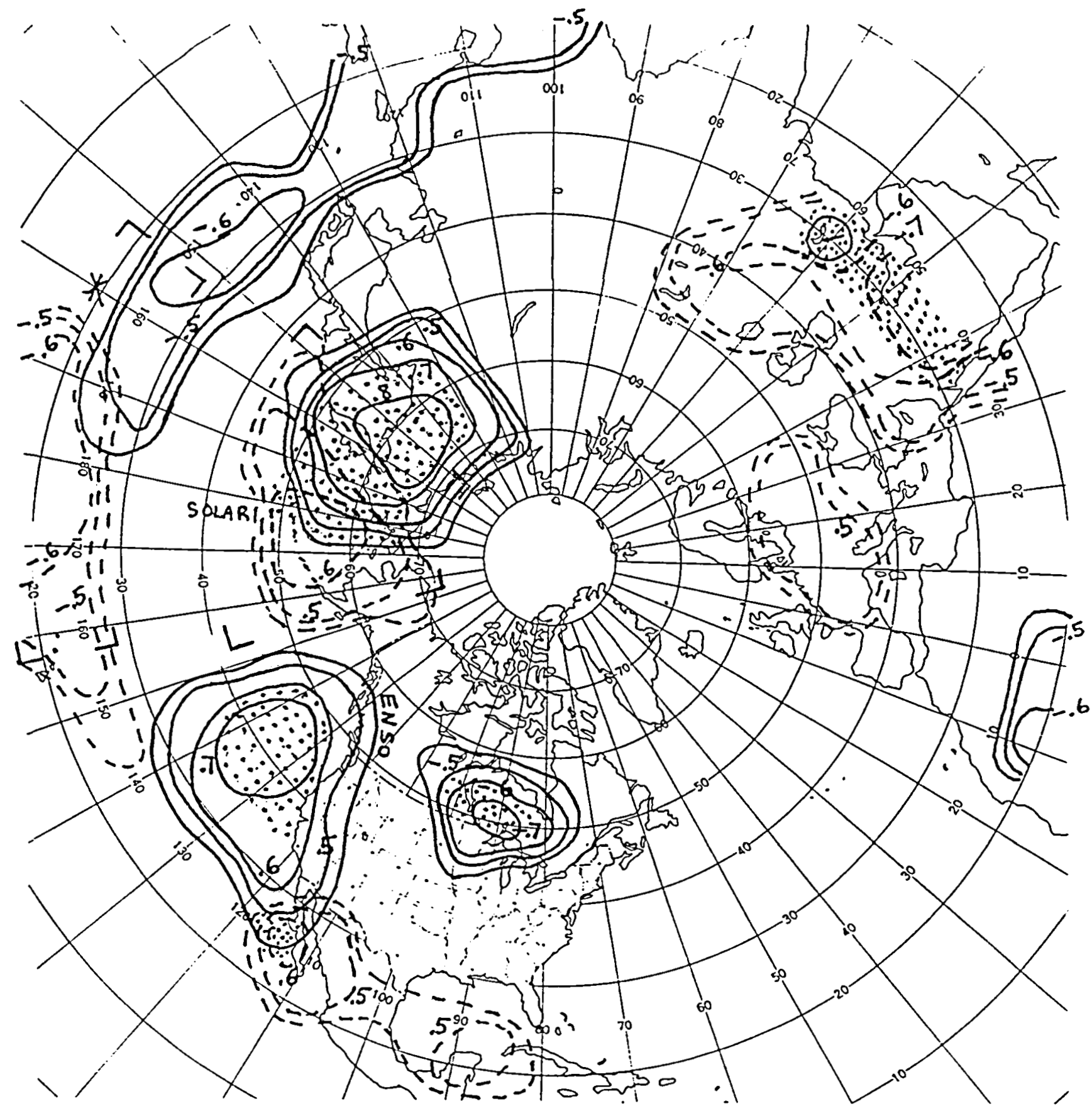

FIG. 9. As in Fig. 5 (multiple regression) except for the 17 east QBO phase years. (FIG. 10 is on previous page..) 


\title{
A MECHANISM INVOLVING SOLAR ULTRAVIOLET VARIATIONS FOR MODULATING THE INTERANNUAL CLIMATOLOGY OF THE MIDDLE ATMOSPHERE
}

\author{
L. L. Hood and J. L. Jirikowic \\ Lunar and Planetary Laboratory, University of Arizona \\ Tucson, Arizona 85721 USA
}

\begin{abstract}
In years of low solar activity, free traveling wave modes in the upper stratosphere are dominated by atmospheric normal modes such as the 16-day wave. However, within a 4year interval centered on the 1980-81 solar maximum, cross-spectral analyses of zonal mean satellite temperature data versus the solar UV flux demonstrate significant power near 27 and 13 days, providing indirect evidence that short-term UV variations were capable of exciting traveling planetary-scale waves in the upper stratosphere. Previous theoretical and observational work has indicated that interference between traveling waves and stationary waves forced from below (and the resulting oscillating latitudinal heat transports) plays a likely role in the initiation of stratospheric warmings. We therefore hypothesize that the initiation of a major stratospheric warming in the upper stratosphere and lower mesosphere may depend to some extent on the amplitude of longer-period 27-day traveling waves in the upper stratosphere. This would represent a new mechanism for solar UV effects on stratospheric climatology that may be relevant to the interpretation of some recent longterm correlative results.
\end{abstract}

\section{INTRODUCTION}

Labitzke [1982] originally noted a tendency for the occurrence of major mid-winter warmings in the northern hemisphere to depend on (a) the phase of the equatorial quasibiennial oscillation (QBO) at 50 mbar and (b) the 11-year solar activity cycle. Labitzke and van Loon [1989] have reported a resulting correlation between January-February average north polar temperature at 30 mbar during QBO west winters (i.e. when 50 mbar equatorial winds are westerly) and the solar $10.7 \mathrm{~cm}$ flux. The latter authors also found that $\mathrm{QBO}$ west years with major mid-winter warmings have occurred only during periods of relatively high solar activity (F10.7 > 160 units) since the mid-1950's when QBO records were begun. Although Baldwin and Dunkerton [1989] and others have argued that the statistical significance of these correlations may not be as high as calculated by Labitzke and van Loon (1989), the preference of major warming years for times of high 10.7 $\mathrm{cm}$ flux during the QBO west phase seems clear from their work. In addition, van Loon and Labitzke (1990) have more recently found a consistent pattern of correlation between 30 mbar temperature (or geopotential height) and the $10.7 \mathrm{~cm}$ flux for separate 2-month epochs in their 31-year data set without sorting according to QBO phase.

One aspect of solar activity changes that may influence thermal and dynamical processes in the middle atmosphere is solar ultraviolet variability through its effects on ozone concentration and radiative heating. The solar flux variation near $200 \mathrm{~nm}$ is important 
for producing changes in molecular oxygen photodissociation (hence, ozone production) and is therefore most relevant for the middle atmosphere. According to available satellite measurements [e.g., Rottman, 1988], the solar flux variation at $205 \mathrm{~nm}$ (usually chosen as a reference wavelength) consists of a long-term solar cycle variation of order 5 to $7 \%$ on which is superposed short-term (mainly 27-day) variations associated with the rotation of active regions on the solar disc. Near solar maximum, the amplitude of the short-term variations exceeds five per cent and is comparable to the solar cycle variation amplitude. Near solar minimum, the amplitude of the short-term variations is typically near one per cent.

Most previous models for effects of solar UV variations on stratospheric dynamics have considered only the solar cycle variation of UV spectral irradiance. In these models, solar cycle changes in ozone (and resulting latitudinal changes in radiative heating) modify the stratospheric zonal wind field. Changes in the zonal wind field then alter the transmissivity of the stratosphere to upwardly propagating planetary waves [Hines, 1974; Springer, 1983]. Numerical studies have indicated that solar cycle UV effects on planetary wave propagation are significant in the upper stratosphere but become negligible below 10 mbar for plausible solar cycle changes in UV flux [Callis et al., 1985]. In this paper, we suggest that the fluctuating component of solar UV variations (occurring mainly on the 27-day time scale) may also have significant perturbing effects on stratospheric dynamics that may be relevant to the long-term statistical results of Labitzke and van Loon. In particular, on the basis of a cross-spectral analysis of zonal mean ozone and temperature measurements versus the solar $205 \mathrm{~nm}$ flux near the 1980 solar maximum, it is suggested that short-term solar UV variations may be capable of exciting or modulating traveling planetary-scale waves in the upper stratosphere. These waves may then increase the probability of occurrence of major stratospheric mid-winter warmings at high northern latitudes under solar maximum conditions.

\section{OBSERVATIONAL BACKGROUND}

Previous work on the stratospheric photochemical and radiative heating effects of short-term solar UV variations has shown that the ozone response at low latitudes maximizes near 3 mbar (about $40 \mathrm{~km}$ altitude) and amounts to about $0.5 \%$ for each $1 \%$ change in the solar flux at wavelengths near $200 \mathrm{~nm}$ [Hood and Cantrell, 1988; Keating et al., 1987; and references therein]. Variations in the solar $205 \mathrm{~nm}$ flux are as large as 6-7\% on the 27 -day time scale near solar maximum so that peak-to-peak ozone mixing ratio changes of as much as $3 \%$ near 3 mbar are observed. The corresponding ozone column changes are in all cases much less than $1 \%$. Zonal mean temperature perturbations at low latitudes in the upper stratosphere derived from satellite radiances have been found to be weakly correlated $(\mathrm{R} \simeq 0.3$ ) with solar ultraviolet variations (Keating et al., 1987; Hood and Cantrell, 1988). The temperature response maximizes near the stratopause and amounts to about $0.06 \%(\sim 0.16 \mathrm{~K})$ for a $1 \%$ change in the solar flux near $200 \mathrm{~nm}$. Actual temperature changes are as large as $1 \mathrm{~K}$ on the solar rotation time scale. The mean temperature phase lags are large, ranging from about 6 days near 1 mbar to about 13 days near 10 mbar. Hood and Douglass [1988] have shown that the observationally derived coupled ozone and temperature responses to short-term solar ultraviolet variations are consistent with the 
odd oxygen continuity equation using currently accepted photochemistry.

Earlier comparisons of theoretical models with observationally derived ozone and temperature responses to solar ultraviolet variations have suggested that a dynamical component of the stratospheric response may be necessary [Hood, 1987; Brasseur et al., 1987]. In particular, the measured temperature phase lags (e.g., 6-9 days at 2 mbar) are larger by a factor of about 2 than expected if radiative heating changes alone are responsible. Ebel et al [1988] have applied a global three-dimensional numerical circulation model to show theoretically that dynamical effects in the middle and lower stratosphere may be produced by weak perturbations at stratopause heights.

Further evidence of the existence of a dynamical component of the upper stratospheric response is obtained by considering the latitude dependence of the ozone and temperature fluctuations and their relation to solar ultraviolet variations. Figure 1 shows an example of 1.5 mbar ozone and temperature deviations for a 7 -month interval compared to the Nimbus 7 solar backscattered ultraviolet (SBUV) solar $205 \mathrm{~nm}$ flux. This interval was chosen because it was characterized by relatively strong and regular 27-day solar ultraviolet variations. All time series were detrended to remove periods greater than about 35 days. At low latitudes, the behavior is generally consistent with the ozone and temperature response measurements summarized above. For instance, between $20^{\circ} \mathrm{S}$ and $20^{\circ} \mathrm{N}$, ozone maxima tend to occur approximately in phase with solar ultraviolet maxima. Temperature variations (dashed lines) also show an approximate 27-day periodicity but occur at substantial lags following the UV flux maxima, as expected from the temperature response measurements. However, at approximately $30^{\circ}$ to $40^{\circ}$ latitude in the winter hemisphere, both the ozone and temperature variations begin to change phase and, by $60^{\circ} \mathrm{N}$, are nearly $180^{\circ}$ out of phase with corresponding fluctuations at low latitudes.

As shown, for example, by Chandra (1985; 1986), out-of-phase temperature oscillations between low and higher latitudes are a common characteristic of the winter hemisphere upper stratosphere even in the absence of solar ultraviolet variations. They are also a common property of temperature changes in the middle and lower stratosphere, including those associated with mid-winter warmings (Fritz and Soules, 1970; van Loon et al., 1975). These oscillations are typically driven by the interaction of stationary upward propagating planetary waves with free traveling Rossby wave modes under the influence of tropospheric forcing (see below and the reviews by Madden [1979] and by Salby [1984a,b]). However, the phase relationships between ozone, temperature, and solar ultraviolet variations evident in Figure 1 suggest also a possible role of solar UV variations during this time period under solar maximum conditions.

Figure 2 summarizes results of a Box-Jenkins cross spectral analysis of the 4 available years of 2 mbar SAMS temperature data averaged between $20^{\circ} \mathrm{S}$ and $20^{\circ} \mathrm{N}$ versus the SBUV solar $205 \mathrm{~nm}$ flux (F205). This analysis is a desirable supplement to earlier cross correlation results for the same data [e.g., Hood and Cantrell, 1988] which did not rigorously determine the statistical significance of the reported correlations. As shown in the top panel, the F205 autospectrum (solid line) contains a large maximum near 27 days and a secondary maximum near 13 days. The 13-day periodicity is not a simple harmonic of the 27-day period but represents an actual forcing component arising when active regions tend to cluster in opposite hemispheres on the solar disk [Donnelly et al., 1986]. The 27-day 
period arises when active regions tend to cluster in a single hemisphere. The averaged SAMS temperature autospectrum (dashed line) exhibits a broad maximum centered on approximately 27 days and a secondary maximum at a period near 16 days (frequency 0.062 days $\left.^{-1}\right)$. The latter period corresponds to the second symmetric $(1,3)$ free Rossby wave mode that is prominently observed in the upper stratosphere [Hirooka and Hirota, 1985]. The largest maximum at $\sim 25-29$ days period appears to be a possible result of solar ultraviolet forcing during this 4-year interval. A visual inspection of Figure 1 confirms the tendency for temperature maxima to occur at $\sim 25$-30 day intervals within the 7 -month time section shown. The second panel shows the coherency square statistic with two maxima exceeding $95 \%$ confidence at periods of 24-28 days and at $\sim 12.5$ days. The derived phase lag (lower panel) is large as expected from the correlative results of Hood and Cantrell [1988]. At a period of 25 days where the coherency square reaches a maximum, the phase lag is $8 \pm 2$ days. Similar applications to other SAMS pressure levels yield coherency maxima that are positive at $95 \%$ confidence between 1 and 3 mbar. The phase lags for the $\sim 27$-day maximum increase with decreasing altitude in agreement with the correlative results of Hood and Cantrell [1988].

Given that statistical evidence exists for a relation between solar ultraviolet variations on the solar rotation time scale and temperature variations in the low-latitude upper stratosphere, it is next of interest to see whether the low-latitude temperature variations are related to those at higher latitudes. Figure 3 shows results of a cross-spectral analysis of the low-latitude average temperature variations versus those averaged for latitudes of $40^{\circ} \mathrm{N}$ to $60^{\circ} \mathrm{N}$ for the October to March epoch of the 4 available years of SAMS data. The autospectra in the top panel show maxima near periods of 25 days and 16 days; these maxima are largest for the higher-latitude data as expected from the increased amplitudes of winter temperature fluctuations at higher latitudes (e.g., Figure 1). The coherency square statistic plotted in the center panel of Figure 3 shows two broad maxima at periods of 23-27 days and $~ 13.5$ days that are positive at $95 \%$ confidence; in contrast, the 16-day period has no significant coherency maximum between the chosen latitude bands during this 4-year time period. The phase plotted in the lower panel is centered on $180^{\circ}$ for both the $\sim 25$-day and the $\sim 13$-day periods, confirming the qualitative interpretation of Figure 1 given above. Since the coherency square between low and higher latitude temperature fluctuations is significantly positive preferentially near 27 and 13 days (Figure 3), and since the low-latitude fluctuations are significantly coherent with F205 (Figure 2), it may be inferred that solar ultraviolet variations are either forcing or modulating latitudinal temperature oscillations in the upper stratosphere during the time interval of this study. Similar applications to other pressure levels between 1 and $3 \mathrm{mbar}$ yield qualitatively similar results.

\section{INTERPRETATION AND PHYSICAL MECHANISMS}

One way of interpreting the occurrence of out-of-phase latitudinal temperature oscillations is in terms of corresponding oscillations in the zonal mean wind field at middle latitudes. As indicated qualitatively in Figure 4 (see also Figure 6.7 of Andrews et al. [1987]), a decrease in the zonal wind velocity $(\mathrm{d} \bar{u} / \mathrm{dt}<0)$ induces a poleward meridional circulation, due mainly to coriolis torques (an easterly flow is deflected northward in the 
northern hemisphere). By mass conservation, the poleward flow results in upwelling at low latitudes and downwelling at higher latitudes in the stratosphere. The reverse occurs in the mesosphere. The downwelling heats the stratosphere at higher latitudes while the upwelling results in a simultaneous adiabatic cooling at low latitudes. Opposite temperature tendencies are induced in the mesosphere, as is observed [Labitzke, 1972]. The cross spectral results of Figures 2 and 3 could be understood if short-term solar UV variations either force or modulate variations of the mean zonal wind velocity at middle latitudes at periods near 27 and 13 days.

Changes in the zonal wind at middle latitudes can be produced by wave-mean flow interactions if there are corresponding variations in the convergence of the planetary wave (Eliassen-Palm) flux $(\nabla \cdot F<0$; see Figure 4). This follows from the zonal momentum equation in the transformed Eulerian mean system (see Andrews et al. [1987]; p. 129). Any initial convergence of $\mathbf{F}$ produces a deceleration of the zonal wind and is followed by a secondary induced meridional circulation.

Salby (1984a,b) (see also Salby and Garcia (1987)) has reviewed evidence that interference between stationary and traveling planetary wave components can produce a modulation of the EP flux and resulting latitudinal temperature vacillations. A qualitative picture of the wave interference process for modulating the EP flux can be seen in Figure 10 of Salby [1984b] or in Figure 3 of Salby and Garcia [1987]. The phase surfaces of the stationary wave tilt with height while simple traveling waves are nearly barotropic. Interference between these waves therefore results in a modulation of the EP flux components in height and in time. At a given height, the EP flux appears as a sequence of pulsations and the divergence of the EP flux is oscillatory with frequency $\sigma$.

Given the cross-spectral results of Figures 2 and 3 combined with the current theory of stratospheric latitudinal temperature vacillations, one is led to hypothesize that solar UV variations may either force or modulate traveling waves with a period near 27 days in the upper stratosphere. Solar ultraviolet variations may be capable of forcing or modulating wave disturbances in the upper stratosphere via their effects on ozone concentration and radiative heating. These waves could then interfere with the time-averaged stationary wave at these levels to produce dynamical oscillations in step with solar UV variations. If so, then this would represent an initial mechanism for dynamical effects of short-term solar UV variability in the middle atmosphere.

\section{IMPLICATIONS FOR THE SOLAR CYCLE TIME SCALE}

Madden $(1975 ; 1983)$ and others have discussed the likely role of interference between free traveling planetary wave modes and forced stationary waves (and the resulting oscillating latitudinal heat transports) in initiating stratospheric warmings. Also, Hirooka and Hirota (1985) have shown that higher-degree (longer-period) atmospheric normal modes are often amplified simultaneously, especially before the occurrence of sudden warmings. It may therefore be suggested that transient wave disturbances in the upper stratosphere driven by short-term solar UV variations may be capable of affecting the initiation of stratospheric warmings. Of course, the primary energy that is involved in generating warmings originates in the troposphere through the forcing of stationary and transient waves that propagate upward through the winter westerlies and interact with the zonal 
flow (e.g., Andrews et al., 1987). However, it may be hypothesized that the initiation of a major warming in the upper stratosphere and lower mesosphere may depend at least in part on the amplitude of "27-day" waves so that more major warmings are produced near solar maximum than near solar minimum.

The QBO, on the other hand, also is capable of modulating warmings such that major warmings occur in the east phase but not in the west phase in the absence of any solar UV variations. This is at least partly due to the weaker (more easily reversed) polar night jet at 500 mbar during the east phase QBO as compared to the west phase QBO (Holton and Tan, 1980; 1982). It is possible that years of the east phase QBO are sufficiently prone to the occurrence of major warmings that the smaller upper stratospheric effects of solar UV variations have a small or negligible influence. However, the initiation of major warmings during years of the west phase QBO may be more sensitive to the amplitude of the 27-day wave in the upper stratosphere, thus yielding the stratospheric temperature correlation with the solar cycle reported by Labitzke and van Loon. Quantitative tests of this hypothesis will require detailed analysis of atmospheric planetary wave statistics in addition to the zonal mean ozone and temperature fields considered in this paper.

Acknowledgment. This work was supported in part by the NASA Upper Atmosphere Theory and Data Analysis Program under grant NAGW-909.

\section{REFERENCES}

Andrews, D. G., J. R. Holton, C. B. Leovy, 1987: Middle Atmosphere Dynamics, 489 pp., Academic Press, New York.

Baldwin, M. P., and T. J. Dunkerton, 1989: Observations and statistical simulations of a proposed solar cycle/QBO/weather relationship, Geophys. Res. Lett., 16, 863-867.

Brasseur, G., A. de Rudder, G. M. Keating, and M. C. Pitts, 1987: Response of middle atmosphere to short-term solar ultraviolet variations: 2. Theory, J. Geophys. Res., 92, 903-914.

Callis, L. B., J. C. Alpert, and M. A. Geller, 1985: An assessment of thermal, wind, and planetary wave changes in the middle and lower atmosphere due to 11-year UV flux variations, J. Geophys. Res., 90, 2273-2282.

Chandra, S., 1985: Solar-induced oscillations in the stratosphere: A myth or reality?, $J$. Geophys. Res., 90, 2331-2339.

Chandra, S., 1986: The solar and dynamically induced oscillations in the stratosphere, $J$. Geophys. Res., 91, 2719-2734.

Donnelly, R. F., D. E. Stevens, J. Barrett, and K. Pfendt, 1987: Short-term temporal variations of Nimbus 7 measurements of the solar UV spectral irradiance, NOAA Tech. Memo. ERL ARL-154, Air Resour. Lab., Silver Spring, M.

Ebel, A., M. Dameris, and H. J. Jakobs, 1988: Modelling of the dynamical response of the middle atmosphere to weak external forcing: Influence of stationary and transient waves, Ann. Geophys., 6, 501-512.

Fritz, S., and S. D. Soules, 1970: Large-scale temperature changes in the stratosphere observed from Nimbus III, J. Atmos. Sci., 27, 1091-1097. 
Hines, C. O., A possible mechanism for the production of sun-weather correlations, $J$. Atmos. Sci., 31, 589-591, 1974.

Hirooka, T. and I. Hirota, 1985: Normal mode Rossby waves observed in the upper stratosphere. Part II: Second antisymmetric and symmetric modes of zonal wavenumbers 1 and 2, J. Atmos. Sci., 42, 536-548.

Holton, J. R., and H.-C. Tan, 1980: The influence of the equatorial quasi-biennial oscillation on the global circulation at $50 \mathrm{mb}, J$. Atmos. Sci., 37, 2200-2208.

Holton, J. R., and H.-C. Tan, 1982: The quasi-biennial oscillation in the northern hemisphere lower stratosphere, J. Met. Soc. Japan, 60, 140-148.

Hood, L. L., 1987: Solar ultraviolet radiation induced variations in the stratosphere and mesosphere, J. Geophys. Res., 92, 876-888.

Hood, L. L., and S. Cantrell, 1988: Stratospheric ozone and temperature responses to short-term solar ultraviolet variations: Reproducibility of low-latitude response measurements, Ann. Geophys., 6, 525-530.

Hood, L. L., and A. R. Douglass, 1988: Stratospheric responses to solar ultraviolet variations: Comparisons with photochemical models, J. Geophys. Res., 93, 3905-3911.

Keating G. M., G. P. Brasseur, J. Y. Nicholson III, and A. De Rudder, 1985: Detection of the response of ozone in the middle atmosphere to short-term solar ultraviolet variations, Geophys. Res. Lett., 12, 449-452.

Keating G. M., J. Y. Nicholson III, D. F. Young, G. Brasseur, and A. De Rudder, 1987: Response of middle atmosphere to short-term solar ultraviolet variations: 1 . Observations, J. Geophys. Res., 92, 889-902.

Labitzke, K., The interaction between stratosphere and mesosphere in winter, J. Atmos. Sci., 29, 1395-1399, 1972.

Labitzke, K., On the interannual variability of the middle stratosphere during the northern winters, J. Meteor. Soc. Japan, 60, 124-138, 1982.

Labitzke, K., and H. van Loon, Association between the 11-year solar cycle, the QBO, and the atmosphere. Part III: Aspects of the association, J. Climate, 2, 554-565, 1989.

Madden, R. A., Oscillations in the winter stratosphere: Part 2. The role of horizontal eddy heat transport and the interaction of transient and stationary planetary waves, Mon. Wea. Rev., 103, 717-729, 1975.

Madden, R. A., The effect of the interference of traveling and stationary waves on time variations of the large-scale circulation, J. Atmos. Sci., 40, 1110-1125, 1983.

Nathan, T. R., 1989: On the role of ozone in the stability of Rossby normal modes, $J$. Atmos. Sci., 46, 2094-2100.

Rottman, G., Adv. Space Res., 8, 53-60, 1988.

Salby, M., 1984a: Survey of planetary-scale traveling waves: The state of theory and observations, Rev. Geophys. Space Phys., 22, 209-236.

Salby, M., 1984b: Transient disturbances in the stratosphere: Implications for theory and observing systems, J. Atmos. Terr. Phys., 46, 1009-1047.

Salby, M., and R. Garcia, 1987: Vacillations induced by interference of stationary and traveling planetary waves, J. Atmos. Sci., 44, 2679-2711.

Springer, B. D., Solar variability and quasi-stationary planetary wave behavior, in Weather and Climate Responses to Solar Variations, edited by B. M. McCormac, Colorado As- 
sociated University Press, Boulder, p. 381-394, 1983.

van Loon, H. and Labitzke, K., 1990: Association between the 11-year solar cycle and the atmosphere. Part IV: The stratosphere, not grouped by the phase of the QBO,J. Climate, 3, in press.

van Loon, H., R. A. Madden, and R. L. Jenne, 1975: Oscillations in the winter stratosphere: Part 1. Description, Mon. Wea. Rev., 103, 154-162.

Figure 1

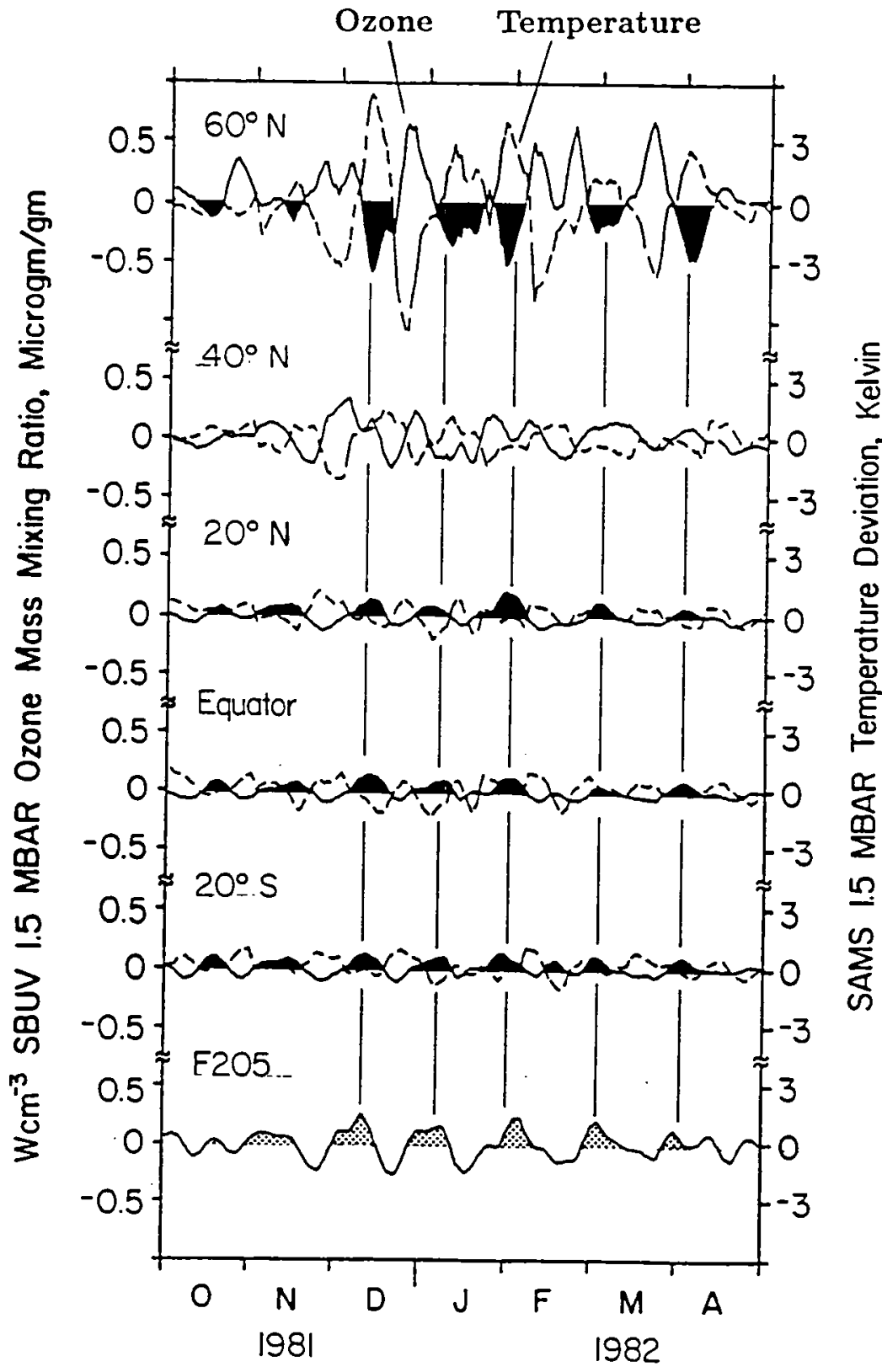


Figure 2

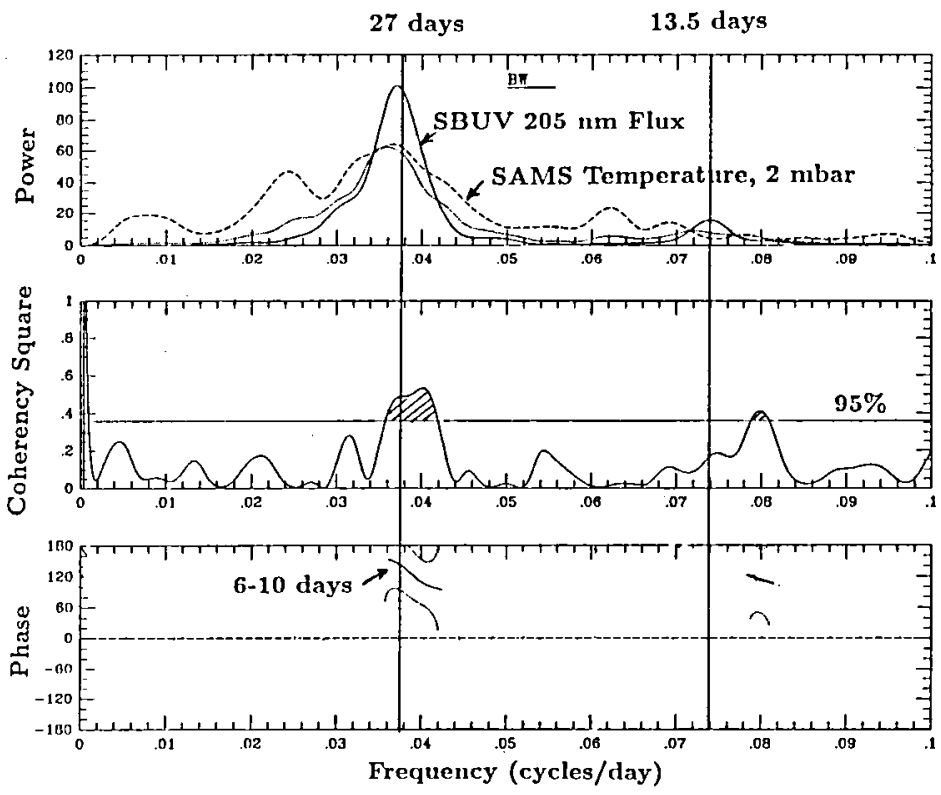

N. H. Winter Only

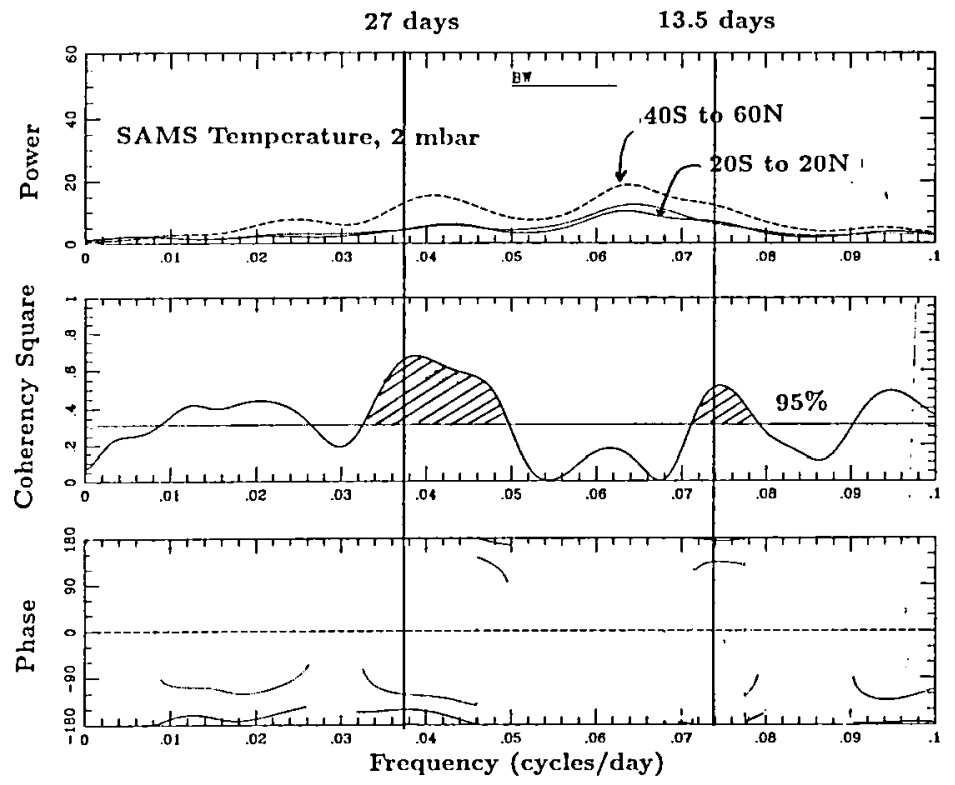

Figure 3

EQUATOR

Figure 4

$\frac{d T}{d t}>0$

Mesosphere $\quad \frac{d T}{d t}<0$

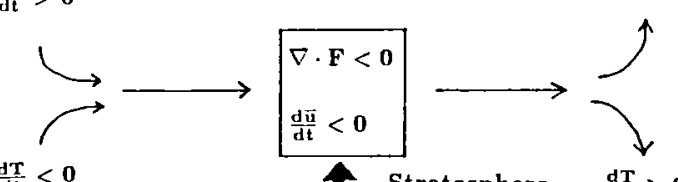

$\frac{\mathrm{dT}}{\mathrm{d} t}<0$

- Stratosphere

$\frac{d T}{d t}>0$

Adiabatic Cooling 
SESSION 6: MODELLING SOLAR VARIABILITY 



\title{
A SOLAR CONSTANT MODEL FOR SUN-CLIMATE STUDIES:1600-2000AD
}

\author{
Kenneth H. Schatten \\ Code 910.1 \\ Laboratory for Atmospheres \\ NASA/Goddard Space Flight Center \\ Greenbelt, MD 20771 \\ and \\ Jerome A. Orosz \\ Dept. of Physics and Astronomy \\ Ohio University \\ Athens, $\mathrm{OH} 45701$
}

\begin{abstract}
This paper discusses the solar constant model published recently (Schatten, 1988), but with a modified the phasing and amplitude. Nevertheless, the asymmetry of the solar constant variations relative to solar activity may be one interesting feature of the present model. This model enables the "known" solar constant variations to be calculated from known active region and quiet region solar parameters. We use the term "known" in the previous sentence to indicate that this modelling effort does not utilize effects such as solar radius - solar luminosity changes, which we believe may be important, but which have not either been well substantiated or generally agreed upon by the scientific community. We do recognize that these other solar constant variations likely do exist, however, they are simply not understood well enough to incorporate them into a solar constant model. The "known" effects we consider are thus only those features which show contrast in white light for which we have some generally recognized data set to allow a modelling effort. The features which can be modelled are sunspots and faculae, the only two features which mark the photospheric continuum with their unusual contrast behavior. We include both the active region features (sunspots and faculae) and the quiet region features (global faculae). Although the direct influences of sunspots upon the solar constant leads to short term decreases, an opposite, nearly in phase, 11 year variation in the solar constant is modelled, thereby agreeing with the ACRIM and ERB secular trends observed. This opposite behavior results primarily from global faculae (polar, network, and active region). The main contributor to the global behavior are the network faculae. The model attributes the observed variations in the solar constant entirely to magnetic features in the solar atmosphere. Hence, the model does not require any indirect effect of the magnetic cycle on the brightness of non-magnetic elements of area on the Sun. The model utilizes the well studied active region influences upon the solar constant, together with the influences of global faculae, asociated with polar and general solar magnetism. The solar constant variations are calculated on a yearly basis and thus detailed comparisons with daily data should not be undertaken. The present model serves purely to model the secular (long term) trend in the solar constant. An advantage of this model is that one need only utilize such long baseline activity parameters as sunspot number or the Carbon 14 record as a base without resorting to modem parameters inaccessible in the distant past. The model suggests a change of $\sim 0.5 \mathrm{~W} / \mathrm{m}^{2}$ for the differences between the late twentieth century solar constant and the 17th century solar constant. This supports Eddy's view that this difference could give rise to the glacial increase during the little ice age of the 17th century. Important for present day climate studies, is that it shows the recent peak activity (peaking in 1958) is associated with an atypically high value of the solar constant, with respect to the past few hundred years.
\end{abstract}




\section{INTRODUCTION}

Recent work by climate modelers (e.g. Hansen et al., 1988) show a startling trend in the atmospheric global temperature. As suggested from "greenhouse" theories of the atmospheric temperature structure, these more elaborate models support a small but highly significant rise in temperature, on the order of $1 \mathrm{C}$, in the Earth's climate over the next decade, associated with the accumulation of trace gases $\left(\mathrm{CO}_{2}, \mathrm{CH}_{4}, \mathrm{~N}_{2} \mathrm{O}\right.$, etc.). The effect is expected to be "at least three standard deviations above the climatology of the 1950's". This model does not consider solar variability, which has time constraints (from the 11 year solar cycle to the 80-100 year Gleissberg cycle) comparable to those being studied in the climate modeling. Although the changes associated with the trace gas constituency rise are likely to be of greater significance than the solar activity variations, it is important to understand the latter influence, since there is evidence (see Eddy, 1977; Grove, 1988) that long-term solar activity variations can also play a significant role in affecting the Earth's climate. Thus, to better understand the influence of anthropogenic changes to the atmospheric temperature structure, we must "remove" or understand as fully as possible those sources of temperature change associated with natural variability. These include volcanic dust veils (see Lamb, 1970), as well as solar activity. It will be the purpose of this paper to provide an update to our understanding of the influence of solar activity upon the solar constant, with a minor comment upon the degree to which this may affect the Earth's climate.

We first briefly review the influences of solar activity upon the solar constant. There are several well known, as well as some contentious, influences which we categorize as follows: 1) active region influences (sunspots and faculae - for which plague data are often employed to serve as a proxy data set (see Chapman, 1987), 2) global contrast features - for which network and polar faculae and the most prominent examples, although pores may also be considered in this category (see Foukal and Lean, 1988, and Schatten, 1988), 3) global size and shape changes for which moderate evidence exists supporting changes in the solar size and less evidence for changes in the oblateness (see Endal and Sofia, 1981, and Sofia, et al., 1985), and 4) other unknown changes which may affect the solar constant (temperature variations in the quiet photosphere, etc.).

Theory and modeling shows the following influence of category 1 features upon the solar constant: a moderate decline in the total irradiance as the cycle progresses, owing to the associated solar cycle reduction in latitude of active region features. The effect is of the order of 1 Watt per meter ${ }^{2}$, due to the reasonably close "energy balance" which appears to exist over the week or fortnight lifetime of active regions.

The primary category 2 features are global faculae. These provide an increase in the total irradiance early in a solar cycle, followed by a decrease as the cycle progresses. These variations are also of the order of 1 Watt per meter ${ }^{2}$ and are associated with the changing number and latitude of global faculae. Since faculae show their greatest contrast towards the Sun's limbs (Schatten et al., 1986), polar faculae and early cycle network faculae (at high latitudes) provide for a marked increase in the solar constant early in the solar cycle associated with the large amount of time that these features spend at large heliocentric angles, where they are bright. As the cycle progresses, the Sun's polar fields and their associated faculae diminish, and thus also does their contribution to the solar constant.

Category 3 features may affect the solar constant. At present however, there exists evidence only that the Sun's diameter is varying by about 1 " in timescales of tens to hundreds of years. Without further knowledge to what degree this may affect the solar constant, it is much too early to include these variations, as well as category 4 variations, in the present modeling effort.

\section{MODEL AND RESULTS}

We choose the solar constant model published recently (Schatten, 1988), but have modified the phasing and amplitude (Schatten and Orosz, 1990), since recently we have seen that the previous global faculae were instituted too early in the cycle. Nevertheless, the asymmetry of the solar constant variations relative to solar activity may be one important feature of the present model. We base the number of sunspots and faculae since 1610, upon the sunspot number shown by Waldmeier (1961). Figure 1 shows this modeled solar constant versus year from 1610 to the year 2000. One small difference exists in this figure from the published model--the phasing of the global faculae has been shifted roughly 1 year. The dashed line shows the smoothed 33-year running mean of the modeled solar constant, allowing the secular trend to be observed more clearly. Data past cycle 21 are based upon the predicted behavior from the dynamo theory model (Schatten et al., 1977; Schatten and Sofia, 1987). A reduction in the solar constant is clearly observed during the Maunder minimum in the late 17th century. Following this, there has been a 
general rise in the modeled solar constant, with slight depressions near the turn of the last two centuries. This 80 100-year undulation is referred to as the "Gleissberg" cycle, although it appears to be more of a persistency than a true periodic cycle. It is unlikely that the general rise in the modeled solar constant over the last few hundred years is the actual source for the global climate warming that has been observed recently (Hansen et al., 1988) for two reasons: 1) the observed heating trend follows the trace gas heating curve closely, and 2) the recent values for the modeled solar constant have not been increasing in the last three decades, since the largest solar cycle to date has been cycle 19, which peaked in 1957. Thus, it does not appear that we can explain the atmospheric global warming to the natural solar variability.

Figure 2 shows the modeled solar constant with recent Nimbus 7 observations (see Hickey et al., 1988). As can be seen, there is a reasonably good agreement between the two. The recent upswing in the solar constant observations in 1987 matches the model quite well, however, the present model was chosen solely for the purpose of yearly comparisons to ascertain whether the secualr trend could be understood, not the daily variations.

\section{CONCLUSIONS}

The solar constant has been modeled from the years 1600 to 2000 . The 11-year solar cycle is seen in the model as well as secular changes in the solar activity record (Gleissberg cycle), supporting Eddy's contention that the little ice age in Europe may have been associated with the dearth in sunspots during the Maunder Minimum. On the other hand, the recent increase in the global temperature of the Earth is not likely fully associated with the solar activity. Recent solar constant observations of a downward trend for the last half of solar cycle 21 followed by an upturn in the solar constant associated with the onset of high latitude faculae in the beginning of cycle 22 (Mecherikunnel $e t$ al., 1988; and Willson and Hudson, 1988) support the model's general trend for the recent solar constant behavior. Using this model, we now calculate a "proxy" solar constant, based upon this model for: 1) the past four centuries, based upon the sunspot record, and 2) the next 20 years, based upon our dynamo theory model for the solar cycle (Schatten and Sofia, 1987). The proxy solar constant data displayed here may be useful for climate modelers studying global climate changes. It would be helpful in disentangling the solar influences from any anthropogenic changes associated with trace gas increases in the terrestrial atmosphere. We have not included short term fluctuations in the present model. 


\section{REFERENCES}

Chapman, G. A., "Solar Variability Due to Sunspots and Faculae," J. Geophys. Res., 22(D1), 809, 1987.

Damon, P. E., "Energetic Particles at One A.U.," in The Solar Output and Its Variations, ed. O. R. White, 429-445, Col. Assoc. Univ. Press, Boulder, 1977.

Eddy, J., "Climatic Change", 1, 173, 1977.

Foukal, P. and J. Lean, Astrophys. J., 328, 347-357, 1988.

Grove, J. M., The Little Ice Age, Methuen, London, ch. 11, 354-378, 1988.

Hansen, J., I. Fung, A. Lacis, S. Lebedeff, D. Rind, R. Ruedy, and G. Russell, "Global Climate Changes as Forecast by the GISS 3-D Model," J. Geophys. Res., to appear, 1988.

Hickey, J. R. et al., "Total Solar Irradiance Measurements by ERB/Nimbus-7: A Review of Nine Years," Space Science Reviews, submitted, 1988.

Mecherikunnel, A. AT., R. B. Lee III, H. L. Kyle, and E. R. Major, "Intercomparison of Solar Total Irradiance Data from Recent Spacecraft Measurements," J. Geophys. Res., 23, 9503-9509, 1988.

Schatten, K. H., "A Model for Solar Constant Secular Changes," Geophys. Res. Lett., 15, 121-124, 1988.

Schatten, K. H., P. H. Scherrer, L. Svalgaard, and J. M. Wilcox, "Using Dynamo Theory to Predict the Sunspot Number During Solar Cycle 21," Geophys. Res. Lett., 5, 411, 1978.

Schatten, K. H. and S. Sofia, "Forecast of an Exceptionally Large Even-Numbered Solar Cycle," Geophys. Res. Lett, 14, 632-635, 1987.

Schatten, K. H. and J. A. Orosz, "Solar Constant Secular Changes," Solar Physics, 125.179, 1990.

Sofia, S., P. De Marque, and A. Endal, "From Solar Dynamo Theory to Terrestrial Climate," American Scientist, $73,326,1985$.

Waldmeier, M., The Sunspot Activity in the Years 1610-1960, Zurich Schultness and Co., 1961.

Willson, R. C., S. Gulkis, M. Janssen, H. S. Hudson, and G. A. Chapman, "Observations of Solar Irradiance Variability," Science, 211, 700, 1981.

Willson, R. C. and H. S. Hudson, Nature, 332, 810-812, 1988. 


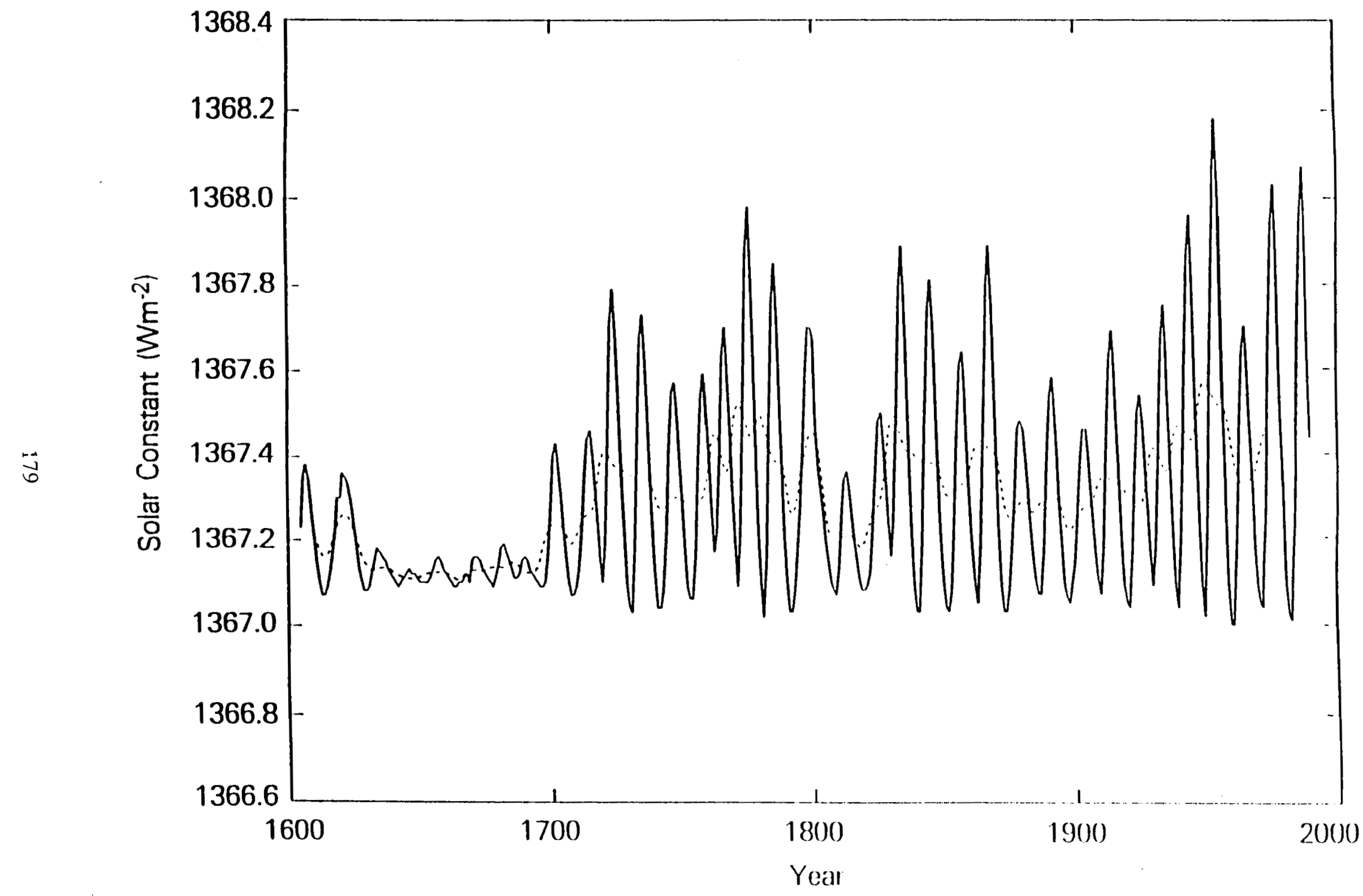

Figure 1. The solid line shows the modeled solar constant (with the Solar Maximum Mission's Active Cavity Radiometer experimental calibration) versus time in years from 1610 to 2000 . The secular trend is seen better from the dashed line, which is a 33-year smoothing of the solid curve. 
MODELED SOLAR CONSTANT VERSUS YEAR

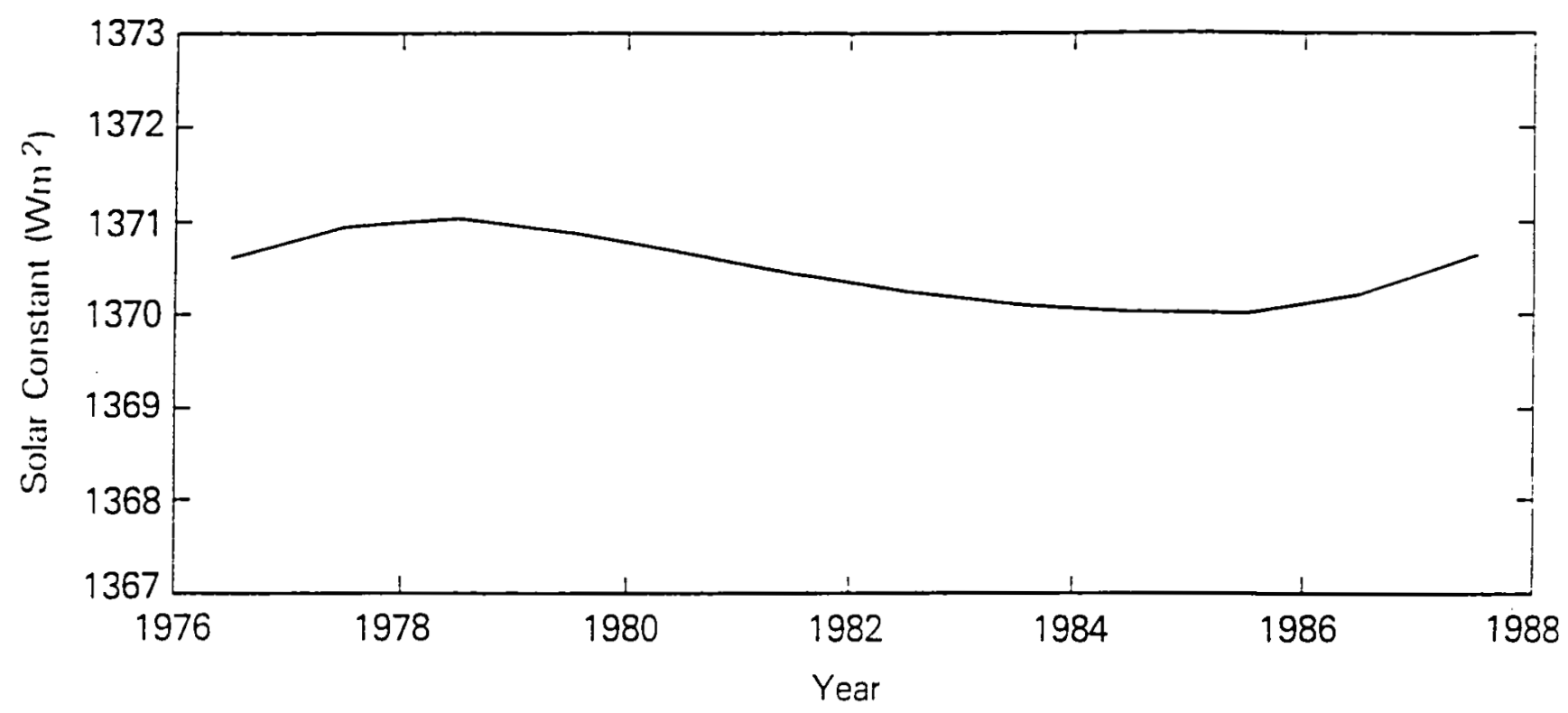

SOLAR CONSTANT - NIMBUS 7 DAILY MEANS

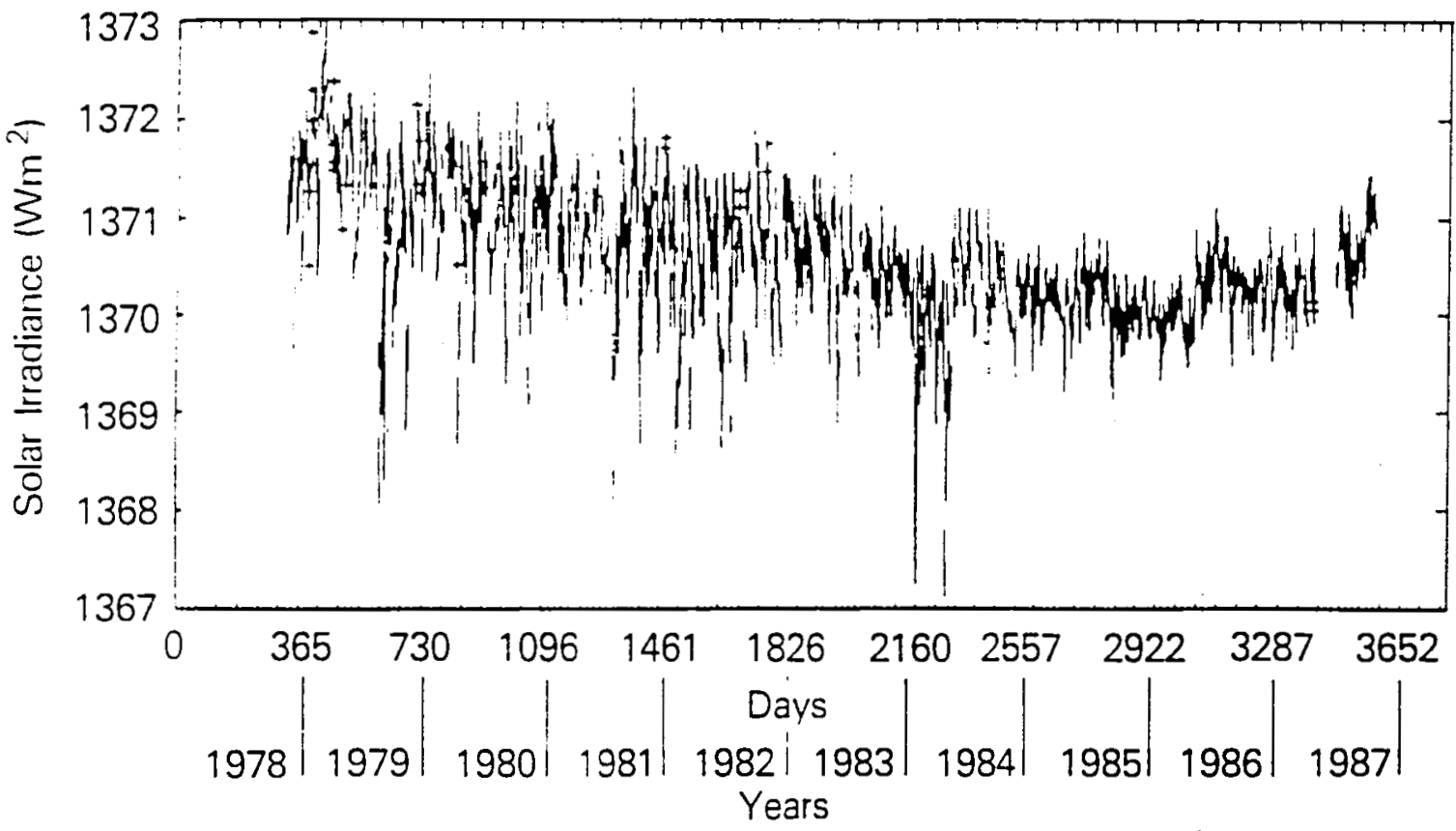

Figure 2. Shown (top) is the modeled smoothed solar constant from mid-1976 to mid-1987 (using the Nimbus 7 calibration), with the solar constant observations fro Nimbus 7 (bottom) (see Hickey et al., 1988). The values agree reasonably well throughout the time period, including the overall peak in 1979 and the valley at the end of 1985 . The observations in 1987 appear to rise slightly faster than the model. 


\title{
A Solar-Luminosity Model and Climate
}

\author{
Charles A. Perry
}

\author{
U.S. Geological Survey, Lawrence, Kansas 66049
}

\begin{abstract}
Although the mechanisms of climatic change are not completely understood, the potential causes include changes in the Sun's luminosity. Solar activity in the form of sunspots, flares, proton events, and radiation fluctuations has displayed periodic tendencies. Two types of proxy climatic data that can be related to periodic solar activity are varved geologic formations and freshwater diatom deposits. A model for solar luminosity was developed by using the geometric progression of harmonic cycles that is evident in solar and geophysical data. The model assumes that variation in global energy input is a result of many periods of individual solar-luminosity variations. The 0.1-percent variation of the solar constant measured during the last sunspot cycle provided the basis for determining the amplitude of each luminosity cycle. Model output is a summation of the amplitudes of each cycle of a geometric progression of harmonic sine waves that are referenced to the 11-year average solar cycle.
\end{abstract}

When the last eight cycles in Emiliani's oxygen-18 variations from deep-sea cores were standardized to the average length of glaciations during the Pleistocene ( 88,000 years), correlation coefficients with the model output ranged from 0.48 to 0.76 . In order to calibrate the model to real time, model output was graphically compared to indirect records of glacial advances and retreats during the last 24,000 years and with sea-level rises during the Holocene. Carbon-14 production during the last millenium and elevations of the Great Salt Lake for the last 140 years demonstrate significant correlations with modeled luminosity. Major solar flares during the last 90 years match well with the time-calibrated model.

\section{INTRODUCTION}

The climate of the Earth has had major variations throughout its geological record and minor fluctuations during its historical record. Although causes of major climatic variations, especially the ice-age cycle, are not completely understood, some of the more important causal mechanisms include continental drift, changes in the Earth's relative position with the Sun, volcanic activity, changes in atmospheric gases, and variations in solar output. The most widely accepted theory for the cause of the ice-age cycles of the Pleistocene is the Milankovitch orbital cycles coupled with positive feedback from the greenhouse effect ${ }^{1}$. Evidence in support of the Milankovitch theory is substantial ${ }^{2,3}$, but there are still problems in its quantitative aspects. Recent work by Ramanathan et al., ${ }^{4}$ which involved satellite measurements of global radiation, indicates that clouds may cool the Earth more than they warm it, thus injecting a source of uncertainty into the models used to study the greenhouse effect.

The most direct mechanism for climate change would be a decrease or increase in the amount of radiant energy reaching the Earth. Because the Milankovitch orbital theory can account for only a maximum of 0.1 -percent change in the total global energy and must rely on a complex redistribution of that energy to force a climatic change, one can look to the Sun as a possible source of larger energy fluctuations. Earth-satellite measurements of the so called "solar constant" in the last decade have revealed that it is far from being a constant. Total energy reaching the Earth decreased between 1980 and 1986 by more than 0.1 percent $^{5}$. If this rate of decrease were to continue, the world would be entering another global ice age within centuries. However, the solar constant has increased since 1987 along with increased solar magnetic activity. Evidence of solar-forced climatic variations on the order of years to centuries is accumulating. This paper presents a simple model for solar luminosity that shows significant correlations with both short- and long-term climatic variations. 


\section{SOLAR ACTIVITY}

Solar activity is quite variable and has displayed periodic tendencies. An obvious indicator of solar activity are sunspots. They have been counted in various ways for nearly 300 years, and several periodicities are evident. The best-known feature of the sunspot record is that the number observed follows an approximate 11-year cycle. G.E. Hale ${ }^{6}$ discovered that the polarity of the sunspots goes through an approximate 22-year cycle. Later, W. Gleissberg ${ }^{7}$ noted that the maximum number of sunspots for each 11-year cycle goes through an approximate 88-year cycle. This group could include a weak cycle of 44 years if the two sunspot maximums within a polarity cycle ( 22 years) are averaged. When the periods of 5.75 years found by Cole ${ }^{8}$ and 179 years found by Cohen and Lintz ${ }^{9}$ are added, an apparent progression of cycles in sunspot data of $5.75,11,22,44,88$, and 179 years appears.

Since the advent of artificial Earth satellites and improvements in ground-based observing instruments, other features of the Sun were discovered to be periodic in nature. Table 1 is a list of some of the observed periodicities of several features of the Sun. A complete list of all periodicities found by each investigator is provided by Perry ${ }^{10}$.

Table 1. Observed periods of solar activity

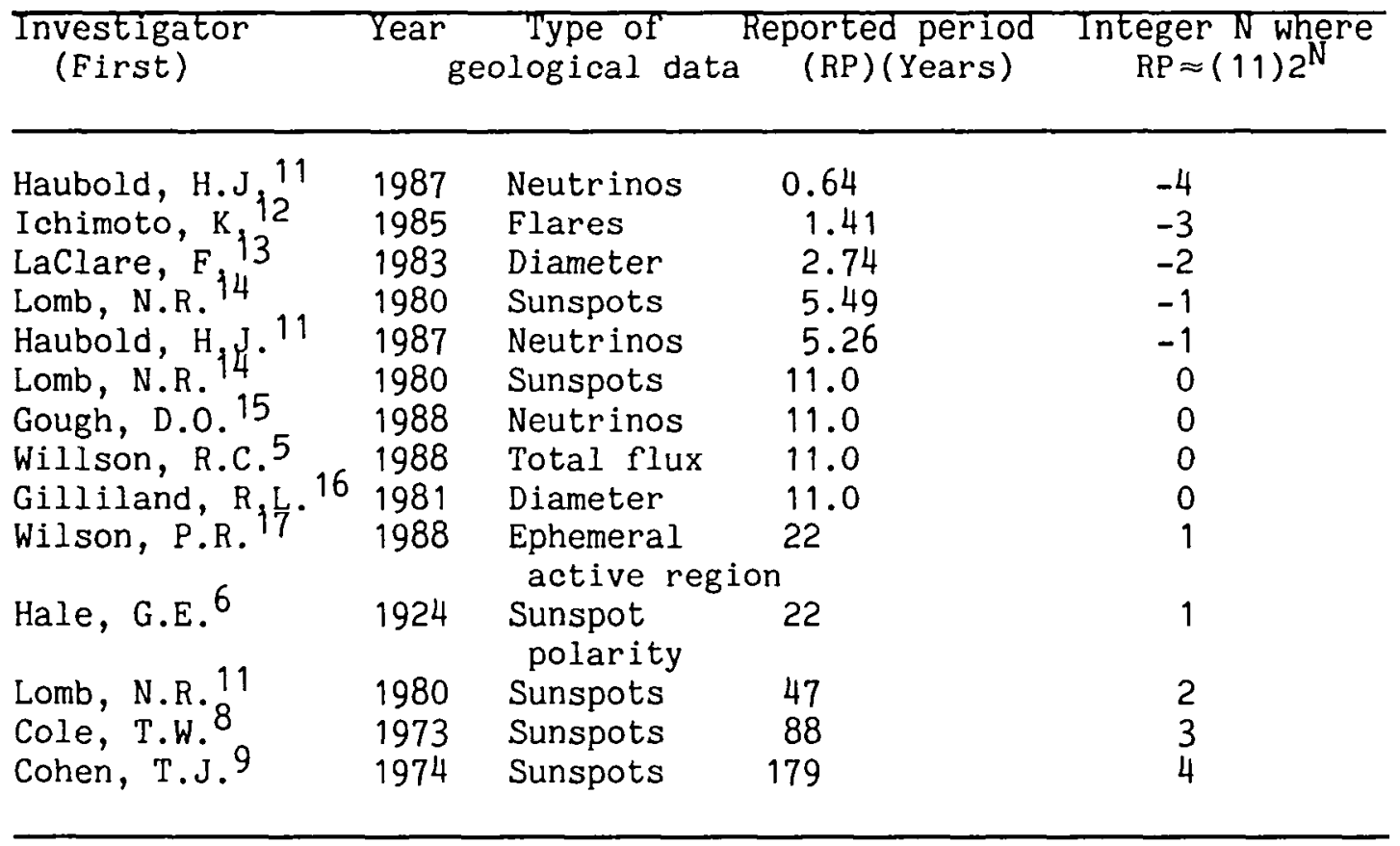

\section{SOLAR CYCLES AND LUMINOSITY VARIATIONS}

The luminosity of the Sun has increased and decreased in phase with the 11-year solar cycle ${ }^{5}$, and this variation has been measured at approximately 0.1 percent of the total flux from sunspot maximum to sunspot minimum. This variation in total energy is as large as that expected from the Milankovitch eccentricity cycle. The total flux from the Sun may have an even greater percentage variation over greater time scales. Proxy-climatic information can provide clues to these variations. Excellent records of sea temperature and sea level preserved in many geologic formations may provide possible indicators of solar luminosity.

Anderson ${ }^{18,19,20,21}$ has provided detailed analyses of several periodicities found in varve thicknesses. Varves are layered sediments that are assumed to be annual deposits in which the layer thickness is a function of climatic factors such as evaporation or precipitation. Another analysis of geologic data that supports and adds to the periodicities found by Anderson was performed by Pokras and Mix ${ }^{22}$. 
They found cycles in oxygen-18 composition of freshwater diatoms deposited on lake beds in tropical Africa. Periodicities that Anderson found in varves and those Pokras and Mix found in freshwater diatoms are listed in table 2.

Table 2. Periodicities in selected geological data

\begin{tabular}{lrccc}
$\begin{array}{l}\text { Investigator } \\
\text { (First) }\end{array}$ & $\begin{array}{r}\text { Year } \\
\text { geological data }\end{array}$ & $\begin{array}{c}\text { Type of } \\
\text { (RP)(Years) }\end{array}$ & $\begin{array}{c}\text { Reported period } \\
\mathrm{RP} \approx(11) 2^{\mathrm{N}}\end{array}$ \\
\hline Anderson, R.Y.18 & 1961 & Varves & 22.1 & 1 \\
$\quad$ Do. & do. & do. & 40.2 & 2 \\
Anderson, R.Y.19 & 1963 & Varves & 90 & 3 \\
Anderson, R.Y.20 & 1989 & Varves & 180 & 4 \\
Anderson, R.Y21 & 1982 & Varves & 2,700 & 8 \\
Pokras, E.M.22 & 1987 & Diatoms & 5,500 & 9 \\
Do. & do. & do. & 11,500 & 10 \\
Do. & do. & do. & 23,000 & 11 \\
Anderson, R.Y.20 & 1982 & Varves & 20,000 & 11 \\
Do. & do. & do. & 100,000 & 13 \\
& & & & \\
\hline
\end{tabular}

The combination of reported periodicities in tables 1 and 2 reveals an interesting geometric progression. Each reported cycle (RP) can be approximated by the equation:

$$
R P \approx 11(2)^{N},
$$

where $\mathrm{N}$ is any integer, positive, negative, or zero. Using the three data sets (solar activity, varves, and diatoms), the geometric progression for the integer values of -4 to +13 lacks examples for $\mathrm{N}$ equal to $5,6,7$, and 12. By including carbon-14 data periodicities of 740 ( $\mathrm{N}$ approximately equal to 6 ) and 1,486 years $^{23}$ ( $\mathrm{N}$ approximately equal to 7 ) and freshwater diatoms from a Mesozoic deposit with a periodicity of 43,000 years ${ }^{24}$ ( $\mathrm{N}$ approximately equal to 12 ), the mathematical progression has supporting physical evidence for 17 of the 18 integers from -4 through +13 . There is evidence that the harmonic progression continues for values of $\mathrm{N}$ less than -4 and greater than 13 in other geophysical data sets that are indicative of climate ${ }^{10}$. Certain reported cycles do not fit the progression ideally, but they do have a systematic variation, quite like the distribution of sunspot cycle lengths since 1700 $A D^{10}$. The variation in the lengths of solar-activity cycles may be a function of seismonuclear processes within the Sun's core. ${ }^{25}$

The geometric progression described by equation 1 can be considered a "fundamental" harmonic progression, with each cycle length being an integer doubling or halving the previous cycle. This is different from "simple" harmonics, which are integer multiples of one-cycle length, such as the progression of $11,22,33,44,55,66$, and 77 years. The equation for this "simple" harmonic progression is $11 \times \mathrm{N}$, with $\mathrm{N}$ equal to $1,2,3,4,5,6$, and 7 . This important differentiation between fundamental and simple harmonics must be maintained throughout this discussion. Any designation of "harmonic progression" in this paper will imply an integer doubling or halving.

\section{SOLAR-LUMINOSITY MODEL}

The total instantaneous energy output of the Sun is hypothesized to be a summation of many individual harmonics. These variations would follow a simple sine-wave function with the amplitude in terms of a plus or minus percentage change. The solar-luminosity variation of 0.1 percent for the 11-year solar cycle provides the basis for the development of a harmonic progression of sine waves used in the summation. The strengths of spectral analyses of the solar and geophysical cycles suggest that the progression of amplitudes for each harmonic sine wave may not increase steadily with each progressively larger harmonic, but instead increases in a stepped manner. For instance, the 22-year cycle is evident in a variety of data, whereas the 44-year cycle has not been detected as often. The 88year cycle is stronger in most data than the 176-year cycle. Of the longer period cycles, greater 
spectral powers were found near 22,000 and 88,000 years than near 11,000 and 44,000 years. It appears that the amplitude of the odd-numbered harmonics (odd-numbered $\mathrm{N}$, for example $\mathrm{N}=1$ for $\mathrm{RP}=22$ ) may be almost as large as the next even-numbered harmonic. The model algorithm was formulated to allow the odd-numbered harmonic cycles to have the same increase in amplitude as the even-numbered harmonic cycles following them. Model output is simply a summation of a series of harmonic sine waves.

\section{CORRELATIONS OF MODEL WITH PROXY CLIMATIC DATA}

Fourteen harmonic sine waves from 11 to 90,112 years were superimposed to generate a sequence of solar-luminosity variations. This sequence was compared cycle by cycle with a composite sequence of oxygen-18 variations in deep-sea cores from approximately 730,000 years ago to the end of the last glacial period ${ }^{26}$. Although more detailed oxygen-18 data are available from other sources, Emiliani's data were chosen for this analysis because they constituted a continuous record and had not been "orbitally tuned." There were eight complete glacial cycles during this time, with an average length of approximately 88,000 years. The first cycle (cycle \#1) used in this analysis began 720,000 years ago, and the last one (cycle \#8) ended about 22,000 years ago with the coldest period of the Wisconsin glaciation. Data from each of the eight cycles were standardized to a cycle length of 88,000 years. Oxygen-18 variations were not changed.

Graphical comparisons between the model and each of the eight cycles are shown in Fig. 1. The numerical correlations between luminosity variations of the model and the standardized oxygen-18 variations were 0.48 for cycle $1,0.56$ for cycle $2,0.59$ for cycle 3 , and improving to 0.67 for cycle 4 . Correlation coefficients for cycles $5,6,7$, and 8 were $0.71,0.76,0.62$, and 0.73 , respectively. The average of the correlation coefficients for the eight cycles was 0.64 , and for the last five cycles, 0.70 . The probability of such good correlations occurring in unrelated data is quite small. A strong connection between cyclic solar activity, as expressed by the superimposed solar-luminosity cycles, and responses of the Earth's climate would be needed to produce such relations.

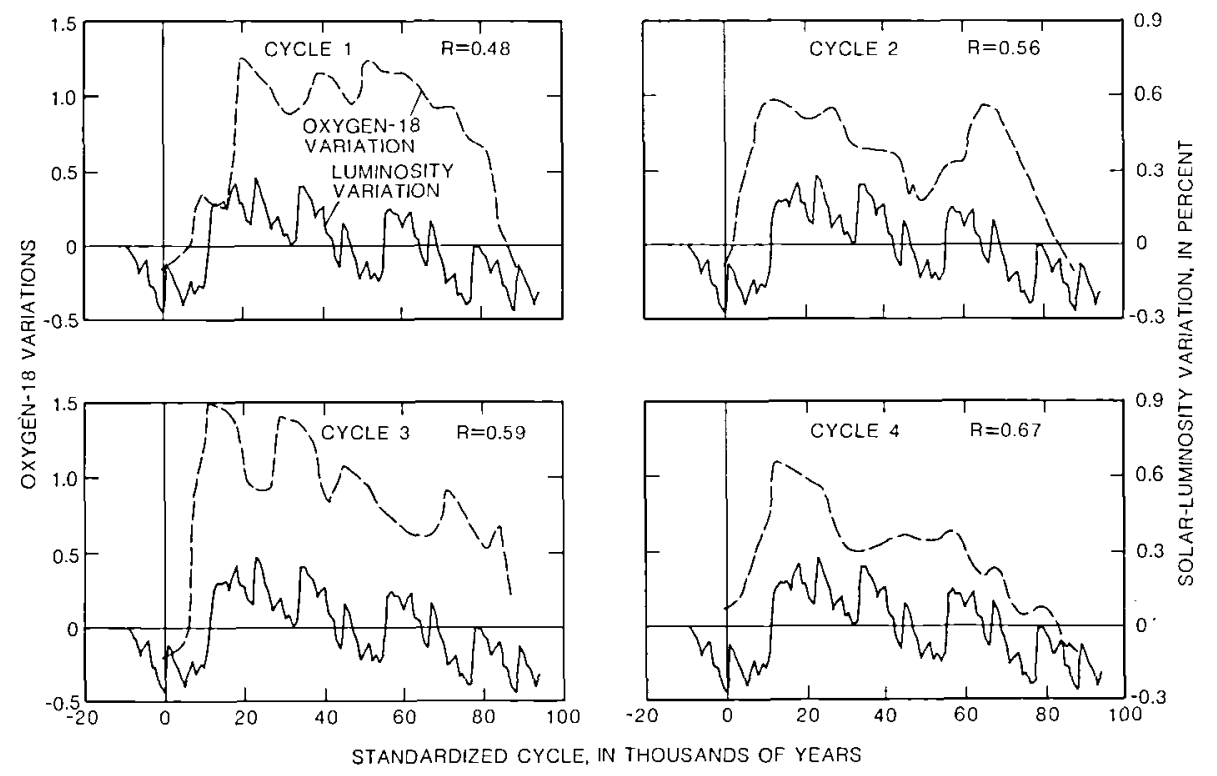

Figure 1. Comparison of standandized cycles of oxygen-18 and solar-luminosity variations for glacial cycles $1-4$. 


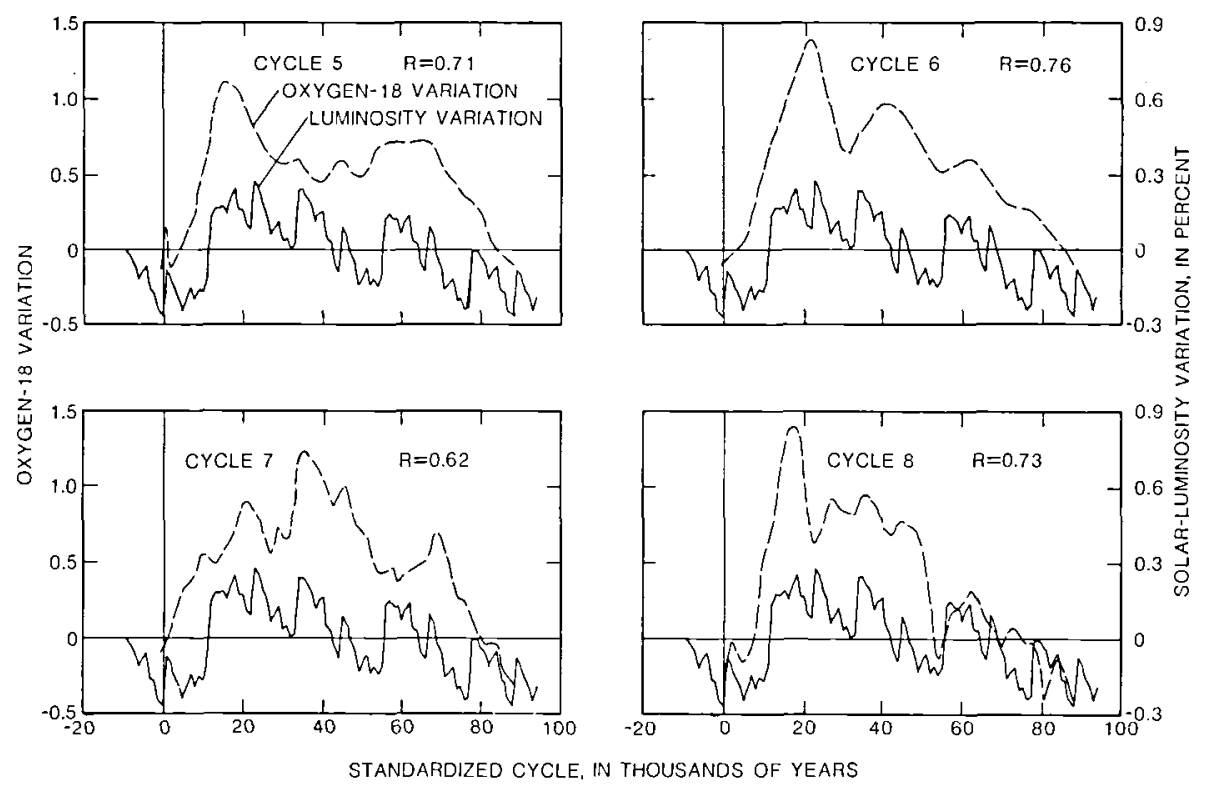

Figure 1 (continued). Comparisons of standardized cycles of oxygen-18 and solar-luminosity variations for glacial cycles 5-8.

\section{CALIBRATION OF MODEL TO REAL TIME}

The model also was evaluated with the timing of recent well-dated climatic events. The solarluminosity model was calibrated to a significant climatic event, and a graphical comparison of the timing of events before and after that match point was made. The most obvious match point occurs when all cycles are coming in phase together to produce a large increase of solar luminosity in a short time. Climatically, this time corresponds well with the very rapid melting of the last continental glacier approximately 10,000 years ago, the Pleistocene-Holocene boundary. This boundary became time 0 , and luminosity variations were generated for a period of 14,000 years before to 12,000 years after this match point. This 26,000-year sequence of luminosity variations is plotted at 176-year intervals in Fig. 2.

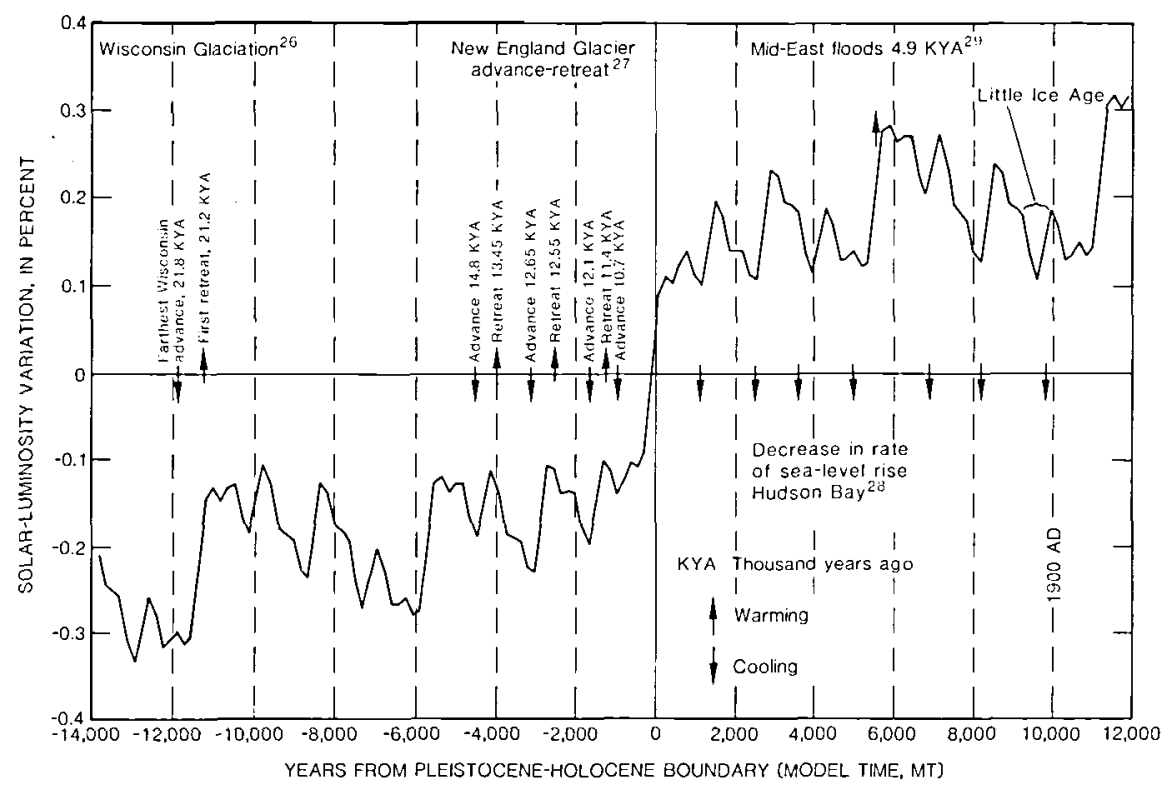

Figure 2. Comparison of solar-luminosity model and climatic events during the last 24,000 years. 
The model output corresponds well with several significant climatic events during the last 24,000 years. The farthest Wisconsinan glacial advance is dated at 21.8 thousand years ago (KYA) followed by the first retreat at $21.2 \mathrm{KYA}^{27}$. Later, readvances and retreats of the glacier in the New England $\operatorname{area}^{28}$, match well with variations in the modeled luminosity. During the Holocene, decreases in the rate of sea-level rise measured on Hudson Bay shorelines ${ }^{29}$ occurred at corresponding small values of luminosity. The "Little Ice Age" corresponded with the smallest luminosity value in the last 10,000 years. A major rise in sea level, chronicled by many Mid-Eastern civilizations ${ }^{30}$, and measured along the French Coast by Ters ${ }^{31}$ may be evidence of rapid melting of mountain glaciers and the remaining ice caps by a large increase in luminosity.

A comparison of the solar-luminosity model with more recent information was made with carbon-14 production in the atmosphere. Carbon-14 is produced by cosmic rays that are modulated by the solar wind. During times of increased solar output (high solar activity), the solar wind is strong, deflecting the cosmic rays away from the Earth ${ }^{32}$. Because the cosmic-ray flux is decreased, the production of carbon-14 decreases. During periods of low solar activity, more carbon-14 is produced. The bottom graph in Fig. 3 shows the relative rate of carbon-14 production during the last millennium as measured in tree rings (data from Damon ${ }^{33}$ ). The graph is inverted for direct comparison with the solar-luminosity model (lower solar output versus greater carbon-14 production) and plotted every 11 years. Evidence can be seen for the Wolf Minimum about $1300 \mathrm{AD}$, the Sporer Minimum about 1500 $A D$, and the Maunder Minimum, which ended with the return of increased solar activity, about 1700 $\mathrm{AD}$. These three minima in solar activity correspond to the Little Ice Age. The solar-luminosity model from 9,000 to 9,950 Model Time [MT] follows the carbon-14 pattern closely. The correlation coefficient for these data is $R=0.82$.
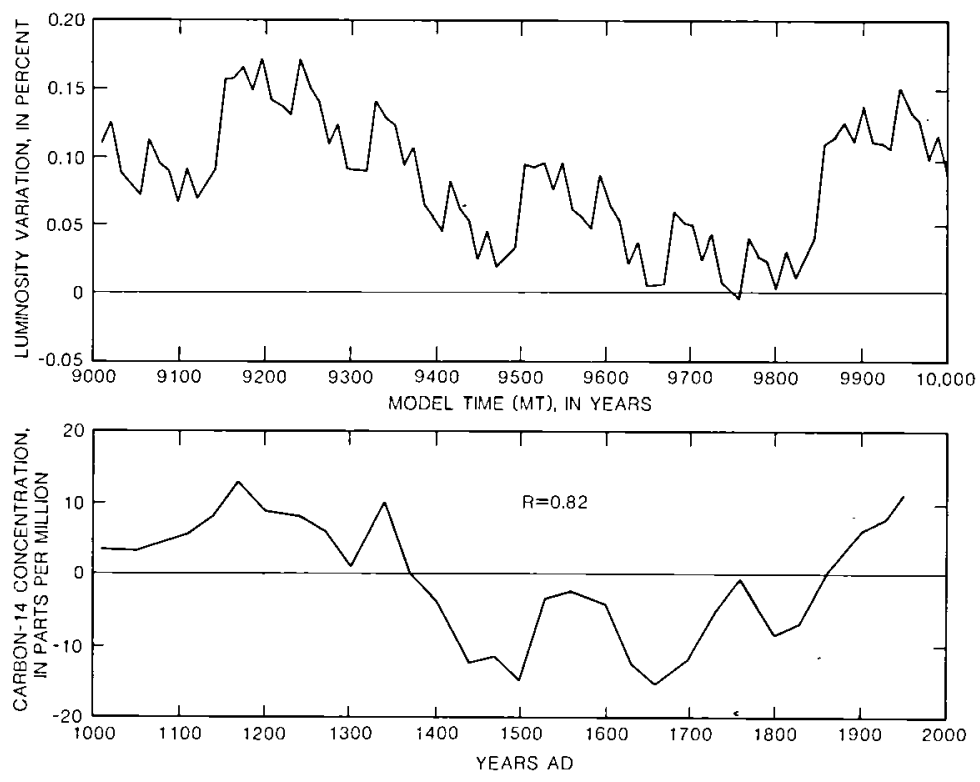

Figure 3. Correlation of solar-luminosity model and carbon-14 concentrations in tree rings.

The model was related also to recent climatic fluctuations. Elevations of the Great Salt Lake in Utah, which represent climatic conditions for a large geographical region, are compared with a detailed plot (yearly) of the modeled values (Fig. 4). Correlation of the model output and lake levels resulted in a correlation coefficient of 0.30 . The correlation of model output with these data has an exciting side note. Major solar flares during the 90 years ${ }^{34}$ match with large increases in modeled luminosity. 

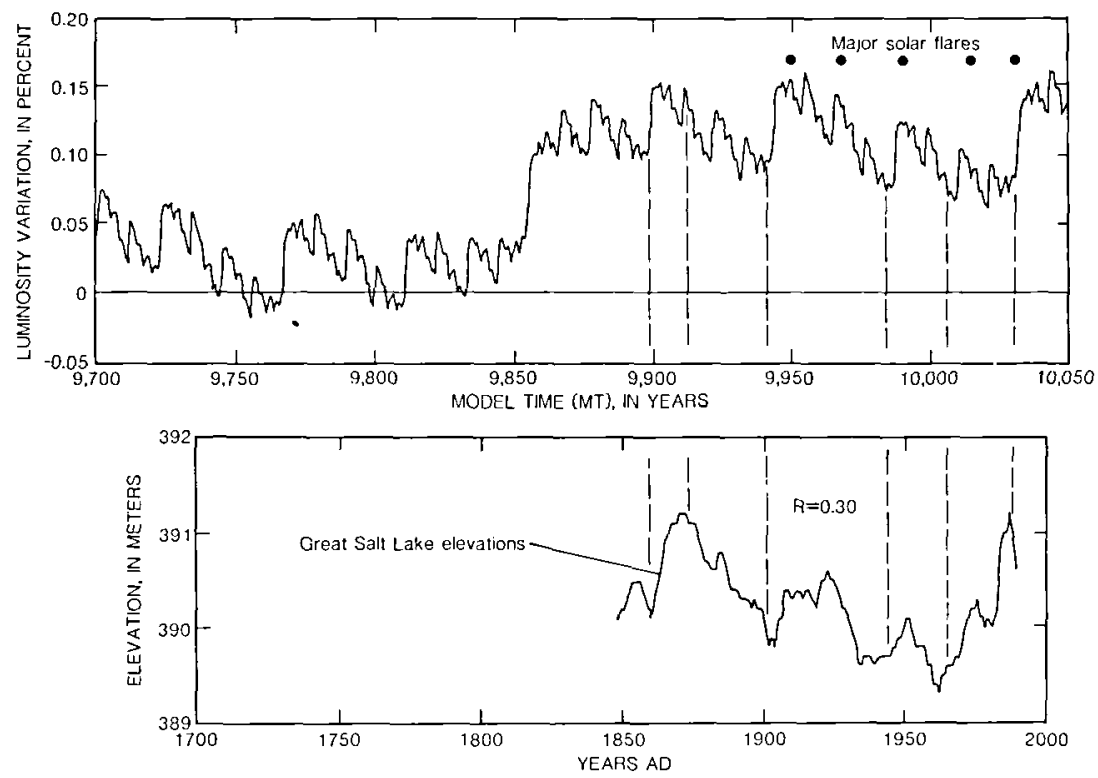

Figure 4. Correlation of solar-luminosity model with elevations of the Great Salt Lake and occurrences of major solar flares.

\section{CONCLUSIONS}

The total energy output of the Sun is hypothesized to be a summation of many periods of individual solar-luminosity variations. One such period is the 0.1-percent variation in solar luminosity that occurs during the 11-year sunspot cycle. Direct and indirect measurements of solar activity and luminosity demonstrate a harmonic progression of cyclicity that has been used to develop a simple model for solar output. Output from the model correlates well with proxy climatic records at different time scales. The model matches well with dated climatic fluctuations, which allowed the model to be calibrated to real time. Dates of advances and retreats of the Wisconsinan glaciation, dates of rates of change in sea-level rise, and sequence of sea levels during the Holocene provided climatic milestones that coincided directly with the solar-luminosity model. Time calibration of the model allows a glimpse at possible future solar-luminosity variations.

\section{REFERENCES}

1. Hays, J.D., J. Imbrie, and N.J. Shackleton, "Variations in the Earth's orbit--Pacemaker of the Ice Ages," Science, 194, 1121-1132, 1976.

2. Imbrie, J., and K.P. Imbrie, Ice Ages--Solving the Mystery, p. 224, Enslow Publ., New Jersey, 1979.

3. Berger, A., J. Imbrie, J.D. Hays, G. Kukla, and B. Saltzman, "Milankovitch and Climate," in Understanding the Response to Astronomical Forcing, p. 894, D. Reidel, The Netherlands, 1984.

4. Ramanathan, V., R.D. Cess, E.F. Harrison, P. Minnis, B.R. Barkstrom, E. Ahmad, and D. Hartmann, "Cloud-radiative forcing and climate--Results from the Earth radiation budget experiment," Science, 243, 57-63, 1989.

5. Willson, R.C., and H.S. Hudson, "Solar luminosity variations in solar cycle 21," Nature, 332, 810-812, 1988.

6. Hale, G.E., "Sunspots as magnets and the periodic reversal of their polarity," Nature, 113, 105, 1924.

7. Gleissberg, W., "The eighty-year cycle in auroral frequency numbers," British Astron. Assoc. J., 75, 227-231, 1965.

8. Cole, T.W., "Periodicities in solar activity", Solar Phys., 30, 103-110, 1973.

9. Cohen, T.J., and P.R. Lintz, "Long term periodicities in the sunspot cycle," Nature, 250, 398$399,1974$. 
10. Perry, C.A., A solar chronometer for climate--Astronomical and geophysical evidence, p. 314, Ph.D. diss., University of Kansas, Lawrence, 1989.

11. Haubold, H.J., and E. Gerth, "Periodic behavior of solar activity and solar neutrino flux," Proc. 20th International Cosmic Ray Conference, Moscow, USSR, 4, 343-346, 1987.

12. Ichimoto, K., J. Kubota, M. Suzuki, I. Tohmura, and H. Kurokawa, "Periodic behavior of solar flare activity," Nature, 316, 422-424, 1985.

13. LaClare, F., "Solar diameter measurements," Austrian Astrophys., 125, 200-203, 1983.

14. Lomb, N.R., and A.P. Andersen," The analysis and forecasting of the Wolf sunspot numbers," Monthly Notes, Royal Astron. Soc., 190, 723-732, 1980.

15. Gough, D.O., "Deep roots of solar cycles," Nature, 336, 618-619, 1988.

16. Gilliland, R.L., "Solar radius variations over the past 265 years," Astrophys. J., 248, 1144-1155, 1981.

17. Wilson, P.R., R.C. Altrock, K.L. Harvey, S.F. Martin, and H.B. Snodgrass, "The extended solar activity cycles," Nature, 333, 748-750, 1988.

18. Anderson, R.Y., "Solar-terrestrial climatic patterns in varved sediments," New York Acad. Sci. Ann., 95, 424-439, 1961.

19. Anderson, R.Y., "Harmonic analysis of varve time series," J. Geophys, Res., 68, 877-893, 1963.

20. Anderson, R.Y., "A long geoclimatic record from the Permian," J. Geophys. Res., 87, 7285-7294, 1982.

21. Anderson, R.Y., "Solar forcing and pacing of climate change--A geologic perspective based on varves," written commun., 1-19, 1989.

22. Pokras, E.M., and A.C. Mix, "Earth's precession cycle and Quaternary climatic change in tropical Africa," Nature, 326, 486-487, 1987.

23. Sonett, C.P., "Very long solar periods and radiocarbon record," Rev. Geophys. and Space Phys., 22(3), 239-254, 1984.

24. Olsen, P.E., "A 40-million-year lake record of early Mesozoic orbital climatic forcing," Science, $234,842-848,1986$.

25. Perry, C.A., "Speculation on a solar chronometer for climate," Proceedings of the Conference on the Climate Impact of Solar Variability, April 24-27, Goddard Space Flight Center, Greenbelt, Maryland, 1990.

26. Emiliani, C., "The cause of the Ice Ages," Earth Planetary Sci. Lett., 37, 349-352, 1978.

27. Larson, B.J., and B.D. Stone, Late Wisconsin glaciation of New England, p. 242, Kendall/Hunt, Dubuque, Iowa, 1982.

28. Schove, D.J., "Sunspot cycles and weather history," in Climate, M.R. Rampino, J.E. Sanders, W.S., Newman, and L.K. Konigsson, eds., p. 355-377, Van Nostrand Reinhold Co., New York, 1987.

29. Fairbridge, R.W., and C. Hillaire-Marcel, "An 8,000-yr palaeoclimatic record of the "Doublehale" 45-yr solar cycle," Nature, 268, 413-416, 1977.

30. Cornfield, G., Archaeology of the Bible, Book by Book, p. 8-9, Harper and Row Publ., New York, 1976.

31. Ters, M., "Variations in Holocene sea level on the French Atlantic Coast and their climatic significance," in Climate, M.R. Rampino, J.E. Sanders, W.S. Newman, and L.K. Konigsson, eds., p. 204-236, Van Nostrand Reinhold Co., New York, 1987.

32. Stuiver, M., "Statistics of the A.D. record of climate and carbon isotopic change," Radiocarbon, 25, 219-228, 1983.

33. Damon, P.E., "Solar induced variations of energetic particles at one A.U." in The Solar Output and Its Variations, O.R. White, ed., p. 429-448, Colorado Associated Press, Boulder, Colorado, 1977.

34. Dreschhoff, G.A.M., and E.J. Zeller, "Evidence of individual solar proton events in Antarctic snow," Solar Physics, (In Press), 1990.

C.A. Perry, U.S. Geological Survey, 4821 Quail Crest Place, Lawrence, KS 66049. 


\title{
MODELING SOLAR LYMAN ALPHA IRRADIANCE
}

\author{
J.Pap, H.S.Hudson, G.J.Rottman, R.C.Willson, R.F.Donnelly, and J.London \\ Univ. of Colorado (CIRES)/NOAA, Boulder CO 80309; Univ. of California, \\ San Diego CA 92109; Univ. of Colorado (LASP), Boulder CO 80309; Jet \\ Propulsion Laboratory, Pasadena CA 91103; NOAA/SEL, Boulder CO \\ 80303; Univ. of Colorado (APAS), Boulder CO 80309
}

\begin{abstract}
Solar Lyman alpha irradiance is estimated from various solar indices using linear regression analyses. Models developed with multiple linear regression analysis, including daily values and 81-day running means of solar indices, predict reasonably well both the short- and long-term variations observed in Lyman alpha. It is shown that the full disk equivalent width of the He line at 1083 $\mathrm{nm}$ offers the best proxy for Lyman alpha, and that the total irradiance corrected for sunspot effect also has a high correlation with Lyman alpha.
\end{abstract}

\section{INTRODUCTION}

For the first time, the total and UV solar irradiances have been measured simultaneously from space during most of a solar cycle. This permits the quantitative study of irradiance variabilities related to the solar cycle. Lean ${ }^{1}$ has shown that variations of UV irradiance are mainly caused by the bright magnetic elements on the solar surface associated with plages and the "active network". A recent study by Barth et al. ${ }^{2}$ has shown that variations of Lyman alpha can be modeled reasonably well during the declining portion of solar cycle 21 with the $10.7 \mathrm{~cm}$ radio flux, but a phase-shift between the time of their respective solar minimum is evident. In this paper we have used statistical methods to examine the variations of Lyman alpha irradiance and its relation to the total solar irradiance and various ground based solar activity indices.

This paper uses observations of Lyman alpha $(121.6 \mathrm{~nm})$ from the Solar Mesosphere Explorer satellite (e.g. Barth et $a_{.}{ }^{2}$ ). Data for the total solar irradiance (S) come from the SMM/ACRIM radiometer (e.g. Willson ${ }^{3}$ ). In order to compare the variations of Lyman alpha and total irradiance, the effect of sunspots has been removed from $\mathrm{S}$. For this purpose, the "Photometric Sunspot Index (PSI)", developed by Hudson et al. ${ }^{4}$, has been used. The total irradiance corrected for sunspot darkening $(\mathrm{Sc})$ is therefore calculated as $\mathrm{Sc}=\mathrm{ACRIM}+\mathrm{PSI}$. The $10.7 \mathrm{~cm}$ radio flux (F10), Fe XIV coronal green line index (CI), Ca-K plage index (PI), the full disk equivalent width of the He line at $1083 \mathrm{~nm}$ (EWHe) and projected sunspot areas (PSSA) are used as indices of solar activity. Daily values of F10 and PI have been published in Solar Geophysical Data catalogue. The Fe XIV coronal index has been calculated from ground-based observations and published through 1986 by Rybansky et al. ${ }^{5}$ These data give the total radiant energy of the coronal emission line at $530.3 \mathrm{~nm}$ and its variation is known to be controlled mainly by solar magnetic activity, as has been shown by Rybansky et al. ${ }^{5}$ The He $1083 \mathrm{~nm}$ line equivalent width is measured at the Kitt Peak National Solar Observatory and Harvey ${ }^{6}$ has found that it correlates well with the full disk Ca-K index. Thus, it is used as a proxy for the emission of bright magnetic elements, including faculae and the active network. Data sets for projected sunspot areas of "active" and "passive" sunspot groups were created using information on age and magnetic structure of sunspots reported in Solar Geophysical Data catalogue and daily area of spots reported 
in Solnechnye Dannye catalogue. The active sunspot groups are defined by Pap ${ }^{7}$ as developing complex groups, and the passive groups are the decaying old sunspots. Note that there is no direct physical relationship between the projected sunspot areas and Lyman alpha variability, since changes in Lyman alpha are primarily associated with bright plages and not with sunspots. The projected areas of active and passive spots provide a reliable discriminator of solar activity, indicating the presence of young and old active regions. Therefore we consider the relation of Lyman alpha to both types of sunspots in order to improve our understanding of the role of the evolution of active regions in UV irradiance variability.

\section{SINGLE VARIABLE LINEAR REGRESSION ANALYSIS}

To reveal the correlation between Lyman alpha irradiance and solar activity indices, including total irradiance, we use simple linear regression analysis as a first step. The solar indices have been scaled to Lyman alpha by means of the equation: $y=a+b \times x$, where $x$ is the selected solar index and $y$ is the estimated Lyman alpha value. The $a$ and $b$ regression coefficients are calculated. from the daily values of Lyman alpha and the different solar indices. Figs. 1(a-h) show the 27-day running means of Lyman alpha observed by the SME satellite (solid lines) and the results of the regressions (dashed lines) against S (a), Sc (b), F10 (c), CI (d), PI (e), EWHe (f), PSSA of active $(\mathrm{g})$ and passive $(\mathrm{h})$ spots. The correlation coefficients $(\mathrm{r} 1)$ between the observed and estimated Lyman alpha flux values as well as the a and $b$ regression coefficients are summarized in Table 1. The units of the selected solar indices are given in the footnote of Table 1. The Lyman alpha flux values are expressed in units of $\mathrm{W} / \mathrm{m}^{2}$.

Table 1

Results of simple and multiple linear regression analyses are summarized. The correlation coeffirients ( $r 1$ and $\mathrm{r} 2$ ) calculated between the observed Lyman alpha and its estimations from the listed solar indices are given. The regression coefficients are also listed for both simple and multiple regressions.

\begin{tabular}{|c|c|c|c|c|c|c|c|}
\hline Indices & $r 1^{S}$ & $g l e a_{a}^{L i}$ & $\begin{array}{c}a r \text { re } \\
\text { able } \\
b\end{array}$ & r & $\begin{array}{l}\text { Mul } \\
\text { a }\end{array}$ & $i_{b}$ & C \\
\hline $\begin{array}{l}\text { S } \\
\text { SC } \\
\text { F10 } \\
\text { CI } \\
\text { PI } \\
\text { EWHe } \\
\& \text { act } \\
\text { \& pas }\end{array}$ & $\begin{array}{l}0.49 \\
0.91 \\
0.90 \\
0.91 \\
0.85 \\
0.96 \\
0.57 \\
0.73\end{array}$ & $\begin{array}{c}-1.0 \\
-1.3 \\
3.2 \mathrm{E}-3 \\
3.7 \mathrm{E}-3 \\
4.2 \mathrm{E}-3 \\
1.4 \mathrm{E}-3 \\
4.7 \mathrm{E}-3 \\
4.5 \mathrm{E}-3\end{array}$ & $\begin{array}{l}7.7 \mathrm{E}-4 \\
9.7 \mathrm{E}-4 \\
1.5 \mathrm{E}-5 \\
1.4 \mathrm{E}-4 \\
3.5 \mathrm{E}-5 \\
6.1 \mathrm{E}-5 \\
3.6 \mathrm{E}-7 \\
9.8 \mathrm{E}-7\end{array}$ & $\begin{array}{l}0.85 \\
0.94 \\
0.92 \\
0.93 \\
0.91 \\
0.96 \\
0.80 \\
0.87\end{array}$ & $\begin{array}{c}-2.8 \\
-1.5 \\
3.0 \mathrm{E}-3 \\
3.6 \mathrm{E}-3 \\
4.1 \mathrm{E}-3 \\
1.4 \mathrm{E}-3 \\
4.3 \mathrm{E}-3 \\
4.2 \mathrm{E}-3\end{array}$ & $\begin{array}{l}3.4 \mathrm{E}-5 \\
4.0 \mathrm{E}-4 \\
7.7 \mathrm{E}-6 \\
5.5 \mathrm{E}-7 \\
1.2 \mathrm{E}-5 \\
5.3 \mathrm{E}-5 \\
7.4 \mathrm{E}-8 \\
1.3 \mathrm{E}-7\end{array}$ & $\begin{array}{l}2 \cdot 1 \mathrm{E}-3 \\
6 \cdot 9 \mathrm{E}-3 \\
9 \cdot 1 \mathrm{E}-6 \\
1.0 \mathrm{E}-6 \\
3 \cdot 4 \mathrm{E}-5 \\
8 \cdot 7 \mathrm{E}-6 \\
8 \cdot 3 \mathrm{E}-7 \\
1.5 \mathrm{E}-6\end{array}$ \\
\hline
\end{tabular}

units: S and Sc: W/m $/ \mathrm{m}^{2}$ F $10: 10^{22} \mathrm{~W} / \mathrm{m}^{2} / \mathrm{Hs} ; \mathrm{CI}: 10^{16} \mathrm{~W} / \mathrm{sr}$; PI: $10^{-6}$ of the hemisphere; EWHe: $\mathrm{mA}$; projected sunspots areas: $10^{-6}$ of the disk.

As can be seen from Fig. 1, the Lyman alpha fluxes estimated from various solar indices sometimes under-, sometimes overestimate its observed variability. The observed Lyman alpha generally decreases until mid-1986, when it reaches a minimum and begins to rise again. The Lyman alpha values estimated from S, Sc, F10, PI and PSSA tend to reach minimum levels in early 1985 and 
then show flat backgrounds during the two years of minimum, with only small changes occurring on the solar rotational time scale. This behavior for F10 has already been shown by Barth et al. ${ }^{2}$. The Lyman alpha modeled from EWHe and CI shows behavior similar to the observed Lyman alpha, both with minima in mid-1986.

To study the variations of Lyman alpha we have subtracted the daily values of estimated Lyman alpha from the data to obtain the residuals plotted in Fig. 2(a-h). We have calculated power spectra for each of the residual time series. In order to concentrate on the high-frequency variability, these time series have been detrended by means of a 4 th degree polynomial fit. The results of Fourier analysis are given in Fig. 3(a-h) for each residual. As can be seen from Figs. 2 and 3 , the irradiance model based on EWHe gives the best fit with the Lyman alpha flux observed by the SME satellite. The power spectrum of the residuals for EWHe strongly resembles white noise, except for the low frequency domain (due to periods above 300-day). This and the high correlation $(\mathrm{r} 1=0.96)$ between the observed Lyman alpha and its model based on EWHe shows that the full disk equivalent width of $\mathrm{He} 1083$ is the best of these proxies for the UV irradiance.

The other residuals still show a significant variability. This indicates that $\mathrm{S}, \mathrm{Sc}, \mathrm{F} 10, \mathrm{CI}, \mathrm{PI}$, and PSSA are less reliable for modeling the UV irradiance with a single variable linear regression analysis. Finally, we note that Figs. 3(g) and 3(h) show quite different power spectra for residuals calculated from models based on PSSA of active and passive spots, respectively. Residuals from passive spot areas show more peaks, and this power spectrum is very similar to the power spectrum of projected areas of active spots (see Pap et al. ${ }^{8}$ ). Likewise, the power spectrum of residuals from active spot areas is very similar to the power spectrum of the projected areas of passive spots ( shown in the paper of Pap et al.$^{8}$ ). This shows that the developing complex active regions and the old decaying active regions cause strong, but qualitatively different signals in the UV irradiance. As Fig. 3(h) shows, in the remaining Lyman alpha variability, after removing the effect of old active regions, a strong 13.5-day period exists that is not present in the spectrum of residuals calculated from the active spot areas. This suggests that the 13.5-day periodicity of UV flux (e.g. Donnelly ${ }^{9}$ ) may tend to arise from the effect of developing complex regions, which are located in opposite hemispheres, as has been shown by $\mathrm{Bai}^{\mathbf{1 0}}$.

\section{MULTIPLE LINEAR REGRESSION ANALYSIS}

In order to improve the statistical models of Lyman alpha, multiple regression analysis has been used instead of single variable linear regression. The estimated Lyman alpha is now calculated from the equation: $y=a+b \times x+c \times z$, where $a, b$, and $c$ are the partial regression coefficients (Table 1). $\mathrm{x}$ is the selected solar index and $\mathrm{z}$ is its 81 -day running mean. The parameter $\mathrm{b}$ characterizes the variation related to the rapid fuctuations of the given index, while $c$ describes the slower fluctuations seen in the 81-day smoothing. Therefore, y gives the best-fit relationship between Lyman alpha and solar activity indices, considering both the short- and long-term variabilities. The multiple correlation coefficients ( $\mathrm{r} 2$ ) are listed in Table 1.

Figs. 4(a-h) show the 27-day running means of SME/Lyman alpha observations (solid lines) and Lyman alpha values estimated with multiple regression analysis from various solar indices (dashed lines). As can be seen, the correlation between the observed Lyman alpha and its models is improved significantly for the total irradiance and the projected sunspot areas. The correlation coefficients are also higher for the models calculated from Sc, F10, CI and PI. The residuals between the observed and modeled Lyman alpha values are calculated again and plotted in Figs. 
5(a-h). After detrending these residuals by means of a 4 th degree polynomial fit, we again Fourier-analyze them. The power spectra of these residual time serjes are shown in Figs. $6(a-h)$. Comparing Figs. 3 and 6, it can be seen that the power spectra of the remaining Lyman alpha flux are simplified in case of multiple regression analysis relative to that for the single variable linear regression. The main features of power spectra of these residuals are the clearly seen peaks at the rotational period and at 13.5-day, plus broad-band power below 0.1 micro $\mathrm{Hz}$ (periods > 100 days). Both of these features are minimal for the EWHe, again establishing it as the best proxy.

Figs. 4, 5, and 6, and Table 1 demonstrate that models of Lyman alpha developed with multiple regression analysis describe the observed irradiance variations better than models calculated with single variable linear regression analysis. Using nultiple regression, the models fit reasonably well both the short- and long-term changes observed in Lyman alpha, with detailed differences in the magnitude of the rotational modulation relative to the slowly-varying components. In case of EWHe, the multiple regression analysis gives only a small improvement compared to the model calculated by a single variable linear regression. Table 1 shows that for EWHe the b partial regression coefficient is larger than c. This also shows that changes in the daily values of EWHe correlate better with the variation of Lyman alpha than the changes in the smoothed EWHe.

\section{CONCLUSIONS}

Our results demonstrate that models of Lyman alpha calculated with single variable linear regression analysis from various solar indices (other than the He $1083 \mathrm{~nm}$ line equivalent width) cannot describe totally the observed variations of Lyman alpha solar irradiance. These models under- or overestimate the short-term changes, and a phase shift is seen between the minimum times of Lyman alpha and its estimates from the total irradiance, total irradiance corrected for sunspot darkening, $10.7 \mathrm{~cm}$ radio flux, $\mathrm{Ca}-\mathrm{K}$ plage index and projected sunspot areas. Multiple linear regression models predict much better both the short- and long-term variations observed in Lyman alpha, but still leave considerable power not modeled at the 27-day solar rotational and 13.5-day periods. In the case of the $\mathrm{He} 1083 \mathrm{~nm}$ line equivalent width the fits between the Lyman alpha values and their estimates are good using both single variable and multiple regression analyses. Residuals are of comparable size and both models explain about 92 percent of the variations observed in Lyman alpha irradiance.

Figs. 2 and 5 show a systematic variation of residuals, especially for the $10.7 \mathrm{~cm}$ radio flux and projected sunspot areas. This variation is caused by a phase shift between Lyman alpha and these solar indices used to produce the model, indicating their systematically different physical origin. The changes in the $10.7 \mathrm{~cm}$ radio flux and sunspot areas are related to the strong magnetic fields. Thus, their variation is the largest during the first appearance of magnetic fields on the solar disk. The plages are more long-lived than sunspots, and their area may be more extended during their second or third rotations than during their first appearance. This means that the peaks in the $10.7 \mathrm{~cm}$ radio flux caused by strong magnetic fields decrease faster than peaks in Lyman alpha which are caused mainly by plages. Our results also show that mainly during solar minimum, the plages themselves cannot account for the observed variations of UV irradiance. One may suppose that the plage remnants, which are neglected from the present $\mathrm{Ca}-\mathrm{K}$ plage data (see Marquette and Martin ${ }^{11}$ ), influence the UV irradiance. This indicates the neccessity of improvements of the present UV-models (e.g. Lean and Skumanich ${ }^{12}$ ) by direct photometric observations of plages and 
plage remnants. It is also shown that the evolution of active regions should be taken into account in the irradiance models. Until the time that new "photometric UV-models" are developed, the He-1083 $\mathrm{nm}$ line equivalent width can be used as the best proxy for the Lyman alpha irradiance.

\section{ACKNOWLEDGEMENTS}

The authors express their gratitude to Dr. J. Harvey for providing the He 1083 data, which are produced cooperatively by NSO/NOAO, NASA/GSFC and NOAA/SEL. This research was supported by a grant of NOAA/Sun-Climate Staff.

\section{REFERENCES}

1. Lean, J., 'Solar UV variations', J. Geophys. Res. Vol. 92, 839-868, 1987.

2. Barth, C.A., Tobiska, W.K., and Rottman, G.A., 'Comparison of $10.7 \mathrm{~cm}$ radio flux with SME solar Lyman alpha flux', Geophys. Res. Let. (1990), in press.

3. Willson, R.C., 'Measurements of solar total irradiance and its variability', Space Sci. Rev. 38, 203-242, 1984.

4. Hudson, H.S., Silva, S., Woodard, M., and Willson, R.C., The effect of sunspots on solar irradiance, Solar Phys. 76, 211-219, 1982.

5. Rybansky, M., Rusin, V., and Dzifcakova, E., 'Coronal index of solar activity', Bull. Astron. Inst. Czechosl. 39, 106-119, 1988.

6. Harvey, J.W., 'Variation of the solar He I 10830 A line: 1977-1980', in Variations of the Solar Constant', (ed.) S. Sofia, NASA-2191, p. 265-272, 1980.

7. Pap, J., 'Variation of the solar constant during the solar cycle', Astrophys. Space Sci. 127, 55-71, 1986.

8. Pap, J., Tobiska, K., and Bouwer D., 'Periodicities of solar irradiance and solar activity indices I.', Solar Phys. in press, 1990.

9. Donnelly, R.F., 'Solar UV variability', in Proceedings of the IAGA Symposium for Solar Activity Forcing of the Middle Atmosphere, (ed.) J. Lastovicka, p. 1-8., 1989.

10. Bai, T., 'Distribution of flares on the Sun: superactive regions and active zones of 1980-1985', Ap.J. 314, 795-807, 1987.

11. Marquette, W.H., and Martin, S.F., Long-term evolution of a high-latitude active region, Solar Phys. 117, 227-241, 1988

12. Lean J., and Skumanich, A., 'Variability of the Lyman alpha flux with solar activity', J. Geophys. Res. 88, 5751-5759, 1983. 


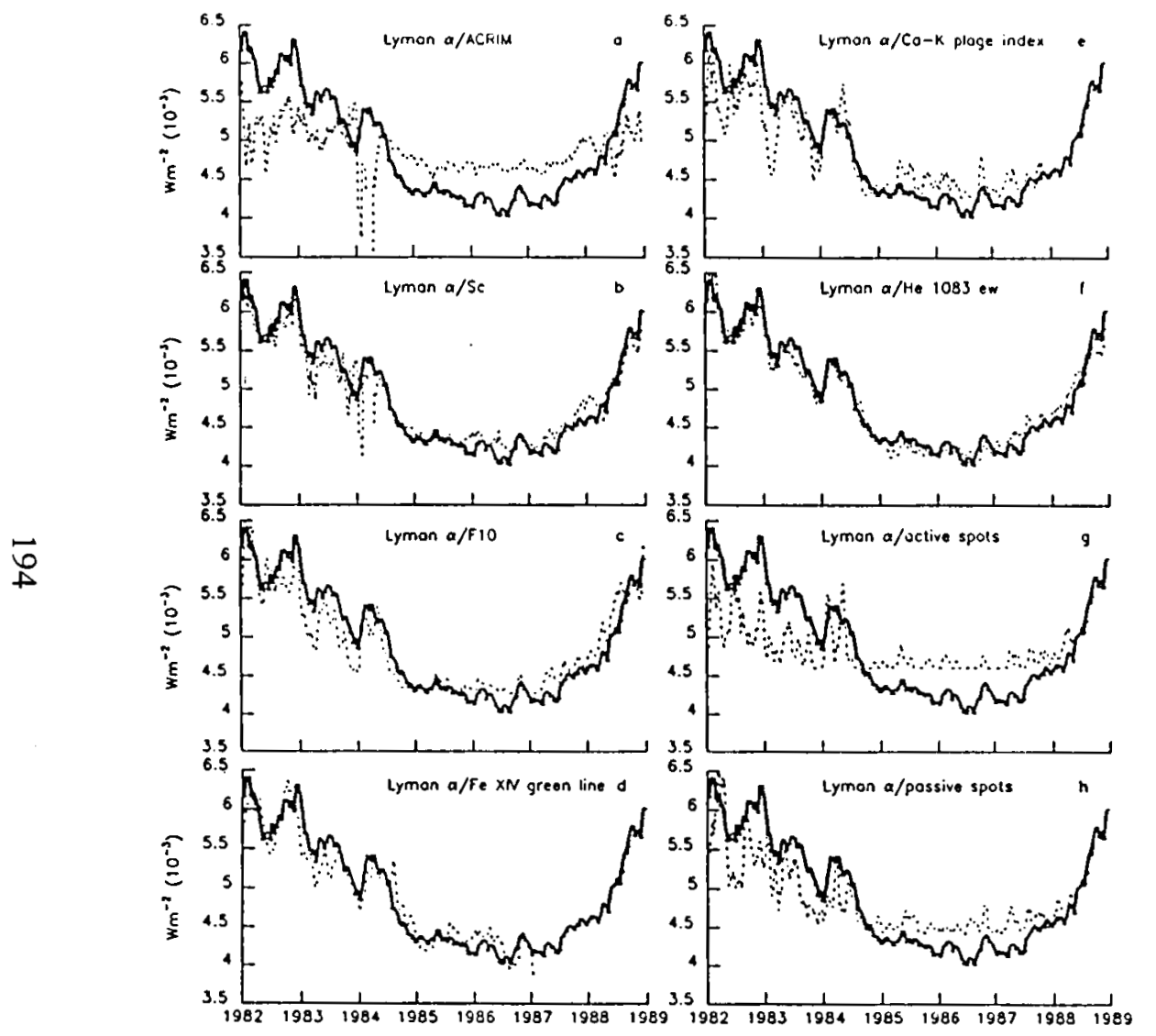

Fig. 1. Solid lines show the 27-day running means of SME Lyman alpha. Dashed lines show the 27-day running means of Lyman alpha estimated with simple linear regression analysis. The appropriate solar indices are printed on each plot.

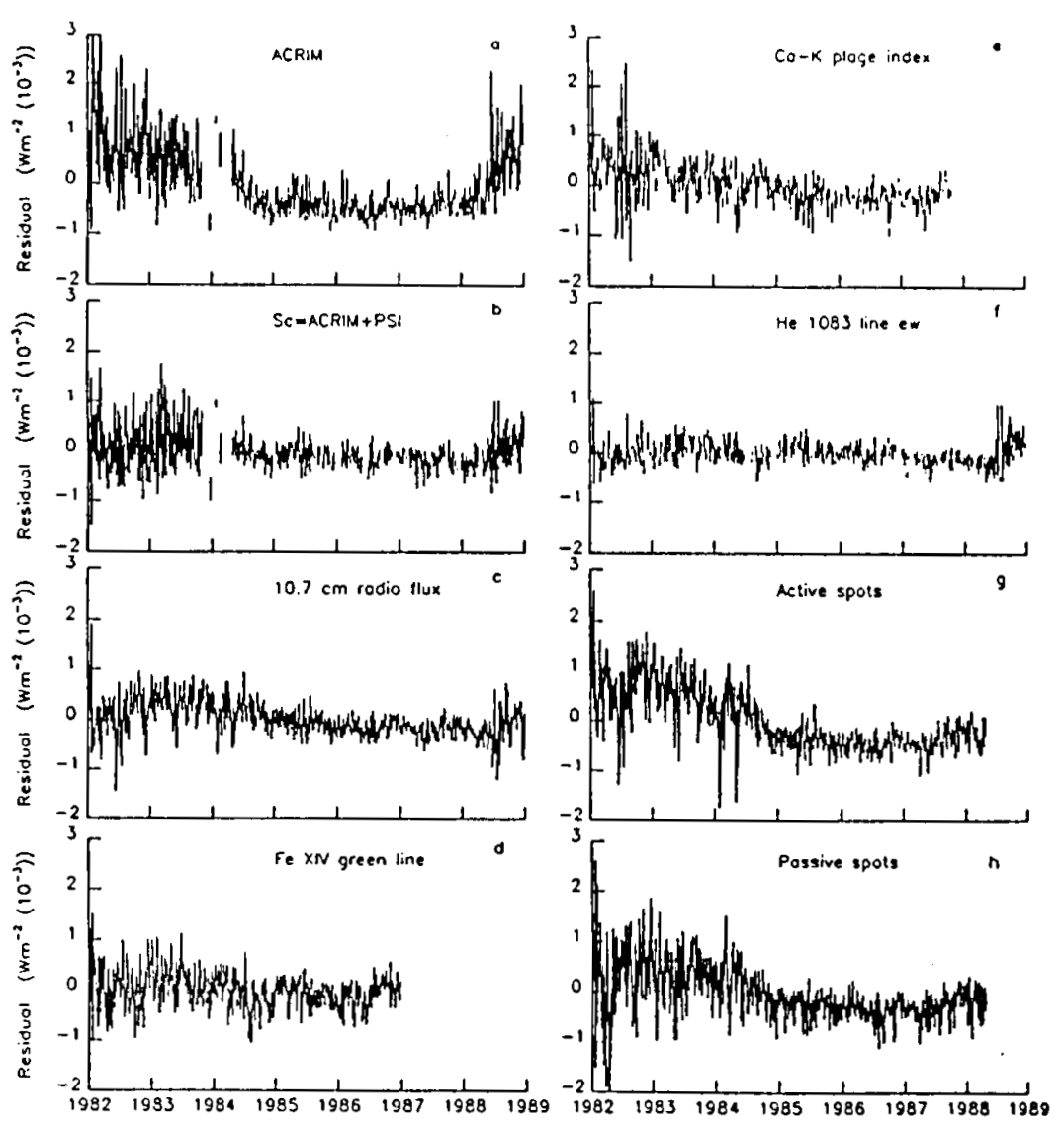

Fig. 2. The residuals after subtracting the daily values of Lyman alpha modeled with simple linear regression from the observed Lyman alpha data. The selected solar indices used for modeling are printed on each plot. 

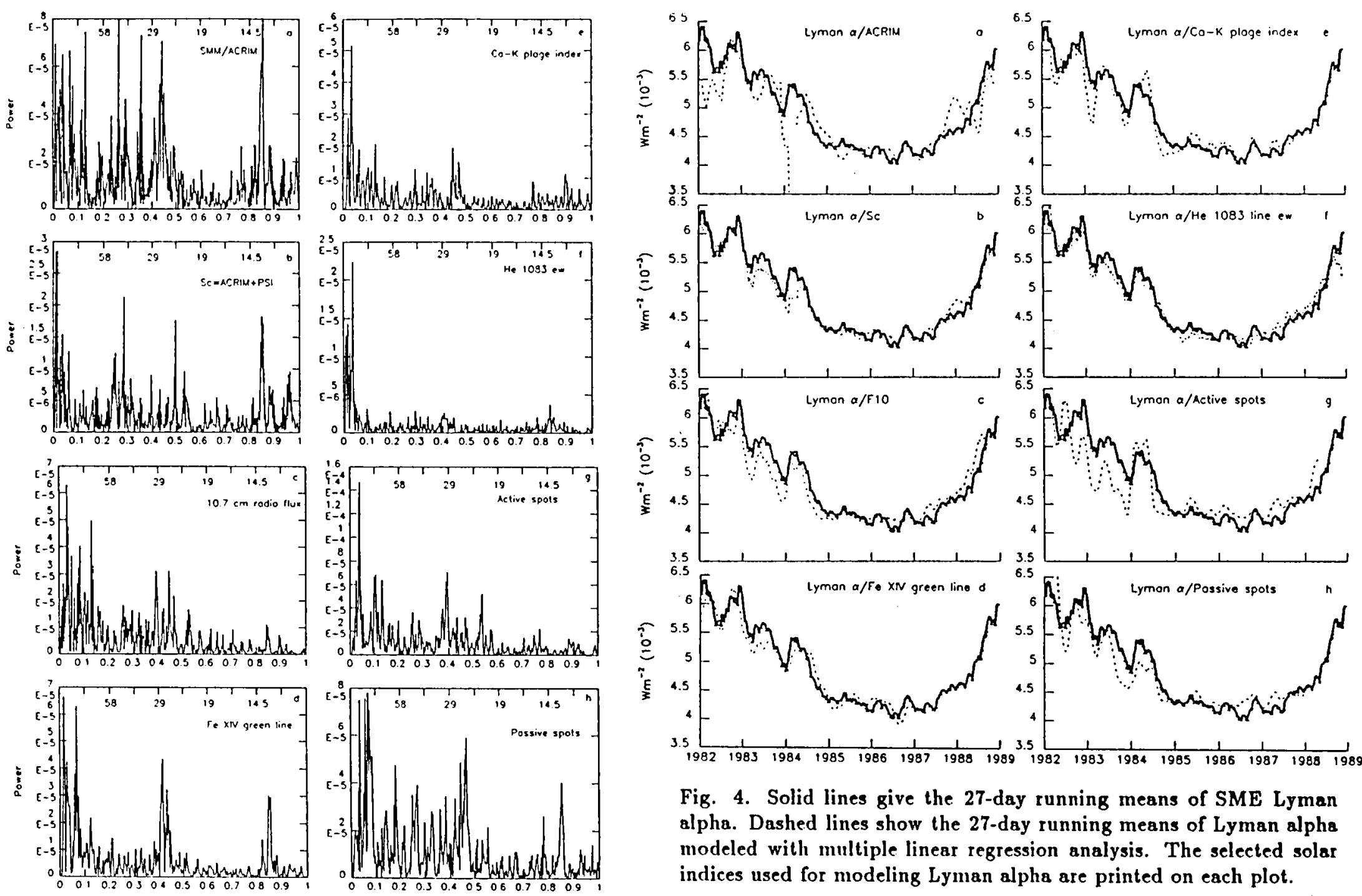

Fig. 4. Solid lines give the 27-day running means of SME Lyman alpha. Dashed lines show the 27-day running means of Lyman alpha modeled with multiple linear regression analysis. The selected solar indices used for modeling Lyman alpha are printed on each plot.

Fig. 3. Power spetra of residuals between the daily values of the observed Lyman alpha and Lyman alpha modeled with simple linear regression analysis. The solar indeces used for modeling Lyman alpha are printed on each spectrum. Periods in days are written on the top of the power spectra. 


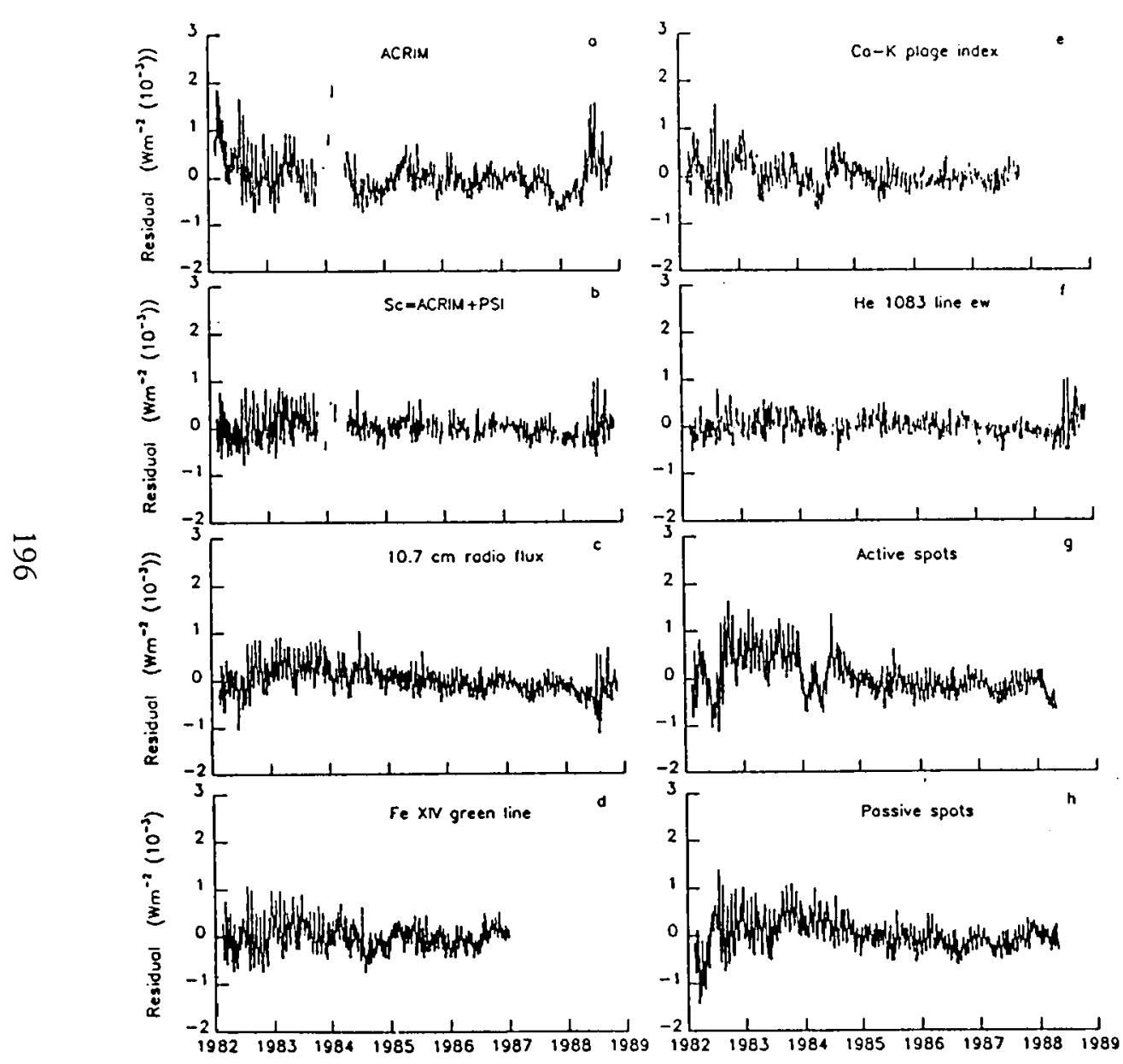

Fig. 5. The residuals between the daily values of the observed Lyman alpha and Lyman alpha modeled with multiple regression analysis are given. The solar indices selected for modeling Lyman alpha are printed on each plot.
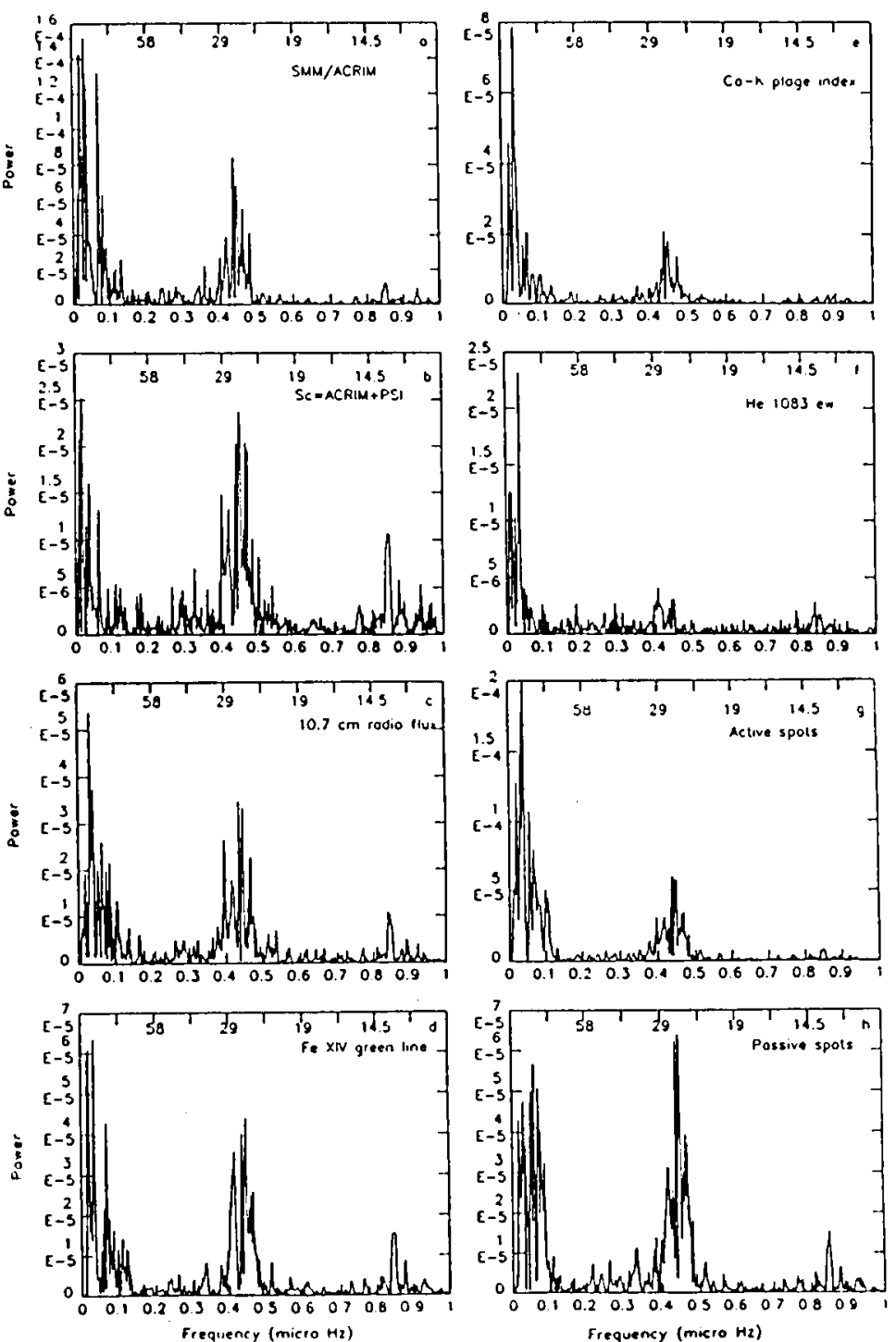

Frequency (micio $\mathrm{Hz}$ )

Fig. 6. Power spectra of residuals between the observed and modeled Lyman alpha values are given. Lyman alpha is modeled with multiple linear regression analysis. The appropriate solar indices used for modeling Lyman alpha are printed on each plot. The periods in days are also printed on each power spectrum. 
SOLAR CYCLE MODULATION OF TOTAL IRRADIANCE:

AN EMPIRICAL MODEL FROM 1874 TO 1988

\author{
J. Lean ${ }^{1}$ and P. Fouka1 2 \\ 1. E. 0. Hulburt Center for Space Research, \\ Naval Research Laboratory, Washington, DC 20375 \\ 2. Cambridge Research and Instrumentation, Inc., \\ Cambridge, MA 02139
}

\begin{abstract}
Evidence acquired during the past decade indicates that over time scales of the solar cycle, enhanced emission from bright solar faculae cause significant variations in the sun's total irradiance even though, on shorter time scales, the most pronounced variations are those resulting from the passage of dark sunspots across the solar disc. An empirical model which accounts for the competing effects of dark sunspots and bright faculae has been developed from the available radiometry in cycle 21 , and extended back to the beginning of solar cycle 12. According to this model, the largest 11-year modulation of total irradiance during the C20th occurred in the most recent cycle 21 .
\end{abstract}

\title{
OBSERVED VARIATIONS IN SOLAR TOTAL IRRADIANCE
}

Measurements of the sun's total irradiance have been made by two independent radiometers during solar cycle 21 and the ascending phase of cycle 22 . These data are illustrated in Figure 1. Data obtained by the Active Cavity Radiometer (ACRIM) $^{I}$ on the Solar Maximum Mission satellite and by the Earth Radiation Budget (ERB) ${ }^{2}$ experiment on the Nimbus 7 satellite were obtained from NASA's Climate Data System (NCDS) on 16 June 1989 and 21 Feb 1990, respectively. Both data sets in Figure 1 exhibit a solar cycle variation in which maximum irradiance coincides with maximum solar activity. Except for 1980, the ACRIM and ERB data are in general agreement during solar cycle 21 , over both short and long time scales. 3 However, important differences between the long term trends are evident in the two measurements from 1987 to 1988, the ascending phase of the new solar cycle 22. These differences are currently being investigated.

A variety of previous studies have identified sites of enhanced magnetic activity on the solar disc as the primary origin of the sun's total irradiance variations, $3,4,5,6,7$ over time scales of both solar rotation 6 and the solar cycle.3,7 Dark sunspots, regions on the solar disc where magnetic flux is strongly concentrated, dominate the short term irradiance variations. Bright faculae, of greater spatial extent than sunspots, but of less concentrated magnetic flux. dominate variations over the 3 to 6 month time scales of active region evolution. Enhanced emission from scattered faculae outside of those active region boundaries identified by CaII $K$ plage has been suggested as the source of additional brightness variations in the total irradiance over the solar cycle.3,7 Global pulsations ${ }^{1}$ and photospheric temperature changes ${ }^{8}$ have also been suggested as alternative causes of the solar cycle modulation of total irradiance. 
Extant observations of the areas, locations and contrast of sunspots on the solar disc enable an independent estimate of the contribution of these active region features to changes in the total irradiance. This quantity, the sunspot blocking function, $P_{S}$, is calculated from Foukal ${ }^{9}$ as

$$
P_{S}=\left(C_{S}-1\right) \sum A_{i} \mu_{i}\left(3 \mu_{i}+2\right) / 2 \quad \ldots \ldots(1)
$$

where $A_{i}$ and $\mu_{i}$ are the area (in units of the solar hemisphere) and heliocentric location of the $i$ th sunspot. $\left(C_{s}-1\right)=0.33$ is a measure of the average bolometric emission deficit in sunspots, relative to the background photosphere and $(3 \mu+2) / 5$ is the bolometric center-to-limb variation.

Other calculations of the sunspot blocking function, also called the photometric sunspot index, PSI, differ from that given in Equation 1. For example, Hoyt and Eddy 10 include separately the blocking by sunspot umbra and penumbra. Willson and Hudsonl employ the same center-to-limb function as in Equation 1 but with $\mathrm{C}_{\mathrm{s}}-1=0.35$. Schatten et a1.5 have adopted a multiparameter expression whose constants are derived by fitting the total irradiance observations. Shown in Figure 2 is a comparison of $P_{s}$ determined from Equation 1 with the calculations of Hoyt and Eddy, 10 for data over three solar cycles. An approximately systematic difference of $\sim 20 \%$, thought to be associated with a different center-to-limb function in the Hoyt and Eddy calculations, is evident.

Fig. 1. Measurements of the sun's total irradiance during solar cycles 21 and 22, measured by the ACRIM and ERB experiments.

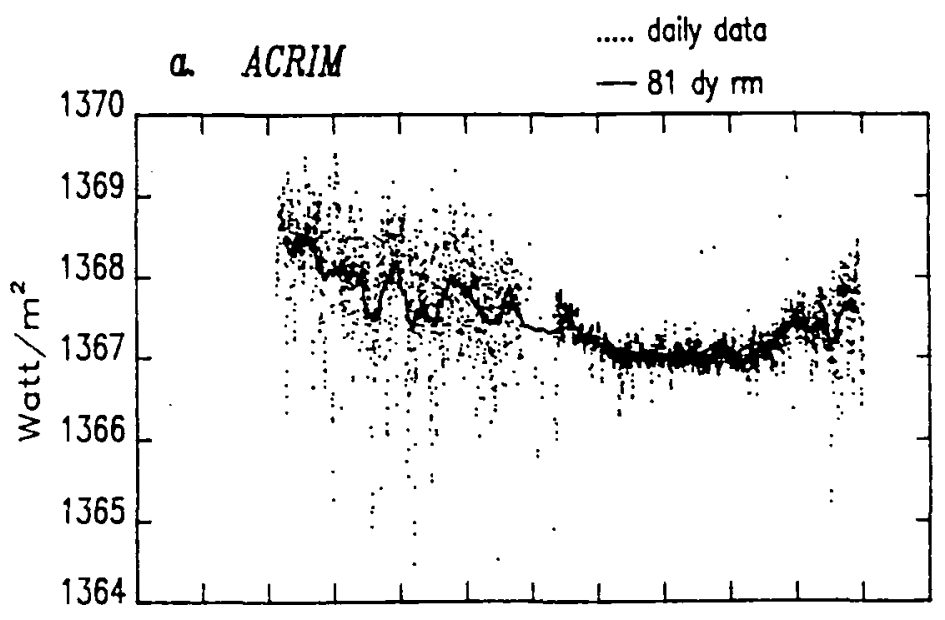

b. $E R B$

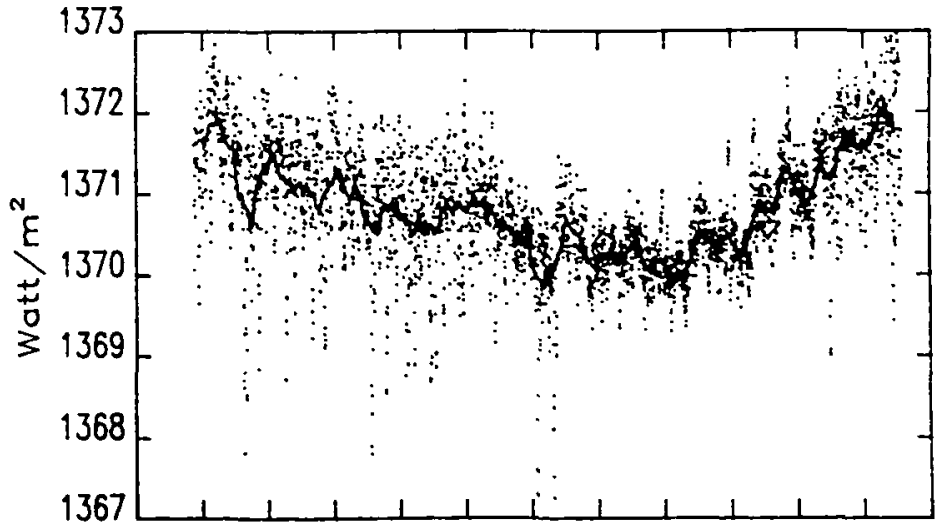

$\begin{array}{llllllllllll}78 & 79 & 80 & 81 & 82 & 83 & 84 & 85 & 86 & 87 & 88 & 89\end{array}$ YEAR 
Standardizing the calculations of the blocking of the sun's total irradiance by sunspots is an important priority for future modelling efforts.

\section{PARAMETERIZING THE FACULAR CONTRIBUTION}

Eaculae have been identified as playing a major role in the variations of the sun's total irradiance. However, a direct, independent estimate of their contribution to the irradiance variations is considerably less reliable than for the sunspot effect because faculae are more difficult to quantify. Their contrast at visible wavelengths is only a few percent, compared with $33 z$ for sunspots and, although their total disc area may be a factor of ten or more larger than that of sunspots, individual faculae, comprising polar faculae, ephemeral regions and network emission, are more dispersed across the solar disc, and less compact than sunspots.

In lieu of adequate observations of the disc coverage of faculae areas and of their contrast, an alternative approach to estimating their contribution to variations in the total irradiance is illustrated in Figures 3 and 4 .

Subtracting the calculated sunspot blocking function, $P_{S}$, and the quiet sun irradiance, $S_{0}$, from the measured irradiance, $S$, yields a residual time series, $\mathrm{S}-\mathrm{P}_{\mathrm{S}}-\mathrm{S}_{\mathrm{O}}$. This residual time series represents the brightness source in the total

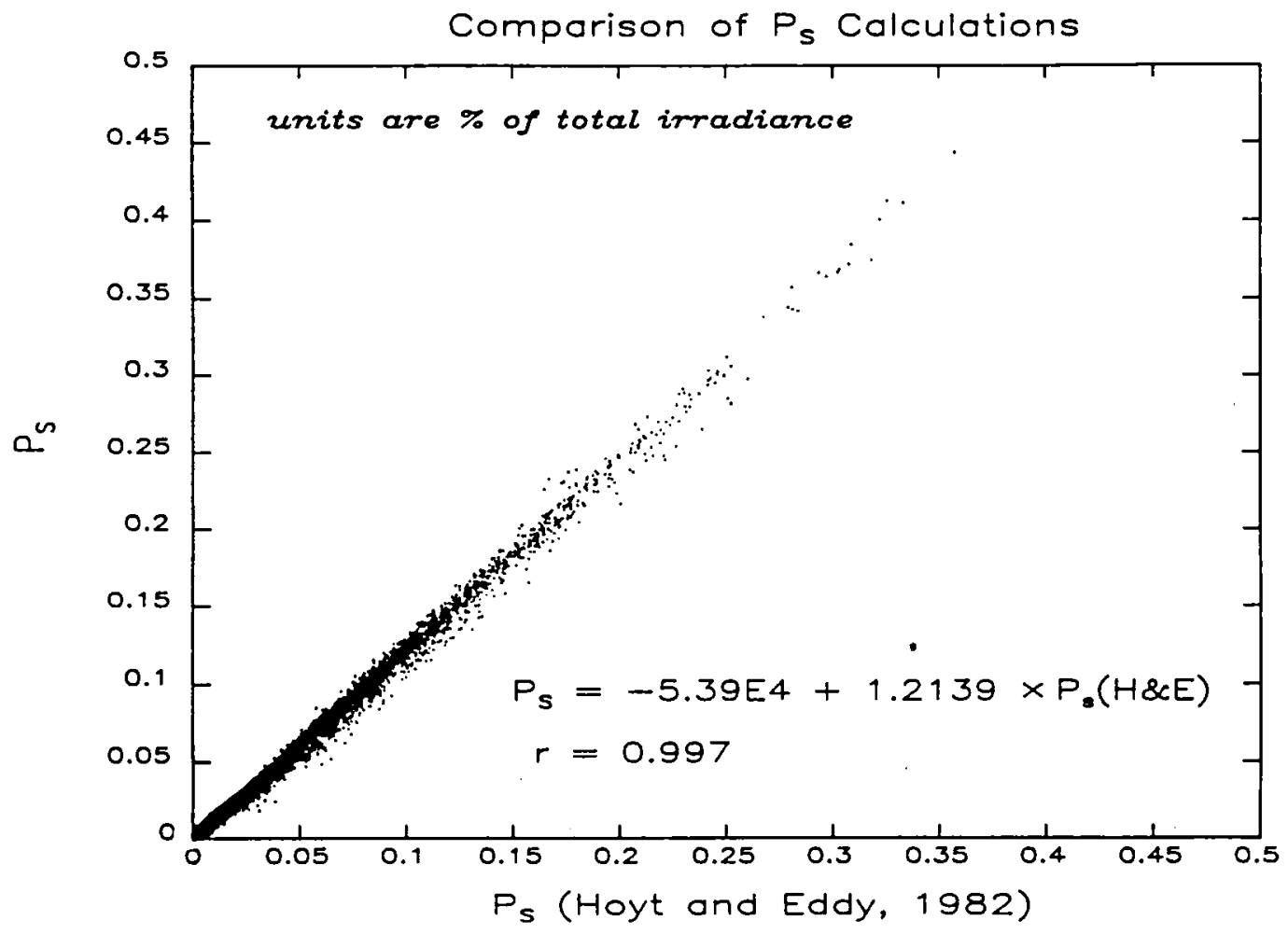

Fig. 2. Scatter plot of the sunspot blocking function, $P_{S}$, calculated by using Equation 1, with that calculated by Hoyt and Eddy, 10 from 1954 to 1984. The $P_{S}$ calculated from Equation 1 are systematically higher, by about $\sim 20 z$, than the calculations by Hoyt and Eddy. 10 
irradiance. Its temporal variations have been shown to track closely the changes in a variety of other emissions from the full solar disc, such as CaII $\mathrm{K}^{12}$, the equivalent width (EW) of the Helium $1083 \mathrm{~nm}$ line 3,12 and HI Lyman $a^{13,14}$, whose variations are known to be dominated by changes in enhanced emission from CaII $\mathrm{K}$ plage, the chromospheric extensions of photospheric faculae. As illustrated in Figures 3 and 4 , the correspondence between $S-P_{S}-S_{O}$ and $L a$ is equally good during the 27 -day time scale of solar rotation, and over longer, solar cycle time scales. Fitting the $S-P_{S}-S_{0}$ residuals to a suitable facular proxy (such as the La data) during the time interval of the $S$ measurements allows the brightness variations in total irradiance to be calculated from that proxy time series during times when $S$ was not measured. 14

Fig. 3. Variations in 1982 associated with solar rotation in $S$, the total irradiance measured by ACRIM (top panel), $P_{S}$, the calculated sunspot blocking function (second panel), $S-P_{S}-S_{O}$ (third panel) and HI $L \alpha$, the Lyman $\alpha$ irradiance at $121.6 \mathrm{~nm}$, measured by the solar spectrometer on the Solar Mesosphere Explorer (SME) satellite 11 (bottom panel).

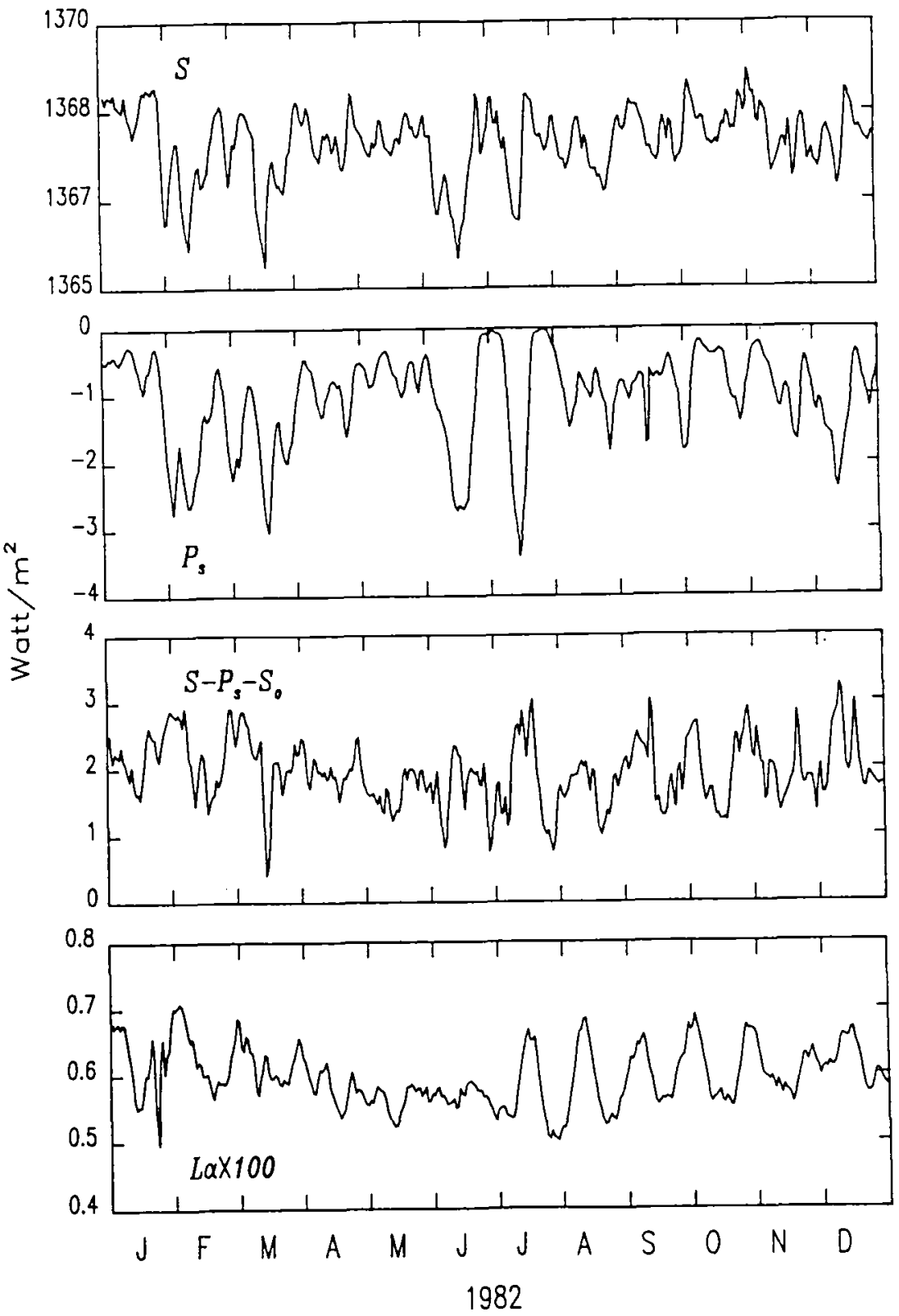


An empirical model of solar total irradiance variations is constructed by using $P_{S}$ from Equation 1 to represent the sunspot blocking, together with a facular proxy transformed to an equivalent facular enhancement via its correlation with the $\mathrm{S}-\mathrm{P}_{\mathrm{S}}-\mathrm{S}_{\mathrm{O}}$ residuals. Such models of the sun's total irradiance variation have been developed by using both the Helium $1083 \mathrm{~nm} \mathrm{EW}$ and the Lyman $a$ irradiance as proxies for the facular emissions during solar cycle $21,3,14$ and by using the $10.7 \mathrm{~cm}$ radio flux as a facular proxy during solar cycles 19, 20 and 21 . 15

\section{ESTIMATED LONG TERM TOTAL IRRADIANCE VARIATIONS}

To calculate historical solar total irradiance variations requires information about both the sunspot blocking and the facular brightening during each solar activity cycle. Observations of sunspot areas and locations made by the Greenwhich observatory allow $P_{s}$ to be calculated from 1874, onwards. Obtaining an appropriate proxy for the facular variations prior to solar cycle 19 is more difficult. Foukal and Lean 14 have demonstrated that during solar cycles 19, 20 and 21 , long term variations in the $10.7 \mathrm{~cm}$ full disk radio emission are highly correlated with long term variations in the sunspot number, $R_{z}$.

Fig. 4. Variations during solar cycle 21 and the ascending phase of solar cycle 22 in 81 -day running means of $S$, the total irradiance measured by ACRIM (top pane 1 ), $P_{S}$, the calculated sunspot blocking function (second panel), $\mathrm{S}-\mathrm{P}_{\mathrm{S}}-\mathrm{S}_{\mathrm{O}}$ (third panel) and HI La, the Lyman alpha irradiance at $121.6 \mathrm{~nm}$, measured by the solar spectrometer on the SME satellite (bottom panel).
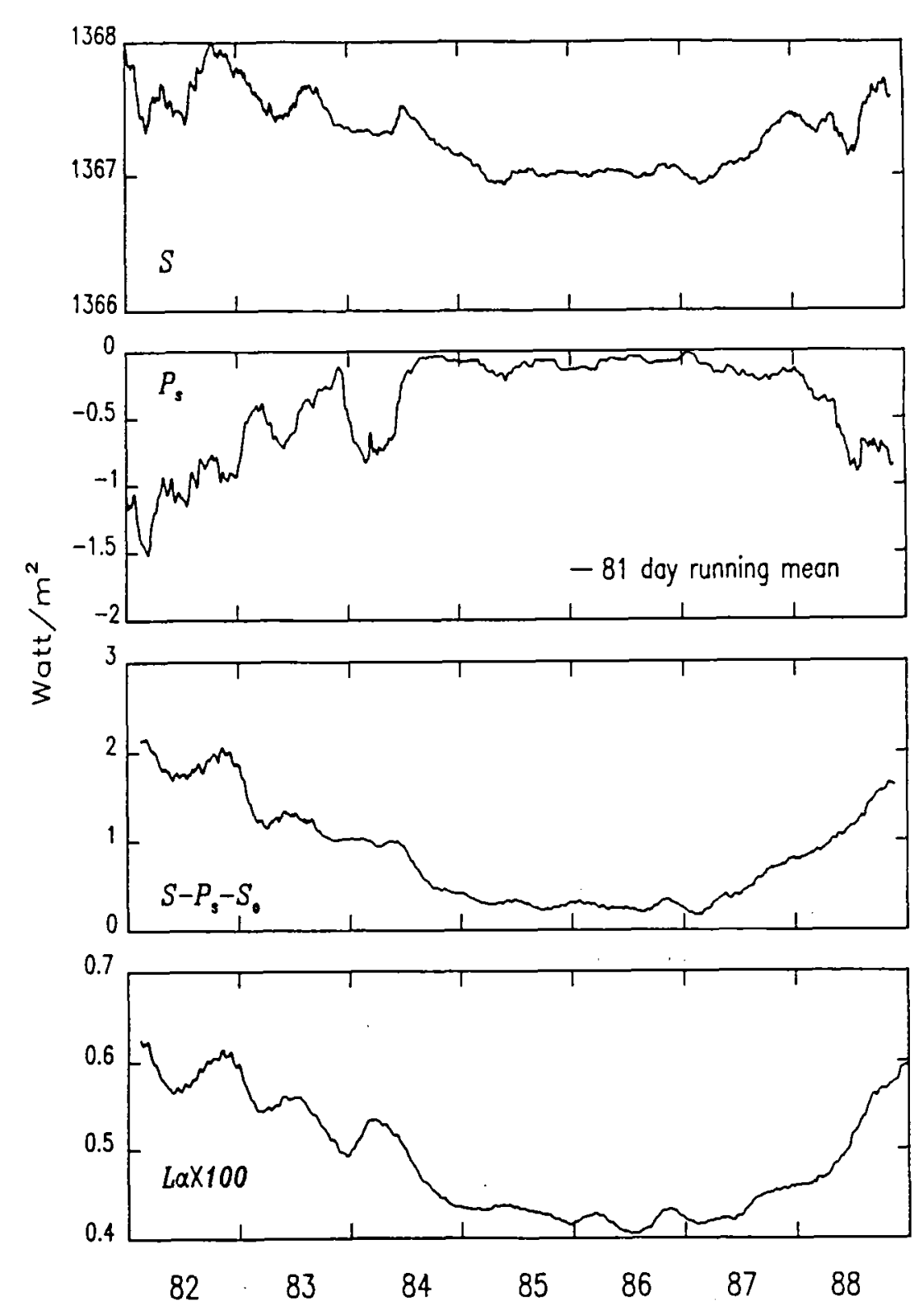
This correlation presumably reflects the weighting of $R_{z}$ by the number of sunspot groups (rather than by individual sunspot numbers), indicative of the occurrence on the solar disk of large scale, complexes of magnetic activity which are sites of enhanced facular emission. Since $R_{z}$ is available since 1874, the use of $R_{z}$ as a facular proxy allows estimates of $S$ variations from solar cycle 12 to the present.

Shown in Figure 5 are scatter plots of monthly mean values of the irradiance residuals $S-P_{S}-S_{0}$ with monthly mean $R_{Z}$, during the interval 1980 to 1988. Figure 6 compares the measured irradiance residuals with reconstructions using the monthly mean $R_{z}$, converted to irradiance units via the linear regression coefficients determined from the scatter plots in Figure 5 . By combining the facular variations estimated in this way from $R_{z}$, with the sunspot blocking calculated by using Equation 1, variations in the total irradiance can be estimated from 1874, onwards. Shown in Figure 7 are the monthly mean values of $S$, as calculated by an empirical model developed from both the ACRIM and ERB irradiance residuals. Note that there are slight differences between the $S$ variations in Figure 7 derived from ACRIM radiometry and the $S$ data in Foukal and Lean. 14 This is because, although the $S$ data published in Foukal and Lean were labelled as being derived from ACRIM radiometry, they were actually derived from ERB radiometry, but using an earlier version of the ERB data than are used here to generate the variations shown in Figure 7 .

Figure 7 illustrates that, according to this empirical model, there has been an overall increase of $\sim 0.1 z$ in the sun's total irradiance throughout the C20th, with the largest variation occurring in solar cycle 21 . When the variations in Figure 7 are used as input for simple climate models, the corresponding change in global temperature is of the order of $0.02^{\circ}$, or 6.77 of the $0.3^{\circ}$ temperature increase that has been detected since 1850.14

\section{ACKNOWLEDGEMENTS}

The ACRIM and ERB data were obtained from NCDS with the help of Jim Closs and Lola Olsen. Dick Willson and John Hickey are responsible for the ACRIM and ERB experiments, respectively. SME La data were provided by Gary Rottman.

\section{REFERENCES}

1. R. C. Willson and H. S. Hudson, "Solar luminosity variations in solar cycle $21^{n}$, Nature, $332,810,1988$.

2. J. R. Hickey, B. M. Alton, H. L. Kyle and D. Hoyt, "Total solar irradiance measurements by ERB/Nimbus-7. A review of nine years", Space Sc. Rev., 48 , $321,1988$.

3. P. Foukal and J. Lean, "Magnetic modulation of solar luminosity by photospheric activity", Astrophys. J. 328, 347, 1988.

4. H. S. Hudson, S. Silva, M. Woodard and R. C. Willson, "The effects of sunspots on solar irradiance", Solar Phys., 76, 211, 1982.

5. K. H. Schatten, N. Miller, S. Sofia and L. Oster, "Solar irradiance modulation by active regions from 1969 through 1980", Geophys. Res. Lett., $9,49,1982$

6. P. Foukal and J. Lean, "The Influence of faculae on total solar irradiance and luminosity", Astrophys. J., 302, 826, 1986. 

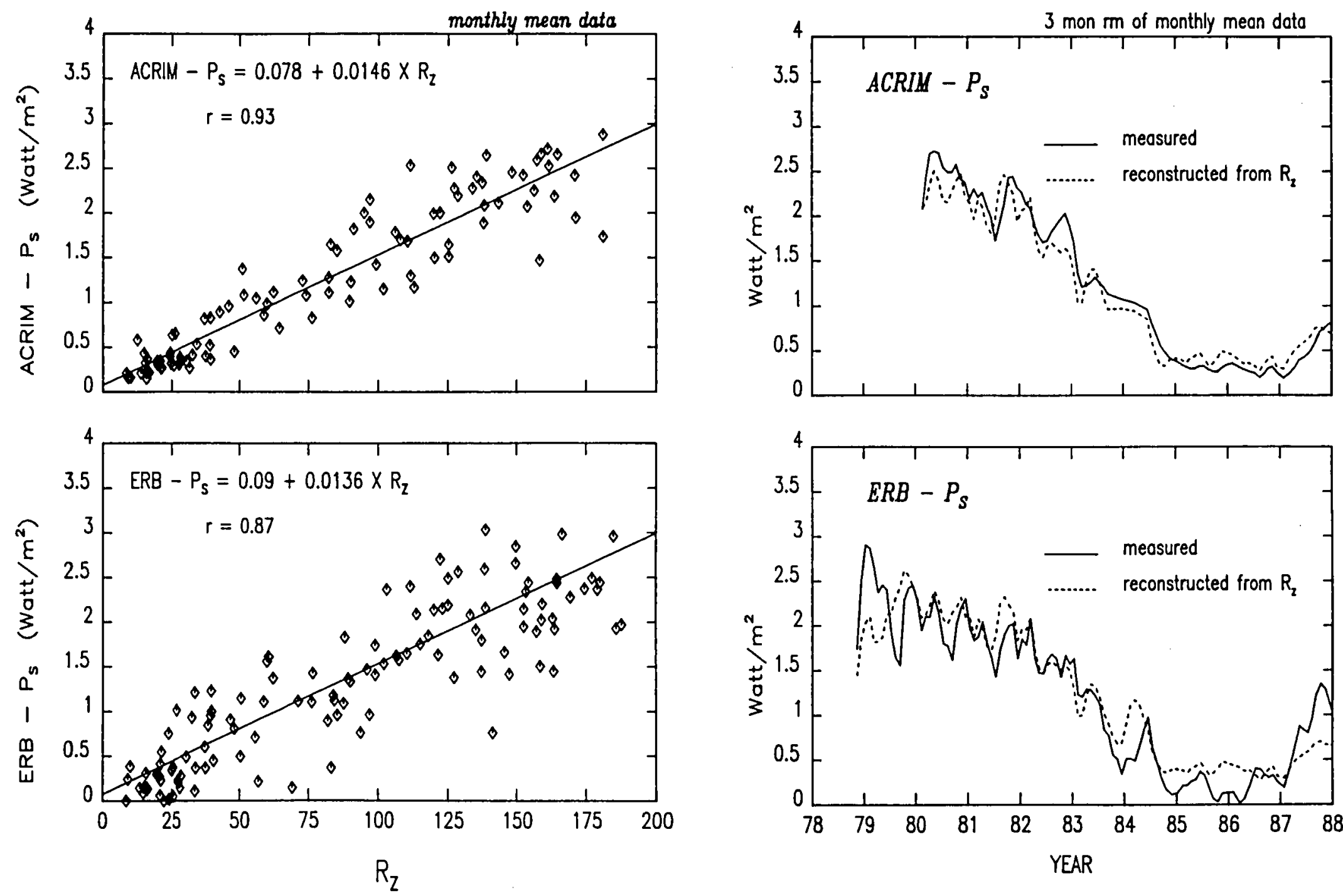

Fig. 5. Scatter plot of monthly mean values of $R_{z}$ with $S-P_{S}-S_{O}$, the residuals obtained by subtracting the sunspot blocking function from the total

irradiance variations measured by ACRIM (upper panel) and ERB (lower panel).

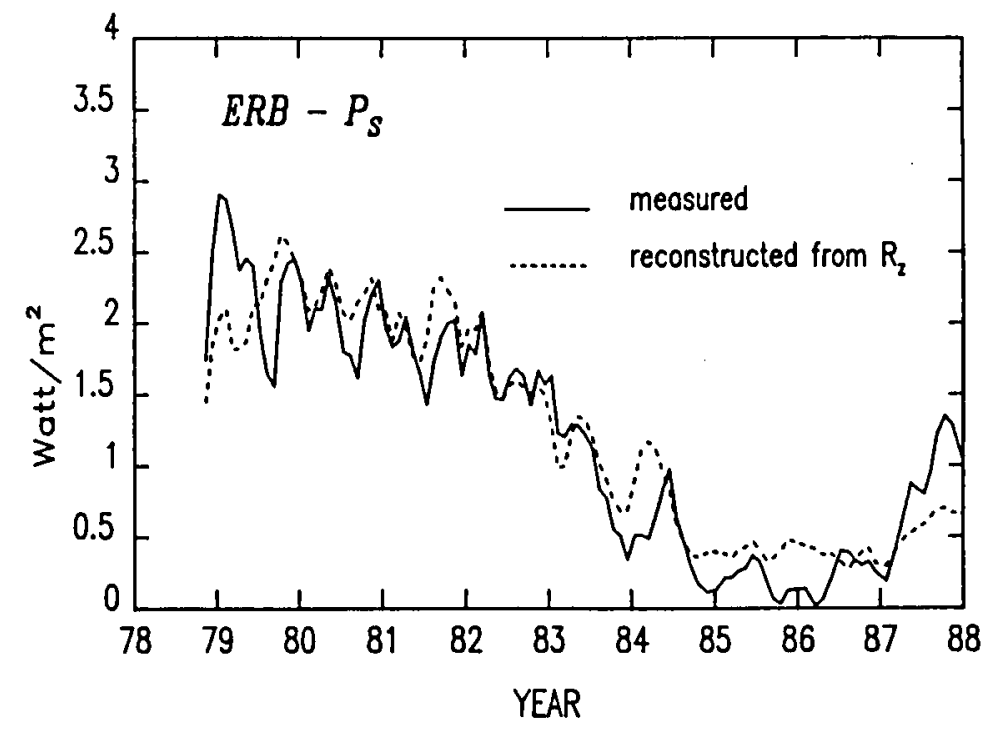

Fig. 6. Variations in 3-month running means of the monthly means of the irradiance residuals $S-P_{S}-S_{0}$ derived from ACRIM (upper panel) and ERB (lower panel), compared with the residuals calculated from monthly $R_{z}$. 


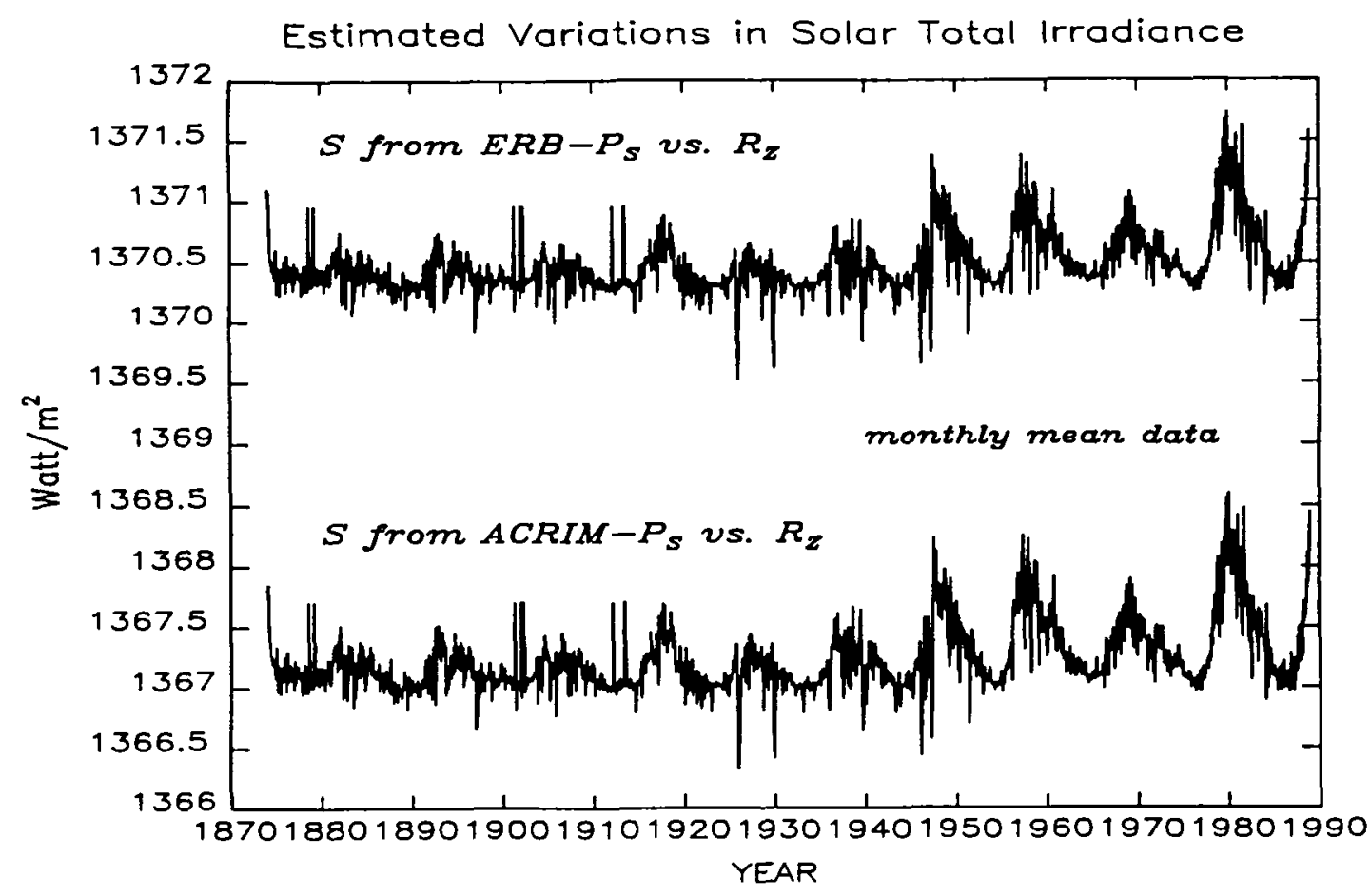

Fig. 7. Calculations of the variations in S during 1874 to 1985 , estimated from both the ACRIM and ERB residuals.

7. K. Schatten, "A model for solar constant secular changes", Geophys. Res. Lett., 15, 121, 1988.

8. J. R. Kuhn, K. G. Libbrechht and R. H. Dicke, "The surface temperature of the sun and changes in the solar constant", Science, 242, 908, 1988.

9. P. Foukal, "Sunspots and changes in the global output of the sun", in The Physics of Sunspots, Proceedings of a Conference held at Sunspot, New Mexico, 14-17 July, 1981, edited by L. E. Cram and J. H. Thomas.

10. D. V. Hoyt and J. A. Eddy, "An atlas of variations in the solar constant caused by sunspot blocking and facular emissions from 1874 to 1981", Tech. Note 194+STR, High Alt. Obs., NCAR, CO, May, 1982.

11. G. Rottman, "Results from space measurements of solar UV and EUV fIux", in Solar Radiative Output Variations, Proceedings of a Workshop held Nov. 911, NCAR, Boulder, Co., edited by P. Fouka1, p. 71, 1987.

12. W. C. Livingston, $L$. Wallace and 0 . R. White, "Spectrum line intensity as a surrogate for solar irradiance variations". Science, $240,1765,1988$.

13. J. Lean, "Contribution of ultraviolet irradiance variations to changes in the sun's total irradiance", Science, 244, 197, 1989.

14. P. Foukal and J. Lean, "An empirical model of total solar irradiance variation between 1874 and 1988", Science, 247, 505, 1990.

15. J. Lean and P. Foukal, "A model of solar luminosity modulation by magnetic activity between 1954 and 1984", Science, 240, 906, 1988. 
SESSION 7: CLIMATE CONNECTIONS II 


\title{
Climate Fluctuations and Climate Sensitivity
}

\author{
Gerald R. North and Kuor-Jier Joscph Yip \\ Climate System Research Program \\ Texas A\&M University \\ College Station, TX 77843 \\ Telephone: $409-845-8083$
}

May 18, 1990

\begin{abstract}
Some evidence is presented that the main part of the atmospheric climate system is such that small forcings in the heat balance lead to linear responses in the surface temperature field. By examining first a noise forced energy-balance climate model and then comparing with a long run of a highly symmetrical general circulation model, one finds a remarkable connection between spatial autocorrelation statistics and the thermal influence function for a point heat source. These findings are brought together to indicate that this particular climatological field may be largely governed by linear processes.
\end{abstract}

\section{Introduction}

The sensitivity of the ensemble averaged (climatological) surface temperature ficld to changes in the outside forcing such as the solar insolation or carbon dioxide loading of the atmosphere is a topic of obvious importance. Similarly, one wants to know as much as possible about the temporal delays in such responses. In this paper it is argued that certain aspects of the climate response, mainly the surface temperature, are controlled by effectively linear processes. By this I mean that say a $2 \%$ change in solar constant will lead to twice the response as a $1 \%$ change. In the following some evidence will be produced indicating that this is so and in turn how a study of the fluctuations in the system can shed some light on the sensititivity issue.

First, consider why such an approach is important. The controversy over the magnitude of global warming to be expected in the next 60 years is well known. Climate models differ markedly in their projections for conditions with an effectively doubled $\mathrm{CO}_{2}$ concentration in the 
atmosphere. The models give a range of from $2^{\circ} \mathrm{C}$ to about $5^{\circ} \mathrm{C}$ for their responses. Much of the uncertainty seems to be traceable to the differing ways the models include the effects of clouds (Cess et al., 1989). When we discuss the transient climate problem, i.e., the response of the system to a gradual (say $1 \%$ per year) increase in the effective $\mathrm{CO}_{2}$ concentration we have the question of how the slower parts of the system (mainly oceans) share and effectively sequester the heat away from the surface and thereby cause a substantial lag in the surface responsc. The problem of cloud feedback and oceanic lag are the first order problems of forced climatic change; after that we must begin to look at the way the general circulation of the atmosphere and the occans might be distorted by small changes in the forcing. These may be amplified in subtle ways by how the system disposes of latent heat through precipitation. Precipitation is notoriously poorly simulated by today's general circulation models (GCMs).

The above problems illustrate the need to occasionally step back and look at the bigger picture in search of general principles that can help us in cross-checking our results. Getting a better feeling for the climate sensitivity has enormous practical implications as well. The difference in policy response to a $2^{\circ} \mathrm{C}$ versus a $5^{\circ} \mathrm{C}$ scenario could be quite dramatic. It might mean the difference between an adaptive versus an interventionist strategy. Hence, it is very important to establish whether we are on the high or low end of the sensitivity range. Is there any evidence from the natural fluctuations in the system to tell us about the climate's sensitivity to cxternal changes?

We are not the first to enter such a program since it was first opened by Leith (1975) and examined in some fluid dynamical model calculations by Bell $(1980,1985)$ in the context of the socalled Fluctuation Dissipation Theorem. The theorem states that in certain nonlinear fluctuating systems the sensitivity to changes in external forcing can be related to temporal autocorrelation propertics. The example discussed in this paper is a trivial application of the theorem to a linear system forced by noise. Our task is to see how the all-important surface temperature field in the real world can be modeled by such a linear noised forced model. We postpone consideration of the real world for lack of data just now and concentrate on the available data set gencrated by a long run of a GCM with highly symmetrical boundary conditions.

In the following, some results from linear energy balance climate models (EBMs) will be reviewed along with a discussion of their sensitivity to changes in external forcing at various frequencies of forcing. Then some interesting properties of a noise forced linear EBM will be discribed as an analog of the real fluctuating climate system. Then I will discuss a long run of a GCM for an idealized planet. By examining the results of the natural fluctuations of this system and some simple experiments a preliminary assessment of the linearity question will be given. 


\section{Linear EBM Review}

The EBM used in the present study is derived from one introduced by North, Mengel and Short (1983) and recently updated by Hyde et al., (1989). It can be completely characterized by the governing energy balance equation

$$
C(\hat{\mathbf{r}}) \frac{\partial}{\partial t} T(\hat{\mathbf{r}}, t)-\nabla \cdot(D(\hat{\mathbf{r}}, t) \nabla T(\hat{\mathbf{r}}, t))+A+B T(\hat{\mathbf{r}}, t)=Q S(\hat{\mathbf{r}}, t) a(\hat{\mathbf{r}})
$$

where

$\hat{\mathbf{r}}=$ point on the sphere

$t=$ time of year

$T(\hat{\mathbf{r}}, t)=$ surface temperature at point $\hat{\mathbf{r}}$ and time $t$

$A, B=$ phenomenological infrared radiation constants, from satellite data

$C(\hat{\mathbf{r}})=$ location dependent heat capacity (large over ocean:60, small over land:1)

$D(\hat{\mathbf{r}})=$ latitude (only) dependent thermal diffusion coefficient

$Q=$ solar constant $/ 4=340 \mathrm{~W} / \mathrm{m}^{2}$

$S(\hat{\mathbf{r}}, t)=$ normalized solar insolation at the top of the atmosphere

$a(\hat{\mathbf{r}})=$ local coalbedo, here given a smooth latitude dependence from satellite data

For a discussion of the parameters and their physical interpretations see the references cited. Suffice it to say that there are a small number of adjustable paraneters (three in $D(\hat{\mathbf{r}})$ and one in $C(\hat{\mathbf{r}}))$ which are adjusted to obtain the best possible fit to the present climate. Since the system is linear as posed, it is convenient to develop the solution into harmonics of the annual cycle, a rapidly converging series. Maps of the modeled and observed annual harmonic and phase show a good degree of similariy. For these and other comparisons the reader is refered to Hyde et al., (1989). The semiannual harmonic is everywhere outside the polar regions less than $2^{\circ} \mathrm{C}$ and its pattern looks like that of the data. This is already extremely strong evidence that the system is behaving linearly at least at time scales in the frequency band around the annual cycle. 


\section{$2.1 \quad$ Noise Forcing}

Next consider the departures $T^{\prime}(\hat{\mathbf{r}}, t)$ from the equilibrium seasonal cycle by fluctuations induced by a noise forcing (see Hasselmann, 1976; North and Cahalan, 1981; Leung and North, 1990). The governing equation of the fluctuations is

$$
C(\hat{\mathbf{r}}) \frac{\partial}{\partial t} T^{\prime}(\hat{\mathbf{r}}, t)-\nabla \cdot\left(D(\hat{\mathbf{r}}, t) \nabla T^{\prime}(\hat{\mathbf{r}}, t)\right)+B T^{\prime}(\hat{\mathbf{r}}, t)=F(\hat{\mathbf{r}}, t)
$$

where $F(\hat{\mathbf{r}}, t)$ is a white noise forcing in both space and time. That is, its autocorrelations vanish unless they are taken at equal time and position

$$
\left\langle F(\hat{\mathbf{r}}, t) F\left(\hat{\mathbf{r}}^{\prime}, t^{\prime}\right)\right\rangle=\sigma_{F}^{2} \delta\left(\hat{\mathbf{r}}-\hat{\mathbf{r}}^{\prime}\right) \delta\left(t-t^{\prime}\right)
$$

The noise forcing may be thought of as a combination of imbalances due to transport related eddies or such local influences as cloudiness fluctuations. In what follows we take $D(\hat{\mathbf{r}})$ and $C(\hat{\mathbf{r}})$ to be independent of position for simplicity.

Next is a list of results that follow directly from the equations. The global average tempcrature is a first order Markov process with autocorrelation time $\tau_{0}=C / B$. One can also show that the higher spherical harmonic mode amplitudes have characteristic times

$$
\tau_{n m}=\frac{\tau_{0}}{(n(n+1) D / B+1)}
$$

which are also the decay times of the unforced system. The spatial statistics for this system are also readily computed. Consider the autocorrelation function in space for equal times (we use the plane tangent to the sphere to obtain closed form solutions with familiar functions)

$$
\rho(s, \tau=0)=K_{0}\left(\frac{s}{\ell}\right)
$$

where $s$ is the distance separating the points, $K_{0}$ is the Bessel function and $\ell^{2}=D / B$ is a characteristic length in these climate models. Its interpretation is as follows: a thermal anomaly is carried away from a point by random walk a distance $\sqrt{D t / C}$; the appropriate time $t$ is the radiation damping time $C / B$; insertion gives for the characteristic distance $\ell$. Another spatial autocorrelation of interest is the low frequency limit (i.e., the time series is low pass filtered before the autocorrelation in space is computed)

$$
\rho(s, f \rightarrow 0)=\frac{s}{\ell} K_{1}\left(\frac{s}{\ell}\right)
$$

The Green's function for a point heat source was given by North (1984). Its form is simply 


$$
G(s)=g K_{0}\left(\frac{s}{\ell}\right)
$$

where $s$ is the distance of the heat source to the point being examined and $g$ is the strength of the heat source. Note that the shape of the Green's function is the same as the equal time spatial autocorrelation function. The latter shape equivalance is a general property of a large class of linear heat transport operators of which diffusion is an example. This property of linear models tells us that we can learn about the Green's function by examining certain spatial autocorrelation propertics of the natural system.

Finally, we show observed data from the paper by Hansen and Lebedeff (1987) for correlations between separated stations of annual average surface temperatures for various latitude bands. Since the real earth contains oceans ( 5 year time constant) as well as land (one month time constant) near the stations considered, these spatial correlations represent something in between the equal time case and the low frequency case. However, we are certainly left with the impression that a length scale between 1000 and $2000 \mathrm{~km}$ is involved. This is in substantial agreement with the EBM calculations described above.

It is important to note that the length scale in the nfEBM is controlled by the diffusion parameter and the radiation parameter. This is different from the length scale often encountered in dynamics, the Rossby Radius of Deformation, which has nothing to do with radiation damping. We argue that the length scale in the Hansen-Lebedeff data is the climate length scale which is controlled by radiation because it comes from annual averages. One way to eliminate the RRD from the present length scale is that the RRD should have a much stronger latitude dependence $(1 / \sin ($ latitude $))$ than is found in the data.

\subsection{Selected GCM Results}

We have conducted a long run (15 years) of the NCAR CCM0 (R15 version) on the Texas A\&M Cray Y-MP computer with the assistance of Dr. Robert Chervin of NCAR. We have saved all output from the run once per model day but are particularly interested at the moment in the surface temperature field. In addition, to facilitate comparison with simpler models and to avoid confusion with too many variables, we have simplified the boundary conditions in the CCM0 as follows: 1) The obliquity is set to zero so that the climate system is forced by perpetual equinox solar insolation. 2) All mountains have been removed so that the planet has only sea level topography. 3) Oceans and other zonal symmetry brealing features have been removed so that it is an all-land planet. The planet has no snow to change the local albedo. Statistically, every month is equivalent to every other month, all longitudes are statistically equivalent and the planet is northsouth symmetric statistically. The longest time scale in the model (autocorrelation time of global 


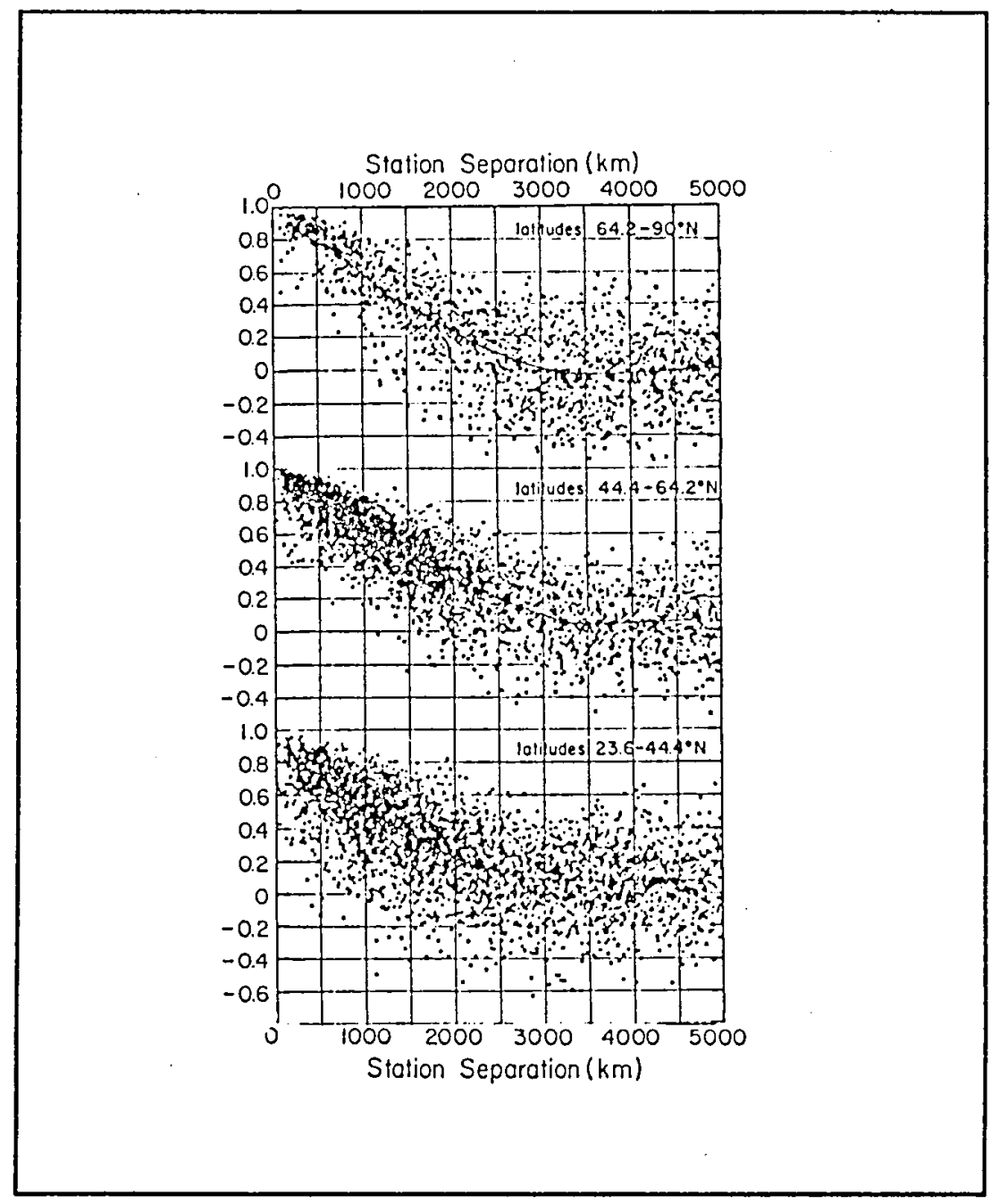

Figure 1: The spatial autocorrelation between sites separated by distance $s$ for annually averaged temperatures. The three panels show the relationship for three different latitude bands. From Hansen and Lebedeff(1987) 
average surface temperature) is about 30 days. Hence, 15 years represents $15 \times 12=180$ such units, an extremely long time series. By contrast the corresponding time scale when a mixed layer ocean is present is 5 years, which would require a 900 year run for equivalent statistics. Furthermore, since each longitude is statistically equivalent and the hemispheres are (statistically) reflection symmetric, the data can be pooled to have an even greater sample. While this configuration has the weakness of ignoring certain important forcing mechanisms (such as mountains and lagging heat sources such as seasonally varying ocean surfaces) it is a physically realizable planet and should yield some interesting insights into the mechanisms of climate dynamics. We refer to the planet as Terra Blanda.

It is important to emphasize from the beginning that the CCM0 version of the climate system (R15, noninteractive soil moisture) is far from realistic in many respects. It is nonetheless close to models being used in routine simulations today and therefore to better understand it is a worthy goal considering the policy implications of present day model simulations. The T42 (CCM2) version is now being tested for experiments by the community, and we intend to employ it in some of our later experiments to check that our conclusions are insensitive to resolution.

As part of our preliminary work with CCMO and its simulation of surface temperatures for Terra Blanda we are working out the complete climatology for the planet. We report here only those aspects of the climatology relevant to the research plan to be discussed in the next section.

First consider the autocorrelation times for various spherical harmonic mode amplitudes of the CCM0 surface temperature data. We obtain these by projecting out these mode amplitudes and examining the corresponding time series. The lowest mode $(0,0)$ has a time constant $\tau_{0}$ of 29 days. The autocorrelation function is reasonably exponential in shape (in agreement with a noise-forced linear model). Higher modes also exhibit exponential autocorrelation functions with a sequence of time scales in rough agrecment with those suggested by the linear model.

Next we turn to the question of spatial correlations in the CCMO data. Consider first the equal time spatial correlations, which are shown in Fig. 2 for a point centered at $60^{\circ}$ latitude. The horizontal scale on the map has been stretched so that distances in either direction are in locally equal length units (a small circle on the sphere should look approximately like a small circle here). The contours in the map show equal correlation lines. Note that the equal time autocorrelation length is about $13^{\circ}$ of latitude on the map $(\approx 1300 \mathrm{~km})$. Note first how remarkably isotropic the correlations are. Figure 3 shows the same contours for a low pass filtered $(\approx 60$ day moving average). We see an east-west elongation of the contours and a small stretching effect not unlike the effect predicted in Fig. 1 for low-pass filtering. The anisotropy is easily identified with advection, since correlations lagged by one day show the maximum of the contours moving to the east at about $2.5 \mathrm{~m} / \mathrm{sec}$. However, the advected correlation decays very quickly with an e-folding time of its peak of only about 2.8 days. Hence, while advection is clearly present, it may be of little practical importance for the surface temperature in climate applications. (It is clearly the 
very essence of weather forecasting).

As another test of linearity in the surface temperatures generated by the CCM0 we consider the case of the response of the temperature field to a steady point (one grid site) heat source injected at the surface. The standard deviation of the surface temperature at a point is about $15^{\circ}$ $\mathrm{C}$ with an autocorrelation time of about 3 days. Hence, in order to graph the spatial climatological response to a steady $400 \mathrm{~W} / \mathrm{m}^{2}$ heat source (local time averaged response $\approx 6.2^{\circ} \mathrm{C}$ ) requires several years of model generated data. After some experimentation, we exploited the symmetry in our model to combine results from a "picket fence" of point sources placed $45^{\circ}$ apart around a latitude circle in both hemispheres. After pooling the data, the resulting contour map of responses can be found and is shown in Fig. 4. Note how nearly isotropic the map is. The distortion from isotropy is surely due to the small advection alluded to earlier. The scale of the contours is in agreement with the scale of the equal-time autocorrelations as predicted by the noise-forced EBM. Finally, we changed the strength of the heat source to $2 / 3$ that used above. The result (not shown) was identical within sampling errors to that shown in Fig. 4 except for the scaling.

\section{Conclusion}

The above preliminary results suggest that forced surface temperature climate change is essentially a linear response to external forcings such as changes in the solar constant. This is remarkable considering that heat transport on the sphere is governed by extremely nonlinear interactions. We are apparently seeing the result of statistical ensemble averaging at its most beneficial. The advection of heat which is dominant for the purposes of weather forecasting is essentially indistinguishable from thermal diffusion for ensemble averaged climate. The linearity has shown up in the spatial and temporal autocorrelation statistics as well as in the scaling of point-heat-source strength/response characteristics. Of course, our analysis leaves out the rather obvious nonlinearity associated with snow-albedo feedback and other simplifications which could also induce nonlinear response such as mountain wave locking are similarly omitted. The main importance of the results is that the sensitivity of the real climate system may be relatable to the natural fluctuation statistics. This could be a key element in a strategy for deciding between competing models whose sensitivities are grossly different.

\section{References}

- Bell, T. L., 1985: Climatic sensitivity and fluctuation-dissipation. in Turbulence and Predictability in Geophysical Fluid Dynamics and Climate Dynamics. Corso. Soc. Italiana di 


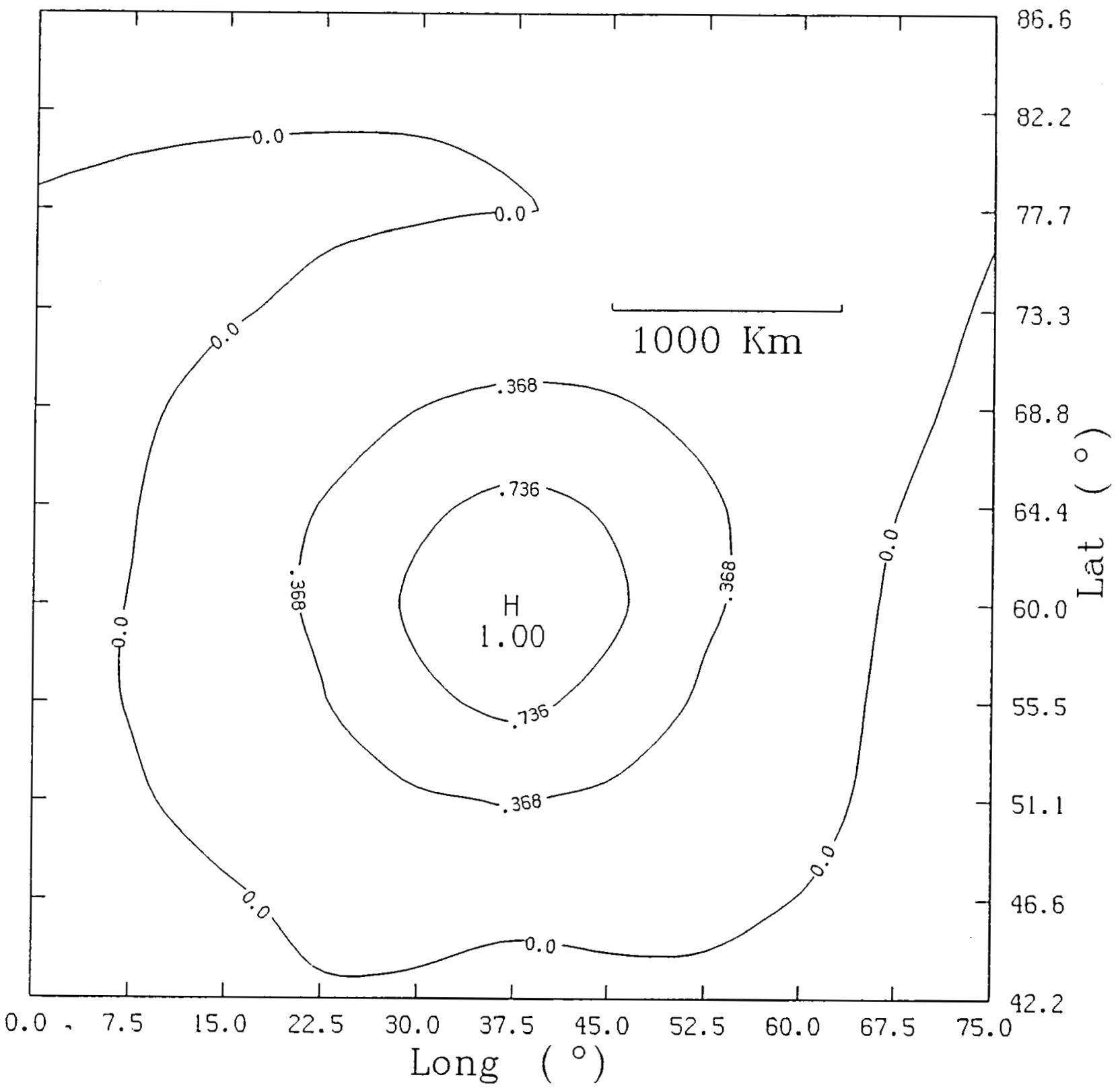

Figure 2: Equal time spatial correlations with the surface temperature at a point located at $60.0^{\circ}$ latitude for Terra Blanda as simulated in the CCMO. Horizontal and vertical scales are approximately equal for linear distances. 


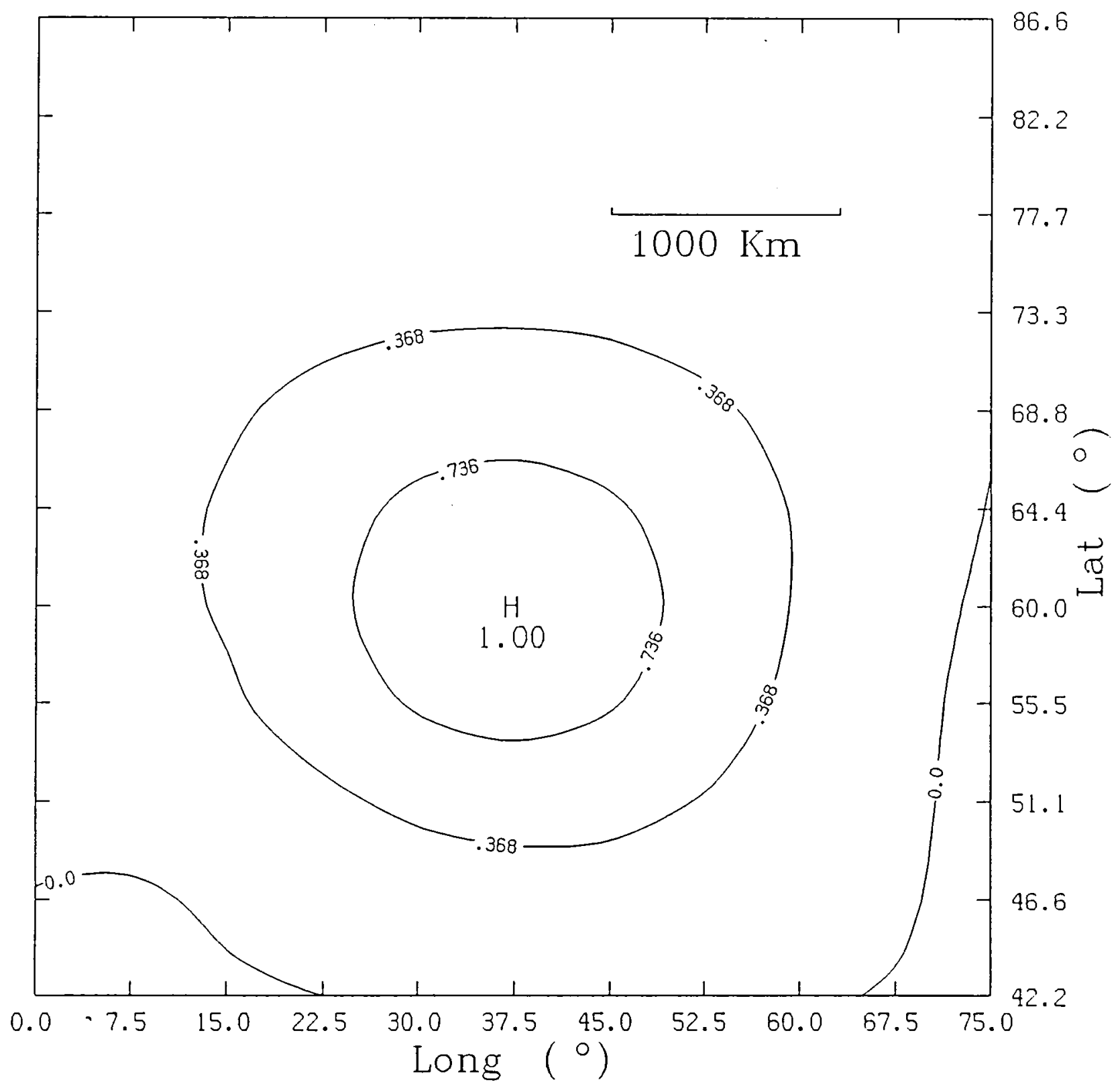

Figure 3: Same as Fig. 2 except that the time series has been low pass filtered (61 day moving average). 


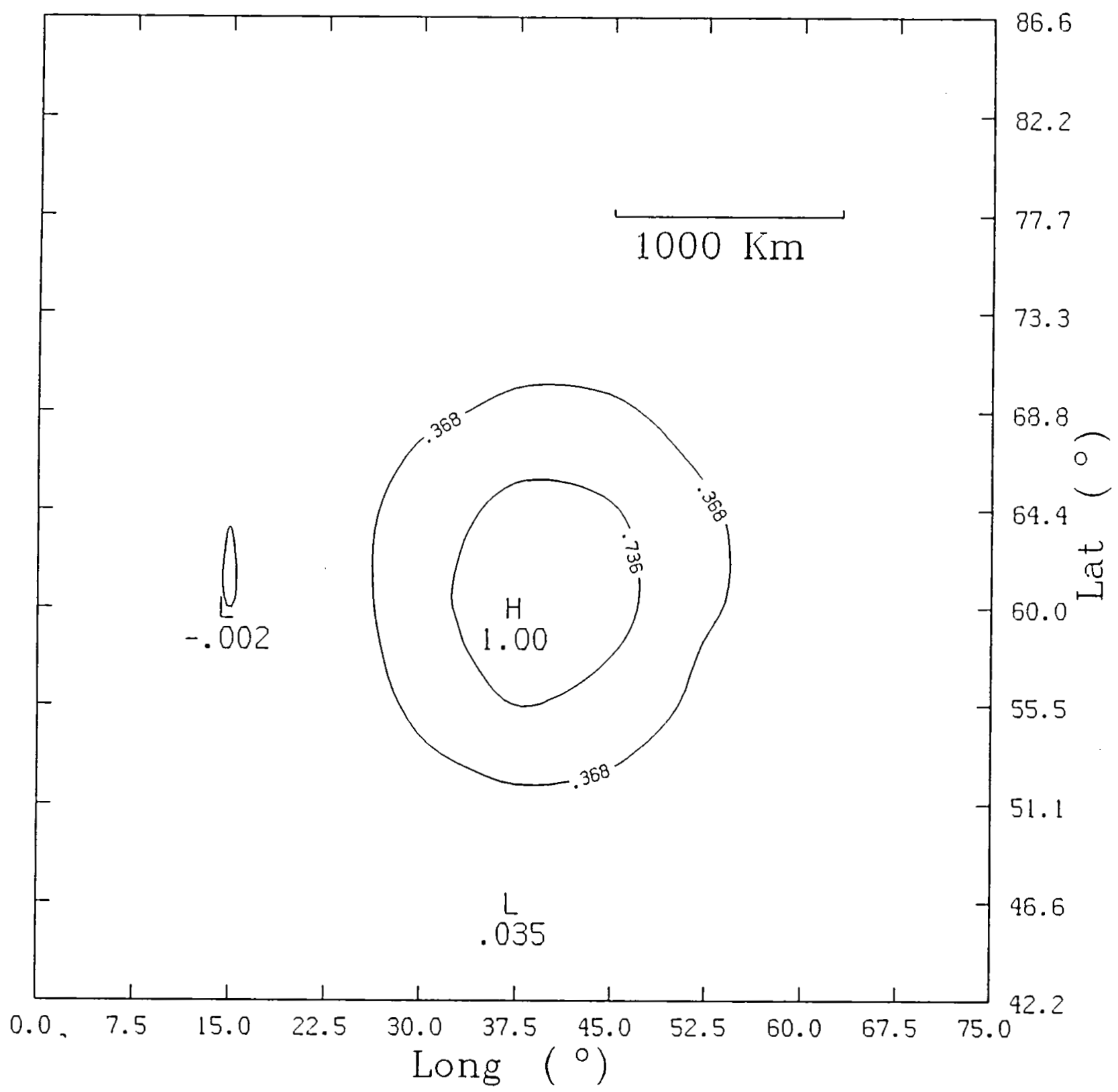

Figure 4: Time averaged surface thermal response to a heat source at a single grid point of strength of $400 \mathrm{~W} / \mathrm{m}^{2}$. 
Fisica. 1985.

$\diamond$ Bell, T. L., 1980: Climatic sensitivity and fluctuation-dissipation: Some simple nodel tests. J. Atmos. Sci., 37, 1700-1707.

- Cess, R. D, (and 19 coauthors), 1989: Interpretation of cloud-climate feedback as produced by 14 atmospheric general circulation models. Science. 245, 513-516.

$\diamond$ Hansen, J. and S. Lebedeff, 1987: Global trends of measured surface air temperature. J. Geophys. Res., 92, 13345-13372.

○ Hasselmann, K., 1977: Stochastic climate models. Part I: Theory. Tellus. 28, 473-484.

- Hyde, W. T., T. J. Crwoley, K.-Y. Kim and G. R. North, 1989: Comparison of GCM and energy balance model simulations of seasonal temperature changes over the past 18000 years. J. Clim., 8, 864-887.

$\diamond$ Leith, C. E., 1975: Climate response and fluctuation dissipation. J. Atmos. Sci., 32, 20222026.

- Leung, L.-Y. and G. R. North, 1990:; Information theory and climate prediction. J. Clim., $3,5-14$.

$\diamond$ Mengel, J., D. A. Short and G. R. North, 1988: Seasonal snowline instability in an energy balance model. Clim. Dynam., 2, 127-131.

- North, G. R. and R. F. Cahalan, 1981: Predictability in a solvable stochastic climate model. J. Atmos. Sci., 38, 504-513.

- North, G. R., J. G. Mengel and D. A. Short, 1983: Simple energy balance model resolving the seasons and the continents: Application to the astrononomical theory of the ice ages. J. Geoplyys. Res. 88, 6576-6586.

$\diamond$ North, G. R., 1984: The small ice cap instability in diffusive climate models. J. Atmos. Sci., 41, 3390-3395. 


\title{
FEEDBACK PROCESSES AND CLIMATE RESPONSE
}

\author{
Albert Arking \\ NASA Goddard Space Flight Center \\ Greenbelt, MD 20771
}

\begin{abstract}
The repsonse of the climate system to an external perturbation--e.g., a change in solar irradiance or a change in atmospheric opacity due to an increase in $\mathrm{CO} 2$--depends rather strongly on feedback processes in the system, which either amplify or dampen the effects of the initial perturbation. A simple representation of the climate system is used to compare several important feedbacks, based upon GCM simulations by various investigators. The models are in general agreement with respect to water vapor feedback, but in wide disagreement with respect to cloud feedback. Because of the arguments raised by Lindzen (1990)--that the processes which determine water vapor mixing ratio in the upper atmosphere are quite different from those which operate in the planetary boundary layer, and that upper tropospheric water vapor might actually decrease even when the boundary layer is getting warmer and more moist--we undertook a study to determine the sensitivity of climate to changes in water vapor at various levels in the troposphere. The result is that climate is just as sensitive to percentage changes in upper tropospheric water vapor, where the mixing ratio is very small, as it is to percentage changes in the boundary layer, which contains the bulk of total column water vapor.
\end{abstract}

\section{INTRODUCTION}

This presentation is intended as a summary of what we know and what we do not know about climate feedback and the response of the climate system to external perturbations. The results come mostly from other investigators, the main contribution here being to interpret them within a common framework.

\section{CLIMATE FEEDBACK}

The importance of climate feedback is well illustrated by looking at successive stages as we allow more and more of the components of the climate system to respond to an external perturbation. For example, if we fix all parameters of the climate system except surface temperature then the change in mean global surface temperature, in response to doubling the $\mathrm{CO} 2$ concentration, will be approximately $1.2 \mathrm{C}$. It is a purely radiative process, and there is not much disagreement in this result.

In the next step we allow the temperature profile to change along with the surface temperature. In that case the effect is no longer purely radiative, and the result now depends upon the dynamical processes 
in the model (e.g., non-radiative energy transport). Allowing the temperature profile to change reduces the change in mean global surface temperature from $1.2 \mathrm{C}$ to $\sim 1.0 \mathrm{C}$. This is the lapse rate feedback, which is always negative.

We continue in this fashion, with the results shown in Table 1. Allowing the water vapor mixing ratio to change brings in a whole slew of hydrological processes--e.g., surface evaporation, moist convection, dynamic transport, cloud formation, and precipitation. In most models the net result of all of these prcocesses is for relative humidity to remain roughly the same, and the change in mean global surface temperature increases to approximately $2 \mathrm{C}$. This is a fairly substantial positive feedback, which will be discussed further in Section 5. Until the issue was raised by Lindzen (1990) there was not much disagreement on water vapor feedback being large and positive. This is a characteristic of almost all GCMs used to determine equilibrium response to perturbations, and is consistent with Cess et al (1989) finding a large measure of agreement amongst many GCMs in the relationship between forcing and response in cloud-free regions.

Where the models do show substantial disagreement is in surface albedo feedback and cloud feedback. Surface albedo feedback, due primarily to changes in ice and snow cover, is positive in all models (warmer temperature, less ice/snow cover, more absorbed solar radiation). But models differ on the magnitude of the effect. There is even wider disagreement on cloud feedback, discussed in Section 4, where even the sign is uncertain. With all the feedbacks in Table 1, the change in mean global surface temperature due to $\mathrm{CO} 2$ doubling, based on GCM simulations, is in the range 2-5C.

\section{A SIMPLE REPRESENTATION OF FEEDBACK}

The combined effect of several feedback processes is definitely non-linear, even if we make the simplifying assumptions that the model responds linearly to any forcing (external forcing or the internally generated forcing associated with a feedback process) and that the processes are independent of each other. These assumptions lead to the schematic representation of the climate system shown in Fig. 1.

The gains in Fig. 1 add linearly as shown, but the effect of the gains on climate system response is non-linear, being proportional to the factor $1 / 1-\Sigma \mathrm{g}_{\text {. }}$ Thus, when the sum of the gains is large--i.e., when $\Sigma g_{i} \rightarrow 1$--each feedback is substantially amplified by the effects of all the other feedbacks. For example, a feedback with a gain of 0.1 has only a $10 \%$ effect if the net gain of all other feedbacks is small (i.e., close to zero), but a $33 \%$ effect if that net gain is 0.6 . 


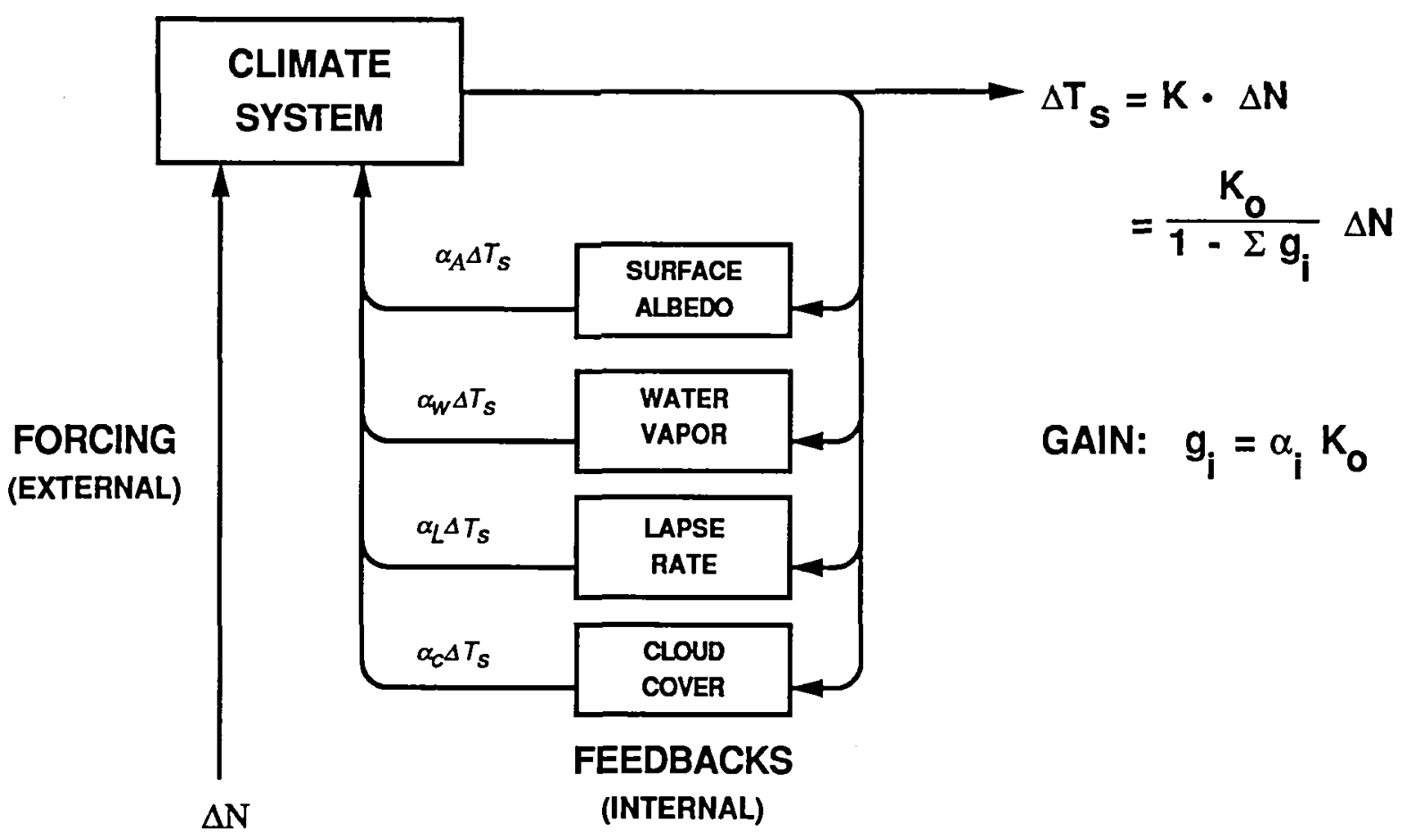

Figure 1. A schematic of the climate system showing how feedback processes contribute to the climate sensitivity coefficient $K$, defined as the change in surface temperature $\Delta T_{s}$ per change in net energy flux at the top of the atmosphere $\Delta N$. The gain $g$ associated with any feedback process is non-dimensional; it is the product of $\alpha$ (the change in $N$ due to that process, per change in surface temperature) times $K_{0}$ (the change in surface temperature that would result from an externally induced change in $N$ without feedback).

TABLE 2. The calculated gain associated with feedback processes in the GFDL and GISS models.

\begin{tabular}{|c|c|c|}
\hline FEEDBACK PROCESS & GFDL & GISS \\
\hline \hline Surface Albedo & .16 & .09 \\
\hline $\begin{array}{l}\text { Water Vapor } \\
\text { \& Lapse Rate }\end{array}$ & .43 & .40 \\
\hline Cloud Cover & .11 & .22 \\
\hline \hline Total Gain & .70 & .71 \\
\hline
\end{tabular}

"Effect of stratospheric response to $\mathrm{CO}_{2}$ change is subtracted from GFDL results to make it consistent with GISS. 


\section{CLOUD FEEDBACK}

The feedback gains of two of the models used to investigate the effects of doubling CO2, GISS (Hansen et al, 1984) and GFDL (Wetherald and Manabe, 1988), are shown in Table 2. The mean global surface temperature response is about the same--4.2C for GISS and 4.0C for GFDL--so that they have nearly the same total gain, $\sim 0.7$. The breakdown of the gains, however, exhibits a large difference in cloud feedback, which is compensated by the difference in surface albedo feedback.

Our simple representation of feedback allows us to play a game with the numbers in Table 2, in which we exchange the cloud parameterization schemes between the two models. The result is an increase in the net gain for the GFDL model to 0.81 and a decrease for the GISS model to 0.60 . In that case the response to a doubling of $\mathrm{CO}_{2}$ in the GFDL model would be 6.3C, and in the GISS model 3.0C. Although this representation of climate system response is highly simplified, it does convey the essential result that cloud processes can have a very large effect on model response.

A further illustration of model sensitivity to the parameterization of cloud processes is provided by the results of a study at the British Meteorological Office (Mitchell et al, 1989). By changing the parameterization of the hydrological cycle, including cloud formation processes, and the parameterization of cloud optical properties, the response to doubled $\mathrm{CO} 2$ changed by almost a factor of three (1.9C versus $5.2 \mathrm{C})$. The $1.9 \mathrm{C}$ result implies negative cloud feedback.

Cloud feedback now stands as the major cause of uncertainty in climate sensitivity studies. One has to conclude at this point that clouds may have a strong influence on climate change, but we are far from knowing the magnitude, and perhaps even the sign, of this influence.

\section{WATER VAPOR FEEDBACK}

While models used up to now for simulating radiative perturbations, such as the effects of changing the solar irradiance or changing $\mathrm{CO}$, are generally in agreement that the water vapor feedback is large and positive, Lindzen (1990) has recently questioned this result. He accepts that water vapor in the boundary layer will increase with increasing temperature, consistent with the models, but argues that water vapor above the boundary layer will actually decrease.

It is generally believed that water vapor in the upper troposphere is controlled primarily by moist convection and precipitation, processes which are only crudely parameterized in climate models. It is conceivable, therefore, that changes in upper tropospheric moisture may not be accurately computed in the models. To determine the relative importance of upper tropospheric moisture versus boundary layer moisture, we employed a one-dimensional radiative equilibrium model with convective adjustment. (See Ramanathan and Coakley, 1978, for a review of these models.)

The model calculates an equilibrium temperature profile such that the net radiative flux is zero above a convective zone. Within the convective zone the temperature follows a moist adiabatic lapse rate. The height of the convective zone is adjusted to be the minimum height for which the temperature 

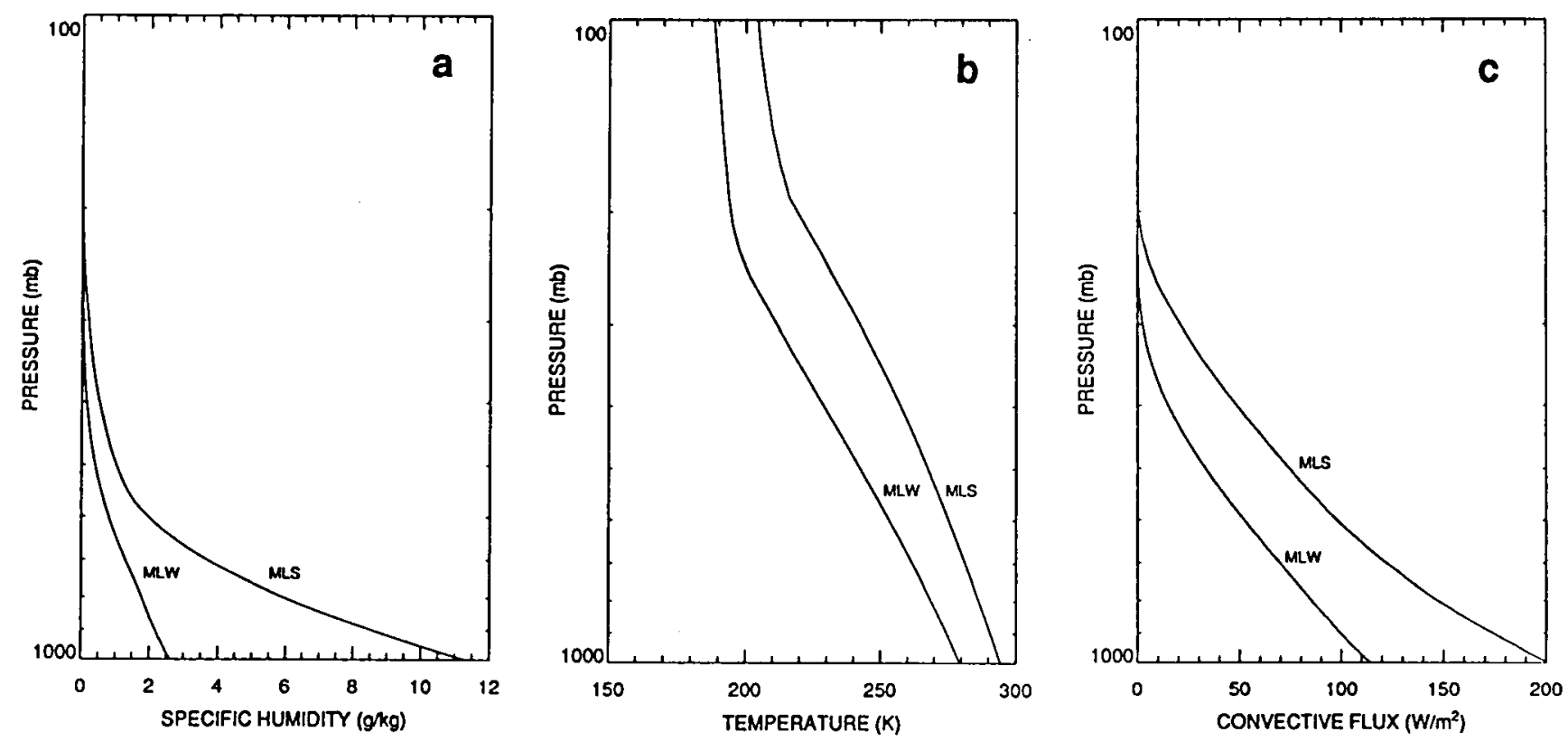

Figure 2. For two humidity models, MLW (mid-latitude winter) and MLS (mid-latitude summer), shown in a, the equilibrium temperature (b) and the convective flux (c). The prescribed parameters are: no clouds, $\mathrm{CO} 2$ concentration 330ppm, and net absorbed solar flux $236 \mathrm{~W} / \mathrm{m}^{2}$ for MLW and $290 \mathrm{~W} / \mathrm{m}^{2}$ for MLS. The height of the convective zone is determined to be $240 \mathrm{mb}$ and $180 \mathrm{mb}$ for MLW and MLS, respectively.
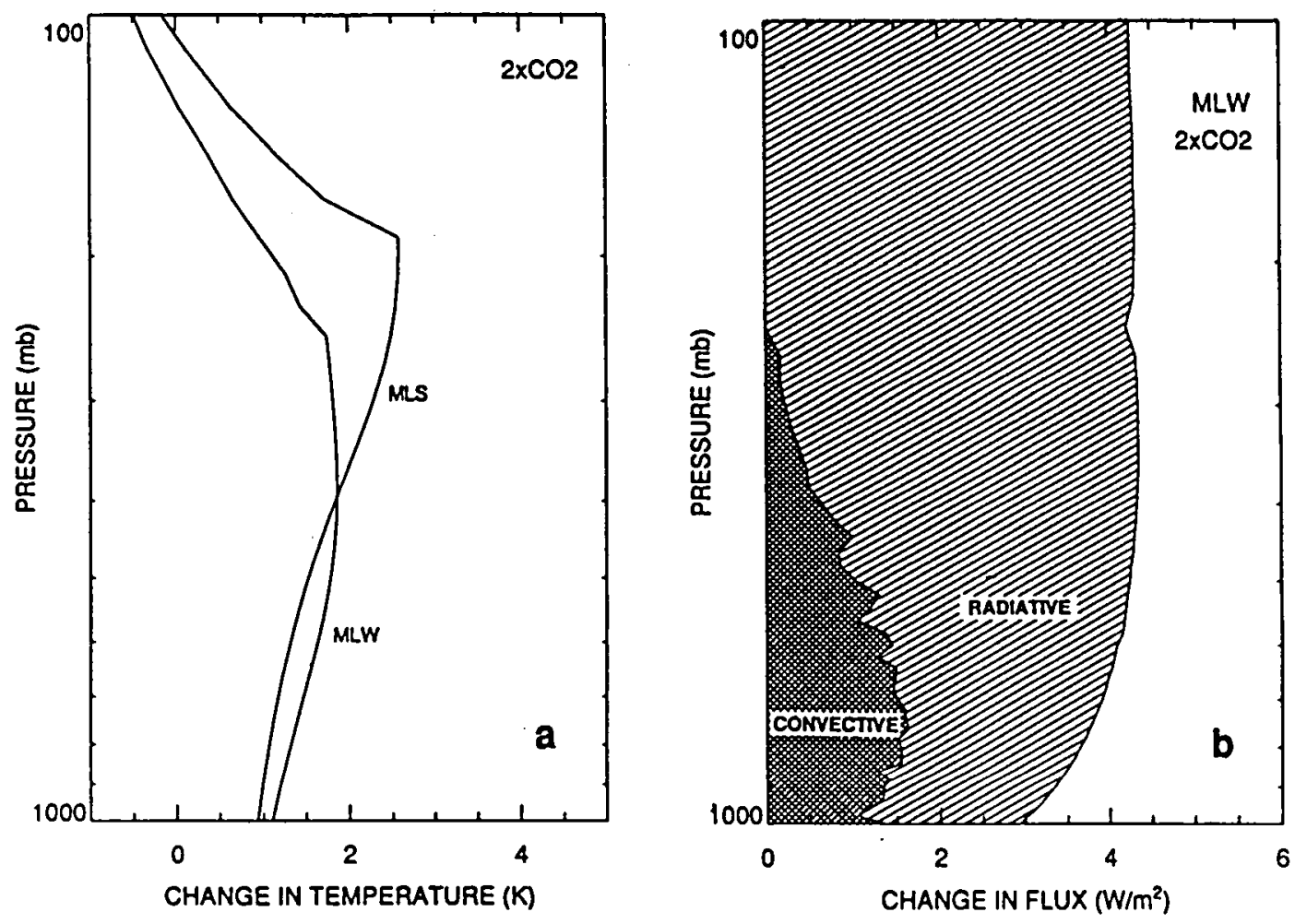

Figure 3. The change in temperature (a) and the change in convective and total flux (b) required to achieve equilibrium after increasing $\mathrm{CO} 2$ from $330 \mathrm{ppm}$ to $660 \mathrm{ppm}$. (Note that the irregular shape of the convective flux is an artifact of the numerical procedure and has no physical significance.) 
lapse rate above the zone is less than adiabatic. The model has 57 layers from the surface to $10 \mathrm{mb}$, and the radiation routines are based on Chou and Arking (1980 and 1981) and Chou and Peng (1983).

An equilibrium solution obtained with this model is shown in Fig. 2 for two humidity profiles: MLW (mid-latitude winter) and MLS (mid-latitude summer). In this example we omit clouds and specify a $\mathrm{CO} 2$ concentration of $330 \mathrm{ppm}$ and a net absorbed solar flux of $236 \mathrm{~W} / \mathrm{m}$ for MLW and $290 \mathrm{~W} / \mathrm{m}$ for MLS. The net upward flux (radiative plus convective) must match the net downward solar flux at every level. Since the model computes the radiative flux, the convective flux is obtained by subtraction (Fig. 2c). The height of the top of the convective zone is found to be $240 \mathrm{mb}$ for MLW and $180 \mathrm{mb}$ for MLS.

If we introduce a perturbation--e.g., doubling the $\mathrm{CO} 2$ concentration--with neither temperature nor any other parameter in the model allowed to change, there would be a net imbalance in the outgoing flux at the top of the atmosphere. For the case of doubling $\mathrm{CO} 2$, this deficit is shown in Fig. $3 \mathrm{~b}$ by plotting the change in radiative flux as a function of height due to the perturbation (the outer boundary of the lightly shaded area). Allowing the model to reach a new equilibrium, the change in temperature is shown in Fig. 3a and the change in convective flux in Fig. $3 \mathrm{~b}$ (boundary of the darkly shaded area). We interpret Fig. $3 \mathrm{~b}$ as showing the change in total flux and the change in convective flux required to restore equilibrium, with the shaded areas depicting the partitioning of the change in total flux between convection and radiation.

In response to the change in temperature and the change in the magnitude of the convective flux required to maintain a stable lapse rate, one would expect the total amount and distribution of water vapor to change. However, water vapor is a prescribed parameter, since the processes of evaporation, condensation, and transport of water vapor are not included in this model. We therefore introduced changes in water vapor mixing ratio in individual layers and let the model calculate the change in surface temperature. The result is shown in Fig. 4, where we plot the change in equilibrium surface temperature due to a $50 \%$ increase of water vapor in a $40 \mathrm{mb}$ layer as a function of the height of the layer in which water vapor is perturbed. For both the MLS and the MLW water vapor profiles the

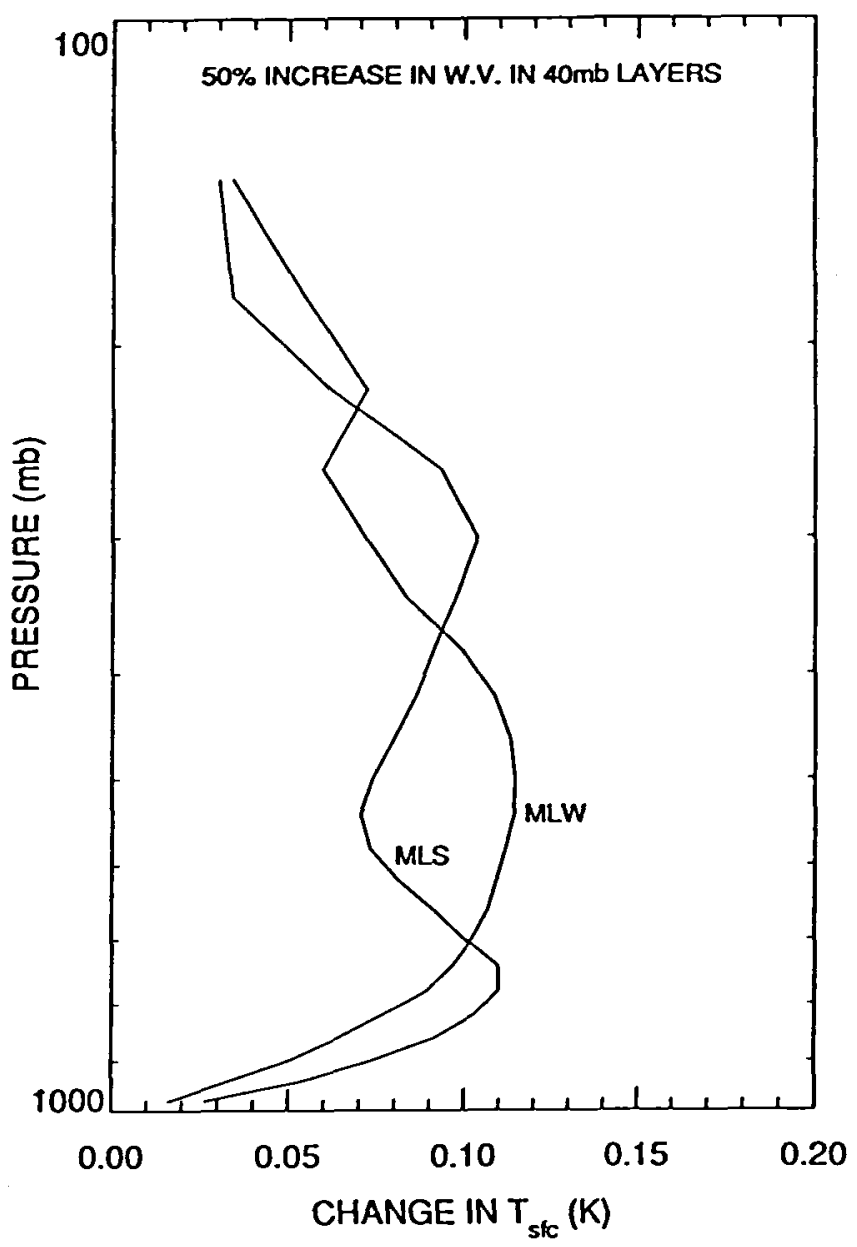

Figure 4. The change in surface temperature due to a $50 \%$ increase in water vapor in a $40 \mathrm{mb}$ layer, as a function of the height of the layer. 
sensitivity varies by less than a factor of two over the range 200-950mb. (The sensitivity must necessarily be zero at the surface.)

From these results we draw the conclusion that the response of the climate system to a percentage change of water vapor is almost independent of the height at which the change takes place. This is so, even though the water vapor mixing ratio varies by a factor of 500 to 1000 within the troposphere. These results imply that climate is just as sensitive to percentage changes in upper tropospheric water vapor, where the mixing ratio is very small, as it is to percentage changes in the planetary boundary layer, which contains the bulk of total column water vapor. Hence, increases in total column water vapor with temperature are not necessarily indicative of a positive water vapor feedback in climate; the changes in the upper troposphere, very small in terms of mixing ratio, are critically important.

\section{CONCLUSION}

A simple representation of the climate system--in which key processes are assumed to have a linear effect on system response and to be independent of each other--is used to show the extent to which climate response to an external radiative perturbation depends upon feedback processes. Examination of the results of GCM simulations of $\mathrm{CO} 2$ doubling shows wide differences in cloud feedback amongst models. The conclusion drawn is that clouds may have a strong influence on climate change, but we are far from knowing the magnitude, and perhaps even the sign, of this influence.

GCM models are consistent in having a large positive feedback associated with water vapor, in that atmospheric water vapor increases with increasing temperature, thereby amplifying the effect of any external forcing. However, upper tropospheric water vapor depends on moist convection and precipitation--processes that may not be well parameterized in the models used for these studies. A study with a one-dimensional radiative equilibrium model with convective adjustment shows that a percentage change in upper tropospheric water vapor, where the mixing ratio is very small, is just as important as a percentage change in the planetary boundary layer, which contains the bulk of total column water vapor. Hence, increases in total column water vapor with temperature are not necessarily indicative of a positive water vapor feedback; the changes in the upper troposphere, very small in terms of mixing ratio, are critically important. Therefore, if the Lindzen hypothesis is correct, that the enhanced convection associated with radiative warming will lead to a drier upper troposphere, then water vapor feedback could be much smaller than in present models, and may even be negative.

\section{ACKNOWLEDGEMENT}

The author would like to thank Dr. William Ridgway for helpful discussions on radiative transfer methods and for modifying the radiation codes, supplied by Dr. Ming-Dah Chou, so that they could be called as external routines by the radiative-convective model used in this investigation. 


\section{REFERENCES}

Chou, M.-D., and A. Arking, 1980: Computation of infrared cooling rates in the water vapor bands, J. Atmos. Sci., 37, 855-867.

Chou, M.-D., and A. Arking, 1981: An efficient method for computing the absorption of solar radiation by water vapor, J. Atmos. Sci., 38, 798-807.

Chou, M.-D., and L. Peng, 1983: A parameterization of the absorption in the $15-\mu \mathrm{m} \mathrm{CO} 2$ spectral region with application to climate sensitivity studies, J. Atmos. Sci., 40, 2183-2192.

Cess, R.D., G.L. Potter, J.P. Blanchet, G.J. Boer, S.J. Ghan, J.T. Kiehl, H. Le Treut, Z.-X. Li, X.-Z. Liang, J.F.B. Mitchell, J.-J. Morcrette, D.A. Randall, M.R. Riches, E. Roeckner, U. Schlese, A. Slingo, and K.E. Taylor, 1989: Interpretation of cloud-climate feedback as produced by 14 atmospheric general circulation models, Science, 245, 513-516.

- Hansen, J. A., Lacis, D. Rind, G. Russell, P. Stone, I. Fung, R. Ruedy, and J. Lerner, 1984: Climate sensitivity: analysis of feedback mechanisms. Climate Processes and Climate Sensitivity, Geophys. Monogr. Ser., 29, Ed. by J. E. Hansen and T.Takahashi, 130-163, AGU, Washington, D.C.

Lindzen, R.S., 1990: Some coolness concerning global warming, Bull. Amer. Meteor. Soc., 71, 288-299.

Mitchell, J.F.B., C.A. Senior, and W.J. Ingram, 1989: CO2 and climate: a missing feedback? Nature, 341, 132-134.

Ramanathan, V., and J.A. Coakley, Jr., 1978: Climate modeling through radiative-convective models, Rev. Geophys. and Space Phys., 16, 465-489.

Wetherald, R.T., and S. Manabe, 1988: Cloud feedback processes in a general circulation model, J. Atmos. Sci., 45, 1397-1415. 


\title{
ON THE DECADAL MODES OF OSCILLATION OF AN IDEALIZED OCEAN-ATMOSPHERE SYSTEM
}

\author{
Vikram M. Mehta* \\ Department of Meteorology \\ and \\ Supercomputer Computations Research Institute \\ The Florida State University, Tallahassee, FL 32306
}

\begin{abstract}
Axially-symmetric, linear, free modes of global, primitive equation, oceanatmosphere models are examined to see if they contain decadal (10-30 years) oscillation time scale modes. A two-layer ocean model and a two-level atmospheric model are linearized around axially-symmetric basic states containing mean meridional circulations in the ocean and the atmosphere. Uncoupled and coupled, axially-symmetric modes of oscillation of the ocean-atmosphere system are calculated. The main conclusion of this study is that linearized, uncoupled and coupled, ocean-atmosphere systems can contain axially-symmetric, free modes of variability on decadal time scales. These results have important implications for externally-forced decadal climate variability.
\end{abstract}

\section{INTRODUCTION}

There are numerous observational studies of decadal (10-30 years) time scale climate oscillations (see, for example, Pittock (1983), Currie and O'Brien (1988), Labitzke and van Loon (1989), and references therein). Most of these studies have tried to correlate decadal climate variations with solar and lunar influences. Although the existence (or otherwise) of these correlations and the mechanisms by which variability of solar and lunar influences might affect terrestrial climate are not yet clear, the observational studies show that decadal climate variations do exist. These variations may be due to internal variability of the climate system, they may be forced externally or a combination of internal variability and external forcing may give rise to these modes. Some results from a research effort to study the natural variability of an idealized ocean-atmosphere system, especially on decadal time scales, are reported here. In addition to the above-mentioned observations, more specific motivation for the work reported here was provided by two observational studies dealing with long-period (years to decades) fluctuations of the axially-symmetric (symmetric around the rotation axis of the earth) components of some climatic variables. Krishnamurti et al. (1986)'s analysis of global sea level pressures (SLP) and Semazzi et al. (1988)'s analysis of global sea surface temperatures (SST) showed that axially-symmetric components of SLP and SST fluctuate with a variety of time scales, including the years to decades time scales. In view of the above observations, the emphasis in the modelling

\footnotetext{
* Current affiliation:Code 613, NASA/Goddard Space Flight Center
} 
work reported here is on axially-symmetric modes of the ocean-atmosphere system oscillating at decadal time scales.

Anderson and Stevens (1987) (hereafter referred to as AS) found that a multilevel model of the tropical atmosphere linearized about a Hadley cell basic state contains wavelike, axially-symmetric modes in thermal wind balance. The oscillation time scales of these modes range from a few weeks to a few months. Advection of perturbation zonal wind and temperature fields by the basic state meridional velocity was suggested as the mechanism giving rise to these modes.

In the ocean, there appears to be a similar but opposite mean meridional circulation (MMC) than in the atmosphere. This can be seen clearly in studies of model ocean circulations by Bryan and Lewis (1979, fig. 10a) and Meehl et al. (1982, fig. 2). This similarity of atmospheric and oceanic MMC raises some interesting questions in light of AS's results. If advection of perturbation fields by the basic state meridional velocity is indeed the mechanism giving rise to the axially-symmetric atmospheric intra-seasonal modes found by AS, then the same mechanism may give rise to axiallysymmetric oceanic modes with time scales of several years and longer. This is because basic state meridional velocities in the ocean are at least 2 orders of magnitude smaller than in the atmosphere. If such long-period, axially-symmetric modes do exist in an idealized ocean model, then within the framework of a coupled ocean-atmosphere system, such oceanic variability might also result in atmospheric variability on long time scales. Moreover, since MMC exist not only in the tropics but also in higher latitudes, such axially-symmetric modes might exist in association with higher latitude MMC also. Therefore, a modelling study was carried out to test the hypotheses outlined above.

\section{MODEL DESCRIPTION AND METHOD OF SOLUTION}

The governing equations of the two-level model of the atmosphere formulated for this study are primitive equations in spherical co-ordinates with sigma (pressure at any level divided by surface pressure) as the vertical co-ordinate. The model atmosphere extends around the earth in longitudinal direction and from the South Pole to the-North Pole in latitudinal direction. The governing equations of the two-layer model of the ocean are primitive equations with height as the vertical co-ordinate. The model ocean has the same horizontal domain as the model atmosphere. Vertically, the ocean is divided into two homogeneous layers separated by a thermocline. The model ocean has a uniform depth of 4000 meters. The model ocean and the model atmosphere interact via heat and momentum exchange. Other physical processes included in the models are entrainment of mass, momentum, and heat between the oceanic layers, thermal infrared radiation, and vertical diffusion within the atmosphere and the ocean. The two models and parameterizations of physical processes are described with details in Mehta (1990a, 1990b).

The two models and parameterizations of physical processes are linearized around an axially-symmetric basic climatic state containing mean meridional circulations in the atmosphere and the ocean. The resulting linearized perturbation climate system is solved as an eigenvalue problem. The eigenvalues are complex-valued, where the real parts are the oscillation frequencies and the imaginary parts are the growth rates of individual modes. The eigenvectors contain vertical and horizontal structures of individual modes. Details of linearization and numerical solution procedure are given in Mehta (1990a, 1990b). 


\section{RESULTS AND DISCUSSION}

In the case of a motionless basic state, the oscillation frequencies of axiallysymmetric modes in a model of the ocean or the atmosphere can be anticipated based on analytical calculations (see, for example, Matsuno (1966) and Lindzen (1967), among others). The two types of axially-symmetric modes which can exist for motionless basic state are fast (oscillation periods of a few hours), inertia-gravity modes and steady Rossby and Kelvin modes at zero frequency. Also, in the absence of a reservoir of energy in the basic state, these two types of axially-symmetric modes are neutrally stable. The two types of modes described above exist (not shown) in uncoupled two-layer ocean model and uncoupled two-level atmospheric model in the absence of basic state motion. In the next step, basic states containing mean meridional circulations were specified based on Meehl et al. (1982) for the ocean and Peixoto and Oort (1984) for the atmosphere. Figures $1 \mathrm{a}$ and $1 \mathrm{~b}$ show eigenvalue spectra of the uncoupled ocean and the uncoupled atmospheric models, respectively. Comparing the oceanic spectrum (fig. 1a) in the presence of mean meridional circulations with the two types of modes described above for the motionless basic state case, it is clear that a new family of free, axiallysymmetric ocean modes with oscillation periods ranging from about two years to several centuries appear when the basic oceanic state contains mean meridional circulations. These modes have practically neutral growth rates. A similar comparison of uncoupled, axially-symmetric atmospheric modes shows that specification of a basic atmospheric state containing MMC gives rise to atmospheric modes (fig. 1b) with oscillation periods ranging from about a week to several years. There are a few atmospheric modes with decadal and longer oscillation periods also. Unlike the decadal oceanic modes (fig. 1a), however, the low-frequency atmospheric modes have substantially large growth rates.

To see if these modes of individual systems (ocean and atmosphere) are modified due to interaction between the ocean and the atmosphere, the two models were coupled by wind stress and heating parameterizations. Coupled, axially-symmetric, oceanatmosphere modes were then calculated for the same oceanic and atmospheric basic states as used for the uncoupled calculations. A representative value of the coupling coefficients was used. The eigenvalue spectrum resulting from this coupled calculation is shown in figure 1c. Comparison with figures $1 \mathrm{a}$ and-1b shows that eigenvalues, especially growth rates, change dramatically due to ocean-atmosphere coupling. This growth rate enhancement is maximum in the decadal time scale range. Ocean modes with periods of about 2 years to several hundred years grow much more quickly because of ocean-atmosphere coupling. Spatial structures of atmospheric and oceanic components of a coupled mode oscillating with 14.6 years period and growing with 3.19 years efolding time are shown in figure $2 a$ and $2 b$, respectively.

A number of sensitivity experiments were carried out to test the robustness of results by varying parameter values. Figure 3 shows coupled eigenvalue spectra from three experiments in which coupling coefficients were varied over a factor of four. As the coupling coefficients are increased, the modes in 1-20 years oscillation period range are slowed down and their growth rates are increased. This can be seen as general displacement of eigenvalues of decadal periods towards higher growth rates and lower oscillation frequencies as the coupling coefficients are increased. Modes with oscillation periods of weeks to months and periods longer than twenty years are also affected significantly by increasing coupling coefficients. Results from other sensitivity experiments are described and discussed in Mehta (1990b). The most important result of the sensitivity studies is that decadal modes exist in the coupled models over a wide range of parameter values and even in the presence of dissipative processes.

Like any model, the ocean-atmosphere models formulated for this study also have their limitations. The most important ones are absence of continents, absence of fully interactive hydrological cycle in the atmosphere, and absence of salinity, ice-snow, and 
high-latitude convection in the ocean. In spite of these limitations, it is felt that the results are reliable enough, computationally and physically, to proceed to experimentation with models of higher complexity. There are two main implications of the results presented here for the Sun-climate relationship. One implication is that if there is external forcing on decadal time scales, there is a possibility of resonant response of the climate system since the system appears to have natural variability on decadal time scales. The other implication is that it will be necessary to separate internal climate variability from externally-forced climate variability, if any. It can be speculated that transient correlations between solar activity and climate, such as solar cycle-level of Lake Victoria and solar magnetic field sector boundary-atmospheric vorticity area index, may be due to internal variability. It is suggested that climate data analysis studies be carried out with an aim of separating internal variability from externally-forced variability.

\section{ACKNOWLEDGEMENTS}

This work was supported partially by a Supercomputer Computations Research Institute Fellowship and partially by NSF grant ATM-8714674 to the Florida State University. The Florida State University Supercomputer Computations Research Institute is partially funded by the U.S. Department of Energy through contract no. DEFC05-85ER250000.

\section{REFERENCES}

Anderson, J.R., and D.E. Stevens, 1987: The presence of linear wavelike modes in a zonally symmetric model of the tropical atmosphere. J. Atmos. Sci., 44, 2115-2127.

Bryan, K., and L.J. Lewis, 1979: A water mass model of the world ocean. J. Geophys. Res., 84, 2503-2517.

Currie, R.G., and D.P. O'Brien, 1988: Periodic 18.6-year and cyclic 10 to 11 year signals in northeastern United States precipitation data. J. Climatol., 8, 255-281.

Krishnamurti, T.N., S. Chu, and W. Iglesias, 1986: On the sea level pressure of the Southern Oscillation. J. Met. Atmos. Phys., 34, 385-425.

Labitzke, K., and $H$. van Loon, 1989: Association between the 11-year solar cycle, the QBO, and the atmosphere. Part III: Aspects of the association. J. Climate, 2, 554-565.

Lindzen, R.D., 1967: Planetary waves on beta planes. Mon. Wea. Rev., 95, 441-451.

Matsuno, T., 1966: Quasi-geostrophic motions in the equatorial area. J. Met. Soc. Japan, 44, 24-42.

Meehl, G.A., W.M. Washington, and A.J. Semtner, Jr., 1982: Experiments with a global ocean model driven by observed atmospheric forcing. J. Phys. Oceanogr., 12, 301 312.

Mehta, V.M, 1990a: On the axially-symmetric modes of oscillation of an idealized ocean-atmosphere system. Submitted to Climate Dynamics. 
----, 1990b: On the decadal modes of oscillation of an idealized ocean-atmosphere system. Ph. D. dissertation, Dept. of Meteorology, The Florida State University, 179pp.

Peixoto, J.P., and A.H. Oort, 1984: Physics of climate. Rev. Mod. Phys., 56, 365-429.

Pittock, A.B., 1983: Solar variability, weather, and climate: An update. Quart. J. Roy. Met. Soc., 109, 23-55.

Semazzi, F.H.M., V. Mehta, and Y.C. Sud, 1988: An investigation of the relationship between sub-Saharan rainfall and global sea surface temperatures. Atmosphere-Ocean, $26,118-138$. 

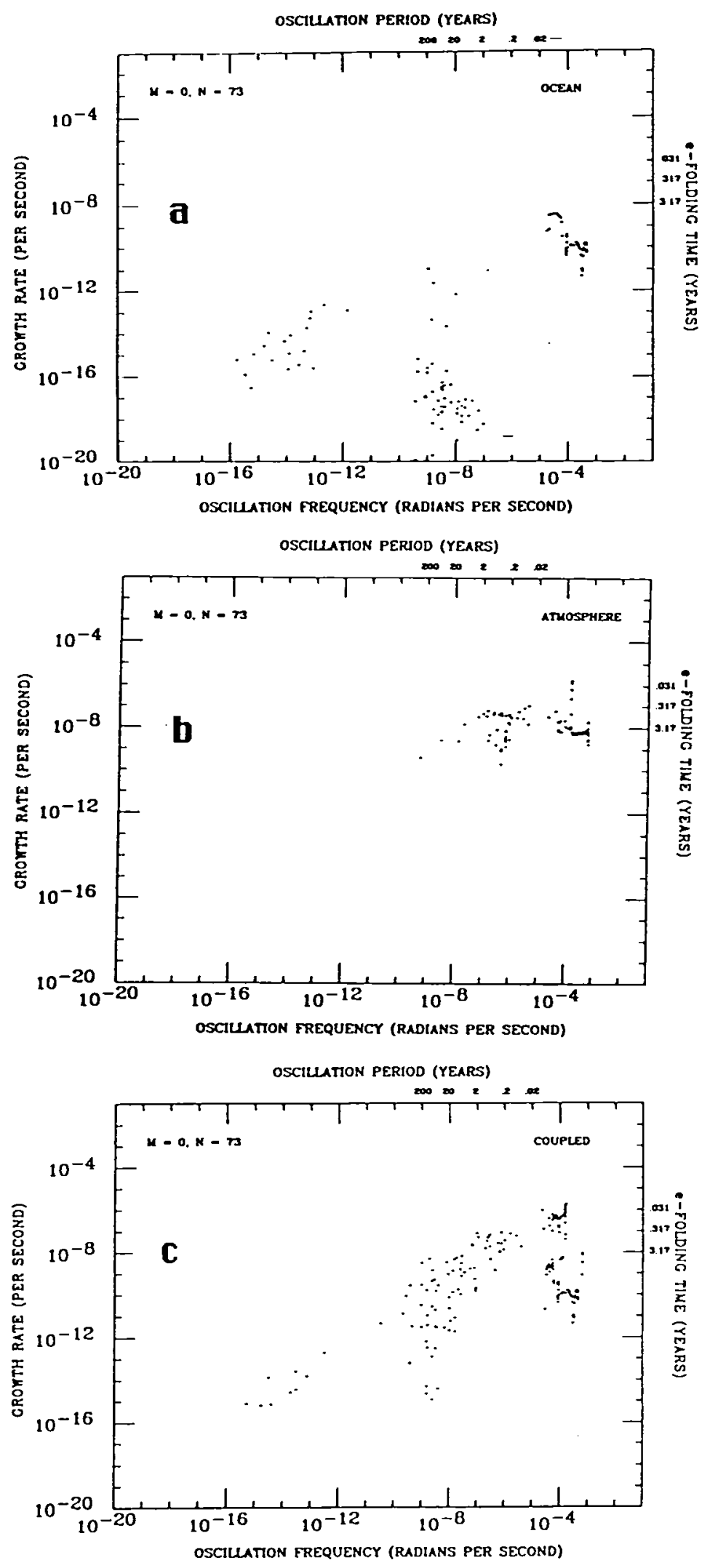

Figure 1. Oscillation frequency $(\mathrm{rad} / \mathrm{sec})$ versus growth rate $(/ \mathrm{sec})$ of free axiallysymmetric modes in the presence of basic state with motion. Only positive values are plotted. $M$ is the zonal wavenumber and $N$ is the number of north-south grid points including the poles. 2.5 degree north-south resolution. (a) Uncoupled ocean (b) Uncoupled atmosphere (c) Coupled ocean-atmosphere with nominal values of coupling coefficients. 

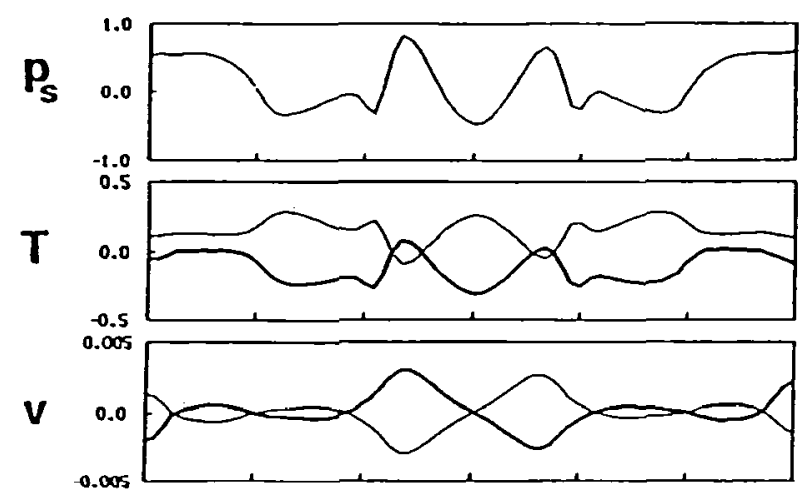

a
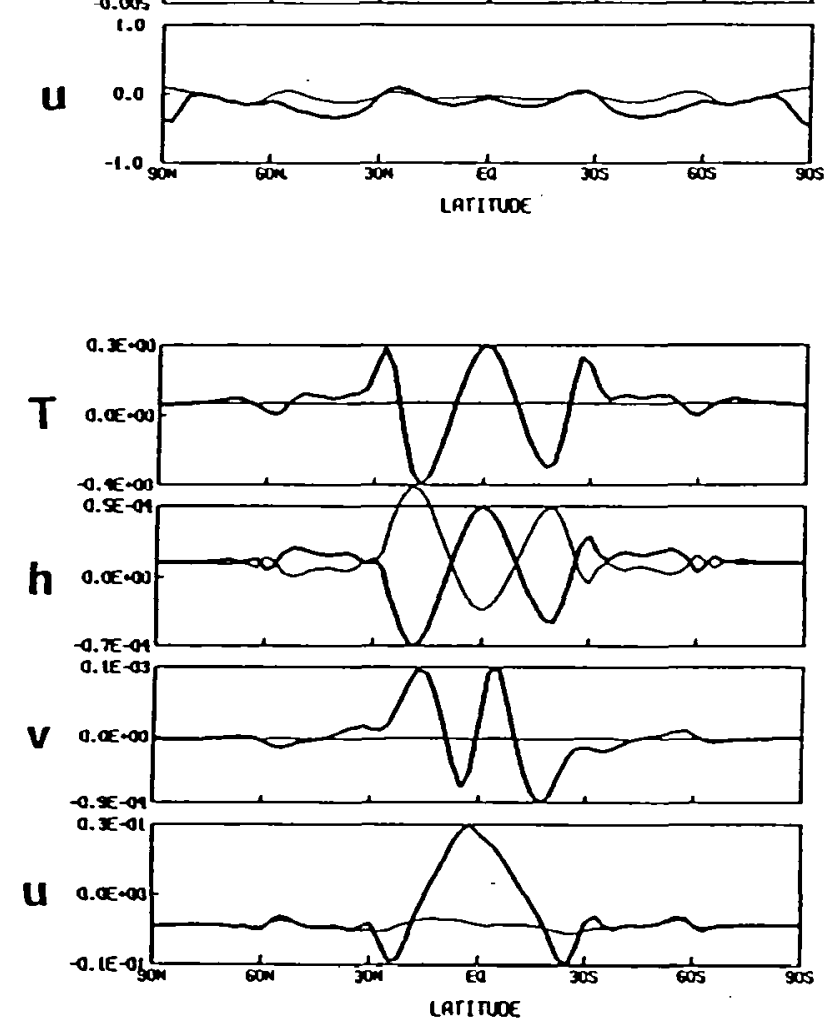

$\mathbf{b}$

Figure 2. Latitudinal structure of an axially-symmetric coupled ocean-atmosphere mode oscillating at 14.6 years period with amplitude e-folding time of 3.19 years. All variables are non-dimensional. Upper (lower) level variables are plotted with heavy (light) solid lines. $u$ and $v$ are zonal and meridional velocities, respectively. $T$ is temperature. (a) Coupled atmospheric component. $p$ is surface pressure. (b) Coupled oceanic component. $h$ is layer thickness. 


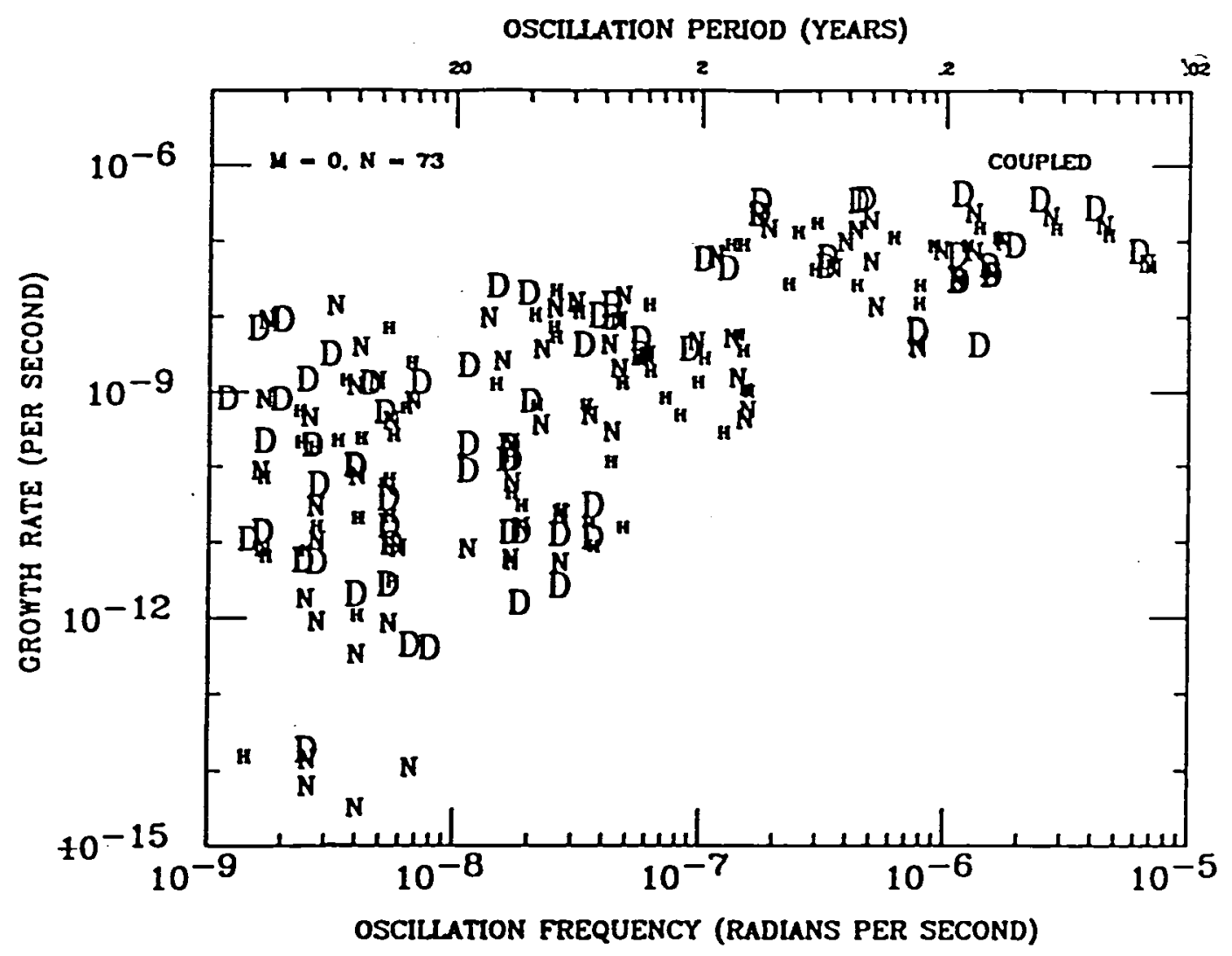

Figure 3. Oscillation frequency ( $\mathrm{rad} / \mathrm{sec}$ ) versus growth rate (/sec) of axiallysymmetric coupled ocean-atmosphere modes in the presence of basic states with motion. Only positive values are plotted. $M$-is the zonal wavenumber and $N$ is the number of north-south grid points including the poles. 2.5 degree north-south resolution. $H, N$, and $D$ denote eigenvalues for half, nominal, and double values of coupling coefficients. 


\title{
WHAT COULD BE CAUSING GLOBAL OZONE DEPLETION?
}

\author{
S. Fred Singer \\ Department of Environmental Sciences, University of Virginia \\ Charlottesville, VA 22903
}

\begin{abstract}
The reported decline trend in global ozone between 1970 and 1986 may be in part an artifact of the analysis; the trend value appears to depend on the time interval selected for analysis--in relation to the 11-year solar cycle. If so, then the decline should diminish as one approaches solar maximum and includes data from 1987 to 1990 . If the decline is real, its cause could be the result of natural and human factors other than just chlorofluorocarbons (CFCs).
\end{abstract}

\section{INTRODUCTION}

The Ozone Trends Panel (OTP) of NASA, after a massive re-analysis of data from ground stations and satellites, announced the existence of a declining trend in northern hemisphere ozone of about $0.2 \%$ per year over the 17 -year period from 1970 to $1986 .^{1}$ The result was announced at a press conference in March 1988, but the underlying analysis has not yet been published. Nevertheless, as a result of the announcement and subsequent press reports, it has become widely accepted that there has already been a global decline of stratosphere ozone caused by the release of CFCs into the atmosphere. Based on this impression, far-reaching international actions are contemplated to phase out quickly the production of CFCs and other chemicals.

The ozone trend analysis must, of course, eliminate the much larger natural variations: the seasonal changes, quasi-biennial oscillations $(\mathrm{QBO}), 11$-year solar cycle effects, major volcanic eruptions, and even the after-effects of atmospheric nuclear testing. One should also be aware that ozone trends, like climate trends, may depend on the choice of time interval selected for analysis. ${ }^{2}$

\section{DEPENDENCE OF THE TREND ON THE METHOD OF ANALYSIS}

While the OTP Report itself is not yet available, a parallel report from the Center for Applied Mathematics of Allied-Signal, Inc. was distributed at the UNEP Ozone Science Meeting at The Hague in October 1988. The Allied study ${ }^{3}$ deals with many of the corrections necessary to establish the existence of a secular trend. It should be noted that the study models the trend as a linear ramp function, beginning in December 1969; the trend is assumed to be zero before that date. (This procedure is suggested by model calculation of CFC effects rather than by any measured stratospheric chlorine concentrations. $)^{4}$ Here we will examine primarily: (1) the solar flux correction and (2) the effect of choice of time period on the ozone trend result.

The Allied study uses the solar 10.7-cm radio flux as a proxy for the solar UV flux, for which a continuous data series is not available. Since the radio flux has no influence whatsoever on atmospheric ozone, the proxy is clearly one of convenience. (The OTP, 
on the other hand, used the sunspot number as a proxy for the "effective" UV flux.) The correlation between the radio flux and the relevant UV flux is problematic ${ }^{5}$, making it difficult to judge the adequacy of the solar flux correlation.

The main results of the Allied study can be derived from their sensitivity analyses, shown in their table $2^{3}$ :

o The estimated change in total ozone (30-64 N) over the 17 years (between 1970-86) is $-1.9 \%$, which is less than the reported OTP result.

o About half of the change, $-0.9 \%$, comes from using a "multiple trend" ramp function (where the coefficient of the ramp function is varied according to the month).

o Another half of the change, $1.0 \%$, comes from the inclusion of post-1982 data; the 13year change between 1970 and 1982 is only $-0.5 \%$, i.e., $-0.04 \%$ per year.

o Excluding pre-1965 data, as was done in the OTP analysis, would make the 17-year decline $(1970-86)$ more negative by $0.6 \%$, i.e., $-2.5 \%$.

To explain the surprisingly strong dependence of the trend result on the choice of time period, the authors suggest natural causes, such as El Nino or volcanism, or unknown man-made causes. ${ }^{3,6}$ Another simpler explanation may be that the $1970-86$ period covers only 12.5 solar cycles and includes two solar flux decreases vs. one increase; figure 1 shows the strong dependence on sunspot number of total ozone observed in different zones ${ }^{7}$.

If this hypothesis is correct and the reported ozone decline ${ }^{1,3}$ is partly due to the analysis procedure, then one would predict a diminished global ozone decrease if the analysis includes the years from 1987 up to 1990 as we reach a solar cycle maximum. An answer should thus be forthcoming soon.

(However, even if the observed ozone trend were then to go to zero, this would not rule out that increased use of CFCs will affect the stratospheric ozone layer sometime in the future. For example, the AER one-dimensional CFC-ozone model predicts change of only $-0.25 \%$ over the period $1970-86 .^{3}$ Since the standard error of this prediction is $1.03 \%$, it would be consistent with a zero observed ozone trend or even a small positive trend.)

\section{POSSIBLE CAUSES OF A REAL TREND}

If, on the other hand, the ozone secular trend is real, then there could be several possible causes, in addition to CFCs; they might be distinguished by measurements of ozone changes with altitude, latitude, and time.

o Anthropogenic factors other than CFCs might decrease ozone levels. One such factor is methane from various human activities. Like CFCs, methane has a long tropospheric lifetime and percolates into the stratosphere, where it participates in ozone chemistry and eventually produces water vapor ${ }^{8}$. Since tropospheric methane has increased by about $100 \%$ in the past century, stratospheric water vapor should have increased also. 
Certainly, there has been an increase in stratospheric $\mathrm{CO}_{2}$ as a result of human activities, such as fossil fuel burning. As a consequence, one would expect increased radiative heat loss from the stratosphere and an effect of these colder temperatures on ozone chemistry.

I have speculated elsewhere ${ }^{9}$ that such cooling, coupled with increased stratospheric humidity, could lead to the formation of polar stratospheric clouds (PSCs) that are believed to be essential in causing the Antarctic ozone hole (AOH). Thus, the AOH may indeed be due to human activity, but controlled now by stratospheric temperature and humidity rather than by increasing $\mathrm{CFC}$ concentrations.

Another source of water vapor (and cirrus) could be commercial jet aircraft that increasingly penetrate into the lower stratosphere. While current theory ${ }^{10}$ does not envisage ozone destruction from aircraft at that altitude, current theory considers only homogenous (gas-phase) reactions and not yet heterogeneous reactions with particulates and ambient aerosols.

o Natural effects related to the variability of solar cycles may also be responsible for an observed ozone decline. The analyses ${ }^{1,3}$ implicitly assume perfect correlation between the relevant solar UV and the proxies (whether sunspot number or radio flux), and are not equipped to deal with long-term changes in the correlation.

This last observation leads to an interesting aside. Solar cycles have varied greatly in the past ${ }^{11}$ (see figure 2). In recent times, sunspot numbers have been as low as 40 (in 1817) and as high as 190 (in 1958) at the peak of the cycle. During the Maunder Minimum (1645-1715) sunspots were essentially absent. This suggests that there could have been substantial changes in average ozone levels in the past ${ }^{12}$, approximating those feared to result from the release of CFCs. It would be interesting, therefore, to search the historical records for any biological consequences to humans, agricultural crops, or marine life, that have been hypothesized as having been caused by low ozone levels.

\section{REFERENCES AND NOTES}

1 NASA Ozone Trends Panel Executive Summary, R.T. Watson, Chm., March 1988, and press release. See summary by R.A. Kerr, "Research News," Science, March 25, 1988.

2. Singer, S.F., "Stratospheric ozone decreases: Doubts about causes and consequences," letter submitted to $\underline{\text { Science, }}$ April 12, 1988 (unpublished).

3. Bishop, L., W.J. Hill, and M.A. Marcucci, "An Analysis of the NASA Ozone Trends Panel Dobson Total Ozone Data over the Northern Hemisphere," Center for Applied Mathematics, Allied Signal, Inc., August 3, 1988. (see also R. Bojkov et al., J. Geophys Res. (1990), in press.) I thank the authors for additional information and critical discussion.

4. Shifting the starting date of the ramp to 1965 reduces the trend by 40\%; a 1975 data steepens it by 50\%. (private communication, March 28, 1989.) 
5. The Allied study refers to the UV-B flux, which is clearly inappropriate. The relevant region is in the far-UV and involves mainly the Runge-Schumann bands and continuum in the generation of ozone, and the Hartley-Huggins bands in its destruction. See also reference 12 .

6. Symonds, R.B., W.I. Rose, and M.H. Reed, "Contributions of Cl- and F-bearing gases in the atmosphere by volcanoes," Nature, 334, 415 (1988); Volcanoes contribute particulates as well as gases, including chlorine compounds.

(See also B.J. Finlayson-Pitts, M.J. Ezell, and J.N. Pitts, Jr., "Formation of chemically active chlorine compounds by reactions of atmospheric $\mathrm{NaCl}$ particles with gaseous $\mathrm{NO}$ and ClONO," Nature, 334, p. 241 (1989). Ocean spray could be another contributor to stratospheric chlorine.)

7. Angell, J.K., "On the relation between atmospheric ozone and sunspot number," J. Climate (1989).

(See also R.J. Angione, E.J. Medeiros, and R.G. Roosen, "Stratospheric ozone as viewed from the Chappuis band," (1976);Stratospheric ozone data have been reported for the period 1912-1950 from stations in California and Chile, showing variations of as much as $20-30 \%$ on time scales changing from months to decades.)

8. Singer, S., "Stratospheric water vapor increase caused by human activities," Nature, 223 , p. $543(1971)$.

(See also D.J. Hoffman, "Increase in the stratospheric background sulfuric acid aerosol mass in the past 10 years," Science, 248, pp. 996-1000 (1990); By an analogous mechanism, anthropogenic activities may contribute atmospheric sulfur compounds and thus stratospheric aerosols.)

9. Singer, S.F., "Does the Antarctic ozone hole have a future?,"Eos. November 22, 1988.

10. Kinnison, D.E., and D.J. Wuebbles, "A study of the sensitivity of stratospheric ozone to hypersonic aircraft emissions," Lawrence Livermore National Laboratory UCRL98314 (preprint), September 1988. Aircraft exhausts may contribute to stratospheric sulfuric acid aerosols, observed to increase at about 5\% per year in the last 10 years. See D.J. Hoffman (reference 8).

11. Eddy, J.A., "The Maunder Minimum," Science, 192, pp. 1189-1200 (1976).

(See also "Historical evidence for the existence of a solar cycle," in Solar Output and its Variation. O.R. White, ed., University of Colorado Press, Boulder, CO, (1977 )).

12. There are not as yet good models on the relation between sunspot number and ozone content. During periods of high solar activity, increased UV fluxes would tend to raise ozone levels; but solar proton events would destroy ozone (C.H. Jackman, NASA-Goddard Space Flight Center) as would particle precipitation into the polar zones (W.R. Sheldon, Univ. of Houston). Nor are there good models relating UV fluxes to ozone content. One problem is that different components of solar UV have quite different 
degrees of variation during the solar cycle; i.e., as a function of sunspot number or of solar flux. For example, in solar cycle 21, Lyman-alpha $(121.6 \mathrm{~nm})$ shows a variation of a factor of two, while UV irradiance in the interval 200 to $250 \mathrm{~nm}$ varies only by a few percent. (See J. Lean, "Contributions of ultraviolet irradiance variations to changes in the

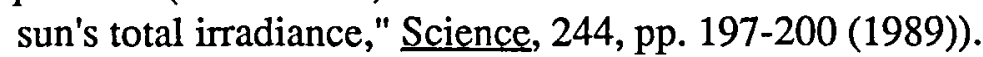




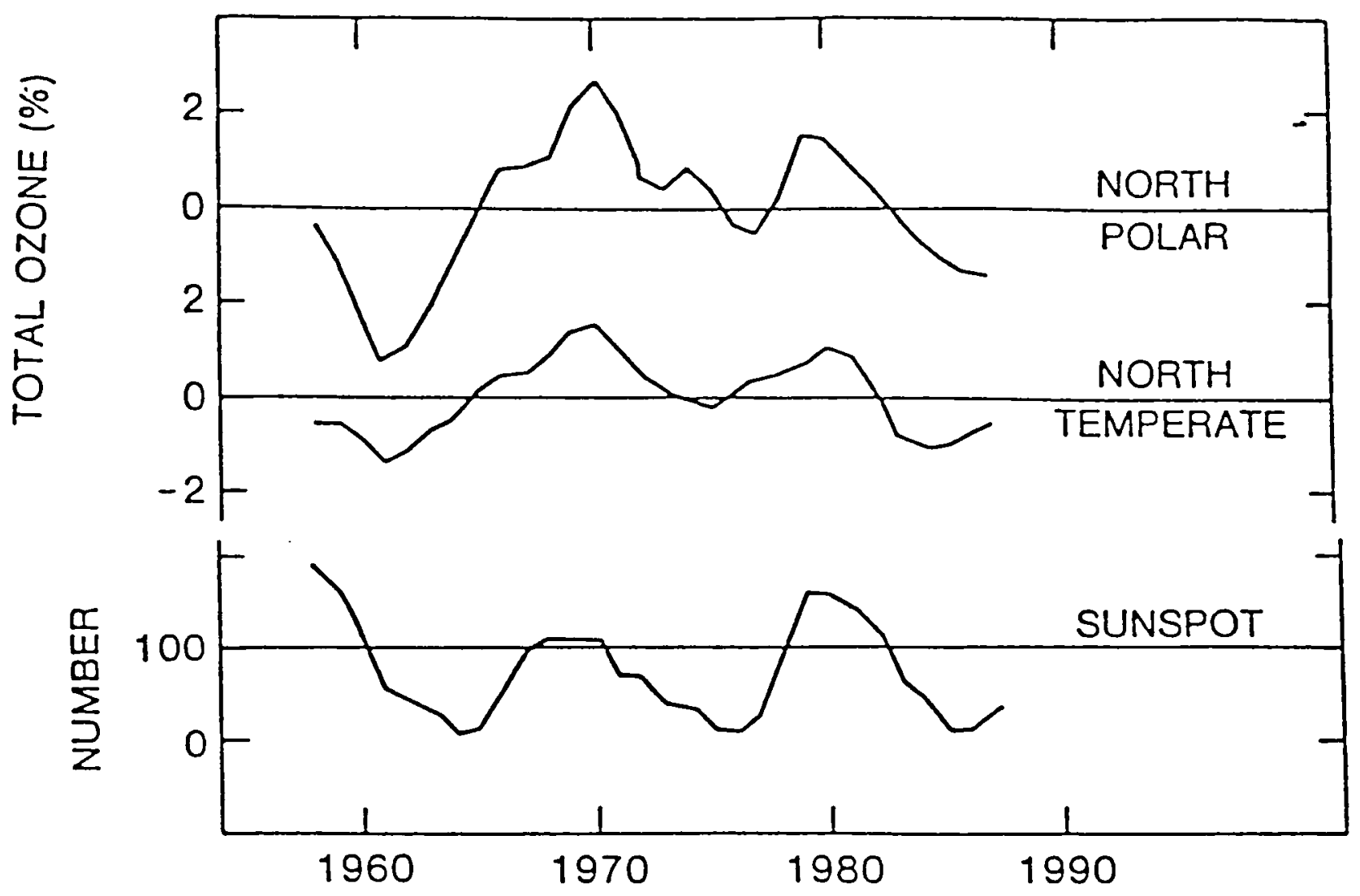

Figure 1. Total ozone change (in \%) and sunspot number (Angell, 1989).

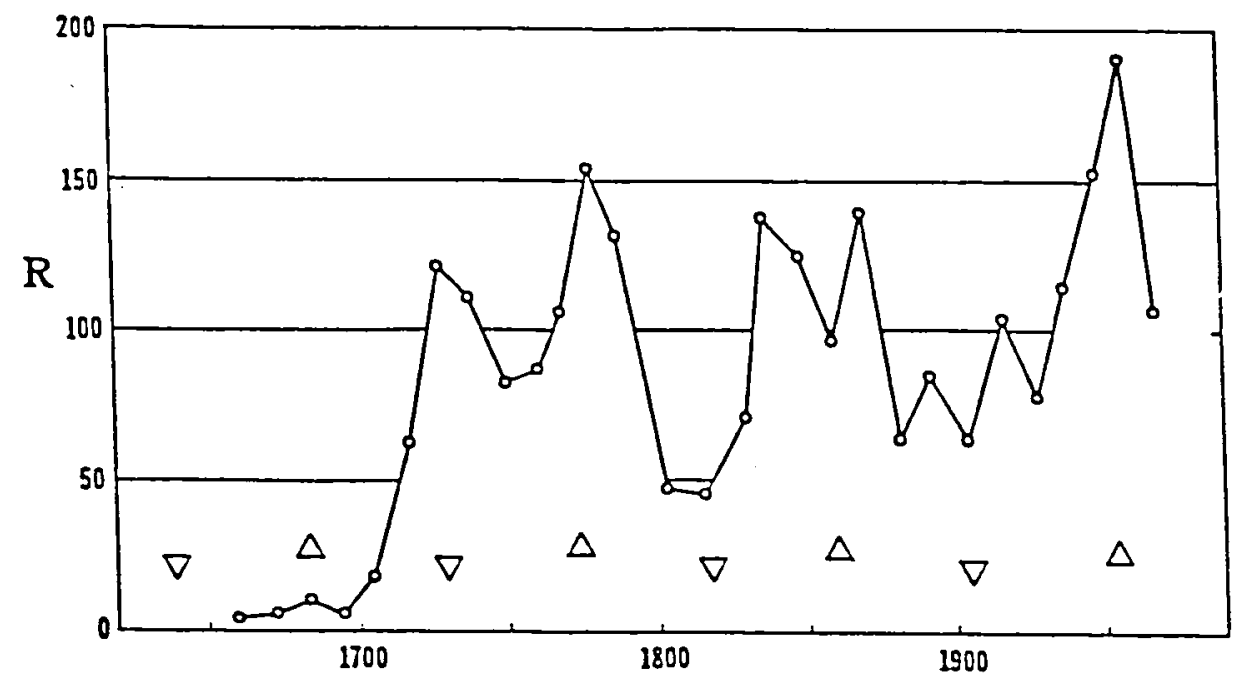

Figure 2. Annual mean sunspot number $R$ at maxima of the 11-y cycle, A.D. 1645 to present, to demonstrate long-term trends in solar activity. Evident is the 80-year "Gleissberg cycle" (extrema shown as triangles) imposed on a persistent rise since the Maunder Minimum. 


\title{
ON THE VARIABILITY OF THE SOLAR INTEGRAL RADIATION CONSTITUENTS
}

\author{
G.A. Nikolsky \\ Atmospheric Physics Department, Institute of Physics, Leningrad University \\ Leningrad-Petrodvorets 198904, U.S.S.R.
}

The results of spectral analysis of series of observations of the equatorial and polar diameters, as well as of series of satellite observations of the $S_{0}$ variations during 1975-1987 presented in papers by Laclare (1987), Delache (1988) and Delache et al. (1988) confirm with confidence the presence of an 11-year modulation in the Sun's radiation and diameter, and consequently, in the effective temperature of the photosphere. The same conclusion has been drawn with regard to the "1000th" and "320th" daily periodicities.

With regard to the manifestation of a 22 -year periodicity in solar and terrestrial processes, there are no final, generally accepted conclusions, in spite of a great number of investigations performed. It should be noted that Gilliland (1981), in his analysis of all basic longperiod series of observations of diameter variations, has not found any substantial evidence in favor of the existence of a 22-year modulation.

Let us consider now the results of papers by Vasilyev and Rubashev (1971- 1972), where combined processing of the Greenwich and Rome series of observations of the solar radius have been made. Due to the presence in the observational data of these Observatories of non-eliminated errors (the random error for the Rome series is about $0.024 " \pm 0.01$ ") and the existence of opposite tendencies in individual regions of the temporal series, the smoothing of the initial data using the third differences has been performed, after which an average curve has been plotted for the period in common (from 1876 to 1937). Therefore, on the temperal variation of the radius thus synthesized, there are three maximums and three minimums with the mean interval of 21 years. Spectral analysis of the synthetic dependence $R_{\odot}(t)$ yielded the basic period (according to split components) of 21.6 years with the magnitude of 0.20 ". The presence of a constituent with the period of 10.8 years and magnitude of 0.03 ' should also be noted. There are no traces of the 76-year constituent anywhere.

Although one of the two observation series of $\mathrm{R}_{\odot}$ (the Greenwich one), which have been included in the statistical analysis made by Vasilyev and Rubashev $(1971,1972)$, served as the main one in many combinations of series used for statistico-harmonic analysis by Gilliland (1981), the conclusions drawn in these two investigations concerning the constituents of the radius variations with the periods of 22 to 76 years are quite opposite. The impression is that the results of the analysis are under a strong influence of the order of priority of the variation periods. Thus, Gilliland states that when the 76-year period is extracted, 
the greater part of the variability in the measured series of the radius values is withdrawn. Also important are the depth of averaging of the series data and the deliberate selection of the series combination. This is indicated by the spread from 56.9 to 99.7 years in the period sought for with various combinations (20 combinations) of series. It is regrettable that the successful (in our opinion) combination of the Greenwich and Rome series has been used in the series analysis to reveal only the 11 -year constituent.

The periods of combinations averaged over 20 series constituted $76 \pm 8$ years, with the phases of maximums at $1911 \pm 4$ years and at $1987 \pm 9$ years, with the magnitude equal to $0.4 " \pm 0.1 "$.

Coming back to the hypothesis of the manifestation in the radius variations of periodicities which are multiples of the basic variation, one can suppose that the 76-year periodicity is a 7 -fold one of the 10.86-year variation. The difference from the 10.56-year period can be easily explained by the slow variation of the basic period of the solar activity cycle (SAC) with time. The acceptance of this hypothesis must result in rejecting the 76-year variation as it is, and in changing the method of analysis of observation series, viz., the singling out (after the withdrawal of the trend [secular] variation) of the 11-year and 22-year variations, and later the harmonics of the 11-year variation. The delay of $-0.8 \pm 1.1$ years between $R_{\odot}$ and $W_{z}$ found from 5 series (1860-1940) is apparently important for the analysis.

Let us consider now the graphic presentation of the above-mentioned periodic constituents of the radius variations on the temporal scale from 1967 to 1986 (Fig. 1), together with the results of direct measurements at Greenwich, Belgrade and CERGA Observatories. The smooth, slightly bent curve (the 76-year constituent) and the 11-year constituent superimposed on it (readings from the left-hand scale) are presented according to Gilliland's (1981) data. The other two smooth curves with the minimum in 1979 represent the main result of the statistical analysis performed by Vasilyev and Rubashev $(1971,1972)$. The 22-year constituent of the Sun's radius variations has been obtained from the data of the Greenwich and Rome (Campidoglio) series and is presented here by two sinusoidal curves with different amplitudes. The curve denoted by the triangles has the mean amplitude for the 1876-1937 period, whereas the second curve (dash-dot) has an amplitude characteristic for the period from 1911 to 1924 , when the 22-year variation had the maximum span ( 0.43 '). The phase of the variation is characteristic of considerable uncertainty.

Before we pass on to the consideration of the $\Delta R(t)$ dependences presented in Fig. 1 , one should clarify the circumstances relevant to certain changes in the Greenwich $\Delta \mathrm{R}_{\mathrm{Gr}}$ taken from the paper by Fröhlich and Eddy (1984), who processed the data from Greenwich Observatory concerning the solar diameter observations. The authors of this work made an interesting attempt to relate the changes in the radius and those of the Sun's luminosity (to which we will return). As follows from the conclusions made by Fröhlich and Eddy, they felt the presence of some long-period variation which was close to that 76 -year variation which was found by Gilliland (1981), or coinciding with the 22-year one. However, its 
phase, which determines the coincidence of maximum values in the variation of $\Delta \mathrm{R}_{\odot}$, with a maximum in the luminosity value (the solar constant) and the maximum of relative solar spot numbers, was obviously outside the scope of the understandable relationships between the above-mentioned solar parameters. Recently, C. Fröhlich informed us that in the course of processing of the Greenwich data, namely, in the calculation of the dependence $\Delta R(t)$, an accidental change of the sign might have taken place, which reverses the sign of the deviations from the mean value. In Fig. 1, the Greenwich dependence $\Delta \mathrm{R}_{\mathrm{Gr}}(\mathrm{t})$ is presented in the corrected form, and as can be clearly seen, it correlates very well not only with the direct measurement data, but also can be fairly well described by the 22-year (average) variation. However, the variation of the direct measurement data distinctly indicates the influence of the 11-year constituent superimposed upon the upward part of the 76-year variation, and the contribution of the latter is not manifested.

If one compares the 11-year variation (see Fig. 1) with the variations of the annual mean data of direct observations, one can easily see the contribution of the 11 -year variation. If one supposes that the amplitude of the presented 11- and 22-year variations corresponds to the period under consideration (1979), then the "mean" 22 -year variation, and not the "maximum" one should be introduced in the calculation. In this case, the measured value of $\Delta R_{\odot}$ for 1979 will be entirely determined by these two constituents.

The presence of several series and the restoration of the sign of deviations of $\left(\Delta R_{\odot}\right)$ found from the Greenwich series of the horizontal diameter make it possible to derive common annual mean values of the $R_{\odot}$ variations, which will, in all probability, be more representative than this or that individual series. The period from 1967 to 1974 is represented only by deviations from the operational mean value $\left(\overline{\mathrm{R}}_{\odot}=959.50\right.$ ") $)$ obtained from the Greenwich series (see Table 1).

The following obvious conclusions can be drawn from the data presented:

- The 76-year variation in the period from 1967 to 1987 is not revealed in the data of observations; the data of the middle series (see Table 1) will doubtless be made more precise after the facsimile from the initial information is obtained.

- The basic and comparable contributions to the radius variability yield the 11 - and 22-year variations.

- The presence can easily be seen (Fig. 1) of harmonics with periods of 2 and 4 years; the 4-year period is revealed up to 1979 only, and the 2-year one, after 1980 only. This is possibly due to the combined contribution of the 11- and 22-year variations (to be more precise, 10.8 and 21.2 years) forming a certain mean 16-year periodicity. In this case, the 4- and 2-year variations can be regarded as the 4 th and 8 th harmonics of such a mean variation.

- Measurements of the horizontal diameter made at Greenwich Observatory have not lost their significance for the analysis of phenomena on the Sun, since they contain data having precision characteristics conforming to the level of the latest ground-based diameter measurements. 
Table 1

Annual mean radius variations obtained by averaging three series of observations: at the Observatories of Greenwich for 1967-1985; Belgrade for 1977-1986; and CERGA for 1975-1986 (in arc sec).

\begin{tabular}{ccccccccccc}
\hline Year & 1967 & 1968 & 1969 & 1970 & 1971 & 1972 & 1973 & 1974 & 1975 & 1976 \\
\hline$\Delta \mathrm{R}$ & 0.09 & 0.24 & 0.17 & 0.08 & -0.01 & 0.03 & 0.07 & -0.03 & 0.22 & 0.02 \\
\hline \hline Year & 1977 & 1978 & 1979 & 1980 & 1981 & 1982 & 1983 & 1984 & 1985 & 1986 \\
\hline$\Delta \mathrm{R}$ & -0.03 & -0.1 & -0.33 & -0.12 & -0.14 & -0.17 & -0.04 & -0.1 & -0.005 & +0.05 \\
\hline
\end{tabular}

Precision measurements of the total solar radiation flux (the solar constant, $\mathrm{S}_{\mathrm{O}}$ ) carried out for almost 10 years on the NIMBUS-7 and SMM satellites (Hickey et al., 1988; Mecherikunnel et al., 1988), as well as measurements of the latitudinal distribution of the Sun's limb brightness from 1983 to 1987 (Kuhn et al., 1988) have opened up the possibility of justification of the selection and numerical confirmation of one of a number of proposed relationships between the variations of luminosity, temperature and radius of the Sun.

The most justifiable, physically, is the relationship $(\Delta \mathrm{L} / \mathrm{L})=4(\Delta \mathrm{T} / \mathrm{T})+2(\Delta \mathrm{R} / \mathrm{R})($ Spiegel, Weiss, 1980), which is the sum of the first terms of the series expansion of the basic law of absolute radiometry and which makes it possible (with the sufficiently large time of data averaging) to estimate the contribution of the variations of temperature and radius to changes in the Sun's luminosity. Since the luminosity variations have been obtained with a high level of accuracy and the accuracy of the annual mean measurements of the solar diameter is also high enough, it is possible, making use of the above-presented relationship, to calculate the variations of the effective temperature of the photosphere and to compare them with the measurements of Kuhn et al. (1988).

Combining the data from the NIMBUS-7 and SMM satellites, it has become possible to provide sufficient data on the luminosity variation for the period from 1979 to 1987. Data on the radius variation have been taken from the Greenwich series of the horizontal diameter measurements (Fröhlich, Eddy, 1984) and the CERGA Observatory series (Delache et al., 1988).

The results of calculations performed agree very well with the temperature variation at heliolatitudes $26.5^{\circ}, 29^{\circ}$ and $32^{\circ}$. The $\Delta \mathrm{S}_{\mathrm{o}}$ data have a temporal variation close to the calculated data for $\Delta \mathrm{T}_{\text {eff }}^{\varphi}$ for the heliolatitude belt from $26.5^{\circ}$ to $29^{\circ}$ over the entire time interval (1983-1987) provided with sufficient information (Kuhn et al., 1988).

The extrapolation of the temporal variation of the heliolatitudinal dependences $\Delta \mathrm{T}_{\mathrm{eff}}^{\varphi}$ for 1979, taking into account the calculated data for 1980-1983, makes it possible to estimate the variations of $\mathrm{T}_{\mathrm{eff}}^{\odot}$ in the 11 -year cycle $(\sim+2.2 \mathrm{~K})$ and to single out the variations of $\mathrm{T}_{\mathrm{eff}}^{\odot}$ connected with the 22 -year cycle $(\sim+0.7 \mathrm{~K})$. Fig. 2 shows the calculated variations of $\mathrm{T}_{\mathrm{eff}}^{\varphi}$ and $\mathrm{L}^{\varphi}$ in the latitudinal belt from $20^{\circ}$ to $32^{\circ}$ in the 11 -year solar activity cycle (SAC). 
The results obtained make it possible to state that there are processes influencing the luminosity and acting in phase or counterphase (or a phase close to it). Above all, this is relevant to annual mean variations of the effective temperature of the photosphere and solar diameter variations, whose contribution in the course of SAC No. 21 is estimated approximately as $0.2 \%$ and $-0.1 \%$, respectively, which leads to $\Delta \mathrm{L} / \mathrm{L}=0.1 \%$ or $\Delta \mathrm{L}=1.37 \mathrm{~W} / \mathrm{m}^{2}$ in SAC. It is obvious that the contribution of the latitudinal belts to the total radiation flux changes substantially in SAC. On the decreasing branch of the 11-year SAC, the basic contribution to the luminosity is made by the near-equatorial region $\left( \pm 20^{\circ}\right)$ of the Sun. On the increasing branch, the zones with $\varphi>20^{\circ}$ make the greatest contribution.

Completing our brief excursion to the area of interrelationships between $L, R$ and $T_{\text {eff }}$, it should be noted that measurements on the NIMBUS-7 satellite yielded during their first 6 months of work the mean value of $S_{o}=1376 \pm 0.73 \mathrm{~W} / \mathrm{m}^{2}$. Later Hickey et al. (1988) reduced this value, but the data of Fig. 1 show that in 1979, an abrupt decrease of the Sun's diameter took place $\left(\Delta \mathrm{R}_{\odot} \approx 0.37\right.$ ") which was accompanied by the corresponding growth of $\mathrm{T}_{\text {eff. }}$. It is possible that Hickey et al. considered that the data of the initial stage of measurements were not reliable enough and introduced the reduction. The actual value of $\mathrm{S}_{\mathrm{O}}$ for 1979 is very important for the agreement between the data of NIMBUS- 6 and NIMBUS-7. The confirmation of a higher $S_{O}$ value for 1979 would be very important for the estimation of the efficiency of the mechanism of solar-terrestrial relations through the channel of the solar constant variations. 


\section{REFERENCES}

Delache, Ph., "Variability of the solar diameter," Adv. Space Res., vol. 8, No. 7, pp. 119-128 (1988).

Delache, Ph., F. Laclare, and H. Sadsaoud, "Long periods in diameter, irradiance and activity of the Sun," In: Advances in Helio- and Astro-Seismology, J. Christensen, Dalsgaard, S. Frandsen, eds., JAU, pp. 223-226 (1988).

Frohlich, C., and J. Eddy, "Observed relation between solar luminosity and radius," $A d v$. Space Res., vol. 4, No. 8, p. 127 (1984).

Gilliland, R.L., "Solar radius variations over the past 265 years," Astron. J., vol. 248, Sept. 15, pp. 1144-1155 (1981).

Hickey, J.R., B.M. Alton, and F.J. Griffin, "Solar variability: Indications from Nimbus 7 satellite data," In: NASA Conf. Proc. 2191, Variations of the Solar Constant, S. Sofia, ed., NASA/GSFC, Greenbelt, MD (1981).

Kuhn, J.R., K.G. Libbrecht and R.H. Dicke, "The surface temperature of the Sun and changes in the solar constant," Science, vol. 242, pp. 908-911 (1988).

Laclare, F., "Sur les variations du diametre du Soleil observees a l'astrolabe solaire du CERGA," C.R. Acad. Sci., t. 305, ser. II, pp. 451454, Paris (1987).

Mecherikunnel, A.T., R.B. Lee, H.L. Kyle, and E.R. Major, "Intercomparison of solar total irradiance data from recent spacecraft measurements," J. Geophys. Res., vol. 93, No. D8, pp. 9503-9509 (1988).

Ribes, E., J.C. Ribes, and J. Vince, "Merlin Ph. Sur l'oscillation du diametre solaire," C.R. Acad. Sci., t. 307, ser. II, pp. 1195-1201, Paris (1988).

Spiegel, E.A., and N.O. Weiss, "Magnetic activity and variations in solar luminosity," Nature, vol. 287, No. 5783, pp. 616-617 (1980).

Vasilyev, O.B., and B.M. Rubashev, "On the solar radius variations," Solar Data, Bull No. 12, Leningrad: Nauka, pp. 93-100 (in Russian) (1971).

Vasilyev, O.B., and B.M. Rubashev, "On the coupling between possible variations of the solar radius and solar cyclicity," Solar Data, Bull No. 1, Leningrad: Nauka, pp. 95-101 (in Russian) (1972). 


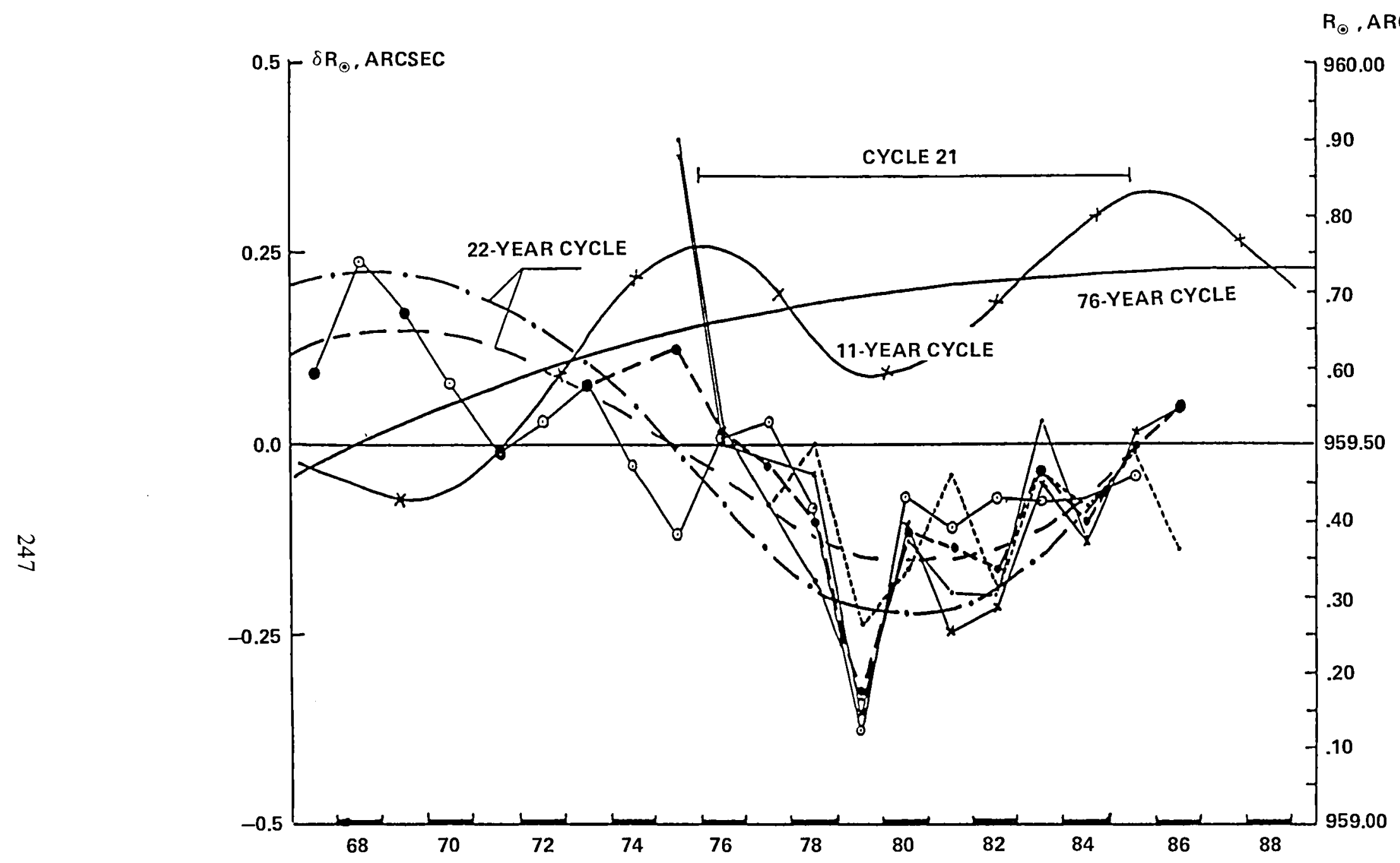

Figure 1. Temporal variations of the Sun's radius from the latest measurement data and periodic constituents of the radius variation from the results of spectral analyisis of long period observation series of the Sun's angular diameter.

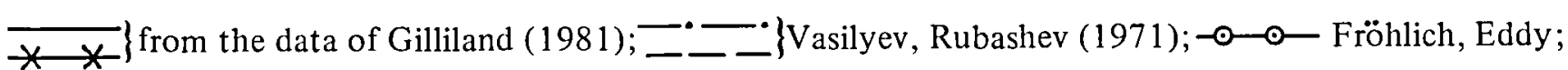

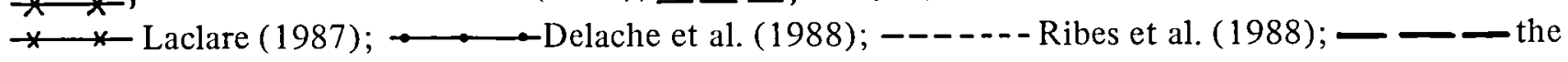
mean dependence. 


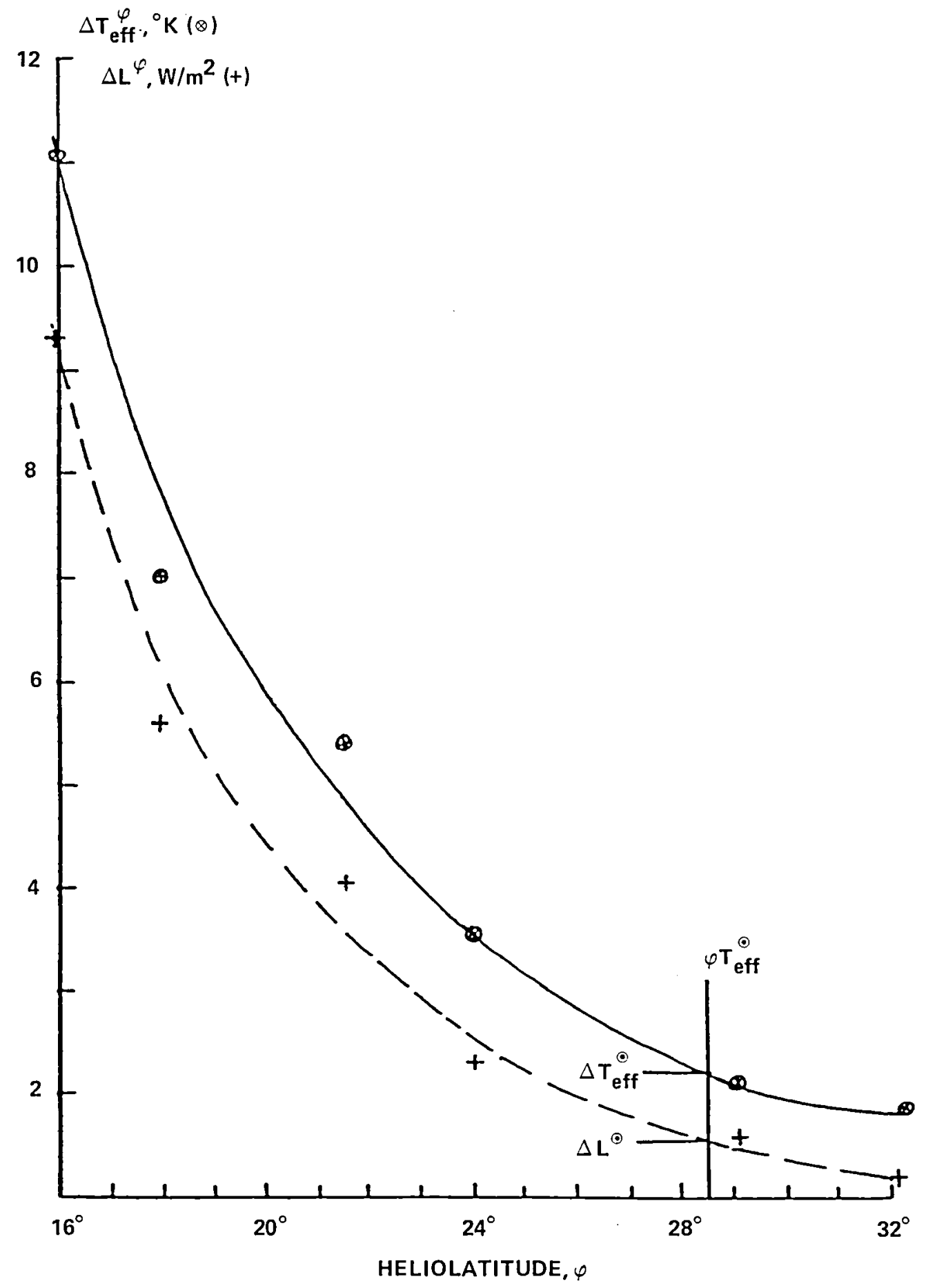

Figure 2. Increments of effective temperature $(\otimes)$, and luminosity $(+)$ of the photosphere at different heliolatitudes in phase of maximum solar activity (SA) (1979) relative to the phase of minimum $\mathrm{SA}^{\prime}(1986)$. The values of $\mathrm{T} \mathscr{e f f}_{\mathrm{ff}}$ and $L^{\varphi}$ have been obtained using the data of Kuhn et al.(1988). 


\title{
FORCING OF CLIMATE VARIATIONS BY MeV-GeV PARTICLES?
}

\author{
Brian A. Tinsley, \\ Center for Space Science, University of Texas at Dallas, \\ MS FO22, Box 830688, Richardson, TX 75083-0688
}

\begin{abstract}
There are three time scales for correlation of $\mathrm{MeV}-\mathrm{GeV}$ particles (galactic and solar) with changes in the sun, and with weather and climate:

A. DECADAL/INTERANNUAL (with 11 and 22 year solar cycles and QBO),

B. CENTURY SCALE (with Maunder minimum etc.),

C. DAY TO DAY (with coronal mass ejections, solar wind sector structure etc.)

Day to day correlations are not understandable in terms of total solar irradiance or UV changes; but are understandable as solar wind/particle forcing. If solar wind/particle forcing can produce short term weather responses, it could also produce decadal and longer term climate responses. A possible chain of amplifying and coupling mechanisms involves: stratospheric ionization, electric fields and chemistry; aerosol nucleation; sublimation, freezing and condensation nuclei; cloud microphysics and particle size distributions; winter storm intensification; and changes in circulation.
\end{abstract}

\section{CORRELATIONS ON THREE TIME SCALES}

Examples of tropospheric correlations with solar and cosmic ray variability on the decadal/interannual time scale are given in Figure 1. Solar variability is represented by sunspot number. Changes in $\mathrm{GeV}$ particles are represented by both surface neutron monitor count rates (NGDC, 1989) and by the lower stratospheric flux of particles above about $500 \mathrm{MeV}$ energy for which data is from Soviet daily balloon measurements at Mirny and Murmansk (Lebedev Inst., 1968-73 and IZMIRAN 1972-89) together with measurements from Thule (Neher, 1971). The change in the MeV flux over the 11 year solar cycle is about $40 \%$. Changes in tropospheric circulation and temperature are represented by the latitude shift in winter storm tracks in the eastem North Atlantic (Brown and John, 1979, and John, 1989, 1990); the frequency of winter cyclonic storms in the western North Atlantic for winters when the equatorial stratospheric winds are from the west (QBO West phase) (Labitzke and van Loon, 1989; John, 1990); and in winter surface temperature at eastern North American stations for QBO west phase (van Loon and Labitzke, 1989). The correlation in smoothed storm track latitudes with sunspot number for six cycles should be enough to rule out the accidental coincidence of decadal scale variations (Pittock, 1978). The storm track latitude shifts in the west phase of the QBO are about twice those in the smoothed data (Tinsley, 1988; John, 1990), but since the QBO phase is only known after 1952, we show the whole time series with east and west phase winters averaged with a five year running mean, with individual west phase winters superimposed after 1952. It is apparent that other sources of variance on the decadal/interannual time scale are of comparable importance to the presumed solar forcing, with candidates being volcanic activity, the El Nino-Southern oscillation, and internal variability. For the winter of 1989 the La Nina forcing appears to have dominated (Barnston and Livesy, 1989), however the 1990 winter (not shown) follows the solar variability trend, with storm tracks well south in the eastem Atlantic. 


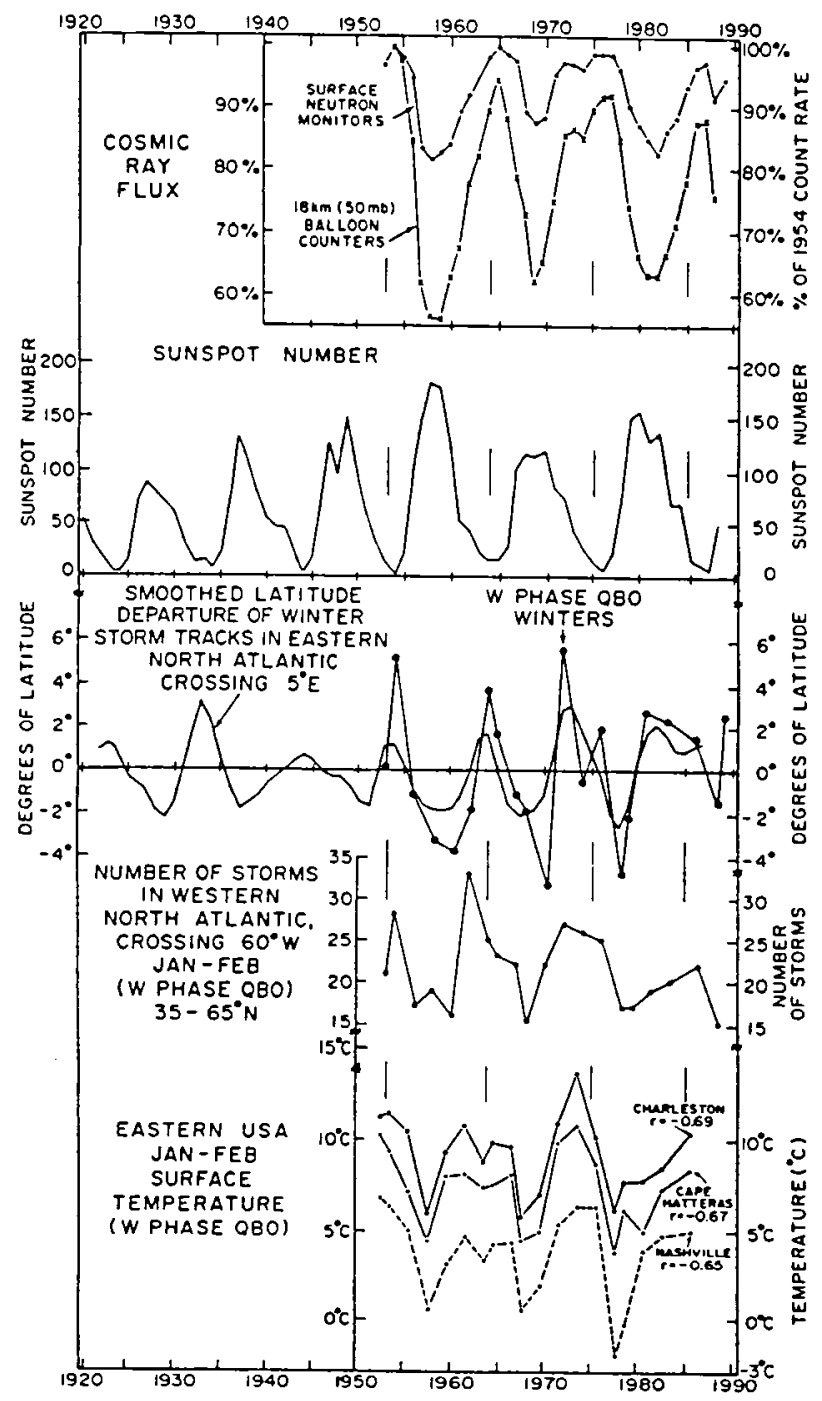

Figure 1. Correlation of solar and cosmic ray variability with tropospheric dynamics and temperature on the decadal/interannual time scale. The cosmic ray flux is from the Climax neutron monitor and daily Soviet Arctic and Antarctic balloon measurements supplemented by Thule measurements. The stom track latitudes are smoothed values for winter storms crossing $5^{\circ} \mathrm{E}$ above $50^{\circ} \mathrm{N}$ latitude, with unsmoothed values for QBO west phase winters superimposed. The storm frequencies are for those crossing $60^{\circ} \mathrm{W}$ between $35^{\circ} \mathrm{N}$ and $65^{\circ} \mathrm{N}$ for QBO West phase. Surface temperatures in Eastem North America are also for QBO west phase winters. See text for details and sources.

Evidence for solar variability on the century time scale and for tropospheric response to it was first given by Eddy (1976) relating solar variability in terms of the Maunder Minimum in sunspots to the "Little Ice Age" in northern Europe. The correlation was extended to earlier epochs with Carbon 14 concentrations as a proxy for solar variations, and indices of winter severity and glacial advance representing climate variations. It should be noted that Carbon 14 is a record of actual solar wind/GeV particle changes, and only by inference an indicator of possible irradiance and UV changes. Measurements of Beryllium 10 concentrations 
from Greenland ice cores (Attolini, et al., 1988) have shown an increase during the Maunder Minimum of $70 \%$ above the levels before and after, demonstrating directly that tens of percent changes occur in the $\mathrm{GeV}$ particle flux on the centennial as well as the decadal time scale.

On the day to day time scale, the first study showing a relationship of tropospheric storm intensification to the magnetic storms produced by coronal mass ejections was made by McDonald and Roberts (1960) for storms in the Gulf of Alaska. Figure 2 is from a recent study by Tinsley et al. (1989) relating high speed plasma streams in the solar wind (from coronal mass ejections) to changes in tropopause pressure over Berlin. The effect is clear over northem Europe,

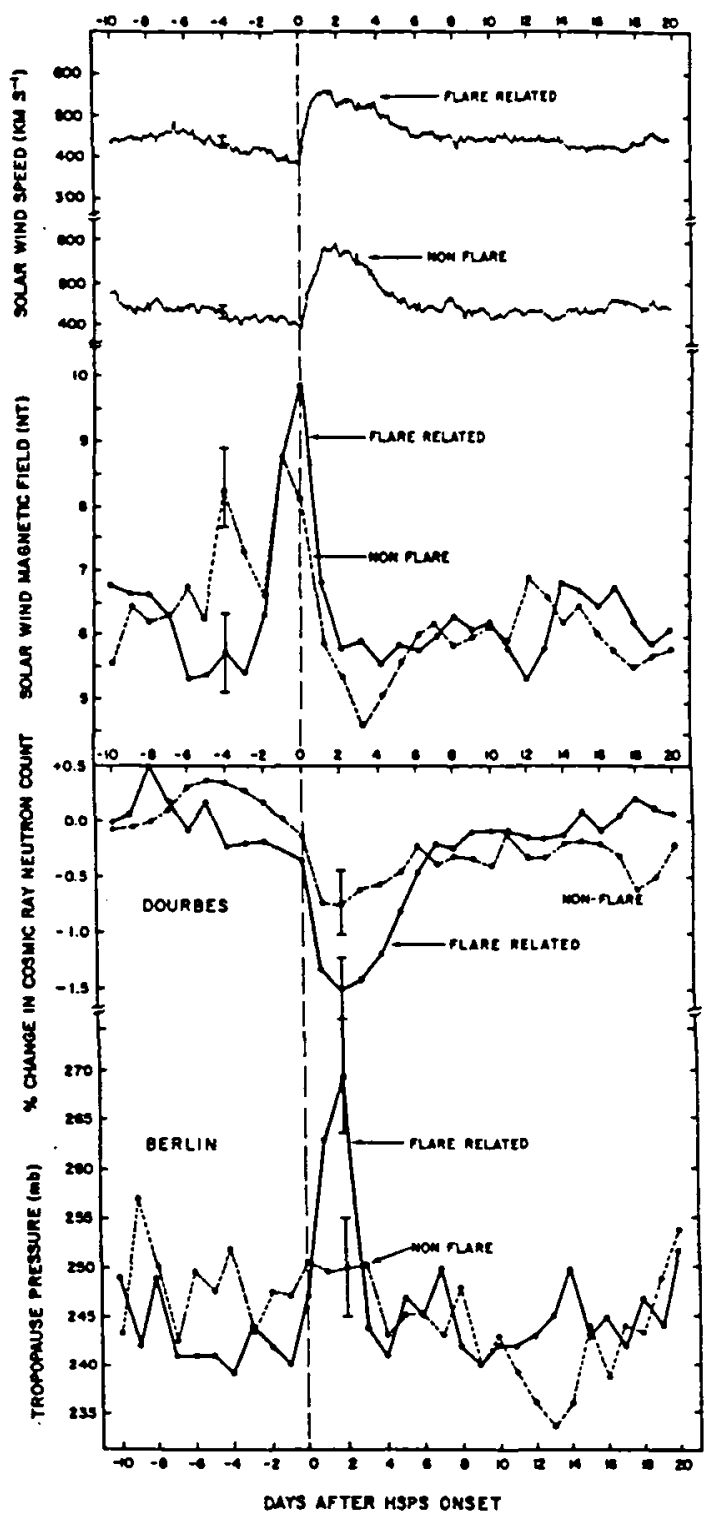

Figure 2. Superposed epoch analysis for variability of soler-terrestrial parameters with key days (day 0) the days of arrival of 55 Aare related and 55 non flare related high speed plasma streams in the solar wind, Jan. 1966 through Feb. 1978. Upper panel, solur wind speed and magnetic field; lower panel, surface neutron monitor count rate from Dourbes, Belgium, and tropopause pressure from West Berlin. Length of error bars is two standard deviations. 
corresponding to an increase in temperature by $2-3^{\circ} \mathrm{C}$ at the $200 \mathrm{mb}$ level, and a decrease in temperature by about the same amount at the $500 \mathrm{mb}$ level. The change on the day-to-day time scale in the $\mathrm{MeV}-\mathrm{GeV}$ particle flux precipitating into the stratosphere that is associated with these events is known as a Forbush decrease in the cosmic ray flux. To test how well the troposphere responds to such particle flux changes in comparison to other inputs related to short term solar variability we have constructed Figures 3 and 4 . Figure 3 contains superposed epoch plots with the key days (day 0 ) being the days of onset of Forbush decreases greater than 3\% (NGDC, 1985). The particle fluxes are represented by the Climax neutron monitor count rate and the polar cap stratospheric balloon particle counters as in Fig. 1. The Ap and F10.7 cm indices represent the variation of geomagnetic activity and solar ultraviolet variations respectively. The tropospheric response is represented by the average over the northern hemisphere of the $500 \mathrm{mb}$ Vorticity Area Index (VAI) (Roberts and Olson, 1973) which is a measure of the strength of cyclonic disturbances. The numbers in parenthesis are the numbers of events, which are different for different parameters on account of missing data. The comparison of the tropospheric variations for winter months (November through March) as compared with non-winter months shows that, consistent with earlier analyses, the characteristic dip in VAI on days 1 and 2 following the key day is a wintertime effect.

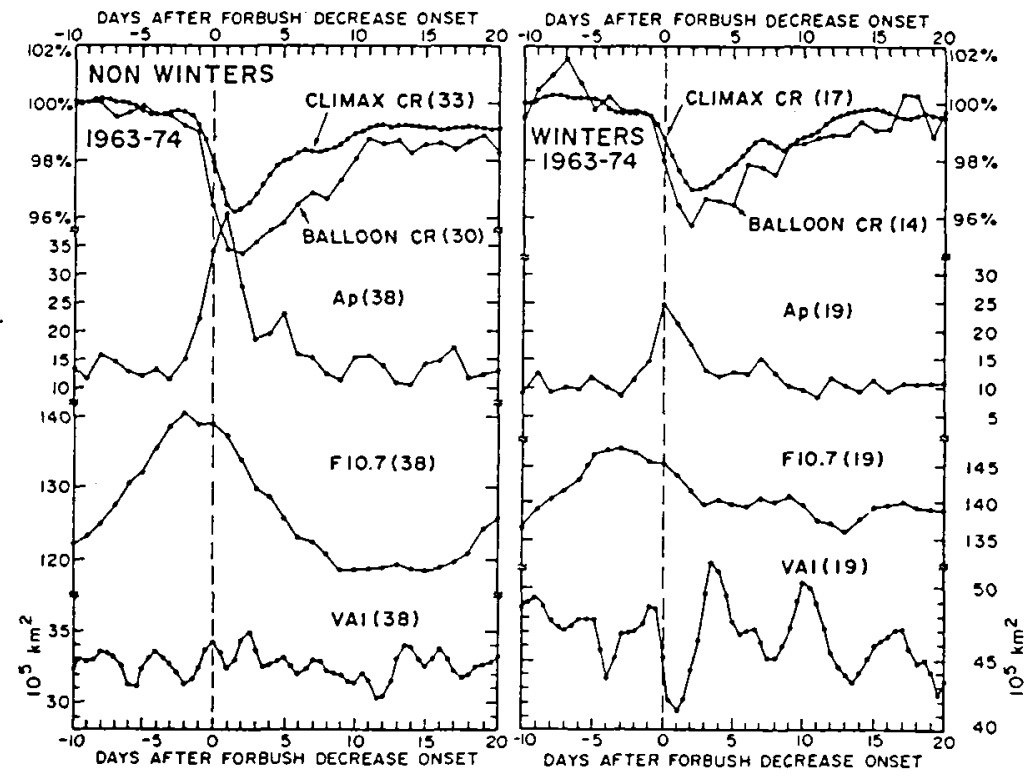

Figure 3. Superposed epoch plots with the key days (day 0 ) being the days of onset of Forbush decreases at Mt. Washington greater than 3\%. The particle fluxes are represented by the Climax neutron monitor count rate and the polar cap stratospheric balloon particle counters as in Fig. 1. The Ap and F10.7 cm indices represent the variation of geomagnetic activity and solar ultraviolet variations respectively. The tropospheric response is represented by the average over the northem hemisphere of the $500 \mathrm{mb}$ Vorticity Area Index.The numbers in parenthesis are the numbers of events, which are different for different parameters on account of missing data. Results for winter months (November through March) in the left panel are compared with non-winter months in the right panel. 
Figure 4 contains superposed epoch plots with the key days in the top two panels being the days of onset of Forbush decrease as before, and in the lower two panels the key days are the days of onset of magnetic storms with a $\mathrm{Ci}$ increase greater than unity (Stolov and Shapiro, 1974). Other quantities are as in Figure 3. The hypothesis that short term tropospheric forcing is due to solar UV irradiance changes (represented by $F 10.7 \mathrm{~cm}$ variations) can be seen from Fig. 3 to be not supported, due to their longer time scale, which is essentially the time scale for solar rotation. While the F10.7 index does not always accurately represent the amplitude of solar UV variations, it does have the same time scale for variations (Barth et al., 1990).
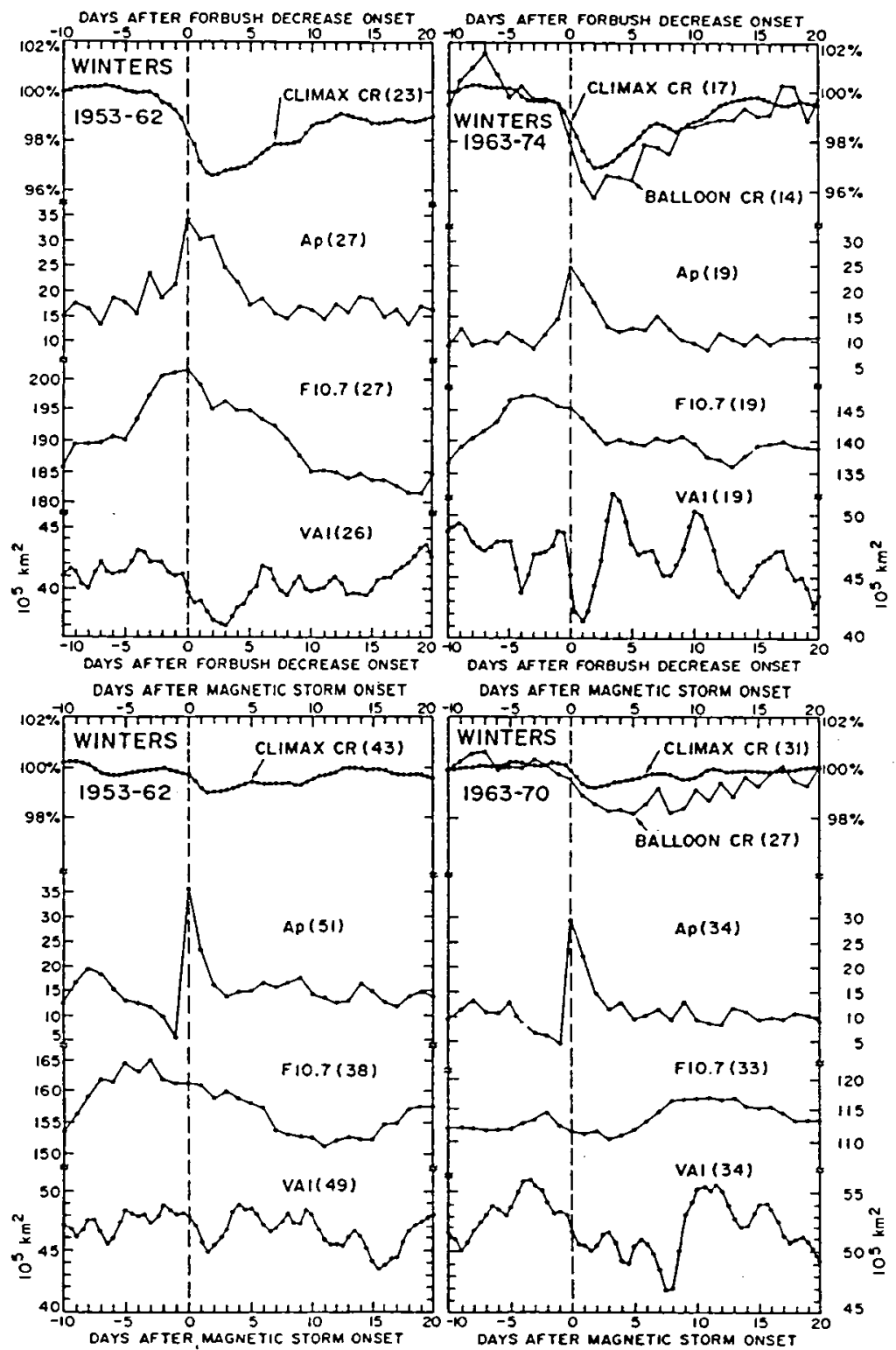

Figure 4. Comparison of superposed epoch plots using Forbush decrease days as the key days (top panels) with plots using days after magnetic storm onset (lower panels) as key days. The left panels are for winter months 1952-62, and the right panels for 1963-74. Otherwise the format is as for Fig. 3. See text for details. 
The Ap index variation provides a test for possible forcing by magneticactivity-related effects associated with the coronal mass ejections, e.g. bremmstrahlung $\mathrm{X}$-ray radiation from the precipitation of $\mathrm{keV}$ electrons, or from large scale magnetospheric convection electric fields. We see that with keying by Forbush decrease there is a significant dip in the hemispheric VAI on days 1 and 2 , for 1953-62 as well as for 1963-74. In the bottom two panels, the selection for and keying on the days of onset of the magnetic storms has strengthened and sharpened the Ap variation, but smeared out and weakened the Forbush decrease variation, compared to the top two panels. It has also weakened the dip in VAI on days 1 and 2 . This is evidence that the forcing is by particles, and not by other inputs related to magnetic activity.

The same transient response of the VAI (a dip on days 1 and 2 following the key day) is found when the key day is the day of a solar wind magnetic sector boundary crossing (Wilcox et al. 1973). There is a transient decrease of about $0.5 \%$ in the cosmic ray flux from day -1 to day +2 (Schuurmans and Tinsley, 1989), making the particle flux to VAI relationship similar to that for the Forbush decreases. Figure 5 compares the variations in superposed epochs for the years 1953-62, 1963-74 and 1975-82. The sector boundary effect on cosmic rays was reduced and of a different character before 1963 and after 1974, as also was the Ap response and the VAI response. Thus any solar wind forcing related to its sector structure must be considered non-stationary, and the weakness or absence of a tropospheric correlation after 1974 should not be used to discount the significance of the correlation for 1963-74.
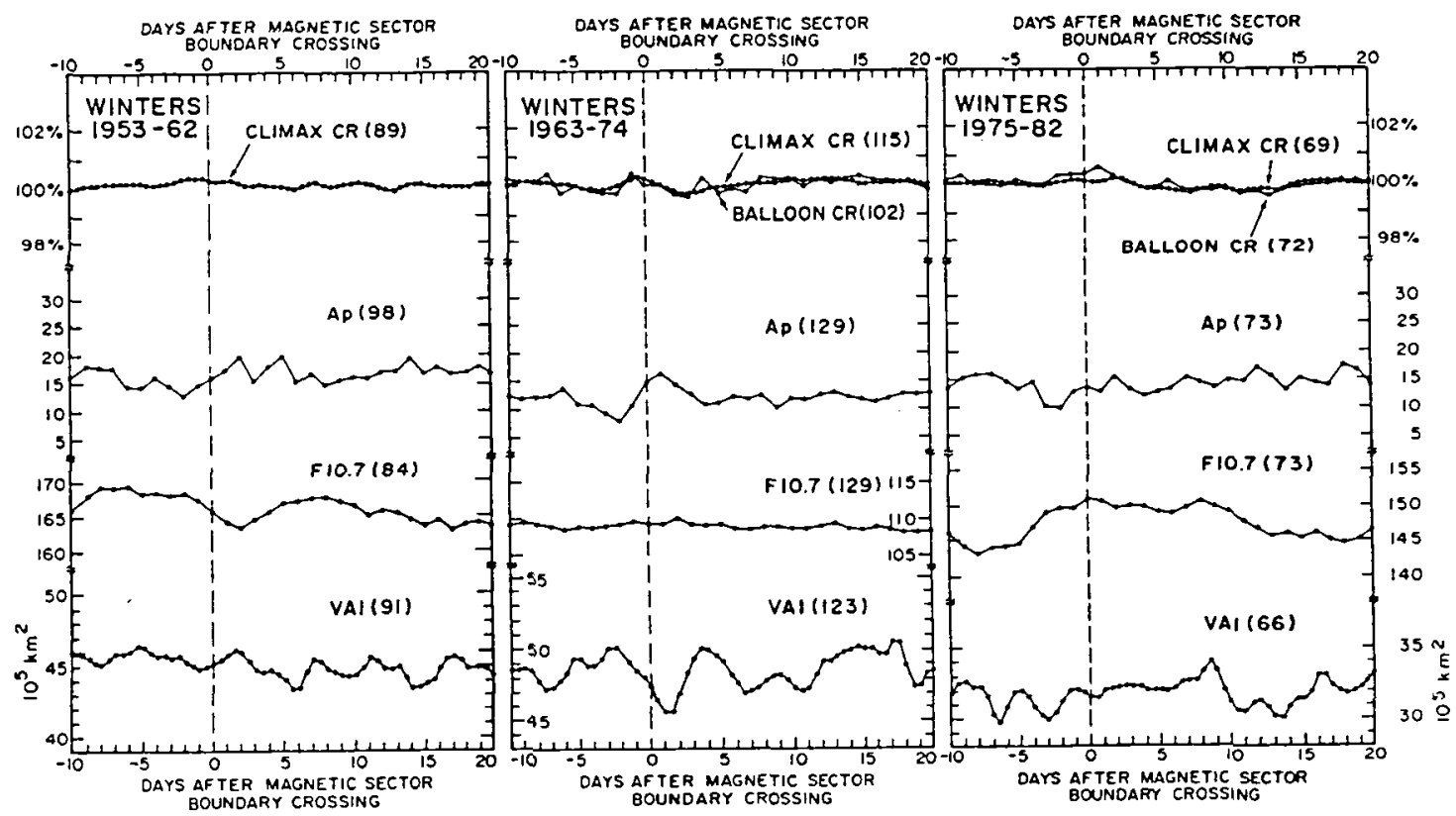

Figure 5. Comparison of superposed epoch plots with key days being the days of solar wind magnetic sector boundary crossing, for winter months. Panels from left to right are for 1953-62, 1963-74, and 1975-82. Otherwise the format is as for Fig. 3. See text for details. 
Tinsley: MeV-GeV particles and climate

\section{DISCUSSION}

The tropospheric changes on a timescale of 1 or 2 days following the arrival of coronal mass ejections at the earth do not seem understandable in terms of forcing by total solar irradiance or UV changes, which have a timescale related to solar rotation and development of active regions of a week or more. If, as seems more likely, solar wind/particle forcing is producing short term weather responses with particle flux changes of less than $10 \%$, then the decadal and century timescale changes in flux by tens of percent should be capable of producing the observed longer term climate changes.

The question remains of the intermediate mechanisms involved. We have been approaching it from two directions; from the sun and from the troposphere, with the aim of converging on a candidate set of coupling and amplifying processes, perhaps in the stratosphere. From the solar end, for short term forcing, we have concluded that solar wind modulation of $\mathrm{MeV}-\mathrm{GeV}$ particle precipitation into the stratosphere is the best supported candidate. From the tropospheric end, the analysis of Hines and Halevy (1977) of the related VAI response to solar wind magnetic sector boundary crossings indicates that the tropospheric effect is an amplification of a pre-existing dynamical variation, rather than the generation of a new variation. The latitude shifts of storm tracks shown in Figure 1 are explainable in terms of the intensification of cyclonic storms (through changes in the parameters of the feedback processes involved) with changes in the momentum radiated out of the jet stream by waves (Tinsley et al. 1989). The observed decreases in VAI on the short time scale are equivalent to the reduction in storm frequency at solar maximum on the decadal time scale, as shown in figure 1. The situation as discussed above is represented in Figure 6.

An enhancement in a positive feedback process for winter storms in the Gulf of Alaska and the North Atlantic (the longitude region at highest magnetic latitude where cold continental air encounters a relatively warm ocean, with significant potential for diabatic heat release) could provide the necessary amplification. Thus we are led to consider whether particle precipitation could affect storm cloud processes. There are two broad categories of mechanisms that have been suggested that could produce changes in clouds. These are represented in the two branches inside the speculative box of Figure 6. Changes in atmospheric electric fields are produced by changes in precipitation affecting conductivity in parts of the global electric circuit. Changes in ions are involved in the suggestion by Dickinson (1975) that ion induced nucleation might compete with homogeneous nucleation of $\mathrm{H}_{2} \mathrm{SO}_{4}$ aerosols from the gas phase. Some support for this has come from the inference that homogeneous and perhaps ion induced nucleation of gaseous $\mathrm{H}_{2} \mathrm{SO}_{4}$ is occurring in the winter Arctic and Antarctic stratospheres (Hoffman, 1990; Hamill et al. 1990; see also Rosen and Hoffman, 1983). In addition, particle precipitation would increase the production of $\mathrm{OH}$ and the conversion of $\mathrm{SO}_{2}$ to $\mathrm{H}_{2} \mathrm{SO}_{4}$ (see Herman and Goldberg 1978, p 262-3). Also, under conditions of severe denitrification, the production of $\mathrm{NO}_{x}$ might increase the production of the nitric acid trihydrate aerosols of type $x_{1}$ polar stratospheric clouds. Since both sulphate and nitrate aerosols act as efficient condensation nuclei, and perhaps also freezing and ice nuclei, they can affect cloud processes when present in potential cloud forming regions. 
Tinsley: MeV-GeV particles and climate

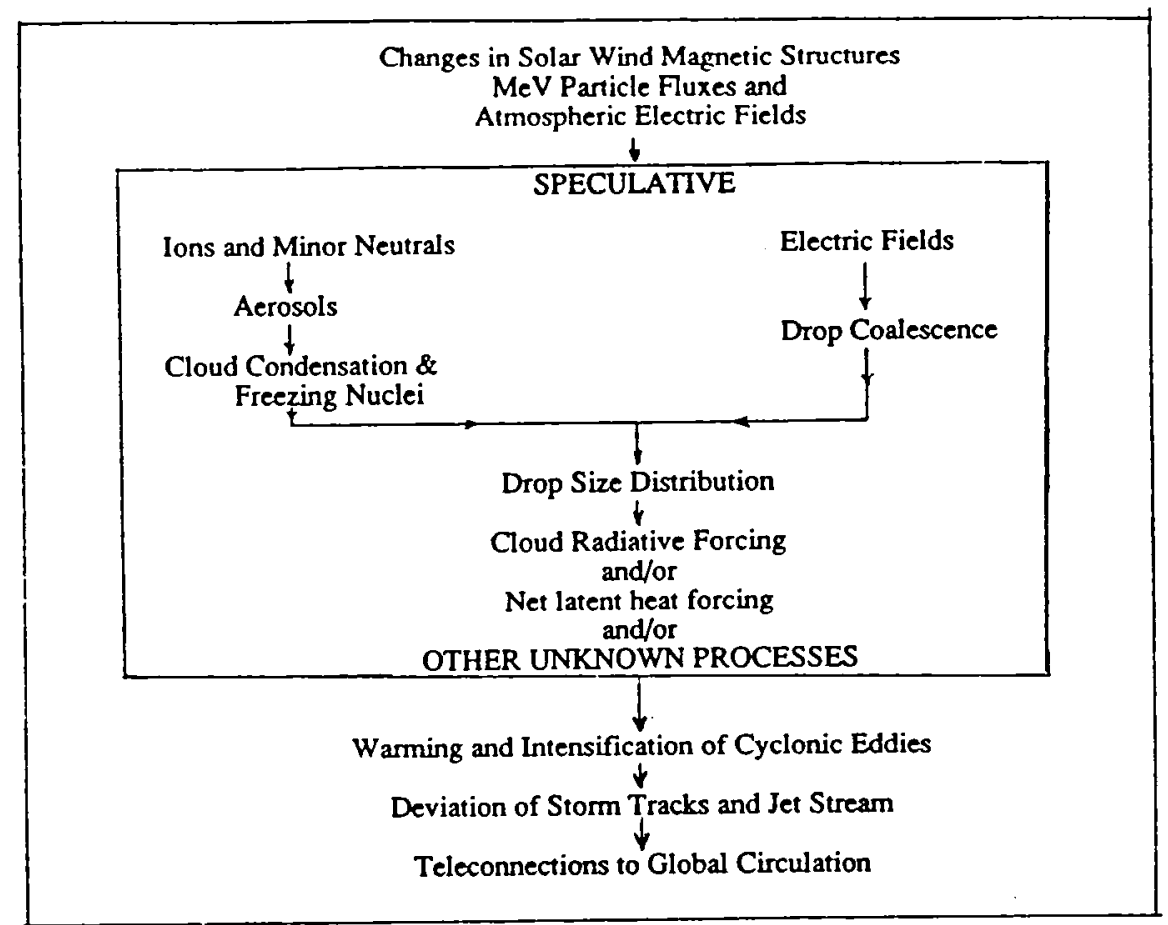

Figure 6. Possible relationships between forcing by solar wind magnetic structures, producing changes in MeV-GeV particle precipitation, atmospheric electric fields, aerosol and cloud microphysical processes, and tropospheric temperature and dynamics.

In the region of winter cyclones tropopause fold events are likely to occur (Browell et al., 1987) with the transport of cold humid tropospheric air into the stratosphere. On the contact surface with the condensation nuclei-rich stratospheric air a layer of cirrus may form that would be enhanced for higher ionization rates. Or merely the horizontal advection of low latitude (high tropopause) air into high latitude (low tropopause) air could provide the same contact. One possibility is that enhanced high cloud opacity would trap heat in the cyclone, which would increase the vertical advection and release of latent heat. Another is that sedimentation from the high cirrus would affect the particle size distribution and precipitation and re-evaporation of cloud droplets, and thus the net release of latent heat at lower levels. Such latent heat would feed back into intensifying the cyclone (Pauley and Smith, 1988). All such mechanisms must remain speculative until data is available on the response of clouds in such storm systems to changes in ionization and electric fields. It is also possible that aerosol transport processes with longer time scales may affect cloud opacity and albedo on longer time scales, and affect regions outside of cyclones. 


\section{CONCLUSIONS}

Changes in ionization production in the lower stratosphere by a few percent during Forbush decreases have been shown to correlate well with changes in winter tropospheric dynamics by a similar relatively small amount. Changes in ionization production by tens of percent on the decadal time scale have been shown to be correlated with changes in winter storm frequencies by tens of percent in the western North Atlantic. Changes in total solar irradiance or solar UV do not have time variations to match the tropospheric variations on the day to day time scales discussed here. Forcing related to magnetic activity is not supported. Thus solar wind/MeV-GeV particle changes appear to be the only viable forcing function for these day to day variations. If solar wind/particle forcing of a few percent amplitude can produce short term weather responses, then observed changes by tens of percent on the decadal and centennial time scale could produce climate changes on these longer time scales. The changes in circulation involved would produce regional climate changes, as observed.

At present the relations between stratospheric ionization, electric fields and chemistry and aerosol and cloud microphysics are poorly known, as also between the latter and storm feedback processes. However, the capability for investigating these relationships now exists, and has recently been most successfully used for elucidating the stratospheric chemistry and cloud microphysics associated with the Antarctic ozone hole. The economic benefits of being able to predict winter severity on an interannual basis, and the extent to which climate change related to solar variability will add to or subtract from the greenhouse effect, should be more than adequate to justify support for research in this area.

Acknowledgements; This work has been supported by the Atmospheric Sciences Division of NSF under grant ATM-8902207. I wish to thank J.I. John for permission to use his updated analyses of North Atlantic storms, and for useful discussions. I thank J. Allen and the National Geophysical Data Center for help in obtaining solar terrestrial data, and $R$. Jenne of NCAR for help with meteorological data.

References;

Attolini, M.R., S.Cecchini, G.C. Castagnoli, M. Galli and T. Nanni, On the existence of the 11-year cycle in solar activity before the Maunder Minimum, J. Geophys. Res., 93, 12729, 1988.

Barnston, A.G., and R.E. Livezey, The northern hemisphere mean January-February flux-climate relationship - 1989 update; p 174 in "Workshop on Mechanisms for Tropospheric Effects of Solar Variability and the Quasi-Biennial Oscillation", S.K. Avery and B.A. Tinsley, eds., Boulder, CO, 1989.

Barth, C.A., W.K. Tobiska, G.J. Rottman and O.R. White, Comparison of $10.7 \mathrm{~cm}$ radio flux with SME solar Lyman alpha flux, Geophys. Res. Lett., 17, 571, 1990.

Browell, E.V., E.F. Danielson, S. Ismail, G.L. Gregory and S.M. Beck, Tropopause fold structure determined by airbome Lidar and in-situ measurements, J. Geophys. Res., 22, 2112, 1987.

Brown, G.M., and J.I. John, Solar cycle influence in tropospheric circulation, J. Atmos. Terr. Physics, 41, 43, 1979.

Dickinson, R.E., Solar variability and the lower atmosphere, Bull. Am. Meteorol. Soc., 56, 1240, 1975.

Eddy, J.A., The Maunder Minimum, Science, 192, 1189, 1976.

Hamill, P., O.B. Toon, and R.P. Turco, Aerosol nucleation in the winter Arctic and Antarctic Stratospheres, Geophys. Res. Lett.,17, 417, 1990. 
Herman, J.R., and R.A. Goldberg, Sun, Weather, and Climate, NASA Spec. Publ., NASA SP-426, 1978.

Hines, C.O., and I. Halevy, On the reality and nature of a certain sun-weather correlation, J. Atmos. Sci., 34, 382, 1977.

Hoffman. D.J., Measurement of the condensation nuclei profile to $31 \mathrm{~km}$ in the Arctic from January 1989 and comparisons with Antarctic measurements, Geophys. Res. Lett., 17, 357, 1990.

IZMIRAN, Cosmic ray intensity maximum in the Stratosphere, in "Cosmic Data" (in Russian) monthly bulletins, Nauka Publishing House, Moscow, 1972-89.

John, J.I., Storm tracks and atmospheric circulation indices over the northeast Atlantic and northwest Europe in relation to the solar cycle and the QBO, p 209 in "Workshop on Mechanisms for Tropospheric Effects of Solar Variability and the Quasi-Biennial Oscillation", S.K. Avery and B.A. Tinsley, eds., Boulder, CO, 1989.

John, J.I., Secular changes in storm tracks over the north Atlantic Ocean, 1920-89, extended abstract, AMS meeting Anaheim CA, 1990.

Labitzke, $\mathrm{K}$, and $\mathrm{H}$. van Loon, Association between the 11 year solar cycle, the $\mathrm{QBO}$, and the atmosphere, III, Aspects of the association, J. Climate, 2, 554, 1989.

Lebedev Institute, Cosmic ray intensity maximum in the stratosphere, data series 1957-71, Acad. Sci. USSR, 1968-73.

McDonald, N.J. and W.O. Roberts, Further evidence of a solar corpuscular influence on large scale circulation at $300 \mathrm{mb}$., J. Geophys. Res., 65, 529, 1960.

Neher, H.V., Cosmic rays at high latitudes and altitudes covering four solar maxima, J. Geophys. Res., 76, 1637, 1971.

NGDC, Climax cosmic ray hourly count rates, 1953-87, National Geophysical Data Center, Boulder CO, 1989.

Pauley, P.M., and P.J. Smith, Direct and indirect effects of latent heat release on a synoptic scale wave system, Mon. Weather Rev., 116, 1209, 1988.

Pittock, A.B., A critical look at long term sun-weather relationships, Rev. Geophys. Space. Phys., 16, 400, 1978.

Rosen, J.M., and D.J. Hoffman, Unusual behavior of the condensation nuclei concentration at $30 \mathrm{~km}, \mathrm{~J}$. Geophys. Res., 88, 3725, 1983.

Roberts, W.O., and R.H. Olson, Geomagnetic storms and wintertime 300-mb trough development in the North Pacific-North America area, J. Atmos. Sci., 30, $135,1973$.

Schuurmans, C.J.E., and B.A. Tinsley, Comparison of lower atmosphere responses to Forbush decreases of cosmic rays; solar proton events; solar flares; and high speed plasma streams, p 237 in "Workshop on Mechanisms for Tropospheric Effects of Solar Variability and the Quasi-Biennial Oscillation", S.K. Avery and B.A. Tinsley, eds, Boulder, CO, 1989.

Stolov, H.L., and R. Shapiro, Investigation of the responses of the general circulation at 700-mb to solar geomagnetic disturbance, J. Geophys. Res. $79,2161,1974$.

Tinsley, B.A., The solar cycle and the QBO influences on the latitude of storm tracks in the North Atlantic, Geophys. Res. Lett., 15, 409, 1988.

Tinsley, B.A., G.M. Brown and P.H. Scherrer, Solar variability influences on weather and climate; possible connections through cosmic ray fluxes and storm intensification, J. Geophys. Res., 94, 14783, 1989.

van Loon, $H$., and $K$. Labitzke, Association between the 11 year solar cycle, the QBO, and the atmosphere, Part II, surface and $700 \mathrm{mb}$ on the northem hemisphere in winter, J. of Climate, $1,905,1988$.

Wilcox, J.M.,P.H. Scherrer, L. Svalgaard, W.O. Roberts and R.H. Olson, Solar magnetic sector structure: relation to circulation in the earth's atmosphere, Science. 189, 185, 1973. 


\title{
Relationship between Rainfall in the Northern Hemisphere and Impulses of the Torque in the Sun's Motion
}

\author{
T. Landscheidt \\ Schroeter Institute for Research in Cycles of Solar Activity \\ Belle Côte, Nova Scotia BOE 1C0, Canada
}

\begin{abstract}
The analysis of major change in the angular momentum of the sun's irregular motion about the barycenter of the solar system, represented by extrema in the running variance of impulses of the torque (IOT), discloses a connection with both extrema in the Gleissberg cycle of secular sunspot activity and maxima in the thickness of varves from Lake Saki,Crimea. This significant relationship can be traced back to the 7 th century. Further inquiries link the running variance in IOT to rainfall over central Europe, England, Wales, eastern United States, and India, as well as to temperature in Europe. This significant correlation covers more than 130 years.
\end{abstract}

\section{Introduction}

There has been permanent controversy over whether or not the sun's varying activity influences weather on earth. Simple relationships discovered in the 1870's and the following decades vanished when examined more critically, or faded in the light of longer records. Furthermore, such ephemeral relationships were limited to special regions and did not cover larger areas subjected to the same or a similar climate. Yet the quest persists. In 1987 Labitzke ${ }^{1}$ reported that mid-winter warmings in the U.S. and Western Europe are well correlated with the 11-yr sunspot cycle over the past 40 years, provided the switch in direction of stratospheric winds (QBO) is taken into account. It has been objected that this connection, covering only some decades, could be explained by internal decadal variations in the atmosphere that happen to be in phase with sunspots for some cycles, but show no actual physical relation. ${ }^{2}$ This kind of criticism does not apply to the results presented here that cover more than 130 years of rainfall and temperature in different parts of the Northern Hemisphere and 12 centuries of Lake Saki varve data. The solidly significant correlation of these data with impulses of the torque in the sun's motion about the center of mass (CM) of the solar system cannot be explained by a complete chain of cause and effect. An acknowledged theory of solar activity does not yet exist, and the atmosphere's response to solar activity remains poorly understood. It is shown, however, that special phases and cycles in the sun's motion, accessible to computation, show a strong relationship both with the sun's varying activity and with synchronous climatic phenomena. ${ }^{3}$ Earlier results based on this relationship were tested by successful long-range forcasts of energetic solar eruptions and strong geomagnetic storms, ${ }^{4}$ and by the correct prediction of the end of the Sahelian drought three years in advance. ${ }^{5}$

\section{Change in the Sun's Angular Momentum and Gleissberg Cycle}

Satellite instruments revealed that the sun's brightness decreased by about .1 percent between the peak of sunspot activity in 1980 and its minimum in fall 1986. Unexpectedly, the sun grew more luminous in the ascending phase of the new cycle. Analysis by Foukal and Lea ${ }^{6}$ indicates that the increase in bright faculae outweighs the increase in dark spots. ${ }^{7}$ According to calculations based on standard climate models, a dip in the solar irradiance of between .2 and .5 percent, acting over several decades, would have been sufficient to cause the Little Ice Age. 
Thus, it is not unimaginable that the secular cycle of solar activity, covering more than eight decades, builds up a sufficient potential of climatic change by steady accumulation of excess radiation, or by continued radiation lower than average over a period of several decades. ${ }^{9}$ If this proved true, techniques based on impulses of the torque in the sun's motion (IOT) could presumably help to forecast the sun's secular behavior and the earth's climatic response. Figure 1 shows the 36-yr running variance $v=s^{2}$ of impulses of the torque in the sun's motion measured by the time integral $\Delta L=\int_{t_{0}}^{t_{1}} T(t) \mathrm{d} t$. In this equation, $L$ represents the sun's orbital angular momentum and $T$ the torque that drives the sun's motion about the $C M$. The running variance $v$, the moving square of the standard deviation, provides an apt representation of the change in the orbital angular momentum $L$ within special time intervals. The epochs of minima in the secular sunspot cycle from A.D. 700 to 1600, defined by Gleissberg $^{10}$ and Link, ${ }^{11}$ are indicated in Figure 1 by arrows. It is obvious that all of the extrema in the running variance $v$ in IOT coincide with the epochs of secular minima. A connection with secular maxima emerges when the smoothed 9-yr running variance of IOT is computed that shows a finer resolution. The resultant complex pattern, shown in Figure 2, conveys the impression of pentadactyl hands. The middle fingers (3) and little fingers (5) point to secular sunspot minima, marked by triangles, while the other fingers indicate secular periods of intense sunspot activity, designated by arrows pointing upwards. Fingers 1 and 2 coincide with periods of protracted higher activity with an intermittent lull, wheras finger 4 is related to secular maxima concentrated on a single spell of secular activity.

This rhythmic pattern is very sensitive to disturbances. Figure 2 shows that in the 15th century one finger was lacking so that instead of two consecutive maximum periods 1 and 2 there was only one. Remarkably, this phase anomaly coincided with the beginning of the Spörer minimum. Figure 3 shows the effect of a different kind of disturbance that did not occur in the centuries A.D. $700-1600$. In 1632, 1811, and 1990 the sun's motion relative to the CM was retrograde, and the orbital angular momentum became negative. The epochs of these very rare events, marked by $R$ in Figure 3, always fell on period 2, the index finger. The retrograde motion in 1632 did not change the rhythmic pattern, but it is conspicuous that the minimum period 3 coincided with the Maunder minimum. The retrogression in 1811 was related to a phase shift. Period 2 should have indicated a maximum, but coincided with the deepest secular sunspot minimum observed after the Maunder minimum. This phase inversion, the only case in 14 centuries, also affected the following periods 3 and 4. Open triangles in Figure 3 label this perturbed period; triangles pointing upwards indicate secular maxima, and those pointing downwards mark epochs of secular minima. Period 5 at the beginning of the 20 th century restored the regular rhythm. The extended period of weak sunspot activity from 1880 to 1930 reflects the circumstance that period 4, irregularly connected with a secular minimum, was followed by period 5 that regularly coincides with secular minima. As yet, the 20 th century has continued to follow the regular cyclic pattern. Period 1 reached its peak in the second half of the 1950's, when the sunspot activity was extremely high. After an intermittent decrease around 1970, there was a steep ascent again. Period 2, the index of the current hand, peaked at the end of the 1980's. This marks the epoch of a further secular maximum, which is in accord with unexpectedly high sunspot activity observed in 11-yr sunspot cycles 21 and 22 . It should not be forgotten, however, that period 2 is subject to instability as it coincides with retrograde solar motion and negative angular momentum. Considering that change in the sun's orbital angular momentum is fundamental to the concept in question, it seems consistent to assume that a switch in sign will have an effect. As a phase switch like that around 1811 did not occur, the anomaly will perhaps affect the dimension of the secular minimum related to period 3. A comparable situation occurred around 1670 when period 3, following retrograde solar motion in 1632, concurred with the Maunder minimum. The coming period 3 will peak around 2026. Period 4 , the next pointer to a secular maximum, will reach its peak at the end of the 2050's.

\section{Lake Saki Varve Thickness and Impulses of the Torque}

A complete pentadactyl hand covers a period of 178.8 years, the fundamental cycle in the sun's 
motion discovered by Jose ${ }^{12}$ and studied by Fairbridge, Sanders, and Shirley. ${ }^{13}$ Dansgaard ${ }^{14}$ has derived a cycle of just 180 years in climate from the Camp Century ice core drilled from the Greenland Ice Sheet. This correspondence begs for an investigation of a possible relationship of climate features with the complex pattern of the pentedactyl hand which reflects both variations in impulses of the torque in the sun's motion and secular sunspot activity. The mean interval between fingers of the hand is 35.76 years, and the average distance from the peaks of fingers - major maxima in the running variance of IOT $\left(V_{\operatorname{Max}}\right)$ - to the deepest points between two neighboring fingers - major minima in the running variance of IOT ( $V$ Min) - covers 17.88 years. In a thorough analysis of Lake Saki varves, Dewey ${ }^{15}$ has found cycles with a corresponding length between 17 and 18 years. Hence, the thickness changes in the varve deposits from Lake Saki, Crimea, were examined with regard to connections with the variance in IOT. Shostakovich, who published the varve data, covering a period of 4189 years (2295 B.C. to A.D. 1894), ${ }^{16}$ also presented evidence that related varve thickness to local precipitation: the thickest varves were linked to very wet years and the thinnest varves to very dry years. ${ }^{17}$ In his analysis, Dewey focused attention on a cycle of 17.3 years because he had found a cycle of nearly the same length in the flood stages of the River Nile. He noted, however, that a concurrent 17.75-yr cycle was also present in the data. As a result of his analysis, Dewey assessed the dates of the ideal crests in the cycle that he had found in the Lake Saki data. Dewey's crest dates do not perfectly fit the actual crests in the varve thickness in all single waves of the cycle. But when he fitted a sinusoid to each of the waves, measured the extent to which the actual crests concentrated around the time of the ideal crest, and calculated average values for groups of 48 waves each, all crests fell within 0.47 year of ideal timing. When the averaging was based on groups of 12 waves, the median irregularity did not go beyond 0.84 year. The Lake Saki varve data are noisy and show a serrated shape. The timing of major crests in a new investigation could be exposed to arguments. Therefore, the independently assessed ideal epochs in Dewey's table were compared with the epochs of extrema $V$ Max and $V_{\text {Min }}$ in the $9-y r$ running variance of IOT.

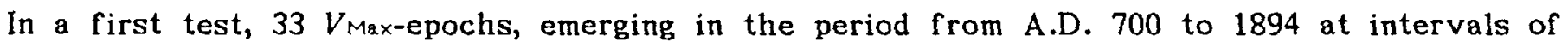
about 36 years, were related to every second ideal crest date from Dewey's list. The ideal crest date next to the first $V_{\operatorname{Max}}$ epoch determined the beginning of the continuous sequence formed by every second ideal crest. This sequence was disturbed only at the start of the Medieval maximum, also a climate optimum, and at the beginning of the Maunder minimum. There was a phase change by $\pi$ radians reflecting synchronous signals in the variance of IOT. Half of the respective interval between consecutive $V_{\max }$ was defined as a range into which associated ideal crest epochs can fall. Relative to $V_{\operatorname{Max}}$ at the center, this range covers \pm 18 years. The evaluation of the deviation of ideal crest dates from $V$ max data becomes easier, when absolute values are considered. In a random distribution, the mean of the deviations of respective ideal crest dates from $V_{\operatorname{Max}}$ epochs in years would then be $\bar{x}_{1}=9$. A Monte Carlo model based on 10,000 iterations yields the standard deviation $\sigma_{1}=5.15$. The parameters of the observed distribution are quite different: $\bar{x}_{2}=4.97$ and $\sigma_{2}=2.81$. Student's $t$-test (Fisher-Behrens formula) yields $t=8.19$ for 33 degrees of freedom. The rules valid for singlesided distributions apply: $P<6 \times 10^{-7}$. The null hypothesis of no significant difference between the means involved can be rejected at a high level of significance. This result is corroborated when $V$ Min epochs in their relation to the rest of the ideal crest dates are subjected to the same process such that the ideal crests that were skipped are now continuously related to $V$ min epochs. This procedure yields $\bar{x}_{3}=4.5$ and $\sigma_{3}=2.34$. The parameters of the control group, $\bar{x}_{1}$ and $\sigma_{1}$, are the same as before. Student's test results in $t$ $=10.96$ for 33 degrees of freedom; $P<6 \times 10^{-8}$. The null hypothesis of no correlation between the epochs of $V_{\text {Min }}$ is rejected at a high level of significance. A $\chi^{2}$-test as well as a nonparametric Mann-Whitney-test yield the same results. The tests of $V_{\max }$ and $V_{\text {Min, }}$ when combined, point to a real difference between the random group and the observed group, and substantiate the working hypothesis of a relationship between extrema in the variance of IOT, connected with solar activity, and Lake Saki varve thickness, related to local rainfall. Further computations, that cover all of the data back to 2295 B.C., are underway; the results will be published soon, as well as the yield of another approach that evaluates actual maxima in 
smoothed varve thicknesses in relation to $V_{\operatorname{Max}}$ and $V_{\min }$.

Shostakovitch's varve data show a mean thickness $\bar{t}=1.2 \mathrm{~mm}$. Thicknesses $t \geq 80 \mathrm{~mm}$ can be considered extreme. There were only nine such events observed in 4189 years. In conformance with the proposed working hypothesis, all of these outstanding events occurred close to $V \max$ and $V$ Min in IOT. When waves running from $V_{\max }$ to $V_{\max }$ are normalized to 100 , all events $t \geq 80$

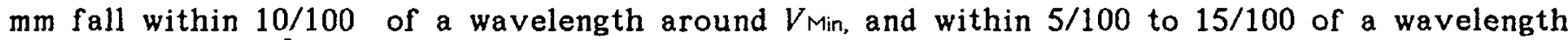
around $V_{\text {Max. }}$ A $\chi^{2}$-test yields the value 13.5 for 1 degree of freedom: $P<0.0003$.

\section{Rainfall and Temperature Connection with Impulses of the Torque}

Lake Saki varves offered an occasion of looking into a special relationship confined to local climate, but covering many centuries. The following connection of IOT with climate covers merely 13 decades, but comprises different areas of the Northern Hemisphere. The IOT features, presented hitherto, constitute the most prominent phenomena related to major maxima and minima. The variance presented in Figures 2 and 3 was subjected to a Gaussian low pass filter eliminating all patterns emerging within a range shorter than 30 years. The unsmoothed data reveal intermittent maxima and minima in the 9-yr running variance $v$ of IOT. Major as well as intermittent minima in $v$ occur, when Jupiter, which contributes most to the sun's motion about the CM, the center of mass itself, and the sun's center (CS) are in line (JU-CM-CS events). Maxima in $v$, including intermittent maxima, develop near the middle between two consecutive JU-CM-CS events. Henceforth, all minima in the 9-yr running variance in IOT are termed $v_{M i n}$, and all corresponding maxima vmax. These extrema seem to be connected to rainfall over Central Europe. Figure 4 shows the smoothed 2-yr running variance $v_{r}$ of yearly rainfall totals $(\mathrm{mm})$ derived from observations of 14 German stations by Baur. ${ }^{18}$ This homogeneous time series, supplemented by data from 'Berliner Wetterkarte', published by the Meteorological Institute of the Free University of Berlin, covers the period 1851-1983. The distribution is Gaussian and free from Markov type persistence. Daily or monthly data show some persistence patterns. These were eliminated, however, by the formation of yearly means. The 2-yr running variance, smoothed by a Gaussian low-pass filter, focusses attention on the change in variance from year to year. Peaks in the plot point to strong contrast in the rainfall of consecutive years - very wet years follow very dry years, or very dry years follow very wet years whereas minima indicate little contrast in this respect. Arrows in Figure 4 mark epochs of vmin, and open circles designate dates of vmax. The plot conveys the impression of a strong

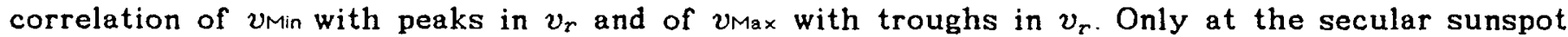
minimum around 1900 the vmax connection is weak or even disappears. Possibly, this is due to the lack of releasable magnetic energy stored in large sunspots. The first impression of a good correlation is confirmed by a series of statistical tests. The probabilities resulting from different approaches in $t$-tests range from $P<0.0002$ to $P<0.00003$. The null hypothsesis of no connection with IOT may be considered disproved. A replication was made by means of yearly rainfall averages in England and Wales for the years $1850-1976 .{ }^{19}$ The probability of false rejection of the null hypothesis of no correlation is low again: $P<0.004$. Another replication makes use of the yearly total rainfall $(\mathrm{mm})$ in the eastern United States, reduced to Philadelphia equivalent values, covering the period $1850-1967 .^{20}$ The outcome is $P<0.009$. Data from the equatorial region, too, were examined. Yearly monsoon season (June - September) rainfall (mm) at Bombay from 1850 to $1960^{21}$ showed a significant correlation $(P=0.014)$ when a phase shift by $\pi / 2$ radians was allowed for. This phase shift, emerging in low latitudes is no ephemeral feature. It was confirmed by the analysis of All-India summer monsoon rainfall (mm) from $1871-1978:^{22} \quad P=0.005$. Therefore, a two-phase system with relation to the geographical latitude seems to be a promising approach.

Temperature opens up a further field for replication. Baur established a time series of yearly temperature averages based on the respective means of the stations Utrecht-de Bilt, Potsdam, Basle, and Vienna. ${ }^{18}$ With extensions taken from the 'Berliner Wetterkarte', the series covers the period $1851-1983$. The higly significant result of a $t$-test yields $P<0.002$. Yearly mean 
temperatures in central England from $1851-1976^{23}$ confirm this result, though on a lower level of significance: $P<0.05$. As was to be expected at this latitude, there are no indications of a phase shift. With regard to temperature, peaks in the 2-yr running variance, connected with $v_{m i n}$, are characterized by alternately hot and cold years, whereas troughs, correlated to $v_{\text {max }}$, point to consecutive years with little contrast in temperature. As for details of the statistical tests, reported in this section, reference is made to the comprehensive paper 'Solar Rotation, Impulses of the Torque in the Sun's Motion, and Climatic Variation. ${ }^{24}$

The first results of this interdisciplinary approach certainly are in need of further corroboration by replications based on new data that cover long time series and various geographical regions. With regard to possible phase shifts, it seems not advisable to investigate global data. An analysis of annual average temperatures of the Northern Hemisphere yielded no significant results. This may be due to phase differences in different geographical latitudes that cancel out. If appropriate replications confirmed the studied connection, this could be of practical importance as, e.g., the probability of a wet year following another wet year or of a drought year following another drought year would then be much higher at $v_{\text {Max }}$ epochs than around vmin. These prospects should be intriguing enough to induce climatologists and meteorologists to engage in further investigations. Hopefully, this could lead, besides longrange predictions of energetic $X$-ray flares and outstanding geomagnetic activity practised already, to progress in forecasting climatic variability.

\section{Acknowledgements}

This work has been supported by the Foundation for the Study of Cycles, Irvine, California.

\section{References}

1. Labitzke, K., 'Sunspots, the QBO, and the Stratospheric Temperature in the North Polar Region.' Geophys. Res. Letters, 14, 535 -537 (1987).

2. Geller, M.A., 'Variations without Forcing.' Nature, 342, 15-16 (1989).

3. Landscheidt, T., 'Swinging Sun, 79-Year Cycle, and Climatic Change.' J. interdiscipl. Cycle Res., 12, 3-19 (1981).

Landscheidt, T., 'Solar Oscillations, Sunspot Cycles, and Climatic Change.' Weather and Climate Responses to Solar Variations, McCormac, B.M., ed., pp. 293-308. Colorado Associated University Press, Boulder (1983).

Landscheidt, T., 'Long-Range Forecasts of Solar Cycles and Climate Change.' Climate History, Periodicity, and Predictability, Rampino, M.R., Sanders, W.S., Newman, W.S. and Königsson, eds., pp. $421-445$. Van Nostrand Reinhold, New York (1987).

4. Landscheidt, T., 'Long-Range Forecast of Energetic X-Ray Bursts Based on Cycles of Flares.' Solar-Terrestrial Predictions: Proceedings of a Workshop at Meudon, France, June 18-22, 1984, pp. 81 -89. National Oceanic and Atmospheric Administration, Boulder.

5. Landscheidt, T., 'Solar Oscillations, Sunspot Cycles, and Climatic Change,' Op. cit. sup., p. 304 (1983). See also Faure, H. and Gac, J.Y., Will the Sahelian Drought End in 1985?' Nature, 268, 475-478 (1981).

6. Foukal, P.V. and Lean, J., 'Magnetic Modulation of Solar Luminosity by Photospheric Activity.' Ap. J., 328, 347-357 (1988). 
7. Kuhn, J.R., Libbrecht, K.G. and Dicke, R.H., 'The Surface Temperature of the Sun and Changes in the Solar Constant.' Science 242, 908-911 (1988).

8. Foukal, P.V., 'The Variable Sun.' Scient. American, 34-41, February 1990.

9. Landscheidt, T., 'Beziehungen zwischen der Sonnenaktivität und dem Massenzentrum des Sonnensystems.' Nachr. Olbers-Gesellschaft, No. 100, 3-19 (1976).

10. Gleissberg, W., 'The 80-Year Sunspot Cycle.' J. Brit. Astron. Ass., 68, 150 (1958).

11. Link, F., 'Solar Cycles between 1540 and 1700.' Solar Physics, 59, 175-178 (1978).

12. Jose, P.D., 'Sun's Motion and Sunspots.' Astron. J., 70, 193-200 (1965).

13. Fairbridge, R.W. and Sanders, J.E., 'The Sun's Orbit, A.D. 750-2050: Basis for New Perspectives on Planetary Dynamics and Earth-Moon Linkage.' Climate-History, Periodicity, and Predictability, Op. cit. sup., pp. 446-471.

Fairbridge, R.W. and Shirley, J.H., 'Prolonged Minima and the 179-Year Cycle of the Solar Inertial Motion.' Solar Physics, 110, $191-220$ (1987)

14. Dansgaard, W., Johnsen, S.J., Moller, J. and Langway, C.C., 'One Thousand Centuries of Climate Record from Camp Century on the Greenland Ice Sheet.' Science 166, 377-381 (1969).

15. Dewey, E.R., 'The 17 1/3-Year Cycle in Lake Saki Varves.' Research Bulletin 1964-1, $1-66$, Foundation for the Study of Cycles, Pittsburg (1964).

16. Shostakovich, W.B., 'Bodenablagerungen der Seen und periodische Schwankungen der Naturerscheinungen.' Memoires de l'Institut Hydrologique, Leningrad, 13, 95-140 (1934).

17. Shostakovich, W.B., 'Geschichtete Bodenablagerungen der Seen als Klima.' Ann. Met. Z., $53,176-182(1936)$.

18. Baur, F., 'Abweichungen der Monatsmittel der Temperatur Mitteleuropas und des Niederschlags in Deutschland, Beilage zur Wetterkarte des Institus für Meteorologie der Freien Universität Berlin vom 24. 6. 1975 (1975).

19. Lamb, H.H., Climate Present, Past, and Future, Vol. 2, 'Climatic History and the Future,' pp. 621-625. Methuen, London (1977).

20. Lamb, H.H., Op. cit. sup., pp. $625-628$.

21. Lamb, H.H., Op. cit. sup., p. 631 .

22. Mooley, D.A. and Parthasarathy, B., 'Fluctuations in All-India Summer Monsoon Rainfall 1871-1978.' Climatic Change, 6, $287-301$ (1984).

23. Manley, G., 'Central England Temperatures: Monthly Means 1659 to 1973.' Quart. J. Roy. Met. Soc., 100, 389-405 (1974).

Lamb, H.H.,, Op. cit. sup., pp. 574-576.

24. Landscheidt, T., 'Solar Rotation, Impulses of the Torque in the Sun's Motion, and Climatic Change.' Climatic Change, 12, 265-295, (1988). 


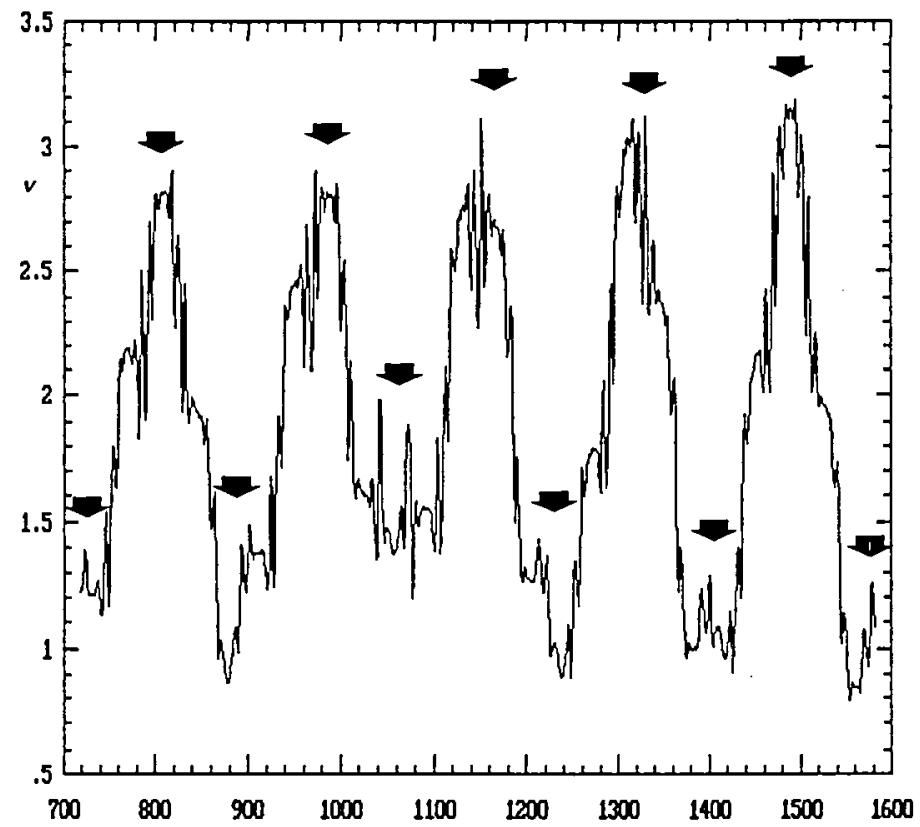

Fig. 1. Correlation between the 3E-yr running variance in the sun's orbital angular momentum ( $v)$ and the 80-yr sunspot cycle A.D. $700-1600$. Extrema in the variance $v$ coincide with minuma in the secular cycle of sunspot activity, the epochs of which are indicated by arrows.

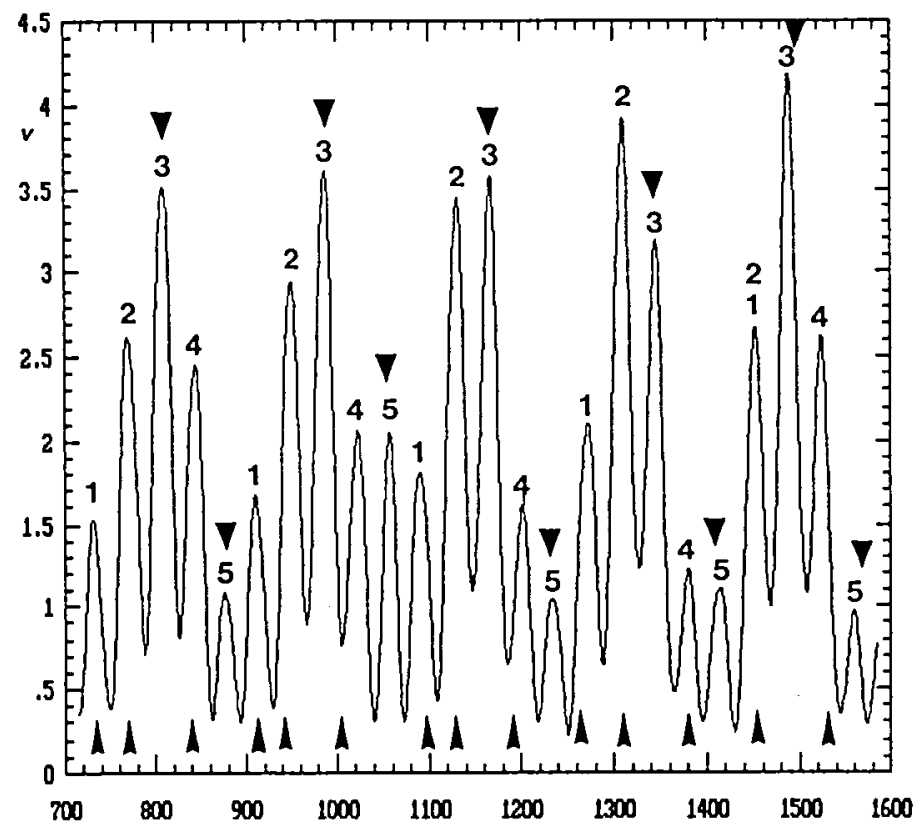

Fig. 2. Smoothed 9-yr running variance in the angular momentum of the sun's motion about the center of mass of the solar system (v) A.D. $700-1600$. The cyclic pattern, formed by the curve, conveys the impression of pentadactyl hands. Fingers are numbered. Fingers 3 and 5 point to minima in the $80-y r$ sungpot cycle, the epochs of which are marked by triangles. Fingers 1, 2, and 4 indicate secular maxima; respective epochs of intense solar activity are indicated by arrows. Perturbation of the penta-rhytm seems to induce instability. The rightmost hand, lacking a finger, coincides with the Spörer minimum. 


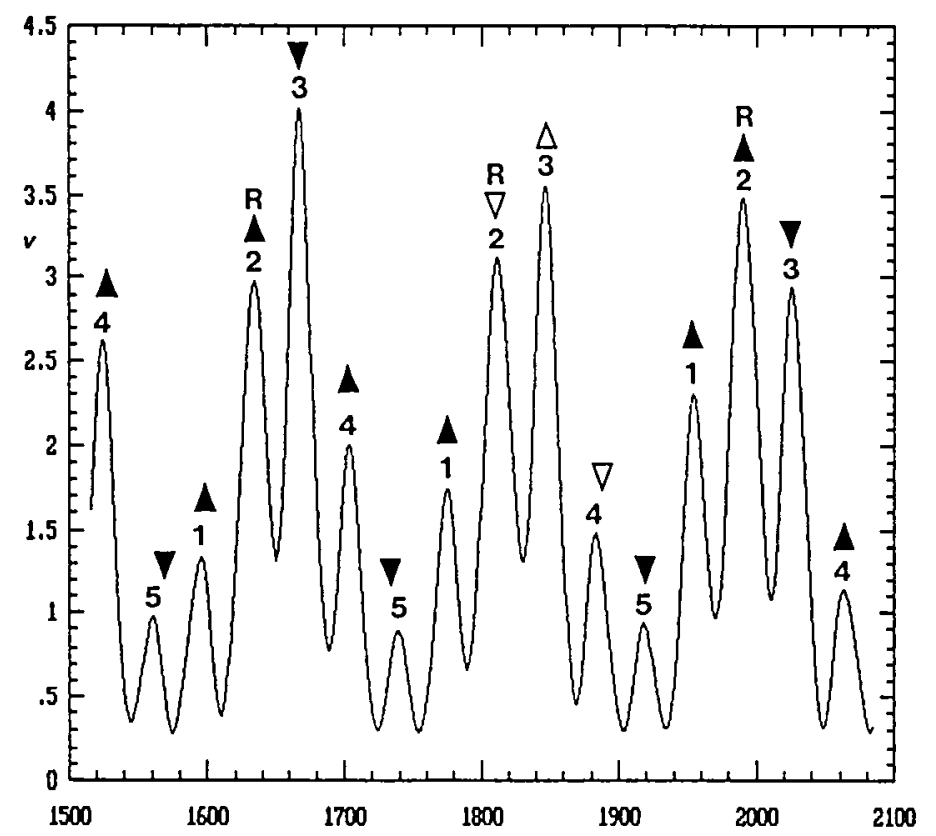

Fig. 3. Pentadactyl pattern, as presented in Fig. 2, covering the period $1500-2100$. Triangles pointing upwards and downwards mark epochs of maxima and minima in the 80-yr sunspot cycle. Perturbing phases of retrograde solar motion, designated by $R$, seem to be linked to instability. After 1811, the extrema in the correlated secular sunspot cycle switched phase. Open triangles focus attention on this anomaly, the only event in 1300 years of data looked into. Retrograde motion in 1632 and 1990 did not perturb the regular rhythm. Period 3 after 1632, however, concurred with the Maunder minimum. Period 3 after 1990. peaking in 2026, could have similar implications.

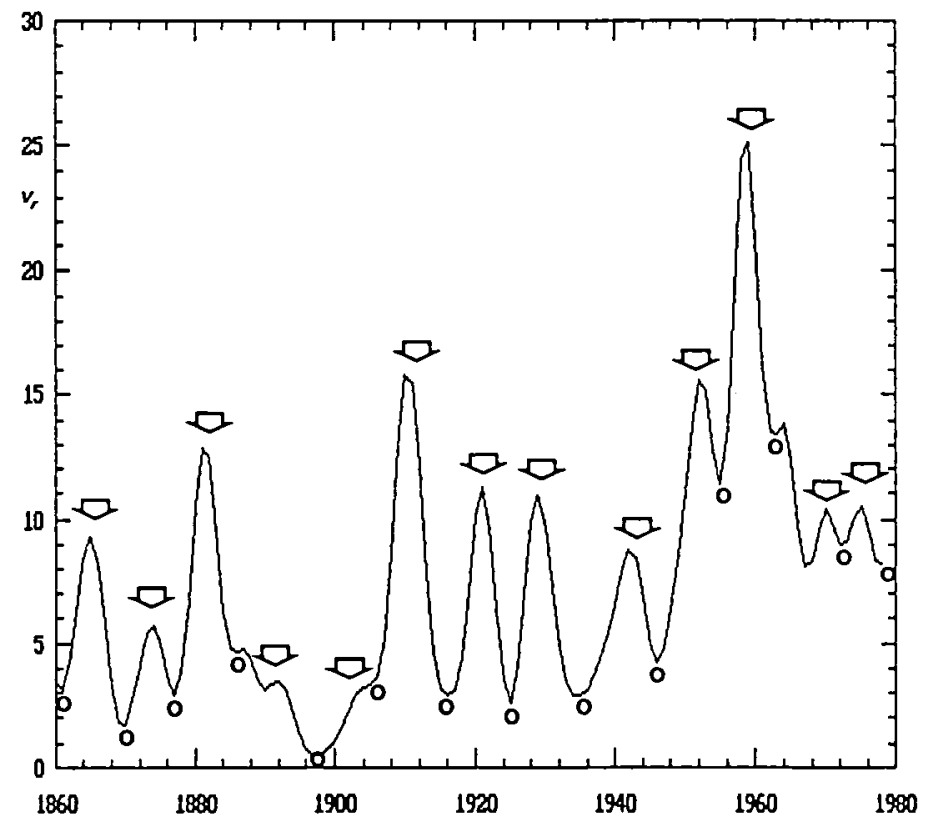

Fig. 4. Smoothed 2-yr running variance $v_{r}$ of yearly rainfall totals $(\mathrm{mm})$ derived from obseryations of 14 German stations by F. Baur. The data cover the period $1851-1983$. Arrows mark epochs of minima in the $9-y r$ running variance $v$ of the sun's orbital angular momentum. Open circles designate respective maxima in $v$. The significant correlation between these solar and terrestrial data is corroborated by rainfall observations from England, Wales, eastern U.S.A., and India. 
SESSION 8: SOLAR IRRADIANCE VARIATIONS I 



\title{
Irradiance Variability of the Sun
}

\author{
Claus Fröhlich \\ Physikalisch-Meteorologisches Observatorium Davos, World Radiation Center, CH-7260 Davos Dorf, \\ Switzerland
}

\begin{abstract}
Direct measurements of the solar constant - the total irradiance at mean Sun-Earth distance - during the last ten years from satellite show variations over time scales from minutes to years and decades. At high frequencies the spectral power is determined by granulation, super- and mesogranulation. In the 5-minute range, moreover, it is dominated by power from the solas p-mode oscillations. Their power and frequencies change with time yielding information about changes in the convection zone. Towards periods of several hours the power is steadily increasing and may be partly due to solar gravity modes. The most important variance is in the range from days to several months and is related to the photospheric features of solar activity: decreasing the irradiance during the appearance of sunspots, and increasing it by faculae and the magnetic network. Long-term modulation by the 11-year activity cycle are observed conclusively with the irradiance being higher during solar maximum. All these variations can be explained - at least qualitatively - by their manifestation on the photosphere. For the long-term changes the simultaneous changes of the frequencies of solar p-mode oscillations suggest a more global origin of the variations. Indeed, it seems that the observed irradiance modulation is a true luminosity change with the magnetic cycle of the Sun.
\end{abstract}

\section{Introduction}

The irradiance from the Sun at the mean Sun-Earth distance, integrated over the energetically important wavelength range (hence total irradiance) is called "solar constant". Observations of the solar constant have a long history, starting with the measurements by the Smithsonian Institution from mountain stations at the turn of the century and including data from the beginning of space era. Many of the early measurements were inconclusive, mainly because of lack of sufficient radiometric precision, but also due to influences of the Earth's atmosphere for observations from ground and airplanes (for reviews see e.g. Fröhlich 1977, Hoyt 1979, Angione 1981, Fröhlich 1987). The first clear evidence of solar constant variability only appeared at the beginning of the 1980's with the data from radiometers on the Solar Maximum Mission (SMM) and on NIMBUS-7, proving that the Sun is indeed a "variable" star. The interest in total solar irradiance variability on all time scales has then very much increased and the modern data on total solar irradiance reveal a broad spectrum of variations (e.g. Hudson, 1988). Not only are the atmospheric physicists and climatologists concerned, because of possible effects on the Earth's energy balance, but also the solar physicists have become interested: the existence of global changes of the solar output had been doubted for a long time and their observation obviously leads to new ways to understand the Sun.

The measurements from satellites discussed in this paper have been performed by the sensors of the Earth Radiation Budget Experiment (ERB) of the NIMBUS-7 satellite in a near-polar orbit since November 16, 1978 (Hickey et al. 1988 and 1989) and by an active cavity radiometer on the Solar Maximum Mission Satellite in a $27^{\circ}$ orbit since February 14, 1980 (Willson 1984). Reference is also made to the data from the ERBE experiment on NASA ERBS in a $57^{\circ}$ orbit and the NOAA9 and 10 satellites in near-polar orbits starting in October 1984, January 1985 and October 1986 respectively (Mecherikunnel et al., 1988). In all 
these experiments the solar irradiance is measured by electrically calibrated cavity radiometers. The time series of the three experiments are plotted in Fig.1 and illustrate the solar irradiance variability on all time scales. The difference in value between the three experiment is due to their absolute calibration which is accurate to "only" about $\pm 0.2 \%$ and does not reflect the precision and stability of the instruments which is obviously much better.

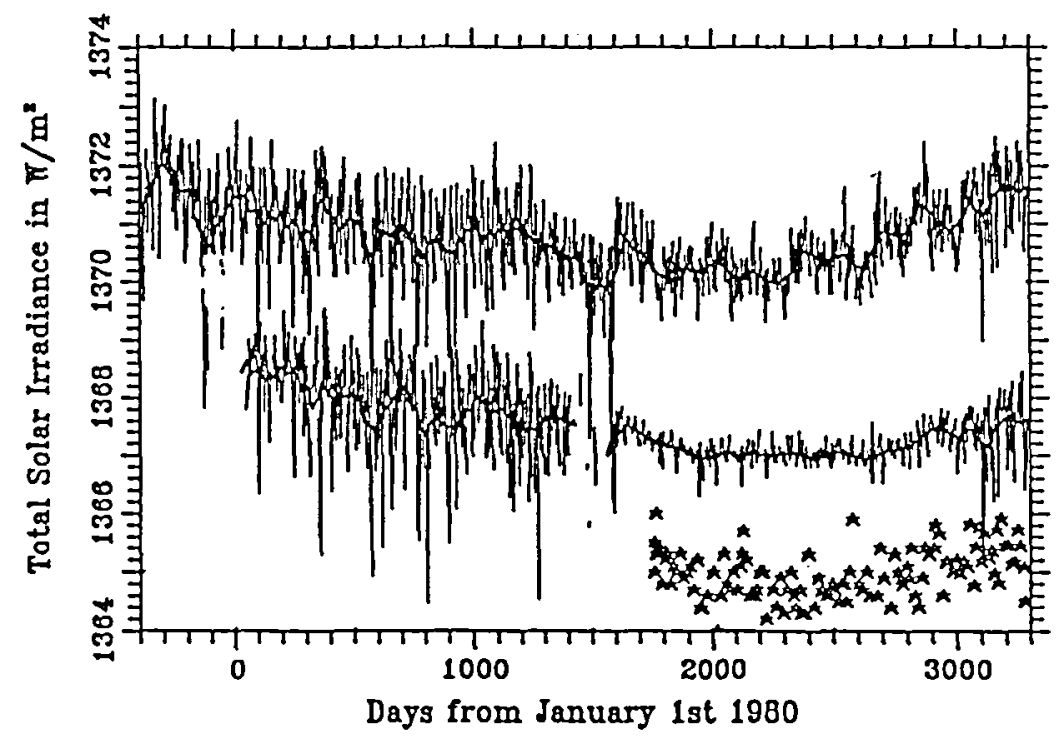

Fig. 1. Time series of ERB (top), ACRIM (middle) and ERBE (bottom) total solar irradiance measurements. For ERB and ACRIM the individual points represent daily mean values, the heavy lines 81 -day running means. The ERBE data points are individual measurements taken at about a bi-weekly rate. (from Fröhlich et al. 1990b)

Furthermore data from the IPHIR investigation (Fröhlich et al. 1990a) on the USSR mission PHOBOS to Mars and its satellite Phobos are used. With this experiment the solar spectral irradiance in three wavelength bands centered at 335,500 and $865 \mathrm{~nm}$ was observed continuously during more than 5 months between July and December 1988 before the spacecraft was injected into the orbit around Mars.

\section{Variations on Time Scales up to Months}

At high frequencies the most important features of the variance are the solar p-mode oscillations manifested as brightness fluctuations (e.g. Woodard and Hudson 1983, Fröhlich et al. 1990a, Toutain 1990). These oscillations are eigen-modes of the Sun and the restoring force is pressure, hence p-modes which are classified by degree $l$, order $n$ and tesseral order $m$. These eigen-modes yield a equi-distant discrete spectrum due to the well defined resonant cavity. The values of the mode frequencies $\nu_{l, n, m}$ have important diagnostic power and are used in helioseimology, which is applying seismic techniques for sounding the interior of the Sun, as it is done with waves from earthquakes on Earth. An example of the p-mode spectrum from the IPHIR investigation is shown in Fig.2. In the context of this paper the long-term changes of these low degree p-mode frequencies and the variations of their amplitudes are important. The former will be discussed together with the solar cycle variations where possibly changes in the upper boundary of the resonant cavity are changed, whereas the latter is important for this section as these changes reveal information about short to medium term global changes in the upper layers of the convection zone. These may influence the luminosity and give a clue to the underlying physical mechanisms.

The solar variance is best illustrated by the power spectral density as shown for the SMM/ACRIM data in Fig.3 for 1980 and '85. It covers the range from about $50 \mathrm{nHz}$ to $86 \mu \mathrm{Hz}$, the Nyquist frequency 


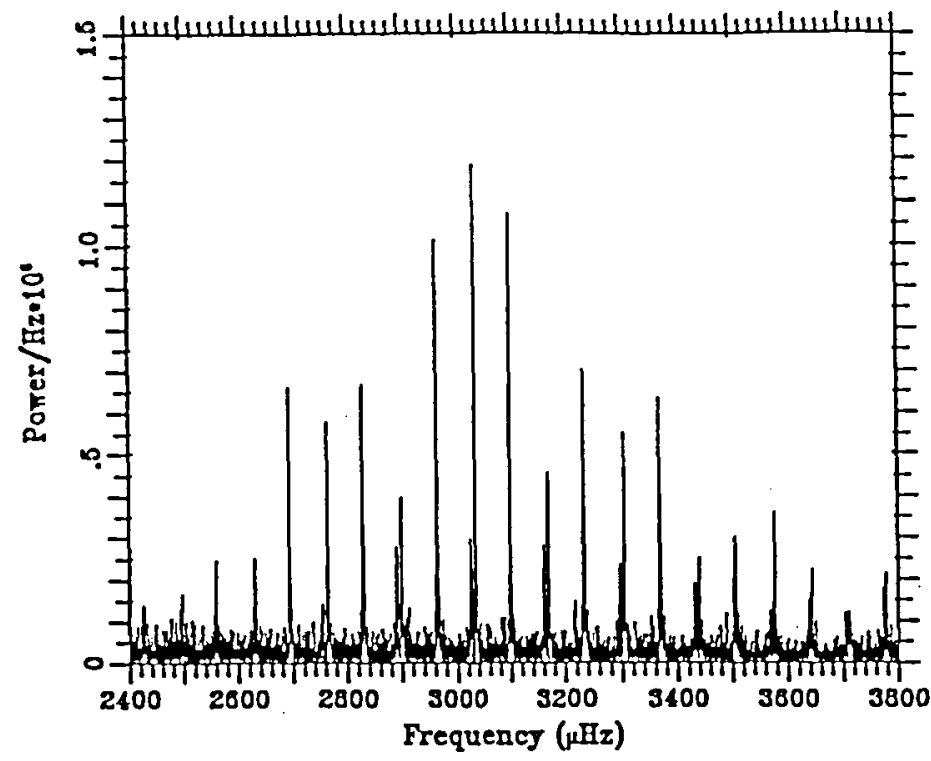

Fig. 2. Spectrum of p-mode oscillations with $l=0 \ldots 2$ and $n=17 \ldots 27$ from the IPHIR investigation (Frölich and Toutain 1990). The highest peak corresponds to an amplitude of a few parts per million in brightness. Thus, solar oscillations are only a very small contribution to the total variance.

corresponding to the orbital period of SMM. At low frequencies the strength of solar activity changes the spectral density by up to a factor of ten (1980 compared to 1985) whereas the density at higher frequencies seems independent of solar activity. In this frequency range the power is mainly due to granulation, superand mesogranulation, which is illustrated by the result of an analysis of simulated time series (Andersen 1990). The simulations are based on a compilation of direct observations of such features on the solar disk both in terms of contrast and behavior with time. In the range above $10 \mu \mathrm{Hz}$ (11.57 $\mu \mathrm{Hz}$ corresponds to a period of 1 day) the behavior of the ACRIM spectrum is very similar to the simulated one; the level of power is, however, three to five times higher. At lower frequencies the difference becomes quite obvious; in this range it is due to the fact that the simulations do not include any effects from solar activity. The difference at high frequencies may be an indication of power from solar gravity oscillations. Searches for these modes have been performed, but the results are not yet conclusive (Kotov et al. 1984, Fröhlich and Delache 1984, Henning et al. 1988, Kroll et al. 1988, Fröhlich 1990, van der Raay 1990). Their detection and identification, however, would be very important for constraining the structure and composition of the solar core.

In the frequency range above $10 \mu \mathrm{Hz}$ the power is mainly due to activity related features on the Sun. The time scales associated with solar magnetic active regions reveal extremely interesting variations of the total solar irradiance. Here we will discuss time scales of a few solar rotations (about $0.1 \mu \mathrm{Hz}$ ) down to two days (the $5.8 \mu \mathrm{Hz}$ Nyquist frequency of daily sampling). The dominant feature in the time series for this range of periods is the "dip", a negative excursion of a few days' length and a depth ranging up to a few tenths of a percent of the normal total irradiance. These dips result from large sunspot groups rotating past the central meridian. Between the dips the total irradiance fluctuates on a wide range of time scales, as demonstrated by the broad-band character of the power spectrum. Prominent dips appeared during the first few months of data from the ACRIM instrument on board SMM, and Willson et al. (1981) described them in terms of the Projected Sunspot Index, the PSI function similar to the models of sunspot darkness noted earlier by Foukal and Vernazza (1979) and Hoyt and Eddy (1982). Hudson and Willson (1982) give the definition of this projected-area inder as follows: 

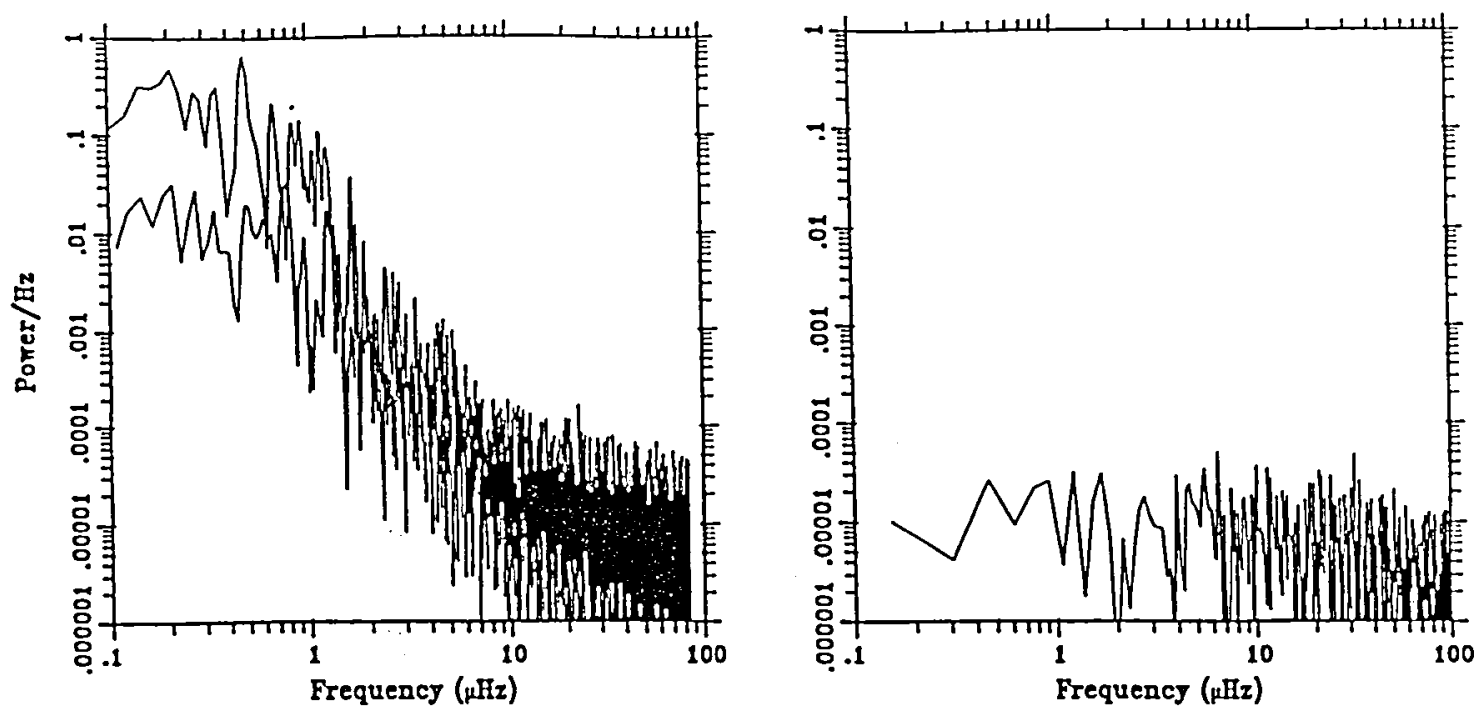

Fig. 3. Powerspectrum of the solar irradiance for 1980 and 1985 observed by SMM/ACRIM (left, Fröhlich et al. 1990b) and of the simulated irradiance at $500 \mathrm{~nm}$ (right, Andersen 1990) due granulation, super- and mesogranulation.

$$
P S I=\alpha \cdot \sum_{i} \frac{3 \mu_{i}+2}{2} \cdot \mu_{i} \cdot a_{i}
$$

where $\mu_{i}$ is the central angle of the sunspot group $i$ and $a_{i}$ its area. The factor $\alpha$ takes into account the umbra/penumbra area ratio and the effective temperature of the sunspot photospheres, in the simplest possible way. This index, calculated from the synoptic data for sunspot areas and with textbook calculation of the value of $\alpha$ (i.e., no adjustment of parameters whatsoever), removed about half of the variance of the total ACRIM time series in the active year 1980. This success was quite surprising in view of the grossly simplifying assumptions involved in the construction of PSI. For the period 1980 to 1988 the PSI function is shown in Fig.4 together with the irradiance data from ACRIM and ERB corrected for spots by this function $(S+P S I)$. The success of the simple $P S I$ explanation of sunspot effects immediately implied that a "bright ring" surrounding the sunspot did not directly re-radiate the blocked convective flux presumed to be welling up continuously through the solar interior. The attempts to improve generally on the naive fit of the PSI have generally failed, owing prehaps to the additional noise in the correlation introduced by random and systematic errors in the synoptic data (Schatten et al. 1985).

Willson (1982) called attention to the effects of faculae in modulating the irradiance and having the potential to compensate the sunspot deficit. More direct photometric approaches have yielded some insight into the relationship between the irradiance excesses due to faculae of an active region and the sunspot deficits. Given the almost simultaneous existence of dark spots and bright plage in active regions, it immediately became interesting to ask if the energy excess and deficit actually balanced. If so, this would imply some mechanism for local storage via the sub-photospheric magnetic field structure. Indeed, at the simplest level both the Ca proxy measure of facular brightness (Hirayama et al. 1984, Chapman et al. 1986) and direct facular photometry (Chapman 1984, Lawrence et al. 1985) show a rough balance (see Chapman, 1987, for a complete review).

Multi-variate spectral analysis is a powerful tool for the investigation of multiple influences on a time series, as for example the quasi-independent spot and facular contributions discussed above. Fröhlich and Pap (1989) have applied this technique to the ACRIM data, both in 1980 and after the repair mission in 1984/85. The results are shown in Fig.5. They find for the former period that more than 90 percent of the variance of the ACRIM data can be explained by the effects of sunspots and magnetic elements, the latter including both active-region and network facular elements (represented by the equivalent width of the $\mathrm{He}$ 


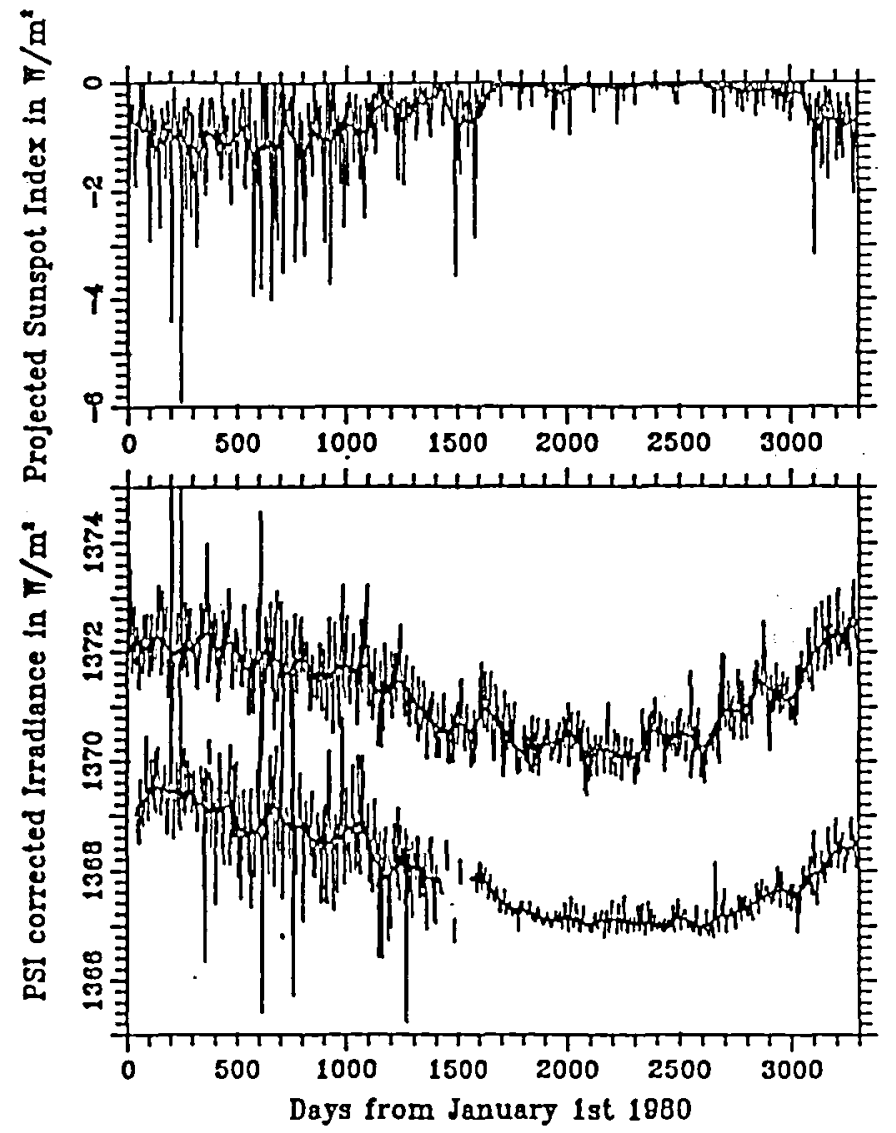

Fig. 4. The influence of sunspots on the irradiance during 1980-88. Top panel: the downward plotted PSI function and bottom panel: the PSI corrected irradiance $(S+P S I)$ of ACRIM and ERB with the solid lines indicating a 81 day running mean (from Fröhlich et al. 1990b).

$1083 \mathrm{~nm}$ line, the HeI index). The analysis reveals the presence of power spectral peaks not explained by spots, faculae or magnetic network near 27 and 9 days, the latter suggestive of heretofore unknown processes within the convection zone.

The long stretch of the IPHIR data allows for the first time to study in detail the time variation of the amplitudes of the p-mode oscillations (Toutain 1990, Fröhlich and Toutain 1990). In Fig.6 the total power as sum of the individual power of 18 lines with $l=0 \ldots 2$ and $n=18 \ldots 23$ are shown as time series and estimates of the spectral power density. Most of the variation comes possibly from stochastic excitation and damping of the modes by convection. It is, however, interesting to note that the power is varying by nearly a factor three during the 160 days observations, although it is the sum of 18 individually excited and damped modes. Moreover, the estimated life time of the modes is of the order of a few days and their are periods of low or high power lasting for $\mathbf{2 0}$ to $\mathbf{3 0}$ days. Some of the variation could also be due to modulation by the irradiance itself, but the comparison with the irradiance variations shows in the time series no apparent correlation. For some periods a positive and for others a negative correlation may be observed. In the frequency domain the correlation is also not obvious. Between 0.5 and $1 \mu \mathrm{Hz}$ similar features are observed in both spectra; the significance of this similarity, however, is not clear. It is interesting to note that the broad peak observed around 9 days as residual in 1980 (Fig.5) is also present in this spectrum with the same width (1.05-1.35 $\mu \mathrm{Hz})$. The peak at $0.4 \mu \mathrm{Hz}$ (25-33 day period) may be related to solar rotation, again it could be of the same origin as the unexplained 27-day peak in the 1980 and 1985 spectrum. The origin of the strong peak 

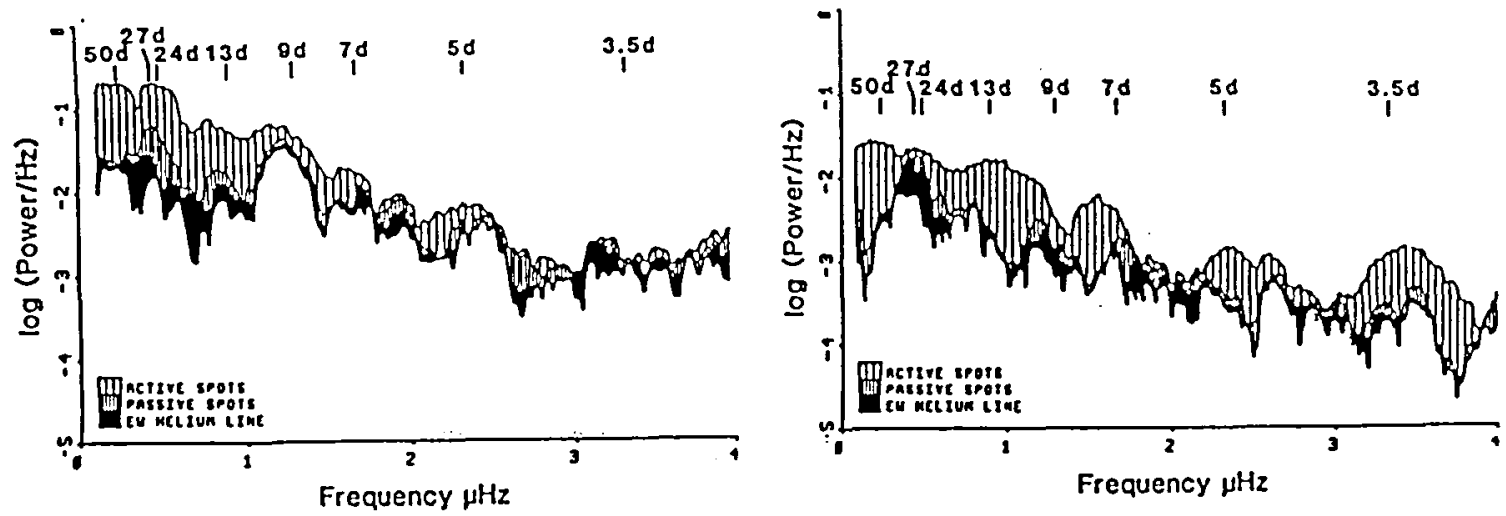

Fig. 5. For 1980 (left) and 1984/85 (right) the power spectra of the SMM/ACRIM data and the results of the multispectral analysis are shown: the;shaded areas indicate the part of the irradiance power explained by the power of active and passive spot areas (similar to PSI, which is the sum of both) and of the equivalent width of the $1083 \mathrm{~nm}$ HeI line (from Fröhlich and Pap 1989).

around $0.2 \mu \mathrm{Hz}$ (50-96 day period) is not obvious, but again it is at the same place and has the same width as the lowest unexplained peak in the 1980 spectrum of Fig.5. All three coincidences may be fortuitous, but it is highly suggestive that they may have the same origin which is related to the activity cycle. 1980 was right after the maximum of the cycle 21 and 1988 is close to the end of the ascending phase of cycle 22; a possible explanation of the better correlation with the 1980 data.

\section{Variations on Time Scales of Months to Years}

After smoothing the time series of the ERB and ACRIM radiometry (solid lines in Fig.1 and 4) both data sets show common variations on time scales of 4-9 months, as well as a general slow downturn, which has reversed during 1986, after passage of the solar minimum.

Several ideas and models have been put forward to explain these variations. One approach is to account for the variations purely in terms of the effects that magnetic flux tubes seem to have on the radiation and convection in the relatively shallow photospheric layers that emit most of the Sun's luminosity. Others have invoked deep seated changes in solar convection, perhaps involving even variations in the nuclear-burning core (e.g. Gough, 1989).

A relatively straightforward approach to both the 4-9 month and 11-year variations has been put forward by Foukal and Lean (1988) and Livingston et al. (1988). In these studies, it was shown that the residual irradiance variations remaining after a correction for sunspot blocking is made to the smoothed ACRIM or ERB data correlate well with indices of facular area such as provided by the $\mathrm{HeI}$ index or the $10.7 \mathrm{~cm}$ flux. This is not surprising since facular area variations were previously shown to account for day-to-day variations in these residuals (Hudson and Willson 1981, Foukal and Lean 1986).

The HeI index and the $10.7 \mathrm{~cm}$ flux represent contributions from all bright magnetic elements on the disc, including the network. Thus, one may conclude that the 6-9 month variations are caused by the tendency of major complexes of activity to persist for about this number of solar rotations (Gaizauskas et al. 1983). This time scale in persistence of solar activity episodes has been documented before in studies of the HeI index time series (Harvey 1984) and may be related to the persistence of certain active longitudes on the solar surface, noted already by Dodson and Hedeman (1970). No well-accepted explanation of this 6-9 month activity time scale (or of the active longitudes) exists as yet, although the recent ideas of Wolff (1984) in terms of Rossby waves and surface pattern of g-mode oscillations and their possible interference are interesting, 

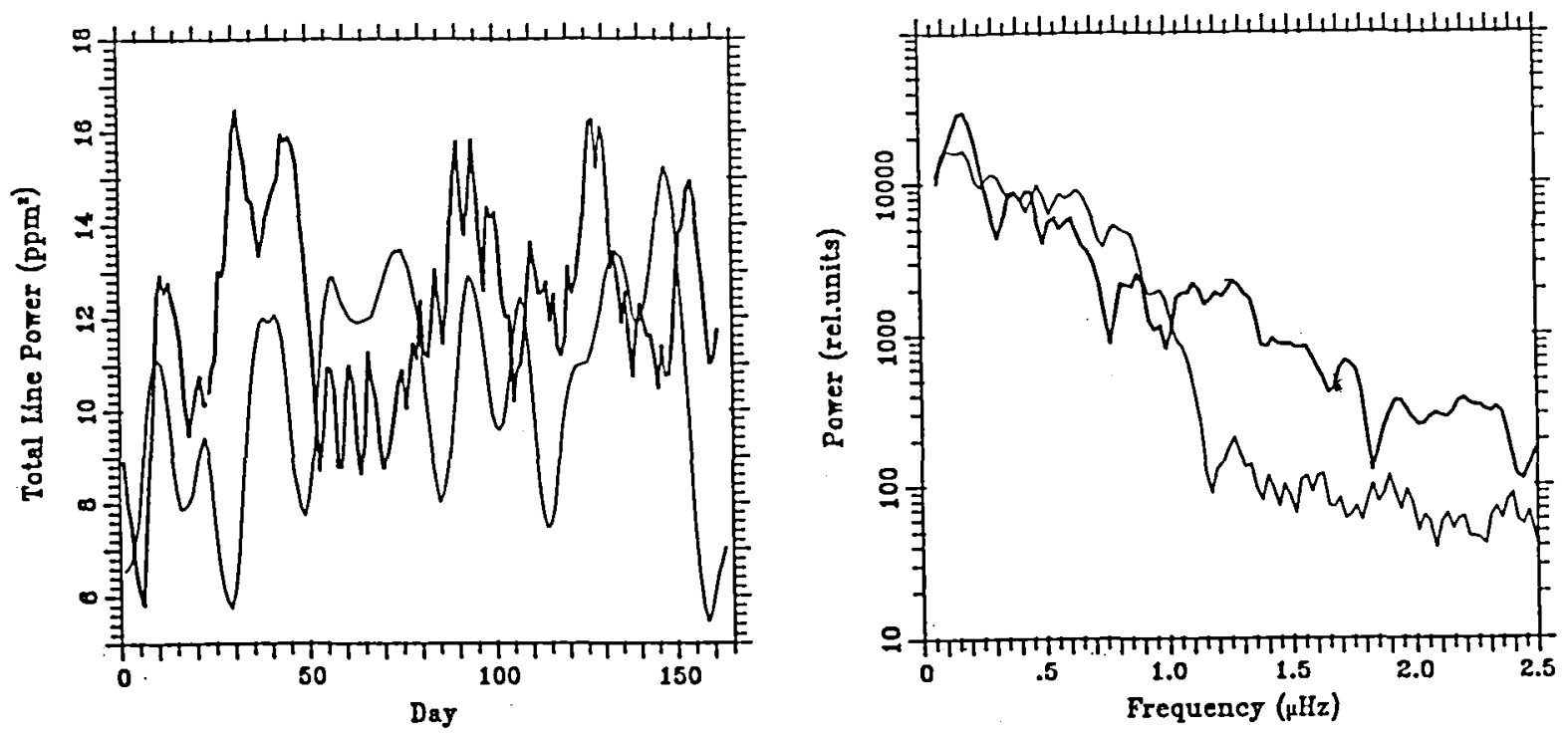

Fig. 6. Variation of total line power of solar p-modes as observed in brightness by the IPHIR investigation. Left: time series of total line power (heavy line) and of solar irradiance from SMM/ACRIM (thin line, the range from bottom to top is 1366.6 to $1368.8 \mathrm{Wm}^{-2}$ ). Both sets are running means over 8 days. Right: power spectral density of the total line power (heavy line) and the solar irradiance (thin line). The irradiance spectrum is shifted so that it is at the same level as the total line power at low frequencies.

and deserve more attention. Wolff and Hickey (1987) have also proposed that such oscillations may modulate heat flow to the photosphere and thus contribute to irradiance variation directly.

The finding that simulation of the downtrend between 1981-1986 requires use of a global index, as mentioned above, indicates that this downtrend is associated with some kind of a slow change in the solar atmosphere well modeled by e.g. the $\mathrm{HeI}$ index or the $10.7 \mathrm{~cm}$ flux. The simplest explanation is a slow decrease in the emission from the bright magnetic network outside active regions. This identification cannot be considered proven until the changes in area (and possibly also intensity) of the network are measured in white light over a solar cycle. Fig.7, lower panel, suggests that the whole solar cycle can possibly be modelled by these indices.

Kuhn et al. (1988) have reported observations of the limb brightness which can be used to explain the total irradiance variation of the solar cycle. The observations are broad-band, two-color photometric measurements of the brightness distribution in a narrow annulus 20 arcsec wide, just inside the solar limb. The solar limb flux observed as a function of latitude can be divided into a "facular" and "temperature" part based on the assumption that the "temperature" part is constant over the 4-month observing summer period and that the "facular" part shows up as intermittent bright regions. The component of excess brightness moves toward the equator between 1983 and 1985, and then reappears at relatively high latitudes again in 1987. The excess brightness responsible for $d T$ is due to features which are not resolved by this observation, and it could be due to the bright network in and outside the active regions. The decreasing contribution of $d T$ (including the facular component) can account for the total irradiance decrease between 1983 and 1987, and its increase in 1988 (Kuhn, 1989) as shown by Fig.7.

Only the zero order term of the polynomial expansion of the latitudinal variation of the $d T$ signal is responsible for the irradiance variation. In a separate paper Kuhn (1988) compared the expansion coefficients needed to explain the latitudinal variation of $d T$, which can be interpreted as an asphericity. This breaks the spherical symmetry and can be observed as splitting and frequency shifts of the p-mode oscillations. He developed a model in which the underlying physical effects are parametrized by a local effective sound speed where $\frac{d c^{2}}{c^{2}}$ is directly related to the surface $\frac{d T}{T}$ observed. He is able with his model to explain consistently 


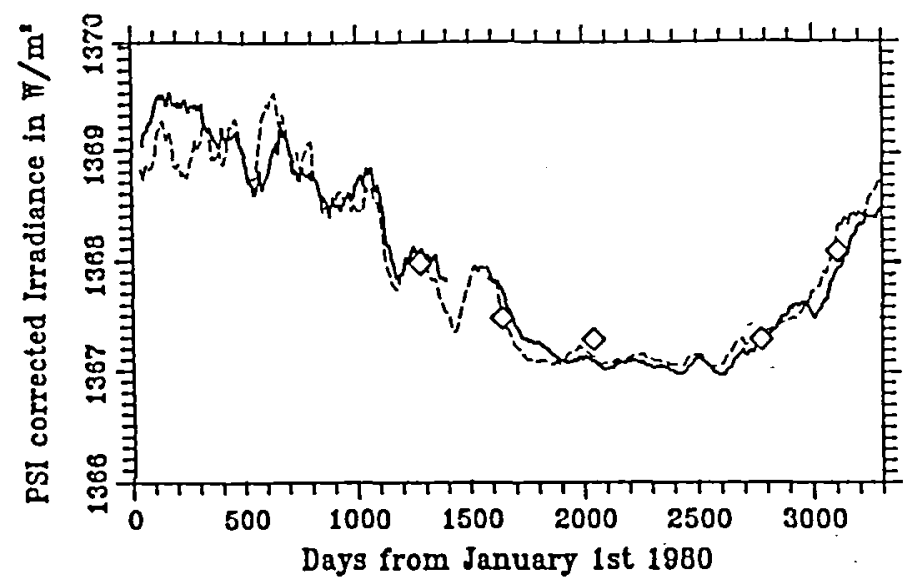

Fig. 7. Comparison of the 81-day running mean of the observed irradiance from ACRIM with two different models: The $P \dot{S} I$ corrected ACRIM irradiance $(S+P S I)$ is shown as broken line, the irradiance residual calculated from the $10.7 \mathrm{~cm}$ flux (the scaling is determined by linear regression of $S+P S I$ and the $10.7 \mathrm{~cm}$ flux) as solid line and the irradiance data deduced by Kuhn et al. (1989) from $d T$ and the simultaneously measured facular contribution as diamonds (from Fröhlich et al. 1990b).

the p-mode splitting coefficients from the corresponding coefficient of the $d T$. In a more recent paper Kuhn (1990) shows that the splitting data from the Big Bear Solar Observatory (Woodard 1990, Libbrecht 1990) are best fit by a shallow $\frac{d c^{2}}{c^{2}}$ perturbation, extending downward less than a few hundred kilometers below the photosphere. Also the observed centroid frequency shifts of the low order p-modes first reported by Woodard and Noyes (1985) fit well to this simple model as well as the frequency dependence of the $l=1$ mode changes (Palle 1990). These independent results strongly support the model that the observed $d T$ is due to a global effect manifested by an asphericity in $c^{2}$ and in $T_{e f f}$ in a shallow layer below the surface which varies with the solar cycle. This also suggests that it is the solar luminosity and not only the observed irradiance that evolves during the solar cycle. Moreover, these results also imply that the fractional radius variations are much smaller than the fractional luminosity changes; thus $\frac{\delta r / r}{\delta L / L} \lessgtr 0.03$ is very small.

How this explanation of the solar cycle modulation of the irradiance by observed $d T$ is physically linked to the explanation by a varying bright network as indicated by e.g. the $\mathrm{HeI}$ index is not yet clear, but we note that the two interpretations are not necessarily inconsistent with each other. A more detailed study of the amplitude variation of p-modes may shed some light on this question.

\section{Conclusions}

The past decade has seen the introduction of radiometers with sufficient precision and sampling to measure a variety of small variations of the total solar irradiance. These variations are interesting to solar physicists in several ways. In addition, the presence of a distinct 11-year modulation of total irradiance suggests that longer-term variations may be significant for the Earth's climate.

For most of the variations at least qualitative explanations are available; the details of the underlying physics, however, is not yet fully understood and more simultaneous studies of the detailed features on the solar disk together with continued solar constant observations are needed. A major result from the present data sets is that the solar cycle modulation of the irradiance is a true luminosity change of the Sun which can be modelled without assuming a simultaneous radius change of any significant magnitude. 
Acknowledgments: As part of this paper and some Figures are from a review recently prepared for the "Sun in Time", a book to be published by the Arizona University Press, Tucson (Frōhlich et al. 1990b), I would like to take the opportunity to thank the co-authors Peter Foukal, John Hickey, Hugh Hudson and Richard Willson for their contributions, yet unpublished data and many helpful discussions. Thanks are extended to Jeff Kuhn for many helpful discussions and for providing material before publication. Furthermore, the continuous support of this work at PMOD/WRC by the Swiss National Science Foundation is also acknowledged.

\section{References}

Andersen, B. (1990): "Simulation of time series for granulation, super- and mesogranulation", private communication Angione, R. (1981): "Review of Ground-Based Measurements" in Variations of the Solar Constant, ed. S.Sofia, NASA Conf.Publ. CP-2191, pp. 11-30

Chapman, G.A. (1984): "On the Energy Balance of Solar Active Regions" Nature 308, pp. 252-254

Chapman, G.A., Herrog, A.D. and Lawrence, J.K. (1986): "Time-Integrated Energy Budget of a Solar Activity Complex", Nature 310, Pp. 654-655

Chapman, G.A. (1987): "Variations of Solar Irradiance due to Magnetic Activity", Ann. Revs. Astron. Astrophys. 25, pp. 633-667

Dodson, H.W., Hedeman, E.R. (1970): "Time Variations in Solar Activity", in Solar Terrestrial Physics, Part I, eds. E.Dyer and C. deJager, (Reidel, Dortrecht) pp. 151-172

Foukal, P.V., Vernazza, J. (1979): "The Effect of Magnetic Fields on Solar Luminosity", Ap.J. 234, pp. 707-715

Foukal, P.V., Lean, J. (1986): "The Influence of Faculae on Total Solar Irradiance and Luminosity", Ap.J. 302, pp. 826-835

Foukal, P.V., Lean, J. (1988): "Magnetic Modulation of Solar Luminosity by Photospheric Activity", Ap.J. 328, pp. 347-357

Frohlich, C. (1977): "Contemporary Measures of the Solar Constant", in The Solar Output and its Variation, ed. O.R.White, Colorado Associated Univ. Press, pp. 93-109

Frōhlich, C. (1987): "Variability of the Solar "Constant" on Time Scales of Minutes to Years", J.Geophys.Res. 02, pp. 796-800

Fröhlich, C. (1990): "Search for g-Modes in the IPHIR Data", in Seismology of the Sun and Stars, ed. H.Sibahashi (Springer, Heidelberg), in press

Frōblich, C., Delache, Ph. (1984): "Solar gravity modes from ACRIM/SMM irradiance data", in Solar Seismology from Space, ed. by R.K. Ulrich, J.Harvey, E.J. Rhodes and J.Toomre, (JPL Publ.84-84, Pasadena), pp. 183-194

Fröhlich, C., Pap, J. (1989): "Multi-Spectral Analysis of Total Solar Irradiance Variations", Astron. Astrophys. 220, pp. 272-280

Fröhlich, C., Toutain, T., Bonnet, R.M., Bruns, A.V., Delaboudinière, J.P., Domingo, V., Kotov, V.A., Kollath, Z., Rashkorsky, D.N., Vial, J.C., Wehrli, Ch. (1990a): "Recent Helioseismological Results from Space: Solar pmode Oscillations from IPHIR on the PHOBOS Mission" in Inside the Sun, ed. by G.Berthomieu and M.Cribier (Klewer, Dordrecht), pp. 279-304

Fröhlich, C., Toutain, Th. (1990): "P-Mode Analysis of the IPHIR Data", in Progress in Seismology of the Sun and Stars, ed. H.Sibahashi (Springer, Heidelberg), in press

Fröhlich, C., Foukal, P.V., Hickey, J.R., Hudson, H.S., Willson, R.C. (1990b): "Solar Irradiance Variability from Modern Measurements", in Sun in Time, (Univ.of Arizona Press, Tucson), in press

Gairauskas, V., Harvey,K.L., Harvey, J.W. and Zwaan, C. (1983): "Large-Scale Patterns formed by Solar Active Regions During the Ascending Phase of Cycle 21", Ap.J. 205, pp. 1056-1062

Gough, D. (1989): "Deep Roots of Solar Cjcles", Nature \$30, pp. 618-610

Harrer, J. (1984): "Helium 10830 A Irradiance: 1975-1983", in Solar Irradiance Variations on Active Region Time Scales, eds. B.J.LaBonte, G.A.Chapman, H.S.Hudson, R.C.Willson, NASA Conf. Publ. CP-2310, Pp. $197-212$

Heaning, H.M., Scherrer, P. (1988): "The Search for solar gravity modes" in Seismology of the Sun and Sun-like Stars, ESA SP-286, (ESA, Noordwijk), pp. 419-423

Hickey, J.R., Alton, B.M., Kyle, H.L., Major, E.R. (1988): "Observation of Total Solar Irradiance Variability from Nimbus Satellites", Adv.Space Res. 8( 7$)$, pp. 5-10

Hickey, J.R., Alton, B.M., Kyle, H.L., Hoyt, D.V. (1989): “Total Solar Irradiance Measurements by ERB/NIMBUS 7. A Review of nine Years", Space Sci. Rer. 48, pp. 321-342. 
Hirayama, T., Okamoto, T., Hudson, H.S. (1984): "Facular Limb Darkening Function for Irradiance Modelling", in Solar Irradiance Variations on Active Region Time Scales, eds. B.J.LaBonte, G.A.Chapman, H.S.Hudson, R.C.Willson, NASA Conf. Publ. CP-2310, pp. 59-72

Hoyt, D.V. (1979): "The Smithsonian Astrophysical Observatory Solar Constant Program", Rev. Geophys. Space Res. 17, pp. 427-458

Hoyt, D.V., Eddy, J.A. (1982): "An Atlas of Variations in the Solar Constant Caused by Sunspot Blocking and Facular Emissions from 1874 to 1981", NCAR Techn.Note, NCAR/TN194+STR, (NCAR, Boulder Co. U.S.A.)

Hudson, H.S. (1988): "Observed Variability of the Solar Luminosity", Ann. Revs. Astron. Astrophys. 20, pp. 473-508.

Hudson, H.S., Willson, R.C. (1981): "SMM Experiment: Initial Observations by the Active Cavity Radiometer", Adv.Space Res. 1(13), pp. 285-288

Hudson, H.S., Willson, R.C. (1982): "Sunspots and Solar Variability", in Physics of Sunspots, ed. L.Cram and J.Thomas, Sacramento Peak Obs.Publ., pp. 434-445

Kotov, V.A., Severny,A.B., Tsap,T. (1984): "160-Minute and other long-period oscillations of the Sun: Analysis and interpretation of 9-year observations", Mem.S.A.It. 55, pp. 117-122

Kroll, R.J., Chen, J., Hill, H.A. (1988): "Properties of solar g- mode signals in total irradiance observations" in Seismology of the Sun and Sun-like Stars, ESA SP-286, (ESA, Noordwijk), pp. 415-418

Kuhn, J. (1988): "Helioseismological Splitting Measurements and the Nonspherical Solar Temperature Structure", Ap.J. 331, pp. L131-L134

Kuhn, J., Libbrecht, K., Dicke, R. (1988): "The Surface Temperature of the Sun and Changes in the Solar Constant", Science 242, pp. 908-911

Kuhn, J. (1989): "Helioseismic Observations of the Solar Cycle", Ap.J. 339, pp. L45-L47

Kuhn, J. (1990): "Measuring Solar Structure Variations from Helioseismic and Photometric Observations", in Progress in Seismology of the Sun and Stars, ed. H.Sibahashi (Springer, Heidelberg), in press

Lawrence, J.K., Chapman, G.A., Herzog, A.D., Shelton, J.C. (1985): "Solar Luminosity Fluctuations During the Disk Transit of an Active Region", Ap.J. 202, pp. 297-308

Lean, J., Foukal, P. (1988): "A Model of Solar Luminosity Modulation by Magnetic Activity Between 1954 and 1984", Science 240, pp. $806-908$

Libbrecht, K.G. (1990), "New Observations of Solar p-Mode Splittings from BBSO" in Progress in Seismology of the Sun and Stars, ed. H.Sibahashi (Springer, Heidelberg), in press

Livingston, W., Wallace, L., White, O.R. (1988): "Spectrum Line Intensity as a Surrogate for Solar Irradiance Variations", Science 240, pp. 1765-1767

Mecherikunnel, A.T., Lee, R.B., Kyle, H.L., Major, E.R. (1988): "Intercomparison of Solar Total Irradiance Data from Recent Spacecraft Measurements", J. Geophys. Res. 83, pp. 8503-9509

Pallé, P.L. (1990): "Frequency Dependence of the Solar Cycle Related Shifts of Low Degree p-Modes", private communication

Schatten, K.H., Miller, N., Sofia, S., Endal, A., Chapman, G.A. (1985): “The Importance of Improved Facular Observations in Understanding Solar Constant Variations", Ap.J. 204, pp. 689-696

Toutain, Th. (1990): "Etude théorique de la visibilité des oscillations non radiales et analyse de l'experience IPHIR", PhD Thesis, Université de Paris VI, Paris.

van der Raay, H.B. (1990), "Solar g-Modes" in Progress in Seismology of the Sun and Stars, ed. H.Sibahashi (Springer, Heidelberg), in press

Willson, R.C., Gulkis, S., Janssen, M., Hudson, H.S., Chapman, G.A. (1981): “Observations of Solar Irradiance Variability", Science 211, pp. 700-702

Willson, R.C. (1982): "Solar Irradiance Variations and Solar Activity", J.Geophys. Res. 87, pp. 4319-4324

Willson, R.C. (1984): "Measurements of Solar Total Irradiance and its Variability", Space Sci. Rev. 38, pp. 203-242

Woodard, M. (1990), "Time Variation in Solar p-Mode Frequncies Observed at BBSO" in Progress in Seismology of the Sun and Stars, ed. H.Sibahashi (Springer, Heidelberg), in press

Woodard, M., Hudson, H.S. (1983): "Frequencies, Amplitudes and Linewidths of Solar Oscillations from Total Solar Irradiance Observations", Nature 305, pp. 589-593

Woodard, M., Noyes, R.W. (1985): "Change of Solar Oscillation Eigenfrequencies with the Solar Cycle", Nature 318 , pp. 449

Wolf, C. (1984): "Solar Irradiance Changes Caused by g-Modes and Large Scale Convection", Sol.Phys. 83, pp. 1-13

Wolf, C., Hickey, J. (1987): "Multiperiodic Irradiance Changes Caused by I-Modes and g-Modes", Sol.Phys. 108, pp. 1-18 
SOLAR IRRADIANCE VARIABILITY DURING SOLAR CYCLES 21 AND 22

\author{
Richard C. Willson \\ Jet Propulsion Laboratory \\ Mail Stop 171-400 \\ 4800 Oak Grove Dr. \\ Pasadena, CA 91109
}

Total solar irradiance observations by the Active Cavity Radiometer Irradiance Monitor on the Solar Maximum Mission, the Nimbus 7/ERB, NASA/ERBS, and NOAA 9/ERBE are combined to demonstrate the characteristics of the variation of solar luminosity over the solar cycles 21 and 22 between 1978 and 1990. Observations of solar Lyman Alpha and 10.7 Mhz fluxes are used to demonstrate a total irradiance variability asymmetry relative to the maximum of solar activity. 


\title{
THE ACRIM DATA IN THE CONTEXT OF STELLAR VARIABILITY
}

\author{
Hugh S. Hudson \\ Center for Astrophysics and Space Sciences C-011 \\ University of California, San Diego 92093
}

\begin{abstract}
The ACRIM total-irradiance data from the Solar Maximum Mission have given us a first comprehensive view of solar variability in the stellar sense. Five types of solar variability have been identified thus far. These have small amplitudes, less than a few tenths of one percent, and are at levels generally not yet detectable on other stars. The possible stellar analogs are interesting physically, and in particular may help us to understand solar behavior on longer time scales. This paper describes the ACRIM data from the stellar point of view and comments on the present state of stellar time-series photometry.
\end{abstract}

\section{INTRODUCTION}

Observations from space have now given us a chance to observe solar luminosity variability in the sense of "the Sun as a star." As described below, the observed variations have been rather small, at or just below the threshold of the best ground-based observations. Nevertheless at least five independent mechanisms of variability have been detected in the analysis of ACRIM data thus far, as shown in Table 1 (ACRIM stands for Active Cavity Radiometer Irradiance Monitor).

Table 1

Types of Solar Variability

\begin{tabular}{lll}
\hline \hline Mechanism & Time scale & Amplitude \\
\hline Oscillations & 5 min & few parts per million \\
Granulation & tens of min & tens of parts per million \\
Sunspots & few days & $\lesssim 0.2 \%$ peak-to-peak \\
Faculae & tens of days & $\lesssim 0.1 \%$ peak-to-peak \\
Solar cycle & 11 years & $\sim 0.1 \%$ peak-to-peak \\
\hline
\end{tabular}

The general approach of the discussion in this review is based on the perspective of the frequency domain. In a power spectrum analysis, one can divide patterns of variability into resonant and broad-band phenomena. The former includes the Hale 22-year magnetic cycle and the p-mode (five minute) oscillations; the latter includes the incoherent variations due to sunspots, granulation, and flares (but note that in Solar Cycle 21 there was a clear tendency for a 155-day periodicity in the occurrence of high-energy flare $\gamma$-ray bursts ${ }^{1}$. It is important to note that non-periodic (broad-band) variations are just as important physically, and require just as much explanation, as periodic variations. The question of periodicity may not be particularly important, unless there is a question of resonance in a driven secondary process (e.g. in the Earth's weather or climate). 
If strongly periodic phenomena do occur (the p-modes being the best solar example) they can be a rich source of precise inputs for reconstructions of interior structure and dynamics via inverse theory.

\section{STELLAR BACKGROUND INFORMATION}

There are many types of rapid stellar variability (e.g. Warner ${ }^{2}$ ). Essentially all of the observations of these variations are at amplitude levels considerably larger than those observed on the Sun, and so no strictly comparable types of variation can be studied yet. However we are quite interested in both avenues of information exchange: We wish to learn about stellar physics from the solar case, where we have more sensitive and complete time series of data, and can often identify the direct causes of variability via imaging observations; and we wish to learn about solar physics from the stars, which allow for widely different parameters (stellar age, gravity, chemical composition, rotation rate, etc.). The solar analogs are strongest in late-type main-sequence stars, i.e. those stars whose parameters approach solar values. These stars may show spottedness (BY Draconis prototype) and flaring (UV Ceti prototype) resembling exaggerated levels of the corresponding solar phenomena. In addition, there are long-term cyclic variations originally discovered by O.C. Wilson ${ }^{3}$. Our interest here centers on how stellar observations can help us to understand the longterm behavior of solar luminosity, since the modern data on total irradiance barely cover one period of the 11-year solar magnetic cycle.

$\mathrm{BD}+26^{\circ} 730$

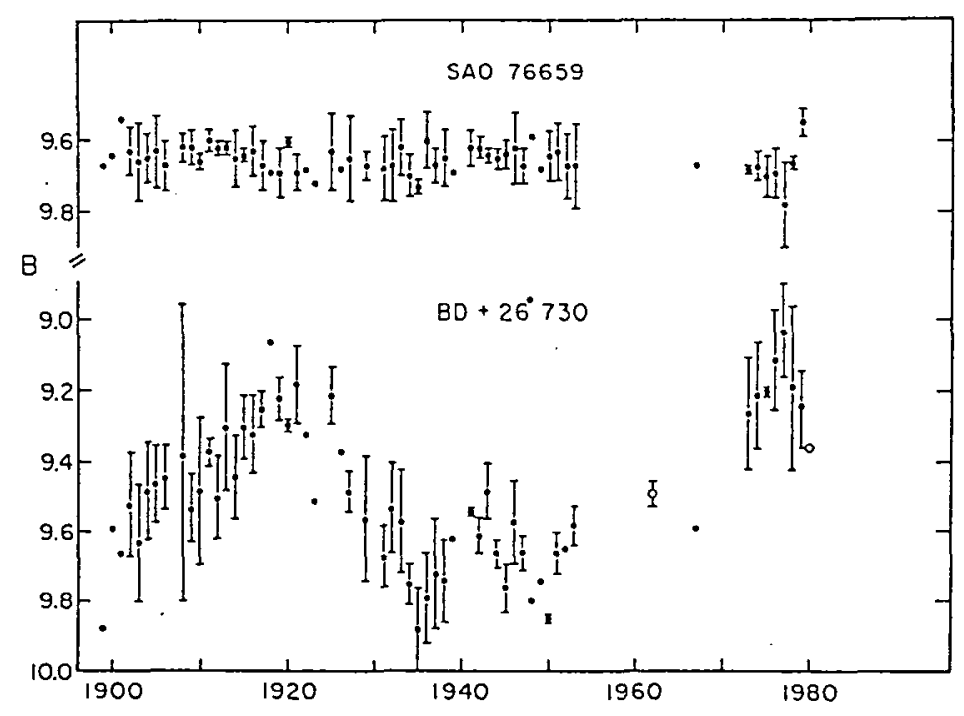

1. Long-term photographic variability of $B D+26^{\circ} 730$ and a nearby comparison star (Figure 1 of Hartmann et al., 1981). Error bars represent standard deviations of annual means, and open circles represent photoelectric data. 
Hartmann et al. ${ }^{4}$ have found a late-type star $\left(\mathrm{BD}+26^{\circ} 730\right.$, dK5e) that shows an enormous cyclic variation apparently associated with a magnetic cycle such as the Sun's; this star shows a full amplitude of some $0.6 \mathrm{mag}$ (roughly doubling in brightness during maximum), whereas the solar amplitude appears to be on the order of $0.1 \%^{5}$. The $\mathrm{BD}+26^{\circ} 730 \mathrm{cycle}^{4}$, data reproduced here as Figure 1, illustrates two key points about stellar photometry, namely the difficulty of getting complete time series (see the discussion below of the solar photometry), and of getting adequate precision on longer time scales. Few astronomers wish to spend their valuable observing time endlessly observing the same star, and unfortunately automated observatories (either on the ground or preferably in space) have not reached broadly productive levels yet. Stellar photometric time series are therefore often incomplete, undersampled, and full of gaps, as well as suffering from the problem of atmospheric seeing. Many of these problems are alleviated for high-speed variations ${ }^{2}$ (see $\mathrm{Kurtz}^{6}$ for an example of some of the best stellar data, the relatively complete studies of the Ap "oblique pulsator" stars).

\section{OVERVIEW OF ACRIM DATA}

The most complete of the solar total-irradiance measurements come from the ACRIM experiment ${ }^{7}$, summarized in Table 2. The standard mode of operation of ACRIM consisted of regular sampling through a mechanical shutter that opened and closed with a 131.072-s cycle. However a long hiatus (December 1980 to May 1984) in the spacecraft's capability for accurate pointing resulted in a period of observation known as the "spin mode." This had greatly reduced sampling and a physically different observing pattern. Another shorter period in late 1989, near the end of SMM's life, was devoted to increased sampling without the shutter in operation. The ACRIM instrument was radiometrically self-calibrated, and contained three identical sensors to permit the monitoring of some kinds of degradation with time of the prime sensor.

Table 2

ACRIM measurements of total irradiance

\begin{tabular}{ll}
\hline Period of Observation & February 1980- December 1989 \\
"Spin-mode" data & December 1980 - April 1984 \\
"No-shutter" data & August, 1989 - November, 1989 \\
Sampling interval & $1.024 \mathrm{~s}$ \\
Shutter cycle & $131.072 \mathrm{~s}(25 \%$ duty cycle) \\
Orbital period & $96 \rightarrow 92$ min \\
Digital resolution & 12 bits (power measurement) \\
Principal time-series gaps & shutter cycles (25\% on) \\
& orbital modulation ( 60\% on) \\
Spectral response & Bolometric \\
\hline
\end{tabular}

The analysis of the ACRIM data is still far from complete, but much has been published already ${ }^{8}$; see several papers in these Proceedings).

It is worth noting that of all of the forms of variability, only the largest individual sunspot groups make individually recognizable features in the time series; all of the others are best observed statistically or via Fourier analysis. In particular, flares have not been detected in the total solar irradiance ${ }^{9}$, whereas they are quite prominent in the $\mathrm{dMe}$ stars of the UV Ceti type. Major solar flares can barely be detected in chromospheric lines in the stellar sense ${ }^{10}$. The ACRIM record also 
does not appear to show prominences projected against the dark sky at the limb, coronal mass ejections, or comets crashing on the Sun (or at least, studies of the data thus far have not revealed good evidence for these or other mechanisms of variability).

Figures 2-4 show a set of time series of the ACRIM data on successively finer scales, in order to give a feeling for the nature of the variations. Each plot shows the range of variation, as indicated by the sample standard deviation, rather than the error of measurement. At each successivelyfiner time scale, variations become more clearly resolved by the ACRIM sensor, an indication that broad-band variability is present on all time scales, a point confirmed by power spectrum analysis. Figure 5 shows several distribution functions of solar flux levels - these may be compared with the scatter of stellar fluxes on the principle that the time series of brightnesses of one star should have the same distribution as the instantaneously sampled brightnesses of many identical stars undergoing the identical types of variation. This principle is limited, of course, by the lack of a very long time series of solar observations; secular variations not reflected in the modern solar data might yet be present and contributing to the scatter of stellar magnitudes.

\section{LIMITS ON LONG-TERM SOLAR VARIABILITY}

We would like to detect or set limits on any secular variability in solar irradiance, i.e. variations on longer time scales than even the 11-year variation seen in the ACRIM data. On the longest time scale (Figure 2) the ACRIM variation appears to consist of a flat minimum, upon which the solar maxima appear to be excess amounts of radiation. This is similar to, but less exaggerated than, the variations seen in UV spectral irradiance or the $10.7 \mathrm{~cm}$ radio flux.

The longer-term variations compete with the five (or more) mechanisms on shorter time scales, which appear as non-random systematic biases in any characterization of a trend. Indeed, Reid ${ }^{11}$ pointed out that the sunspot numbers, over the duration of the Greenwich sunspot record, contains a clear secular trend. Foukal and Lean ${ }^{12}$ note that this implies a secular trend in the total irradiance if calibrated against the total irradiance data of the past decade.

Our best hope for measuring the long-term variability of the Sun directly is to apply model corrections for the known effects. This can only work if the models are accurate, and do not introduce more (or less-well-understood) errors than they remove. Irradiance modeling based upon sunspot areas, $10.7 \mathrm{~cm}$ flux, the Ca plage index, and He $10830 \AA$ was quite instrumental in elucidation of the solar-cycle component ${ }^{5,13}$.

The key to accurate modeling lies in getting better diachronous (synoptic) data. Ground-based data must be improved, and we must urge that NOAA and NASA establish space-based systems for diachronous data collection. At present, the ACRIM data obtained over the "standstill" at solar minimum can only limit any secular trend only at approximately $0.1 \%$ per year, not competitive with limits established by ground-based observations. The careful application of total-irradiance models to the ACRIM data can provide stronger limits to secular trends from this same data set, but analysis along these lines has not yet been carried out. Improvements in the diachronous data collection (plus of course a continuation of total irradiance measurements from space) would make this approach quite attractive for the next solar minimum period.

\section{CONCLUSIONS}

From the 27-day means of Figure 2, we can already infer that the cyclic variation of a star such as $\mathrm{BD}+26^{\circ} 730$ differs from that of the solar type, in that the cyclic term appears much larger relative to the short-term contributions of the activity. It is also known that more rapidly rotating stars exhibit an anticorrelation of the cyclic and short-term modulations, rather than a direct correlation 

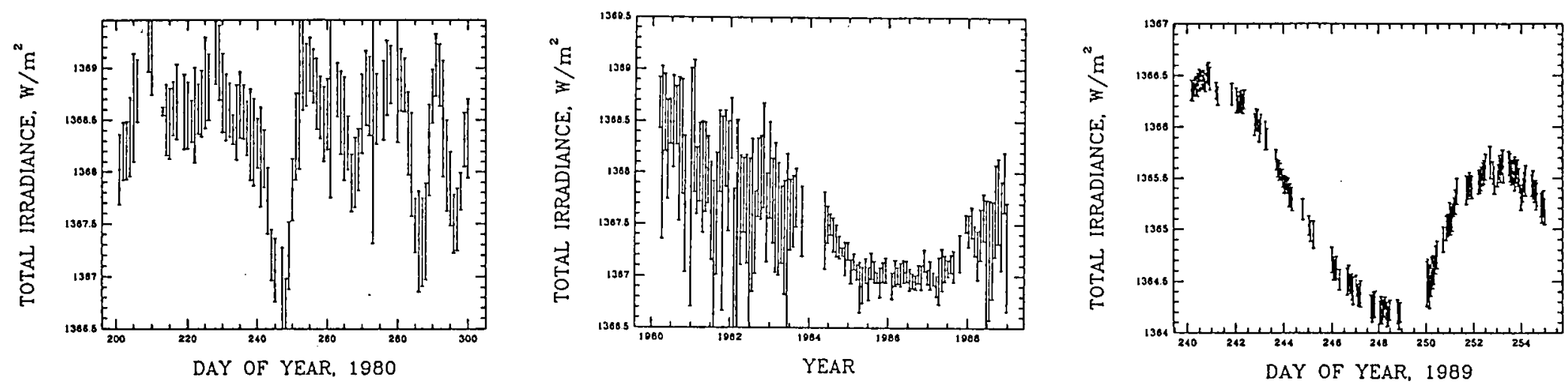

2. (Left) Time series of ACRIM data in 27-day intervals. As in Figure 1, the error bars represent standard deviations of the data, rather than true error estimates, and might better be called "range bars." Much of the variation in the solar maximum periods is due to the direct effects of sunspots.

3. (Center) Representative time series of ACRIM daily data. Again, the error flags are sample standard distributions showing the full range of fluctuation. Figures $2-4$ have the same amplitude range of $3 \mathrm{~W} / \mathrm{m}^{2}$.

4. (Right) Representative time series of orbital means of ACRIM data (preliminary version; the final analysis of these no-shutter data will differ from the values shown here). Again, the error flags are sample standard distributions showing the full range of fluctuation. The data show a high degree of variability associated with the disappearance at the limb of still-growing sunspots of active region \# 5669, but the data distribution is approaching a normal distribu of errors on this one-orbit ( $\sim 55$-minute) time scale. 


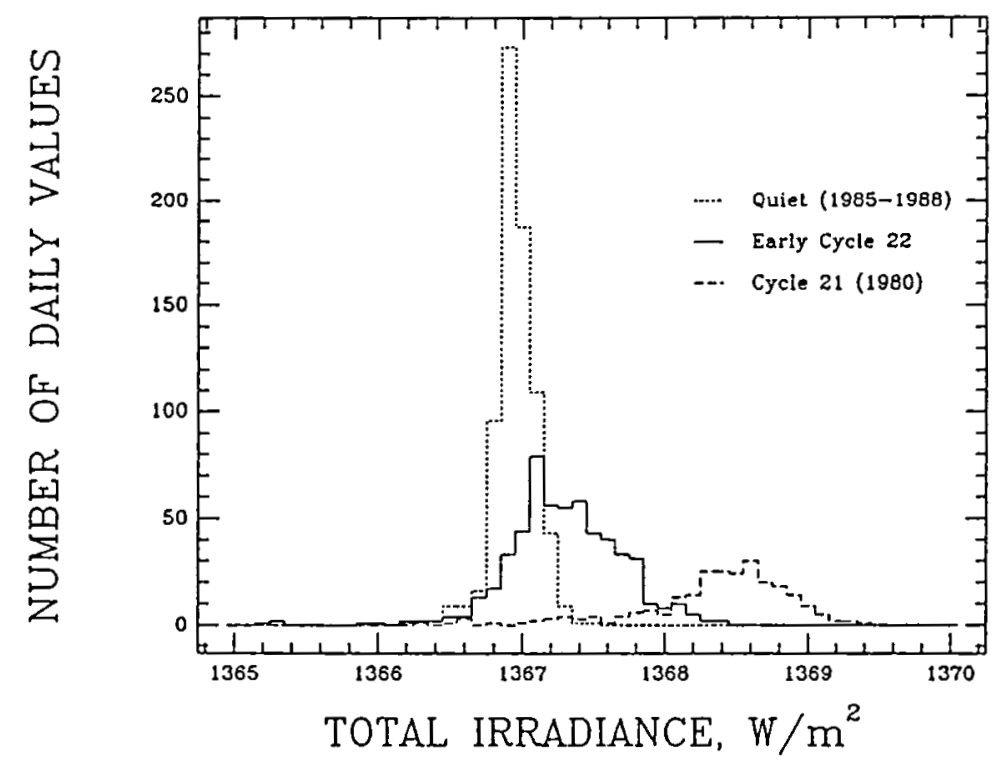

5. Distributions of solar total irradiance values from the ACRIM data for three different epochs, excluding the special data (spin mode and no-shutter mode). The 1980 data show a well-defined skew due to the dips in the time series resulting from sunspots. Such distributions can be compared with the results of photometry of solar-type stars under the "equivalence principle" (e.g. Baliunas, 1990) of time-series and multiple-star photometry. Because the solar variations are so small, no direct photometric comparison has yet been possible.

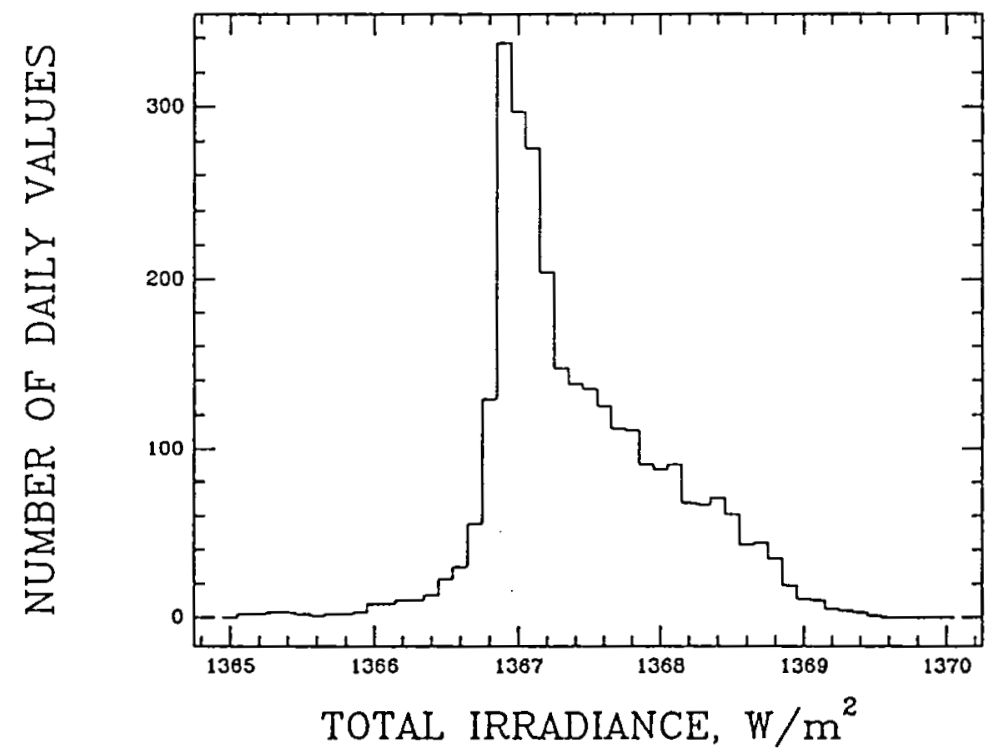

6. Distribution of all daily values of ACRIM total irradiance. This distribution includes the spin-mode data. Note the prominent narrow peak in "bolometric luminosity" values produced by the standstill of total irradiance during the sunspot minimum. 
as shown by the Sun.

The histograms in Figures 5 and 6 can also be compared with stellar photometry in the $\mathrm{Ca}$ $\mathrm{K} 1-\AA$ index or other chromospheric indicator ${ }^{14}$, but only if appropriate calibration can be made from solar observations. Such comparisons will always be uncertain to the extent that the physics may differ, thus destroying the calibration based upon solar processes.

Better stellar time-series photometry clearly would make all the difference in exploiting the analogous forms of variability, since this would allow us to make direct comparisons rather than going through a chromospheric proxy of uncertain calibration. This will require drastic improvements in sensitivity and observational duty cycle; this suggests observations from space ${ }^{15}$ or else substantial improvements in ground-based photometry. The use of frame normalization of CCD sensors is very promising in this regard ${ }^{16,17,18}$.

Acknowledgments. This research has been supported by NASA under SMM Guest Investigator grant NAG 5-1321. I thank R.C. Willson for all his encouragement and assistance, and B.M. Fisher for help with Figure 4 and with the analysis of the ACRIM no-shutter data.

\section{REFERENCES}

1. Rieger, E., Reppin, C., Kanbach, G., Forrest, D.J., Chupp, E.L., and Share, G.H., 1983, Proc. Int. Cosmic Ray Conf. 10, 338.

2. Warner, B., 1988, High Speed Astronomical Photometry (Cambridge University Press).

3. Baliunas, S., and Vaughan, A.H., 1985, Ann. Revs. Astron. Astrophys. 23, 379.

4. Hartmann, L., Bopp, B.W., Dussault, M., Noah, P.V., and Klimke, A., 1981, Astrophys. J. 249, 662).

5. Willson, R.C., and Hudson, H.S., 1988, Nature 332, 810.

6. Kurtz, D.W., 1990, Ann. Revs. Astron. Astrophys. (to be published).

7. Willson, R.C., 1984, Space Sci. Revs. 38, 203.

8. Hudson, H.S., 1988, Ann. Revs. Astron. Astrophys. 26, 473.

9. Hudson, H.S., and Willson. R.C. 1983, Solar Physics 86, 123.

10. Livingston, W.C., and Ye, B, 1982, Publ. Ast. Soc. Pac. 94, 713.

11. Reid, G.C., 1987, Nature 329, 142.

12. Foukal, P.A., and Lean, J., 1990, preprint.

13. Foukal, P.A., and Lean, J.L., 1988, Astrophys. J. 328, 347.

14. Baliunas, S., 1990, these proceedings.

15. Hudson; H.S., 1984, in W.J. Borucki and A.T. Young (eds.), Proc. Workshop on Improvements to Photometry (NASA CP-2350), p. 43.

16. Walker, A.R., 1984, Mon. Not. R. astr. Soc. 209, 83.

17. Gilliland, R.L., and Brown, T.M., 1988, Publ. Ast. Soc. Pacific 100, 754.

18. Buffington, A., Hudson, H., and Booth, C., 1990, Publ. Ast. Soc. Pacific, to be published. 


\title{
THE VARIATION OF SOLAR FE XIV AND FE X FLUX OVER 1.5 SOLAR ACTIVITY CYCLES
}

\author{
Richard. C. Altrock \\ Geophysics Laboratory (AFSC) \\ National Solar Observatory/Sacramento Peak \\ National Optical Astronomy Observatories† \\ Sunspot, NM 88349 \\ SPAN: NOAO::RALTROCK
}

\begin{abstract}
This paper presents a new source of data on the solar output, namely "limb flux" from the one- and two-million degree corona. This parameter is derived from data obtained at the National Solar Observatory at Sacramento Peak with the $40 \mathrm{~cm}$ coronagraph of the John W. Evans Solar Facility and the Emission Line Coronal Photometer. The limb flux is defined to be the latitude-averaged intensity in millionths of the brightness of disk center from an annulus of width $1.1^{\circ}$ centered at a height of $0.15 \mathrm{R}_{\odot}$ above the limb of emission from lines at $6374 \AA$ (Fe X) or $5303 \AA$ (Fe XIV). Fe XIV data have been obtained since 1973 and Fe X since 1984. Examination of the Fe XIV data shows that there is ambiguity in the definition of the last two solar activity minima, which can affect the determination of cycle rise times and lengths. There is an indication that a constant minimum or basal corona may exist at solar minimum. Cycle 22 has had a much faster onset than Cycle 21 and has now overtaken Cycle 21. The rise characteristics of the two cycles were very similar up until Jul-Aug 1989, at which time a long-term maximum occurred in $\mathrm{Fe} \mathrm{X}$ and Fe XIV, which could possibly be the "solar maximum". Another maximum is developing at the current time. Cycle 21 was characterized in Fe XIV by at least 4 major thrusts or bursts of activity, each lasting on the order of a year and all having similar maximum limb fluxes, which indicates that coronal energy output is sustained over periods in which the sunspot number declines significantly. Dramatic increases in the limb fluxes occur from minimum to maximum, ranging from factors of 14 to 21 in the two lines. Two different techniques to predict the epoch of solar maximum have been applied to the Fe XIV data, resulting in estimates of Apr 1989 ( $\pm 1 \mathrm{mo})$ and May $1990( \pm 2 \mathrm{mo})$.
\end{abstract}

\section{INTRODUCTION}

There is currently a great deal of interest in evaluating various ground-based obscrvations of the Sun that may be of use as inputs to models of the Earth's atmosphere. Until such time as full-spectrum solar observations are available from space, these ground-based observations will be the main source of information on how the solar output affects the atmosphere. This paper presents a new source of data on the solar output, namely parameters related to flux from the one- and two-million degree solar corona. I will present information on the obscrvations and their calibration, their behavior throughout the solar cycle, comparison with other similar observations and how such observations may be used to predict the epoch of solar-activity maximum.
Observations are made at the National Solar Observatory site at Sacramento Peak with the FisherSmartt Emission Line Coronal Photometer (ELCP) (Smartt, 1982). This instrument photoelectrically records the solar corona when fed with the John W. Evans Solar Facility $40 \mathrm{~cm}$ Coronagraph. It operates at high precision due to its ability to subtract the sky background from the signal in emission lines through use of a lockin amplifier oscillating at a rate of $100 \mathrm{kHz}$ between the continuum and the lines at $6374 \AA$ (Fe X) and $5303 \AA$ (Fe XIV), which are formed at approximate temperatures of 1 and $2 \mathrm{MK}$, respectively. Reproducibility of successive scans is frequently to less than 1 millionth of the brightness of the solar disk at the given wavelength. Scans are routinely made in skies as bright as 200-400 millionths. A $1.1^{\circ}$ aperture is scanned around the limb daily at $1.15 \mathrm{R}_{\odot}$ (data at other heights and in Ca XV $5694 \AA$ will not be discussed in this paper). The output of the ELCP is sensed by a photomultiplier, digitized and recorded every $3^{\circ}$ of latitude.

\section{OBSERVATIONS}

† Operated by the Association of Universities for Research in Astronomy, Inc., (AURA) under cooperative agreement with the National Science Foundation (NSF). The National Solar Observatory is partially supported by the USAF under a Memorandum of Understanding with the NSF. 
Scans in Fe XIV have been made since 1973, and in Fe $X$ since 1984 (scans of Fe X were made in 1983 but have not yet been examined in detail).

Absolute intensities in millionths of the brightness of the center of the disk at $6374 \AA$ and $5303 \AA$ are obtained by calibrating the system through a neutral density filter. Scans in latitude at $1.15 \mathrm{R}_{\odot}$ may be redefined to coronal "limb flux", F, by averaging over the entire 360 degrees of position angle. The limb flux is therefore the latitude-averaged intensity in millionths of the brightness of disk center from an annulus of width $1.1^{\prime}$ centered at a height of $0.15 R_{\odot}$ above the limb. I will denote the Fe XIV limb flux by F5 and that for Fe $X$ by F6. Such fluxes are of considerable interest because they are related to true $1 \mathrm{MK}$ and $2 \mathrm{MK}$ coronal fluxes.

This study is proceeding in parallel with a similar study by Yasukawa, Sime, Fisher and myself, which is using pseudo-full-disk fluxes from the same data.

\section{CALIBRATIONS}

In Jan 1983 the original birefringent $5303 \AA$ filter was replaced by 3 independent mica etalon filters. At this time the calibration procedures were re-evaluated, and a new calibration procedure was adopted. As a result, the earlier $5303 \AA$ calibrations were called into question. In order to investigate this uncertainty, a statistical study of the relationship between 27-day averages of the sunspot number, $R$, and the coronal limb flux F5 was done. Values of $R$ were obtained from the NOAA National Geophysical Data Center CD-ROM NGDC01. A least-squares fit was calculated between F5 and R for (i) 1975-1982 and (ii) 1983-1986 (R has not been obtained yet for 1987-1989). Figure 1 shows the fit, $F R=f(R)$, for (i). The fit for (ii) is similar, and the squares in Figure 1 show FR(ii). A statistical correction value, $c(R)$, may be calculated for $F R(i)$ such that the corrected values for (i), $F R^{\prime}(i)$, are given by $F R^{\prime}(i)=c(R) F R(i)$. An integral average value of $c(R)$, $c^{\prime}$, may be calculated for the observed range, $0<R<200$, from the least-square fits to (i) and (ii) by assuming that $F^{\prime}(\mathrm{i})=c(R) F R(i)=F R(i i)$. The result is $c^{\prime}=0.995$, thus indicating that no correction is required for the earlier results (in fact, a second correction factor of 0.74 needs to be applied to all of the data, but the figures do not reflect this correction).

\section{SOLAR MINIMA IN FE XIV}

Figure 2 shows 27-day-averaged values of F5 for 1976 and 1977. The "official" solar-cycle minimum of $\mathrm{R}$ was Jun 1976. The F5 minimum occurred for data

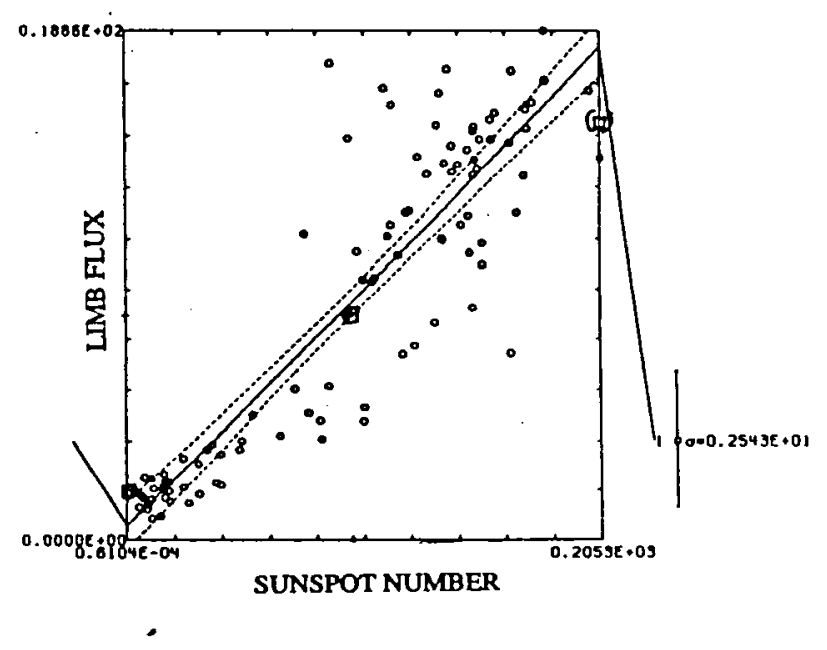

Fig. 1. Circles and lines: Values of Fe XIV "limb flux" (F5) (See text for definition) plotted vs. and linearlyfitted to values of the sunspot number $\mathrm{R}$ for 1975 to 1982. Squares: linear fit of F5 vs. R for 1983-1986 (the square in parentheses is extrapolated beyond the range of $\mathrm{R}$ for that period).

mostly from the month of Aug 1976 (location 2). Note the near-minimum that occurred in Mar 1976 (location 1) $(\mathrm{F} 5=1.0$ vs. 0.8 in Aug 1976). This may be considered an alternative minimum for F5.

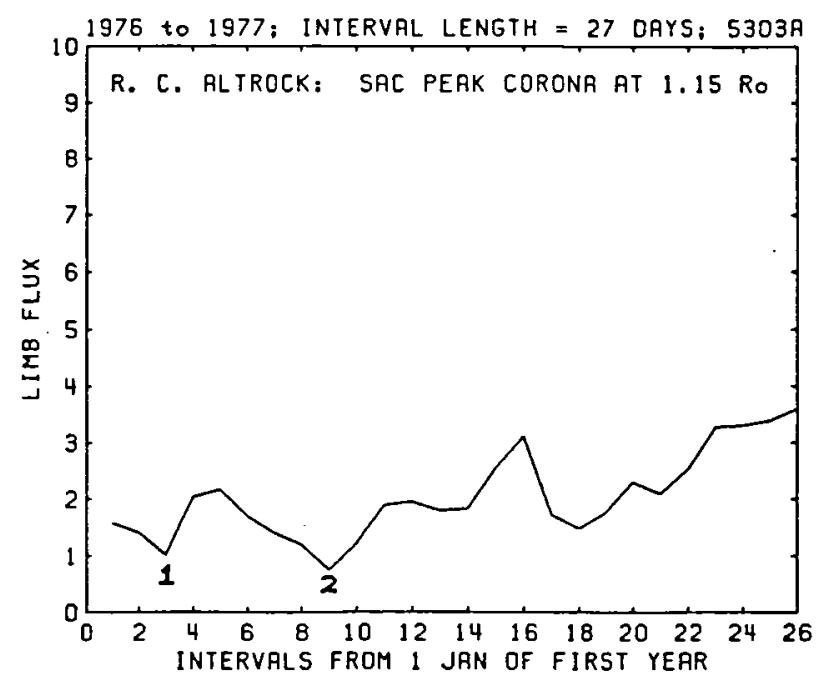

Fig. 2. 27-day-averaged Fe XIV "limb flux" (F5) (Sec text for definition) for 1976 and 1977.

Figure 3 is the same as Figure 2, but for 1986 and 1987. The official minimum of $\mathrm{R}$ was Sep 1986. The coronal minimum occurs in Aug 1986 (location 3) or Sep 1986. Note the secondary minimum in Jan 1987 (location 4) (F5 = 1.4 vs. 1.3 carlier). Comparing Figures 2 and 3 shows that the 1986 minimum had only 
slightly higher F5 than the 1976 minimum, perhaps indicating the presence of a constant minimum or "basal" corona. The resurgence of activity between Aug 1986 and Jan 1987 is due to a combination of one old-cycle region near the equator and one new-cycle region near $30^{\circ}$ latitude. Soon after minimum the Cycle 22 flux began to increase rapidly over that of Cycle 21 .

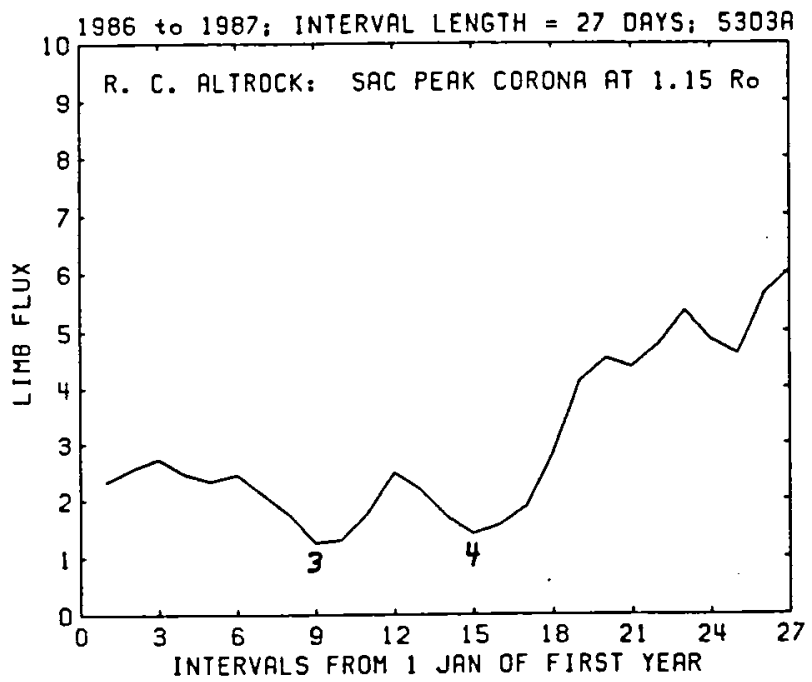

Fig. 3. 27-day-averaged F5 for 1986 and 1987.

\section{THE SOLAR CYCLE VARIATION OF FE $X$ AND FE XIV FLUX}

Figure 4 shows daily values of F5 from 1973 to 3 Apr 1990. Note the expansion of the ordinate scale by a factor of 4 over that of Figures 2 and 3 . This figure shows the rotational modulation as well as solar-cycle variation. Some of the variation is no doubt due also to noise from observational errors. However, it is clear that Cycle 22 has already overtaken Cycle 21.

Figure 5 is the same as Figure 4, but with 27day-averaged F5. The dashed line shows the onset of Cycle 22 overlaid on the onset of Cycle 21 solely to show the similarity of the rise characteristics of the two cycles. The 27-day-averaged F5 has also surpassed the maximum of Cycle 21. Note the rather flat-topped nature of the F5 distribution relative to $\mathrm{R}$ during Cycle 21 and the multiple surges of activity lasting on the order of 1 year. The numbers below the curve indicate the respective minima as defined in Figures 2 and 3. The superimposed curves 1 and 2 are discussed below.

Figures 4 and 5 show that there was a large spike in Fe XIV flux in Jan 1989, reaching a daily value higher than at any other time in Cycles 21 and 22. Since that time, Figure 5 shows that the flux levels slowly rose to 20 to $30 \%$ higher than those of Cycle 21

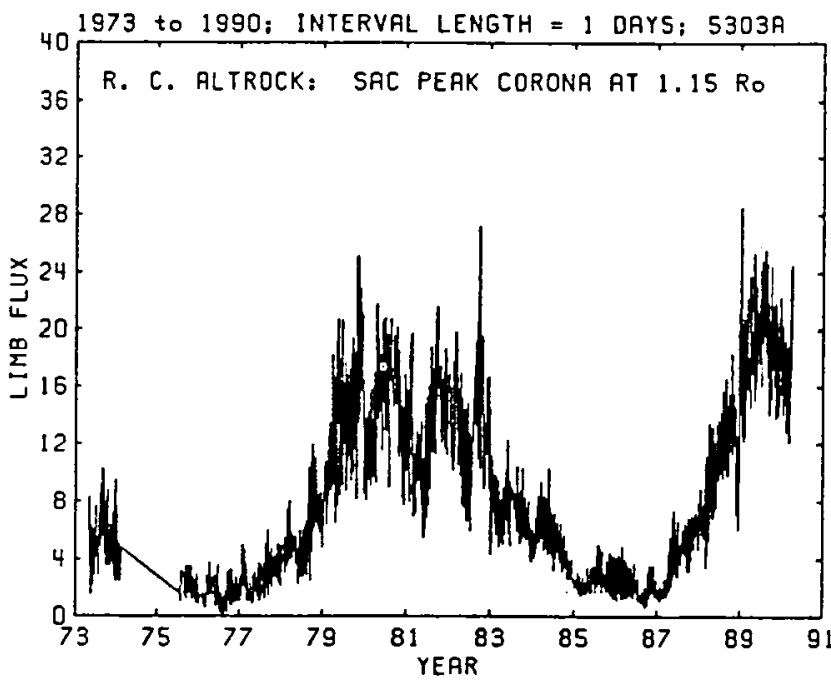

Fig. 4. Daily values of F5 from 1973 to 3 Apr 1990. Note the expansion of the ordinate scale by a factor of 4 over that of the two-year plots.

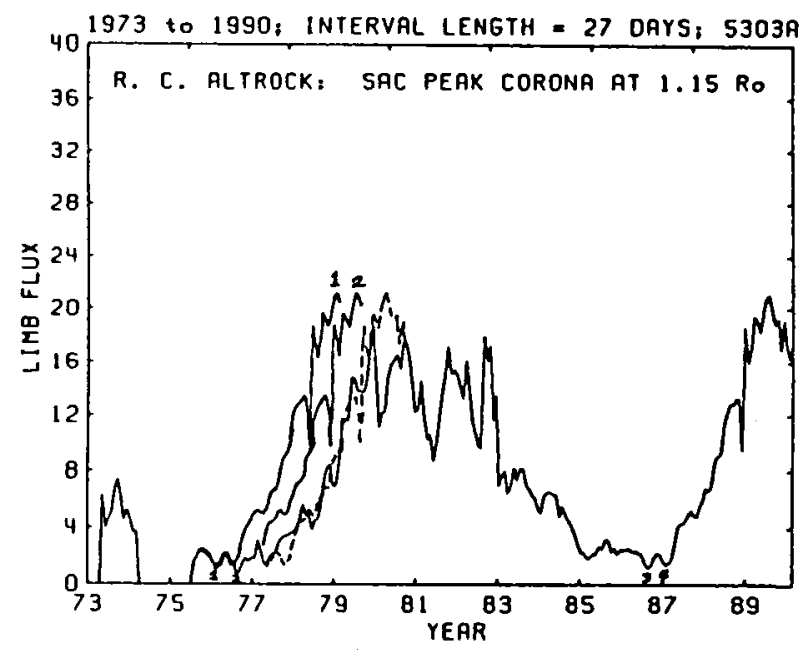

Fig. 5. 27-day-averaged F5 from 1973 to $3 \mathrm{Apr} 1990$. See text for details. Zero values denote no data.

in Jul and Aug 1989 and then slowly declined until Mar 1990. Figure 6 shows that the solar minimum and onset phases in Fe X have been similar to Fe XIV, with F6 showing an even more rapid onset than F5. A spike of activity in F6 occurred also in carly 1989 (Jan to Fcb) and the 27-day-averaged F6 reached a maximum slightly earlier than Fe XIV, in Jun 1989. After that major maximum, $\mathrm{F} 6$ has also been slowly declining. Figure 7 shows that since mid-Mar 1990 F5 has been increasing and has reached levels similar to those in last Jul and Aug. The value of 27-day-averaged F5 (19.6 as of 4/19/90) is still below the 1989 maximum value of 21.1. A resurgence to mid-1989 levels has also been 


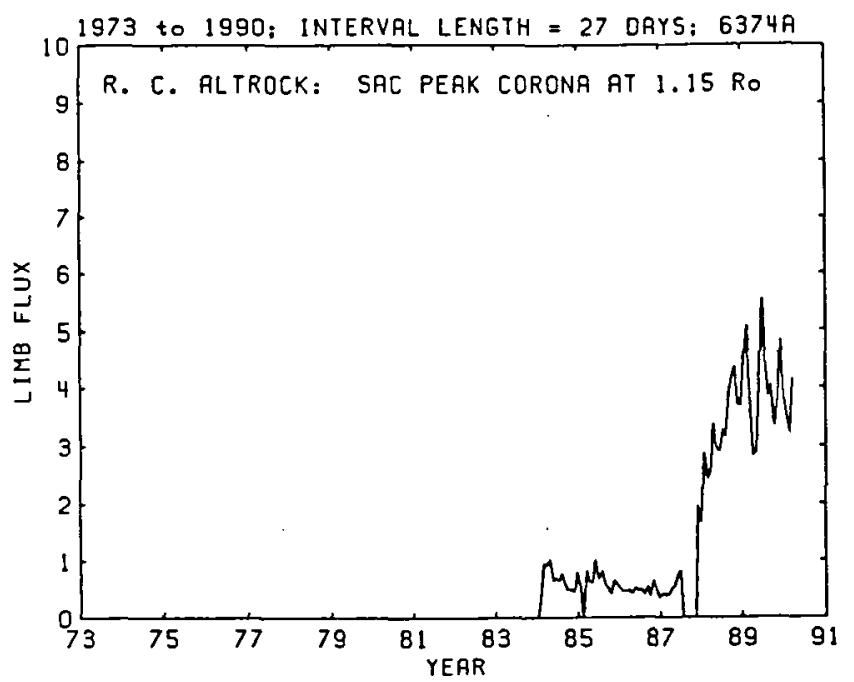

Fig. 6. 27-day-averaged Fe X limb flux (F6) from 1984 to $3 \mathrm{Apr} 1990$. Zero values denote no data. Note the factor of 4 difference in the ordinate scales of figures 5 and 6.

recently seen in F6. The 27-day value has reached 4.3, vs. 5.6 in 1989.

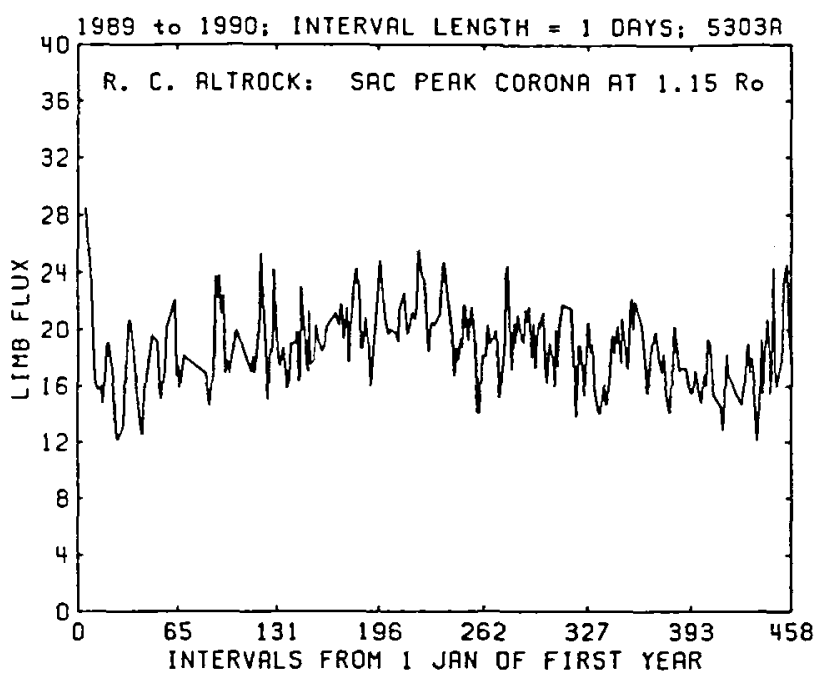

Fig. 7. Daily values of F5 from 1989 to 3 Apr 1990.

\section{CALCULATION OF THE EPOCH OF SOLAR MAXIMUM}

\section{Method 1: Cycle 22 Lead Time Implies an Early Maximum}

Curve 2 of Figure 5 shows the onset of Cycle 22 compared with Cycle 21 when minimum 3 (Aug 1986) is lined up with minimum 2 (Aug 1976)*. If curve 2 and the onset of Cycle 21 are compared, Cycle 22 appears to be leading Cycle 21 by approximately 9 months. If the "official" minima (Jun 1976 and Sep 1986) were aligned, the lead time would be 12 months. Since the rise time (minimum to maximum) for Cycle 21 was 42 months, and if the above lead times are interpreted as evidence for an earlicr maximum, then this implies that the Cycle 22 rise time should be 42 months minus the lead time, which is 33 months from Aug 1986 or 30 months from Sep 1986. This results in cstimates for the maximum of Cycle 22 of May 1989 and Mar 1989.

Curve 1 shows the situation when minimum 4 (Jan 1987)** is aligned with minimum 2. This aligns the "double minima" in 1976 and 1986-1987 referred to above and demonstrates how closely the two solar minimum epochs follow each other in this case. IF this 5-month shift were allowed, THEN Cycle 22 is leading Cycle 21 by 14 months. If the error were in 1976 , then the Cycle 21 rise time would be increased to 45 months (Mar 1976 to Dec 1979), and the estimated Cycle 22 rise time would be 31 months from Aug 1986, for a solar maximum in Mar 1989. If the error were in 1986-87, then the current rise time would be 28 months from Jan 1987, for a solar maximum in May 1989.

We thus obtain estimates for Cycle 22 maximum of between Mar and May of 1989. However, we must also recognize that these calculations are based on the (necessarily-unproven) assumption that any lead of Cycle 22 over Cycle 21 may simply be subtracted from the Cycle 21 rise time. Note that a major maximum for Cycle 22 occurred in Jul-Aug of 1989.

\section{Method 2: Cycle 22 Shape Will Be Similar to Cycle 21 Shape}

The dashed line shows how similar Cycles 22 and 21 have been in the shape of their early rise phases. If this is taken as evidence that this similarity will continue (which now appears to be less likely), then we could hypothesize that the Cycle 21 rise time (42 months) will apply to Cycle 22, and that maximum will occur 42 months from Aug 1986 or Jan 1987 (sec above); i.e., between Feb and Jul 1990. If, as suggested above, there was an error in the time of solar minimum in 1976 of 3 months, then the Cycle 21 rise time was 45 months, and Cycle 22 maximum would occur in Jun 1990. Note the possible resurgence in

1976 (F5 = 0.9)

**Actually a combination of two points containing data mostly from Dec 1986 to Jan 1987 (F5 = 1.6) and Jan to Feb 1987 (F5 = 1.5). These values are slightly different from those quoted earlier due to different starting dates for the 27-day intervals.

*Actually a combination of two points containing data mostly from Jul to Aug 1976 (F5 = 0.8) and Aug to Sep 
activity that began in Mar 1990 (see Figure 7). Of course, if solar maximum was about to occur, we would not necessarily know it for 6.5 months afterwards, since the Cycle 21 rise time, on which these calculations are based, is calculated from weighted 13-month running means.

These two methods lead to widely disparate estimates for the time of solar maximum. Method 1 (Mar to May 1989) depends on Cycle 22 having historicallylow values of the rise time of from 28-33 months. Since no values below 35 months have ever been observed, perhaps this estimate should be rejected out of hand. In addition, an early maximum would necessarily accept a value of maximum flux not much greater than that of Cycle 21, whereas other methods indicate historicallyhigh values of all other parameters. If, in fact, solar maximum did occur in Aug 1989, the above information indicates a rise time of between 31 and 36 months, which would indeed be near an all-time record low!

Method 2 (Mar to Jul 1990) is more in step with other estimates (cf. NASA Working Group report), does not violate historical rise-time limits and allows for considerable increase from the current value of the flux. It now appears that another maximum will occur in the coming months. We must await the decision of Sol to see if it will be a primary or secondary maximum.

\section{COMPARISON WITH OTHER OBSERVATIONS}

Previous investigators such as Gentili de Giuseppe et al. (1966) and Sýkora (1980) found that in Cycles $18 \& 19$ the Fe XIV flux was double-peaked, with one peak occurring near the maximum of $R$ and the second 2-3 years later. However, the results for Cycles 20 and 21 [Sjikora (1980), and Rusin et al. (1988)] show a multipeaked variation with surges of activity every 2-3 years, and some of the data do not show any clear single maximum, similar to our F5. Coronal processes show a much more continuous level of activity than does $\mathbf{R}$.

Fleck and Keil (1990, in preparation) report a steep drop in early 1983 in some of the full-disk parameters (most noticeably their $\mathrm{Ca} \mathrm{K}$ emission index [EI] and other $\mathrm{Ca} \mathrm{K}$ parameters), followed by a sharp recovery or spike in late spring (cf. Figure 8, from Lcan (1987)). I confirm in our F5 (Figure 9) the steep drop near the beginning of 1983 , which marks the end of the high fluxes that occurred during the epoch of solar maximum. There is no indication in the coronal data of sustained recovery from that low level in 1983 but only a brief burst of activity in May of that year (Figure 10), similar to the X-Ray spike seen in the NOAA GOES data presented by Fleck and Keil (cf. Wagner, 1988).
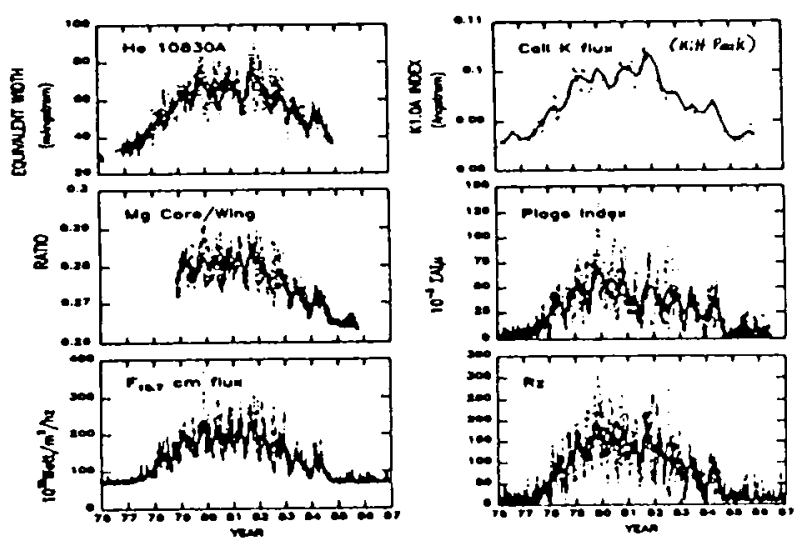

Fig. 8. Solar Cycle 21 as observed in several full-disk parameters (Lean, 1987).

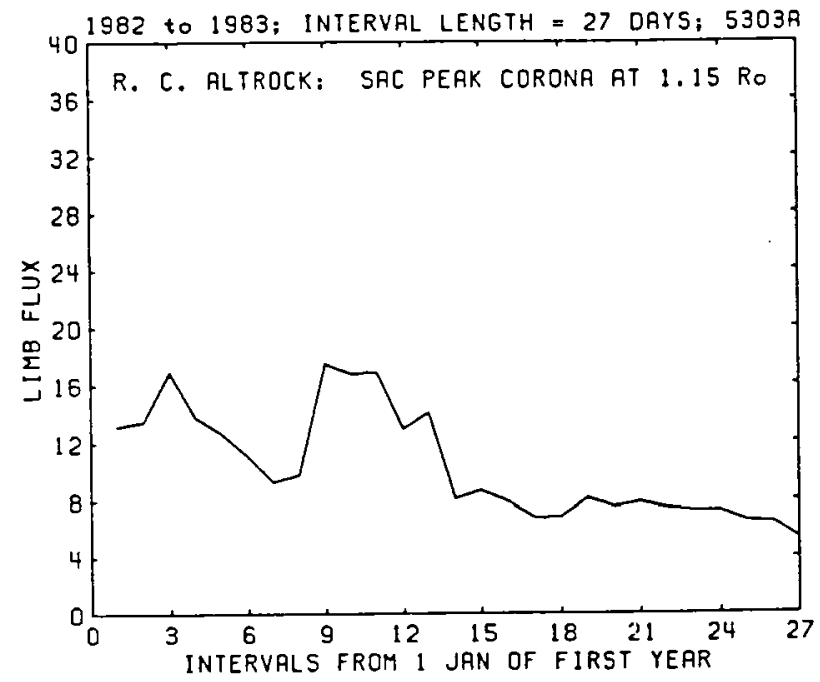

Fig. 9. 27-day-averaged F5 for 1982 and 1983.

Almost all of the data sets show a sharp rise to a plateau beginning in Jan 1984, and F5 also shows a $36 \%$ increase in the 27-day-average values during during the first half of 1984 (Figure 11). There is also a hint of the double maximum seen in many of the parameters, with F5 27-day-average values maximizing in Mar and May of that year. The large spike in F5 seen in Jan 1989 is matched by a similar peak in the Ca K EI that rises for the first time to "solar maximum" levels. In addition, the largest spike of Cycle 22 is seen in the GOES soft $\mathrm{X}$-ray data at that time, a pattern similar to that of the $\mathrm{Fe}$ XIV data. However, the preceding sharp dip in F5 is not matched by any other parameter and may be due to instrumental problems occurring at that time in the Fe XIV filter. The overall rise from solar minimum in 


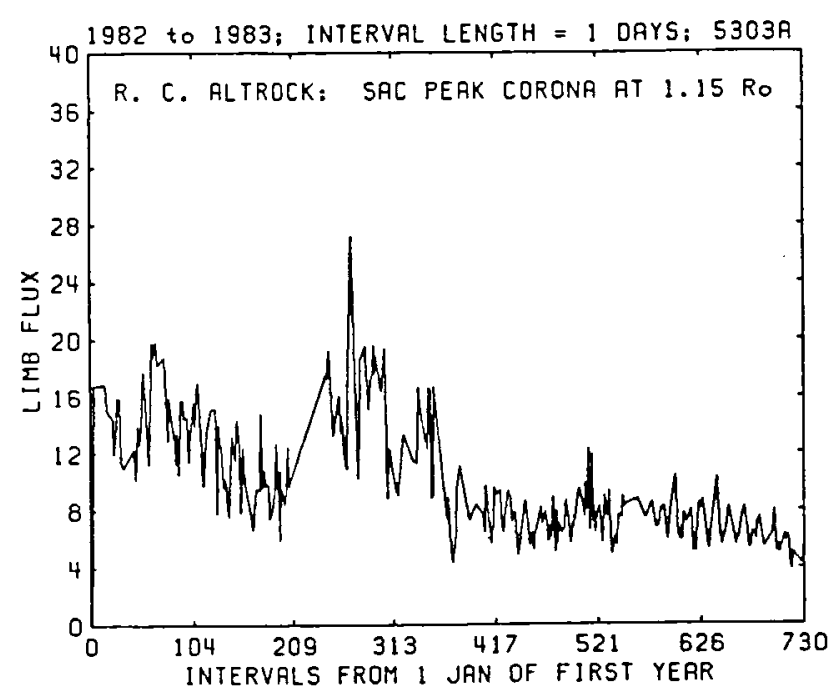

Fig. 10. Daily values of F5 for 1982 and 1983.

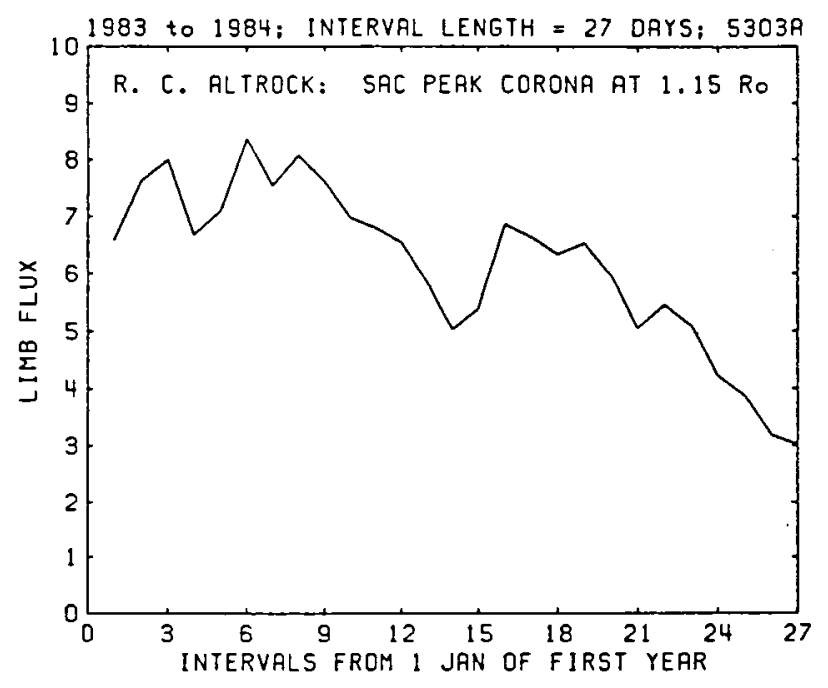

Fig. 11. 27-day-averaged F5 for 1983 and 1984.

1976 to solar maximum in late 1979 is $2184 \%$ in the 27-day-average values of F5, compared to an average rise of $19 \%$ in the Ca K EI. So far in Cycle 22, the increase from 1986 to 1989 in F5 has been 1564\%, and in $\mathrm{F} 6,1371 \%$.

\section{REFERENCES}

Gentili de Giuseppe et al:: Ann. d'Ap. 29, 43, 1966.

Lean, J.: Solar Radiative Output Variation, ed. P. Foukal, Cambridge Research and Instrumentation, Cambridge, MA, 113, 1987.
Rusin, V., Rybansky, M., and Strecko, J.: Solar and Stellar Coronal Structure and Dynamics, ed. R. Altrock, National Solar Observatory, Sunspot, NM, 392, 1988.

Smartt, R.N.: Proc. SPIE 331, 442-447, 1982.

Sjykora, J: Solar and Interplanetary Dynamics, cd. M. Dryer and E. Tandberg-Hanssen, D. Reidel, Dordrecht, Holland, 87-104, 1980.

Wagner, W. J.: Adv. Space Res. 8, No. 7, 67-76, 1988. 


\title{
AN ALTERNATIVE DERIVATION OF THE NIMBUS 7
} TOTAL SOLAR IRRADIANCE VARIATIONS

\author{
by \\ Douglas V. Hoyt \\ Research and Data Systems, Corp. \\ 7855 walker Drive, Suite 460 \\ Greenbelt, MD 20770 \\ and \\ H. Lee Kyle \\ Goddard Space Flight center \\ Greenbelt, MD 20771
}

\begin{abstract}
Nimbus 7 solar irradiance values have been made available to the scientific community through the open literature (e.g., Hickey et al., 1988) and through NASA data centers. A comparison of these measurements to the SMM/ACRIM time series indicated differences which might be caused in part by the method of converting the Nimbus 7 raw data counts to solar irradiance values. In an effort to see if the derivation of the solar irradiance could be improved, the raw counts were extracted from the tapes and analyzed to see how a new algorithm could be constructed. The basic form of the calibration remains the same as in the previous solar irradiance derivations. However, the input values to the equation differ from what was used before. In particular, improved values of the Earth-sun distance are incorporated and new temperature sensitivities were derived. Several problems with the instrument were uncovered which previously had not been noticed: 1) The sun did not appear to cross the center of field of the radiometer but was systematically off by 1.5 to 2.5 degrees. 2) The A/D convertor changed its properties in July 1980. 3) The gain of the electronics apparently increased by $0.03 \%$ in September 1987. Applying these and other changes in the processing, the day to day variations appear much more like the SMM observations. In fact, the Nimbus 7 observations are sufficiently stable that a problem with the SMM observations in the spin mode period of 1981-84 can be detected when the two time series are compared.
\end{abstract}


The major highlights of the method of reducing the instrument influences upon the measurements are described in section 2 . Because of space limitations some of these descriptions will be limited. A paper describing these techniques in more detail is now being prepared for publication in the refereed literature. The third section provides a comparison of some of the newly derived Nimbus 7 solar irradiance values to the SMM/Acrim measurements. The final section sums up our present status in understanding the instrument.

2. The Removal of Instrument Influences

The basic equation used to convert counts to irradiance is:

$$
S_{0}=\frac{0.998}{1.3013} r^{2} \frac{\left(C_{\text {sun }}-C_{\text {space }}\right)}{\cos (G)} \frac{1}{[1 .+A(T-22)]}
$$

where 1.3013 is the calibration constant, 0.998 is a correction constant to account for spurious reflections from the baffles into the cavity, $r$ is the Earth-sun distance, $c_{\text {sun }}$ is the mean on-sun counts, $C_{\text {space }}$ is the mean space look counts, A is the coefficient for the temperature sensitivity of the radiometer, $T$ is the temperature of the radiometer in degrees celsius, and $G$ is the offset angle between the normal vector to radiometer cavity and the vector to the sun. This equation is an attempt to remove all the portions of the counts signal which arises from the instrument and geometry so that only a pure signal arising from solar behavior remains.

If the Nimbus 7 radiometer had no pointing problems, then each time the sun drifted across its field of view it would pass through the center of the radiometer's field of view. Unfortunately, the sun normally appears to drift through the field of view on an off center cord or path. The angle between the radiometer-sun vector and the normal vector of the plane of the detector is known as the off-axis angle.

The off-axis angle is generally assumed to be measured by taking the difference between the so-called beta and gamma angles. The beta angle is angle between the plane of the satellite's orbit and the plane defined by the ecliptic. The beta angle is measured by the Digital Solar Aspect Sensor (DSAS). The gamma angle, on the other hand, is the angle between the axis of the solar telescope and the orbital plane and it can be varied only in one degree steps. If the two angles are equal, then the radiometer is assumed to be on-axis. In most cases the difference between these two angles is measured as less than one degree. The difference in the two angles provides a measure of the off-axis angle. If these offaxis angles are kept less than 0.5 degrees, then the cosine of 
these angles will be very close to one so the pointing correction will be negligible (e.g., 0.05 watts per square meter or less).

To derive what the off-axis angle really is, it is assumed that the solar constant and radiometer properties remain stable just before and just after the gamma angle change. It follows then that the ratio of the counts, corrected to one astronomical unit, will equal the ratio of the cosine of the off-axis angle prior to the change to the cosine of that angle plus one degree after the change. The only unknown is the off-axis angle, $G$, which can be solved for, using the following equation:

$$
G=\operatorname{atan}\left[\frac{-\frac{C_{1}}{C_{2}}+\cos (1)}{\sin (1)}\right]
$$

where $c_{1}$ is the mean on-sun counts prior to the gamma angle change and $\mathrm{C}_{2}$ is the mean on-sun count after the gamma angle change. In practice, we use $5,6,7,8$, or 9 orbits prior and after to form the two means of the counts. These five determinations are averaged together to derive an average off-axis angle. In effect, the observations closest to the gamma angle changes are more heavily weighted.

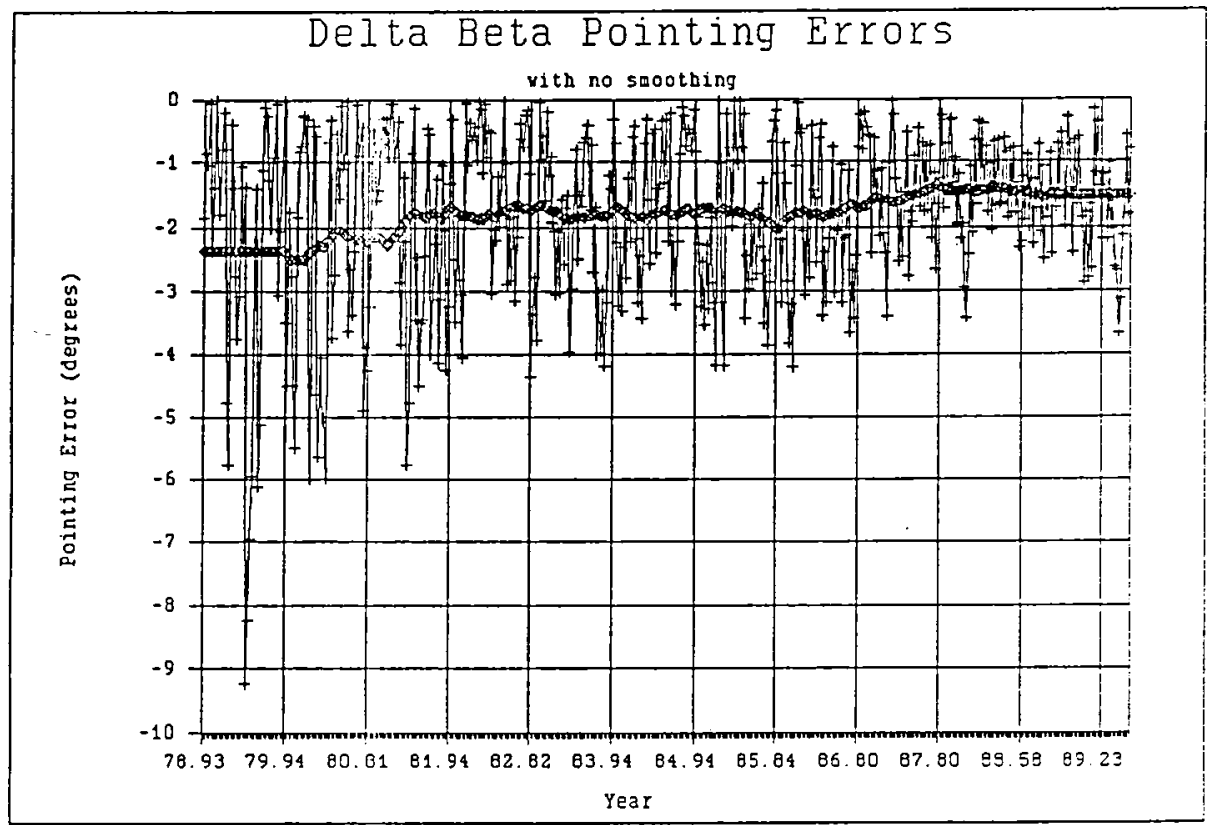

Figure 1. The off-axis angle vs. time using the change in gamma angles and change in counts to calculate the off-axis angle. The individual values show considerable scatter due to the limited resolution of the $A / D$ convertor, the limited number of useful measurements on each side of the gamma angle change, and spurious influences caused by high solar activity. An 81 point running mean is drawn through the points. 
Figure 1 shows the 236 determinations of the off-axis angle. An 81 point running mean is drawn through the points which shows the slow component of the off-axis angle variations. There is considerable scatter in the raw values but a systematic off-axis angle slowly comes closer to the zero as the experiment precedes.

The scatter in Figure 1 is reduced by forming a running mean of values. This procedure is justified since any individual offaxis angle is uncertain due to 1 ) the limited resolution of the A/D convertor, 2) possible variations in the solar irradiance during each determination, and 3) some small changes in radiometer temperature. Various lengths for the running mean were tried, but a length of 81 points was chosen since there are about 20 gamma angle changes per year and 81 covers 4 years reducing any signal from an annual variation. The radiometer's gamma angle was systematically too low by about 2.5 degrees early in the measurement period, but more recently it is off by only about 1.5 degrees.

Because the A/D convertor provides only a 0.7 watt per square meter resolution for any one measurement, when looking at space to determine the offset of the instrument, a large number of measurements is required to accurately determine the offset. It was decided that large blocks of time, usually one year's worth of data could be used to provide a measure of the offset. This procedure considerably reduces the day to day scatter in the solar irradiance measurements.

On July 20, 1980 the A/D convertor changed its properties such that it tended to round off its count values to the next higher digit more frequently than it would if this bias had not developed. Because both the on sun and zero counts were equally effected, the result is not readily evident in most derivations. However, if we use large blocks of time to measure the zero offset, the zero offset must be determined for the period January 1 to July 20 and from July 21 to December 31,1980 separately. Otherwise, an artificial trend could be introduced into the solar irradiance values for 1980 .

Assuming the calibration constant of channel $10 \mathrm{c}$ remained unchanged throughout the mission and calculating the solar irradiance, it varies smoothly except for an upward jump of $0.03 \%$ in irradiance on september 26, 1987. The change in sensitivity is confirmed by the electrical calibrations, so its effect is removed by having a new calibration constant after this date.

The radiometer is slightly sensitive to temperature since the cavity sees the field-of-view limiters which have different temperatures for each solar irradiance measurement. The temperature sensitivity can be determined by two techniques: 1) Assume the solar irradiance is a constant and the unexplained variations arise from the radiometer temperature variations and 2) use the space look observations and the temperature measurements and derive a fit between the counts and the temperature. Both 
techniques were, tried and gave nearly the same answers. The coefficient " $A$ " in the calibration is found to be 0.001998 for temperatures above $18 \mathrm{C}, 0.0002713$ for temperatures between 15 and $18 \mathrm{C}$, and 0.0003086 for temperatures below $15 \mathrm{C}$ based upon the first technique and using all observations between November, 1978 and December, 1988. Using the second technique, the temperature sensitivity above $18 \mathrm{C}$ for 1988 is 0.000196 . The second technique is actually less accurate than the first technique, because of the limited resolution of the A/D convertor. Therefore, the temperature coefficients from the first technique are used.

Previously published solar irradiance measurements assumed a single value of 0.000524 for temperatures of the radiometer. This value was based upon a few determinations in 1978 and 1979. Smith et al. (1983) previously pointed out that a linear relationship of the radiometer is not valid since the transfer of radiation from the field-of-view limiters to the cavity is done radiatively and is thus proportional to the blackbody flux which depends upon temperature raised to the fourth power. By using a linear relationship and splitting it into three ranges, much of this nonlinearity is captured. However, there are several methods which may be used to derive the temperature sensitivity of the instrument and the problem continues to be studied. Future studies may improve our understanding of the temperature sensitivity of the instrument.

Finally, the derived solar irradiance measurements were improved by using more precise Earth-sun distance calculations. This distances are now accurate to eight significant figures and are calculated for each orbit rather than for each day as was previously done.

\section{A Comparison to the SMM/ACRIM Measurements}

Daily solar irradiance values from the SMM are available for the period February 1980 to December 1988 (e.g., Willson and Hudson, 1988). These independent solar irradiance measurements are numerous enough to provide a good comparison to the Nimbus 7 measurements. Figure 2 shows the measurements for 1988. The correlation between these two measurements is 0.87 for this year. overall these two independent sets of measurements show good agreement. This increases our confidence that the true solar behavior is being tracked.

Figure 3 provides a plot of the Nimbus 7 and SMM monthly mean solar irradiance values. The two curves are quite similar with both instruments indicating a rise in solar irradiance in the latest cycle to a value less than that in the previous cycle. In Figure 4, the differences in monthly means is plotted. These differences are about 4 to 5 watts per square meter when the SMM pointing is known to be correct and slightly less than 4 watts per square meter during the period 1981 to 1984 when the SMM had 
N-7 and SMM 1988 Solar Irradiances

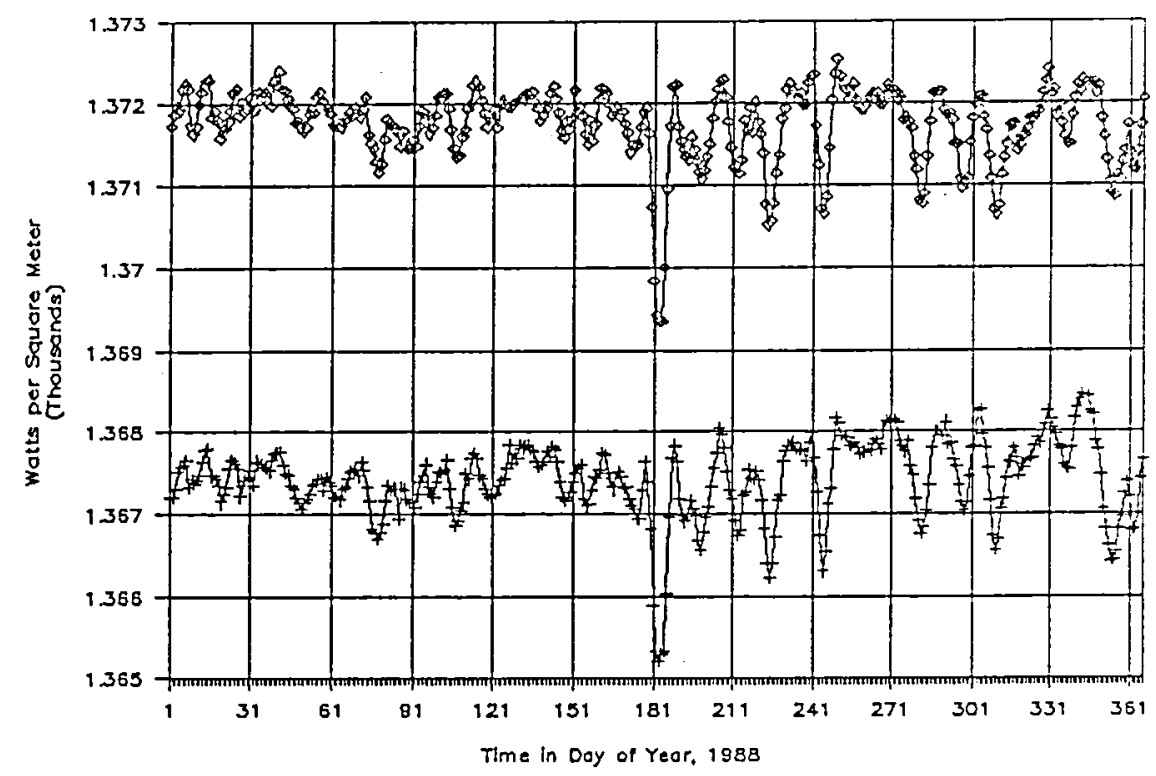

Figure 2. A comparison of the Nimbus 7 and SMM solar irradiance values for 1988 on the same plot. Day to day variations have a correlation of 0.87 for this year.

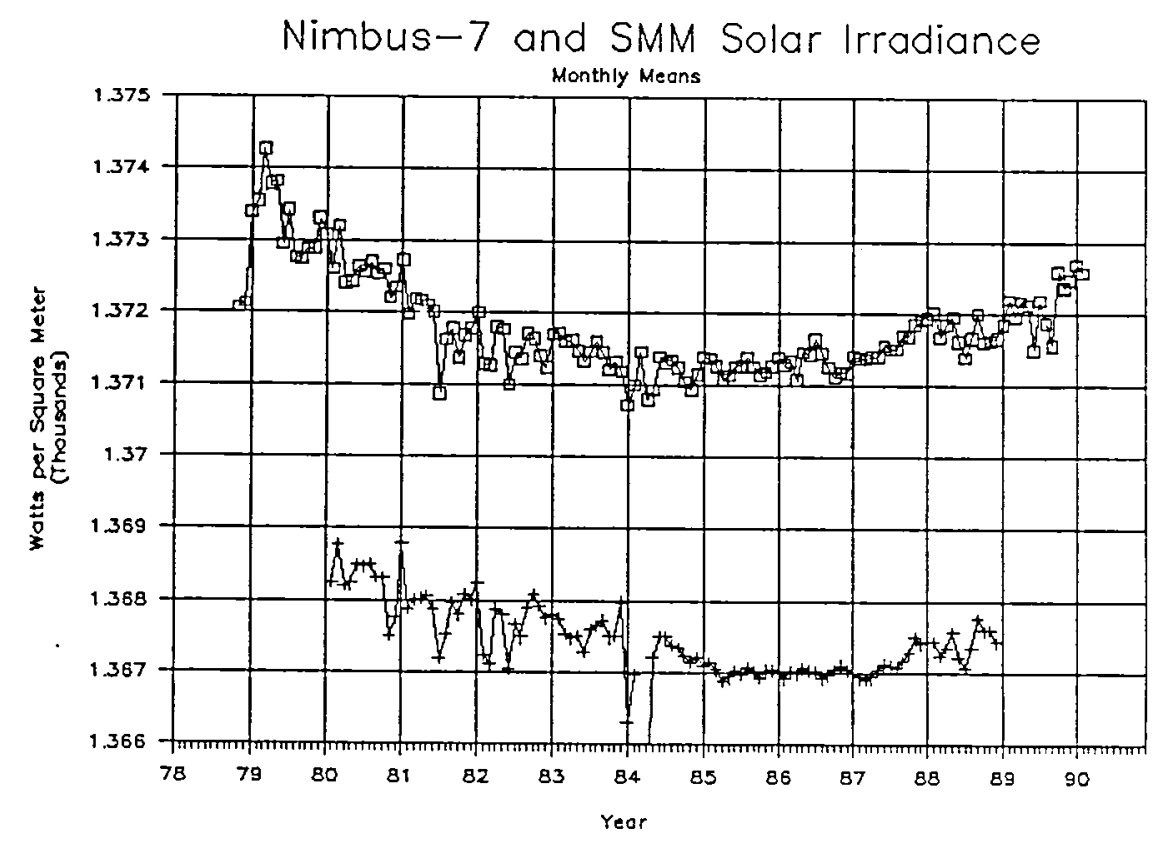

Figure 3. The Nimbus 7 and SMM monthly means for 1978 to 1989. Both instruments are indicating similar solar behavior except for a few periods such as early 1980, late 1983, mid-1986, and late 1988 . 
pointing problems. During the quiet sun, the SMM radiometer has smaller month to month variations than does the Nimbus 7 radiometer. This may indicate that some residual pointing errors still exist in the Nimbus 7 measurements. The rise in Nimbus 7 measurements in August 1987, which does not occur in the SMM measurements, could be caused by an undetected 0.1 degree error in the Nimbus 7 pointing. Alternatively, since this rise was evident just after a special operations period, the Nimbus 7 radiometer's thermal properties may have changed for a time and relaxed back to normal after several months. In late 1988 there is a small divergence between the two measurements for as yet unknown reasons.

Despite these divergences the agreement is still rather good. The correlation of the monthly means is 0.867 for 1980 to 1983 , 0.014 from 1984 to 1986 after the SMM pointing was restored but the sun remained quiet, and 0.816 in 1987-88 when solar activity resumed. The low correlation in 1984-86 arises because the variability in the solar irradiance became less than the noise in the measurements, particularly in the Nimbus 7 measurements. As a comparison, the older published Nimbus 7 irradiances had correlations with SMM of $0.863,0.000$, and 0.836 respectively for the three periods. Much of these correlations are arising from common long-term trends. Using daily values for 1988, the new algorithm has a 0.87 correlation with SMM whereas the old algorithm has a 0.75 correlation.

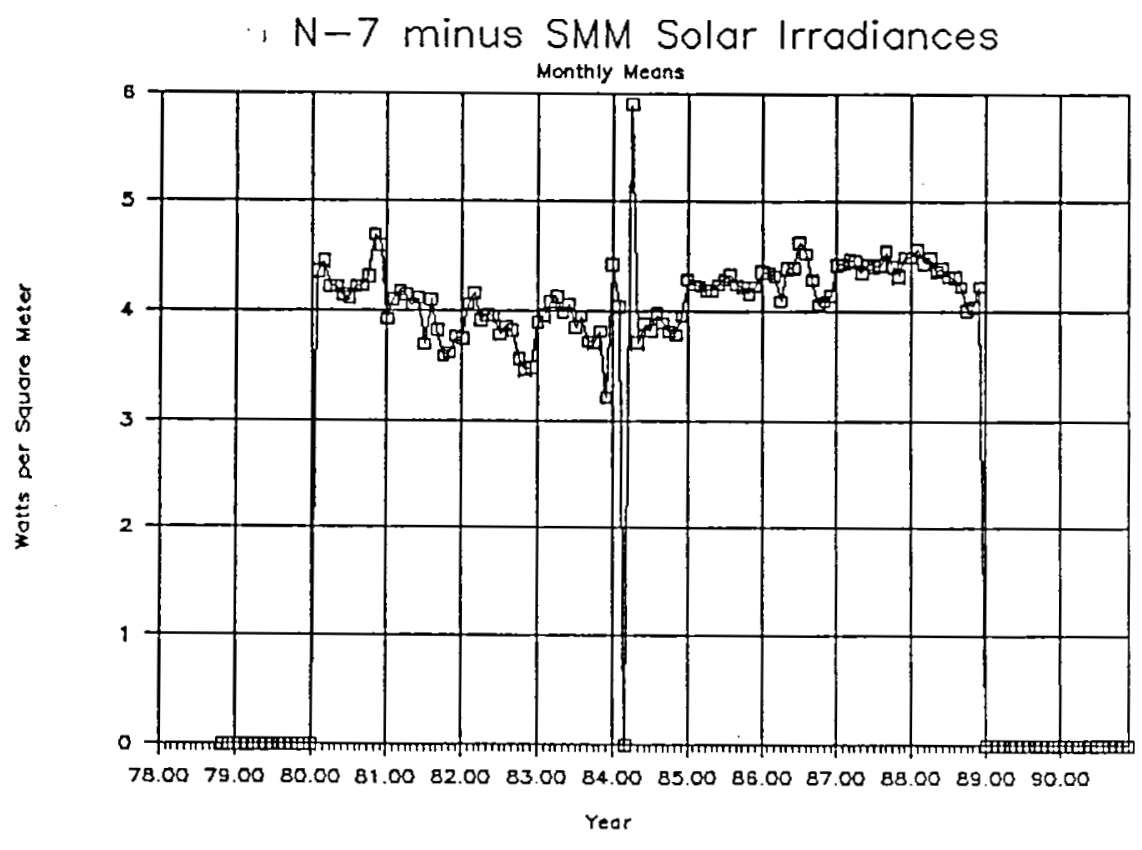

Figure 4. The Nimbus 7 monthly mean solar irradiance minus the monthly mean SMM solar irradiance. Most differences are between 4 and 5 watts per square meter except for 1981 to 1984 when the SMM had pointing problems. 


\section{Concluding Remarks}

A new algorithm to derive the solar irradiance from the Nimbus 7 raw data is reported upon here. The algorithm removes more of the instrumental and geometrical influences upon the measurements than did the old algorithm so that the true solar behavior becomes more apparent. Changes in the analysis include: 1) better Earthsun distance determinations, 2) improved temperature sensitivity coefficients, and 3) re-calculated off-axis angles. These algorithm changes give substantially smoother day to day variations in the solar irradiance and a greater correlation with the SMM observations. The Nimbus 7 long-term trends in solar irradiance are different then previously calculated and now generally closer to the SMM observations. These changes increase our confidence that both satellites are measuring a real solar signal. Despite nearly equal solar activity for the last two solar cycles based upon Wolf sunspot numbers, the solar irradiance for the two cycles are not equal. Both the Nimbus 7 and SMM satellites appear to confirm this behavior.

Considering that the SMM measurements suffered from pointing problems between 1981 and 1983, it is reasonable to suggest that the Nimbus 7 solar irradiances derived for this time period are a better measure of true solar behavior. Most of the differences between the two measurements in the 1981-83 time period may be arising from random errors in the SMM measurements which has a greater day to day variability than the Nimbus 7 measurements.

Although some of the problems with the Nimbus 7 measurements are removed in this analysis, insufficient information exists to remove all the instrument influences on the solar irradiance determinations. In particular, the off-axis angle corrections remove the short-term and long-term variations in the off-axis angle, but the intermediate variations (months to two years) may not be fully removed. With the limited number of independent measurements of the off-axis angle (236) and the limited accuracy of each off-axis angle determination, it does not appear that these intermediate term variations can be removed completely.

\section{References:}

Hickey, J. R., B. M. Alton, H. L. Kyle, and D. V. Hoyt, 1988 . Total solar irradiance measurements by ERB/Nimbus-7. A review of nine years. Sp. Sci. Rev., 48, 321-342.

Smith, E. A., T. H. Vonder Haar, and J. R. Hickey, 1983. The nature of the short period fluctuations in solar irradiance received by the Earth. Climatic Change, 5, 211-236.

Willson, R. C. and H. S. Hudson, 1988, Solar luminosity variations in solar cycle 21 . Nature, $332,810-812$. 
LONG-TERM SOLAR IRRADIANCE VARIABILITY: 1984-1989 OBSERVATIONS

\author{
Robert B. Lee III \\ Atmospheric Sciences Division, NASA Langley Research Center \\ Hampton, Virginia 23665-5225
}

\begin{abstract}
Long-term variability in the total solar irradiance has been observed in the Earth Radiation Budget Experiment (ERBE) solar monitor measurements. The monitors have been used to measure the irradiance from the Earth Radiation Budget Satellite (ERBS) and the National Oceanic and Atmospheric Administration NOAA-9 and NOAA-10 spacecraft platforms since October 25, 1984, January 23, 1985, and October 22, 1986, respectively. Before September 1986, the ERBS irradiance values were found to be decreasing -0.038 per year. This period was marked by decreasing solar magnetic activity. Between September 1986 and mid-1989, the irradiance values increased approximately $0.1 \%$. The latter period was marked by increasing solar activity which was associated with the initiations of the sunspot cycle number 22 and of a new 22-year Hale solar magnetic cycle. Therefore, long-term solar-irradiance variability appears to be correlated directly with solar activity. The maximum smoothed sunspot number occurred during September 1989, according to the Sunspot Index Data Center. Therefore, the recent irradiance increasing trend should disappear during early 1990 and change into a decreasing trend if the observed irradiance variability is correlated more so with the 11-year sunspot cycle than the 22 -year Hale cycle.
\end{abstract}

The ERBE solar monitors are the most recent pyrheliometers, active cavity radiometers, to be placed into orbit to continuously measure the irradiance. They are sensitive to irradiance in the 0.2 to $50+$ micrometer spectral broadband region. The precision of a single ERBS irradiance measurement has been estimated to be less than 0.028 . It is expected that at least one of the three monitors will extend the ERBE irradiance time series into the year 1998.

In this paper, the ERBE irradiance values are presented and compared with sunspot activity for the 1984-1989 period. The ERBE values are compared with those available from the Nimbus -7 and Solar Maximum Mission spacecraft experiments.

\title{
1. INTRODUCTION
}

At the top of the atmosphere, the Earth Radiation Budget Experiment (ERBE) ${ }^{1}$ is measuring the three components of the Earth radiation budget: the incoming total solar irradiance, the Earth/atmosphere-reflected solar irradiance, and the Earth/atmosphere-emitted radiant exitance. The irradiance measurements are designed to detect long-term changes in the irradiance magnitude and to produce reference measurements for the calibration of the ERBE scanning and nonscanning radiometers. The reference measurements are obtained using the solar monitor ${ }^{2}$. The monitors are located on the Earth Radiation Budget Satellite (ERBS), NOAA-9, and NOAA-10 spacecraft platforms which were launched October 5, 1984, December 12, 1984, and September 17, 1986, respectively. The monitors are active-cavity-type radiometers and recent versions of the active-cavity radiometer irradiance monitors (ACRIM) which were flown on the Solar Maximum Mission (SMM) spacecraft. 
The monitors' total solar irradiance measurements are performed every 14 days during a single orbit for a 128 - to 640 -second period. The measurement period is divided into 64-second cycles. Each cycle consists of a 32-second solar irradiance measurement phase and a 32-second reference (near zero irradiance) phase. During the irradiance phase, the shutter of the monitor is opened and the solar irradiance is sensed by the monitor. During the reference phase, the shutter is closed and the near zero irradiance, which is emitted by the back of the aluminum shutter, is sensed. During the measurement period, the Sun drifts through the unobstructed field of view of the monitor which is \pm 4.6 angular degrees. The angular position of the Sun with respect to the optical axis has to be considered since the response of the monitor varies as the cosine of the angular position. The data reduction model and solar monitors are described by Lee et al. ${ }^{2}$

\section{ERBE SOLAR IRRADIANCE MEASUREMENTS}

In Figure 1, the ERBS, NOAA-9, and NOAA-10 irradiance values, normalized to 1 astronomical unit, are presented as a function of time. The irradiance values are available from the National Space Science Data Center (NASA Goddard Space Flight Center, Greenbelt, MD 20771) and the NOAA Solar Terrestrial Data Center ${ }^{3}$. The ERBS time series covers the October 25, 1984, through August 30, 1989, period, while the NOAA-9 one covers the January 23, 1985, through August 30, 1989, period. The NOAA-10 data set covers the October 22, 1986, through April 1, 1987, period. The mean irradiance values for the ERBS, NOAA-9, and NOAA-10 time series are 1365.1

$\pm 0.6,1364.8 \pm 0.8$, and $1363.2 \pm 0.3 \mathrm{Wm}^{-2}$, respectively. The bars on each time series denote the measurement precisions of $0.2,0.5$, and $0.8 \mathrm{Wm}^{-2}$ for the ERBS, NOAA-9, and NOAA-10 monitor data sets, respectively. The absolute uncertainty of the monitor measurements has been estimated to be $2.7 \mathrm{Wm}^{-2}(0.28)$.

In Figure 1, three features are significant. The first feature is that the NOAA-10 irradiance data set covers only 6 months. On April 1, 1987, its shutter mechanism failed. During pre-launch tests, the mechanism exhibited operational problems. The ERBS and the NOAA-9 monitors have not exhibited any operational problems with the shutter. The second feature is that the NOAA-10 mean irradiance value is approximately $2 \mathrm{Wm}^{-2}$ lower than the mean for either the ERBS or NOAA-9 data set. The absolute uncertainty of the monitor has been estimated to be $2.7 \mathrm{Wm}^{-2}$. Therefore, the agreement among the three data sets is excellent and well within 0.28 . The third most obvious feature is the long-term decreasing and increasing trends. In the following paragraphs, the long-term trends are discussed using the ERBS and NOAA-9 irradiance data sets.

In Figure 2, the NOAA-9 irradiance values are presented for the 1985 through 1989 period. The previously mentioned decreasing and increasing trends are highlighted by the second order polynomial fit through the data. Applying least squares analyses to the data, the irradiance was found to be decreasing at a rate of $-0.05 \%$ per year before September $1986^{4}$ and increasing at a rate of +0.038 after August $1986^{5}$. The correlation coefficients for the decreasing and increasing trends are 0.52 and 0.42 , respectively. In Figure 1, it can be seen that the NOAA-9 data set exhibited more scatter than the data sets for the ERBS and NOAA-10, especially after 1986. It is believed that the increased scatter was caused by the spacecraft solar panels occasionally interfering with the solar monitor field of view. In Figure 1 , the measurement precision bars indicate that the ERBS data set is the best one to evaluate the irradiance trends which are prominent in both ERBS and NOAA-9 data sets. 
In Figure 3, the ERBS irradiance values are presented for the 1984 through 1989 period. The second order polynomial fit accents the long-term decreasing and increasing trends which were noticeable in the NOAA-9 data. Since the precision of the ERBS set is better than that for NOAA-9, the ERBS fit can be used to establish mid-1986 as the time of irradiance minimum. Applying the method of least squares, the slope of the ERBS decreasing trend was found to be -0.038 per year 4,6 before September 1986. After August 1986, the slope of the increasing trend was found to be approximately $+0.038^{5}$. For the two periods, the correlation coefficients were found to be 0.56 and 0.63 , respectively. In the ERBS and NOAA-9 data sets, the magnitude of the irradiance increased approximately $1.3 \mathrm{~W} / \mathrm{m}^{-2}(0.18)$ between 1986 and 1989. Both the increasing and decreasing trends have been observed in the Nimbus $-7^{7}$ and $\mathrm{SMM}^{8}$ irradiance data sets. These trends were not caused by instrument degradation ${ }^{5}$. They indicate solar variability.

In Figure 3, the ERBS irradiance values are compared with smoothed international sunspot number ${ }^{9}$, an index of solar magnetic activity. The smoothed sunspot number represents a 12 -month running mean. On a long-term scale, the comparison emphasizes that the irradiance varies directly with the sunspot number. The minimum sunspot number occurred during September $1986^{10}$ which marked the end of sunspot cycle 21. Thereafter, cycle 22 started with rapidly increasing sunspot activity. September 1986 also marked the beginning of a new 22-year Hale magnetic cycle. Cross correlating the ERBS data set with the sunspot numbers, the method of least squares yields a positive correlation coefficient of +0.57 for the decreasing trend and a positive correlation coefficient of +0.59 for the increasing trend between irradiance variability and sunspot activity. It is believed that the resulting correlation coefficients are reasonable when one considers that the irradiance varies inversely $2,7,8$ with sunspot number over periods of the order of the 27 -day rotational period of the Sun. In Figure 3, the two isolated, low ERBS irradiance values for December 21, 1988, and June 16, 1989, were caused by large sunspot groups crossing the solar disk. The sunspot blocking is considered to cause the irradiance to decrease as large numbers of sunspots drift across the solar disk into the effective field of view center and to cause the irradiance to increase as the sunspots drift out of the field-of-view 11 . Sunspots emit less energy than the surrounding photosphere because they are approximately 2000 degrees Celsius cooler. In the vicinity of sunspots, faculae are the bright features which emit more energy than the surrounding photosphere. On the short-term scale, sunspot blocking dominates faculae brightening 11,12 . On the long-term scale, faculae dominate because they have a significant longer lifetime than sunspots. The reader is referred to Willson and Hudson $^{8}$, Schatten ${ }^{13}$, and Foukal and Lean ${ }^{14}$ for reviews of efforts to model the long-term and short-term irradiance variability.

\section{COMPARISONS AMONG ERBS, NIMBUS-7, AND SMM IRRADIANCE DATA SETS}

In Figure 4, the ERBS, Nimbus-7 channel 10c, and SMM ACRIM irradiance data sets are presented. The bars denote the estimated absolute accuracy of each set. The Nimbus-7 and SMM data sets were obtained from the National Space Science Data Center. There is reasonable agreement among the three data sets since they are found to be within approximately 0.58 of each other. The best accuracy characterization of any room temperature radiometer is its response in measuring the theoretical value of the Stefan-Boltzmann constant. The best room temperature experimental measurement of the Stefan-Boltzmann constant differed from the theoretical value by 0.3815 . The ERBS, Nimbus -7 , and SMM radiometers are room temperature devices. At the solar irradiance value of $1365 \mathrm{Wm}^{-2}$, the absolute accuracy would be about $4 \mathrm{Wm}^{-2}$. Therefore, the differences between the radiometers which were characterized independently would not 
be expected to agree much closer than $0.3 \%$. It should be pointed out that the long-term measurement precision and the bias constancy among the data sets are just as important as the absolute accuracy in characterizing the long-term variability of solar irradiance. The 5 years of overlap among the three experiments will allow additional precise comparisons to be performed among the irradiance time series as was done by Mecherikunnel et a1 ${ }^{16}$ for the 1984-1987 period.

The ERBS data set exhibited comparably more scatter than the Nimbus-7 and SMM because the ERBS values represent instantaneous values and not daily averages. On a single day, the Nimbus-7 data sampling is 7 times greater than the ERBS sampling.

Therefore, Nimbus-7 scatter statistically should be approximately one-third of that for the ERBS. The SMM daily sampling is 200 times greater than that for the ERBS. Therefore, the SMM scatter should be statistically one-fourteenth of that for the ERBS. The sampling rates are important when the magnitude of the scatter in the data is considered. For example, the SMM experiment did not track the Sun between November 1980, when its solar tracking mechanism failed, and April 1984 when the mechanism was repaired. During this period, its measurement sampling was reduced by approximately $99.958^{17}$ compared to the samplings before and after the non-tracking period. Accordingly, the SMM scatter was statistically higher during the November 1980 to March 1984 period as indicated in Figure 4. Sampling affected the Nimbus-7 data set also. For example, the Nimbus-7 scatter decreased noticeably after September 1983 when its sampling rate was increased to daily.

The ERBS and NOAA-9 data sets, with considerably lower sampling rates, exhibited similar long-term irradiance trends which are found in the Nimbus-7 and SMM sets. Applying the method of least squares, the Nimbus-7 and SMM data sets yielded decreasing irradiance trends of -0.0138 and $-0.016 \%$ per year before September 1986 with correlation coefficients of 0.56 and 0.63 , respectively. After August 1986, the rates for the increasing irradiance trends were found to be +0.048 and +0.028 per year for the Nimbus-7 and SMM data sets with correlation coefficients of 0.69 and 0.48. With lower sampling rates, the ERBS and NOAA-9 sets were found to have similar trend results which were described earlier.

According to Sunspot Index Data Center, a smoothed maximum sunspot number of 165 occurred during December 1979, maximum for sunspot cycle 21. A maximum value of 161 occurred during September 1989, maximum for sunspot cycle 22. If irradiance variability can be correlated strongly with sunspot number, then the magnitude of the irradiance should be essentially identical for December 1979 and September 1989. To explore this concept, the ERBS irradiance variability was characterized with cosine functions for 11 - and 22-year cycles. The cosine parameters for the 11-year characterization are described in the following sentences. During the periods of decreasing sunspot numbers for sunspot cycles 21 and 22 , I have assumed that (1) the period of the cosine function is equal to twice the time differential between December 15, 1979, and September 15, 1986; (2) the amplitude of the cosine function is equal to 0.058 of the mean irradiance magnitude; and (3) there is zero phase angle between the irradiance and sunspot variability. During the periods of increasing irradiance for sunspot cycles 21 and 22, I have assumed that (4) the period is equal to twice the time differential between September 15, 1986, and September 15, 1989; (5) the amplitude is equal to $0.05 \%$ of the mean irradiance value; and (6) the phase angle is equal to zero. For the 22-year characterization, the period is assumed to be twice those for the 11-year ones with an amplitude which is equal to $0.15 \%$ of the mean irradiance value.

The resultant cosine characterizations of the ERBS data are presented in the lower portion of Figure 5. Both the 11-year and 22-year characterizations fit the ERBS 
data. The ERBS data set does not extend back to the 1979 through 1984 period. It cannot be used to evaluate the characterizations for that period. Therefore, the characterizations are compared with the longer Nimbus-7 and SMM data sets. Both the 11-year and 22-year characterizations provided good fits to the SMM data set. The SMM data set cannot be used to clearly identify the period of the long-term variability as 11 or 22 years. However, the comparisons with the Nimbus-7 data set suggest that the irradiance variability correlates closer with the 11 -year cycle than the 22-year one. Therefore, it is reasonable to assume that the irradiance variability follows an 11-year cycle. Since the SMM experiment failed during late 1989, the Nimbus-7 and ERBE data sets will have to be relied upon to verify the 11-year irradiance variability and to detect possible 22-year variability.

\section{CONCLUSIONS}

The ERBS, NOAA-9, and NOAA-10 solar monitors have been used to derive the magnitude of the total solar irradiance, normalized to 1 astronomical unit, between 1365 and $1363 \mathrm{Wm}^{-2}$ which fall within the $0.28\left(2.7 \mathrm{Wm}^{-2}\right)$ estimated measurement accuracy. The long-term measurement precisions of the monitors were demonstrated by their ability to detect irradiance variability in the 0.028 to $0.05 \%$ per year rate range. The observed 1984-1986 decreasing and 1986-1989 increasing trends are of solar origins and represent irradiance variability which is directly correlated with solar magnetic activity associated with the 11 -year sunspot cycle.

The variability has been measured at the 0.18 level between sunspot minimum for sunspot cycle 21 and maximum for cycle 22. Comparisons of the Nimbus-7 irradiance magnitudes near sunspot maxima for cycles 21 and 22 indicated that the irradiance variability may differ during the remainder of cycle 22 and during cycle 23 from that which was observed during the 1979-1989 period. Therefore, it is important that the ERBE irradiance measurements be extended into the late 1990's in order to refine the characterization of long-term irradiance variability. The extension of the irradiance data set will have to be carried out by at least one of the three ERBE monitors because the SMM experiment was terminated in late 1989 and because the aging Nimbus-7 experiment may experience a failure in the near future similar to the one which occurred during September 1989.

\section{REFERENCES}

1. B. R. Barkstrom, E. F. Harrison, and R. B. Lee III, "The Earth Radiation Budget Experiment (ERBE) Preliminary Seasonal Results," Eos, vol. 71, pp. 297-305, 1990.

2. R. B. Lee III, B. R. Barkstrom, and R. D. Cess, "Characteristics of the Earth Radiation Budget Experiment Solar Monitor," Applied Optics, vol. 26, no. 7, pp. 3090-3096, 1987.

3. H. E. Coffey, Solar-Geophysical Data Comprehensive Reports, National Oceanic and Atmospheric Administration (Boulder, Colorado), report no. 538, part II, pp. 101-113, June 1989.

4. R. B. Lee III, M. A. Gibson, S. M. Natarajan, "Total Solar Irradiance Values Determined Using Earth Radiation Budget Experiment (ERBE) Radiometers, " $\mathrm{New}$ Developments and Applications in Optical Radiometry, editors N. P. Fox and D. H. Nettleton, Institute of Physics Conf. Series no. 92, Bristol and Philadelphia, pp. 129-132, 1989. 
5. R. B. Lee III, M. A. Woerner, M. A. Gibson, S. Thomas, and R. Wilson, "Total Solar Irradiance Variability: 5 Years of ERBE Date," Proc. of July 23-27, 1990 Seventh Conf. on Atmospheric Radiation, American Meteorological Society, Boston, MA, 1990 (In Press).

6. R. B. Lee III, B. R. Barkstrom. E. F. Harrison, M. A. Gibson, S. M. Natarajan, W. L. Edmonds, A. T. Mecherikunnel, and H. L. Kyle, "Earth Radiation Budget Satellite Extraterrestrial Solar Constant Measurements: 1986-1987 Increasing Trend," Advances in Space Research, vol. 8, no. 7, pp. 11-13, 1989.

7. J. R. Hickey, b. M. Alton, H. L. Kyle, and D. Hoyt, "Total Solar Irradiance Measurements by ERB/Nimbus-7: A Review of Nine Years," Space Science Reviews, vo1. 48, pp. 321-342, 1988.

8. R. C. Willson and H. S. Hudson, "Solar Luminosity Variations in Solar Cycle 21," Nature, vol. 332, pp. 810-812, 1988 .

9. A. Koeckelenberg, Sunspot Bulletin, Sunspot Index Data Center, Brussels, Belgium, May 1, 1984 through February 1, 1990.

10. A. Koeckelenberg, Sunspot Bulletin, Sunspot Index Data Center, Brussels, Belgium, March 1988 .

11. H. S. Hudson, "Energy Balance in Solar Active Regions: the Dip of Apri1 1985," Advances in Space Research, vol. 6, no. 8, pp. 81-83, 1987.

12. R. C. Willson, S. Gulkis, M. Jansen, H. S. Hudson, and G. A. Chapman, "Observations of Solar Variability," Science, vol. 211, pp. 700, 1981.

13. K. H. Schatten, "A Model for Solar Constant Secular Changes, " Geophysical Research Letters, vol. 15, pp. 121-124, 1988.

14. P. Foukal and J. Lean, "An Empirical Model of total Solar Irradiance Variation Between 1874 and 1988," Science, vo1. 247, pp. 556-558, 1990.

15. J. M. Kendal1, and M. Berdah1, "Two Blackbody Radiometers of High Accuracy," Applied Optics, vol. 9, no. 5., pp. 1082-1091, 1970.

16. A. T. Mecherikunnel, R. B. Lee III, H. L. Kyle, and E. R. Major, "Intercomparisons of Solar Total Irradiance Data from Recent Spacecraft Experiments," J. Geophysical Research, vol. 93, no. D8, pp. 9503-9509, 1988.

17. R. C. Willson, H. S. Hudson, C. Frohlich, and R. W. Brusa, "Long-term Downward Trend in total Solar Irradiance," Science, vol. 234, pp. 1114-1117, 1986.

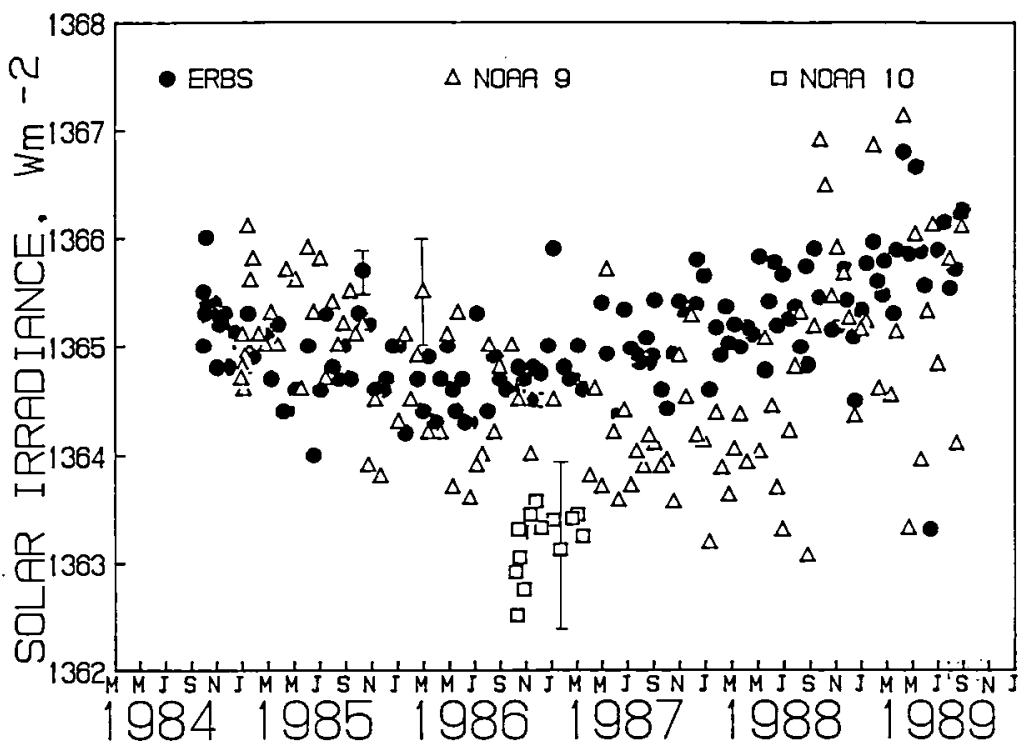

Figure 1. ERBS, NOAA-9, and NOAA-10 solar monitor irradiance measurements presented for the October 1984 through August 1989 period. The bars denote the measurement precisions for each data set. 


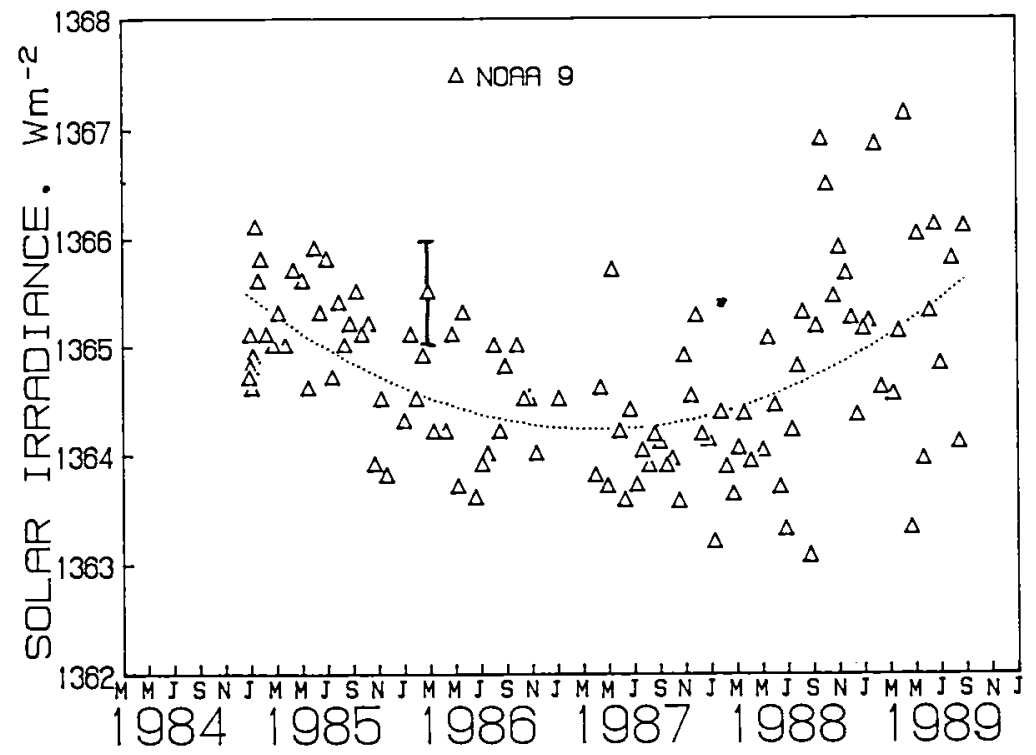

Figure 2. NOAA-9 solar monitor irradiance values for the January 23, 1985, through August 30, 1989, period. The curve represents a second order polynomial fit through the values. The bar denotes the typical measurement precision of $0.5 \mathrm{Wm}^{-2}$.

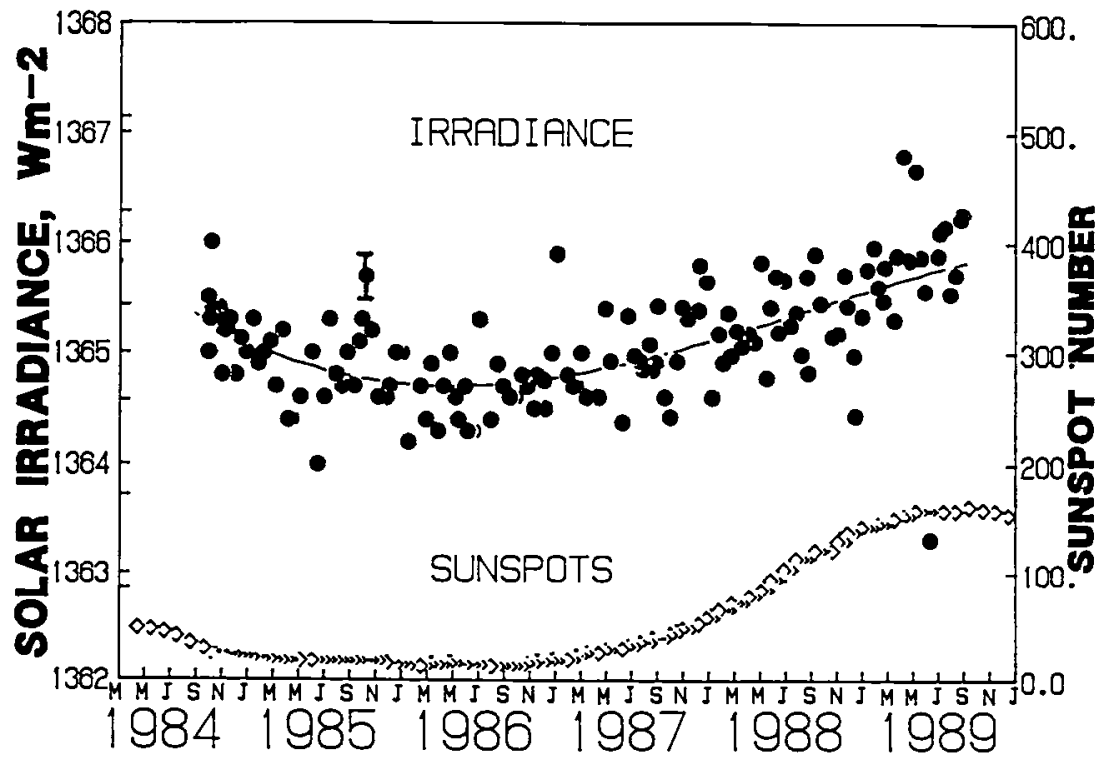

Figure 3. ERBS solar monitor irradiance values are presented for the October 25 , 1984, through August 30, 1989, period. The bar denotes a typical measurement precision of $0.2 \mathrm{Wm}^{-2}$. ERBS irradiance values are compared with smoothed international sunspot number. 


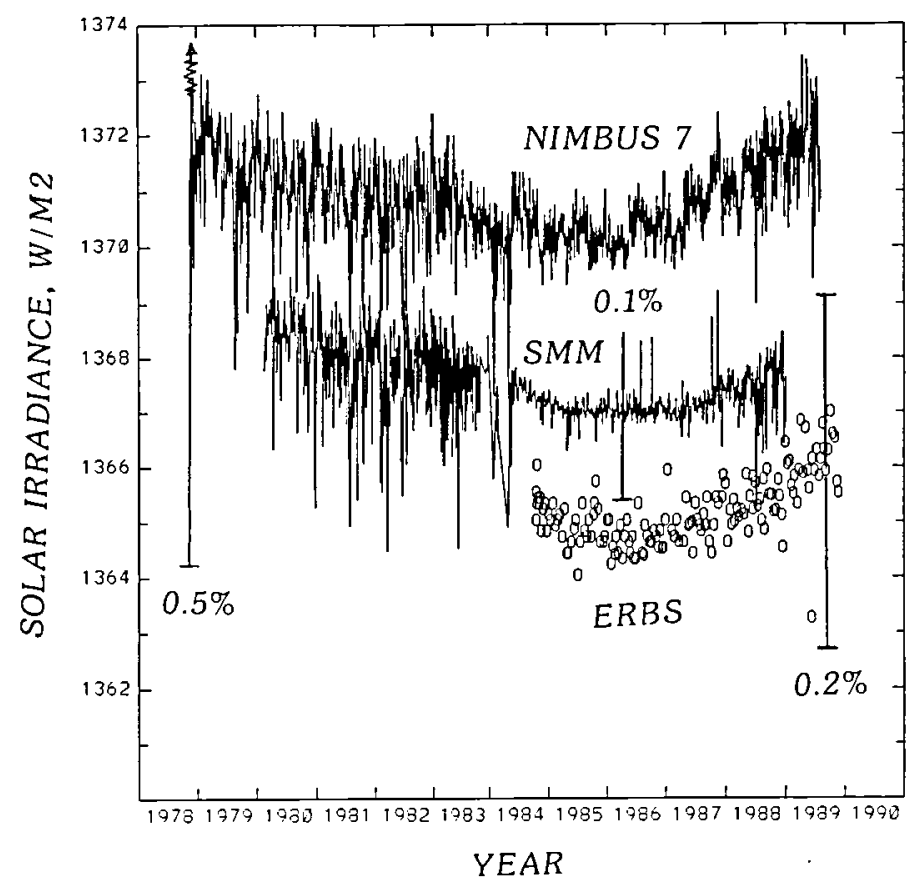

Figure 4. Solar irradiance values from the ERBS, Nimbus-7, and SMM experiments are compared. The bars denote the absolute accuracies of the irradiance data sets.

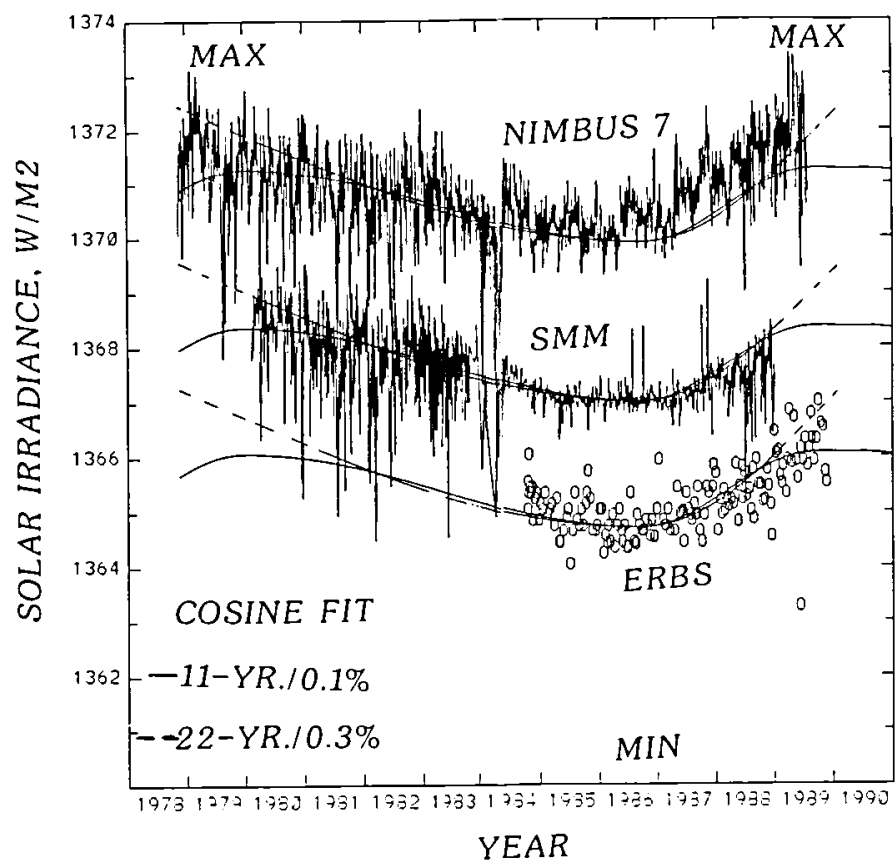

Figure 5. Cosine characterizations of irradiance variability are compared with the ERBS, Nimbus-7, and SMM irradiance data sets. The 11-year characterization assumes a 0.18 change in the irradiance between sunspot minimum and maximum, while the 22-year characterization assumes a 0.38 change in irradiance between minimum and maximum Hale magnetic activity. 
A. T. Mecherikunne1, H. L. Kyle, NASA/GSFC, Greenbelt, Maryland 20771 and

R. B. Lee III, NASA/Langley Research Center Hampton, Virginia 23665

\section{ABSTRACT}

At present, solar total irradiance measurements are made from four satellites using electrically self calibrating pyrheliometers, as a part of the earth radiation budget measurement programs. The Earth Radiation Budget mission onboard Nimbus-7 spacecraft (Nimbus-7/ERB) started solar total irradiance measurements in November 1978, and is still obtaining irradiance data on every orbit, daily. The Earth Radiation Budget Experiment (ERBE) solar monitors onboard Earth Radiation Budget Satellite (ERBS), NOAA-9 and NOAA-10 started solar total irradiance measurements in October 1984, January 1985, and October 1986, respectively. The ERBE solar monitors are active cavity radiometers of similar design and mode of operation to the Active Cavity Radiometer Irradiance Monitor-1 (ACRIM-1) operated onboard Solar Maximum Mission (SMM) during 1980-1989. In the past, the ERBE missions obtained solar total irradiance measurements biweekly, mainly for the calibration check of earth viewing ERBE sensors. But, after the loss of SMM/ACRIM-1 in December 1989, the solar total irradiance data are taken daily from ERBE onboard NOAA-10, and weekly from NOAA-9. Our knowledge of solar total irradiance and its variability has grown remarkably during the past few years, as a result of the above measurements, and the high precision data obtained from SMM/ACRIM-1. The results from a comparative study of the solar constant data available from the above missions will be presented. The solar constant value derived from the sensors agree within the uncertainty associated with absolute pyrheliometers available at present. An attempt will be made to correlate the solar irradiance variability with other solar parameters. The measurements from Nimbus-7/ERB started November 1978, as the solar cycle 21 was increasing in activity. The solar luminosity reached a maximum in the spring of 1979. The irradiance then decreased slowly to a minimum which lasted from 1984 through 1986. The irradiance is presently increasing towards a new maximum. It appears that the solar constant value follow an eleven year cycle.

\section{INTRODUCTION}

The solar constant of radiation, is the most important of all physical quantities because of its influence on the life in the solar system. By the term Solar constant, we infer the intensity of the solar beam, in energy units per unit area normal to the beam, and at earth's mean distance (one Astronomical unit). The Earth's weather and climate are determined by the incoming solar radiation, and its interaction with the atmosphere, land surface $-i$ oceans. The radiation balance or the net 
radiation at the top of the atmosphere, resulting from the radiative exchange between the incoming solar radiation and the outgoing earth emitted thermal radiation, is the central determinant of the earth's climate. The radiation balance $(R)$ can be expressed mathematically as follows.

$$
\mathrm{R}=\mathrm{S}(1-\mathrm{A})-\mathrm{F} \quad-\ldots-.-\mathrm{P} \quad 1
$$

where, $\mathrm{S}$ is the Solar Constant, $\mathrm{A}$ is the planetary albedo, and $\mathrm{F}$ is the thermal radiation emitted to space from the earth-atmosphere system. The absolute value of the solar constant, is the key term that determines the Earth's albedo and the radiation budget. The determination of solar constant and its temporal variations is one of the prime goals of climate research.

\section{NIMBUS-7 Earth Radiation Budget Mission}

The Earth radiation budget measurement programs determine the Earth Radiation Budget Components: the solar total irradiance, the earth reflected short wave, and the earth emitted thermal radiation. Because of its significance to weather and climate studies, the measurement of the Earth's radiation budget components from spacecraft platforms dates back to the early days of the NASA space program (House et a1., 1986). The Nimbus-7/ERB, launched in November 1978, is the first ERB mission to make solar total irradiance measurements using an electrically self calibrating pyrheliometer (H-F or Hickey-Friedan sensor, also known as Channel 10C). The electrical self calibration provided an absolute radiance scale for comparison, while the cavity design reduced the sensitivity to degradation of the solar absorbing surface. The details of the sensor characteristics, data reduction and the results are published by Hickey et al, 1982, 1984, 1987), and by Hoyt and Kyle, 1990 (in this publication). The Nimbus-7 is a sunsynchronous polar orbiter, and the solar total irradiance measurements are made as the satellite crosses the southern terminator. There are 14 orbits in a day and the orbital period is about 104 minutes. The solar disk is completely within the field of view (10 degrees) of the pyrheliometer for about 175 seconds, during each orbit. During the first four years, the Nimbus-7/ERB solar sensor operated on a 3 days on, 1 day off, duty cycle. In later years, the sensor has operated on a full time basis. The solar total irradiance measurements onboard Nimbus-7 continue to the present day, and the data set used in this study cover a period of 11 years and 3 months. The mean value of $1371.89 \mathrm{~W} \mathrm{m-2}$ is derived from the Nimbus-7/ERB for the period November 1978 through February 1990.

\section{The Earth Radiation Budget Experiment (ERBE)}

The Nimbus-7 spacecraft, being a sunsynchronous polar orbiter, the earth radiant exitance measurements are confined to two local times, near noon and midnight; and the earth reflected data are confined to local noon only. This reduces the usefulness of the data for studies of the temporal variability of earth radiation budget components. The ERBE mission of the $1980^{\prime} \mathrm{s}$ is designed to make improved measurements of the radiation budget components at various local times, to study the temporal and spatial variations of these components. In order to achieve this objective, the ERBE sensors make measurements from three spacecraft plat- 
forms: the National Aeronautics and Space Administration (NASA) Earth Radiation Budget Satellite (ERBS), and the two National Oceanic and Atmospheric Administration satellites NOAA-9 and NOAA-10, at different equator crossing times and orbital inclinations. The first of the three satellites carrying the ERBE sensors, the NASA ERBS, was launched into a 57 degree inclination orbit by Space Shuttle Challenger in October 1984. The NOAA operational meteorological satellites NOAA-9 and NOAA-10 were launched into near polar orbits in December 1984, and September 1986, respectively (ERBE Science Team, 1986). The ERBE solar monitors are identical electrically self calibrating pyrheliometers of active cavity type; and are similar in design and mode of operation to the SMM/ACRIM-1. In the ERBE measurement approach, the sun is allowed to drift through the field of view (13.6 degrees) of the solar monitors. The sensor characteristics, and mode of operation are given in detail by Lee et al (1987), and Lee (1990) in this publication. The Earth Radiation Budget Experiment (ERBE) sensor package consists of a solar monitor, and the earth viewing radiometers. The solar monitors make direct measurements of solar total irradiance, every two weeks, to provide data for earth radiation budget computations and to serve as a check on the radiometric calibration of earth viewing sensors. A mean value of 1365.15 $\mathrm{W} \mathrm{m}^{-2}$ for is obtained from ERBS/ERBE for the period October 1984 through December 1989. NOAA-9/ERBE gives a mean value of $1364.75 \mathrm{~W} \mathrm{~m}^{-2}$ for the solar constant for the period January 1985 through August 1989. The solar total irradiance data from NOAA-10/ERBE are processed for a period of six months, October 1986-April 1987, and the mean solar constant value $1363.24 \mathrm{~W} \mathrm{~m}^{-2}$, is obtained for this period. On April 1, 1987 the shutter mechanism of the solar monitor failed and the solar sensor remain open at all times. However, the solar total irradiance measurements are obtained daily from NOAA-10 spacecraft, from January 19, 1990, and from NOAA- 9 every week.

\section{Intercomparison of Solar Constant Data}

Figure 1 shows the multiyear data set from the four satellites. The Nimbus-7/ERB solar data set covers a period of 11 years and 3 months (November 16, 1978 through February 1990). The SMM/ACRIM-1 data runs for a period of about 9 years from February 1980 through December 1988. The SMM/ACRIM-1 data is described by Willson et al $(1984,1988)$ ) and Willson (1990) in this publication. The ERBS/ERBE data set is for a period of 5 years and 2 months (October 1984 through December 1989), while the NOAA-9/ ERBE data covers the January 1985 through August 1989. It should be remembered that the data points consists of an average of 14 measurements per day for Nimbus-7/ERB; hundred of measurements for SMM/ACRIM-1, while ERBE measurements are the mean of two to three measurements on that particular day. The absolute calibration accuracy of the Nimbus-7/ERB Channe 1 10C, is quoted as $+/-0.5 \%$, while the other experiments claim accuracy of 0.28 or better. The daily fluctuations in the irradiance values are observed in all the data sets. The maximum in the Nimbus-7/ERB irradiance value $1374.29 \mathrm{~W} \mathrm{~m}^{-2}$ occurs in March 1979 , and shows a gradual decrease with time. It reached a minimum during 1984-1986 and then, increases gradually with time up to February 1990, the time the data sets are available at present. SMM/ACRIM-1 data set also shows a decreasing trend in irradiance values during 1985-1986, and then increases from the latter part of 1986. Both the ERBE measurements also show the decrease and increase observed for Nimbus-7/ERB and SMM/ACRIM-1. The increases and 
decreases are of the order of 0.1-0.28 (2-3 $\left.\mathrm{W} \mathrm{m}^{-2}\right)$. The NOAA-9/ERBE data shows more scatter than the other data sets. The results of an earlier intercomparison of the Nimbus-7/ERB, SMM/ACRIM-1, ERBS/ERBE, NOAA-9/ERBE, and NOAA-10/ERBE data sets are available in Mecherikunnel et al (1988).

Figures 2 and 3 present the monthly and yearly mean value of the solar constant respectively, for the same period as shown for the daily mean values. The monthly mean irradiance value shows the same increasing and decreasing trend in all the four data sets. The minimum in solar constant value occurs in 1984-1985 for Nimbus-7/ERB. The SMM/ACRIM-1 and ERBS/ERBE show the minimum during 1985-1986. NOAA-9/ERBE shows a decrease in irradiance with time during 1986 to 1987, and then shows the increase starting 1987. The anomaly observed in the NOAA-9/ERBE data set is caused by factors other than solar, and needs further investigations. (Mecherikunnel et al, 1990).

During the period 1985 through 1989 solar constant data are available from all four satellites for 71 days. It is to be considered that the data samplings vary for the 4 data sets. Table 1 lists the mean solar constant value, the standard deviation and the maximum and minimum observed during the 71 observation days. The solar constant value agree within the uncertainty associated with each solar sensor.

TABLE 1

SOLAR CONSTANT (IN $\mathrm{WM} \mathrm{M}^{-2}$ ) FROM N-7ERB, SMM, ERBS AND NOAA-9 ON FOUR WAY MATCHING DAYS 1985-1989

$\begin{array}{lcccc}\text { SENSOR } & \begin{array}{c}\text { SOLAR CONSTANT } \\ \text { (MEAN VALUE) }\end{array} & \text { STD.DEVIATION } & \text { MAXIMUM } & \text { MINIMUM } \\ & & & & \\ \text { NIMBUS - 7/ERB } & 1371.47 & 0.34 & 1372.24 & 1370.72 \\ \text { SMM/ACRIM-1 } & 1367.16 & 0.40 & 1369.21 & 1366.54 \\ \text { ERBS/ERBE } & 1364.98 & 0.42 & 1365.91 & 1364.00 \\ \text { NOAA-9/ERBE } & 1364.61 & 0.85 & 1366.90 & 1363.07\end{array}$

Figure 4 presents the Nimbus-7/ERB yearly mean solar constant, and the yearly mean sunspot number. Yearly mean value closely follow the 11 year sunspot cycle, as shown in the figure. The sunspot cycle 21 began in June 1976, reached the maximum in 1979. Nimbus-7/ERB data shows the maximum value of solar constant in 1979 (March), and gradually decrease through the years reaching the minimum in 1984 with a gradual increase to 1990 . But, the minimum of sunspot number occurs in 1986 . It should be noted that the long term stability of the Nimbus-7/ERB data system is about $0.3 \mathrm{~W} \mathrm{~m}^{-2}$. This is the first experimental evidence of an 11 year solar constant variability, which several investigators have speculated about for centuries.

Solar Total Irradiance Variability and the Extraterrestrial Solar Spectral Irradiance

The experimental results clearly indicate that there are fluctuations in the daily, monthly, and yearly mean solar total irradiance data. The irradiance variability follow the 11 year sunspot cycle as evidenced from 
Figure 4. How do the observed variations in the total irradiance affect the spectral distribution of the total irradiance? The solar spectral irradiance variations in the UV and microwave regions are known and are being extensively studied. But very little is known about the variability in the visible and near infrared solar spectral region that contains more than 928 of the sun's energy. Almost all the radiation effects in the earth and atmosphere are wavelength dependent. Accurate data on solar constant and its spectral distribution in the UV, Visible, IR regions are important in understanding various interactions in the earth-atmosphere system. This is especially significant in the Earth Observation System (EOS) investigations.

\section{CONCLUSION}

The comparative study of the solar constant data from the recent spacecraft measurements indicate that the results are within the absolute radiometric accuracy of each sensor used in the measurements. The similar trends in solar irradiance variability is observed in all the data sets. The increases and decreases in the total irradiance observed are in step with the 11 year sunspot cycle. The results shows beyond any doubt that the variations observed are solar in nature. The Nimbus-7 ERB solar constant data set gives the first experimental evidence of an 11 -year solar cycle in the total irradiance, analogous to the 11 -year sunspot cycle. 


\section{REFERENCES}

House, F. B., A. Gruber, G. E. Hunt, A. T. Mecherikunne1, History of Satellite Missions and Measurements of the Earth Radiation Budget (19571984), Reviews of Geophysics, Vol. 24, Pp. 357-377, 1986.

Hickey, J. R., B. M. Alton, F. J. Griffin, H. Jacobowitz P. Pelegrino, R. H. Maschhoff, E. A. Smith and T. H. Vonder Haar, Extraterrestrial Solar Irradiance Variability two and one-half years of measurements from Nimbus7, Solar Energy, Vol. 29, Pp. 125-127, 1982.

Hickey, J. R., E. R. Major, and H. L. Kyle, User's Guide for the Nimbus-7 ERB Solar Analysis Tape (ESAT), TM 86143, NASA/Goddard Space F1ight Center, Greenbelt, MD 20771, 82pp., 1, 1984.

Hickey, J. R., B. M. Alton, H. L. Kyle and E. R. Major, The Solar Irradiance Measurements by the Nimbus-7 ERB Experiment, An Update of 100 months, in Proceedings of a Workshop on Solar Radioative Output Variation, edited by P. Foukal, Pp. 189-194, Cambridge Research and Instrumentation, Cambridge, Massachussettes, 1987.

Hoyt, D.V., and H. L. Kyle, An Alternative Derivation of the Nimbus-7 Total Solar Irradiance Variations, 1990 (in this publication).

ERBE Science Team, First data from the Earth Radiation Budget Experiment (ERBE), Bull. Am. Meteorol. Soc., Vol. 67, Pp. 818-824, 1986.

Lee, R. B., B. Barkstrom, and R. D. Cess, Characteristics of the Earth Radiation Budget Experiment Solar Monitors, Applied Optics, Vol. 26, Pp. 3090$3046,1987$.

Lee, R. B., Long-Term Solar Irradiance Variability: 1984-1989 Observations, 1990 (in this publication).

Mecherikunnel, A. T., R. B. Lee III, H. L. Kyle, and E. R. Major, Intercomparison of Solar Total Irradiance Data From Recent Spacecraft Measurements, Journal of Geophysical Research, Vo1. 93, No. D8, Pp. 9503$9509,1988$.

Mecherikunnel, A. T., H. L. Kyle, C. R. Kondragunta, Principal Investigator Report, 27th ERBE Science Team Meeting, Orlando, Florida, April 19-20, 1990.

Willson, R. C., Measurements of Solar Total Irradiance and Its Variability, Space Sciences Reviews, Vo1. 38, Pp. 203-242, 1984.

Willson, R. C., Solar Irradiance Variability During Solar Cycles $21 \& 22,1990$ (in this publication). 


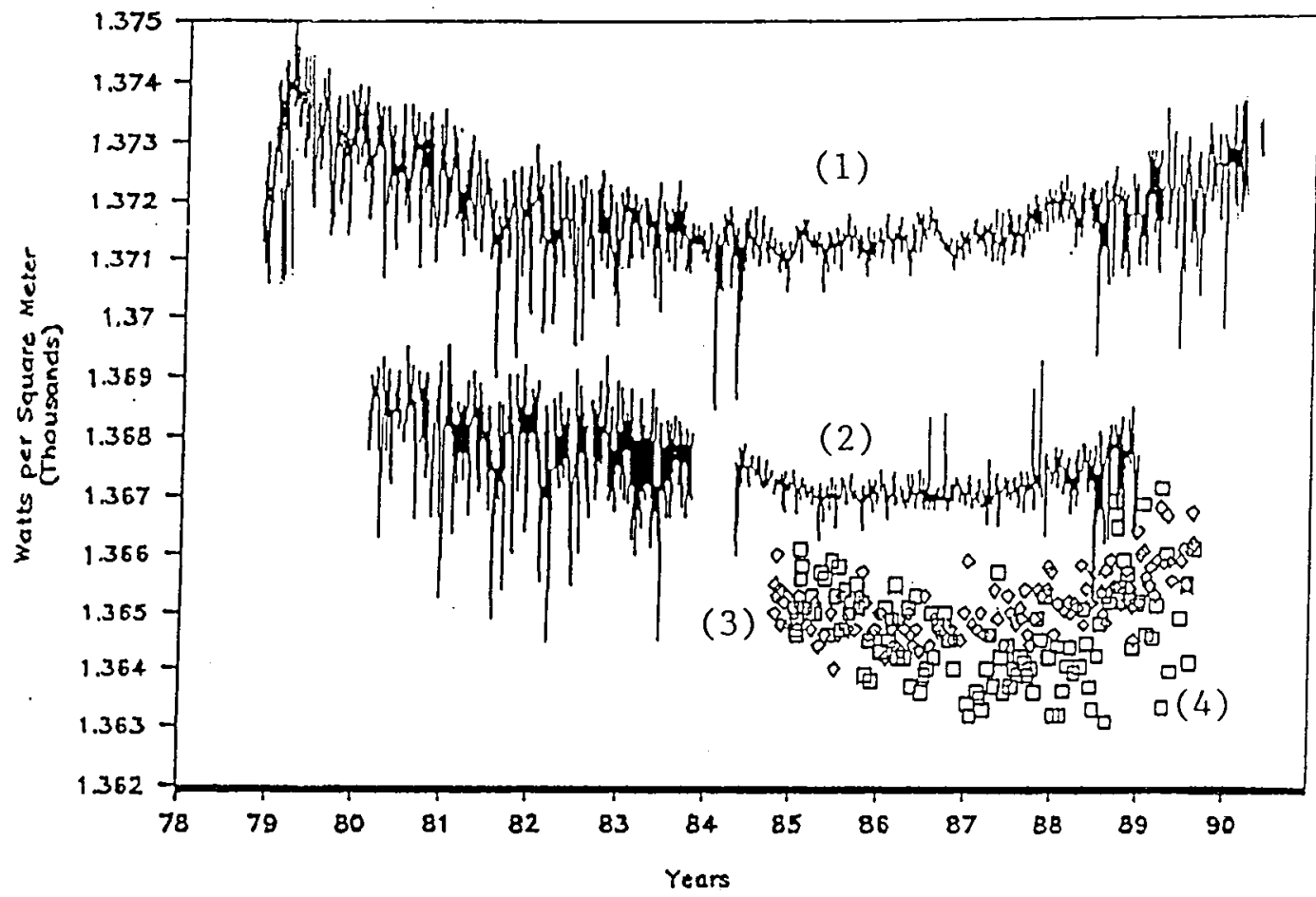

Fig. 1. Daily Mean Solar Constant from: 1) Nimbus-7/ERB Nov. 1978Feb. 1990; 2) SMM/ACRIM-1 Feb. 1980-Dec. 1988; 3) ERBS/ERBE Oct. 1984Dec. 1989; and 4) NOAA/ERBE Jan. 1985-Aug. 1989.

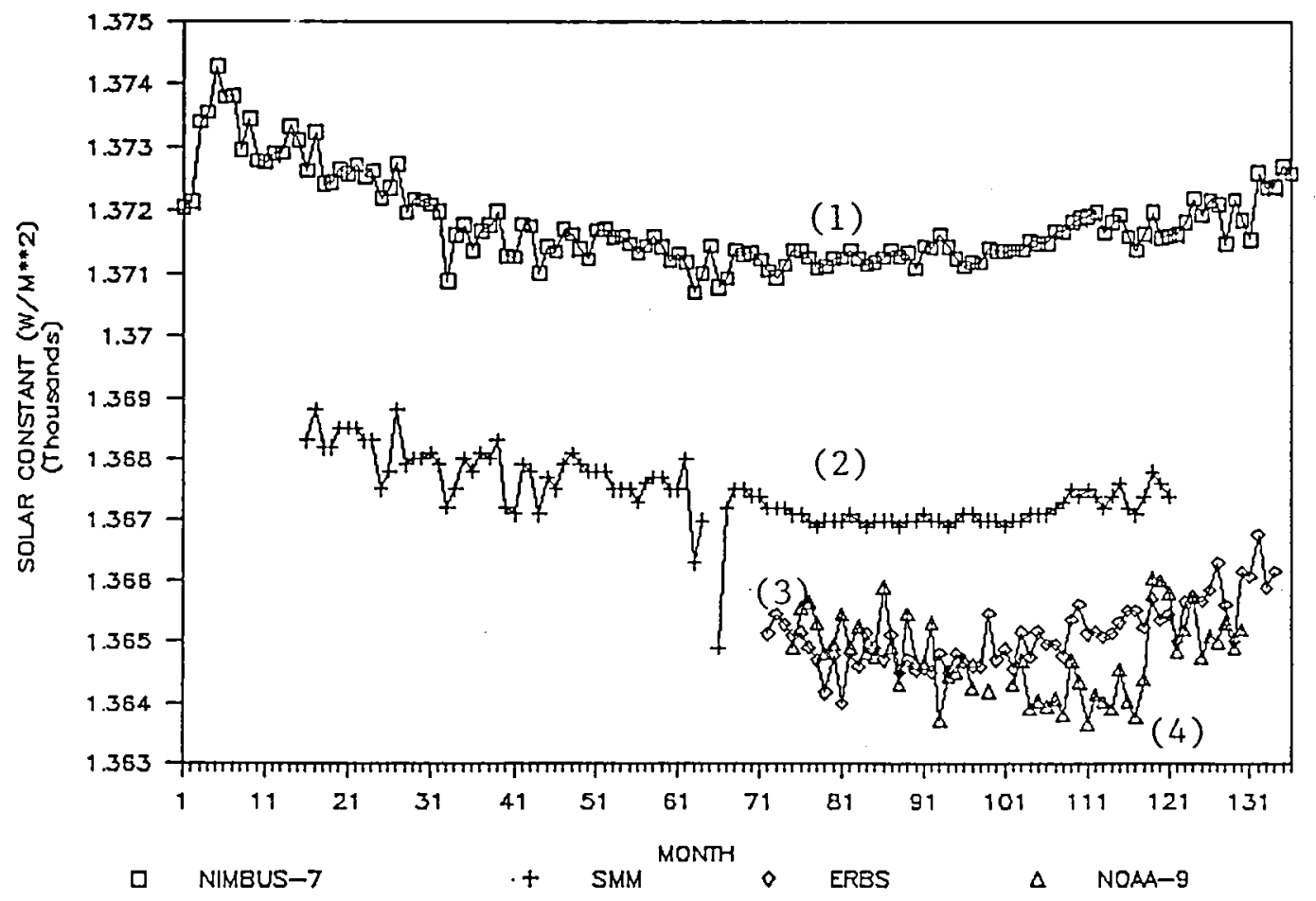

Fig. 2. Monthly Mean Solar Constant from: 1) Nimbus-7/ERB Nov. 1978Feb. 1990; 2) SMM/ACRIM-1 Feb. 1980-Dec. 1988; 3) ERBS/ERBE Oct. 1984Dec. 1989; and 4) NOAA/ERBE Jan. 1985-Aug. 1989. 


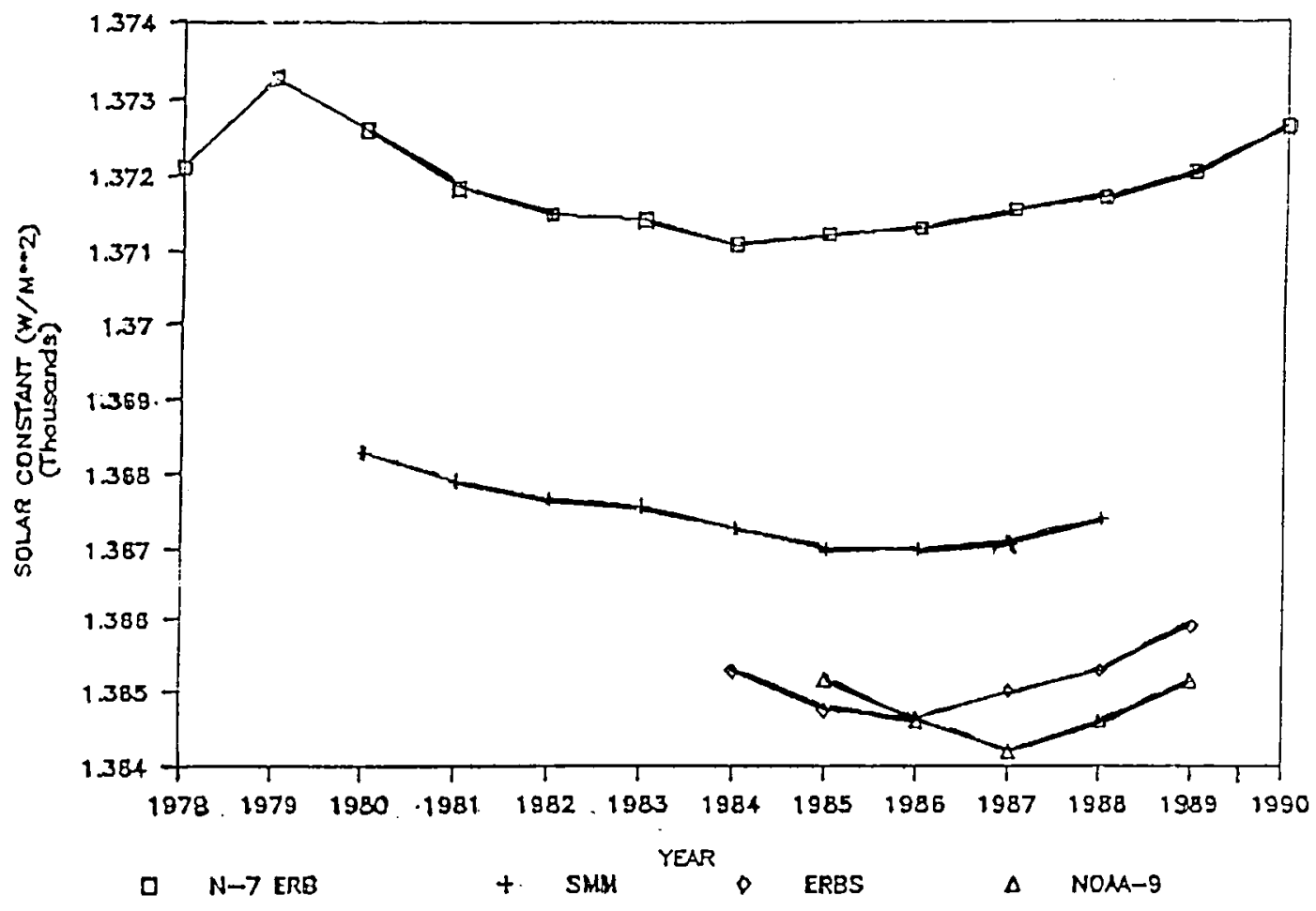

Fig. 3. Yearly Mean Solar Constant from: 1) Nimbus-7/ERB Nov. 1978Feb. 1990; 2) SMM/ACRIM-1 Feb. 1980-Dec. 1988; 3) ERBS/ERBE Oct. 1984-DeC. 1989; and 4) NOAA/ERBE Jan. 1985-Aug. 1989.

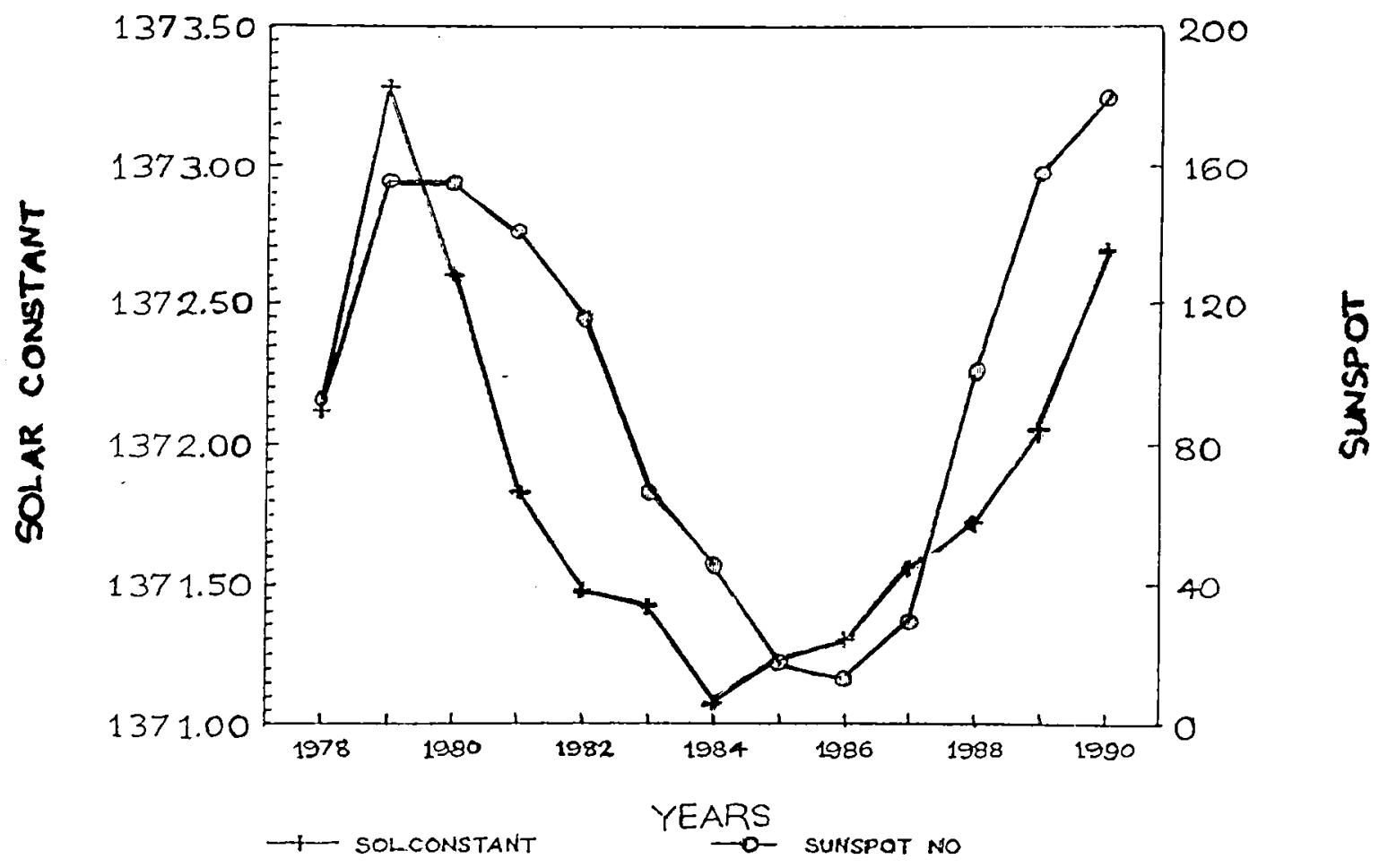

Fig. 4. 11-year Solar Constant Data from Nimbus-7/ERB and the 11year sunspot Cycle. 
SESSION 9: SUN-CLIMATE OVERVIEW II 



\title{
A REVIEW AND REFLECTIONS ON THE SUN-CLIMATE CONNECTION
}

\author{
Richard A. Goldberg \\ National Science Foundation \\ Division of Atmospheric Sciences \\ 1800 G. St., NW \\ Washington, DC 20550
}

The field of sun-climate is beset with an extraordinary number of numerical correlations attempting to relate various periodicities of solar activity with changes in the Earth's weather and climate. Signatures representing climatological variability have been sought for cycles as short as the solar 28-day rotational period up to Milankovich periods of thousands of years, although a majority of correlations have concentrated on the 11-year sunspot and 22-year Hale double sunspot cycles. For the shorter term, parameters including temperature, pressure, winds storm tracks, rainfall, and water levels in rivers and lakes, etc. have been correlated with solar variability. For longer periods, it has been necessary to seek more indirect evidence in ice cores, tree rings, and geologic deep sea cores. Other atmospheric parameters relating to atmospheric electricity and the global electric circuit have also been correlated in similar fashion. Unfortunately, few, if any, of this wide spectrum of numerical correlations have been associated with any viable physical explanation, making most studies in the field an exercise in numerical statistics. More recently, a few suggestions for plausible coupling processes have begun to appear. These, coupled with new and stronger correlations involving selective binning of climatological data sets, e.g. (QBO), have injected new life and hope to this field. This review presents an overview of the historical past and current perspectives, to evaluate possible avenues for defining physical linking processes in the future. 


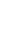


SESSION 10: SOLAR IRRADIANCE VARIATIONS II 



\title{
WAVELENGTH DEPENDENCE OF SOLAR ROTATION AND SOLAR CYCLE UV IRRADIANCE VARIATIONS
}

\author{
Julius London* and Gary J. Rottman+ \\ *Astrophysical, Planetary and Atmospheric Sciences \\ + Laboratory for Atmospheric and Space Physics \\ University of Colorado \\ Boulder, CO 80309
}

\begin{abstract}
It is shown that for the 5-year period 1982-87 the solar irradiance decrease is estimated to be about 5-7 percent over the spectral interval $195-225 \mathrm{~nm}$. This change becomes progressively smaller with increasing wavelength. For the 2-1/3 year period, January 1987 to April 1989, the irradiance increases about 6 percent at $195-205 \mathrm{~nm}$ and about 2 percent between $215-250$ $\mathrm{nm}$. Both 27-day and 13.5-day relative amplitudes peak at the time near solar maximum (1982) but remain comparatively small between 1983 and the onset of solar cycle 22. An average 280 day oscillation is noted for wavelengths up to $230 \mathrm{~nm}$. No physical mechanism is offered for this variation.
\end{abstract}

\section{INTRODUCTION}

Solar radiation in the wavelength interval $195 \mathrm{~nm}-300 \mathrm{~nm}$ is deposited and largely absorbed in the earth's stratosphere and mesosphere. This absorbed energy is responsible for most of the photodissociation of molecular oxygen, ozone, and minor gases in that region, and the radiative heating in the layer $20-70 \mathrm{~km}$. It is therefore reasonable to expect that upper atmosphere ozone and temperature variations would respond to variations in solar irradiance in this critical wavelength interval. Indeed, both models and observations provide support for such responses at different time scales (see, for instance, Gille, 1984'; Garcia et al., $1984^{2}$; Hood, $1989^{3}$ ). The models are generally based on preliminary estimates of solar UV variability derived from occasional rocket observations, satellite measurements or solar proxy data. The following analysis makes use of the data set derived from the Solar Mesosphere Explorer (SME) solar UV measurements in the spectral range of 195-300 nm for the period 1 Jan 1982-13 Apr 1989. A preliminary discussion of some of the early results is contained in London et al. (1984). ${ }^{4}$ Details of the observation technique, estimated accuracy and precision of the instruments are available in Rottman $(1988)^{5}$. The present analysis summarizes the wavelength dependence of solar irradiance variations over an approximate solar cycle, solar rotation, and half solar rotation, and an estimated average 280 day period.

\section{SOLAR CYCLE VARIATIONS}

Solar middle UV irradiance exhibited an extended maximum during solar cycle 21 from 1980-82 (e.g., Hood, 1989) ${ }^{3}$. Irradiance minima given by the SME observations occurred very early in 1987 at all wavelengths in the range 195-300 $\mathrm{nm}$. The percent decrease of the solar irradiance over the 5-year period (1982-1986) is shown in Fig. 1a for the spectral interval $195-300 \mathrm{~nm}$. The maximum change of -7.2 percent (the equivalent of $\sim 1.4 \% \mathrm{yr}^{-1}$ ) was found at $220-225 \mathrm{~nm}$ decreasing to approximately -1 percent (i.e., 0.2 percent $\mathrm{yr}^{-1}$ at $300 \mathrm{~nm}$ as the radiance source gets closer to the lower photosphere. The irradiance at all wavelengths in the interval $200-300 \mathrm{~nm}$ increased after early Jan 1987, somewhat steeply up to about $230 \mathrm{~nm}$ and more slowly at longer wavelengths. The largest increase for the 
period Jan 1987-Apr 1989 (see Fig. 1b) was almost 6 percent at the shorter wavelengths (2.5 percent $\mathrm{yr}^{-1}$ and about 2 percent (less than 1 percent $\mathrm{yr}^{-1}$ ) between 215 and $250 \mathrm{~nm}$. The long-term change beyond $250 \mathrm{~nm}$ is difficult to estimate because of instrument and observing problems at these wavelengths toward the end of the SME period. It should be noted that although the values shown in Figs. 1a,b are probably the best estimates available at present, they are still subject to a possible relative error of a few percent (see, for instance, Rottman, 1988 $)^{5}$. Calibration procedures set up for the SOLSTICE and SUSIM instruments planned for a fall 1991 launch are expected to minimize such a long term possible drift error. Solar irradiance variations with harmonics associated with solar rotation (i.e., 27-day and 13.5-day periods) have been reported by Lean, $1984 ;^{6}$ Lean and Repoff, $1987,{ }^{7}$ and others.

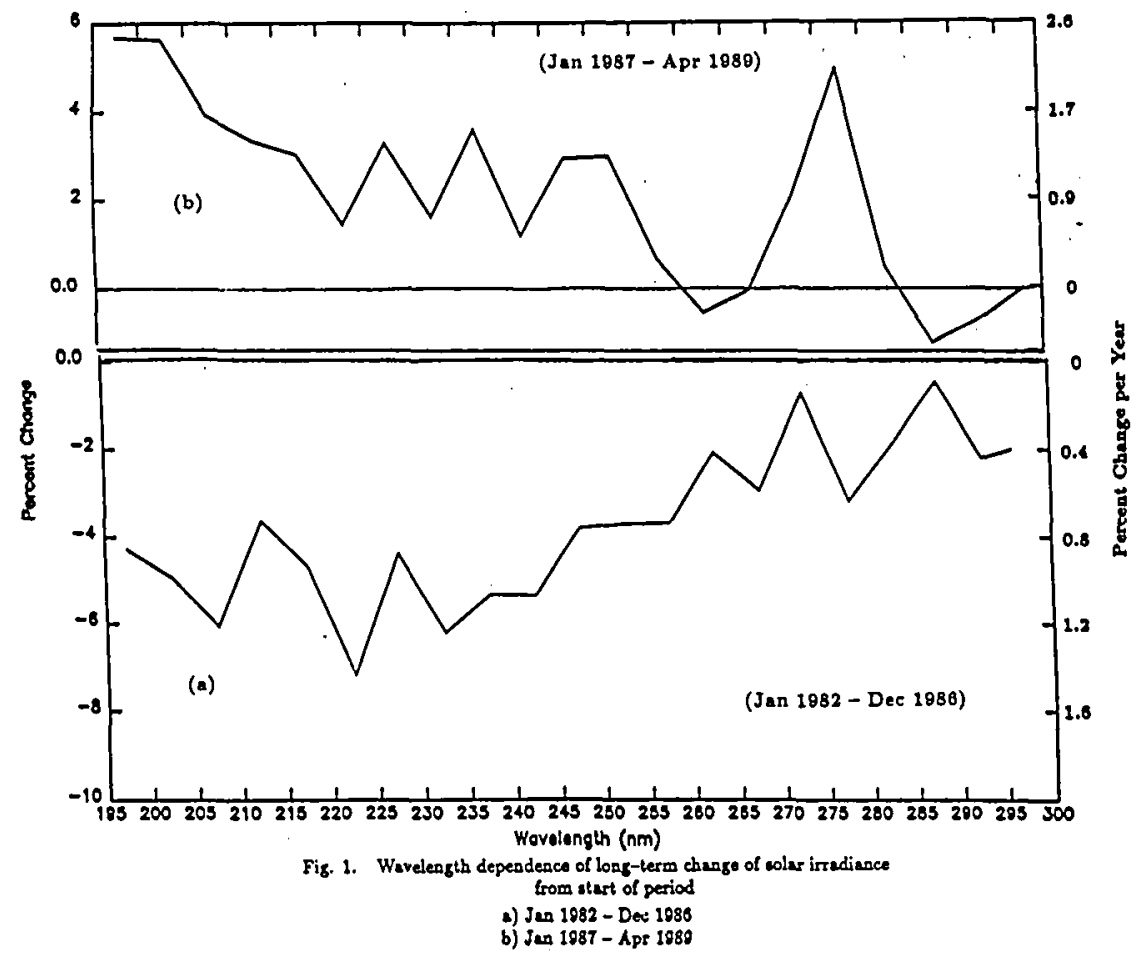

SOLAR ROTATION VARIATIONS

The time variation of the 27-day and 13.5-day relative amplitudes of the SME observed solar irradiance at 200-205 $\mathrm{nm}$ are shown in Fig. 2. The peaks shown for mid-July 1982 corresponding to $2.2 \%$ for 27 -day and $\sim 1 \%$ for 13.5 -day variations occur at a time near solar maximum for cycle 21. Note that the relative amplitudes decrease significantly after 1982 and remain relatively constant over the period 1983-88 before they increase with increase with enhanced activity after the onset of cycle 22 . The 13.5 -day relative amplitude has, on average, about half the amplitude of the 27-day variation as shown in Fig. 2. The wavelength dependence of the 27- and 13.5-day relative amplitudes for the period of maximum solar irradiance observed by SME (8 Jul 1982-4 Aug 1982) is given in Figs. 3a,b. The 27-day relative amplitude maximum $(2.2 \%)$ is found at $200-205 \mathrm{~nm}$, just short of the aluminum edge where the irradiance originates from the upper photosphere. The relative amplitude decreases sharply to $1 \%(210-215 \mathrm{~nm})$, remains at that level to $250 \mathrm{~nm}$ and then continues to decrease to less than $0.5 \%$ beyond $250 \mathrm{~nm}$. For the 13.5 -day rotation period, the relative amplitude during the time near solar maximum is about $1 \%$ at $200 \mathrm{~nm}$ and decreases to a few tenths percent at $300 \mathrm{~nm}$. Thus, at the time of solar maximum, one would expect a direct upper atmosphere response to the solar rotation irradiance signal at wavelengths below below $250 \mathrm{~nm}$ (see, for instance, Ekman, $1986^{8}$ ). 


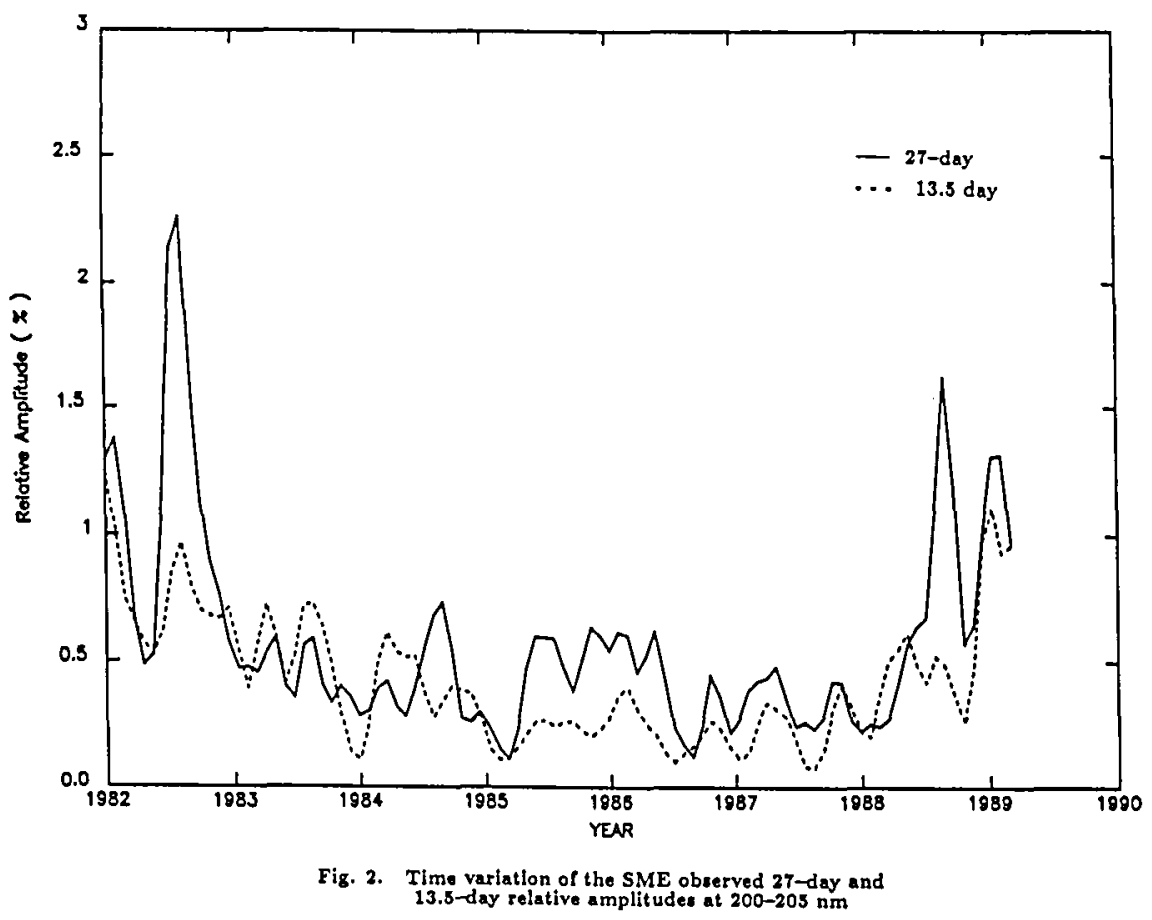

The short period irradiance variations during the 5 years 1983-88 were relatively small at all wavelengths. During this time the 13.5-day relative amplitude harmonic was about twothirds of that for 27-days at almost all wavelengths as shown in Fig 4 . The average 27-day relative amplitude for this period was $0.35 \pm 0.24$ percent. That for the 13.5-day variation was $0.25 \pm 0.13$ percent.

The average standard deviation given here represents the average over wavelength of the time standard deviation of each $5 \mathrm{~nm}$ interval over the 5-year period 1983-88. The ratio of the amplitudes of the two periods indicates the relative strength of the single vs. quasisymmetric double solar active regions ( 180 longitude phase difference). During the time of peak activity (Jul-Aug 1982) this ratio was less than one-half at $\lambda \leq 235 \mathrm{~nm}$ but approached unity at longer wavelengths. However, during the descending and minimum phase of solar cycle 21 there was little significant difference between the 27-day and 13.5-day oscillation in this spectral interval.

Fig. 3.

Wavelength dependence of the relative amplitudes of 27-day and 23.5-day variations for the time near peak solar activity (8 Jul 1882 - 4 Aug 1882)

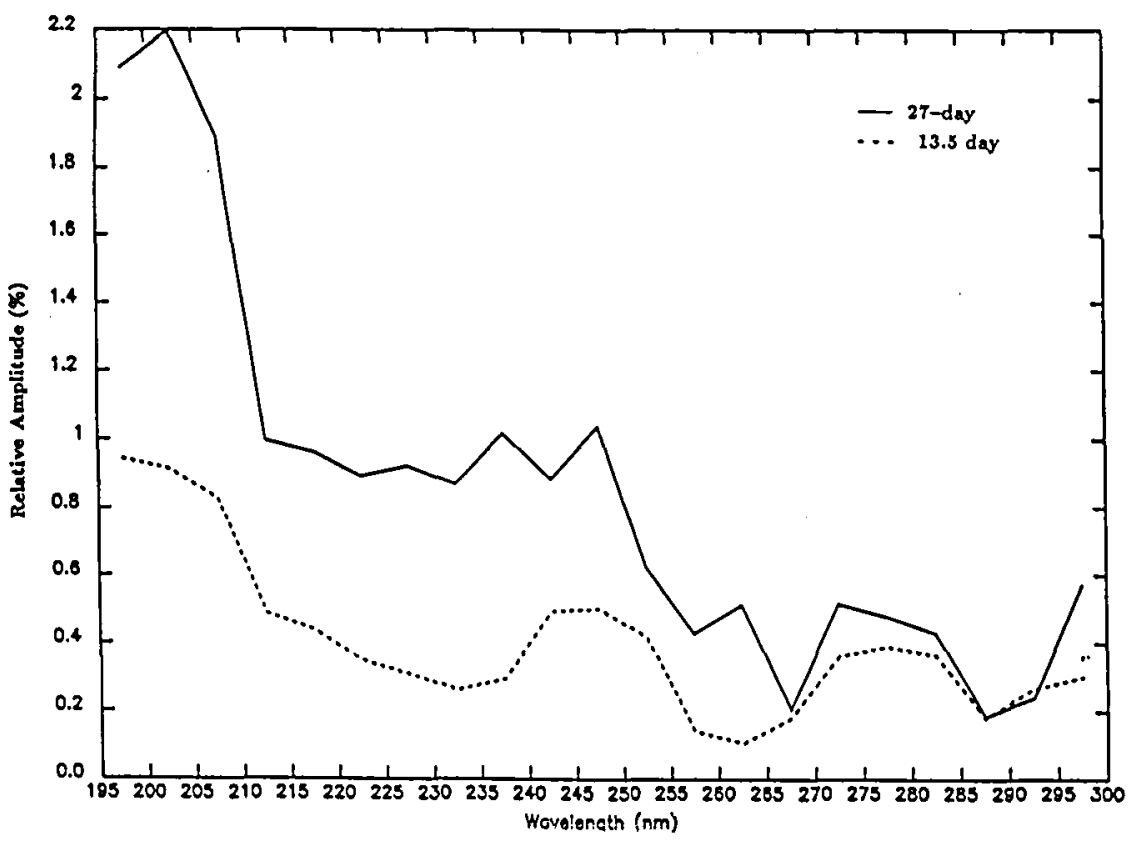




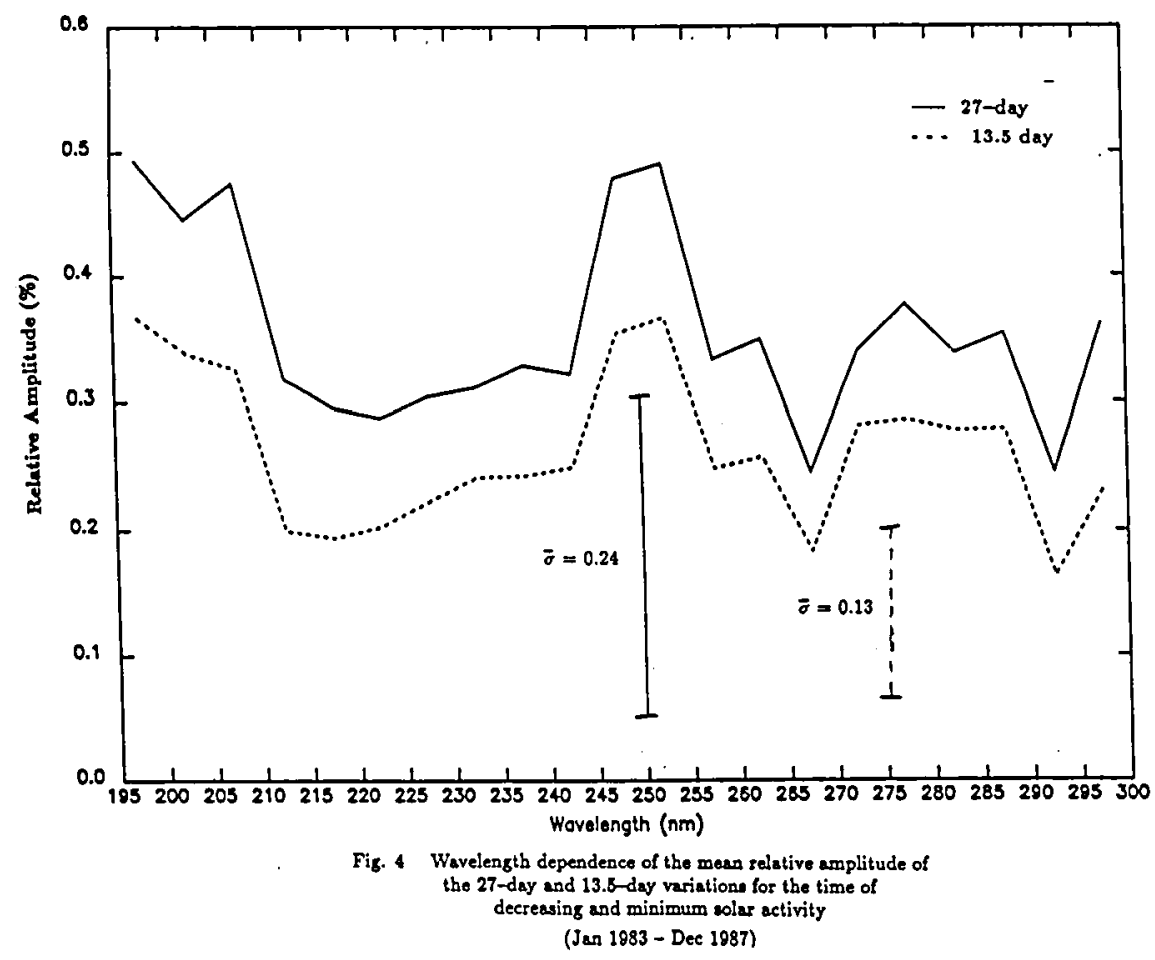

INTERMEDIATE PERIOD VARIATIONS

In addition to solar rotation and solar cycle irradiance variations, there appears to be a quasi-periodic oscillation with an average period of 280 days. Until recently, however, there were no long-term continuous observations to verify this oscillation at wavelengths in the middle UV (see, for instance, Hood, $1988^{3}$; Rottman, $1988^{5}$ ). An average 280 day variation can also be seen from SME data for wavelengths from $195 \mathrm{~nm}$ up to about $230 \mathrm{~nm}$. As an example we show such a variation for $\Delta \lambda=200-205 \mathrm{~nm}$.

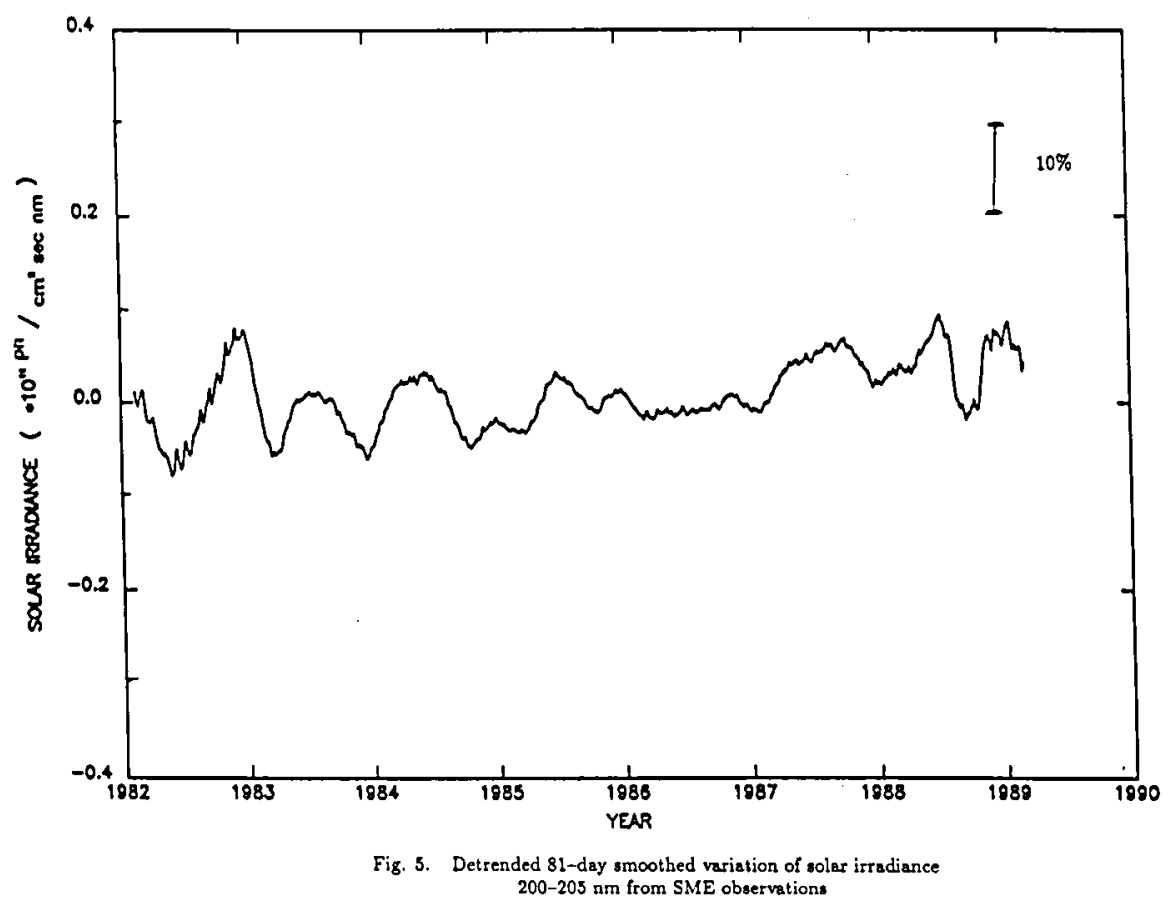

A four term Fourier series was used to detrend the 200-205nm irradiance data for the entire period of observations. The residuals were then subject to an 81-day running mean. The resulting curve is shown in Fig. 5. Again, the largest irradiance range (about 15\%) occurs 
in mid-1982. The oscillation goes to zero in 1987 and then apparently increases by the end of the observed period. We do not know of any physical mechanism for this oscillation, but since it is found at shorter wavelengths, including $\mathrm{Ly}-\alpha$ and at $\mathrm{F} 10.7 \mathrm{~cm}$ irradiance (Pap et al., 1990), ${ }^{9}$ it is likely to be related to plage area enhancement of quasi-periodic behavior in the convection zone. It is not known how the upper atmosphere would (or does) respond to such an oscillation.

\section{ACKNOWLEDGEMENTS}

We would like to thank Barry Knapp and Kristin Hoyer for reprocessing some of the SME data and for programming assistance. Support is acknowledged from the Upper Atmosphere Research Program of the National Aeronautics and Space Administration under grant NAS527263.

\section{REFERENCES}

1. Gille, J. C., C. M. Smythe, and D. F. Heath, "Observed ozone response to variations in solar ultraviolet radiation," Science, 225, 315-317, 1984.

2. Garcia, R. R., S. Solomon, R. G. Roble, and D. W. Rusch, "A numerical response of the middle atmosphere to the 11-year solar cycle," Planet. Space Sci., 32, 411-423, 1984.

3. Hood, L. L., "Stratospheric effects of solar ultraviolet variations on the solar rotation time scale," Middle Atmosphere Program, Handbook, 29, International Symposium on Solar Activity Forcing of the Mid-Atmosphere, 79-81, September 1989.

4. London, J., G. G. Bjarnason, and G. J. Rottman, "18 months of UV irradiance observations from the Solar Mesosophere Explorer," Geophys. Res. Lett., 11, 54-56, 1984.

5. Rottman, G. J., "Observations of solar UV and EUV variability," Adv. Space Res., , (7) $53-(77) 66,1988$.

6. Lean, J. L., "Estimating the variability of the solar flux between 200 and $300 \mathrm{~nm}$," $J$. Geophys. Res., 89, 1-9. 1984.

7. Lean, J. L. and T. P. Repoff, "A statistical analysis of solar flux variations over time scales of solar rotation: 1978-1982," J. Geophys. Res., 92, 5555-5563, 1987.

8. Eckman, R. S., "The response of ozone to short-term variations in the solar ultraviolet irradiance. 2. Observations and interpretation," J. Geophys. Res., 91, 6705-6721, 1986.

9. Pap, J., J. London, and G. J. Rottman, "Variability of solar Lyman-alpha and total solar irradiance." (Submitted to Astronomy and Astrophysics Main Journal), 1990. 
SOLAR UV TEMPORAL VARIATIONS DURING SOLAR CYCLE $22 \&$ THE TWENTIETH CENTURY

\author{
Richard F, Donnelly \\ Space Environment Lab., NOAA ERL \\ Boulder, Colorado 80303 , U. S. A.
}

\begin{abstract}
Solar ultraviolet measurements of the $11 \mathrm{~g}$ II core-to-wing ratio from the NOAA9 satellite show a fast rise for solar cycle 22 from the minimum in september 1986. The high values in late 1989 are comparable to the maximum values for cycle 21 . Estimates of earlier solar UV variations are made back to 1947 using a combination of the $10 \mathrm{cn}$ solar radio flux (F10) and the sunspot blocking function. The latter is interpreted to partially remove the gyroresonance component from F10, which is not present in the UV flux.
\end{abstract}

\title{
INTRODUCTION
}

Solar ultraviolet (UV) variations are important to research of the climatic impact of solar variability because the UV flux does the following: (1) influences the solar radiation input to the troposphere; (2) photodissociates the major atmospheric constituents in the stratosphere, which subsequently induce changes in minor constituents that affect atmospheric chemistry and radiation absorption and emission; and (3) heats the stratosphere, which may influence tropospheric as well as stratospheric dynamical processes. In case 1 , the solar UV flus variations contribute between one fifth to one third of the solar cycle variation of the total solar irradiance (Lean, 1989; London et al., 1989). This reduces the amplitude of the solar cycle variation of solar radiation reaching the ground compared to the total solar irradiance variations observed from satellites. Also, solar UV intensifications increase the atmospheric columar ozone content, which enhances atmospheric absorption and further decreases the solar cycle variation of solar radiation reaching the ground.

Heath and Schlesinger (1986) showed that the core-to-wing ratio of the Mg II $h \& k$ absorption 1 ines near $230 \mathrm{~nm}$ provides a measure of solar UV variability that was insensitive to instrunent drift for the Solar Backscatter Ultraviolet (SBUV) observations from the NIMBUS7 satellite. This paper discusses similar Mg II core-to-wing ratios for the rise of solar cycle 22, based on measurements from the SBUV2 monitor on the NOAA9 satellite.

The Ottawa $10.7 \mathrm{~cm}$ solar radio flux measurements (F10) have long been used to estimate the temporal variations of solar UV fluxes. Recent studies have shown that the short-term (days, weeks) UV variations differ markedly from those of Fl0. This paper shows that the long-term variations of $F 10$ and $R(M g I I c / w, t)$ are closely related with a divergence from linearity during the end of the cycle decay into solar minimum. Separating F10 into long-term and short-term variations improves the estimate of UV variations. Including the sunspot blocking function of Hoyt and Eddy (1982) further improves the estimates of short-term UV variations. Their sunspot blocking function sums over the visible solar disk the sunspot umbral and penumbral areas weighted by a contrast function that varies with the angle between the solar radial through the center of the spot and our line of observation. Preliminary estimates for the longterm variations of $\mathrm{R}(\mathrm{MgIIC} / \mathrm{w}, \mathrm{t})$ for solar cycles $18-21$ are presented. 


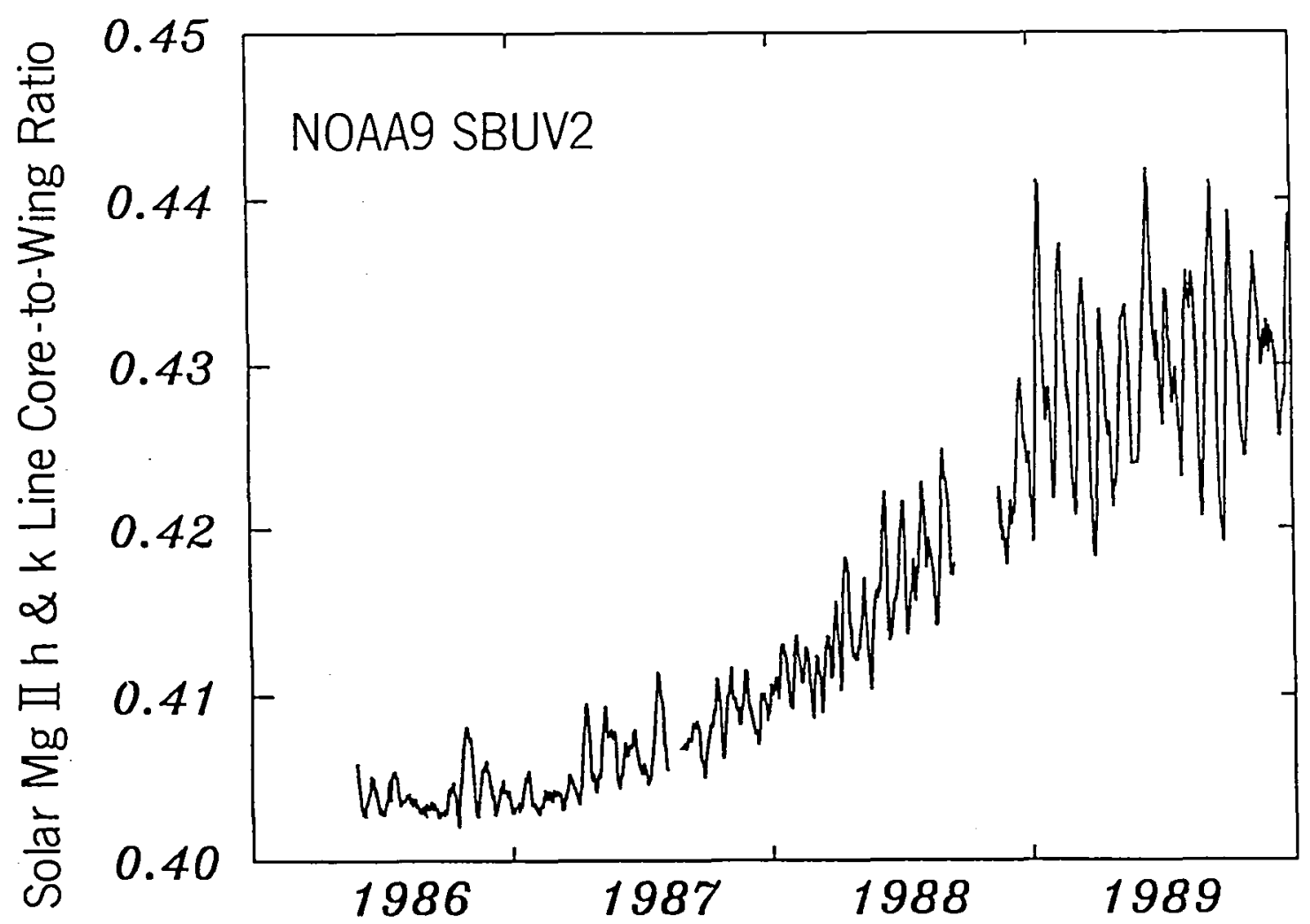

Fig. 1. Daily core-to-wing ratio for the unresolved solar Mg II h \& $k$ absorption lines from solar cycle minimum in September 1986 through the rise of solar cycle 22.

\section{THE RISE OF SOLAR CYCLE 22}

The Mg II core-to-wing ratio derived from the discrete wavelength measurements of the NOAA9 SBUV2 monitor are shown in Fig. 1 from the solar minimum in 1986 through the rise of solar cycle 22. The ratio shown has been modified with respect to that used by Heath and Schlesinger (1986) for NIMBUS7 in order to 1imit the NOAA9 measurements to the second of three gain ranges and thereby reduce the day-to-day jitter and long-term drift in the ratio (Donnelly et al., 1990). These NOAA9 results are related to the NIMBUS7 ratios by a simple linear relation that shows the 1989 maximum daily and 81-day average values are approximately equal to the corresponding maximum values from NIMBUS7 for solar cycle 21 . Note the very strong solar rotational variations with 27 to 28 days periodicity in 1989 after the main rise of the cycle. These short-term variations have roughly half the amplitude of the long-term rise from the $1986 \mathrm{minimum}$ to the average 1989 level, but are not as large as the solar rotational variations observed by the NIMBUS7 satellite in July and August 1982. An episode of two peaks per solar rotation with weak amplitude occurred in early 1988 . The rotational peak in 0ctober 1986 resulted from the first episode of strong newcycle activity for solar cycle 22.

\section{SOLAR CYCLE 21}

Fig. 2 shows monthly values of 13 -month soothed (half weight for first and last months) Mg II core-to-wing ratios from the NIMBUS7 satellite. Note how similar are the curves for the chromospheric $\mathrm{Mg}$ II ratio $\mathrm{R}(\mathrm{MgIIc} / \mathrm{w}, \mathrm{t})$ and the $10.7 \mathrm{~cm}$ solar radio flux (F 10) and how those two curves differ greatly from the photospheric sunspot number, coronal green line $(G)$, and soft $X$-ray flux $(X)$. This similarity between the long-term variations of FlO and R(MgIIc/w,t), which was first noted by Heath and 


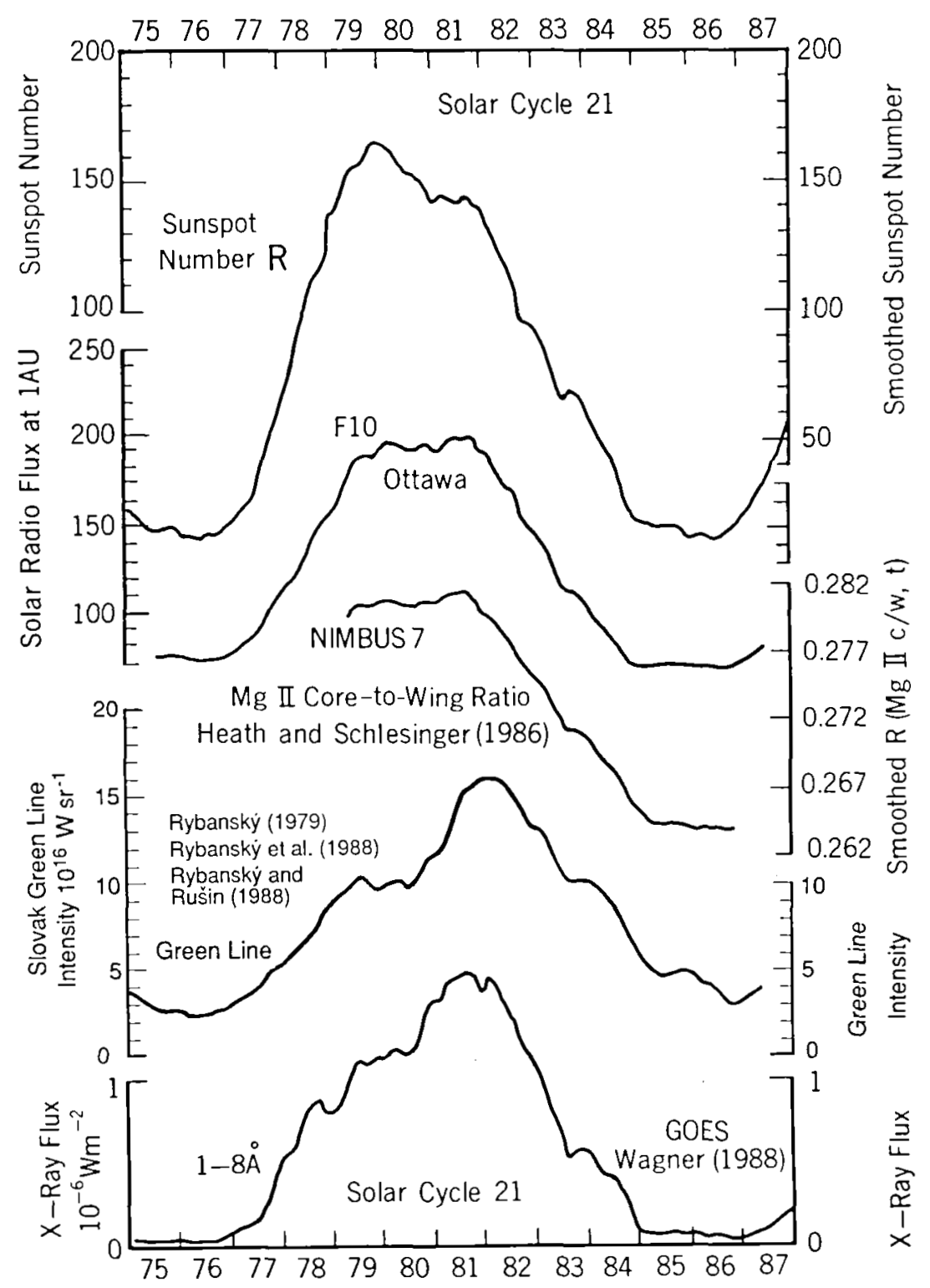

Fig. 2. Smoothed solar cyle 21 variations for the photospheric sunspot number, F10 solar radio flux, chromospheric Mg II core-to-wing ratio, coronal green line effective intensity, and the very hot and active coronal $1-8 \mathrm{Aflux}$. The $10.7 \mathrm{~cm}$ solar radio flux units (sfu) are $10^{-22} \mathrm{~W} \mathrm{~m}^{-2} \mathrm{~Hz}^{-1}$.

Schlesinger (1986), is in stark contrast to the marked differences in their shortterm (days, weeks) variations (Donnelly et al,, 1983; Donne11y, 1987). However, these long-term variations are not identical. R(MgIIc/w,t) peaks higher in late 1981 relative to the late 1979 level and decays slower in 1985 and early 1986 than F10. Compared to $R(M g I C / w, t)$ and $F 10$, the sunspot number sharply peaks early and has a long decay while $G \& X$ have a strong late peak. F10, $R(M g I I c / w, t)$ and $X$ decay in three years to a flat minimum while $G$ decays slowly until late 1986. The differences in decay and minima behavior of $X$ and $G$ imply corresponding differences in the emission measure in the $1-2 \times 10^{6}$ range, represented by $G$, and that at terperatures above $3 \times 10^{6} \mathrm{~K}$, represented by $\mathrm{x}$.

Differences in short-term variations of daily F10 and $R(M g I I c / w, t)$ are evident in Fig. 3. The range in amplitude of the short-term solar rotational variations is much 
larger for F10 relative to the long-term solar cycle amplitude than for $R(\operatorname{MgIIc} / \mathrm{w}, t)$. The high short-term peaks for Flo tend to be on the first rotation of episodes of major groups of active regions, while $R(M g I I c / w, t)$ tends to peak on the second or sometimes third rotation. During 1985 and 1986, F10 shows fewer rotational peaks than R(MgIIc/w,t), which is a consequence of the UV emission of active regions and their remnants having a much greater persistence (Donnelly, 1937).

The 81-day running averages shown in Fig, 4 suppress the short-term variations and illustrate better the intermediate (months) and long-term variations. The short-term solar-rotational variations are still evident in Fig. 4 in two ways. They contribute the small unimportant 27-day ripples in the curves. Because 27-day variations occur in a series where the peak amplitude per solar rotation quickly rises to a maximum in one or two rotations and then decays over several months, the 81-day running average of these short-term variations will also rise and fallover several months. This second effect contributes part of the intermediate-term (several months) variations shown in Fig. 4. Note that the intermediate-term variations in 1980 and 1981 have a period of roughly half a year and those in 1983 and 1984 are less than a year and may be related to the 155 day and 323 day periodicities in solar activity discussed by Lean and Brueckner (1989) and Pap et al. (1990). The half-year variations in 1980 and 1981 appear to be a little larger, rise faster, peak earlier and decay faster in F10 than in $R(M g I I c / w, t)$, which is consistent with the slower episodic evolution found in the solar-rotational variations in $\mathrm{R}(\mathrm{MgIIc} / \mathrm{w}, \mathrm{t})$. The local valley in $\mathrm{Fl0}$ near the start of 1982 appears to be filled in by the slower decay in $R(M g I c / w, t)$, In conclusion, the intermediate-term variations of $F 10$ and $R(M g I c / w, t)$ are fairly similar, with the shorter periods showing some small differences due to the evolution of major groups of active regions (Donne11y, 1987).

How can the temporal variations of $F 10$ be so different from those of $R(M g I c / w, t)$ for short-term variations and yet so similar for long-term variations? Consider F10 to consist of three parts, namely: (1) chromospheric thermal bremsstrahlung emission, (2) transition-region and coronal bremsstrahlung emission, and (3) gyroresonance absorption and emission, which are related to the strong magnetic fields from sunspots (Kundu et a1., 1980). Because of the greater persistence of plages and their remnants than for sunspots or for hot coronal emissions ( $\mathrm{T} \geq 3 \times 10 \mathrm{~K}$ ), long-term averages are dominated by the chromospheric component. The long-term average of the coronal and gyroresonance components in F10 would produce similar temporal results by reflecting the simple build up and decline of the total amount of activity, except the peak would occur earlier. This shift is caused by their more rapid evolution for each active region. It is consistent with the earlier peak, faster decay and larger amplitude of the half-year variations in $1980-1981$ in F10 than in $\mathrm{R}(\mathrm{MgIIc} / \mathrm{w}, \mathrm{t})$ in Fig. 4. Considering the much higher peak in late 1981 than the flux level in late 1979 for the coronal green line and 1 - 8 A X-rays in Fig, 2, the combination of the chromospheric bremsstrahlung and gyroresonance components of Flo must be much stronger than the coronal bremsstrahlung,

\section{ESTIMATES FOR SOLAR CYCLES $18-21$}

Given the similarity of long-term variations of $F 10$ and $R(M g I I c / w, t)$ and our current knowledge of their short-term differences, we should be able to make good estimates of $\mathrm{R}(\mathrm{MgIIc} / \mathrm{w}, t)$ back to the beginning of the F10 measurements in 1947. These ratios may then be used with the Heath and Schlesinger's (1986) wavelength scaling function to determine the UV flux variations in the $170-290 \mathrm{~nm}$ range.

Fig. 5 shows the intensity relation between $\mathrm{R}(\mathrm{MgIIc} / \mathrm{w}, \mathrm{t})$ and $\mathrm{F} 10$, where the correlation coefficient $(r)$ is 0.95 . There are more points further from the main trend on 


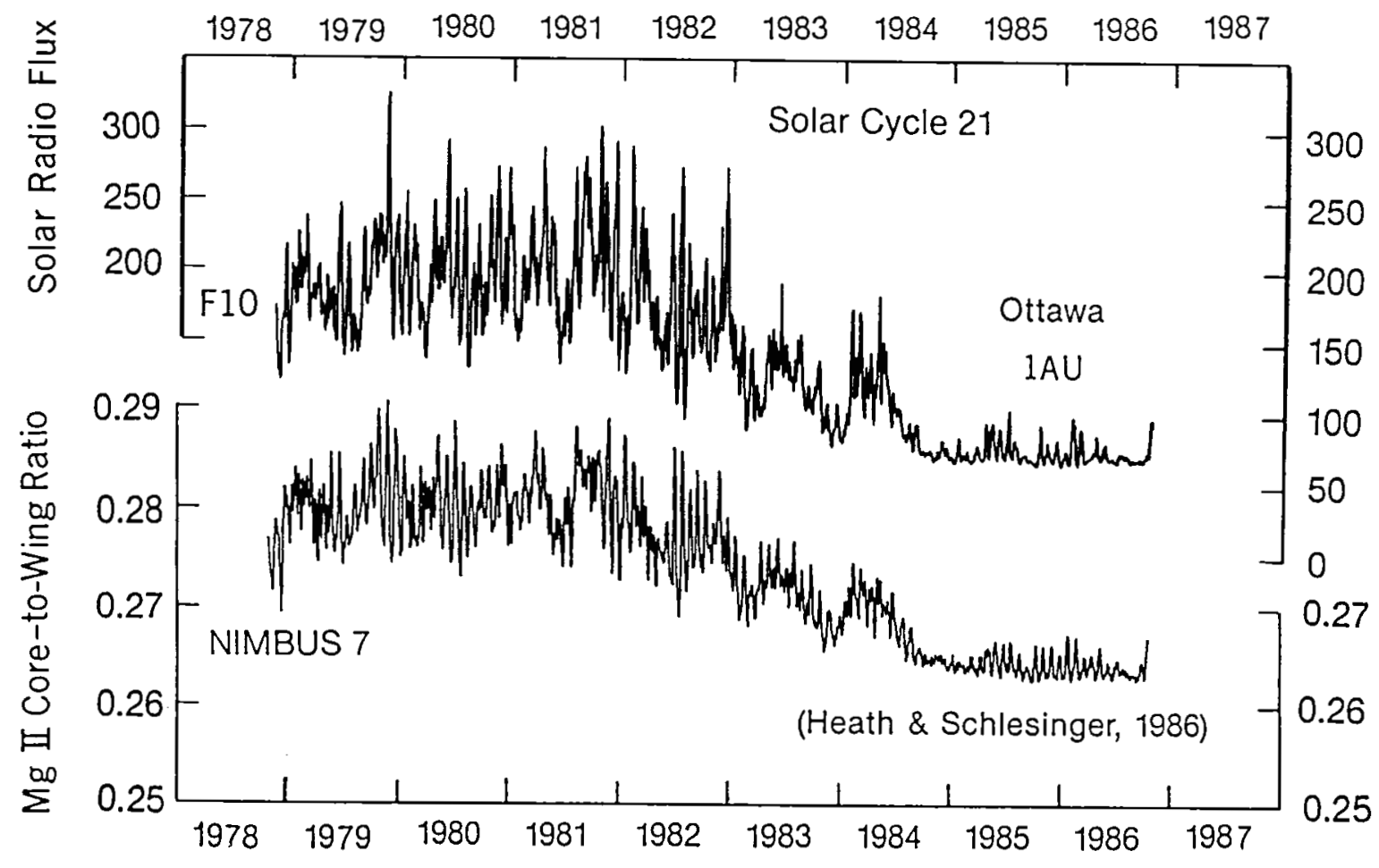

Fig. 3. Daily values of the solar $10.7 \mathrm{~cm}$ radio $f l u x$ and $\mathrm{Mg}$ II core-to-wing ratio.

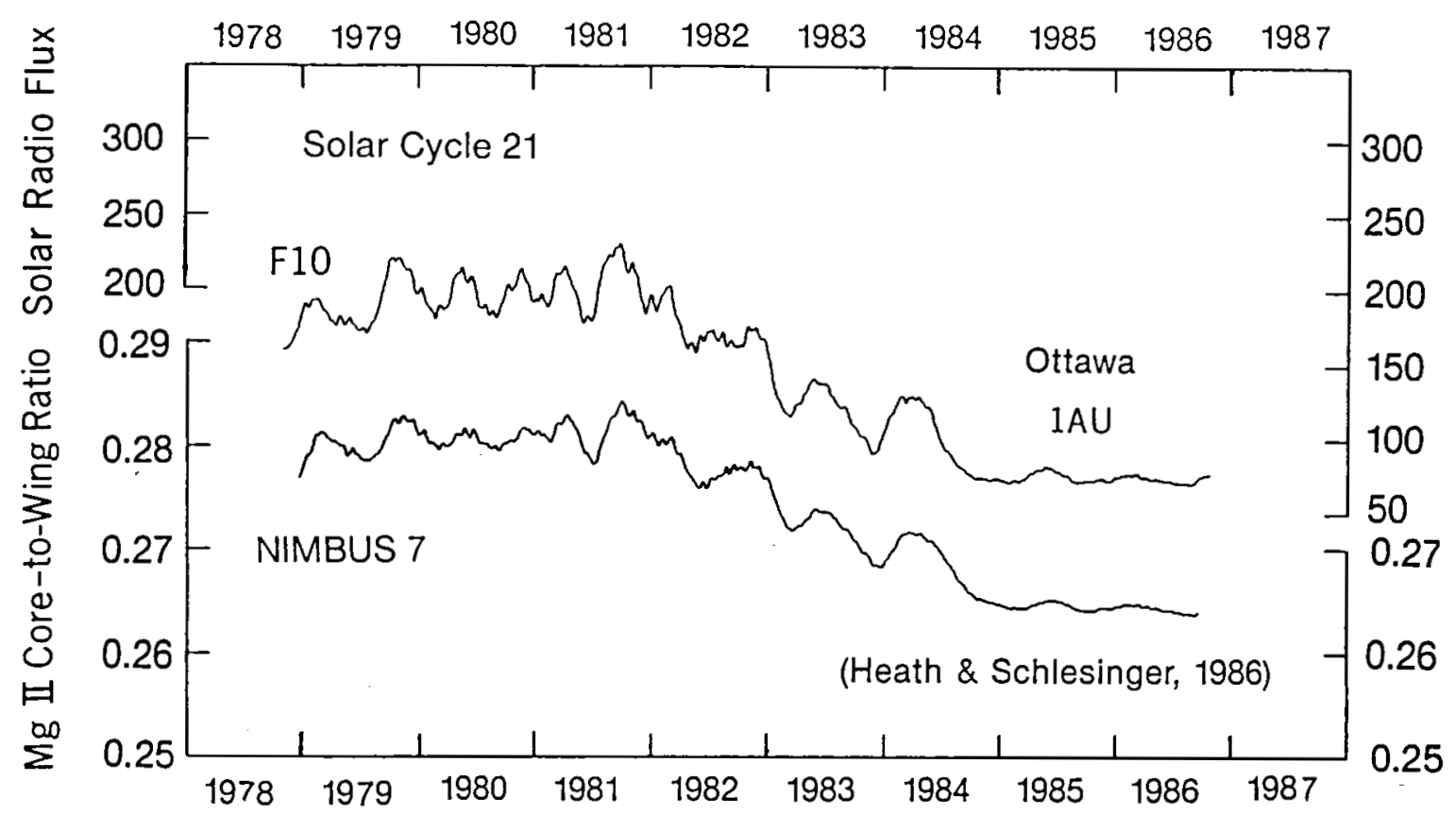

Fig. 4. Daily values of 81-day running averages of Flo and $M g$ II core-to-wing ratio. 
the right side of the diagram, which is caused by the early high solar-rotational peaks in $\mathrm{F} 10 \mathrm{re-}$ lative to $\mathrm{R}(\mathrm{MgIIc} / \mathrm{w}, \mathrm{t})$ during episodes of major groups of active regions. The most important feature is the break from a linear trend near Flo equal to $94 \mathrm{sfu}$ (solar radio flux units $=10^{-22} \mathrm{~W}$ $\mathrm{m}^{-2} \mathrm{~Hz}^{-1}$ ), The same type of change in trends near solar minimum was found in Lyman alpha versus F10 by Barth et al. (1990) and is evident in the equivalent width of He I 10830 A (Harvey, 1984) versus F10, except the change is even larger in these two cases. This effect is caused by the slow decay in $\mathrm{R}(\mathrm{MgIIc} / \mathrm{w}, \mathrm{t})$ in 1985 and early 1986 while the minima in F10 per solar rotation are nearly constant. Considering the slow decay in $G$ in Fig. 2 in 1985, we interpret this change in trend to be caused by the gyroresonace component of F10 rather than by the transition-region and coronal component. The data in Fig. 5 were fit with polynomials of order 1 to 7. The best fit was made with two straight lines that join at F10 $=94 \mathrm{sfu}$. These linear trends were then used with the 81-day average of Flo to make preliminary estimates of the long-term trends of R(MgIIc/w,t) shown in Fig. 6. The maximum estimated values for cycles 18 and 19 are higher than the observed $\mathrm{R}(\mathrm{MgIIc} / \mathrm{w}, \mathrm{t})$ for cycles 21 and 22 , at least through 1989.

In addition to estimating the long-term variations of $\mathrm{R}(\mathrm{MgIIc} / \mathrm{w}, \mathrm{t}$ ), we have derived techniques for improving estimates of the short-term solar-rotational variations. Simply by separating Fl0 into long-term and short-term variations improves the estimate because the regression slopes differ for these two time scales. Secondly, including the sunspot blocking function (Hoyt and Eddy, 1982; Hudson et a1., 1982) reduces the effect of the large F10 peaks on the first rotation of a major new group of active regions. Oster (1990) has found that the $10 \mathrm{~cm}$ enhancement due to an active region is better correlated with that region's sunspot area than with the associated plage area. His result is consistent with the sunspot blocking function improving the fit of $\mathrm{F} 10$ with $\mathrm{R}(\mathrm{MgIIc} / \mathrm{w}, \mathrm{t})$. Apparently, the sunspot blocking function provides an approximate correction for the gyroresonance component in Fl0. This component boosts the radio emission on the first rotation of episodes of major groups of active regions (Donnelly, 1987), when the net sunspot area is large. Another technique for improving the estimate of short-term variations is currently being evaluated. This involves filtering the full-disk $\mathrm{Ca}-\mathrm{K}$ plage index and sunspot number to identify and estimate episodes of 13-day periodicity, which are strong at UV wavelengths but absent in F10.

Comparing the predicted and observed $R(M g I I c / v, t)$ during the rise of solar cycle 22 has shown that the long-term $\mathrm{FlO}$ and $\mathrm{R}(\mathrm{MgIIc} / \mathrm{w}, \mathrm{t})$ trends are related by a small hys- 


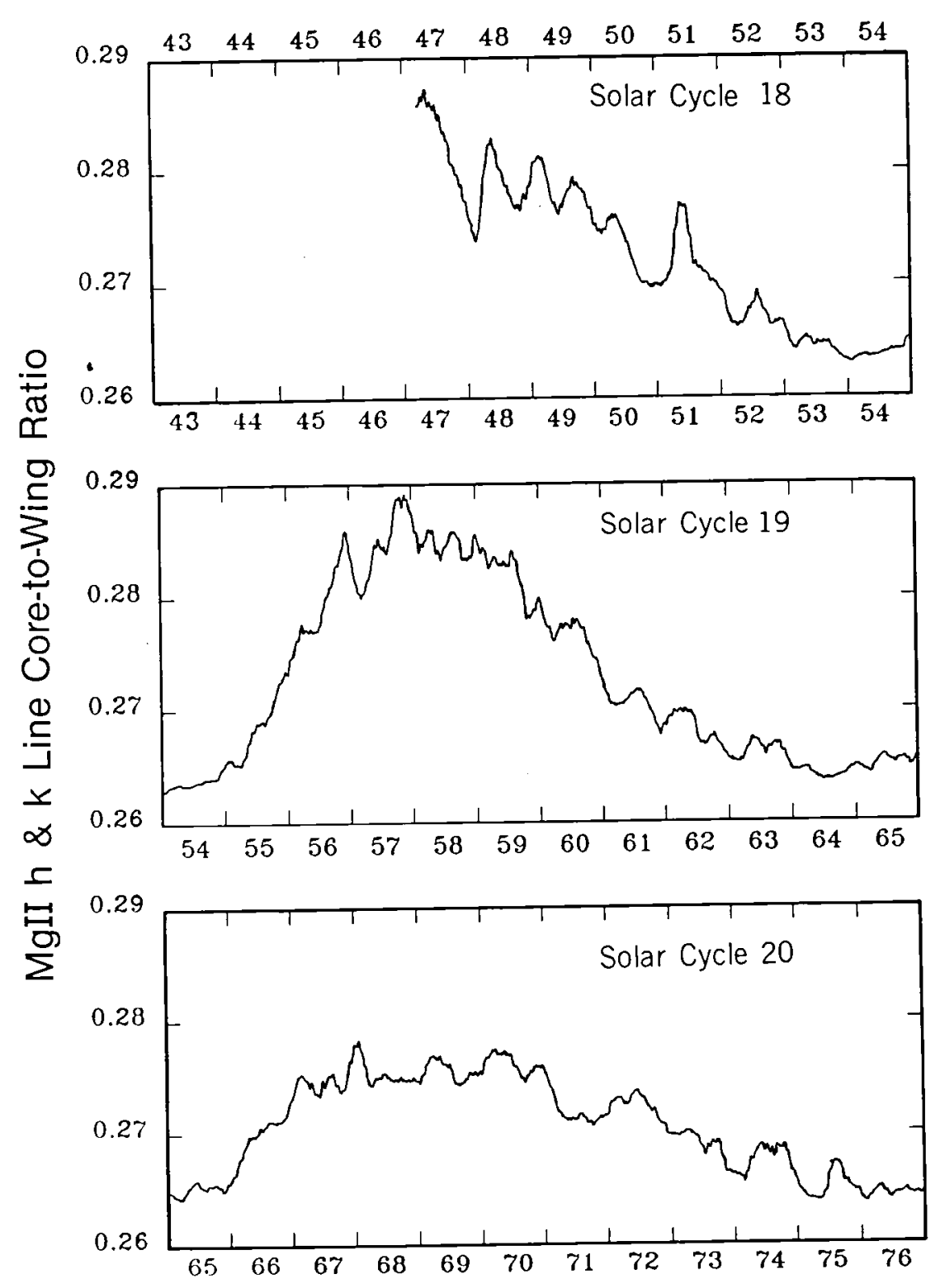

Fig. 6. Preliminary estimates of daily values of 81 -day averaged $M g$ II core-to-wing ratios derived from Flo.

teresis effect at low F10 values, which can be accounted for in future estimates. Secondly, using 81 -day averages to estimate the long-term variations in $R(M g I I c / w, t)$ from F10 leads to overestimating the intermediate-term variations, especially those with periods near half a year. This can be improved by using annual averages.

\section{CONCLUSIONS}

Solar cycle 22 through 1989 at UV wavelengths, as represented by the Mg II core-towing ratio, has already reached the peak intensities observed in solar cycle 21 , but has not yet reached the peak intensities estimated for solar cycles 18 and 19. Estimates of R(MgIIc/w,t) back to 1947 are currently being developed and improved. Separating $F 10$ into long-term and short-term variations improves the fit to $\mathrm{R}(M \mathrm{gIIC} / \mathrm{w}, \mathrm{t})$. Including the sunspot blocking function improves the estimates of 
short-term variations by correcting for the high peaks in F10 relative to $R(\mathrm{MgIIc} / \mathrm{w}, \mathrm{t})$ during the first solar rotation of an episode of a major group of active regions. These high Flo peaks are interpreted as resulting from the gyroresonance component associated with the strong magnetic fields of sunspots. The sunspot blocking function serves as a measure of the net area of these strong field regions.

\section{REFERENCES}

Barth, C. A., W. K. Tobiska, G. J. Rottman, and O. R. White, "Comparison of $10.7 \mathrm{~cm}$ Radio Flux with SME Solar Lyman Alpha Flux, Analysis", to be published in Geophys. Res. L., 1990 ,

Donnelly, R. F., D. F. Heath, J. L. Lean and G. J. Rottman, "Differences in the Temporal Variations of Solar UV Flux, 10.7-cm Solar Radio Flux, Sunspot Number, and $\mathrm{Ca}-\mathrm{K}$ Plage Data Caused by Solar Rotation and Active Region Evolution", J. Geophys. Res., 88, 9883-9888, 1983.

Donne11y, R. F., J. Barrett, S. D. Bouwer, J. Pap, L. Puga and D. Stevens, Solar UV Flux Measurements from the SBUV2 Monitor on the NOAA9 Satellite, Part I. Mg II $\& \underline{\mathrm{k}}$ Line Core-to-Wing ratios for $1986=198$, to be published as a NOAA ERL SEL Tech. Memo., 1990.

Donnelly, R. F., "Temporal Trends of Solar EUV and UV Full-Disk Fluxes", Solar Phys., $109,37-58,1987$.

Harvey, J., "Helium 10830A Irradiance: 1975-1983", Solar Irradiance Variations on Active Region Time Scales, NASA Conference Publ. 2310, eds, B. J. LaBonte et a1, , 197-211, 1984.

Heath, D. F., and B. M. Schlesinger, "The Mg 280-nm Doublet as a Monitor of Changes in Solar Ultraviolet Irradiance", J. Geophys. Res., 91, 8672-8682, 1986.

Hoyt, D. V., and J, A. Eddy, An Atlas of Variations in the Solar Constant Caused by Sunspot Blocking and Facular Emissions from 1874 to 1981, NCAR Tech. Note 194+STR, NCAR, Boulder, Colorado, $106 \mathrm{pp}, 1982$.

Hudson, H. S., S. Silva, M. Woodard, and R. C. Willson, "The effects of sunspots on solar irradiance", Solar Phys., 76, 211-219, 1982.

Kundu, M. R., E. J. Schmah1 and M. Gerassimenko, "Microwave, EUV, and X-ray Observations of Active Region Loops: Evidence for Gyroresonance Absorption in the Corona", Astron. Astrophys., 82, 265, 1980,

Lean, J. L., and G. E. Brueckner, "Intermediate-Term Solar Periodicities: 100 to 500 days", Astrophys. J., 337, 568-578, 1989.

Lean, J., "Contribution of Ultraviolet Irradiance Variations to Changes in the Sun's Total Irradiance", Sci., 244, 197-200, 1989.

London, J., J. Pap, and G. J. Rottman, "Observed Solar Near UV Variability: A Contribution to Variations of the Solar Constant", Handbook for MAP, 29, 9-12, 1989.

Oster, L., "Reconstructing the Emission at $10.7 \mathrm{~cm}$ from Individual Active Regions in Terms of Optical Data", to be published in J. Geophys. Res., 1990.

Pap, J., W. K. Tobiska, and S. D. Bouwer, "Periodicities of Solar Irradiance and Solar Activity Indices I.", submitted for publication in Solar Phys., 1990.

Rybansky, M., and V. Rusin, private communication, 1988.

Rybansky, M., "Coronal index of solar activity III (Years 1971-1976)", Bull. Astron. Inst. Czechos 1., 30, 104-113, 1979.

Rybansky, M., V. Rusin and E. Dzifcakova, "Coronal index of solar activity, V. Years 1977-1986", Bu11. Astron. Inst. Czechoslovakia, 39, 106-119, 1988.

Wagner, W.J., "Observations of 1-8A solar x-ray variability during solar cycle 21 ", Adv. Space Res., 8, (7)67-(7)76, 1988. 


\title{
SECULAR CHANGE IN EQUIVALENT WIDTH OF C 5380, 1978 - 90.
}

\author{
William C. Livingston \\ National Solar Observatory, Tucson, Arizona 85726
}

\begin{abstract}
The equivalent width of the high excitation photospheric line of $\mathrm{C} 5380 \AA$ has been measured 3-4 times monthly in the solar irradiance spectrum since 1978. C 5380 behaves differently than other spectral lines in that it is unmodulated by the activity cycle, yet has increased in strength by $0.081 \pm .008 \mathrm{~m} \AA$ in 12 years. Nominal equivalent width is $22.25 \mathrm{~m} \AA$. Implied is a temperature increase of $4.6 \mathrm{~K}$ which is an order of magnitude greater than constraints allowed by ACRIM results.
\end{abstract}

\section{Introduction}

Twelve years of monthly measurements of selected Fraunhofer lines in the full disk irradiance spectrum now provide us with a good picture on the response of various spectral features to a changing Sun. Chromospheric lines such as $\mathrm{Ca} \mathrm{K}, \mathrm{H}$ alpha and $\mathrm{Ca} 8542$ all weaken a percent or so with an increase of solar activity, Fig. 1. Resolved disk observations suggest this weakening is due to active region plage. In such areas chromospheric lines become shallow or even display emission components.

He 10830, on the other hand, is a maverick that strengthens in plage. From solar minimum to maximum the depth of this line can double in the irradiance spectrum. ${ }^{1}$ It has been demonstrated that the strengths of these chromospheric lines correlate well with Ly alpha emission and thus can serve as surrogates for the EUV-UV solar output. $^{2}$

At the photospheric level the pattern is not so simple. One might expect strong photospheric lines to show the greatest variability. Eclipse "flash" spectra, ${ }^{3}$ which clearly represent the chromosphere, tell us that emission strength goes hand-in-hand with disk equivalent width. If we think of this emission component as "filling in" the cores of strong lines, then they should weaken at solar maximum. The central depths of such lines do display a slight chromospheric weakening with activity (not reproduced herein). Equivalent width, on the other hand, seems constant in our data, for example Fe 5250.6 in Fig. 2. Probably this is a matter of line saturation ${ }^{4}$ combined with insufficient instrumental sensitivity.

The equivalent widths of most moderate to weak photospheric lines track nicely the chromospheric lines in cyclic variability, see Mn 5394 in Fig. 2. In terms of percentage change they are in fact comparable indicators, although observational error is greater owing to their relatively small central depths.

Like He 10830, the "photospheric" oxygen triplet at $7774 \AA$ is another loner. Unlike all other photospheric lines O 7774 increases in strength with activity. Probably there is some NLTE effect at work here.

Finally we come to the behavior of C $5380 \AA$. The equivalent width of this line shows no detectable cycle variability, but curiously has increased in strength over our observing time interval of 12 years.

\section{5380 on the Resolved Disk}

CI $5380.3231 \AA$ has an excitation potential of $7.65 \mathrm{~V}$. The species has an ionizing potential of $11.26 \mathrm{~V}$ and is always completely neutral in the photosphere (compared to $\mathrm{Fe}$, which is not). At disk center $\mathrm{C} 5380$ has a central depth with respect to the continuum of about 0.155 and an equivalent width of $26.3 \mathrm{~m} \AA$. At the limb $(\mu=0.1)$ the central depth reduces to about 0.04 . According to the HSRA model atmosphere, the peak in the physical height of the response function for $\mathrm{C} 5380$ lies only slightly above $(\sim 25 \mathrm{~km})$ the contribution function for the $\lambda 5380 \AA$ 

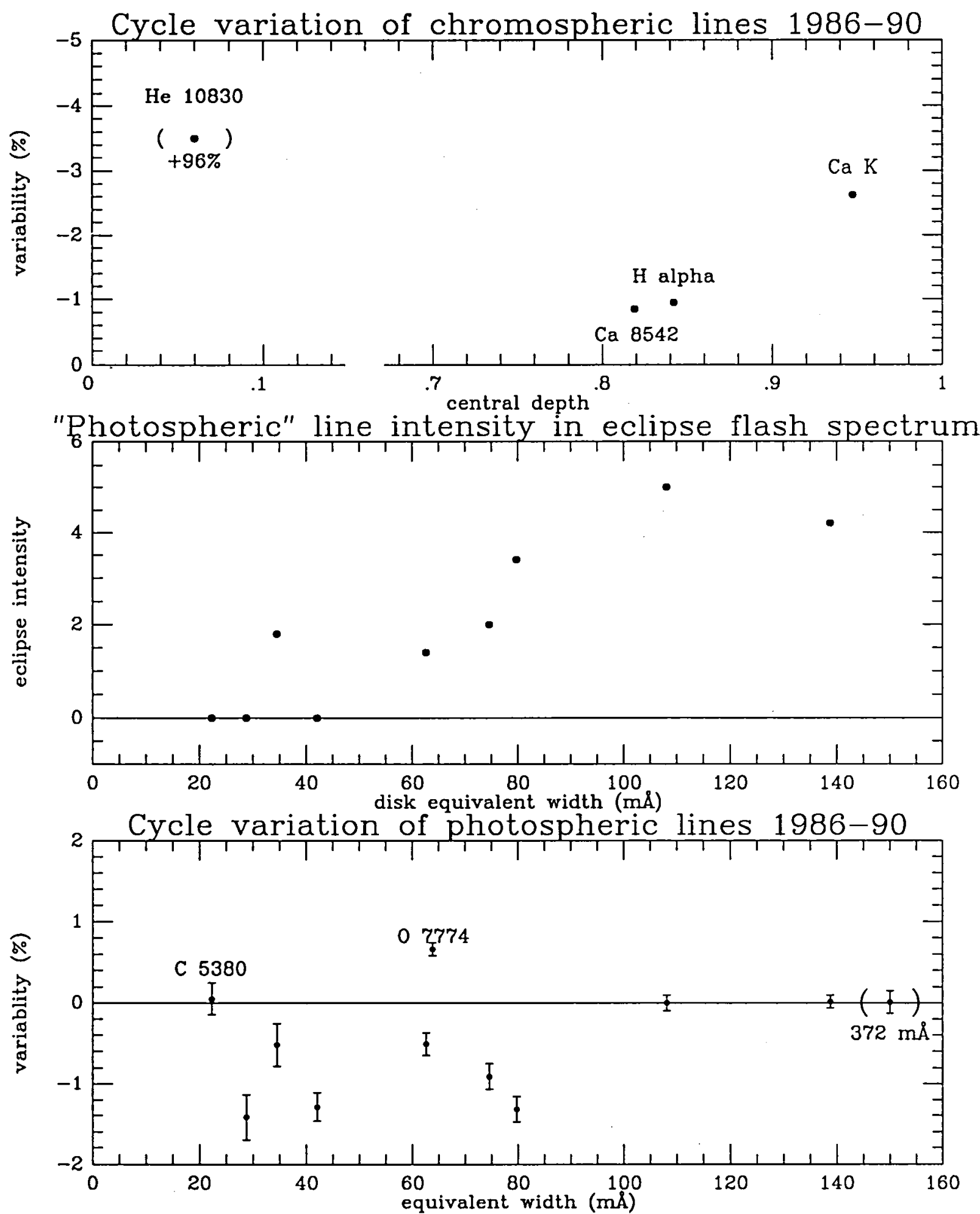

Fig. 1. Observed cycle modulation of a variety of chromospheric (top panel) and photospheric lines (bottom).

Strength of the photospheric lines in an eclipse flash spectrum is indicated in the middle panel. Eclipse intensity of zero means the line is not seen. The lines shown are the same as in the lower panel except for $O 7774$, which is off scale, and the $372 \mathrm{~m} \AA$ line which was outside the wavelength coverage. All chromospheric lines are off scale in this representation. 
continuum. At disk center $\Delta \mathrm{W} / \mathrm{W}=+.00086 / \mathrm{K}$, i.e. the line should strengthen with increasing temperature. Compare this with $\Delta \mathrm{W} / \mathrm{W}=-.00063 / \mathrm{K}$ for the typical moderate strength Fe line such as Fe 5250.6. Sec Livingston, et. al., ${ }^{5}$ and Livingston and Holweger, ${ }^{6}$ for more details and references. From 105 days of observation we obtain at disk center, $\mathrm{W}_{\lambda}=25.024+.0117 \pm .0025$ (year) $\mathrm{m} \AA$, or an increase of $0.14 \pm .03 \mathrm{~m} \AA / 12$ year.
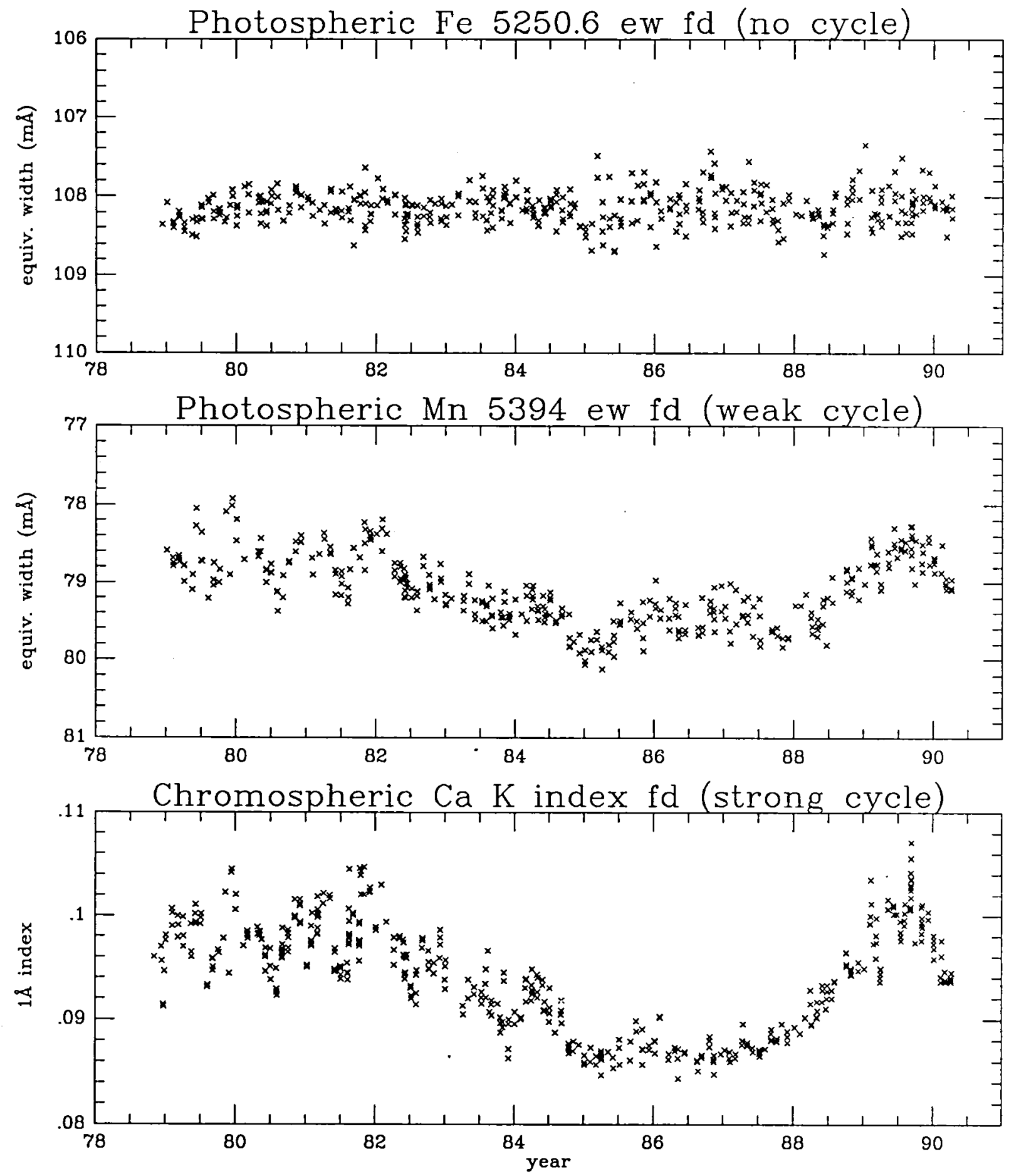

Fig. 2. Examples of solar cycle behavior of selected lines in Fig. 1. The Mn 5394 plot is inverted to agrec in sense with $\mathrm{Ca} \mathrm{K}$. 


\section{5380 Observed Full Disk}

Although our C 5380 full disk archives extend back to 1976, various changes in the instrument and observing technique contaminate the data before November 1978. Since then, however, all aspects of the observations and data reduction have remained fixed. Annually the telescope mirrors are recoated and once, in April 1980, the spectrograph mirrors were also re-aluminized. Otherwise the system has remained immutable (a beneficial aspect of poor funding).

Fig. 3 displays the temporal record for individual observations. A least square fit to the 487 days of data gives $\mathrm{W}_{\lambda}=21.67+.00675 \pm .00066$ (year) $\mathrm{m} \AA$. The formal probable error in the slope is one-tenth the value. Implicd is an increase of $0.081 \pm .008 \mathrm{~m} \AA / 12$ years. This is slightly smaller than the center disk value, and for various reasons the probable error is less. If the HSRA model were applicable, a temperature increase of $4.6 \mathrm{~K}$ is deduccd. Assuming the Sun is a black body this leads to an increment in the solar constant of $0.3 \%$, an amount incompatable with ACRIM results. Evidently the HSRA model is inappropriate here.
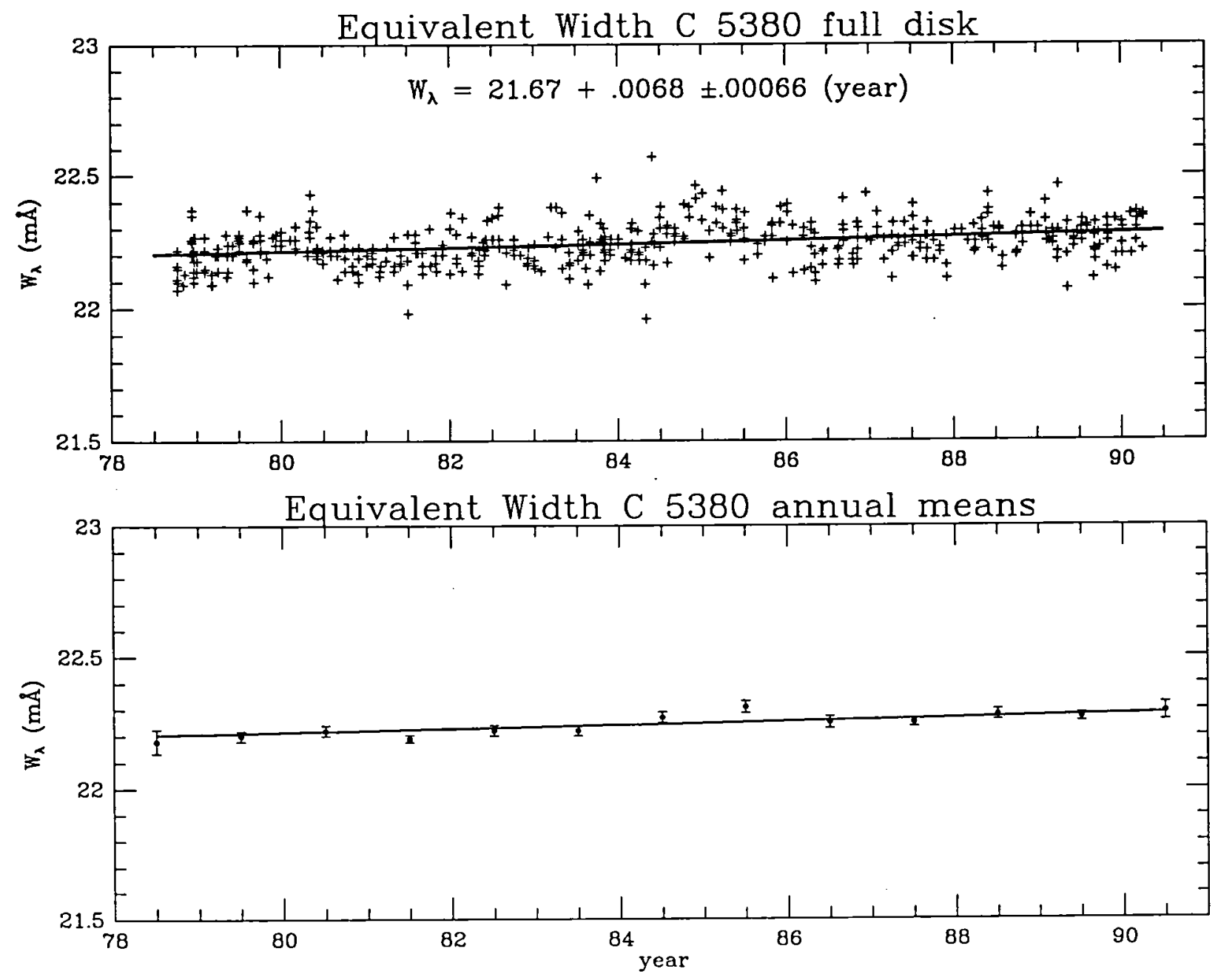

Fig. 3. Temporal record of C 5380. Top panel is individual days, each an average of 6 observations. Bottom, the same data averaged into one year bins. The straight line fit is from the un-bined data. 


\section{Summary and Conclusions}

A comparison between a wide variety of Fraunhofer lines, and how they empirically respond to the solar activity cycle, leads us to conclude that C 5380 is a unique indicator of conditions in the low photosphere. Model calculations suggest it is more temperature sensitive (and should respond in an opposite sense) compared with other lines, yet it displays no significant cycle modulation. Its equivalent width appears to have increased by $0.081 \pm .008$ $\mathrm{m} \AA$ over a time span of 12 years. Formal statistics suggest this trend is real, and implies a $4.6 \mathrm{~K}$ increase in surface temperature. Such a temperature rise translates to $0.3 \%$ in the solar constant, and is much too large for compatibility with ACRIM results.

What is needed to understand C 5380? Obviously patience and a longer archival time base is desirable. We may also search for clues on the resolved solar disk. How does C 5380 behave in plage and faculae? Earlier work was inconclusive. Or perhaps the Sun is evolving in a non-thermal fashion on a time scale of 22 years, or longer. Other evidence has been proposed for long term excursions in solar temperature, but such ideas are speculative at present. ${ }^{7,8,9}$

\section{References}

1. Foukal, P., and Lean, J., 1988, "Magnetic Modulation of Solar Luminosity by Photospheric Activity", Astrophys. J., 328, 347 - 357.

2. White, O. R., Rottman, G. J., and Livingston, W. C., 1990, "Estimation of the Solar Lyman Alpha Flux From Ground Based Measurements of the Ca II K Line", Geophysical Res. Let., (in press).

3. Dunn, R. B., Evans, J. W., Jefferies, J. T., Orrall, F. Q., White, O. R., and Zirker, J. B., 1968, "The Chromospheric Spectrum at the 1962 Eclipse", Astrophys. J. Suppl. 1 5, $275-458$.

4. Mitchell, W. E. Jr., and Livingston, W. C., 1990, "Line-blanketing Variations in the Irradiance Spectrum of the Sun from Maximum to Minimum of the Solar Cycle", Astrophys. J. (submitted).

5. Livingston, W., Milkey, R., and Slaughter, C., 1977, "Solar Luminosity Variation. I. CI 5380 as a Temperature Indicator and a Search for Global Oscillations", Astrophys. J. 2 11, 281 - 287.

6. Livingston, W., and Holweger, H., 1982, "Solar Luminosity Vairation. IV. The Photospheric Lines, 1976 1980", Ap. J. 252, 375 - 385.

7. Kuhn, J. R., Libbrecht, K. G., and Dicke, R. H., 1988, "The Surface Temperature of the Sun and Changes in the Solar Constant", Sci. 242, 908 - 911.

8. Schatten, K. H., and Orosz, J. A., 1990, "Solar Constant Secular Changes", Solar Phys., 12 5, 179.

9. Kroll, R. J., Hill, H. A., and Beardsley, B. J., 1990, “A Correlation Between Changes in Solar Luminosity and Differential Radius Measurements. (this conference). 


\title{
TEN YEARS OF SOLAR CHANGE AS MONITORED BY SBUV AND SBUV/2
}

\author{
B. M. Schlesinger \\ Science Applications Research \\ Lanham, MD 20706
}

R. P. Cebula, D. F. Heath, and M. T. DeLand

ST Systems Corporation

Lanham, MD 20706

and

R. D. Hudson

NASA/Goddard Space Flight Center

Greenbelt, MD 20771

\begin{abstract}
Observations of the Sun by the Solar Backscalter Ultraviolet (SBUV) instrument aboard Nimbus 7 and the SBUV/2 instrument aboard NOAA-9 reveal variations in the solar irradiance from 1978, 10 1988. The maximum (1) minimum solar change estimated from the Heath and Schlesinger ${ }^{1} \mathrm{Mg}$ index and wavelength scaling factors is about $4 \%$ from $210-260 \mathrm{~nm}$ and $8 \%$ for $180-210 \mathrm{~nm}$; direct measurements of the solar change give values of $1-3 \%$ and $5-7 \%$, respectively, for the same wavelength range. Solar irradiances were high from the start of observations, late in 1978, until 1983, declined until early 1985, remained approximately constant until mid-1987, and then began to rise. Peak-to-peak 27-day rotational modulation amplitudes were as large as $6 \%$ at solar maximum and 1-2\% at solar minimum. During occasional intervals of the 1979-1983 maximum and again during 1988, the dominant rotational modulation period was 13.5 days. Measurements near 20(0)-205 $\mathrm{nm}$ show the same rotational modulation behavior but cannot be used to track long-term changes in the Sun because of uncertainties in the characterization of long-term instrument sensitivity changes.
\end{abstract}

\section{INTRODUCTION}

Since November 7, 1978, one week after the launch of the Nimbus 7 satellite, Solar Backscatter Ultraviolet (SBUV) instruments have been continually measuring the solar irradiance in the wavelength range from 160110 $400 \mathrm{~nm}$. The original SBUV instrument aboard Nimbus 7 provided measurements from November 7, 1978 until February 13, 1987, when the chopper that was used to obtain an estimate of the dark current contribution to the observed signal began to go out of synchronization. The fraction of data with unacceptably high noise levels began to increase rapidly, until, by mid-March of that year, all the data were too noisy to be useful. Eight years of SBUV solar irradiance data have been archived at the National Space Science Data Center. The first in a series of SBUV/2 instruments was launched aboard NOAA-9 on December 12, 1984 and began solar measurements on March 12,1985. These measurements continue to the present day. Unlike Nimbus 7, whose Sun-synchronous orbil passes over the ground near local noon, the original NOAA-9 orbit was launched into a 2:30) P.M. orbit. Since launch, the orbit has precessed so that it now passes over the Earth at about 5:30 P.M. local time, so that at times the solar angle of incidence is outside the range for which the system was calibrated. In addition, at some times of the orbit, the solar detector is shadowed by other parts of the satellite. Data from March 12, 1985 to September 13, 1988 have been processed. Later data are still being reduced. A second SBUV/2 instrument aboard NOAA-11 began measurements in December, 1988, but these measurements also are still being reduced. 
While the primary function of the SBUV instruments is to measure solar radiation backscattered by the Earth's atmosphere for the purpose of monitoring total column ozone and its vertical profile, the instrument can measure solar irradiance by deploying a diffuser plate into the optical path. The measurements of solar irradiance discussed in this paper are taken in a mode in which the instrument scans the solar spectrum between 160 and $400 \mathrm{~nm}$. The bandpass is triangular, with a width of $1.1 \mathrm{~nm}$. For SBUV, values are available at central wavelength intervals of $0.2 \mathrm{~nm}$; for SBUV/2, the separation between adjacent points varies from approximately $0.154 \mathrm{~nm}$ at $160 \mathrm{~nm}$ to $0.137 \mathrm{~nm}$ at $400 \mathrm{~nm}$.

\section{RESULTS FROM NIMBUS 7 SBUV}

\section{Magnesium Index}

The uncertainties in the characterization of the change of instrument sensitivity with time motivated the search for a measure of solar variability that would be independent of these uncertainties. To this end, Heath and Schlesinger ${ }^{1}$ (hereafter HS) developed an index based on the ratio of the average irradiance over three wavelengths at the center of the $\mathrm{Mg}$ II 280-nm $h$ and $k$ doublet, which is not resolved by the instrument, to the average over four wavelengths, two each on either side in the far wings at local relative maxima of solar irradiance as a function of wavelength. The wing wavelengths were chosen to be at equal distances from the center; thus, use of this ratio eliminates not only any wavelength-independent changes in the instrument sensitivity, but also any wavelength-dependent changes with time that can be represented by a linear function of wavelength. Instrument characterization studies verify that the wavelength dependence of the changes can indecd be so) represented at these wavelengths.

The lower panel of Figure 1 shows the $\mathrm{Mg}$ index as a function of time for the first 8 years of SBUV measurements. Short-term variability arising from rotational modulation is clearly apparent. HS found that although the period of rotational modulation was generally 27 days, corresponding to domination of the solar variability by a single active region, there were times when the period was 1.3 .5 days, arising from two active regions on either side of the Sun. The $\mathrm{Mg}$ index had high values from launch until the end of 1983 and then declined gradually but not steadily until mid-1984, when it leveled off. Pcak-to-peak 27-day rotational modulation amplitudes were as large as $6 \%$ at solar maximum, decreasing to $1-2 \%$ at solar minimum. The failure of rotational modulation to vanish entirely suggests that some residual activity persists even at solar minimum. The near constancy of the lower envelope for 1985-1987 suggests that days where the $\mathrm{Mg}$ index has its minimum value correspond to the least activity during a cycle. Also noteworthy is the fact that the $\mathrm{Mg}$ index is higher at rotational minimum during the period of solar maximum than at any time during solar minimum. This result implies that during solar maximum, even when the dominant active region is on the hemisphere that is turned away from the Earth, more activity is present on the visible hemisphere of the Sun than at solar minimum.

\section{Scaling}

HS showed that variations from 1978 through 1983 of the irradiance ratio across the Al edge near $210 \mathrm{~nm}$ (Kjeldseth Moe and Milone ${ }^{2}$ have noted that the discontinuity in solar irradiance associated with the Al edge is at a longer wavelength than the laboratory edge; a possible explanation is overlap of many lines just longward of the true edge) could be reproduced by multiplying the variations of the $\mathrm{Mg}$ index by the ratio of the amplitude of rotational modulation at the $\mathrm{Al}$ edge to that of the $\mathrm{Mg}$ index. They used this result to derive a method for estimating solar variations at all wavelengths measured by SBUV. They selected 23 rotations with strong modulation in the $\mathrm{Mg}$ index. For each rotation, they derived the rotational modulation at every wavelength and the $\mathrm{Mg}$ index by calculating the ratio of the irradiance at maximum to the average over the two surrounding minima to eliminate the effect of changes in instrument sensitivity. For each wavelength, they derived a scaling factor by a linear fit to the 23 values of modulation as a function of the $\mathrm{Mg}$ index modulation, plus two points 

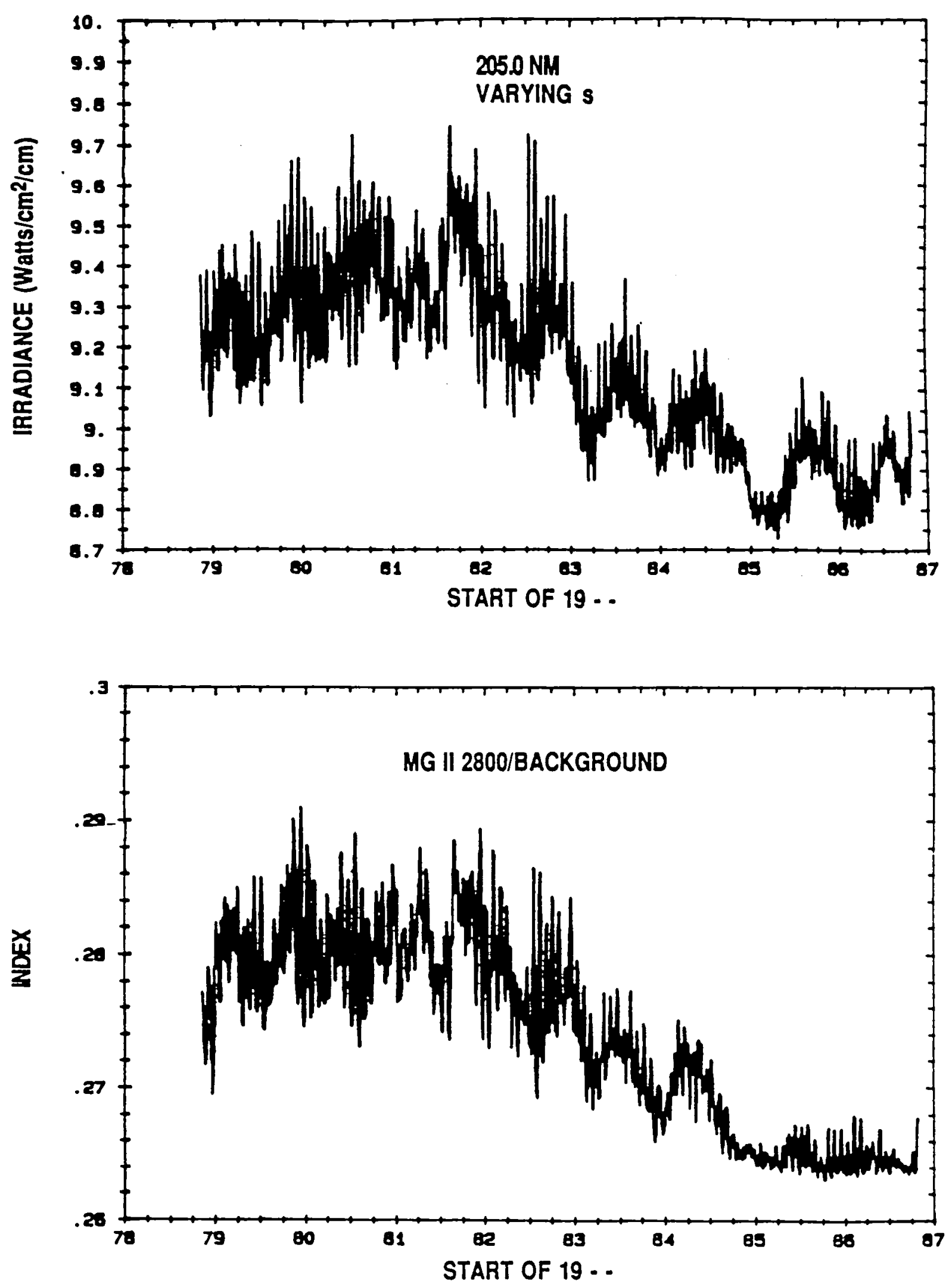

Figure 1. (top) Time scries of solar irradiance at $205.0 \mathrm{~nm}$ using improved instrument characterization. (bottom) Time series of $\mathrm{Mg}$ index. 
at no modulation, a ratio of 1 . Using this method, solar variations between two days are estimated by multiplying the ratio of the $\mathrm{Mg}$ index for the two days by the scale factor for each wavelength.

To derive changes over the solar cycle, two 27 -day periods were chosen: one during which the sustained values were near maximum, October 27 - November 22, 1979, and one near minimum, September 15 - October 11, 1986. Longer-term atmospheric changes will be sensitive to the sustained level of the solar irradiance rather than (1) extreme daily values. An additional constraint was that the two rotations be at the same time of year, to eliminate the effect of a $1 \%$ annual cycle in the later years of data probably arising from an imperfect correction for the angular dependence of the diffuser plate reflectivity.

The lower panel of Figure 2 shows the derived change. Longward of about $260 \mathrm{~nm}$, the variation exceeds $1 \%$ only near the center of strong lines. From 210 to $260 \mathrm{~nm}$, the change is on the order of $3-4 \%$; it rises to $8 \%$ immediately shortward of the Al edge near $210 \mathrm{~nm}$ and $1010-11 \%$ at $180 \mathrm{~nm}$. At wavelengths shorter than 170 $\mathrm{nm}$, the signal is too weak and the data are too noisy to derive useful results.

\section{Improved Instrument Characterization}

Schlesinger and Heath ${ }^{3}$ and the User's Guide to the solar irradiance tapes ${ }^{4}$ have shown that not all instrumental eflects have been removed from the archived SBUV solar irradiance values. Cebula $e t a .^{5}$ and the User's Guide describe how instrument changes were characterized in producing the archived data. Wavelenglh-dependent degradation was assumed to be proportional to two exponential decays, one with diffuser exposure, and one with time. The decay rates were derived by a fit to the data for an interval during 1980-1981. This interval was selected because exposure was not linear with time and overall degradation was dominated by the exposuredependent term. Rotational modulation was removed, and the total elapsed time was sufficiently short that longterm solar change would be small compared to the instrumental effects. The same method has since been applicd to derive the degradation rate at one period during 1984 and one during 1986 . While the decay parameter for exposure agrees to within the errors for all periods, the fits suggest that the time-dependent degradation rate increased between 1981 and 1984. This additional degradation implied by the change in the time-dependent decay parameter has the same magnitude and wavelength dependence as the instrumental component estimated to remain in the archived data. ${ }^{4}$ A new instrument characterization has been derived by Schlesinger and Ccbula, ${ }^{6}$ assuming that the increase in time-dependent decay derived from the fits represents the true instrument behavior.

The upper panel of Figure 1 shows the time series at $205 \mathrm{~nm}$, chosen because of its importance to stratospheric chemistry. Fortuitously, the variations at this wavelength have a scale factor close to unity and thus are predicted to be approximately equal in percentage amplitude to the SBUV Mg index variations shown in the lower panel. Both plots are on a scale with a range of approximately $14 \%$ from top to bottom. With the new characterization, the observed variations at $205 \mathrm{~nm}$ show the same overall short-term and long-term patterns as those of the $\mathrm{Mg}$ index. An additional $1 \%$ amplitude annual cycle is believed to be of instrumental origin.

The upper panel of Figure 2 shows the ratio of irradiances derived using the new characterization for the periods used 10 represent solar maximum and minimum in the $\mathrm{Mg}$ index estimate shown in the lower panel. The solar cycle variation on the figure is approximately $5 \%$ immediately shortward of the Al edge, and about $1 \%$ from 210 to $260 \mathrm{~nm}$. However, around $290 \mathrm{~nm}$, the plot shows the irradiance to be $2 \%$ lower at solar maximum than at solar minimum. Measurements of the solar constant, representing longer wavelengths, ${ }^{7}$ show higher irradiances at maximum, indicating that the overall change is dominated by bright active regions. The dominance of the bright regions becomes even more pronounced at the shorter wavelengths. ${ }^{8}$ Thus, the difference at $290 \mathrm{~nm}$ is probably a residual instrumental change. If we assume that this instrumental effect persists at shorter wavelengths, then the derived change must be increased by $2 \%, 103 \%$ at $210-260 \mathrm{~nm}$ and $7 \%$ just shortward of the $\mathrm{Al}$ edge. These values are in excellent agreement with the $\mathrm{Mg}$ index estimate. 
The structure near the $\mathrm{Mg} \mathrm{II} h, k$ and $\mathrm{Ca}$ II $\mathrm{H}, \mathrm{K}$ doublets is the result of wavelength drift and jitter. In the wings of these strongest lines where the irradiance changes steeply with wavelength, even a small change in the wavelength can lead to a noticeable change in irradiance. The variations shortward of $200 \mathrm{~nm}$ probably include a large component arising from limitations of the instrument characterization scheme at these wavelengths.
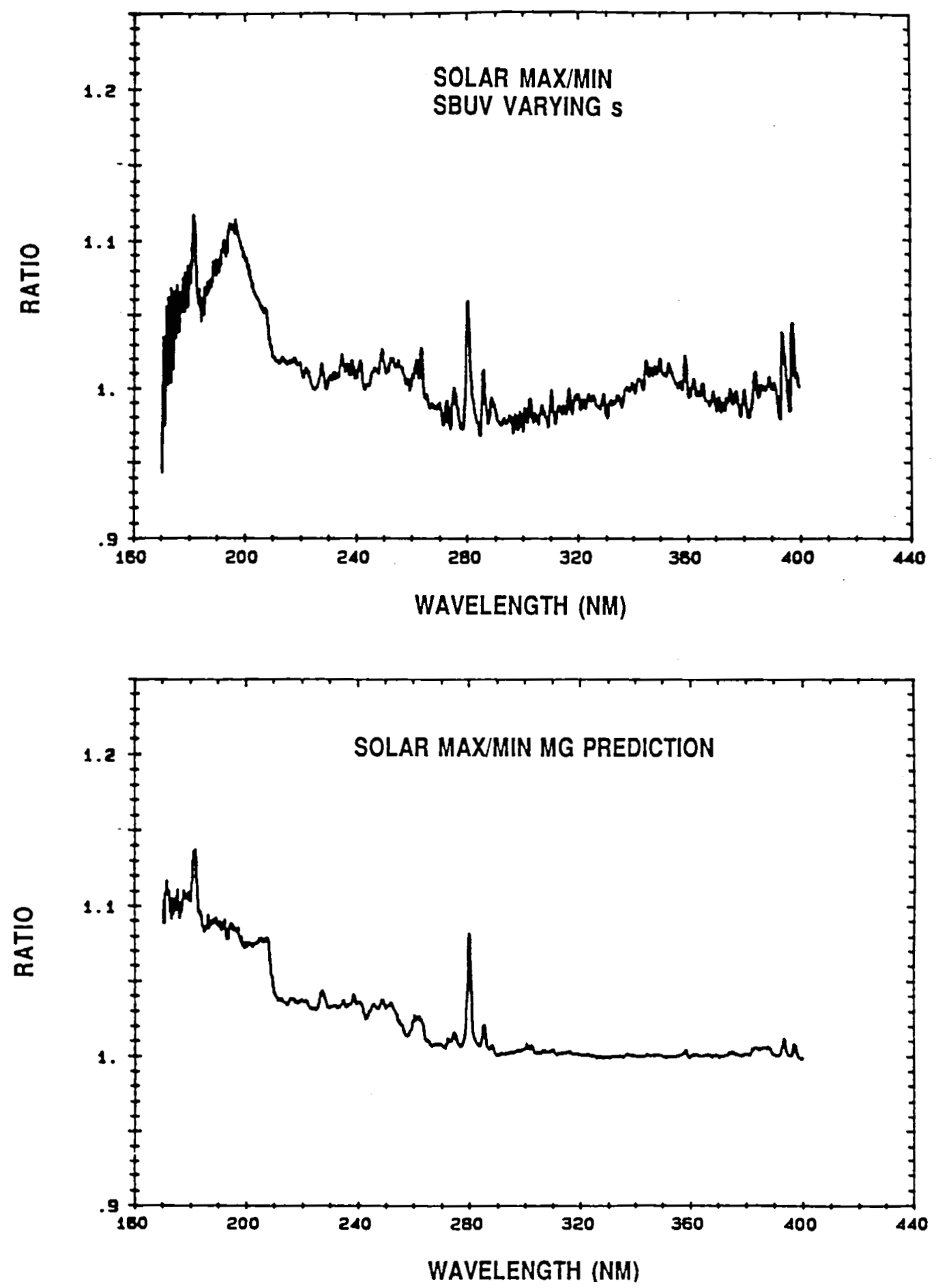

Figure 2. Ratio of solar irradiances at solar maximum (October 27 - November 22, 1979) to irradiances at solar minimum (September 15 - October 11, 1986). (top) Direct measurements using improved instrument characterization. (bottom) Estimated from $\mathrm{Mg}$ index. 


\section{RESULTS FROM NOAA-9 SBUV/2}

\section{Magnesium Index}

For the NOAA-9 SBUV/2, the irradiances at wavelengths in the wing of the Mg doublet are, by an unfortunate chance, measured at the lowest signal for the lowest gain range and consequently have noise of about $2-4 \%$. This problem was recognized before launch. Consequently, the $\mathrm{Mg}$ index for SBUV/2 is measured using a special mode with measurements at carefully selected wavelengths; results of these measurements are being reported by Donnelly. ${ }^{9}$ However, use of running averages reduces the noise sufficiently that long-term trends and qualitative features of the rotational modulation can be examined.

Figure 3 shows the results of applying a 5 -day boxcar running average to the SBUV/2 $\mathrm{Mg}$ index derived from the scans. The plot shows the overall constancy near minimum through 1985 and 1986 seen in the Nimbus results, the beginnings of a rise in spring 1987 (just after the failure of the SBUV chopper!), and a much steeper rise starting at the beginning of 1988 . The rise from minimum has been about $4-5 \%$. Note that these results have not been plotted on the same graph as the SBUV Mg index. That choice is deliberate. The wavelengths measured to derive the $\mathrm{Mg}$ index for SBUV/2 are not exactly the same as for SBUV; furthermore, the $\mathrm{Mg}$ index derived from the discrete measurements uses slightly different wavelengths than those from the scans. The three index values will be different even for simultaneous measurements; compare the 1985-6 values in Figures 1 and 3. If one wishes to generate a single $\mathrm{Mg}$ index plot, the relation among the $\mathrm{Mg}$ indices from the different measurements must first be derived. In addition, in order to apply the HS scaling technique, either the index from the new measurement must be converted to the SBUV value, or the scale factors must be derived independently for the new index. Efforts in this direction are under way. ${ }^{10}$

Figure 3 also shows the presence of rotational modulation of the $\mathrm{Mg}$ index; however, the detailed structure is modified by the averaging procedure.

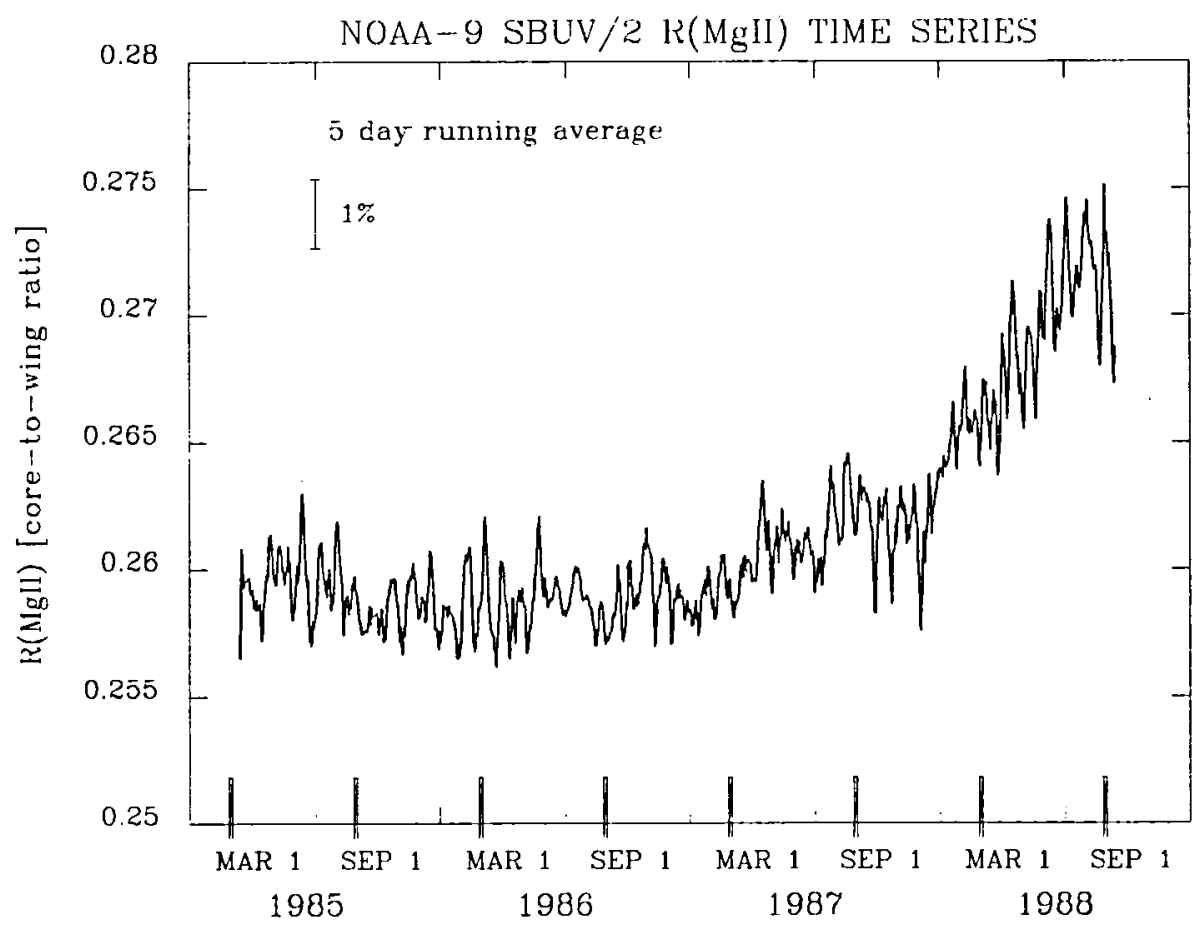

Figure 3. Five-day running average of $\mathrm{Mg}$ index derived from SBUV-2 sweep mode measurements. 


\section{Direct Measurements}

Rotational modulation can be examined by direct examination of the measurement values at shorter wavelengths. In the raw measurements, there is a day-to-day variation, nearly independent of wavelength, in the sense that the data appear to be in one of two discrete "states", one with a higher value and one with a lower value. The most likely origin is a $0.25^{\circ}$ non-repeatability in the discrete-valued dilfuser plate deployment angle. The $\mathrm{Mg}$ index, as noted carlier, would not be affected by such a variation. To eliminate this effect in the values for individual wavelengths, the irradiances at $391.3 \mathrm{~nm}$, a wavelength at which solar variability is expected to be less than $0.2 \%$ long-term and $0.5 \%$ short-term, are used to identify days with anomalous values. Values on these days are corrected by decreasing them by $1.3 \%$. Preliminary data show that this problem has been eliminated for NOAA11.

Figure 4 shows the irradiance at $202.2 \mathrm{~nm}$ with the anomalous values corrected. An insel contains the dala for 1988 with dashed lines appearing at 27-day intervals on the abscissa to show the rolational modulation more clearly. The most striking feature is that the period is 13.5 days, one-half rotation, during much of this period of the rise to maximum. The amplitudes are about $2 \%$. Combined with the Nimbus measurements, this result shows that rotational modulation can be at half the rotation period at any time during solar maximum -- rise or decline.

Because a characterization of the changes with time of the instrument sensitivity has not yet been defined, the long-term change in Figure 4 should not be regarded as describing the actual solar variations. However, it is interesting that the pattern changes from a gradual decline to a steady rise early in 1988 , the time when the $\mathrm{Mg}$ index shows a sharp increase in solar activity. The magnitude of the increase is what would be expected if the SBUV scaling factors were applied and there were no sudden changes in the pattern of instrument sensitivity change.

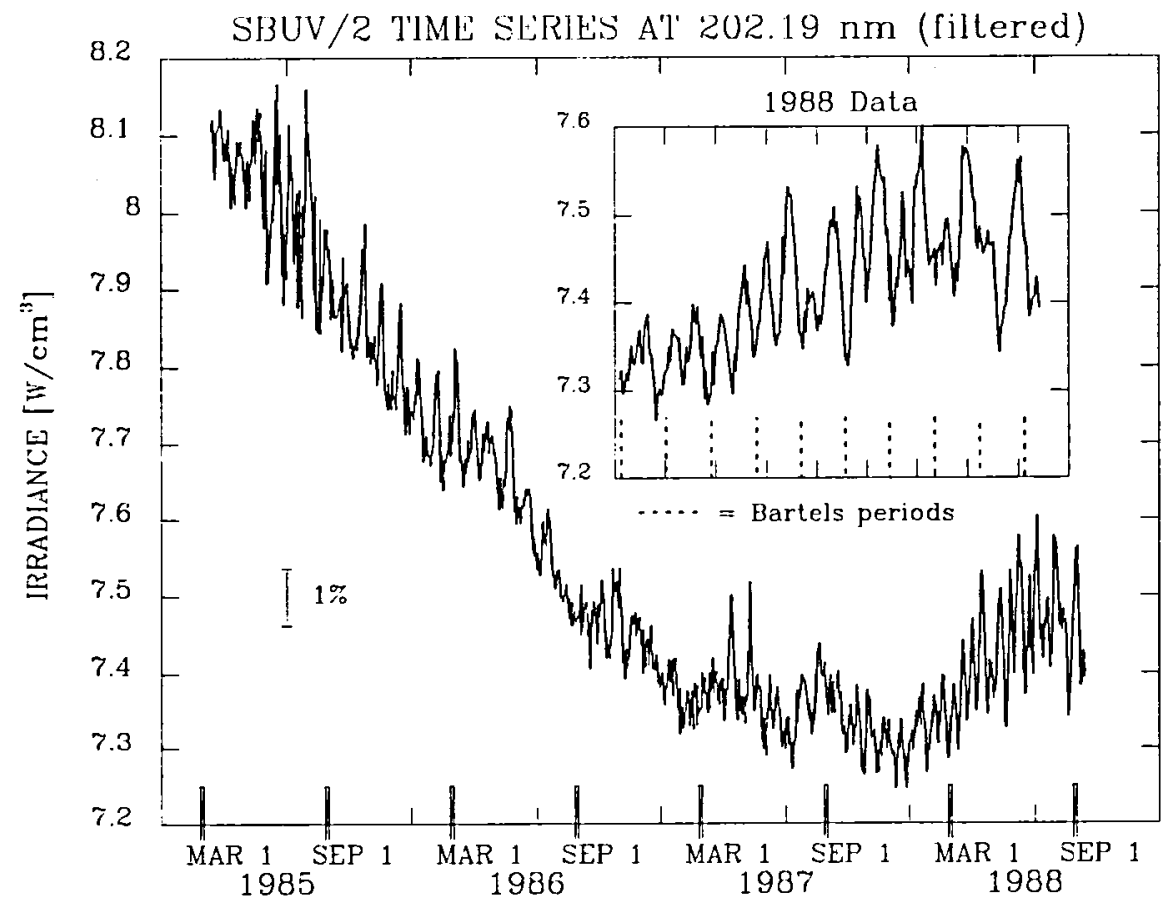

Figure 4. Irradiance measurements at $202.2 \mathrm{~nm}$ from SBUV/2. (inset) Values for 1988. 


\section{CONCLUSIONS}

The Heath and Schlesinger ${ }^{1} \mathrm{Mg}$ index provides a powerful tool for estimating monitoring solar variahility in the near ultraviolet and interpreting the direct measurements. Solar irradiances were high from late 1978 until 1983, declined until early 1985, remained constant until mid-1987, and then began to rise. Time spans when the rotational modulation period was 13.5 days occurred during and after the peak of cycle 21 and during the rise of cycle 22. Using the $\mathrm{Mg}$ index and direct measurements reduced using an improved characterization for changes in instrument sensitivity, variations from solar maximum to minimum of cycle 21 have been derived. Near 200 $\mathrm{nm}$, the estimated variation is $5-8 \%$; for $210-260 \mathrm{~nm}$, the estimate is $1-4 \%$, with the higher values in the range favored for both cases. Because the $\mathrm{Mg}$ indices measured by different instruments are not identical and must be transformed to a common scale, there should be a significant period of overlap between consecutive instruments measuring the solar ultraviolet if continuity of values is to be maintained.

\section{ACKNOWLEDGEMENTS}

The work described in this paper was supported by the NASA Space and Earth Sciences directorate under Task NAS5-29.386. The SBUV data were processed by the NASA Goddard Space Flight Center Ozone Processing Team.

\section{REFERENCES}

$1_{\text {Heath, D. F., and B. M. Schlesinger, "The Mg 280-nm doublet as a Monitor of Changes in the Solar Ultraviolet }}$ Irradiance," J. Geophys. Res., 91, 8672-8682, 1986.

${ }^{2}$ Kjeldseth Moe, O., and E. F. Milone, "Limb Darkening 1945-3245 A for the Quiet Sun from Skylab Data," Astrophys. J., 226, 301-314, 1978.

${ }^{3}$ Schlesinger, B. M., and D. F. Heath, "A Comparison of Solar Irradiances Measured by SBUV, SME, and Rockets," J. Geophys. Res., 93, 7091-7103, 1988.

${ }^{4}$ Schlesinger, B. M., R. P. Cebula, D. F. Heath, and A. J. Fleig, "SBUV Spectral Scan Irradiance and Earth Radiance Product User's Guide," NASA Ref. Publ. NASA-RP-1199, 1988.

${ }^{5}$ Cebula, R. P., H. Park, and D. F. Heath, "Characterization of the Nimbus 7 SBUV Radiometer for the Long Term Monitoring of Atmospheric Ozone," J. Ocean Atmos. Technol., 5, 215-227, 1988.

${ }^{6}$ Schlesinger, B. M., and R. P. Cebula, "Changes with Time in the Sensitivity of the Solar Backscalter Ultraviolet Instrument as Deduced from Measurements of the Solar Irradiance," in preparation.

${ }^{7}$ Willson, R. C., H. S. Hudson, C. Frohlich, and R. W. Brusa, "Longterm Downward Trend in Solar Irradiance," Science, 234, 1114-1117, 1986.

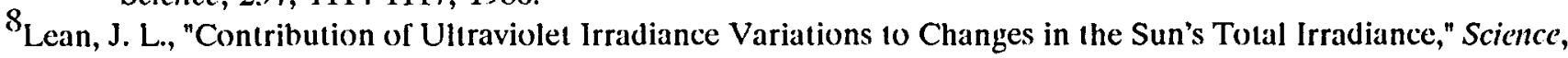
244, 197-200, 1989.

${ }^{9}$ Donnelly R. F., "The Solar UV Mg II Core-10-Wing Ratio from the NOAA-9 Satellite During the Rise of Solar Cycle 22," Adv. Sp. Res., 8, (7) 77-80, 1988.

${ }^{10}$ DeLand, M. T., R. P. Cebula, B. M. Schlesinger, and R. D. Hudson, "Solar Activity Measured by the NOAA-9 SBUV/2 Instrument as Determined from the Mg II Core-1o-Wing Ratio," EOS Trans. AGU, in press, 1990. 
PAPERS ACCEPTED BUT NOT PRESENTED 



\title{
SOME RESULTS FROM STUDIES ON RELATIONSHIPS BETWEEN THE OPTICAL-METEOROLOGICAL PARAMETERS AND SOLAR ACTIVITY. 2. DEVELOPMENT OF THE PROBLEM OF SOLAR FORCING
}

\author{
K.Y. Kondratyev \\ USSR Academy of Sciences \\ Institute for Lake Research \\ G.A. Nikolsky \\ Leningrad State University \\ Institute of Physics
}

\begin{abstract}
A set of complex spectral, actinometric and meteorological data obtained in the periods of heightened solar activity (1981 and 1988) has been considered in order to reveal the atmospheric component affected by solar emissions in the troposphere and lower stratosphere. For the first time, it has been found out that water vapour molecules can be transformed, under the impact of corpuscular and microwave solar emissions, from the free state to the bound one (association into clusters), and vice versa. The transition of water vapour molecules into the bound state results in a decrease of spectral optical thickness in the visible, near IR and IR spectral regions, and an appearance and deepening of the cluster absorption bands at wavelengths $330-340,365,380-390$, and $480 \mathrm{~nm}$.
\end{abstract}

In a brief paper (STP Newsletter, 1989-I) a possibility has been discussed of the effect of a rapid corpuscular component emitted during the sun flares on the microphysical state of water vapour molecules in the middle and upper troposphere. The results of complex spectral measurements have shown that the total water vapour content ( $\mathrm{W} \mathrm{cm}$ of precipitated water), under the influence of solar emissions, can change substantially (by 30\% and more) every 15-20 minutes either after an optical flare at geoeffective solar longitudes and latitudes or after a powerful radioburst at wavelengths 2 to $10 \mathrm{~cm}$. However, assessments of the scale of ionization processes needed for water vapour molecules to transform into $0.35 \mathrm{~cm}$ of precipitated water, have shown that the solar corpuscular component does not ensure a needed abundance of the processes of ionization of nitrogen molecules and processes of association of water vapour molecules into multi-molecular clusters, and furthermore, the processes of a rapid dissociation of the latter.

Interesting phenomena in the microphysical state of water vapour were observed during observations on July 29, 1981 on the last day of a 9-day period of a heightened solar activity. The absence of strong flares and different times of sub-flares and radiobursts have ensured a reliable referencing of the events on the sun to the events in the troposphere. A set of radiobursts at 11:06 local time (08:06 UT) was followed by a substantial decrease of $W$ from $2.2 \mathrm{~cm}$ to $1.6 \mathrm{~cm}$ of precipitated water. A synchronous decrease has taken place in the spectral optical density. A second set of radiobursts at 14:13 local time (11:13 UT) raised $W$ up to $2.2 \mathrm{~cm}$. Radiobursts at wavelengths 2 to $5 \mathrm{~cm}$ corresponded to $\mathrm{H}_{2} \mathrm{O}$ molecules to be released from the cluster. These events in the diurnal change of $\mathrm{W}$ show that radiobursts govern, apparently, the state of a considerable amount of water vapour in the middle and upper troposphere and in the lower stratosphere.

As seen from the recent studies of the spectrum of radiobursts in the frequency interval 1 to $18 \mathrm{GHz}$ (M. Stahly, D.E. Cary, G.J. Hurford, BAAS, 1988, vol. 20, p. 678), in $80 \%$ of the cases the radiobursts have several peaks in the frequency spectrum simultaneously, whose location on the frequency scale is practically constant during the radioburst's lifetime. Bearing all this in mind, the radiobursts may, presumably, excite the molecules due to the presence of two or more components at close and definite frequencies. The phenomenon of broad-band excitation, found out by A.V. En'shin and S.D. Tvorogov (Atmospheric Optics, 1989, N5, p. 456--in Russian) in studies using the laser sources of biharmonic emission, has made it possible to look at the interaction between microwave emission and the medium from a new angle. 
In connection with the fact that, upon the fixation of the event on the sun, the process of water vapour transformation continues 15 to 20 minutes, quasi-relativistic protons and neutrons of solar origin can be supposed to take part in a rapid change of water vapour concentration in the upper troposphere and hence, a rapid change in the optical characteristics of the atmosphere in the UV, visible, and IR spectral regions.

Note that synergism in the effects of solar emissions on the microphysics! state of water vapour is not, apparently, confined to the effect of radiobursts and corpuscular fluxes but is supported by UV-emission bursts which follow the solar flares.

The second period of observations in October 1-12,1981, was also rich in flares and radiobursts. The data for 11 and 12 October demonstrate still stronger effects of the flares and radiobursts on the troposphere, compared to 27 and 28 July. Extremely interesting are data for October 12, 1981, which testify to the strongest perturbation of active components of the troposphere and, naturally, its optical characteristics. A combined effect of strong flares (2B and $3 \mathrm{~B}$ ) and multiple powerful radiobursts have led to anomalous optical characteristics of the troposphere. Before noon, the total content of water vapour remained $0.15 \mathrm{~cm}$ of precipitated water. An interesting fact never observed before is a reversed location of $\tau_{411}, \tau_{471}, \tau_{557}$ on the scale of residual (aerosol) optical densities and extremely low values of $\tau_{411}$ ( 0.01$)$ and $\tau_{471}$ at noon. Such a super-anomalous transparency in the UV should be connected either with practically complete removal of submicron aerosol fraction from the atmosphere or with a $20 \%$ flare of UV solar emission. Results of measurements at the Station Solnechnaya on 16 October 1988

demonstrate not only the effect of anomalous reduction in the spectral interval $330-380 \mathrm{~nm}$ but also show the diurnal dynamics of the spectral maximum of reduction. A sharp decrease of spectral thicknesses with a rapid increase of total water vapour content between 10:00 and 11:00 is associated with a disintegration of clusters with a high degree of association, which makes it possible to assess the cross-section of absorption for large clusters in the wavelength interval $330-380 \mathrm{~nm}$ (see the table).

Between 11:30 and 13:00, the reduction with a maximum at $378 \mathrm{~nm}$ is in-phase with the change in $W$, which can be explained by large amounts of water vapour getting to the atmosphere upon the sublimation of a thick layer of frost covering the objects and plants.

Table The Absorption Cross-Sections of Water Clusters in the Interval $330-380 \mathrm{~nm}$ (estimates).

\begin{tabular}{lllll}
\hline$\lambda, \mathrm{nm}$ & $\sigma \times 10^{21}$ & $\lambda, \mathrm{nm}$ & $\sigma \times 10^{21}$ & Remarks \\
\hline 331 & $4.0 \pm 2.5$ & 352 & $3.4 \pm 2.5$ & \\
333 & $4.3 \pm 2.5$ & 360 & $3.1 \pm 2.0$ & \\
336 & $4.1 \pm 2.5$ & 370 & $3.0 \pm 2.0$ & $\mathrm{n}=50$ \\
340 & $3.9 \pm 2.5$ & 380 & $2.4 \pm 2.0$ & \\
347 & $3.7 \pm 2.5$ & & & \\
\hline
\end{tabular}

Unfortunately, the lack of spectral data between 12:30 and 15:30 did not allow one to monitor variations in the reduction in the period of $W$ approaching a maximum and a subsequent decrease.

Anomalies in the values and in the diurnal change of solar emission reduction on October 16, 1988 are explained by a specific meteorological situation on that day. With a high transparency in the visible and IR 
spectral regions (high integral fluxes) and with negative air temperatures at morning hours, additional water vapour from the surface provided ideal conditions for the development of large clusters in the early morning, for their afternoon disintegration, the afternoon growth of clusters with a lesser degree of association, and an appearance of a third maximum of reduction at about 16:00, characterized by special spectral features.

Summing up, one can state that the effect of solar activity on the tropospheric radiation fields has been found for the first time to be mainly manifested through the processes of transition of a part of water vapour mclecules ( $30 \%$ and more) from the free state to the bound one, and vice versa. In these processes, their contribution to radiation transfer in the UV, visible and IR solar radiation spectrum changes substantially as well as the transfer of longwave emission of the Earth's surface and atmosphere.

In connection with the complexity and variety of the effects of solar emissions on the troposphere, combined efforts are needed to carry out observations at various locations over the globe (using instruments with close measuring parameters).

Our observations show that the solar-tropospheric effects intensify in mountain regions. In this connection, it would be useful to organize coordinated observations at the Caucasus, in the Alps, in the Pyrenees, as well as in the Rocky Mountains, and at Mauna Loa Station.

It is rather perspective to include portable instruments in the measuring complexes for the microwave radiosounding of the atmosphere, used now by the U.S. Meteorological Service. 

POSTER PAPERS 



\title{
SPECULATION ON A SOLAR CHRONOMETER FOR CLIMATE
}

\author{
Charles A. Perry \\ U.S. Geological Survey, Lawrence, Kansas 66049
}

\begin{abstract}
Solar activity has been correlated to climatic fluctuations and has been postulated as a major factor in quasi-periodic global climatic change. However, correlations are not explanations of physical mechanisms and do not couple cause with effect. A mechanism for a chronometer for solar output variability is proposed based on relations between properties of thermonuclear fusion, nuclear magnetic moment, and nuclear magnetic resonance.

A fundamental oscillation of a nucleus with a net nuclear magnetic moment (NMM) is the precession of its axis of rotation when subjected to a magnetic field. Nuclear magnetic resonance (NMR) is the preferred frequency of precession for a nucleus of a particular isotope when placed in a magnetic field of specific intensity. The NMM for those isotopes involved in the proton-proton ( $p-p)$ chain pathway for solar fusion varies from strong positive to strong negative. Individual fusion events, for hydrogen and helium isotopes which release varying amounts of energy, may be controlled by NMR frequencies. The pulses of energy from fusion events occurring at NMR frequencies in the solar interior may be transformed into pressure or gravity waves that emerge as gravity or acoustic waves at the surface. Dictated by spherical harmonics, certain wavelengths may be reinforced and reenter the solar interior to modulate the fusion process. Qualitative analysis of solar and climatic data support the interaction of the three basic components of the chronometer, magnetic activity, oscillation frequency, and solar energy output.
\end{abstract}

\section{INTRODUCTION}

Thermonuclear fusion within the Sun is the major source of energy for the solar system. However, only the outermost layers of the Sun can be studied directly from Earth. The interior is hidden from view, and only indirect methods of observation, such as solar seismology ${ }^{1}$, give clues to its structure and processes. The "standard" models of solar evolution indicate that the solar output has increased with time, being 30 percent greater now than 4.6 billion years ago ${ }^{2}$. Calculations using this increased solar output indicate that the Earth would have been totally covered with ice for nearly one-half of that time, preventing the development of life in the oceans. Nonstandard models of the Sun, which involve periodic mixing of helium and hydrogen, indicate that mixing episodes may have set off major glacial advances ${ }^{3,4}$.

The most well known solar periodicities are the approximate 11-year sunspot cycle, and the Hale double-sunspot cycle in which the polarity of the sunspots goes through an approximate 22-year cycle. Also, the maximum sunspot number for each 11-year cycle, follows an approximate 80 - to 90 -year cycle $^{5}$.

Sunspot disturbances were once considered to be inherently only roughly periodic, with observed intervals of maximum activity ranging from 7.3 to 17.1 years ${ }^{6}$. Later, Kiepenheuer ${ }^{7}$ described the rise and fall of the number of sunspots as an eruption phenomenon, the end result of a number of practically independent individual processes. The idea that each cycle represents an independent eruption of the Sun taking about 11 years to die down implies a random walk in the cycle phase. This random walk seems to agree with the Babcock theory and subsequent modifications in which the poloidal magnetic field remnant of one half-cycle (approximately 11 years) provides the seed field for the generation of the toroidal magnetic field of the next half-cycle by differential winding ${ }^{8}$. Dicke found no support for the existence of a large random walk in the phase of the sunspot cycle. Instead, he noted that "both sunspots and the $(\mathrm{D} / \mathrm{H})$ weather indicator seem to be paced by an accurate clock inside the Sun." 
Could this accurate clock or chronometer be paced by internal oscillations of the Sun? The relatively new field of helioseismology has revealed that the Sun apparently oscillates throughout a range of discrete frequencies. Analysis of these oscillations shows very distinct patterns in frequency-spacial scale diagrams which indicate that the longer the period of oscillation, the deeper the Sun is being penetrated ${ }^{9}$. Observed oscillations of the Sun range in frequency from minutes to decades. Recent work has shown possible oscillations of approximately 80 years $^{10,11}$.

The well documented 160-minute solar oscillation has been described as "a g-mode driven by a seismonuclear process in the solar core"12. This hypothesis is supported by an apparent solar cycle dependence in Davis's solar neutrino experiment ${ }^{13,14}$. It appears that the inverse correlation between the 11-year sunspot cycle and neutrino flux may be linked to activity within the Sun ${ }^{15}$.

Could it be that internal oscillations of the Sun are manifested as gravity waves at the surface, which are reinforced or destroyed as a function of spherical harmonics? In turn, the reinforced modes could penetrate the solar interior to modulate the fusion process, systematically varying the production of neutrinos and energy.

What is the basic oscillation and how is it related to thermonuclear fusion and magnetic activity? The answer may be found in nuclear scale vibrations.

\section{THE SOLAR CHRONOMETER}

Solar activity may be driven by a high-frequency oscillation on the nuclear scale. There are three interrelated physical processes that are presented in this paper that could control a chronometer within the Sun's interior--(a) the difference in energy production between hydrogen fusion and helium fusion, (b) the interaction of nuclear magnetic resonance frequencies of hydrogen and helium in varying magnetic fields, and (c) the nuclear magnetic moments of the hydrogen and helium isotopes.

The first process considered is the difference of energy production in the stages of solar fusion. Hydrogen and helium are the predominate constituents of the Sun. The conversion of mass into approximately 99 percent of the energy in thermonuclear fusion within the Sun is believed to follow the proton-proton $(\mathrm{p}-\mathrm{p})$ chain pathway given below ${ }^{16}$ :

(1) ${ }^{1} \mathrm{H}+{ }^{1} \mathrm{H}>2 \mathrm{H}+$ positron + neutrino

(2) $2 \mathrm{H}+{ }^{1} \mathrm{H}>3 \mathrm{He}+$ gamma ray

(3) $3 \mathrm{He}+3 \mathrm{He}>4 \mathrm{He}+(2)^{1} \mathrm{H}$

where

$1 \mathrm{H}$ is hydrogen-1,

$2 \mathrm{H}$ is hydrogen-2,

$3 \mathrm{He}$ is helium ${ }^{-3}$, and

4 He is helium ${ }^{-4}$.

Steps 1 and 2 of the conversion pathway for 6 initial hydrogen-1 nuclei release 13.870 million electron volts of energy. Step 3 releases 12.859 million electron volts of energy ${ }^{17}$. About 52 percent of the total energy in the proton-proton chain pathway is released in steps 1 and 2--the "hydrogen-fusion" phase. The remaining 48 percent is released in step 3 , the "helium-fusion" phase. 
Hydrogen fusion can occur at a lower temperature and pressure than helium fusion because the force holding the two hydrogen nuclei apart is less than the force holding the two helium-3 nuclei apart. (The coulomb force is less for lighter nuclei.) Therefore, either a higher temperature or greater pressure is required for helium fusion than for hydrogen fusion.

The second process considered is that of nuclear magnetic resonance. Nuclear magnetic resonance (NMR) is the ability of the nucleus of an atom to vibrate at a unique frequency when subjected to a certain magnetic field ${ }^{18}$. This vibration is actually a precession of the axis of spin of the nucleus at an angle also dependent upon the magnetic field intensity. The predominate nuclei involved in solar thermonuclear fusion are hydrogen-1, hydrogen-2, helium-3, and helium-4. Of these four isotopes, all are active in NMR except helium-4, which has magnetic symmetry and does not resonate. However, hydrogen-1 and helium-3 are the most sensitive resonators of all the elements involved in solar fusion ${ }^{19}$, with hydrogen-1 being more than twice as sensitive as helium-3, and 100 times more sensitive than hydrogen-2. Sensitivity could be interpreted as the relative strength of vibration.

One characteristic of NMR is that the resonant frequency of oscillation is dependent upon the magnetic field in which the atom is placed. If the magnetic field is increased from 1,180 gauss to 10,000 gauss, the NMR frequency for hydrogen-1 increases from 5 to 42 megahertz $^{18}$. The response is a linear function. Helium-3 has a similar response with a slightly slower increase of NMR frequency with an equal increase in magnetic field.

The third process involves the nuclear magnetic moments (NMM) of the hydrogen and helium nuclei, and their effect on the composite magnetic field of a parcel of solar material. The magnetic moment of hydrogen-1 is +2.79268 nuclear magnetons $(\mu \mathrm{n})$. Hydrogen-2 has a value of +0.8574 and helium- 3 , $-2.1274 \mu \mathrm{n}^{19}$. A nuclear magneton is equal to $5.0505 \times 10^{24} \mathrm{erg}$ per gauss. The magnetic moment of helium-4 is zero because its nucleus is magnetically symmetric.

Consider a parcel of hydrogen-1 in a uniform magnetic field proceeding through the three steps of the proton-proton chain. The composite magnetic moment of that parcel will vary according to the concentration of each isotope. A schematic diagram of the proton-proton chain is shown in figure 1.

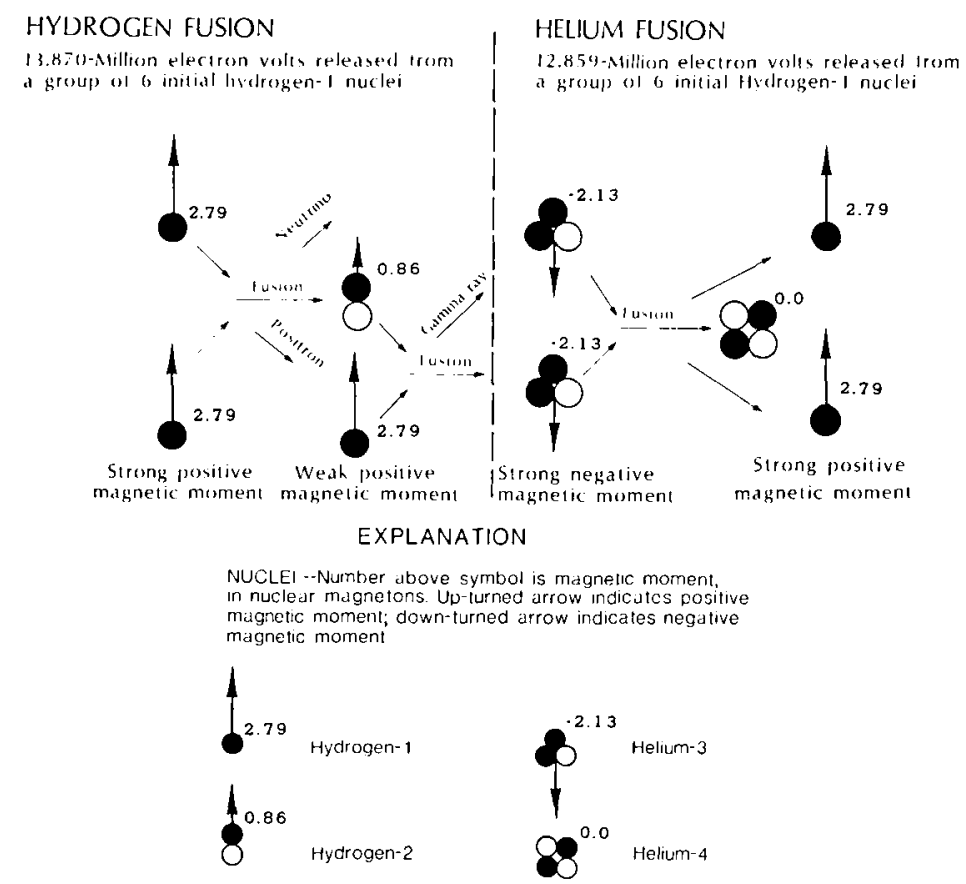

Figure 1. Energy production and relation among nuclear magnetic moments for hydrogen-1, hydrogen-2, helium-3, and helium-4 in solar thermonuclear-fusion process ${ }^{17,19}$. 
For illustrative purposes, positive magnetic moment is depicted by arrows pointing up, negative pointing down. As step 1 begins, the abundant hydrogen-1 nuclei are responsible for the strong positive composite magnetic moment of the parcel. The high composite magnetic moment should allow the nuclei to vibrate at a high NMR frequency. As hydrogen-2 is produced, the composite magnetic moment begins to weaken and NMR frequencies begin to lower. When temperature and pressure increase to the point as step 2 proceeds, the addition of helium-3 nuclei further decreases the parcel's composite magnetic moment, and NMR frequencies continue to decrease. As step 3 begins and the helium-3 is at its greatest concentration, the parcel's composite magnetic moment reaches its minimum value, which can be negative. This not only affects NMR frequencies within the parcel, but also affects the magnetic field and the resultant frequencies in the neighboring parcels. As step 3 progresses, the helium-3 nuclei are fused into helium-4, having no magnetic moment, and hydrogen-1, having a high moment, increasing the composite magnetic moment of the parcel and increasing NMR frequencies. Therefore, when hydrogen nuclei are abundant during hydrogen fusion (steps 1 and 2), NMR frequencies are higher than during helium fusion (step 3 ), when there is a higher concentration of helium-3 nuclei and NMR frequencies are lower.

\section{CONVERTING NUCLEAR VIBRATIONS TO SOLAR CYCLES}

To support the solar chronometer theory, an explanation of the mechanism for the progression from high-frequency NMR vibrations to lower-frequency solar-activity oscillations must be provided. This explanation is based on NMR properties and the assumption that a spinning hydrogen or helium nucleus is not a true sphere but is instead a rounded disk. Recent work has shown structural anomalies in the nucleus of helium $-3^{20}$.

Consider a group of nuclei in the core of the Sun that are subject to a magnetic field, and are very near the temperature and pressure needed for fusion. The distance between the centers of mass of the nuclei would remain nearly constant through an NMR oscillation cycle. However, the disk edges have a minimum distance $(\mathrm{dm})$, which occurs twice during each precession cycle (fig. 2). As the centers of mass of the nuclei become closer as a result of higher temperature and pressure, fusion would occur when $\mathrm{dm}$ reaches the critical value.

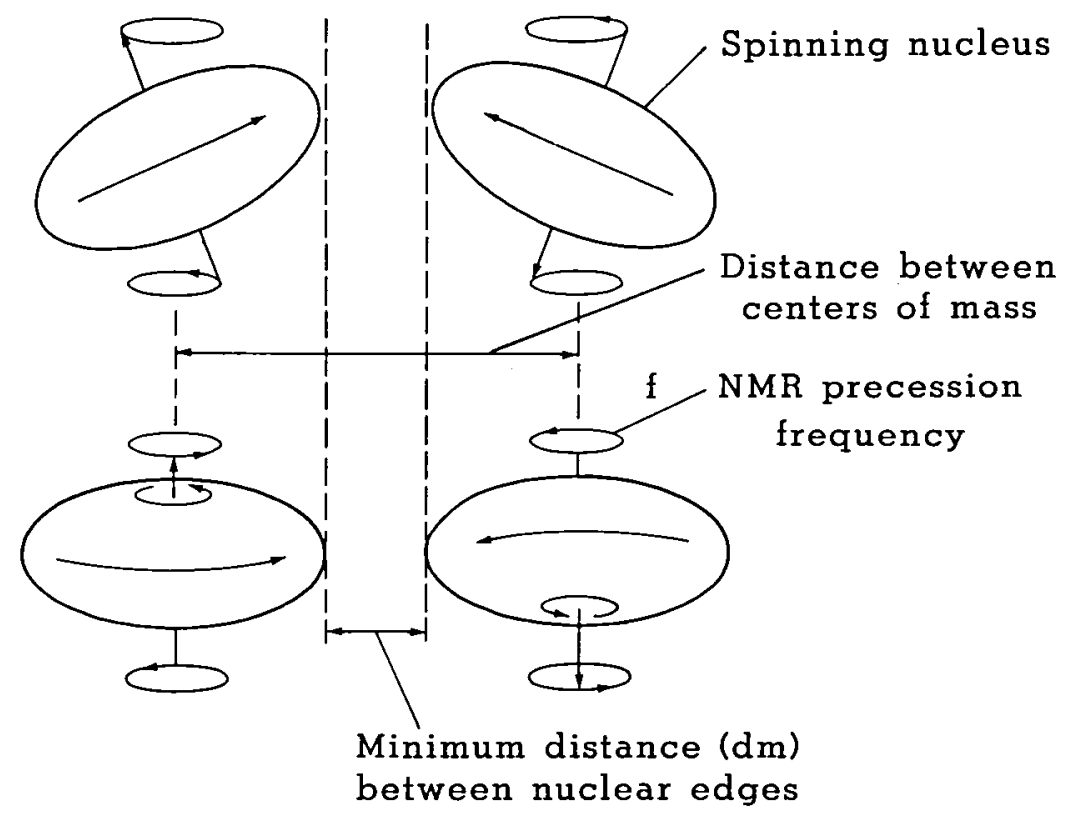

Figure 2. Nuclear magnetic resonance precession of nuclei and relation of minimum distance between nuclear edges. 
After the first fusion event in that group of nuclei, thermodynamic adjustments would be made in the group of nuclei to accommodate the energy released and the presence of a heavier nucleus. The next fusion event for the group could be several precession oscillations later. If these fusion events proceeded at frequencies lower than the NMR frequency within a group of nuclei, pressure waves could develop, further enhancing fusion at the lower frequencies. The interference of pressure waves created by hydrogen-1 fusion and helium-3 fusion at different locations within the solar core would result in even lower frequency pressure waves, which could propogate to the Sun's surface. NMR frequencies of hydrogen-1 and helium-3 nuclei are hypothesized to be responsible for the basic frequency on which the other solar-activity cycles are based. Hydrogen-2 is a much weaker resonator ${ }^{19}$ than hydrogen-1 by a factor of 100 , and its effect on the beat frequency should not be significant.

As the pressure waves emerge from the solar interior, they may be transformed into acoustic or gravity waves at the surface. Dictated by spherical harmonics, certain gravity waves are reinforced and can reenter the solar interior. Gravity waves reaching the solar core modulate the hydrogen and helium fusion, completing the circle. The interaction of NMR frequencies of hydrogen-1 and helium-3 could be the chronometer that sets the tempo of the Sun and all stars that are burning hydrogen and helium.

The linear relationships for hydrogen-1 and helium-3 NMR frequencies are shown in figure 3 . Beat frequencies between hydrogen-1 and helium-3 are also plotted in figure 3 , and the equations are given below:

$$
\begin{aligned}
& \mathrm{f}(1 \mathrm{H})=4.2552 \mathrm{~B}+23.440 ; \\
& \mathrm{f}(3 \mathrm{He})=3.2429 \mathrm{~B}+4.0811 ; \text { and } \\
& \mathrm{f}(\text { Beat })=1.0124 \mathrm{~B}+19.3595 ;
\end{aligned}
$$

where

$\mathrm{f}$ is in kilohertz, and $\mathrm{B}$ is in gauss.

Unlike the individual frequency-magnetic intensity relations for hydrogen-1 and helium-3 which have steep slopes, the slope of the beat frequency is relatively flat. A doubling of the magnetic intensity from 2 to 4 gauss increases the frequency by only slightly more than 10 percent, and doubling from 6 to 12 gauss increases the frequency by about 25 percent. As magnetic intensity increases, the period of oscillation should become shorter, and as magnetic intensity decreases, the period of oscillation should become longer. Increased magnetic field intensity would be associated with hydrogen fusion and greater energy production. Decreased magnetic fields would be associated with helium fusion and less energy production.

\section{IMPLICATIONS OF THE SOLAR CHRONOMETER THEORY}

The three interrelated components of the solar chronometer involving nuclear properties of hydrogen and helium can be used to qualify observed solar activity. With increasing magnetic fields, the NMR frequency increases and the reciprocal, period (cycle length), decreases. The hypothesis of the solar thermonuclear chronometer theory is that NMR governs solar cycles of all time periods. Therefore, if the magnetic field within the Sun increases, the NMR frequency increases, and the solar cycles should become shorter. This feature has been noted in the sunspot record. The sunspot cycles with a larger number of spots, which indicate greater magnetic activity, are usually shorter than cycles with small numbers and less magnetic activity. 


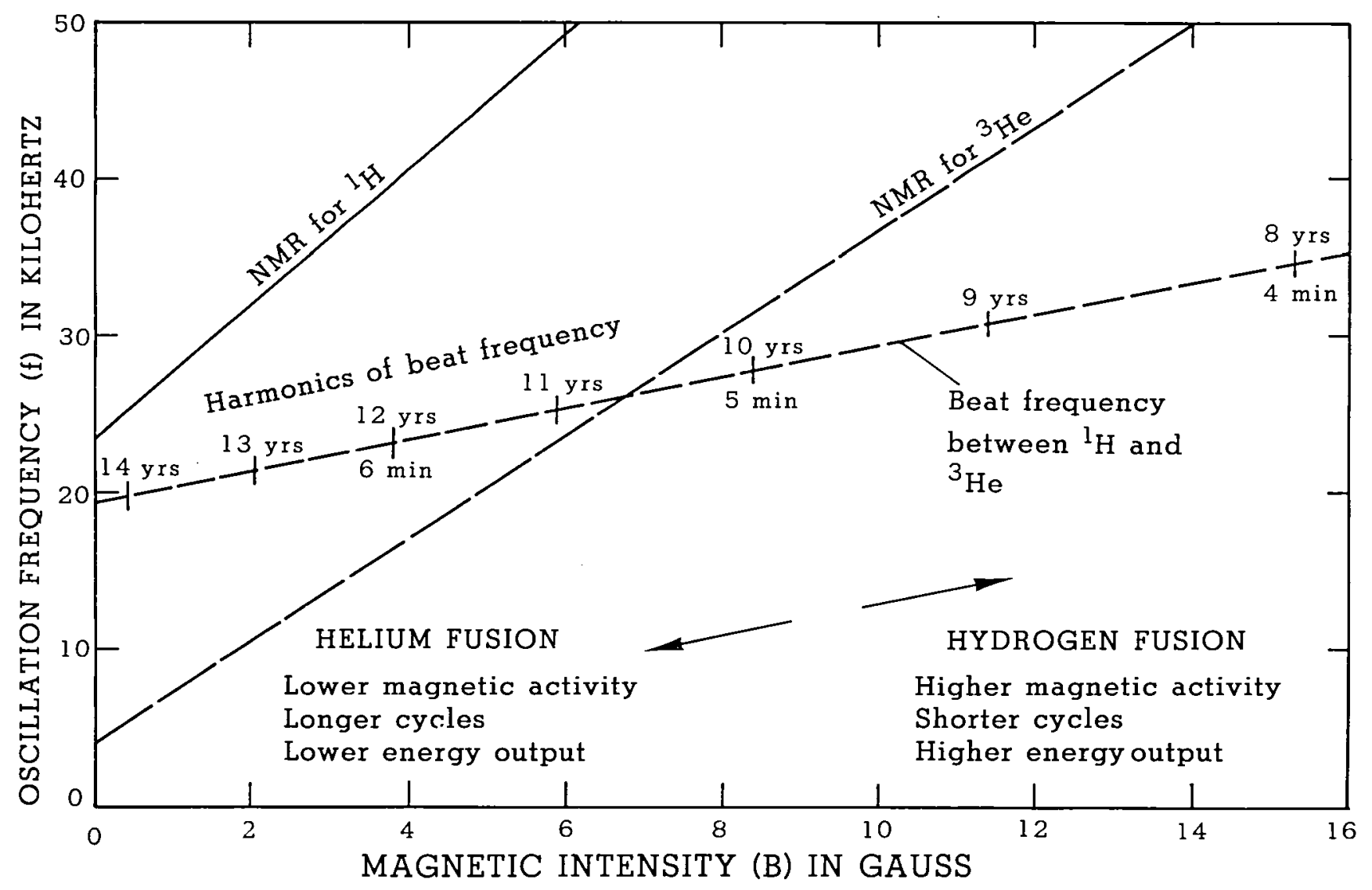

Figure 3. Nuclear magnelic frequencies for hydrogen-1 and helium-3 and "beat" frequencies for various magnetic intensities.

The same relationship between magnetic activity and solar oscillation period has been noted in the last two centuries. From 1810 to 1913 the average sunspot cycle length was 11.4 years with an average cycle maximum sunspot number of 91.3 . Between 1913 and 1986, the average cycle length was 10.4 , and the average cycle maximum was 127.9. A more magnetically active Sun corresponds to shorter solar-cycle lengths. The association of solar energy output to this fluctuation may be inferred from North American annual temperature departures reconstructed using tree rings ${ }^{21}$. The interval 1810 to 1913 had an average departure of nearly -0.4 degrees Celsius from the period 1913 to 1986.

A similar relationship between magnetic activity, oscillation period and solar output can be demonstrated on a shorter time scale. Woodward and Noyes ${ }^{22}$ have reported a slight but systematic decrease in frequencies of low-degree solar p-modes (acoustic modes in the 5 minute band of oscillation) from 1980 to 1984 . Roberts and Campbell ${ }^{23}$ argued that the frequencies of both p- and $g$ modes are modified by a magnetic field within the solar interior which evolves over the solar cycle. From 1980 to 1984 the sun was becoming magnetically less active as the last 11-year solar cycle was ending. During the same period, a general decrease in the solar constant was measured by the Solar Maximum Mission's active cavity radiometer experiment ${ }^{24}$.

The range of the sunspot cycle and the p-mode, 5 minute band has been inserted on the beal frequency line in figure 3 . The 5 minute oscillation coincides with a sunspot cycle length of 10 years, the value of the last full cycle (cycle 20). These values are both harmonics of the nuclear beat frequency. If the nuclear vibrations of the solar thermonuclear chronometer do control the frequency of all solar oscillations, then periods in which the sunspot cycle lasts, for example 11 years, the preferred p-mode oscillations would be expected to be closer to 5.5 minutes. 


\section{CONCLUSIONS}

The Sun and its total output of electromagnetic energy, charged particles and magnetic force fields are not static. The standard models of solar evolution can not adequately describe its quasi-periodic behavior which has been evident in the geologic record and in recent measurements of solar activity. Helioseismology is revealing the many modes of oscillation possible within the Sun.

This paper presents a mechanism for the genesis of a basic vibration for a solar chronometer, which can set the tempo of the other solar oscillations. That vibration frequency is a function of: (1) nuclear magnetic resonance of hydrogen and helium nuclei, (2) the composite magnetic moment of those nuclei through the proton-proton chain solar-fusion steps, and (3) the difference of energy of fusion for each step. The interaction of these three components of the solar thermonuclear chronometer allow a range of energy production and oscillation period length. Hydrogen fusion releases a greater total amount of energy and results in a stronger composite magnetic field which dictates a higher frequency nuclear vibration than does helium fusion. The higher vibrational frequency associated with hydrogen fusion results in shorter period solar oscillations than does helium fusion.

These high frequency oscillations may originate in the solar interior and propagate to the surface and emerge as gravity or acoustic waves. According to spherical harmonics, certain wavelengths may be reinforced and penetrate the solar interior to modulate the fusion process.

Qualitatively, observations of the sun support the solar thermonuclear chronometer. Shorter sunspot cycles tend to be more active magnetically with a greater number of sunspots. A greater number of sunspots is believed to result in greater solar output. Also, the frequency of the 5-minute band of acoustic waves on the Sun tend to decrease as the Sun becomes less active magnetically, accompanied by a decrease in solar output. The Sun is constantly changing and adjusting itself to preferred modes of oscillations. Total instantaneous output is probably a summation or superimposition of many cycles of output. Earth intercepts this seemingly noisy output and climatically resonates at its preferred frequencies.

\section{ACKNOWLEDGMENTS}

I thank G. Cobb for the inspiration for this paper, J.A. Eddy for his critical comments and encouragement on the early draft of the manuscript, and the publication section of the U.S. Geological Survey, which provided support for this independent research.

\section{REFERENCES}

1. Hudson, H.S., "Solar variability and oscillations," Rev. Geophys., 25, 651-662, 1987.

2. Newman, M.J., and R.T., Rood, "Implications of solar evolution for the Earth's early history," Science, 198, 1035-1037, 1977.

3. Ezer, D., and A.G.W. Cameron, "Effects of sudden mixing in the solar core on solar neutrinos and Ice Ages," Nature Physical Sci., 240, 178-182, 1972.

4. Dilke, F.W.W., and D.O. Gough, "The solar spoon," Nature, 240, 262-292, 1972.

5. Gleissberg, W., "The eighty-year cycle in auroral frequency numbers," British Astron. Assoc. Jour., 75, 227-231, 1965.

6. Russell, H.N., R.S. Dugan, and J.Q. Stewart, Astronomy, 205-207, 1926.

7. Kiepenheuer, K., The Sun, University of Michigan Press, Ann Arbor, Michigan, 1959.

8. Dicke, R.H., "Is there a chronometer hidden deep in the Sun?," Nature, 276, 676-680, 1978.

9. Krimigis, S.M., "The Sun and the Sun-Earth connection," Jour. of the British Interplanetary Soc., 41, 63-80, 1988.

10. Gilliland, R.L., "Solar radius variations over the past 265 years," Astrophys. Jour., 248, 1144$1155,1981$.

11. Parkinson, J.H., "New measurements of the solar diameter," Nature, 304, 518-520, 1983. 
12. Kopysov, Y.S., Preprint P-0317, Institute of Nuclear Research, USSR Academy of Science, 1983.

13. Rowley, J.K., B.T. Cleveland, and R. Davis, Jr., Solar Neutrino Astronomy, M.L. Cherry, W.A. Fowler, and K. Lande, eds., Conference Proceeding No. 126, American Institute of Physics, New York, 1-21, 1985.

14. Raychaudhuri, P., "Solar neutrino data and the 11-year solar-activity cycle," Solar Physics, 93, $397-401,1984$.

15. Lerner, Z., and K. O'Brien, "Atmospheric radioactivity and variations in the solar neutrino flux," Nature, 330, 353-354, 1987.

16. Rowley, J.K., B.T. Cleveland, R. Davis, Jr., W. Hampel, and T. Kirsten, "The present and past neutrino luminosity of the Sun," in Proceedings of the Conference on the Ancient Sun, R.O. Pepin, J.A. Eddy, and R.B. Merrill, eds., Pergamon Press, New York, 45-62, 1980.

17. Parker, P.D., "Thermonuclear reactions in the solar interior," in Physics of the Sun. Volume 1, P.A. Sturrock, ed., D. Reidel Publ. Co., Dordrecht, 15-32, 1986.

18. Paudler, W.W., Nuclear Magnetic Resonance, John Wiley and Sons, New York, 2-13, 1987.

19. Weast, R.C., CRC Handbook of Chemistry and Physics, 62nd ed., CRC Press, Boca Raton, E-67, 1981.

20. Cerny, Joseph, ed., Nuclear Physics, National Acadamy Press, Washington, D.C., 152, 1986.

21. Jacoby, G.C., "Long-term temperature trends and a positive departure from the climate-growth response since the 1950's in high elevation lodgepole pine from California," in ClimateVegetation Interactions, C. Rosenzweig and R. Dickinson eds., University Corporation for Atmospheric Research, Boulder, Colorado, 81-83, 1986.

22. Woodward, M.F., and R.W. Noyes, "Change of solar oscillation eigenfrequencies with the solar cycle," Nature, 318, 449-450, 1985.

23. Roberts, B., and W.R. Campbell, "Magnetic field corrections to solar oscillation frequencies," Nature, 323, 603-605, 1986.

24. Rust, D.M., "The solar constant," in The Solar Wind and the Earth, S.I. Akasofa and Y. Kamide, eds., Terra Scientific Publ. Co., 1987. 
TRACKING ATMOSPHERIC TRACE GASES BY SPECTROSCOPY FROM KITT PEAK

\author{
Lloyd Wallace \\ Kitt Peak National Observatory \\ William Livingston \\ National Solar Observatory \\ Box 26732 \\ Tucson, AZ 85726
}

We have analyzed solar spectra obtained with the 1-m FTS for absorption by $\mathrm{CO}_{2}, \mathrm{CH}_{4}, \mathrm{~N}_{2}, \mathrm{CO}$, $\mathrm{HF}$, and $\mathrm{HCl}$. During the period 1979-1985, we find that the $\mathrm{CO}_{2}$ total column amount was increasing $0.4 \%$ /year, $\mathrm{CH}_{4}$ was up $1.1 \%$ /year, while $\mathrm{N}_{2} \mathrm{O}$ shows no detectable trend. $\mathrm{CO}$ undergoes a large seasonal variation that masks any long-term trend within our observational time span. Stratospheric HF doubles in strength, displaying a conspicuous single-point increase that coincided with the "mystery" volcanic event of that era. We are now attempting to resume the observations as resources permit. 

APPENDIX A 



\section{Appendix A \\ List of Attendees}

Dr. Richard C. Altrock

Solar Research Branch, AFGL

Sacramento Peak Observatory

Sunspot, NM 88349

Dr. Stephen Arendt

Laboratory for Astrophysics \& Space Research University of Chicago, 933 E. 56th Street

Chicago, IL 60637

Dr. Albert Arking

Climate/Radiation Branch, Code 913

NASA/Goddard Space Flight Center

Greenbelt, MD 20771

Joan Backer

Atmospheric Environmental Services

Bedford Towers, 5th Floor

Nova Scotia B4A 1ES

CANADA

Dr. Sallie Baliunas

Center for Astrophysics

60 Garden Street, Mail Stop 15

Cambridge, MA 02138

Dr. Tony Barnston

NOAA

W/NMC51, WWB, Room 604

Washington, DC 20233

Dr. B. J. Beardsley

Photometrics Limited

Tuscon, AZ 85721
Y. P. Borsienkov

USSR
Mr. S. Dave Bouwer

CIRES, UOC, NOAA

Mail Code R/E/SE, 325 Broadway

Boulder, CO 80303

Guenter Brueckner

Naval Research Laboratory

4555 Overlook Ave., SW

Washington, DC 20375

Dr. R. P. Cebula

ST Systems Corporation

4400 Forbes Boulevard

Lanham, MD 20706

Dr. Gary Chapman

CSU/Dept. of Physics \& Astron.

18111 Nordhoff Street

Northridge, CA 91330

Dr. Hong-Yee Chiu

NASA/Goddard Space Flight Center Code 910.2

Greenbelt, MD 20771

James W. Closs

Code 934

NASA/Goddard Space Flight Center Greenbelt, MD 20771 
Dr. Thomas J. Crowley

ARC Technologies

305 Arguello Drive

College Station, TX 77840

Matthew T. Deland

Code 916

NASA/Goddard Space Flight Center

Greenbelt, MD 20771

Dr. Pierre Demarque

Center for Solar \& Space Research

Yale University/PO Box 6666

New Haven, CT 06511

Dr. Richard F. Donnelly

CIRES, UOC, NOAA

R/E/SE, 325 Broadway

Boulder, CO 80303

Maurice Dubin

Code 916

NASA/Goddard Space Flight Center

Greenbelt, MD 20771

Dr. David Dunham

Computer Science Corporation

8728 Colesville Road

Silver Spring, MD 20910

Dr. Paul Dussenberg

National Science Foundation

Washington, DC 20550
Dr. Igor J. Eberstein

Code 916

NASA/Goddard Space Flight Center

Greenbelt, MD 20771

Dr. Jack Eddy

UCAR

Office for Interdisciplinary Earth Sciences Boulder, CO 90307

Dr. Peter Foukal

Cambridge Research Instrument, Inc.

21 Erie Street

Cambridge, MA 02139

Mr. Peter A. Fox

Center for Solar and Space Research

Yale/P.O. Box 6666

New Haven, CT 06511

Dr. Claus Frohlich

PMOD/World Radiation Center

CH-7260 Davos Dorf

Postfach 173, Dorfstrasse 33

SWITZERLAND

Dr. Marvin A. Geller

Director, Institute for Atmospheric Science State University of New York

Stony Brook, New York 11794-3800

Dr. Richard Goldberg

National Science Foundation

1800 G Street, NW

Washington, DC 20550 
Mr. Peter Grimm

8859 Thomas Lee Terrace

Gaithersburg, MD 20879

Dr. Bruce Guenther

NASA/Goddard Space Flight Center Code 973

Greenbelt, MD 20771

Dr. James E. Hansen

Code 980 , GISS

2880 Broadway

New York, NY 10025

Dr. Donald F. Heath

ST Systems Corporation

4400 Forbes Boulevard

Lanham, MD 20706

Dr. Alan E. Hedin

NASA/Goddard Space Flight Center

Code 914

Greenbelt, MD 20771

Dr. A. D. Herzog

CSU/Dept. of Physics \& Astron.

18111 Nordhoff Street

Northridge, CA 91330

Prof. H. A. Hill

Faculty of Science, Department of Physics Building \#81, The University of Arizona Tucson, AZ 85721
Dr. Ernest Hilsenrath

NASA/Goddard Space Flight Center Code 916

Greenbelt, MD 20771

Dr. Walter R. Hoegy

Code 914

NASA/Goddard Space Flight Center

Greenbelt, MD 20771

Dr. L. L. Hood

Lunar and Planetary Laboratory

The University of Arizona

Tucson, AZ 85721

Richard Horowitz

Code 933

NASA/Goddard Space Flight Center

Greenbelt, MD 20771

Dr. Matthew K. Howard

ARC Technologies

305 Arguello Drive

College Station, TX 77840

Dr. Douglas V. Hoyt

Research and Data Systems Corporation 7855 Walker Drive, Suite 460

Greenbelt, MD 20770

Dr. Hugh Hudson

Center for Astrophysics and Space Sciences

C-011, University of California

San Diego, CA 92093 
Dr. Robert D. Hudson

Code 916

NASA/Goddard Space Flight Center

Greenbelt, MD 20771

Donald Hunt

National Research Council

2001 Wisconsin Avenue, NW

Washington, DC 20418

Dr. Robert Jastrow

Department of Earth Science

Dartmouth College

Hanover, NH 03755

Dr. J. L. Jirikowic

Lunar and Planetary Laboratory

The University of Arizona

Tucson, AZ 85721

Dr. Robert S. Kandel

Lab. de Météorologie Dynamique/CNRS

Ecole Polytechnique

F-91128 Palaiseau Cedex

FRANCE

Dr. Richard Kerr

Science Magazine

1515 Massachusetts Avenue, NW

Washington, DC 20005

Dr. Joseph H. King

Code 933

NASA/Goddard Space Flight Center

Greenbelt, MD 20771
Dr. David Kratz

Code 913

NASA/Goddard Space Flight Center

Greenbelt, MD 20771

Mr. Ronald Kroll

Faculty of Science, Department of Physics Building \#81, The University of Arizona

Tucson, AZ 85721

Dr. H. Lee Kyle

Code 936

NASA/Goadard Space Flight Center Greenbelt, MD 20771

Dr. Andrew A. Lacis

Code 980 , GISS

2880 Broadway

New York, NY 10025

Mr. Theodor Landscheidt

Schroeter Institute for Research

in Cycles of Solar Activity

Belle Cote, Nova Scotia BOE 1CO

CANADA

Dr. Jurate Landwehr

USGS/Mail Stop 431

National Center

Reston, VA 22092

Dr. J. K. Lawrence

CSU/Dept. of Physics \& Astron.

18111 Nordhoff Street

Northridge, CA 91330 
Dr. Judith Lean

Code 4165

Naval Research Laboratory

Washington, DC 20375

Mr. Robert B. Lee , III

Mail Stop 420

NASA/Langley Research Center

Hampton, VA 23665-5225

Dr. Robert E. Livezey

NOAA

W/NMC51, WWB, Room 604

Washington, DC 20233

Dr. William Livingston

Kitt Peak National Observatory

PO Box 26732

Tucson, AZ 85726

Dr. G. Wesley Lockwood

Lowell Observatory

1400 W. Mars Hill Road

Flagstaff, AZ 86001

Prof. Julius London

APAS

University of Colorado

Boulder, CO 80309

Thomas Lydon

Center for Solar \& Space Research

Yale University/P.O. Box 6666

New Haven, CT 06511
Dr. Hans G. Mayr

Code 914

NASA/Goddard Space Flight Center

Greenbelt, MD 20771

Mr. Andrew McIntyre

Lamont-Doherty Geological Observatory

Columbia University

Palisades , NY 10964

Mr. John A. McKinnon

National Geophysical Data Center

325 Broadway

Boulder, CO 80303

Dr. Ann T. Mecherikunnel

Code 971

NASA/Goddard Space Flight Center

Greenbelt, MD 20771

Dr. V. M. Mehta

Climate and Radiation Branch, Code 913

NASA/Goddard Space Flight Center

Greenbelt, MD 20771

Dr. Shrinivas Moorthi

Code 911

NASA/Goddard Space Flight Center

Greenbelt, MD 20771

Prof. Gerald R. North

Department of Meteorology

Texas A \& M University

College Station, TX 77843 
Lola M. Olsen

Code 934

NASA/Goddard Space Flight Center

Greenbelt, MD 20771

Dr. Kazem Omidvar

Code 914

NASA/Goddard Space Flight Center

Greenbelt, MD 20771

Dr. Jerome A. Orosz

Dept. of Physics and Astronomy

Ohio University

Athens, $\mathrm{OH} 45701$

Dr. Ludwig Oster

NSF/Division of Astronomical Science 1800 G Street, NW

Washington, DC 20550

Dr. Judith Pap

CIRES, UOC, NOAA

Mail Code R/E/SE

Boulder, CO 80303

Mr. Dennis Peacock

National Science Foundation

1800 G Street, NW

Washington, DC 20550

Dr. Charles Perry

USGS

4821 Quail Crest Place

Lawrence, KS 66049
Dr. Bob Rabin

Room 211

1225 W. Dayton

Madison, WI 53706

Dr. Richard R. Radick

Solar Research Branch

Sacramento Peak Observatory, P.O. Box 62

Sunspot, NM 88349

Dr. George Reid

Aeronomy Laboratory/Mail Code R44

NOAA/325 Broadway

Boulder, $\mathrm{CO} 80303$

Dr. Garry Rottman

LASP/Campus Box 392

University of Colorado

Boulder, CO 80309

Dr. Reto A. Ruedy

Code 980, GISS

2880 Broadway

New York, NY 10025

Mr. Herbert H. Sauer

NOAA Space Environment Laboratory

Boulder, CO 80303

Dr. Bradley E. Schaefer

Code 661

NASA/Goddard Space Flight Center

Greenbelt, MD 20771 
Dr. Kenneth H. Schatten

Code 910.1

NASA/Goddard Space Flight Center

Greenbelt, MD 20771

Dr. Robert A. Schiffer

Code EET

NASA Headquarters

Washington, DC 20546

Dr. Barry M. Schlesinger

Science Applications Research

4400 Forbes Boulevard

Lanham, MD 20706

Dr. Fred Singer

Department of Environmental Sciences

University of Virginia

Charlottesville, VA 22903

Dr. B. A. Skiff

Lowell Observatory

1400 W. Mars Hill Road

Flagstaff, AZ 86001

Dr. Sabatino Sofia

Center for Solar \& Space Research

Yale University/Box 6666

New Haven, CT 06511

Dr. Charles P. Sonett

Department of Planetary Sciences

University of Arizona

Tucson, AZ 85721
Mr. Bruce Springer

ASGWC/DOP

Offutt AFB, NE 68113

Dr. Yogesh C. Sud

Code 911

NASA/Goddard Space Flight Center

Greenbelt, MD 20771

Harry A. Taylor, Jr.

RR\#1 Box 2495

Beaver River, Nova Scotia B5A-4A5

CANADA

Dr. Brian Tinsley

FO22, University of Texas at Dallas

Box 830688

Richardson, TX 75083-0688

A. V. Tsvetkov

USSR

Dr. Sastri Vemury, Ph.D.

SMART

9 Hutchinson Court

Silver Spring, MD 20906

Dr. Lloyd Wallace

Kitt Peak National Observatory

P.O. Box 26732

Tucson, AZ 85726 
Dr. S. R. Walton

CSU/Dept. of Physics \& Astron.

18111 Nordhoff Street

Northridge, CA 91330

Dr. Oran R. White, Ph.D.

Solar Astronomer

7590 Road 39

Mancos, CO 81328

Dr. Richard Willson

Jet Propulsion Laboratory

4800 Oak Grove Avenue, Mail Stop 171-400

Pasadena, CA 91103

Dr. C. L. Wolff

Code 910.1

NASA/Goddard Space Flight Center

Greenbelt, MD 20771

Chung-Yu Wu

Code

NASA/Goddard Space Flight Center

Greenbelt, MD 20771

Prof. Kuor-Jier Joseph Yip

Department of Meteorology

Texas A \& M University

College Station, TX 77843

Mr. Jack Zirker

NSO

NOAO

Sunspot, NM 88349 



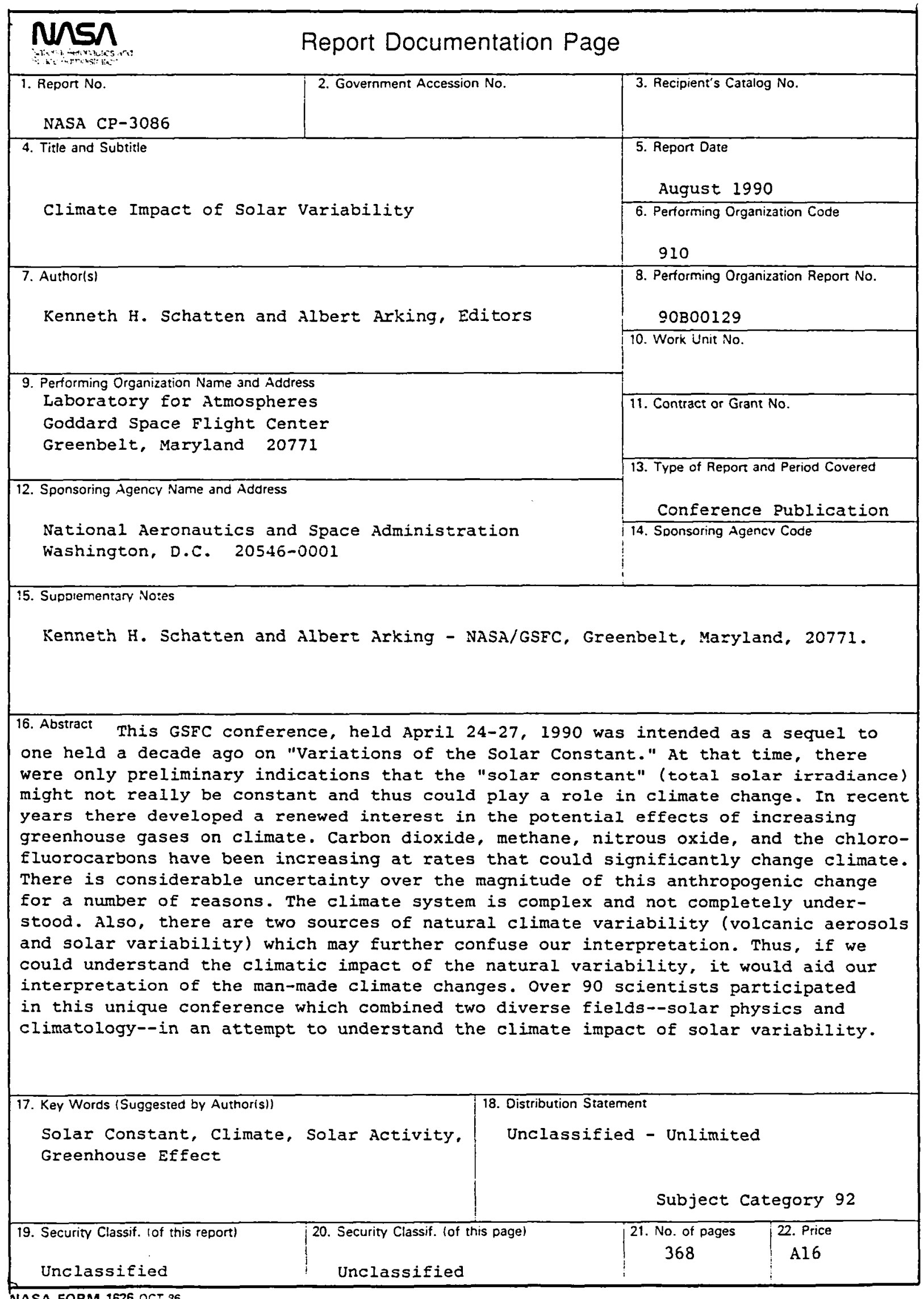



National Aeronautics and Space Administration

Code NTT-4

Washington, D.C.

20546-0001

SPECIAL FOURTH-CLASS RATE

POSTAGE \& FEES PAID

NASA

Permit No. G-27

Otficial Business

Penalty lor Private Use, $\$ 300$ 